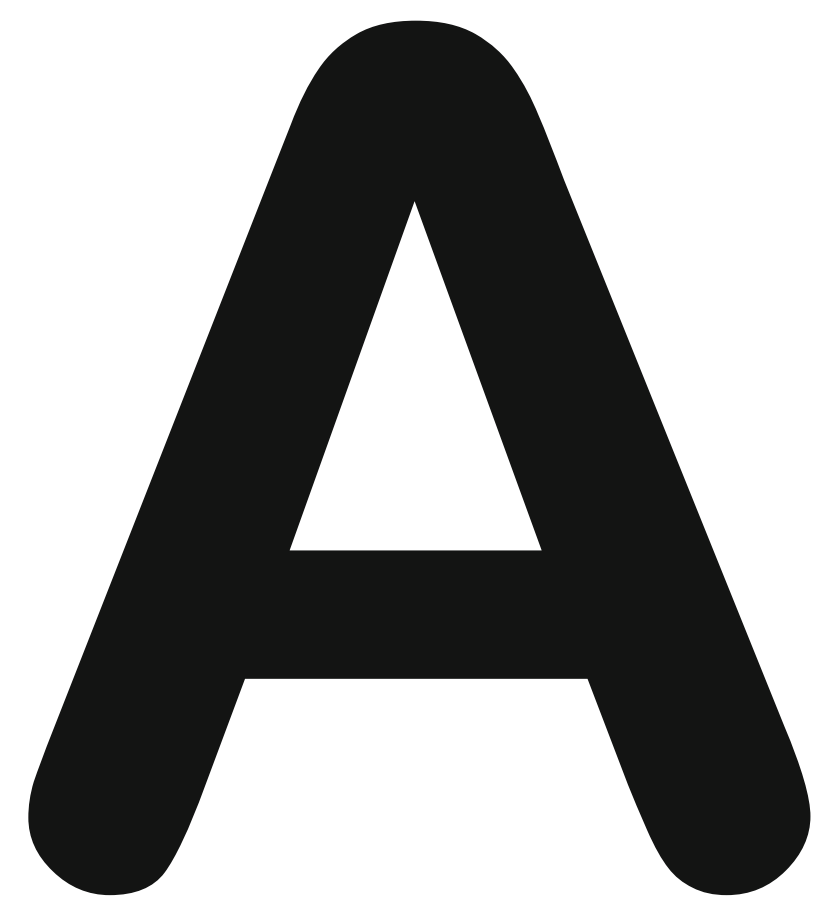

\title{
Postgraduate Educational Programme
}

\author{
Categorical Courses (CC) \\ EFOMP Workshop (EF) \\ ESR meets Sessions (EM) \\ European Excellence in Education $\left(E^{3}\right)$ \\ Honorary Lectures $(\mathrm{HL})$ \\ Mini Courses (MC) \\ Multidisciplinary Sessions (MS) \\ New Horizons Sessions $(\mathrm{NH})$ \\ Opening Lecture $(\mathrm{OL})$ \\ Professional Challenges Sessions (PC) \\ Refresher Courses (RC) \\ RTF - Radiology Trainees Forum (TF) \\ Special Focus Sessions (SF) \\ State of the Art Symposia (SA)
}

Thursday, March 6 .................2

Friday, March 7 ................... 15

Saturday, March 8................ 46

Sunday, March 9 .................. 75

Monday, March 10.............. 113 
Thursday, March 6 
$12: 30-13: 30$

Room E2

The Beauty of Basic Knowledge: Skeletal Radiology

MC 26 A

\section{Plain radiographs: analysis and interpretation}

Moderator:

V.N. Cassar-Pullicino; Oswestry/UK

A-001 12:30

Plain radiographs: analysis and interpretation

I. McCall; Devon/UK (mccalli832@btinternet.com)

The radiograph remains a quick and inexpensive imaging modality. Factors to evaluate include the thickness and outlines of cortical bone, the density and pattern of trabecular bone and the presence or absence of periosteal new bone. The position of lesions within the bone, their outline, size and margin and whether they are single or multiple also provide important diagnostic information. Adjacent soft tissues are affected by many conditions, particularly trauma and infection and are integral to image interpretation. While MRI is essential for internal joint structures, radiographs demonstrate subchondral bones, alignment, erosions and surrounding soft tissues. Knowledge of normal radiographic anatomy and variants is essential. In trauma, subtle changes in bone alignment, discontinuity of bone outline or alteration in density of trabecular bone may indicate injury. Generalised increased or decreased bone density indicates imbalance in bone creation or loss due to systemic diseases and may be difficult to recognise at an early stage, but is recognisable when localised in comparison to the unaffected bone. Localised reduced density may result from hyperaemia, disuse or from replacement by cysts and sclerotic reaction by trauma and infection, while primary and secondary bone tumours may result in either response. Other features including cortical expansion or scalloping and marginal bone response internal calcification will refine the diagnosis. Periosteal new bone in differing patterns results from local insult or more generalised metabolic and haemodynamic changes, while soft tissue swelling, muscle and ligament outline alterations and presence of calcification provide vital clues to disease recognition and diagnosis.

Learning Objectives:

1. To learn about the current role of plain radiographs.

2. To appreciate the strengths and weaknesses of plain radiographs in musculoskeletal disease.

3. To understand methods for analysing radiographic abnormalities and an approach to their correct interpretation.

12:30 - 13:30 Room F1

The Beauty of Basic Knowledge: Interventional Radiology

MC 25 A

\section{Needles and samples: a practical} approach to image-guided biopsy

Moderator:

M. Bezzi; Rome/IT

\section{A-002 12:30}

Needles and samples: a practical approach to image-guided biopsy M. Bezzi; Rome/IT (mario.bezzi@uniroma1.it)

Percutaneous needle biopsy has been a mainstay of oncologic diagnosis for almost three decades, since the advent of ultrasound and CT. The basic principles of fine-needle aspiration and core needle biopsy can be applied to almost any site in the body, with subtle differences in technique depending on the organ being investigated and the imaging modality utilised. While excisional biopsy is still appropriate in certain cases, percutaneous needle biopsy has become the standard of care in the diagnosis of most tumors throughout the body and is also used to diagnose noncancerous conditions, such as infection. Percutaneous biopsy is also beneficial in the staging of patients with cancer, particularly when another treatment method may be more appropriate than surgical resection. The advantages of percutaneous biopsy over surgical excisional biopsy include time and cost savings and reduction in morbidity. The aim of this course is to discuss the practical aspects of biopsy, needle selection, and guidance techniques and to show how to approach difficult lesions and avoid complications.
Learning Objectives:

1. To understand the indications for percutaneous image-guided biopsy.

2. To learn about the practical aspects of biopsy, needle selection and guidance techniques.

3. To learn how to approach difficult lesions and avoid complications.

14:00 - 15:30 Room C

Interactive Teaching Session

\section{$E^{3} 220$}

\section{The treated breast: what you need to know}

\author{
A-003 14:00 \\ A. Imaging after treatment of breast cancer \\ M.H. Fuchsjäger; Graz/AT (michael.fuchsjaeger@medunigraz.at)
}

Imaging after treatment of breast cancer is for confirmation of lesion removal, identification of postprocedural fluid collections, detection of residual or recurrent cancer and screening for metachronous cancers. Posttherapy changes - which include fluid collections, edema, skin thickening, architectural distortion, scarring and calcifications - are mainly due to surgery, axillary dissection and radiotherapy. The greatest treatment-related changes occur 612 months after therapy, and mammographic stability is achieved after two to three years. For mammography, pre- and all posttherapeutic images have to be compared. Ultrasound is the method of choice for evaluation of fluid collections. MRI is for problem solving (i.e. differentiation between scar and relapse) and should not be performed prior to 12 months after therapy to avoid false-positive diagnoses. Dystrophic calcifications may develop in areas of fat necrosis mimicking malignancy. Fat necrosis predominantly occurs at the treated site; however, it can develop anywhere in the ipsilateral breast. Its appearance may be indistinguishable from cancer at all imaging modalities. To differentiate between fat necrosis and other common post-treatment changes from relapse, it is important to know the timeline when these changes occur and schedule follow-up imaging accordingly. Mammography serves as the basis for postoperative surveillance. Ultrasound is helpful in the early postoperative phase, whereas MRI is the method of choice, especially for differentiation of scar and relapse in the later postoperative phases.

Learning Objectives:

1. To understand common features related to breast surgery.

2. To recognise changes related to non-surgical treatments.

\section{A-004 14:45}

B. Imaging after treatment of benign breast conditions

J. Camps Herrero; Valencia/ES (juliacamps@gmail.com)

Breast radiologists need to be familiar with post-treatment imaging findings in patients with breast cancer, but often patients are also imaged after a diagnosis of benign entities which are treated surgically (fibroadenomas, radial scars, papillary lesions) or after a percutaneous diagnosis of a high-risk lesion which has undergone a surgical biopsy to avoid underestimation (atypical ductal hyperplasia, lobular neoplasia or flat epithelial atypia). These procedures will leave an imprint on the breast which can be a cause of concern due to the surgical scar. Furthermore, imaging findings after plastic surgery for mastopexy, reduction mastoplasties, implants or auxiliary techniques (lipofilling, hyaluronic acid) are becoming frequent in our daily practice and have their peculiarities that can overshadow breast cancers. Interventional percutaneous procedures can also be a cause of tissue distortion. There are advanced systems for biopsy (BLES) and for percutaneous removal of benign lesions (fibroadenomas, papillomas), which use large-gauge needles and have to be accounted for due to their trace in the breast tissue.

Learning Objectives:

1. To understand common features related to breast surgery.

2. To recognise changes related to non-surgical treatments.

Author Disclosure:

J. Camps Herrero: Advisory Board; Bayer. Speaker; Bayer. 
16:00 - 17:30

Room B

\section{GI Tract}

\section{RC 301}

\section{Cross-sectional imaging of colitis}

\section{A-005 16:00}

Chairman's introduction

S.A. Taylor; London/UK (csytaylor@yahoo.co.uk)

Cross-sectional imaging is playing an increasing role in the diagnosis, staging and follow-up of colitis. Often, the radiologist diagnoses colitis on crosssectional imaging, and the clinician requires the most likely diagnosis. Alternatively, imaging is used to assess the severity of a known colitis and monitor treatment response. This session will describe state-of-the-art protocols for assessing the inflamed colon using CT, MRI and USS. The common aetiologies of colitis will then be discussed and tips and tricks as to how to limit the differential diagnosis presented. Finally, the role of crosssectional imaging in the management of inflammatory bowel disease (IBD) will be described with emphasis on disease activity. An integrated approach to imaging patients with colonic IBD will be presented.

Author Disclosure:

S.A. Taylor: Grant Recipient; NIHR HTA. Other; Study reader for Robarts.

\section{A-006 16.05}

\section{A. Which protocol to use?}

S. Schmidt; Lausanne/CH (sabine.schmidt@chuv.ch)

The role of cross-sectional imaging in the diagnosis of colitis is increasing following recent technologic advances. Prior colonic cleansing is usually not required, unlike in CTC. However, careful attention to the examination technique is essential, since collapsed colon may suggest colonic wall thickening, thus mimicking colitis. When using CT or MRI, adequate luminal distension is necessary, while this is optional in US where direct compression of the bowel aids diagnosis. Recommended imaging protocols will be discussed. CT remains the workhorse for imaging suspected colitis, especially in emergency settings. It is widely available and facilitates rapid data acquisition with excellent spatial resolution. Intravenous contrast medium administration can reveal the typical target sign of colitis, which can easily be differentiated from other wall patterns, such as diffuse enhancement seen in cancer. MRI does not expose patients to radiation and provides excellent tissue contrast facilitating the detailed analysis of the inflamed colonic wall. Both conventional and diffusion-weighted sequences provide functional noninvasive assessment of the severity of colitis and monitoring of treatment responses, essential in Crohn patients. Colonic US is safe, well tolerated and in general, freely available, although it requires specific operator expertise. It simultaneously provides morphological and functional information. Absent peristalsis and wall thickening in acute colitis usually reduce intraluminal air, thus facilitating colonic assessment compared to healthy subjects. Akin to CT and MRI, the three colonic wall layers are differentiated and pericolonic findings can be assessed. However, US can be limited by the patient's body habit and the radiologist's practical experience.

Learning Objectives:

1. To become familiar with the role of colonic distension prior to imaging of suspected or known colitis.

2. To understand typical CT, MRI and US protocols.

3. To learn about the pros and cons of CT, MRI and US.

\section{A-007 16:28}

B. Differentiating between the causes of colitis

B. Gallix; Montreal, QC/CA (b-gallix@chu-montpellier.fr)

The final diagnosis in determining the type of colitis is based on clinical and laboratory data and colonoscopic and biopsy findings. However, information provided by endoscopy is limited to the mucosal surface. Cross-sectional imaging techniques, such as ultrasound, computed tomography, and magnetic resonance, are widely used to assess patients with non-specific abdominal pain and are gaining acceptance for the assessment of patients with suspected acute or chronic colitis. Cross-sectional studies are useful, alternative tools not only in the assessment of bowel wall abnormalities, but also for the assessment of extraluminal alterations. Differentiating between the causes of colitis is based on the location of involvement, extent and appearance of colonic wall thickening, extraluminal changes, and type of complications encountered. When chronic inflammatory colitis is suspected, imaging helps to distinguish ulcerative colitis from Crohn's colitis. Infectious types of colitis share the features of wall thickening, pericolonic stranding, and various degrees of ascites according to the severity of the disease. Pseudomembranous colitisdemonstrates marked wall thickening and, occasionally, skips areas, but is associated with broad-spectrum antibiotic treatment or chemotherapy. Neutropeniccolitis is characterised by right-sided colonic and ileal involvement, whereas ischaemic colitis is characterised by a vascular distribution pattern and history. Diverticulitis is a focal, asymmetric process with fascial thickening and inflamed diverticula. The diagnosis of ischaemic colitis is suspected when the distribution of the colon wall involvement follows the vasculature. The ischaemic changes are commonly segmental, especially in the left colon in elderly patients.

Learning Objectives:

1. To learn about the most common cause of colitis through cross-sectional imaging in both immune-competent and immune-compromised patients. 2. To become familiar with differentiating infectious, inflammatory, ischaemic and autoimmune conditions based on cross-sectional imaging criteria. 3. To understand the limitations of cross-sectional imaging in differentiating between its causes.

\section{A-008 16:51}

C. The role of cross-sectional imaging in colonic inflammatory bowel disease

J. Rimola; Barcelona/ES (jrimola@clinic.ub.es)

Endoscopy is currently considered the reference standard for the evaluation of colonic disease activity in patients with inflammatory bowel disease (IBD). However, it only allows evaluation of the mucosal surface and is not always complete. It cannot, therefore, help to estimate the depth of involvement of transmural inflammation and extraluminal complications, both characteristics of IBD. An evolving role for cross-sectional imaging in the evaluation of patients with IBD is increasingly recognised, especially in the setting of Crohn's disease (CD) since the cross-sectional imaging has been demonstrated to have a high diagnostic accuracy not only for assessing the presence and extension of luminal disease, but also for evaluating the CD-related acute or chronic complications. Available evidence suggests that ultrasound, computed tomography and magnetic resonance have similarly high diagnostic accuracy in the detection of disease activity, location, severity and complications: in particular, the penetrating and stricturing lesions which are characteristic of $\mathrm{CD}$. Thus, the choice of the technique for assessing $C D$ may be influenced by local availability or expertise. In the case of ulcerative colitis, cross-sectional imaging, although less evaluated, may also be helpful in certain circumstances. There is evidence indicating that cross-sectional imaging is an alternative problem-solving tool to endoscopy whenever tissue sampling is not required and can provide valuable guidance for performing medical and surgical treatment with maximised efficacy and safety. Overall, findings from crosssectional imaging accurately reflect disease activity and provide reliable information for decision-making and patient care optimisation.

Learning Objectives:

1. To learn about optimised examination protocols for ulcerative colitis and colonic Crohn's disease in the acute, subacute and chronic disease setting. 2. To become familiar with the criteria for the assessment of disease activity through CT, MRI and ultrasound.

3. To learn about an integrated approach to the use of cross-sectional imaging in colonic inflammatory bowel disease.

\section{Panel discussion:}

The role of cross-sectional imaging in the diagnosis and follow-up of colitis 17:14

\begin{tabular}{ll}
\hline $16: 00-17: 30$ & Room C
\end{tabular}

Interactive Teaching Session

$E^{3} 320$

\section{The treated liver}

A-009 16:00

A. Imaging of liver transplantation

J.B. Karani; London/UK (johnbkarani@hotmail.com)

Liver transplantation is the accepted treatment of patients with irreversible liver cell failure and some metabolic disorders and in a selected group of patients with hepatocellular carcinomas. Over the last decade, major transplant centres have reported improving survival rates, though during this period they have developed more complex surgical techniques, including split-liver, auxiliary and live-related transplantation, and have treated more marginal higher risk patients. This successful outcome has been dependent on appropriate recipient selection, robust surgical technique, improvements in immunosuppression and intensive care management and the prompt recognition and treatment of complications. Diagnostic and interventional radiology have been core specialties in achieving the goals of improved graft and patient survival. Improvement in surgical techniques has decreased the 
more common vascular and biliary complications, but the newer techniques present differing diagnostic and interventional challenges, particularly in paediatric recipients. Developments in MR and MDCT allow many of these vascular, biliary and infective complications to be diagnosed non-invasively. Vascular techniques of angioplasty and stent placement may reverse the sequel of graft ischaemia or portal hypertension. MRC allows the diagnosis of biliary strictures that may be treated by dilatation or stent placement. Imaging is also important in the diagnosis of recurrent disease and the acquired diseases of prolonged immunosuppression including atypical infections and the post-transplant lymphoproliferative disorders (PTLD). This interactive session will present these appearances by case example and provide guidance of the appropriate diagnostic and treatment paradigm.

Learning Objectives:

1. To understand the common imaging findings after liver transplantation.

2. To recognise significant complications following liver transplantation.

\section{A-010 16:45}

\section{B. Imaging of treated liver tumours}

I. Bargellini; Pisa/IT (irenebargellini@hotmail.com)

Imaging findings after systemic and loco-regional treatments vary greatly depending on baseline features of the treated tumour and treatment modality. Conventional uni-dimensional and bi-dimensional criteria (such as RECIST 1.1 and WHO criteria) have been extensively validated in metastatic lesions treated with conventional chemotherapy. However, their prognostic value is limited in patients treated with new molecular targeted therapies and after locoregional treatments. In the setting of hepatocellular carcinoma (HCC), specific response criteria (such as EASL and mRECIST) have been proposed that take into account variation in the size of the viable tumour. These criteria have been extensively validated, although their interpretation could be troublesome after some specific treatments, such as molecular targeted agents and Y90radioembolisation. On the other hand, there is no consensus regarding tumour response criteria for metastatic lesions after loco-regional treatments. The role of morphological criteria and several imaging biomarkers (such as those provided by diffusion and perfusion imaging, FDG activity, dual source CT) is under investigation, being able to provide additional information on tumour activity and biology. While new drugs with different mechanisms of action and new treatments are becoming available, the work of radiologists is changing and there is increasing evidence that tailored radiological response criteria are required for these new targeted and tailored treatments.

Learning Objectives:

1. To understand the common imaging findings after chemotherapy for liver tumours.

2. To recognise common imaging findings after radiofrequency ablation of liver tumours.

3. To be aware of the common imaging findings following transarterial treatment of liver tumours.

\begin{tabular}{ll}
\hline $16: 00-17: 30$ & Room D
\end{tabular}

\section{Chest}

\section{RC 304}

\section{Pulmonary vasculitis and systemic diseases \\ Moderator: \\ A. $\underline{\text { Persson; }}$ Linköping/SE}

\section{A-011 16:00}

A. Pulmonary manifestations of systemic diseases

C. Engelke; Göttingen/DE

"No abstract submitted"

\section{Learning Objectives:}

1. To learn about the different systemic diseases that affect the lung.

2. To become familiar with pulmonary abnormalities due to systemic disease.

A-012 16:22

B. Large-vessel vasculitis

J. Vilar; Valencia/ES (vilarsamper@gmail.com)

Pulmonary vasculitides are a challenge for radiologists for two reasons: vasculitides are rare diseases and at the same time the pathologic and radiological manifestations are very diverse. Primary vasculitides present with different clinical scenarios and often confusing situations that mimic other diseases. By definition, vasculitides are disorders affecting the blood vessels that may be primary or secondary. Classification of vasculitis according to the vessel size is practical, since it is related to the pathological changes as well as to the clinical and radiological presentation. The two main large vesse vasculitides are giant cell arteritis and Takayasu arteritis. Behcet disease may combine large and small vessel vasculitis. Imaging plays an important role in primary vasculitis. Chest radiographs are not especially useful in large vessel vasculitis. Contrast CT and MRI further detect and especially help in the characterisation of large vessel vasculitis. Vessel wall changes are well detected with both techniques. Contrast enhancement, distribution of vessel involvement and morphological vascular changes should be considered. Today, PET/CT is the recommended imaging technique in the assessment of vessel wall inflammatory changes and in the evaluation of treatment response. Although the clinical scenario differs usually between Takayasu and giant cel arteritis, the radiologist should combine the imaging findings with clinical and laboratory data to suspect a specific vasculitis. Therefore, this presentation will concentrate on the basic signs and associated findings in large vessel vasculitis, pathologic correlation, imaging protocols and the differential diagnosis.

Learning Objectives:

1. To learn about the different types of large-vessel vasculitis.

2. To become familiar with histopathological correlates in vasculitis.

3. To appreciate the different manifestations and imaging appearances of large-vessel thoracic vasculitis.

A-013 16:44

C. HRCT patterns in pulmonary vasculitis

C.M. Schaefer-Prokop; Amersfoort/NL (cornelia.schaeferprokop@gmail.com)

The term vasculitis refers to a variety of clinico-pathological entities. The most widely used Chapel-Hill classification divides the vasculitis syndromes into three groups based on the size of vessels primarily involved. Small vessel vasculitis such as ANCA- associated granulomatous vasculitis (Wegener's disease), Churg-Strauss granulomatosis and microscopic polyangitis are most often associated with pulmonary abnormalities. The spectrum of HRCT findings is quite variable and differs by entity. The HRCT findings will be discussed together with clinical and laboratory findings to be integrated into a multidisciplinary diagnostic approach. The typical findings in Wegener's granulomatosis include solitary or multiple, often cavitary nodules or masses, or focal or diffuse consolidations, Churg Strauss is dominated by interlobular thickening or transient multifocal and nonsegmental consolidations frequently in subpleural distribution. All types of pulmonary vasculitis may present with focal or diffuse pulmonary haemorrhage that produces pulmonary ground glass or consolidations in various distributions. A number of collagen vascular diseases (e.g., lupus erythematosus) or other granulomatous diseases (e.g. sarcoidosis) may affect the small pulmonary vessels causing haemorrhage or pulmonary hypertension. Per disease entity, the course will review typical and more rare HRCT features and key features that allow for diagnosis and classification, discuss side-to-side overlapping of morphologic features important for differential diagnosis and illustrate the findings of other diseases that represent the most challenging differential diagnosis, e.g. oedema, infection or malignant diseases.

Learning Objectives:

1. To learn when HRCT is of value in investigating pulmonary vasculitis.

2. To appreciate the different appearances of pulmonary vasculitis on HRCT.

A-014 17:07

D. Inflammation and remodeling

A.A. Bankier; Boston, MA/US (abankier@bidmc.harvard.edu)

The presentation will lay out the pathological and pathophysiological basis for the complex processes of inflammation and remodelling. The implications of these processes for imaging will be discussed and illustrated by selected pathologies. Finally, the presentation will indicate how the imaging reflections of these processes could be used in the future as imaging biomarkers for the diagnosis, follow-up, and outcome evaluation of disease.

Learning Objectives:

1. To learn about the different causes of pulmonary inflammation.

2. To understand how the lung responds to inflammation.

3. To become familiar with pulmonary changes following inflammation. Author Disclosure:

A.A. Bankier: Author; Elsevier. Consultant; Olympus. Research/Grant Support; NIH. Speaker; ATS.

\section{.}




\section{Cardiac}

\section{RC 303}

\section{Hybrid cardiovascular imaging: where should we go? \\ Moderator: \\ K. Gruszczynska; Katowice/PL}

A-015 16:00

\section{A. PET/CT: present state and future prospects}

M. Schwaiger; Munich/DE (markus.schwaiger@tum.de)

Multimodal cardiac imaging with PET/CT has gained increasing clinical acceptance, since the combination of structural definition of coronary anatomy as well as quantification of regional myocardial perfusion allows very comprehensive workup of patients with suspected coronary artery disease. Independently of detection of coronary artery disease the quantification of regional and global myocardial perfusion has to be shown of prognostic value. The combination of extent of coronary artery disease as well as restriction of macro and micro circulation provides an integrative measure of the ischemic burden to patients with known or suspected coronary artery disease. The diagnostic and prognostic value of this combined PET/CT approach competes successfully with invasive diagnostic workup. However, there are no prospective randomised studies to document the relative value of invasive vs non-invasive strategies in the workup of patients with coronary artery disease, which has to be related to patient workup.

Learning Objectives:

1. To become aware of the present state of PET/CT in cardiovascular imaging.

2. To become familiar with appropriate indications for PET/CT studies of the

heart.

3. To learn about technical innovations in PET/CT imaging

Author Disclosure:

M. Schwaiger: Consultant; Navidea. Research/Grant Support; Siemens PET.

\section{A-016 16:30}

\section{B. SPECT/CT: is it just PET/CT's little brother?}

M. Hacker; Vienna/AT (marcus.hacker@meduniwien.ac.at)

Appropriate diagnosis and therapy of coronary artery disease (CAD) frequently require information about both the morphological and functional status of the coronary artery tree. Thus, combined imaging consisting of invasive coronary angiography (ICA) plus SPECT myocardial perfusion imaging (MPI) is practiced in clinical routine diagnostic of patients with stable angina since many years and can therefore be accepted as the reference standard in the diagnosis of hemodynamically relevant coronary artery stenoses. Both morphological and functional information are mandatory for the decision of performing an interventional therapy or initiating/maintaining medical treatment in numerous symptomatic patients. The hemodynamically relevance of coronary artery lesions is a major condition to decide whether an interventional therapy should be performed or not. A non-invasive concept providing both information could provide accurate allocation of perfusion defects to their determining coronary lesion and specific morphological and functional classification of patients with coronary artery disease. In symptomatic patients, a normal stress MPI confers a very low short-term risk for cardiac death and/or acute myocardial infarction. However, a normal MPI does not exclude the presence of underlying coronary atherosclerosis, which may be extensive although not yet flow-limiting. In this regard, CT will unmask a sizeable subgroup of patients with coronary atherosclerosis who should receive more intensive antiatherosclerotic intervention than would have been indicated by MPI results alone. Knowledge regarding the presence and extent of subclinical coronary atherosclerosis in patients who do not have ischemia by MPI can be of importance in patient management.

Learning Objectives:

1. To appreciate the scope of information a SPECT/CT cardiac study can deliver.

2. To become familiar with protocols of SPECT/CT studies.

3. To learn a structured approach to performing and reporting a SPECT/CT

study.

A-017 17:00

\section{MR/PET: do we really need it?}

H.H. Quick; Erlangen/DE (Harald.Quick@imp.uni-erlangen.de)

Following PET/CT and SPECT/CT, MR/PET hybrid imaging is the most recent addition to the palette of hybrid imaging modalities. MR/PET synergistically combines the excellent soft tissue contrast and detailed image resolution of
MR with metabolic information provided by PET. Integrated MR/PET systems furthermore offer the ability to acquire hybrid imaging data simultaneously. This can be applied to MR-based motion correction of PET data. These features open up several cardiac applications, e.g. evaluation of cardiac function and viability, diagnosis of cardiac inflammatory diseases and tumorous diseases. To fully assess the diagnostic potential of MR/PET, however, several technical challenges have to be considered: attenuation correction (AC) of the patient tissues in MR/PET has to be based on MR-images and is currently hampered by a limited number of tissue classes and undercorrection of bone tissue. Cardiac radiofrequency coils and ECG gating equipment are currently not considered in AC. Consequently, quantification of PET data therefore might be biased. The clinical workflow is rather complex and needs to be tailored to cardiac examinations. Few research groups currently explore this new hybrid imaging modality in selected cardiac applications. Cardiac MR/PET: Do we really need it? Considering the sparse clinical experience that is available today, it is quite early to answer this question yet. However, once the remaining technical hurdles are overcome and the diagnostic potential can be fully exploited clinically, the answer is most likely positive.

Learning Objectives:

1. To learn about technical requirements for performing cardiac PET/MRI studies

2. To learn if cardiac PET/MRI can be successfully performed in clinical routine.

3. To become familiar with the principal advantages/disadvantages of PET/MRI compared to other hybrid imaging technologies.

Author Disclosure:

H.H. Quick: Equipment Support Recipient; The Biograph mMR sytsem at the Institute of Medical Physics, Erlangen, was funded by a research agreement between the University of Erlangen and Siemens AG, Healthcare Sector, Erlangen.

$16: 00-17: 30$ Room E1

\section{Molecular Imaging}

RC 306

\section{Functional and multimodality neuroimaging}

\section{Moderator:}

O. Clément; Paris/FR

\section{A-018 16:00}

\section{A. Clinical applications of PET/CT in neurology}

A.H. Jacobs; Münster/DE

Positron Emission Tomography (PET) is being used together with various radiotracers to investigate alterations of metabolism and neurotransmission in various neurological diseases. Target structures for PET imaging comprise neurons (e.g. glucose or oxygen consumption; enzyme activity; receptor density); glial and endothelial aminoacid transporters; microglial function; and interstitial protein deposits. Together with MRI, PET helps to delineate (i) the penumbra in stroke; (ii) inflammatory activity in multiple sclerosis; (iii) epileptogenic foci in epilepsy; (iv) protein deposits and altered glucose consumption in Alzheimer's disease; (v) altered dopaminergic transmission in Parkinson's disease; and (vi) increased uptake of radiolabeled amino acids in gliomas. The lecture will outline the additional value of PET/CT imaging in the various most common neurological diseases to specifically increase the diagnostic accuracy and to help in the establishment of imaging-guided therapy paradigms. In addition, an outlook will be given from the clinical research programme of the 7th FW EU INMiND project (Imaging Neuroinflammation in Neurodegenerative Diseases; http://www.uni-muenster.de/InMind/).

Learning Objectives:

1. To become familiar with the role of PET/CT in neurology.

2. To learn about radiotracers that can be used in neuroimaging.

3. To understand PET/CT applications in relationship to disease presentations. Author Disclosure

A.H. Jacobs: Grant Recipient; 7th FW EU Programme QuicConcept and INMIND 


\section{Postgraduate Educational Programme}

A-019 16:30

\section{B. MR/PET changes and challenges}

B.J. Pichler; Tübingen/DE

"No abstract submitted"

Learning Objectives:

1. To understand the fundamentals of MR physics relevant to MR/PET imaging.

2. To appreciate the advantages of MR/PET and its complementary role in diagnostic neuroimaging.

3. To learn about the benefits and challenges of combined MR/PET.

A-020 17:00

C. Advanced MR neuroimaging techniques

M. Smits; Rotterdam/NL (marion.smits@erasmusmc.nl)

Functional MR imaging ( $\mathrm{fMRI}$ ) and diffusion tensor imaging (DTI) are used extensively in the research arena to study an infinite number of questions regarding the brain's function and structure under normal conditions, as well as with neurological and psychiatric disease. The clinical use of these techniques, however, is by comparison fairly limited. The current main indication is the presurgical assessment of the relationship between the brain tissue to be resected and functionally eloquent brain tissue. In the context of brain tumour surgery, the aim is maximum tumour resection, while at the same time avoiding functional deficit. With tumour localisation in or near presumed eloquent brain areas, such as the motor or language areas, additional fMRI and DTI may be advantageous to guide the neurosurgical approach, shorten surgery duration and obtain prognostic information prior to surgery. fMRI is used to localise eloquent cortex, which is particularly useful when normal anatomy is obscured by tumour mass effect or in cases of cortical plasticity. With DTI the anatomy and involvement of white matter tracts may be evaluated. Inadvertent transection of white matter tracts during surgery leads to severe neurological deficit. DTI-tractography offers attractive visualisation of the major white matter tracts, such as the corticospinal tract and the arcuate fasciculus, and offers valuable preoperative information on their relationships with the brain tumour to be resected. As well as providing such anatomical information, colour-coded eigenvector maps obtained with DTI can be used to categorise involvement of the white matter tracts by brain tumour.

Learning Objectives:

1. To learn about functional MR imaging ( $\mathrm{fMRI})$ and diffusion tensor imaging (DTI).

2. To understand the application of these techniques in the study of the healthy and diseased human brain.

3. To learn about the brain's activity and its connections.

16:00 - 17:30 Room E2

Multidisciplinary Session: Managing Patients with Cancer

\section{MS 3}

\section{Multidisciplinary approach for detection and treatment of malignant primary bone tumours}

\section{A-021 16:00}

\section{Chairman's introduction}

K. Verstraete; Ghent/BE (koenraad.verstraete@ugent.be)

Malignant bone tumours are rare and have poor prognosis, unless a multidisciplinary approach is applied. First, the radiologist plays an important role in diagnosis, staging and treatment follow-up of bone tumours. Plain radiography and routine and dynamic contrast-enhanced MRI are the most important imaging techniques. In this session, we will focus on osteosarcoma and Ewing sarcoma. Once the diagnosis is proven by biopsy, neoadjuvant chemotherapy will be given by the oncologist during a preoperative period of about 3 months. Then, a new MR study is required for preoperative staging and assessment of response. Whenever possible, a limb-saving surgery will be performed, preferably by a dedicated oncologic surgeon, in a referral centre for bone tumours. After surgery, tumour response will be measured by the pathologist, and adjuvant chemotherapy will be given for about one more year. Close follow-up by the multidisciplinary team is required for 5 more years. Two case presentations will illustrate how the multidisciplinary approach works and clarify the role of the radiologist, oncologist and oncologic surgeon.
Session Objectives:

1. To learn about the task of the radiologist in diagnosis, staging and treatment follow-up of bone tumours.

2. To understand the oncologic and surgical treatment of bone tumours. 3. To become familiar with the multidisciplinary approach to diagnosis staging and treatment of bone tumours.

\section{A-022 16:05 Imaging malignant primary bone tumours K. Verstraete; Ghent/BE (koenraad.verstraete@ugent.be)}

The majority of malignant bone tumours can be detected on plain radiography. The age of the patient, location of the tumour in a bone and history of a preexisting bone abnormality should be included in determining the likely diagnosis. Careful analysis of the pattern of bone destruction, periosteal reaction and matrix mineralisation allow for characterisation of most cases of osteosarcoma, Ewing's sarcoma, chondrosarcoma and adamantinoma. MRI is the best imaging technique for staging by displaying 1 . the extent of bone marrow involvement, including epiphyseal infiltration and skip lesions, 2. the presence and extent of extraosseous soft tissue mass, 3. involvement of the neurovascular bundle, muscle compartments and adjacent joint. This allows to find the best biopsy approach and establish the feasibility of limb salvage as opposed to amputation. Longitudinal non-contrast T1 sequences are the most accurate for determining intraosseous extent. Axial fat-suppressed PD or T2 sequences optimally demonstrate soft tissue extension, and dynamic contrastenhanced MRI is useful for differentiation of extraosseous tumour from edema and for assessment of tumour response to chemotherapy. Chest CT is the most sensitive modality for detection of pulmonary metastases, and technetium skeletal scintigraphy is still frequently used for detection of osseous metastases. The most valuable, readily available and easy-to-use techniques to assess response to radiation therapy and chemotherapy are dynamic contrast-enhanced MR imaging, diffusion MRI and colour-Doppler ultrasound. To evaluate local control of disease and for detection of local recurrence, MRI is usually the best imaging technique. Plain radiography may detect complications of prosthesis.

Learning Objectives:

1. To consolidate knowledge on malignant primary bone tumors on plain radiography, CT and MRI.

2. To understand the principles of tumour staging and monitoring chemotherapy.

3. To become familiar with imaging findings following chemotherapy and surgery.

\section{A-023 16:25}

\section{Oncologic approach to malignant primary bone tumours}

C. Dhooge; Ghent/BE (Catharina.Dhooge@UGent.be)

The most common bone sarcomas are osteosarcoma (OS) and Ewing sarcoma. OS occurs primarily in long bones, Ewing sarcoma occurs also in the pelvis, spine or chest wall. Localised disease confers a $65 \%$ cure rate, and initially metastatic disease $25 \%$. A multidisciplinary approach which includes neo-adjuvant systemic chemotherapy and local control measures for primary and metastatic sites followed by adjuvant chemotherapy has become the standard of care. Complete surgical excision is mandatory in OS; in Ewing, radiation can also be used. Standard chemotherapy for OS is based on a combination of cisplatin, doxorubicin and high-dose methotrexate. Ifosfamide and etoposide will be further considered (EURAMOS). Systemic therapy for Ewing includes vincristine, doxoribicin, cyclofosfamide, ifosfamide and etoposide. High-dose chemotherapy with autologous stem cell reinfusion is evaluated in intermediate prognosis and metastatic disease (euro-EWING). Among the different prognostic factors, response to neo-adjuvant chemotherapy by measuring chemotherapy-induced necrosis is a powerful indicator of outcome in bone sarcomas. Patients who achieve a good histological response to pre-operative chemotherapy, defined as $<10 \%$ viable tumour at the time of tumour resection, have a better survival than those who show poor response $(>=10 \%$ viable tumour). Five-year survival for good responders is $70-75 \%$ compared to $45-50 \%$ for poor responders. However, the outcome for malignant bone tumours has improved little in the last 25 years. Large international studies exploring new drugs such as biologic agents (interferon) or mechanisms conferring drug resistance (topoisomerase inhibitors) in randomised controlled trials will hopefully lead to therapeutic innovation.

Learning Objectives:

1. To become familiar with the principles of chemotherapy in malignant primary bone tumours.

2. To understand what the oncologist expects from the radiologist. 3. To recognise the value and limits of chemotherapy in malignant primary bone tumours. 
A-024 16:45

Surgical approach of malignant primary bone tumours

G.M.L. yㅗㅇ; Ghent/BE (Gwen.Sys@UGent.be)

In primary malignant bone tumours, three surgical procedures are necessary: biopsy, resection and reconstruction. Whether a biopsy is performed in an open or a percutaneous way is a matter of preference, but in each case it should provide sufficient and representative material for pathological investigation without compromising the following treatment. The area of interest and the trajectory should be determined preoperatively in a multidisciplinary meeting. As the biopsy tract has to be removed during the resection surgery, it should be close to the planned approach for the subsequent resection. If a biopsy is performed improperly, the diagnosis may be wrong or the following resection may be impaired because of extensive contamination of compartments, resulting in a severe functional deficit. Surgery planning is based on clear imaging and a multidisciplinary discussion of tumour extension, aiming for a wide resection of the tumour with clear margins. Correct perioperative measurements are based on predefined fixed bony landmarks that should preferably be visible on the same image as the tumour. An amputation is performed if a surgical limb-salvage procedure is expected to result in a non-functional limb. Nowadays, several reconstruction techniques for bones (bone grafts or prostheses), tendons (artificial or human ligaments) and skin (skin flaps or grafts) are available. Invasion of the soft tissues such as the neurovascular structures, muscles and skin, will determine which reconstruction technique (s) are necessary to restore the patient's function. Each reconstruction technique bears inherent complications requiring a regular follow-up.

Learning Objectives:

1. To become familiar with the basic principles of oncologic surgery in malignant primary bone tumours.

2. To understand what the surgeon expects from the radiologist.

3. To recognise the value and limits of limb-saving surgery.

Case presentation and discussion

17:05

$16: 00-17: 30$ Room F1

Professional Challenges Session

PC 3

\section{Radiology: opportunities and threats}

\section{A-025 16:00}

\section{Chairman's introduction}

J.A. Reekers; Amsterdam/NL (j.a.reekers@amc.uva.nl)

We outline the current opportunities and threats in diagnostic radiology: Traditional diagnostic radiology has been rapidly replaced by clinical radiology and the role of the radiologist is changing from image interpreter to clinical manager of imaging data. To care for the patient's medical problem and not only his images is important. This contains great opportunities for radiology to develop and for the radiologist to become a central player in patient management. However, this requires not only clinical knowledge and involvement, but also visibility and sometimes even $24 / 7$ availability. The need for " only" image reporting is declining as clinical subspecialisation easily brings image interpretation into the domain of non-radiologists. To understand the importance of identity, visibility is very important. Visibility can be achieved by being part of MDT $s$ and also in many other ways. Clinical radiology is an idea which, after all these years, has not been very well embedded in current radiology and radiology training. To understand the importance of certified training, maybe the most important action we have to undertake now is to capitalise on our radiological expertise. Image interpretation in a clinical context can only be done or franchised (to non-radiologist) under conditions of certification and accreditation by radiology. We should never let the primate of education and certification slip to non-radiologists. Radiology certification should be a quality standard, recognised by international and national medical bodies, such as medical chambers and specialist societies. The latter should have high priority.

Session Objectives:

1. To learn about the current opportunities and threats in diagnostic radiology.

2. To understand the importance of identity.

3. To acknowledge the importance of certified training.

\section{A-026 16:05}

Where are the turf battles in diagnostic imaging?

G.M. Bongartz; Basle/CH (georg.bongartz@usb.ch)

Turf battles in radiology are a foreseeable development for most imaging techniques which evolve push-button methods and readily interpretable imaging results. We as radiologists must learn to accept competition. Turfs should rather be seen as challenge than as threat. Predominantly, nonirradiating techniques like ultrasound and MRI have become progressively easier to apply (US) or to understand (MRI), where the final image in some standardised areas (eg joints) can readily be interpreted by medical specialists in this field. But also x-ray diagnostics in dental medicine or orthopedic imaging is largely applied outside the radiologic department. CT today seems stil relatively excluded because of its complexity. But with increasing resolution, optimal 3D reconstruction and fast accessibility, it is only a question of time when the added value of the radiologist will be under question. Radiology has changed. We must become experts with respect to patho-anatomy and radiological differential diagnosis. This requires profound education, both initial training and ongoing education. Radiology must be advertised internally and externally. In radiological publications, outcome studies are still underrepresented. Demonstration of our dedicated skills, fostering our special expertise and offering this as support to our medical colleagues will preserve radiology for the future. Cooperation with partners seems the only way out of a turf battle - we need to search for alliances and integrate our partners to create a win-win situation.

Learning Objectives:

1. To know about turf battles with clinicians around imaging.

2. To learn which imaging modalities are most at risk.

3. To learn how to create win-win situations.

Author Disclosure:

G.M. Bongartz: Research/Grant Support; Siemens Medical Systems Germany, Bayer, Switzerland.

\section{A-027 16:23 \\ Beating threats in Europe with radiological training A.K. Dixon; Cambridge/UK (akd15@radiol.cam.ac.uk)}

By introducing radiology to medical students, it is hoped that they will begin to appreciate how difficult it is! Likewise, when teaching residents, they must come to understand the basic anatomy and principles better than their clinical colleagues; this is very much the tenet of subspecialty training. They should also appreciate that patient care is optimal when there is close collaboration between clinicians and radiologists. Passing of an examination (national or EDiR) offers some proof that a radiologist has attained a certain standard, but the principle of testing oneself formally or informally at regular intervals again provides a measure of continuing competence. Such certification can be of value in the case of litigation. Radiology is one of the fastest growing specialties and the techniques that we learn during training are quickly outdated; hence, the need for life-long learning and effective continuing professional development.

Learning Objectives:

1. To understand how to teach radiology to undergraduate medical students.

2. To become familiar with the principles of self-assessment

3. To appreciate the necessity for life-long learning.

\section{A-028 16:41 \\ History of ultrasound in radiology: lessons learned \\ L.E. Derchi; Genoa/IT (derchi@unige.it)}

The relationship between radiology and US has never been an easy love story, and still isn't. In the early 1970s this new imaging field was not readily covered by radiologists and many other colleagues established US sections within their departments. Then, important fields of US (cardiology, obstetrics, gynecology) became almost exclusive domain of non-radiologists. In the 1980s, the increasing success of US initiated turf battles with other clinicians. Nowadays, clinical specialists perform $>50 \%$ of US examinations wordwide. In Europe, most in-hospital US examinations is still performed in radiology, but this is not the case in all countries. A strong practice in US is quite important since this is often the first approach to patients. If initial US referrals are kept, radiologists will continue to guide decisions in subsequent imaging workup. Furthermore, US keeps close to patients; in Europe the examination is usually done directly by radiologists or, if by sonographers, the radiologist usually checks the case with limited directed US imaging. This clearly shows our role as physicians. To maintain a key role in US radiologists need to: 1) Have the best specialists in US; attention must be given to US in residency programs. 2) Invest in US technology (as examples: CEUS, elastography, 3D/4D). 3) Invest in US research. 4) Have high visibility in the US community, keeping close contact with all clinical colleagues. 5) Present higher visibility of US within radiology, with leaders supporting US within the radiological community and each radiologist promoting US in his/her environment.

Learning Objectives:

1. To understand why ultrasound has moved out of the hands of radiology in some subspecialties.

2. To learn what this means for daily radiology practice and the quality of work

3. To know about the threats and how we should deal with them. 
A-029

Interdisciplinary cooperation without losing identity

M.F. Reiser; Munich/DE

Recently, major shifts in the paradigms of patient care took place: interdisciplinary counseling and including the patient in the decision making process for diagnostic and therapeutic measures. The new concept is that the patient no longer comes to the specialist, but the specialists come jointly to the patient. This is reinforced by the competition among health care providers, political decision makers and the public. In many hospitals this general trend has resulted in the establishment of various centers and boards such as the breast cancer centers, vascular centers and tumor boards for several cancer entities to name only few. At the university hospital of Munich 46 centers and boards have been implemented up to now. In almost all of them the participation of radiologists is required. This results in a major challenge in terms of personal resources. Together with the regular clinical-radiological rounds the time necessary for these activities equals 2 full-time radiologist posts. The participation of radiologists has many beneficial aspects: Acknowledgement of radiology as an important clinical discipline and of the radiologist as a clinical consultant; participation in and influence on the creations of SOPs and (internal) guidelines; opportunity to advocate appropriate radiological methods for diagnosis and interventions; increase of knowledge in clinical medicine and new concepts of therapy; close personal links with our clinical partners. In order to prevent radiologists from losing their identity as radiologists it is mandatory that they remain firmly integrated in the radiology department and to strengthen the position of radiology as the central institution for providing cost-effective imaging and interventional services. Moreover it must be taken care to offer attractive career options within the field of radiology.

Learning Objectives:

1. To appreciate how radiologists can work closely with clinicians without losing their identity.

2. To understand what the pitfalls and threats are and how we should deal with them.

3. To learn the central role of radiologists in clinical management of patient care.

Panel discussion:

How to stay in the driver's seat as radiologist 2.0? $\quad 17: 17$

$16: 00-17: 30$

Room F2

\section{Breast}

\section{RC 302}

\section{Breast ultrasound}

Moderator:

M. Müller-Schimpfle; Frankfurt a. Main/DE

A-030 16:00

A. Physics and practical aspects of high-quality hand-held US and automated breast US

M.H. Fuchsjäger; Graz/AT (michael.fuchsjaeger@medunigraz.at)

Ultrasound (US) is the adjunct method of choice to mammography in breast imaging. Over the past two decades, US technology has undergone profound advancements and refinements. US has, therefore, become an assessment tool with a defined field of indications as well as a unique set of diagnostic descriptors for breast lesion differentiation. The ACRIN 6666 study has demonstrated the special benefit of US in patients at risk with dense breast tissue. The BI-RADS Lexicon for Ultrasound, in its second edition, expanded the role of breast US. Further enhancement of image quality as well as the recent advent of automated breast US has even fueled scientific discussions on the potential role of US for breast cancer screening. Automated breast US, which is based on computed generation of a $3 D$ imaging data set obtained from many parallel 2D images, offers a different approach with a variety of benefits. Images are obtained by the sonographer in standardised fashion, whole breast data sets can be reviewed at any time after the examination, reducing operator dependence, and image fusion with, i.e. MRI is easily possible. The basic physical background, the significance as well as important aspects of practical use of handheld and automated breast US will be explained and illustrated by the respective imaging examples. Emphasis will be laid on strengths and potential weaknesses of both US technologies with regard to breast imaging.
Learning Objectives:

1. To understand the physics of hand-held US and whole breast US.

2. To become familiar with the practical techniques of hand-held US and whole breast US

3. To appreciate the clinical applications of hand-held US and whole breast US, including automated screening.

A-031 16:30

B. Complicated cysts and complex-cystic lesions: differentiation and management

C.F. Weismann; Salzburg/AT (christian.weismann@inode.at)

The terms "complicated cyst" and "complex-cystic lesion" are based on breast ultrasound (US) terminology. The simple cyst is a fluid-filled, clearly defined, anechoic lesion without any suspicion of a solid intracystic mass. If a simple cyst is complicated by echogenic fluid, it is called complicated cyst. This echogenic fluid can be caused, e. g., by cell debris. Thin echogenic septa are common in complicated cysts. Vascularisation can never be found in these echogenic components. A complicated cyst has no solid component and no thickened wall or thickened septa. The expected probability of malignancy is $2 \%$. The US criteria of a complex-cystic lesion following Berg et al. categorise it into 4 types: type 1, thick outer wall and thick internal septa (alone or in combination of both); type 2 , one or more intracystic masses; type 3 , mass of mixed cystic and solid components (at least $50 \%$ cystic); type 4 , predominantly solid with eccentric cystic foci (at least $50 \%$ solid). Complex means there is a suspicion of a tumour inside a cyst or a solid tumour with cystic components. A complicated cyst will usually be followed by US after 6 months. A complexcystic lesion needs a correlation with mammography. US-guided large core needle biopsy in combination with a US and mammography visible marker placement is useful in type 3 and in type 4 complex-cystic lesions. In type 1 and type 2 complex-cystic lesions, a preoperative hook wire placement and excision surgery is the typical procedure.

Learning Objectives:

1. To learn about the US appearance of complicated cysts and complex-cystic lesions.

2. To consolidate knowledge on differential diagnosis for these respective lesions

3. To understand the diagnostic algorithm for a work-up of these lesions.

A-032 17:00

C. The use of ultrasound in the evaluation of the nipple-areolar complex

R. Salvador; Barcelona/ES (rafasalvador@imaginebarcelona.com)

The nipple and areola can be well examined by any clinician. Ultrasonography can add new findings mostly related to the main ducts merging at the retroareolar region. A thorough description of the anatomical aspects of the region, and its pathological benign and malignant conditions will be described. Duct ectasia, infection, ductal papillomas, hyperplasia, and DCIS, as well as Paget disease of the nipple and some other skin pathologies can arise in this area of the breast. A brief description is presented of the US techniques to review the region, including the use of Doppler US, elastography, 3D ultrasound, the so-called automated breast ultrasound scan (ABVS) and other techniques that have been used not only to diagnose, but also to treat some diseases. US ductography and pecutaneous sampling or excision of papillomas will also be explained.

Learning Objectives:

1. To understand the normal anatomy of the nipple-areolar complex.

2. To become familiar with conditions commonly affecting the nipple-areolar complex.

3. To appreciate the value of US for diagnosis and management of these conditions.

Author Disclosure

R. Salvador: Advisory Board; Member of the Medical Advsory Board of Philips Healthcare. 
16:00 - 17:30

Room G/H

\section{Genitourinary}

RC 307

\section{Stone disease: new concepts}

Moderator:

A. Magnusson; Uppsala/SE

A-033 16:00

A. From the Stone Age to the New Age

N.C. Cowan; Oxford/UK (nccowan.uro@gmail.com)

Urinary tract stones have affected humans since civilisation began. Stones were first reported in the Aphorisms of Hippocrates and are still a major health problem. Their aetiology is multifactorial. Recent advances in diagnosis and treatment have proved significant. The usual presentation is with loin pain vomiting, fever, and nonvisible or visible haematuria. They may also be asymptomatic. Standard evaluation includes a detailed medical history, physical examination, diagnostic imaging, blood analysis and urinalysis. Principal imaging techniques are ultrasonography and unenhanced CT with judicial use of contrast medium enhancement and plain abdominal x-ray. Secondary imaging techniques include excretory urography, magnetic resonance imaging and antegrade or retrograde pyelography. The advantages and disadvantages of these techniques will be discussed. The imaging technique used depends on diagnostic accuracy, patient acceptability, availability, cost and patient safety. Diagnostic imaging is used to determine if stones are present and if present to measure the size, number, location and possible composition. Imaging may also be used to determine whether there has been an increase in size over a relatively short time period, indicating metabolically active stone disease, or a decrease in size, indicating a response to therapy. Imaging may find an underlying anatomical abnormality predisposing to stone formation such as pelviureteric junction obstruction or tubular ectasia and may also provide essential information regarding the function of the other kidney. Construction of smart pathways of investigation uses ultrasonography and unenhanced $\mathrm{CT}$ as principal diagnostic imaging techniques. Various risk factors are used to determine the selection and order of diagnostic imaging investigations. The clinical scenarios are explored. Learning Objectives:

1. To become familiar with the symptoms, signs, risk factors and significance of stone disease in the urinary tract.

2. To understand the strengths and weaknesses of Stone Age to New Age imaging techniques for stone disease.

3. To be able to construct a state-of-the-art diagnostic imaging pathway for urinary tract stone disease.

\section{A-034 16:30}

B. The contribution of imaging in planning urinary stone therapy

U. Patel; London/UK (Uday.Patel@stgeorges.nhs.uk)

Urinary stone treatment is influenced by stone size, density and composition, stone location and the collecting system anatomy. 1. Stone size: renal stones, $<1.5 \mathrm{~cm}$ in size, are suitable for surveillance. Larger stones, $>2 \mathrm{~cm}$, are better treated surgically by PCNL. $68 \%$ of ureteric stones, $<5 \mathrm{~mm}$ in diameter, will spontaneously pass compared to $47 \%$ of $>5 \mathrm{~mm}$. Very small opacities may represent Randall plaques. The diagnostic appearance of Randall's plaques on CT has not been defined, but opacities $<2 \mathrm{~mm}$ are likely to be stones. 2 . Stone density and composition: stone density can vary from $100 \mathrm{HU}$ up to $>1400 \mathrm{HU}$. Calcium containing stones can vary from $200-1400 \mathrm{HU}$, uric acid are 200 $500 \mathrm{HU}$, struvite (or matrix) calculi lie in the 100-700 HU range and cystine stones are around 300-400 HU. Dense stones are less likely to fragment and a threshold of $<800-1000 \mathrm{HU}$ is the cutoff point for selecting patients for ESWL. Cystine and calcium oxalate monohydrate stones are relatively resistant to ESWL. Dual-energy CT is able to differentiate uric acid stones from other varieties, and to a lesser extent can also predict other stone composites (such as cystine). 3. Stone location: stones located in the lower third of the ureter are more likely to drain $(75 \%$ vs. $50 \%)$. Location in the kidney is also of importance. 4. Collecting system anatomy: appreciation of the collecting system anatomy by $3 \mathrm{D}$ CT pyelography is the ideal modality for surgical planning.

Learning Objectives:

1. To understand how stone morphology, constituency and intrarenal anatomy influence treatment of urinary tract stones

2. To know how to carry out 3D CT reconstruction of the renal collecting system, and understand key technical factors necessary for recreating accurate anatomical or surgical maps.

3. To understand what information the interventionalist or urologist needs for planning urinary stone therapy.

\author{
A-035 17:00 \\ C. Imaging of stone disease in pregnancy \\ G. Masselli, G. Gualdi; Rome/lT (gabrielemasselli@libero.it)
}

Renal colic is the most frequent non-obstetric cause for abdominal pain and subsequent hospitalisation during pregnancy. Intervention is necessary in patients who do not respond to conservative treatment. Ultrasound (US) is widely used as the first-line diagnostic test in pregnant women with nephrolithiasis, despite that it is highly nonspecific and may be unable to differentiate between ureteral obstruction secondary to calculi and physiologic hydronephrosis. Magnetic resonance imaging (MRI) should be considered as a second-line test, when US fails to establish a diagnosis and there are continued symptoms despite conservative management. Moreover, MRI is able to differentiate physiologic from pathologic dilatation. In fact in the cases of obstruction secondary to calculi, there is renal enlargement and perinephric oedema, not seen with physiological dilatation. In the latter, there is smooth tapering of the middle third of the ureter because of the mass effect between the uterus and adjacent retroperitoneal musculature. When the stone is lodged in the lower ureter, a standing column of dilated ureter is seen below this physiological constriction. MRI is also helpful in demonstrating complications such as pyelonephritis. In the unresolved cases, computed tomography remains a reliable technique for depicting obstructing urinary tract calculi in pregnant women, but it involves ionising radiation. Nephrolithiasis during pregnancy requires a collaboration between urologists, obstetricians, and radiologists.

Learning Objectives:

1. To list the US, MR and CT imaging findings of urolithiasis and urinary tract infections.

2. To discuss the role and the appropriate uses of US, MR and CT for imaging these suspected conditions in pregnancy.

3. To discuss how imaging can influence the management of nephrolithiasis during pregnancy.

$16: 00-17: 30$

Room I/K

\section{Special Focus Session}

\section{SF 3}

\section{Pitfalls in FDG PET/CT imaging}

\section{A-036 16:00}

Chairman's introduction

K. Ahlström Riklund; Umea/SE (katrine.riklund.ahlstrom@diagrad.umu.se)

Pitfalls in FDG PET/CT studies will give an overview of what information you really get from an FDG PET/CT study and which pitfalls to avoid. Are all uptakes bad or are they also good and how to judge them? The following talks aim to cover pitfalls in the use of FDG in various fields of medicine. Session Objectives:

1. To learn about imaging with FDG PET/CT.

2. To learn about drawbacks and pitfalls

Author Disclosure:

K. Ahlström Riklund: Board Member; Swedish Society of Radiology, CMIV Linköping; Sweden, Örebro University, Sweden. Grant Recipient; ALF Väserbotten County Council. Speaker; GE user meeting Sweden 1/year (nonprofit).

A-037 16:05
Oncology
T.F. Hany; Zurich/CH (thomas.hany@gmail.com)

In malignant tumours, PET/CT imaging using 18-fluoro-deoxyglucose (FDG) is widely used nowadays. FDG-uptake is an unspecific process and results in physiological as well as pathological malignant and non-malignant tissue uptake. To properly understand and interpret PET/CT imaging, knowledge of the mechanism and pathways of FDG in different tissue types like brain and the gastrointestinal tract is key. Intrinsic as well as extrinsic factors influence uptake pattern not related to oncological disease like uptake in brown fat in patients exposed to cold ambient temperature and psychological stress or increased muscular uptake in nonfasting patient. Typical treatment-related changes occur during and after treatment and must be recognised correctly so as not to overstage patient disease. A systematic analysis and knowledge of all these factors facilitate and improve the reading of oncological clinical cases. Learning Objectives:

1. To understand pattern of physiological FDG-uptake.

2. To learn about the pattern of non-pathological uptake in several tissue types. 3. To understand the influence of chemotherapy and other agents on FDGuptake in the body. 
A-038 16:23

\section{Cardiology}

J. Knuuti; Turku/FI (juhani.knuuti@utu.fi)

The most common clinical situation for FDG PET/CT is the detection of myocardial viability. Also, FDG PET is increasingly used for detection of inflammatory cardiac diseases such as cardiac sarcoidosis. Cardiac tissue utilizes various energy substrates and the selection of substrate is mainly based on availability of these and on the metabolic conditions of the patient. When myocardial viability is studied with FDG PET, the patient must be studied in conditions when glucose is the preferred substrate. This can be achieved by using glucose loading or preferably by using insulin-glucose infusion. Nicotinic acid derivatives such as Acipimox can also be used. Diabetic patients are challenging, since tracer remains in the blood for a longer time. Blood glucose needs to be monitored and insulin may be needed. The reporting of viability studies is usually combined with the assessment of perfusion, allowing classifying the myocardial regions into normal, dysfunctional but viable or scarred myocardium. With a hybrid PET/CT system, coronary CT angiography can also be performed in the same session. However, in many patients invasive coronary angiography has been already performed. In addition, many of these patients have kidney problems, making the use of CT contrast challenging. In inflammatory cardiac diseases the aim is to suppress the myocardial glucose uptake as much as possible and patients should be studied in the fasting state or after special low-carbohydrate diet. The challenge is the physiological heterogeneity of glucose utilization in the fasting state. In most cases the localized tracer uptake by inflammation can be separated from more diffuse physiological uptake.

Learning Objectives:

1. To understand the benefits and limitations of anatomical and functional imaging in cardiology.

2. To learn about the quantitative analysis and limitations of the current methodology.

3. To become familiar with typical and challenging findings in cardiac FDG $\mathrm{PET} / \mathrm{CT}$.

Author Disclosure:

J. Knuuti: Advisory Board; Lantheus Inc. Grant Recipient; Cardirad.

\section{A-039 16:41}

Neurology

K. Van Laere; Leuven/BE (koen.vanlaere@med.kuleuven.be)

FDG PET (/CT) is in the CNS mainly used in the routine evaluation of dementia, movement disorders, epilepsy and brain tumours. In dementia, the main goal of FDG PET is differential diagnosis (e.g. Alzheimer's disease (AD), frontotemporal dementia, primary progressive aphasia, posterior cortical atrophy, etc)., as well as for the early diagnosis of $A D$ and prediction of conversion in the pre-dementia stage of mild cognitive impairment $(\mathrm{MCl})$. FDG PET allows differential diagnosis of parkinsonism (idiopathic versus MSA, PSP and CBD) with good accuracy. In refractory epilepsy, FDG PET is used for definition of the functional deficit zone and lateralisation of the epileptogenic focus and carries high prognostic value in determining surgical outcome. In brain tumours, FDG PET is used for grading and detection of recurrence, best used in combination with anatomical information. Methodologically, normal patterns need to be discerned, which are age dependent. Although underlying CT information is able to provide atrophy assessment, proper partial volume correction needs more detailed MRI information. When hybrid scanning is performed with CT, movement artefacts need to be prevented to avoid attenuation artefacts. Semiquantitative analysis and surface rendering techniques with voxel-based comparison to normal datasets should be applied routinely, to obtain maximal accuracy on the individual patient level. Learning Objectives:

1. To understand the benefits and limitations of FDG PET/CT in neurological disorders.

2. To become familiar with the normal and abnormal uptake patterns of FDG in the brain and potential pitfalls.

3. To learn about the value of semiquantitative analysis of brain FDG PET/CT in comparison to normal data.

\section{A-040}

\section{Inflammation}

J. Sorensen; Uppsala/SE (Jens.Sorensen@medsci.uu.se)

Glucose transport is increased in inflammatory lesions due to increased metabolic needs of activated inflammatory cells and proliferating connective tissue cells. Reports on the use of FDG PET/CT for visualising inflammation provide some evidence for routine clinical use in selected scenarios such as evaluation of patients with prolonged fever of unknown origin, infections after joint replacement surgery and other osteomyelitic conditions, graft infections after endovascular surgery, vasculitis in autoimmune disorders and patients with granulomatous diseases. This paper covers the documented indications and provides an overview of the common pitfalls related to each. Learning Objectives:

1. To learn about the role of FDG PET/CT in the evaluation of inflammatory diseases.

2. To understand the shortcomings and pitfalls of FDG PET/CT in inflammatory diseases.

3. To appreciate the difficulties of differentiating between inflammation, infection and malignancy.

Panel discussion: Is bright always bad? $\quad 17: 17$

$16: 00-17: 30$

Room N/O

Head and Neck

RC 308

\section{Head and neck emergency: for the general radiologist or the patient?}

Moderator:

A. Trojanowska; Lublin/PL

\section{A-041 16:00}

A. Pseudolesions mimicking disease

C. Karaman; Aydin/TR (cankaraman@hotmail.com)

Radiologists mostly prefer to use the comparative technique while evaluating radiological images, especially when the anatomy is identical for both sides. Comparative analysis mostly works if the anatomic details are not complex or the right resembles the left. However, the complex anatomy and relatively high incidence of individual variations in head and neck may hinder this way of interpretation. Variable pneumatisation of paranasal sinuses and tempora bone may cause difficulty. Vascular system, especially venous structures may be sources for pseudolesions. For example, asymmetrically enlarged jugular vein or venous plexuses at any location may be misinterpreted as mass of any origin. Veins may also become problematic on MR imaging due to entry slice phenomenon, in-plane flow, and flow turbulence effects and can have variable enhancement. Normal anatomical structures such as facial nerve may enhance and can be mistaken to be pathologic on MRI. Technical issues can be considered as a group that may cause pseudolesions. Images of the improperly positioned patient may become problematic while evaluating tiny structures. PET and PET-CT carry their own risks for pitfalls. Normal structures may confound interpretation and result in false-positive findings. The situation may become much more complicated when there is asymmetry. Similarly, atrophy and unilateral absence of a structure may be misread as a mass on the contralateral side. Because of the complex anatomy and relatively high incidence of individual variations, head and neck imaging demands much more attention and careful analysis. The appropriate imaging technique and detailed knowledge of anatomy are essential to recognise pseudolesions.

Learning Objectives:

1. To learn how to choose and tailor imaging techniques according to the clinical presentation.

2. To become familiar with common artefacts

3. To be able to recognise typical pseudolesions.

A-042 16:30

B. Where medical history and previous images help to rule out tumour

D. Farina; Brescia/IT (nappaje@yahoo.it)

As a general rule, detailed knowledge of the patient's clinical history is essential before any imaging study is performed, to evaluate the appropriateness of the indication, tailor the acquisition protocol and correctly interpret the study. In head and neck imaging, this rule particularly fits in the emergency setting. In certain circumstances, the swelling and enhancement of soft tissues produced by infectious lesions may mimic a neoplasm. Even more so, in patients already treated with surgery or chemoradiation. In these patients, the challenge is double: to identify any abnormality in the new anatomy produced by treatment and to discriminate between inflammation, complication and relapse. These can be very difficult without knowledge, for example, of the reconstructive procedure after surgery. Previous images are extremely useful, not only because they help detect abnormalities, but also because through indirect information on the growth rate of the lesion they are crucial for correct interpretation of findings. 
Learning Objectives:

1. To understand the importance of medical history and previous images.

2. To become familiar with typical postsurgical and post-radiation imaging findings.

3. To learn about less common anatomical variants without clinical consequence.

\section{A-043 17:00}

\section{Findings that can't wait for follow-up}

M.G. Mack; Munich/DE (m.mack@radiologie-muenchen.de)

Head and neck emergencies which cannot wait for follow-up are variable. Of course, all traumatic injuries affecting vascular structures have to be diagnosed and treated immediately. Due to the close anatomical relationships, nerves and vascular structures can direct inflammatory and tumorous lesion to the skull base and can cause severe clinical complications. It is also important to know all the dangerous spaces downwards to the mediastinum, which can also cause severe clinical problems. During this lecture, efficient imaging protocols and the most relevant traumatic, vascular and infectious emergencies will be discussed.

Learning Objectives:

1. To learn about vascular head and neck emergencies.

2. To understand main pathways of skull base invasion.

3. To be familiar with infections in dangerous spaces

16:00 - 17:30 Conf. Room M3

\section{Computer Applications}

RC 305

\section{Mobile IT in radiology}

\section{A-044 16:00}

\section{Chairman's introduction}

E. Neri; Pisa/IT (emanueleneri1@gmail.com)

Tablets in radiology represent a novelty. Since the introduction of the tablets on the market, the radiological field has been probably the first medical discipline to discover the many advantages of these devices. In fact, many applications for image management have been made available on the app stores (Apple and Android), and allow radiologists and non-radiologists to handle DICOM images on the tablet, as part of the patient's record. However, the emerging applications are driving the process from the simple DICOM image viewing to the full integration of the tablet with the PACS, allowing the handling of a patient's full record and presumably the possibility to report. In view of this rapid technological development, again radiology falls in the middle of a storm and is asked to find a solution to problem: are tablets suitable to read and report DICOM images? And if so, which kinds of images (CT, MRI, x-ray, etc).? How can we manage the portability of patients' data (security issues, data loss, etc).? What will be the impact on teleradiology? These issues will be addressed by the panel of experts who will speak in the refresher course. Session Objectives:

1. To appreciate the current state of tablet technology and its practical use in radiology.

2. To understand the pros and cons of the use of tablets.

3. To learn about specific critical areas of utilisation (DICOM images reading and teleradiology).

\section{A-045 16:05}

A. Tablet-computers: a technical overview

J. Fernandez-Bayo; Sabadell/ES (jFernandezB@cspt.es)

Since their introduction in 2001, tablet PCs have evolved extensively. They have become very popular, filling the gap between laptop computers and smart mobile phones. We will review technical aspects like the processors, storage space and memory, size and weight, connectivity and networking, software, autonomy, and battery life in different devices. Special focus will be on the displays and different possible uses in radiology. For diagnostic purposes, different technical aspects must be taken into consideration, like the display size, resolution, pixel size (pixel pitch or pixels per inch), luminance, and contrast. Displays in radiology normally have sizes between 20 " and 24" and resolutions up to $5 \mathrm{Mpx}$, with luminance that ranges from $250 \mathrm{~cd} / \mathrm{m}^{2}$ to 600 $\mathrm{cd} / \mathrm{m}^{2}$. By contrast, tablets have screen sizes between $9^{\prime \prime}$ and $10^{\prime \prime}$ and resolutions up to $3 \mathrm{Mpx}$, with luminance that ranges from $370 \mathrm{~cd} / \mathrm{m}^{2}$ to 480 $\mathrm{Cd} / \mathrm{m}^{2}$. Due to their screen size, tablets score better in pixel pitch, with around 250 ppi versus $150 \mathrm{ppi}$ in medical displays. The two systems have similar contrast ratios. Other technical aspects of display that we should take into consideration are the number of distinguishable grays that can be represented, which in tablets correlates with the number of colours that can be displayed
(Color Gamut) and colour accuracy (Delta-E). Additionally, we should take into consideration that displays should be calibrated for medical diagnostic purposes and tools to calibrate tablets have recently become available. Learning Objectives:

1. To learn about the PC evolution: from desktops, to laptops and tablets.

2. To appreciate the versatility features of a tablet.

3. To understand the hardware features with a specific focus on display.

4. To understand the hardware features with a specific focus on networking

\section{A-046 16:28}

B. Reading DICOM images on the tablet

O. Ratib; Geneva/CH

Touch screen tablets are becoming widely available, providing convenient mobile solutions for physicians and health-care providers. This is particularly attractive in medicine to "nomad" physicians, who need to be able to access relevant patient data and images anywhere-anytime in their daily practice where they are rarely at a single location. While they may not always be adequate for routine diagnostic tasks, they provide a convenient mobile solution for on-call and remote consultations. There are different types of software architecture that can be implemented for such tasks. Two different major designs are: (1) online web-based applications where the device serves as a "thin-client" to display images rendered and manipulated on a remote computer and (2) local applications that reside on the mobile device and can run independently after images have been downloaded on the device. The first solution requires the user to be constantly connected to the network, while the second solution can continue to function after being disconnected from the network. Most PACS vendors are starting to provide web access to their imaging solutions that can be accessed from mobile devices. Web access can however be slow and dependent on reliable access to wireless network. We chose to develop a stand-alone companion application to our Open Source imaging platform OsiriX. With the increasing capacity and computing power of mobile devices, users will soon be able to perform most of the processing and image manipulation functions that are today only feasible on desktop or laptop computers.

Learning Objectives:

1. To learn which DICOM readers are available for tablets

2. To appreciate the different approaches to DICOM reading (local vs remote) and the PACS/tablets integration.

3. To understand the pros and cons of DICOM image reading with the tablet in regards to image quality and displays.

\section{A-047 16:51}

C. Mobile teleradiology: radiological features of the tablet-computer

E.R. Ranschaert; 's-Hertogenbosch/NL (ranschaert@telenet.be)

Over the past few years, several applications and viewers have been developed for tablet computers to enable remote viewing and interpretation of radiological images. When connected to a wireless network, the images can be viewed from any location within or outside the hospital. Both inside and outside the hospital, tablets seem most useful in radiological emergency settings. Within the hospital, tablet computers may have several other purposes such as teaching, clinical reviewing and communication with referring doctors or even patients. Nevertheless, tablet computers still have limitations both in hardware and software. The implementation of mobile devices in the hospital environment also introduces new questions about security and safety of patient information. These issues and potential solutions will be discussed.

Learning Objectives:

1. To learn about mobile teleradiology within the hospital.

2. To learn about mobile teleradiology outside the hospital.

3. To appreciate the potential risks of mobile teleradiology (data security, confidentiality, etc).

Panel discussion:

Confidence in the use of tablets in our clinical practice 
16:00 - 17:30

Board Room A

\section{Vascular}

\section{RC 315}

\section{How to perform venous sampling for endocrine disease \\ Moderator:}

W.R. Jaschke; Innsbruck/AT

\section{A-048 16:00}

A. Role of venous sampling in localising endocrine disease

C. Degenhart; Munich/DE (christoph.degenhart@med.uni-muenchen.de)

Among the selective venous sampling procedures for the evaluation of endocrine disease, adrenal vein sampling (AVS) and bilateral inferior petrosal sinus sampling (BIPSS) are used most frequently. AVS is recommended by current guidelines to identify surgically curable causes of hyperaldosteronism. However this procedure is regarded as a challenging procedure and the results may be difficult to interpret. Moreover, there is a lack of generally accepted standards for the performance of AVS. The key issues consist in the selection and preparation of the patients for AVS and the procedure for an optimal performance and interpretation of laboratory findings. Cushing's disease (CD) is the most common cause of endogenous hypercortisolism and it is caused by an adrenocorticotrophin (ACTH) secreting pituitary adenoma. In patients with a negative MRI, a positive BIPSS test is associated with a high rate of postoperative biochemical remission. When high suspicion of Cushing's syndrome (CS) exists, recommended screening tests include the overnight dexamethasone suppression test, the low-dose dexamethasone suppression test, or late night salivary cortisol. Corticotrophin releasing hormone $(\mathrm{CRH})$ test helps to distinguish pituitary CD from ectopic ACTH-dependent CS, BIPPS. In special cases, less common selective venous sampling procedures are indicated for the evaluation of parathyroid diseases, the localisation of pancreatic endocrine tumors or the evaluation of ovarian endocrine disease. Learning Objectives:

1. To learn about the diagnostic accuracy of venous sampling.

2. To become familiar with the indications for venous sampling.

3. To learn about the impact of sampling results on patient management.

\section{A-049 16:30}

\section{B. Adrenal venous sampling}

M. Matson; London/UK (matthew.matson@mac.com)

Adrenal venous sampling is an essential diagnostic tool in the investigation of primary hyperaldosteronism as it can provide information to distinguish unilateral from bilateral hyper secretion and thus direct therapy. The place of AVS in the diagnostic algorithm will be discussed in relation to cross-sectional imaging, and the technicalities of the procedure explained, including common reasons for failure and how they can be avoided. Interpretation of the biochemical results will be demonstrated.

Learning Objectives:

1. To learn how to perform adrenal venous sampling.

2. To learn some tips and tricks for difficult procedures.

3. To learn about the complications.

\section{A-050 17:00}

\section{Inferior petrosal sinus sampling}

D.K. Tsetis; Iraklion/GR (tsetis@med.uoc.gr)

Bilateral Inferior petrosal sinus sampling (BIPSS) is considered by many centres to be the gold standard for confirming the origin of ACTH secretion in patients with Cushing's syndrome. The procedure is technically challenging and includes the following steps: a) conscious sedation, b) sterile preparation of both groins with bilateral insertion of femoral venous sheaths, c) heparin infusion, d) fluoroscopically guided placement of catheters into bilateral inferior petrosal sinuses, e) contrast-enhanced fluoroscopy to confirm reflux into ipsilateral cavernous sinus, f) collection of baseline blood samples, g) CRH injection, h) collection of post-CRH injection blood samples, i) catheter removal and groin pressure until venous haemostasis. Occasionally, it is not possible to perform BIPSS via a femoral approach (due to aberrant anatomy, inferior vena cava filters, thrombosis, or inability to cross the internal jugular valve) and alternative access, such as direct internal jugular access is used. The most common complication of BIPSS is groin haematoma, occurring in $3-4 \%$ of cases. Injection of iodinated contrast material may deteriorate renal function, so measurement of BUN and creatinine is recommended prior to the procedure. Pre-procedure assessment of the coagulation system, platelets, and haematocrit is recommended as heparin is frequently infused following the insertion of sampling catheters to protect against cavernous sinus thrombosis
The placement of the catheter may cause discomfort, headache, ear pain, or hearing noises. Very rare complications include deep venous thrombosis and pulmonary thromboembolism, pontocerebellar junction stroke, brain stem injury, cranial nerve palsy, venous subarachnoid haemorrhage and obstructive hydrocephalus.

Learning Objectives:

1. To learn how to perform sinus venous sampling.

2. To learn some tips and tricks for difficult procedures.

3. To learn about the complications.

16:00 - 17:30 Room P

BIA Session

\section{Biomedical image analysis: novel tools in} neurodegenerative disease and breast

\section{cancer}

Moderator:

W.J. Niessen; Rotterdam/NL

\section{6:00}

Introduction

W.J. Niessen; Rotterdam/NL (w.niessen@erasmusmc.nl)

\section{A-051 16:10}

VPH-PRISM: opening doors in breast cancer care

H. Hahn; Bremen/DE (horst.hahn@mevis.fraunhofer.de)

The optimisation of clinical breast cancer care comprises the prevention of over-diagnosis, reduction of over-treatment, and avoiding unsuccessfu treatments. These goals are targeted in the VPH-PRISM project by seven European and two US partner institutions. From the prospectively collected data, we will derive optimised imaging protocols that comprehensively take a woman's history and risk factors into account. The tight integration of radiological and histopathological images enables joint assessment of quantitative tissue parameters from microscopic and macroscopic imaging More informed therapy decisions can be taken by such enhanced multidisciplinary data, backed by a powerful case database. The reduction of overtreatment, and highly individualised diagnosis and therapy decisions are closely related. We ultimately aim at deriving predictive parameters from the multi-modal and multi-disciplinary database to select the treatment option with the best chance of enduring recurrence-free survival. Prospectively collected data, personal risk factors, and corresponding imaging data fed into the database from three large cohort studies are expected to provide insights into the individual preconditions and factors affecting disease progression. To optimise therapy, the decision between therapy options is key. This will be addressed by a decision support system using the project database. For surgical excision planning, VPH-PRISM will aim at providing better estimates of tumour size, and with visual planning aids supporting radiologists and surgeons. For chemotherapy, quantitative lesion parameters, tracked over time during therapy, may help to detect success or failure of the treatment early on, such that a chemotherapy regime can for a non-responder be switched to a different regime in due time.

\section{A-052 16:30 \\ Quantitative imaging biomarkers in dementia; the FP7 VPH-Dare@IT project \\ W.J. Niessen; Rotterdam/NL (w.niessen@erasmusmc.nl)}

The number of individuals suffering from dementia today is roughly 36 million. Due to ageing societies, this number is predicted to increase to 115 million by 2050. Worldwide annual costs are estimated to be $€ 450$ billion. In 2012, the WHO declared dementia a global health priority, highlighting the urgent need for improvements in this area. VPH-DARE@IT's aim is to enable more objective, earlier, predictive and individualised diagnoses and prognoses of dementias to cope with the challenge of an ageing European society. The expected impact of VPH-DARE@IT will influence the scientific, clinical and industrial communities across Europe and internationally to improve health care of dementia patients. In this presentation we will introduce the VPHDARE@IT project. We will then focus on the development, validation and implementation of quantitative imaging biomarkers for the early detection and differential diagnosis, which will take place as part of the project. Author Disclosure

W.J. Niessen: Advisory Board; part-time detached to Quantib BV as Scientific Director. Shareholder; co-founder and shareholder of Quantib BV. 
A-053 16:50

Horizon 2020: Improving diagnosis and medical interventions and support to medical imaging

J.-L. Sanne; Brussels/BE (Jean-Luc.Sanne@ec.europa.eu)

Research and innovation contribute to increasing Europe's competitiveness. At the same time, research and innovation help make people's lives better by improving things like healthcare. In January 2014 the European Union launches Horizon 2020, the biggest EU research and innovation programme ever, with a budget of $€ 79$ billion (current prices) over seven years (2014 2020). EU support for research and innovation helps drive international projects across the European Union and beyond, and promotes the progress of knowledge and technology. One of the three key pillars of Horizon 2020 will be tackling Societal Challenges (Priority 3 ) such "Health, demographic change and well-being". One feature is the development of new and more effective diagnostics. Relevant tools and technology innovations will be supported in view to improve disease outcomes through earlier, more accurate diagnosis and by allowing for more patient-adapted treatment. Support will be provided through the Work Programme 2014 - 2015 of Societal Challenge 1 "Health, demographic change and wellbeing". Additional opportunities are offered in the Work Programme 2014 - 2015 of Priority 2 (Industrial leadership) "LEIT Information and Communication Technologies".

Discussion

$17: 10$

18:30 - 18:50

Room A

Plenary Session

\section{$\mathrm{OL}$}

Presiding:

V.E. Sinitsyn; Moscow/RU

A-055 18:30

Etudes of space radiology

O. Atkov; Moscow/RU (cz-oa@css-rzd.ru)

Practically, all imaging modalities have been used in space research since a long time ago. First of all, radiology is targeted in research activities, because by definition all cosmonauts and astronauts are healthy persons. The biggest volume of examinations had been performed on crew members for studies of organs and functions of the human body before and after long-term flights. Health effects of zero gravity were of special interest. For example, multiple studies with involvement of both Russian and international specialists were done on bone mineralisation (bone densitometry), muscle volume (MRI) and metabolism (MR-spectroscopy) both in astronauts and healthy control subjects. First, ultrasound (US) examinations were conducted in space onboard Russian orbital stations in 1982. Complex US studies were done by the author himself during his flight as a crew member of "Salut-7" orbital station in 1984. The author had found some complex changes in blood and fluid dynamics under weightlessness. Teleradiology was used for data transfer to medical specialists on the Earth. Today, we are continuing to use radiology for research on astronauts. For example, applications of sophisticated brain MRI and fMR methods are of special interest for studies of subtle brain changes in space travellers. Among our targets are flights to the moon, Mars and large asteroids. To be better prepared for that task, we need better diagnostic tools. New versions of mobile US systems, miniature X-Ray and CT machines suitable for use inside spaceships are under development.

Learning Objectives:

1. To learn about the history of imaging experiments on board of space stations.

2. To understand the need for use of radiological examinations in astronauts. 3. To become familiar with future perspectives of diagnostic imaging in space biomedical research. 
Friday, March 7 


\section{Interactive Teaching Session}

\section{$E^{3} 420 a$}

\section{Pitfalls in female pelvic imaging}

\section{A-056 08:30}

\section{A. Pitfalls in MRI}

E. Sala; New York, NY/US (salae@mskcc.org)

There are several MRI pitfalls that should be recognised when imaging the female pelvis. MRI appearances of uterus and ovaries are dependent on the phase of menstrual cycle/use of exogenous hormone therapy. Normal postsurgical and post-radiation appearances of the pelvis can sometimes mimic tumour recurrence. It is important to become familiar with these appearances to avoid potential pitfalls. One very common pitfall is differentiation of transient myometrial contraction from adenomyosis. Interrogation of all imaging planes over the duration of the entire MRI examination can be useful to distinguish between the two, although myometrial contractions can last up to $45 \mathrm{~min}$. The choice of the correct imaging plane is crucial for the precise classification of uterine anomalies (coronal oblique) and accurate evaluation of parametrial invasion (axial oblique) in patients with cervical cancer. Both dynamic contrast-enhanced MRI and diffusion-weighted MRI improve the accuracy of MRI in the evaluation of malignant pelvic conditions. However, certain pitfalls related to each technique should be recognised to avoid misinterpretation. It is crucial to be familiar with the anatomy of the uterovesical (UV) ligament, as it is often the site of pelvic lymphoma (such as bladder or cervix lymphoma). However, some benign conditions such as endometriosis can involve the UV fold and invade both bladder and uterine wall. Certain MRI features can be helpful in making the correct diagnosis

Learning Objectives:

1. To become familiar with normal variations in MRI appearances of female pelvis resulting from physiological conditions (e.g. different phases of menstrual cycle) and treatments (including exogenous hormone therapy, surgery and radiation) potentially mimicking disease.

2. To consolidate knowledge on the role of the correct MR imaging plane in avoiding potential mis-classification of uterine anomalies and parametrial invasion in patients with cervical cancer.

3. To learn about certain pitfalls related to dynamic contrast-enhanced MRI and diffusion-weighted MRI

\section{A-057 09:15}

\section{B. Pitfalls in ultrasound}

K. Kinkel; Chêne-Bougeries/CH (karen.kinkel-trugli@wanadoo.fr)

Pitfalls of sonographic findings in the pelvis can be related to technical issues, interpretation errors or to the patient's specific condition or pathology. Common problems consist of insufficient bladder filling, misinterpretation of posterior enhancement or shadowing according to the anatomical structure and pathology of a size that goes beyond the field of view of the probe. Organspecific problems will be illustrated in interactive questions, particularly for the uterus and the ovaries.

Learning Objectives:

1. To become familiar with anatomical variants potentially mimicking disease.

2. To describe common pitfalls in pelvic ultrasound.

Author Disclosure:

K. Kinkel: Research/Grant Support; Clinique des Grangettes.

08:30 - 10:00 Room B

\section{Gl Tract}

\section{RC 401}

\section{Misses and difficulties in abdominal imaging}

\section{A-058 08:30}

\section{Chairman's introduction}

J. Stoker; Amsterdam/NL (j.stoker@amc.uva.nl)

Identification of diseases can be difficult in many areas of abdominal imaging and therefore misses are easily made, also by the more experienced radiologist. It is difficult in the mesentery and peritoneum, covering a large area but being a very thin structure where abnormalities are easily missed. Therefore, knowledge of the peritoneal anatomy and pathophysiology of peritoneal diseases is not widespread and differential diagnosis can be difficult. Hence, special attention should be paid to prevent misses. Another difficult area is diagnosing occult gastrointestinal bleeding. Here, the choice of the proper imaging method is crucial as well as understanding the pros and cons of the method. The technique used should be optimised, as otherwise sometimes subtle signs of bleeding are missed. The presence of bowel dilatation itself is often correctly diagnosed, but the extent of the obstruction, the cause of the obstruction and especially complications (ischaemia) might be misdiagnosed. CT is the preferred technique for evaluation of bowel dilatation. Care should be taken to scrutinise the examination for principal findings in patients with bowel obstruction.

A-059 08:35

A. Mesentery and peritoneum

D. Akata; Ankara/TR (dakata@hacettepe.edu.tr)

Imaging findings of neoplastic or inflammatory diseases within the peritoneal cavity and the mesentery sometimes overlap and cause difficulties in interpretation. Even disease processes in the peritoneum, mesentery or omentum may not be recognised on radiological examination, causing major difficulties in the management. Peritoneal anatomy and physiopathology of peritoneal diseases must be well understood for better evaluation. CT is the best modality to assess the whole cavity. MR is equally sensitive with better contrast resolution; however, both modalities have advantages and limitations. Ultrasound has a complementary role in evaluating the peritoneal fluid content. The presence of lacelike mobile thin septa is highly significant for tuberculosis. For better diagnosing the pathology, systematic approach is needed such as assessing the presence or absence of fluid in the peritoneal cavity, its character and location, accompanying soft tissue densities and their location. Some inflammatory or infectious causes, such as acute pancreatitis and tuberculosis, involve typically parietal peritoneum and certain peritoneal reflections. Peritoneal carcinomatosis involves typically both visceral and parietal peritoneum as well as subdiaphragmatic space. Contrast enhancement patterns of the peritoneal membranes and the mesentery also have a complementary role in differentiating a variety of diseases.

Learning Objectives:

1. To learn about the imaging characteristics of peritoneal and mesenteric masses and their differentials.

2. To appreciate the potential and limitations of imaging techniques in the detection of such lesions.

3. To understand the common pitfalls in diagnosis.

A-060 08:58

B. Occult GI bleeding

A. Filippone; Chieti/IT (a.filippone@rad.unich.it)

Patients with persistent, recurrent, or intermittent bleeding from the gastrointestinal tract for which no definite cause has been identified by initial oesophagogastroduodenoscopy, colonoscopy, or conventional radiologic evaluation are considered to have an occult gastrointestinal bleeding (OGIB). The management of OGIB is clinically challenging, since the causes of such a bleeding frequently arise in the small bowel (SB), until now considered as the "dark continent". Conventional barium contrast studies allow only a limited evaluation of SB, whereas angiographic diagnosis is strictly related to active bleeding. The introduction of capsule endoscopy (CE) as well as of crosssectional imaging dedicated to the SB visualisation, such as multidetector-row computed tomography enteroclysis (CTE) and magnetic resonance enteroclysis (MRE), represents significant technological advances that have overcome the limitations of older diagnostic tests. Although $\mathrm{CE}$ is recommended as a first-line investigation in OGIB patients, CTE or MRE are alternative diagnostic tools when $\mathrm{CE}$ is contraindicated due to suspected/known obstruction or stricture. Moreover, in patients in whom a tumour is suspected, CTE or MRE may be the preferred initial test. Therefore, radiologists have to be familiar with CTE and MRE techniques, in terms of bowel distension, scanning parameter selection, contrast administration as well as with specific imaging findings. Similarly, they have to be aware of the potential pitfalls such as suboptimal bowel distension, artefacts due to peristalsis or breathing, intraluminal food debris and previous surgery. Learning Objectives:

1. To understand the causes of $\mathrm{GI}$ bleeding and underlying pathophysiology. 2. To appreciate the strengths and limitations of the imaging techniques used in diagnosis.

3. To learn about common pitfalls in diagnosis.

\section{A-061 09:21}

C. Bowel dilatation

E. Danse; Brussels/BE (etienne.danse@uclouvain.be)

Patients with bowel dilatation frequently require imaging procedures, mainly in case of admission in the emergency room as well as in the early post-operative period. Plain films, sonography and CT contribute to the management of bowel 
dilatation. Presently, CT takes a major place in this setting with a reduced role for plain films. Sonography is an alternative method when CT is not recommended. The questions of Mondor in 1947 were pointed before the advent of cross-sectional imaging; these are still valid and have to be answered: 1) Is this a true occlusion (how to differentiate it from adynamic ileus)? 2) Does the obstruction concern the small bowel or the colon (impacting on the decision to operate or not)? 3) What is the cause of the obstruction? 4) Are there signs of bowel ischaemia? The key points to avoid missing imaging diagnoses in bowel dilatation are to make the distinction between true mechanical obstruction and adynamic ileus (an early sign of mesenteric infarct) and to detect signs of strangulation leading to ischaemia (such imaging findings are present despite normal blood tests). In this setting, CT is the preferred technique; if it is not available, plain films and sonography can help in an optimal medical decision.

Learning Objectives:

1. To become familiar with the normal appearances of the bowel and the physiological causes of bowel dilatation.

2. To learn about the different imaging techniques to diagnose bowel dilatation and their limitations.

3. To understand common pitfalls in diagnosis.

Panel discussion:

What have I learned from misses and can improvements be made to reduce misses?

09:44

$08: 30-10: 00$

Room C

\section{Controversies in Chest Imaging}

\section{424}

\section{Computed tomography screening for lung} cancer: is it time to implement individual lung cancer screening in clinical practice?

\section{Moderator:}

D.M. Hansell; London/UK

Teaser:

S. Diederich; Düsseldorf/DE

\section{A-062 08:30}

A. Implications of the national lung screening trials (NLST)

D.R. Aberle; Los Angeles, CA/US (daberle@mednet.ucla.edu)

The National Lung Screening Trial (NLST) found a $20 \%$ reduction in lung cancer mortality with three annual screens using helical CT relative to chest $x$ ray. The US Preventive Services Task Force has issued draft recommendations to provide annual CT screening to high risk individuals defined by age and smoking criteria. Questions remain regarding the implementation of CT screening: the determination of risk to identify those who should be screened, the frequency and duration of screening, definitions of screen positivity based on detected nodule characteristics, diagnostic algorithms for the follow-up of positive screens, overall societal costs of screening, and methods to adequately diffuse this technology across all socioeconomic groups at risk. Answers to these questions are being addressed by several groups through ongoing research and secondary analyses of trial data. Several models exist to identify individuals at highest risk of lung cancer (and lung cancer mortality), which may be enhanced if validated molecular markers are included. Ongoing analyses of nodule features and likelihood of lung cancer will better inform screening interpretation guidelines and diagnostic algorithms. Estimates from the NLST suggest that screening as performed in the NLST will be cost effective: the base case estimates $\$ 67,000$ per quality adjusted life year gained (QALY). Subset analyses suggest that screening is much more cost-effective in women than men, in higher versus lower risk groups, and in current versus prior smokers. While several variables influence cost-effectiveness, major drivers of cost are the cost of CT screening exams as well as the number of follow-up CT scans per positive screen $(1.26$ in NLST).

Learning Objectives:

1. To learn about the design and findings of the trial.

2. To understand the harms associated with screening.

3. To understand the need for better determination of appropriate risk cohorts for screening.

Author Disclosure:

D.R. Aberle: Advisory Board; Member - LUNGevity Science Advisory Board (Unpaid), Consultant - Siemens Medical Advisory Board (Unpaid). Grant Recipient; $\mathrm{NIH} / \mathrm{NCI}, \mathrm{U} 01 \mathrm{CA} 80098$, American College of Radiology Imaging Network (ACRIN), ACRIN / NIH-NCI Prime 20080299.

\section{A-063 08:55 \\ B. Implications of the European Trials \\ M. Prokop; Nijmegen/NL}

While the National Lung Screening Trial (NLST) had shown a significantly reduced lung cancer-specific mortality and all-cause mortality, the current results from the various European trials do not show such positive results. The Dutch-Belgian NELSON trial, the German LUSI trial and the British UKLS have not yet published their results. So far, more cancers were detected in the screening arm of published Danish and Italian trials, but the positive effect on cancer mortality could not yet be shown. The Italian MILD trial even showed higher mortality in the yearly screening arm, and no difference between 2yearly screening and the control arm. This presentation discusses the implications of these findings and the consequences on implementation on screening in clinical practice. While limited in number of participants, European trials used advanced approaches that are most likely to be used if screening will ever be implemented. This presentation will discuss issues such as growth assessment and volumetry as critical factors for nodule assessment, handling of sub-solid lesions, computer aided detection and evaluation, and use of nonradiologists for reading and standardised follow-up. The goal is to provide an insight into how the European trials have affected our idea on practical implementation of lung cancer screening.

Learning Objectives:

1. To learn what positive screening is, what the optimal management algorithms are for the interpretation of scans showing solid or non-solid nodules, and how positive subjects should be investigated.

2. To learn what the optimal follow-up duration and screening interval are. Author Disclosure:

M. Prokop: Research/Grant Support; Toshiba Medical Systems. Speaker; Bracco, Bayer, Toshiba.

A-064 09:20

Discussion

D.M. $\underline{\text { Hansell }}^{1}$, S. Diederich ${ }^{2} ;{ }^{1}$ London/UK, ${ }^{2}$ Düsseldorf/DE

The discussion will address the following issues:

1. Are there sufficient data to support the implementation of individual lung cancer screening in clinical practice?

2. If yes, how to move from efficacy to effectiveness:

What are the most optimised risk profiles for screening?

What is the minimum level of infrastructure support and organisation required? How should subjects be informed about screening?

What are the optimal follow-up duration and screening interval?

What are the technical and positive screen management requirements?

3. What about the alternatives of waiting for additional data from ongoing trials or the validation of new biomarkers of lung cancer, permitting better selection of screened population before implementing individual lung cancer screening? Attendees' opinions will be collected interactively. The speakers will be asked to comment and debate.

08:30 - 10:00 Room D

Oncologic Imaging

RC 416

\section{Multiparametric MRI for prostate cancer management: the essential guide for radiologists}

\author{
A-065 08:30 \\ Chairman's introduction \\ H.-P. Schlemmer; Heidelberg/DE (h.schlemmer@dkfz.de)
}

Prostate cancer is the most frequent cancer in men with high medical and socioeconomic impact. Cancer detection and treatment decision making is frequently based on PSA serum measurement and subsequent TRUS-guided biopsy, although considerable limitations are well known. Multiparametric MR imaging (mpMRI) on the other hand provides comprehensive morphologicbiologic information useful as clinically valuable biomarker for: (1) cancer detection and patient stratification according to individual tumour aggressiveness, (2) localisation of the most aggressive cancer spot (index lesion) in this remarkably multifocal and biologically heterogeneous disease, (3) guidance of biopsy and therewith establishing the ultimate diagnosis, (3) selection and planning of the individually well-adapted therapy, (4) active surveillance and if necessary guidance of re-biopsies. The method may considerably contribute to the urgently required separation of clinical significant from clinical insignificant prostate cancer as overtreatment is still suspected in 
a significant number of patients. This course gives an overview of the currently existing knowledge about diagnosis and treatment of prostate cancer and the role of multiparametric MR imaging for individually optimised patient management.

\section{A-066 08:35}

A. The clinical arena: when and how to treat prostate cancer F.H. Schröder; Rotterdam/NL (f.schroder@erasmusmc.nl)

Treatment of prostate cancer depends strongly on the stage of the disease at the time of detection. However, treatment is controversial, even in prostate cancers found through early detection. This presentation will be based on a careful recent literature review. Early detection of prostate cancer by use of PSA testing leads to a significant reduction of at least $30 \%$ of disease-related mortality in screened men. This is however at the price of $40-50 \%$ of overdiagnosis, usually resulting in overtreatment. Diagnostic developments which reduce overdiagnosis and the proper identification of cases which may not require treatment are central issues of current research and will be addressed by referring to available evidence. Active observation of potentially nonaggressive cancers is an option in the management of this disease until, preferably, the diagnosis of such cases can be avoided all together. Radical prostatectomy and radiotherapy are the options for treating those cancers which are judged to be aggressive.

Learning Objectives:

1. To learn how the diagnosis of prostate cancer is established through PSA testing and biopsy.

2. To understand the various treatment options available to the patient.

3. To become familiar with the role of imaging in clinical decision-making.

\section{A-067 08:58}

B. The radiologist's contribution: capabilities of multiparametric MRI in clinical routine

H. Hricak; New York, NY/US

Treatment recommendations for prostate cancer continue to evolve and are affected by technological advances in surgery, new discoveries in tumour biology, and the development of predictive and prognostic biomarkers. With the increased complexity of treatment decision-making, the role of $\mathrm{MRI}$ is also evolving. The more individualised and targeted the treatment approach, the greater is the role of imaging in treatment selection, planning and follow-up. $\mathrm{MRI}$ is especially crucial for planning technologically sophisticated treatment approaches such as robotic surgery, IGRT, or focal therapy and in assessing patients' eligibility for active surveillance. Though needs for uniform interpretation and standardised reporting remain unresolved, the value of MR in pretreatment staging of prostate cancer, particularly for evaluating extracapsular extension and seminal vesicle invasion, has been documented. Not only the tumour stage, but also its size, volume and grade (aggressiveness) are important factors that influence treatment selection. Therefore, the introduction of functional MR is essential. When added to T2weighted MRI, diffusion-weighted MRI, dynamic-contrast-enhanced MRI, and MR spectroscopy, in addition to improving tumour detection, can provide an indication of tumour aggressiveness. MRI performance on all sequences is dependent on lesion size/volume and grade, and this should be considered when applying MRI results to treatment selection and planning. While it has been shown that multiparametric MRI is generally more accurate and informative than anatomic MRI alone, evidence-based guidelines specifying which combinations of sequences are essential for specific indications in patients with prostate cancer are yet to be developed and validated in welldesigned studies with robust outcome measures.

Learning Objectives:

1. To learn how to perform and interpret multiparamteric MRI.

2. To become familiar with the PiRADS classification system.

3. To become familiar with the role of imaging for patient stratification and treatment planning.

\section{A-068 09:21}

\section{The joint action: MR-guided, MR/TRUS-fusion biopsy}

F. Cornud; Paris/FR (frcornud@imagerie-tourville.com)

The accuracy of multiparametric MRI has greatly improved the ability of localising tumour foci of prostate cancer. This property can be used to perform a TRUS-MR image registration, a new technological advance, which allows for an overlay of an MRI onto a TRUS image to target a prostate biopsy towards a suspicious area. Three types of registrations have been developed: cognitivebased, sensor-based and organ-based registration. Cognitive registration consists of aiming at a suspicious area during biopsy with the knowledge of the lesion location identified on multiparametric MRI. Sensor-based registration consists of tracking in real time the TRUS probe with a magnetic device, achieving a global positioning system which overlays in real time prostate image on both modalities. Its main limitation is that it does not take into account prostate and patient motion during biopsy Two systems (Artemis and
Uronav) have been developed to partially circumvent this drawback. Organbased registration (Koelis) does not aim at tracking the TRUS probe, but the prostate itself to compute in a 3D acquisition the TRUS prostate shape, allowing for a registration with the corresponding 3D MRI shape. This system is not limited by prostate/patient motion and allows for a deformation of the organ during registration. Pros and cons of each technique and the rationale for a targeted biopsy only policy are discussed.

Learning Objectives:

1. To understand the clinical challenges and techniques of prostate biopsy.

2. To become familiar with the MR-guided and MR/TRUS fusion approach.

3. To learn how MRI improves the diagnosis and characterisation of prostate cancer.

Panel discussion:

Is multiparametric MRI essential for the management of prostate cancer patients? 09:44

\begin{tabular}{l}
\hline 08:30 - 10:00 \\
\hline Emergency Radiology \\
RC 417 \\
Vascular: emergent, not urgent!
\end{tabular}

A-069 08:30

Chairman's introduction: highlighting the benefits of minimally invasive procedures in vascular emergencies

G. Schueller; Bülach/CH (gerd.schueller@spitalbuelach.ch)

In major trauma, it is essential to immediately recognise life-threatening conditions and to initiate early treatment. The majority of these patients succumb at the site of the injury to severe injuries of the central nervous system, heart and great vessels. However, there is another peak of early deaths within the first four hours after the injury. In the majority, these patients are at risk due to haemorrhage which is basically controllable by early minimally invasive treatment. Consequently, this means that the vast majority of internal injuries can be survived as long as bleeding control is achieved within the first four hours after an injury. Minimally invasive interventional techniques are already in use at a very early stage of the clinical decisionmaking process. Particularly, we have witnessed a major paradigm shift in the treatment of blunt aortic injuries. Endovascular repair has replaced open repair in many trauma centres. In appropriately selected patients, this adoption of endovascular stent grafts has resulted in a reduction in perioperative mortality, stroke and paraplegia, as compared to open repair. This entire session will provide knowledge about indications, requirements, standard procedures, and outcome of vascular emergencies, pardon me, urgencies.

\section{A-070 08:40 \\ A. Aortic emergencies \\ M. Cejna; Feldkirch/AT (manfred.cejna@Ikhf.at)}

Aortic emergencies can be classified into several categories. "Acute aortic syndrome": Acute aortic syndrome is an entity of changes of the aortic wall. These include aortic dissection, intramural thrombus, and penetrating atherosclerotic aortic ulcer. Besides coronary malperfusion and aortic valve insufficiency (type A dissection), emergency presentation is often due to visceral/limb ischaemia. Sequelae of aortic syndromes are pseudoaneurysm formation and potentially aortic rupture. Endovascular treatment has been established as the treatment option in at least type B dissections and penetrating aortic ulcers. Aortic aneurysm rupture: With increasing diameter, aneurysms in the thoracic, thoraco-abdominal and abdominal aorta are prone to rupture. Signs of impending aortic rupture are pain and imaging signs are sometimes subtle just like blurred contours of the aorta and (blister like) vascular wall deformation. Endovascular treatment is an alternative to vascular surgery. Aortic trauma: In severe thoracic trauma (mostly deceleration trauma), pseudoaneurysm formation and aortic rupture occur at the level of the aortic isthmus. Endovascular treatment is considered the treatment of choice. Inflammation, mycotic aneurysms and aorto-enteric fistulae: With acute and chronic inflammation, aneurysm and pseudoaneurysm formation and fistulae to enteric structures can occur. Endovascular treatment is more often considered as bridging to vascular surgery instead of being a definitive cure. latrogenic aortic injuries: Besides accidental aortic injury during operations or interventional procedures, transaortic valve replacement and placement of aortic balloon pumps can result in iatrogenic trauma. Endovascular treatment may be a minimally invasive approach. The focus will be on systematic presentation of aortic emergencies and the role of endovascular treatment. 


\section{Postgraduate Educational Programme}

Learning Objectives:

1. To become familiar with the most important imaging findings and their impact on patient management.

2. To understand common classification systems and their impact on patient management.

3. To learn about typical interventional radiological procedures.

Author Disclosure:

M. Cejna: Speaker; ABBOTT, COOK, BIOTRONIK.

\section{A-071 09:00}

B. Visceral emergencies

G. Goh; London/UK (gerard.goh@stgeorges.nhs.uk)

There are a number of vascular visceral emergencies that may be traumatic or nontraumatic in origin. The degree of urgency or potential for urgency varies with each condition and the subsequent management and intervention techniques will vary accordingly. An understanding of the natural history, pathophysiology, diagnostic tests and interventional techniques for these conditions will aid the diagnostic and interventional radiologist in identifying the pathology and knowing when to refer or initiate definitive treatment. Learning Objectives:

1. To become familiar with the most important imaging findings and their impact on patient management.

2. To understand common classification systems and their impact on patient management.

3. To learn about typical interventional radiological procedures.

Author Disclosure:

G. Goh: Consultant; Boston Scientific, Covidien. Research/Grant Support; Covidien. Speaker; Cook Medical, St Jude Medical.

\section{A-072 09:25}

\section{Peripheral emergencies}

R. Omidi; Bülach/CH (reza.omidi@spitalbuelach.ch)

I talk about embryonal development and anatomical differentiation of cardiovascular system, with special attention to the peripheral vascular system. The goal of this part of presentation would be to understand the anatomical structure and architecture of arteries and capillaries. We will discuss the Casual Classification of different kinds of peripheral arterial emegencies; e.g congenital, posttrauma, arteriosclerotic and iatrogenic. The audience will learn about different therapy approaches, interventional and non-interventional, with accent on PAD (classification, pathophysiology, Risk factors, statistical facts and therapy). At the end, we will review different studies about combination therapy (conventional and interventional) with different possibilities and outcomes.

\section{Learning Objectives:}

1. To become familiar with the most important imaging findings and their impact on patient management.

2. To understand common classification systems and their impact on patient management.

3. To learn about typical interventional radiological procedures.

Panel discussion

Cooperation with clinicians: what still needs to be done?

09:45

08:30 - 10:00 Room E1

\section{New Horizons Session}

\section{NH 4}

\section{Imaging the hallmarks of cancer}

\section{A-073 08:30}

Chairman's introduction: importance of cancer hallmarks for radiologists A.R. Padhani; Northwood/UK (anwar.padhani@stricklandscanner.org.uk)

Modern cancer research and increasing therapy are mechanism based with the development of disease-modifying therapies that target the hallmarks of cancer. Modern imaging tools enable the visualisation and quantitative assessment of the expression of molecular targets, of their interaction with potential ligands, as well as of the functional consequences of interactions at a molecular, cellular, metabolic, physiological, and morphological levels in a temporo-spatially resolved manner. The ability to gather such information from the intact organism renders imaging highly attractive for biomedical research and drug development.

\section{Session Objectives:}

1. To understand that human cancers acquire functional capabilities, which allow cancer cells to survive, proliferate, and disseminate acquired during a multistep development process.

2. To become familiar with established hallmarks (sustained proliferative signalling, evading growth suppressors, resisting cell death, enabling replicative immortality, inducing angiogenesis, and activating invasion and metastasis) and enabling hallmarks (genomic instability and inflammation). 3. To understand that cancer hallmark development creates vulnerabilities, providing new means to develop treatments for human cancer.

4. To consolidate knowledge of imaging techniques, which allow for serial observation of the heterogeneous development of hallmark distribution and monitoring of adaptive changes in response to therapy interventions.

\section{A-074 08:35}

\section{Cell proliferation and death}

U. Haberkorn; Heidelberg/DE (Uwe_Haberkorn@med.uni-heidelberg.de)

The determination of cell proliferation with radiolabelled thymidine is a wellestablished method in various life science branches where $[3 \mathrm{H}]$ thymidine has been used for more than 40 years and still represents the gold standard for the assessment of tumour cell proliferation. Since non-invasive determination of this parameter is required in clinical studies, considerable efforts have been made in radiopharmaceutical research. The synthesis of a large series of proliferation markers resulted therefrom mostly focussing on pyrimidine nucleosides. The labels used were gamma as well as positron emitting radionuclides. Here, [18 F]FLT (3'-deoxy-3'-fluorothymidine) is examined and discussed with respect to its preclinical and clinical application. Since apoptosis is an important mechanism of cell death in tumours responding to treatment, the non-invasive assessment of apoptosis with tracers for the detection of phosphytidyl-serine presentation and/or caspase activation could be used as surrogate marker for therapeutic efficacy. Several approaches have been followed during the last years; their potential and limitations will be presented.

Learning Objectives:

1. To understand the biochemical processes in apoptosis and proliferation.

2. To learn about the changes in apoptosis and proliferation that can be used as targets for the development of tracers.

3. To learn about the challenges in apoptosis and proliferation imaging.

4. To consolidate knowledge of preclincial and clinical applications.

\section{A-075 09:00}

Angiogenesis

F.M.A. Kiessling; Aachen/DE (fkiessling@ukaachen.de)

Angiogenesis is one of the hallmarks of cancer that is considerably easy to characterise with many different imaging modalities and methods. It is not only crucial for invasive and metastatic tumour growth, but is also a prerequisite for the accumulation of anticancer drugs and highly impacts the success of radiotherapy. This talk summarises our experiences in microstructural, functional, and molecular imaging of tumour angiogenesis. The microarchitecture of vessels can longitudinally be studied using high-resolution $(\mu) C T$. DCE MRI and HF-US are introduced as favourable tools to characterise perfusion, vessel permeability and vessel maturation during antiangiogenic treatments. Using targeted probes, it is shown that "fluorescence molecular tomography (FMT)", MRI and US are capable of estimating the expression of angiogenic marker molecules on tumour vessels and of matrix-associated enzymes in the interstitial space during vascular remodelling. Additionally, examples are also given for EPR-based nano-sized theranostics and it is shown how US can aid in better accumulating them at the target site by inducing vascular permeation. Many of these novel imaging concepts and tools can relatively easily be translated into clinics. Thus, one can expect them to play a major role in the clinical management of tumour treatments soon. Learning Objectives:

1. To understand the basic molecular and physiological processes during angiogenesis.

2. To learn about important imaging techniques for characterising vesse function.

3. To become familiar with techniques and contrast agent methods for molecular imaging of angiogenesis.

4. To learn about its potential for basic research and clinical use.

\section{A-076 09:25}

\section{Dysregulated metabolism}

K. Brindle; Cambridge/UK (kmb1001@cam.ac.uk)

Tumour cells display high rates of aerobic glycolysis (Warburg effect). While the tumour microenvironment, which is frequently hypoxic, might select for cells that are glycolytic, and thus can generate ATP in the absence of oxygen, it has become increasingly clear that this and the other metabolic changes observed in tumour cells are driven by oncogene activation and tumour suppressor loss. 
Moreover, while these metabolic changes are important for generating ATP under the often anaerobic conditions found in the tumour microenvironment, they also have other important functions, such as the generation of metabolic intermediates for biosynthetic pathways. This altered metabolism has provided new ways of detecting tumours and their responses to treatment using metabolic imaging. Nuclear spin hyperpolarisation can increase sensitivity in the magnetic resonance experiment by $>10,000 \mathrm{x}$. This has allowed imaging of injected hyperpolarised $13 \mathrm{C}$ labelled cell substrates in vivo and, more importantly, their metabolic conversion into other metabolites. Exchange of hyperpolarised $13 \mathrm{C}$ label between lactate and pyruvate has been shown to decrease post-treatment and hyperpolarised [1.4-13C] fumarate has been shown to detect tumour cell necrosis. Tumour $\mathrm{pH}$ can be imaged using hyperpolarised $\mathrm{H}_{13} \mathrm{CO}_{3}^{-}$and redox state can be determined by monitoring the oxidation and reduction of [1-13C] ascorbate and [1-13C] dehydroascorbate, respectively. Tumour glycolysis can be monitored by measuring the conversion of hyperpolarised [U-2H, $\mathrm{U}-13 \mathrm{C}]$ glucose to lactate and this flux was shown to decrease post-treatment. This new technique, which has recently transitioned to the clinic, has provided new ways of investigating tumour metabolism and the tumour microenvironment and for detecting treatment response. Learning Objectives:

1. To learn about metabolic imaging using hyperpolarised $13 \mathrm{C}$ MR spectroscopic imaging and to appreciate its limitations with respect to the short half-life of the polarisation.

2. To understand how this technique can be used to study tumour metabolism.

3. To become familiar with applications of the technique to monitor tumour responses to treatment.

4. To consolidate knowledge of this technique in relation to other imaging methods for imaging tumour metabolism and for detecting treatment response. Author Disclosure:

K. Brindle: Patent Holder; A number of patents held jointly with GE Healthcare on the use of hyperpolarised $13 \mathrm{C}$ magnetic resonance spectroscopic imaging. Research/Grant Support; Research agreement with GE Healthcare covering the development of hyperpolarised $13 \mathrm{C}$ magnetic resonance spectroscopic imaging in oncology.

Panel discussion:

Cancer hallmarks as therapeutic targets $\quad 09: 50$

08:30 - 10:00 Room E2

\section{Foundation Course: Breast Imaging}

\section{$E^{3} 420 b$}

\section{Mammography}

Moderator:

P. Skaane; Oslo/NO

A-077 08:30

A. Digital mammography: technique and quality assurance

M.G. Wallis; Cambridge/UK (matthewwallis492@btinternet.com)

Mammography technique from the point of view of radiographic compression and positioning is no different from film screening and radiologists ignore this at our peril. We will discuss the danger areas at the edges and the back of the breast and help you to find lesions only seen on one view. With digital capture the technologist can view images immediately, but the temptation is for them to repeat exposures to obtain radiographic perfection on otherwise clinically acceptable images. However, the task of assessing quality cannot be left to them, as viewing conditions on the 'lower resolution' acquisition monitor in a bright $x$-ray room is not enough. There is now evidence from 2 population screening programmes that poor image quality can reduce cancer detection from between $15 \%$ in France to $24 \%$ in Ontario. 'Quality' can be lost at 3 keys points in the imaging chain: the x-ray beam and dose, the detector and finally display. In Flanders, poor detector performance was compensated for by increasing the $\mathrm{x}$-ray dose by $60 \%$ and by meticulous quality control. Postprocessing is less well understood, but reader studies now show different algorithms will affect performance particularly for the detection and classification of micro-calcification. Finally, it does not matter how good all the equipment is if we try and read the images with the lights on full.

Learning Objectives:

1. To become familiar with basic digital mammography techniques.

2. To appreciate radio-anatomical considerations

3. To understand what can go wrong if the quality is not adequate.

\author{
A-078 09:00 \\ B. Tomosynthesis and contrast-enhanced digital mammography \\ J. Barkhausen; Lübeck/DE (joerg.barkhausen@uksh.de)
}

Mammography is the most important breast imaging technique allowing the visualisation of masses and micro-calcifications. However, in conventional mammography the three-dimensional breast tissue is reduced to a twodimensional image. Therefore, small lesions may be undetectable due to superimposed glandular tissue. Digital breast tomosynthesis (DBT) has emerged as a new imaging modality to overcome this limitation. Several lowdose mammographic projections are performed over a limited angle of up to 50 degree using a standard mammography system. The average glandular dose of a tomosynthesis scan is somewhat higher compared to one-view mammography, but lower compared to a standard mammography in two projections. Based on the low-dose projections, a stack of cross-sectional images covering the entire breast is reconstructed with an interslice distance of about $1 \mathrm{~mm}$. Several studies have shown that tomosynthesis improves both tumour detection and the characterisation of focal masses. Due to the crosssectional nature of DBT, the techniques allow on the one hand the reliable differentiation of true focal masses from summation artefacts. On the other hand, tomosynthesis improves the detailed analysis of the lesions' border to differentiate benign lesions and carcinomas. Additionally, contrast-enhanced spectral tomosynthesis may allow analysing signal-intensity time curves. In conclusion, DBT adds important information to standard two-projection mammography and can replace spot compression and rolled views as problem solver for difficult cases. However, the impact of tomosynthesis on breast cancer screening has to be investigated in larger clinical trials.

Learning Objectives:

1. To understand the implications of switching to $3 \mathrm{D}$ (technique, dose, reading).

2. To become familiar with the pros and cons of $2 \mathrm{D} v \mathrm{vs}$. $3 \mathrm{D}$ reading.

3. To learn about the effects of digital tomosynthesis on productivity.

\section{A-079 09:30 \\ C. Screening mammography \\ E. Azavedo; Stockholm/SE (edward.azavedo@ki.se)}

Reading screening mammograms is not the same as reading diagnostic mammograms. The vast majority of women attending a screening programme are usually asymptomatic and do not have abnormalities associated with a malignancy. The task of a mammogram reader is to scan a mammogram and perceive any possible abnormalities that could be associated with a malignancy. In other words, we are talking about perception and not diagnosis of a finding. Examples will be shown about this crucial step. In modern population-based screening programmes, we use computerised reading and recording systems. Since the work flow is rather quick, we have to ensure basic steps such as identity control and see that both RIS and PACS are synchronised so that decisions of screening readings are recorded in both the systems of one and the same patient. In computerised screening programmes, we use standard reports informing a woman that no signs of malignancy have been detected on her screening mammograms. In case of findings that need further assessment, there are several ways to deal with this situation. The most common way is via a phone call, but this can also be done with a letter. A screening report should be short and concise and should only inform whether we see signs of a possible abnormality that can be associated with a malignancy. Extensive reports describing the contents of a breast or findings of no clinical relevance should not be part of a screening report.

Learning Objectives:

1. To understand the basics of human vision.

2. To become familiar with the key components of a computerised

mammography reading system.

3. To learn about reporting strategies. 
$08: 30-10: 00$

Room F1

Joint Course of ESR and RSNA (Radiological Society

of North America): Emergency Radiology

MC 422

\section{General principles: paediatric and ENT emergencies \\ Moderators:}

S.E. Mirvis; Baltimore, MD/US

A. Palko; Szeged/HU

\section{A-080 08:30}

A. General principles

U. Linsenmaier, S. Wirth; Munich/DE

(Ulrich.Linsenmaier@kliniken-pasing-perlach.de)

Emergency radiology services are very much on the rise in the last decade. Medical emergencies and trauma are of enormous importance and a leading cause of death in all age groups. The use of radiological imaging in emergency departments showed an exponential increase since the 1990s. The annual growth rate in CT is $5-15 \%$ per year depending on the institution. Even for advanced level $3^{\circ}$ medical centres running an own emergency radiology unit, it is a challenge to integrate advanced radiology services in an interdisciplinary team treating patients with acute traumatic and non-traumatic emergencies. General principles: radiology and radiological imaging procedures should be integrated in an interdisciplinary team. Imaging should be guided by interdisciplinary clinical algorithms or guidelines (e.g. NEXUS or Canadian Cspine Rule). Many clinical diagnoses relay today on a early and thorough initial radiological diagnostic workup - mostly based on MDCT. Emergency radiology (ER) is still a relatively young subspecialty in European radiology - it deserves specific training. The lecture will cover: the development of emergency radiology and its future perspectives; the use of conventional radiography, ultrasound and MDCT; logistics and management of the patient; clinical guidelines and MDCT in the primary patient survey (e.g. using ATLS for trauma) and advanced scanning protocols for MDCT.

Learning Objectives:

1. To learn about general principles of diagnostic imaging in emergency radiology in traumatic and non-traumatic emergencies.

2. To understand the etiology, background and management of common radiological emergencies

3. To appreciate the role, indications and protocols for US, CR, MDCT in modern emergency radiology.

\section{A-081 09:00}

B. Challenges of imaging paediatric abdominal emergencies

S.D. John; Houston, TX/US (Susan.D.John@uth.tmc.edu)

Infants and children with abdominal emergencies present special diagnostic challenges because of similarities in clinical findings between various paediatric conditions. Imaging is frequently a critical part of the diagnostic process, but the choice of the best imaging modality can be controversial. Patient age and safety issues such as radiation exposure must be taken into consideration when planning imaging. Because certain pathologies are more likely to occur in specific age groups, patient age is a primary determinant of the imaging approach. This presentation will illustrate the differences in pathology between several paediatric age groups and discuss effective imaging algorithms for congenital and developmental, inflammatory, and obstructive conditions. The optimal use of radiographs, ultrasound, CT, and MRI will be discussed. Pitfalls that should be avoided in ultrasound and CT will be emphasised

Learning Objectives:

1. To learn how to plan safe and effective imaging strategies for evaluating abdominal pain in infants and children.

2. To understand the common and unusual causes of abdominal pain in different paediatric age groups.

3. To become familiar with potentially confusing variations in the appearance of these conditions with imaging.

\section{A-082 09:30}

\section{Imaging in ENT emergencies}

D. Nunez; New Haven, CT/US (Diego.Nunez@ynhh.org)

Non-traumatic emergent conditions involving the ear, nose and throat comprise a variety of disease entities, some of which may become life threatening if not readily recognised and treated. They typically present as bleeding, or as difficulty in breathing or swallowing. Occasionally, imaging studies may aid in the appropriate localisation of airway obstructing foreign bodies or in the diagnosis and treatment of epistaxis by guided interventional procedures. It is however in the assessment of head and neck infections and their complications that cross-sectional imaging plays a fundamental role. Facial and cervical infections are common clinical problems and although a presumptive diagnosis can be made clinically, imaging studies are often requested to confirm the diagnosis, to localise the infectious process and importantly to exclude the possibility of abscess formation or other complications. The contribution of imaging becomes more relevant in patients with clinical suspicion of deep neck infection where access to appropriate clinical evaluation may be limited. This presentation will analyse the imaging findings of head and neck infections using a systematic a spatial approach, as well as the indications for emergent CT and MR when necessary. A variety of complications will be discussed including intracranial extension of disease, airway compromise, vascular lesions and osseous involvement. Learning Objectives:

1. To understand imaging findings in patients presenting with acute head and neck conditions using a systematic spatial approach.

2. To get an understanding of the role and indications of CT and MR in acute non-traumatic ENT case management.

3. To learn how to identify the extent of disease and recognise specific complications of cervicofacial infections.

08:30 - 10:00

Room F2

\section{Radiographers}

\section{RC 414}

\section{Child protection issues: raising awareness among medical imaging professionals}

\author{
A-083 08:30 \\ Chairman's introduction \\ J. McNulty; Dublin/IE (jonathan.mcnulty@ucd.ie)
}

In diagnostic imaging, we tend to focus on radiation protection, justification and optimisation, as the primary opportunity for radiographers and radiologists to ensure the safety of patients, both adults and children; however, this ignores some other important issues. This session aims to raise awareness amongst medical imaging professionals of some key considerations in relation to a number of child protection issues. When imaging a child in case of suspected non-accidental injury, every detail of that examination from conversations with the child and their parents or guardians, technical aspects of the examination, observations during the examination and the language used following the examination need to be carefully considered. Another area of medical imaging requiring attention is how we communicate any radiation risks associated with examinations or procedures with children and their parents. There is evidence that while consent processes for invasive procedures are usually in place, these often lack focus on the risks associated with a high exposure to ionising radiation. These two medico-legal areas, suspected non-accidental injury and risk communication, are commonly encountered by radiographers and radiologists and as such it is important to make sure that we operate at the highest possible professional standards and in keeping with both regulations and best practice including the production of witness statements/contemporaneous notes. On rare occasions, we may find ourselves involved in legal proceedings as a witness or expert witness and it is thus important that we are all aware of the key considerations in this regard. Session Objectives:

1. To appreciate the need for radiographers to focus on more than just the imaging procedure in paediatric examinations.

2. To understand the extremely vulnerable nature of paediatric patients and the need for all health professionals to pay particular attention to this.

\section{A-084 08:35}

A. Professional responsibilities: an international perspective M. Davis; Dublin/IE (michaela.davis@ucd.ie)

Radiographers play a vital role in imaging children with suspected nonaccidental Injuries. This session will also explore the wider role that radiographers have in child protection. Current legislation and its implications will be explored from a variety of countries including those outside Europe. In addition, case studies will be presented focussing upon child protection issues that have arisen in the x-ray department during imaging procedures. Several of the barriers to radiographers becoming involved in child protection will be explored. Radiographers and their contribution to child protection will be discussed and practical examples given. 


\section{Postgraduate Educational Programme}

Learning Objectives:

1. To become familiar with current legislation and guidelines which have particular relevance to radiographers from an international perspective.

2. To appreciate best practice in child protection and the need for all radiographers to be aware of this.

3. To learn about child protection case reports involving imaging, radiologists and radiographers.

\section{A-085 08:58}

\section{B. Risk communication in paediatric examinations}

J.L. Portelli; Msida/MT (jonathan.portelli@um.edu.mt)

Medical imaging plays a vital role in the diagnosis and treatment of numerous medical conditions for millions of patients worldwide. However since most medical imaging examinations utilise ionising radiation, the associated risks of radiation exposure also need to be acknowledged, especially since high doses of radiation are known to cause adverse biological effects. Medical imaging professionals should have a good understanding of the benefits and risks associated with the imaging examinations they perform, so as to ensure a high benefit-risk ratio for all patients undergoing diagnostic imaging procedures, and to be able to appropriately communicate such information to patients, their families and other healthcare professionals. This may be particularly more important when imaging paediatric patients, who are relatively more susceptible to the effects of radiation and receive a higher effective dose per unit of radiation when compared with adults undergoing the same medical imaging examination. Indeed, following worldwide media attention about radiation incidents and their adverse effects, some parents/legal guardians may be concerned and reluctant to pursue important medical imaging examinations requested for their child, as they may have a misconception of the risks involved. In this regard, this lecture will seek to enhance awareness about the radiation doses associated with common paediatric imaging examinations; highlight the importance of medical imaging professionals having an appropriate understanding of benefits and risks associated with these examinations; and encourage such professionals to improve patient care by making use of this knowledge to have better discussions with referring clinicians, patients and their families.

Learning Objectives:

1. To appreciate the frequency and radiation dose associated with certain paediatric imaging examinations.

2. To become familiar with the current status quo in the communication of radiation dose-related risks.

3. To understand best practice and potential considerations in providing accurate information to referring clinicians, patients and their families.

\section{A-086 09:21}

C. What to do if you find yourself being called to give evidence M.D. Viner; London/UK

Radiology is a powerful tool in the investigation of cases of suspected nonaccidental injury and suspicious death in children. The evidence obtained can be pivotal in bringing successful prosecutions against suspects and protecting the child and others from further harm. It is essential that evidence is obtained, recorded and presented in accordance with applicable rules of evidence in order to be admissible in court. It is thus important that those undertaking such examinations are appropriately trained, conversant with standards of evidence and maintain continuity of evidence throughout the investigation. This presentation will review rules of evidence for cases of suspected child abuse and their application to imaging examinations. It will examine the role of the radiographer and the importance of training and familiarity with legislation and guidelines that underpin forensic practice, highlighting the pitfalls that lawyers may exploit if procedures have not been followed. Drawing on examples, it will discuss best practice from the radiographer's perspective, detailing the importance of continuity of evidence and maintenance of contemporaneous notes and witness statements. It will address key considerations for radiographers and others who provide evidence or act as an expert witness. Imaging professionals play a pivotal role in the investigation of suspected child abuse. It is essential that this evidence is obtained, recorded and presented with regard to the applicable standards of evidence. For those called to give evidence, the importance of appropriate imaging protocols and procedures, detailed documentation and thorough preparation cannot be understated. Learning Objectives:

1. To understand the importance of contemporaneous notes and witness statements if involved in any forensic or suspected non-accidental injury examination.

2. To become familiar with the importance of continuity of evidence in forensic examinations.

3. To appreciate the key considerations if you are asked to provide evidence or act as an expert witness in court.

\author{
Panel discussion: \\ Is there a need for further education and training for radiologists and \\ radiographers? \\ 09:44
}

\begin{tabular}{l}
\hline 08:30 - 10:00 \\
\hline Neuro \\
RC 411 \\
The paediatric brain: not just a small brain \\
Moderator:
\end{tabular}

N. Girard; Marseille/FR

A-087 08:30
A. Neurocutaneous syndromes: more than neurofibromatosis
B. Ertl-Wagner; Munich/DE (Birgit.Ertl-Wagner@med.uni-muenchen.de)

Neurofibromatosis type 1 is the most common, but certainly not the only neurocutaneous syndrome. In children, white matter signal alterations are a common neuroimaging finding. The incidence of optic gliomas and other gliomas has increased. Neurofibromas can occur in almost any body region. Plexiform neurofibromas show a diffuse and often locally destructive growth. Neurofibrosarcomas are malignant nerve sheath tumours. Neurofibromatosis type 2 is characterised by "acoustic neuromas" (mostly vestibular schwannomas), which can be uni- or bilateral. Schwannomas can also affect other cranial nerves and spinal nerves. The incidence of meningiomas and to a lesser extent of gliomas is also increased. The hallmark feature of tuberous sclerosis is the combination of cortical or subcortical tubers and subependymal nodules. Tubers are hyperintense on $\mathrm{T} 2 \mathrm{w}$ and FLAIR sequences. Subependymal nodules are NOT isointense to grey matter (vs. subependymal heterotopias). Giant cell astrocytomas are located at the foramina of Monroi, increase in size over time and show a contrast enhancement. Sturge-Weber disease (encephalotrigeminal angiomatosis) is characterised by a leptomeningeal angioma and facial naevus flammeus. There is increased leptomeningeal enhancement and hypertrophy of the ipsilateral choroid plexus. Atrophy of the affected region and tramtrack calcifications typically ensue. Von Hippel-Lindau disease is not a neurocutaneous syndrome per se, but classically considered a phakomatosis. Typical features include haemangioblastomas of the brain, spinal cord or retina, and papillary cystadenomas of the endolymphatic sac. Most neurocutaneous syndromes have manifestations outside the central and peripheral nervous systems as well. Moreover, there are multiple rare neurocutaneous syndromes. Learning Objectives:

1. To become familiar with the typical clinical presentations of neurocutaneous syndromes.

2. To consolidate knowledge of the typical imaging patterns of the major neurocutaneous syndromes.

3. To become familiar with some less common features of neurocutaneous syndromes.

\section{A-088 09:00}

B. Patterns of white matter disease in children

A. Rossi; Genoa/IT (andrearossi@ospedale-gaslini.ge.it)

Pediatric white matter disorders can be distinguished into well-defined leukoencephalopathies and undefined leukoencephalopathies. The first category may be subdivided into: (a) hypomyelinating disorders; (b) dysmyelinating disorders; (c) leukodystrophies; (d) disorders related to cystic degeneration of myelin; and (e) disorders secondary to axonal damage. The second category, representing up to $50 \%$ of leukoencephalopathies in childhood, requires a multidisciplinary approach in order to define novel homogeneous subgroups of patients, possibly representing '"new genetic disorders". An integrated description of the clinical, neuroimaging, and pathophysiological features is crucial for categorising myelin disorders and better understanding their genetic basis. A review of the MR imaging findings in the main genetic disorders affecting white matter in the pediatric age, including some novel entities, will be provided with a highlight on the concept of pattern recognition.

Learning Objectives:

1. To become familiar with the most common white matter diseases in children.

2. To learn how to differentiate between white matter diseases in children.

3. To consolidate knowledge of appropriate imaging protocols for MRI of

children with white matter disease. 
A-089 09:30

\section{Paediatric brain tumours}

M. Bekiesinska-Figatowska; Warsaw/PL (m.figatowska@gmail.com)

Brain tumours are the second most common neoplasms in children after leukaemia. As "paediatric brain is not just a small brain", paediatric brain tumours are not the same as tumours in adults. MRI is a method of choice in their diagnosis in accordance with the principle ALARA in the paediatric population. By the age of approximately 3 years, supratentorial tumours dominate; later (4-10 years of age), the majority of children show infratentorial neoplasms which are pilocytic astrocytomas, most often found in the hemispheres, and medulloblastomas or ependymomas - in the fourth ventricle. After the age of 10 , both groups are diagnosed with equal frequency. Cerebral hemispheric tumours include astrocytoma which is the most frequent, teratoma in neonates, and in older children atypical teratoid/rhabdoid tumour, ependymoma, PNET, ganglioglioma and DNET. In the midline, we deal with chiasmatic or hypothalamic glioma, craniopharyngioma and germ cell tumours of the pineal region. Extraparenchymal tumours are uncommon in the paediatric population. Apart from the location, imaging pattern in various sequences, and contrast enhancement of the tumour, advanced MRI techniques are helpful in establishing the diagnosis. DWI helps to differentiate between tumours of high and low cellularity showing low ADC values in case of medulloblastoma and high in astrocytoma. MRS, PWI and DTI also add important information to the diagnosis. MRI of the spinal canal is obligatory in case of these primary brain neoplasms that have a high propensity to metastasise to other parts of the central nervous system such as medulloblastomas, ependymomas, high-grade gliomas, cerebral neuroblastomas, and pineal region neoplasms.

Learning Objectives:

1. To learn about the difference between paediatric and adult brain tumours.

2. To understand the imaging strategy for the paediatric population.

3. To become familiar with the most common paediatric brain tumours.

08:30 - 10:00

Room I/K

Multidisciplinary Session: Managing Patients with Cancer

\section{MS 4}

\section{Pancreatic neuro-endocrine tumours}

\section{A-090 08:30}

\section{Chairman's introduction}

R. Pozzi-Mucelli; Verona/IT (roberto.pozzimucelli@univr.it)

Pancreatic endocrine tumours (p-NETs) include both pancreatic neuroendocrine tumours associated with a functional syndrome (functional $p$ NETs) and those associated with no distinct clinical syndrome (non-functional p-NETs). Non-functional p-NETs may show immunohistochemical positivity for hormones which may be produced, but not secreted, which are clinically inert and whose serum concentrations are insufficient to induce symptoms. Among functional p-NET, the most common are gastrinomas and insulinomas, whereas those secreting vasoactive intestinal peptide, glucagon, somatostatin and other hormones are considered together as a group called rare functional p-NETs. Non-functional p-NETs are classified, according to the WHO 2010 classification, in well-differentiated neuroendocrine tumours and poorly differentiated neuroendocrine carcinomas (NECs) of small or large cell type. Well-differentiated neuroendocrine tumours are then divided according to a grading scheme based on mitotic count or Ki67 index in NETs-G1 (with a mitotic count $<2$ per 10 high-power fields (HPF) and/or $\leq 2 \% \mathrm{Ki} 67$ index), and NETs-G2 (with a mitotic count 2-20 per $10 \mathrm{HPF}$ and/or 3-20\% Ki67 index). All poorly differentiated neuroendocrine carcinomas are graded G3 (with a mitotic count $>20$ per $10 \mathrm{HPF}$ and/or $>20 \% \mathrm{Ki} 67$ index). Most pancreatic nonfunctional p-NETs are well-differentiated tumours, whereas poorly differentiated neuroendocrine carcinomas are uncommon.

Session Objectives:

1. To understand the biology, clinical presentation and recent classification of pancreatic NET.

2. To recognise the role of multimodality imaging in detecting, staging and characterising biological patterns of lesions.

3. To become familiar with the different treatment options.

\section{A-091 08:35}

\section{Radiologist's point of view}

\section{R. Pozzi-Mucelli; Verona/IT (roberto.pozzimucelli@univr.it)}

Pancreatic endocrine tumours ( $p-N E T$ ) are divided into functional p-NETs and non-functional p-NETs. Diagnostic imaging of these tumours includes detection, characterisation and staging. There are significant differences when dealing with functional p-NETs and non-functional p-NETs. In fact, in the first case the main aim of diagnostic imaging modalities is the detection of the tumour, while for non-functional p-NETs the main aim is the characterisation and the differential diagnosis with other tumours of the pancreas, mainly with pancreatic adenocarcinoma. Staging is also important for both tumours, but mainly for non-functional p-NETs. A large number of imaging modalities are available, both "morphological" and "functional". We can arbitrarily divide the imaging modalities for the diagnosis of p-NETs into three levels: the first level includes US (with contrast-enhanced US), MDCT, MRI and nuclear medicine techniques; the second, endoscopic US, angiography and venous sampling; the third, intra-operative US. Functional p-NETs are represented mainly by insulinomas and gastrinomas which are small in size in most cases. Their detection is still difficult, but significant improvements have been made with US, MDCT and MRI, so that these tumours are detected in most cases if appropriate imaging techniques are performed. However, still, no single modality is $100 \%$ effective. Non-functional p-NETs can be differentiated from pancreatic adenocarcinoma, since they are hypervascular and usually large in size. Since they are potentially malignant, they require accurate staging by imaging modalities, both morphological (MDCT, MRI) and functional (PET/CT) to plan surgery and chemotherapy.

Learning Objectives:

1. To learn how to recognise the specific imaging features of pancreatic NET and how to differentiate NET from other pancreatic tumours.

2. To understand the strengths and weaknesses of the different imaging modalities.

3. To learn about the optimal imaging protocol for the diagnosis, staging and follow-up of NETs.

\section{A-092 08:55}

\section{Surgeon's point of view}

C. Bassi; Verona/IT (claudio.bassi@univr.it)

There is worldwide consensus about surgical treatment of resectable p-NETs in the following clinical situations: proven malignancy, whenever it is possible to remove the primary tumour and at least $90 \%$ of liver metastases (debulking); p-Nets associated with a clinical syndrome caused by hormonal hypersecretion (insulinomas and gastrinomas mainly); non-functional p-NETs with a size larger than $2 \mathrm{~cm}$. Vice versa, there are different expert opinions about the surgical treatment of functional and non-functional p-NETs in MEN1 syndromes and in sporadic non-functional p-NETs less than $2 \mathrm{~cm}$ in size. In MEN1 all insulinomas should be resected, while for gastrinomas the extent of resection is patient tailored, deserving standard Whipple for selected cases. In the most common sporadic small incidentalomas, where long-term prognosis is favourable, surgical treatment has to be weighted with co-morbidities and morbidities of the patient and the procedure planned. These tumours have a risk of node metastases at the time of diagnosis in less than $10 \%$ of patients. Whenever possible, mini-invasive approach with pancreas-sparing operations should be recommended. In elderly patients, a first period of follow-up should be preferable and surgery should be offered only if the size of the tumour demonstrates increasing trends.

Learning Objectives:

1. To understand the rationale of surgical indication.

2. To consolidate knowledge on the resectability criteria and extension of the radical resection.

3. To learn about the role of surgery within multimodality treatment.

\section{A-093 09:15}

Oncologist's point of view

G. Tortora; Verona/IT (giampaolo.tortora@univr.it)

G1-G2 Pancreatic NETs show moderate responsiveness to chemotherapy. While single agents have limited activity in advanced pNETs, doublet containing streptozotocin with adriamycin or 5-fluorouracil are considered standard regimens, leading to RR from 6 to $36 \%$, median PFS from 3.9 to 16 months and OS of 20-24 months. Intensification with three drug regimens did not increase the efficacy. Recently, a combination of temozolomide and capecitabine showed $70 \%$ of partial remission, median PFS of 18 months and OS of $92 \%$ at 2 years. PI3K-Akt-mTOR is a key pathway in NETs. In advanced pNETs, the phase III registrative RADIANT-3 study randomised patients to the anti-mTOR everolimus versus placebo; pretreatment with chemotherapy was a stratification criteria and treatment with SSA was allowed. PFS, the primary end point was 11.4 months with everolimus versus 5.4 months in the placebo arm, with a reduction in the risk of progression of nearly $65 \%$. NETs are highly vascularised tumours and high VEGF expression is a negative prognostic factor in pancreatic NET, related to higher microvessel density, higher incidence of metastases and shorter PFS. A phase III registrative study randomised advanced pNET patients to receive the antiangiogenic drug sunitinib or placebo together with best supportive care. A statistically significant difference in PFS, the primary end point, was observed with everolimus $(11.4$ months) versus placebo (5.5 months), along with increased RR $(9.3 \%$ vs. $0 \%$, respectively). Recent studies have identified critical alterations in the signaling 
pathways of NETs that may be translated into new therapeutic strategies with novel targeted agents.

Learning Objectives:

1. To learn about the relationship between NET biology, signalling pathways and available therapeutics.

2. To understand current treatment options.

3. To learn how to contextualise the clinical management of NETs.

Case presentation and discussion

09:35

08:30 - 10:00 Room N/O

Interventional Radiology

RC 409

\section{Endovascular treatment of acute thoracic aorta syndrome}

\section{A-094 08:30}

\section{Chairman's introduction}

T. Pfammatter; Zurich/CH (thomas.pfammatter@usz.ch)

The "acute aortic syndrome" includes three closely related emergency entities of the thoracic aorta: aortic dissection, intramural hematoma and penetrating atherosclerotic ulcer. As these conditions present with similar symptoms, cross-sectional aortic imaging is essential for diagnosis, triage and treatment planning. Initial imaging features of these pathologies including typical early complications as well as their differential diagnosis will be reviewed. As nowadays several of these patients will undergo early endovascular thoracic aortic repair, an update on its indication, the different technical options and their clinical results will follow. Finally, the focus will be on repair-related late complications and progression of aortic disease. Imaging follow-up protocols, features of chronic complications and their interventional repair will be discussed.

\section{A-095 08:35}

A. Pre-therapeutic radiological evaluation

J. Ferda; Plzen/CZ (ferda@fnplzen.cz)

The acute thoracic aorta syndrome covers patients suffering from acute chest pain and those injured during high-energy trauma. Despite the similar symptoms, the aortic pathologies are heterogeneous, including acute aortic injury, intramural haematoma, aortic dissection, and ruptured thoracic aortic aneurysm and mycotic aneurysms. The diagnostic approaches could include the whole pallete of the imaging modalities: chest X-ray, transesopheageal echocardiography (TEE), multidetector CT angiography (MDCTA), MRI and $\mathrm{PET} / \mathrm{CT}$. Because of some disadvantages of TEE, like problems with intubation in injured patients or problems of MRI with seriously ill patient monitoring, MDCTA has become the most used modality due to its ability of discrimination between aortic and non-aortic pathologies, and also due to its high sensitivity, specificity, availability and especially superior geometrical resolution and potential of 3D imaging. The proper pre-therapeutic MDCTA protocol is using sub-millimeter isotropic resolution covering the entire aorta with pelvic arteries. When the pathology of thoracic aorta is found during the triple rule-out triage protocol, additional MDCTA of the rest of the aorta and pelvic arteries has to be completed. ECG triggering could be an important advantage in the reduction of pulsation-derived artefact. The volume of applied contrast materia used should be as small as possible to plan applications during endovascular treatment. 3D reconstruction of the thoracic aorta with its side branches and advanced vessel analysis have a crucial role in the measurement of the aortic diameter, diameter profile and lengths in the selection of proper stent graft type and size.

Learning Objectives:

1. To learn about the best pretreatment imaging modality.

2. To understand planning and sizing of appropriate stent-graft.

3. To become familiar with critical criteria and vessel morphology. Author Disclosure:

J. Ferda: Speaker; Invited speaker on Somatom World Summit organised by Siemens HealthCare 2013.

\section{A-096 08:58}

B. EVAR techniques and results

F. Fanelli; Rome/IT (fabrizio.fanelli@uniroma1.it)

Endovascular abdominal aortic aneurysm repair (EVAR) has gained acceptance as a minimally invasive alternative to open surgery in selected patients. Many technical improvements have been obtained in the last years with the introduction of new-generation devices. Presenting a lower profile, they can be accommodated even in patients with small vessels and tortuous anatomy. Calcified arteries can be no longer considered absolute exclusion criteria. Some devices are designed to reduce the incidence of endoleaks, improving sealing capabilities by the use of a polymer to better adjust to the variable morphology of the proximal neck or to fill the aneurysmatic sac completely. More importantly, the introduction of fenestrated devices has resolved the problem of facing difficult anatomies when the aneurismatic pathology involves renal or visceral arteries. Obviously, the use of these new devices requires a learning curve. Beyond an increase of cost, they permit treating those patients who in the past were considered unfit candidates for EVAR. The EVAR new frontier requires performing the procedure in an outpatient fashion. A multicentre study was conducted on 100 patients who were discharged $4 / 6$ hours after uncomplicated stent-graft implantation. Cases $(4 \%)$ with access vessel complications required additional procedures and patients were hospitalised overnight. The 30 -day readmission rate was $4 \%$ for access vessel stenosis or false aneurysm. From the time of the first EVAR procedure, significant improvements have been achieved but there is still room for further progress.

Learning Objectives:

1. To understand indications and contraindications for EVAR.

2. To learn more about the advantages of different types of stent-graft.

3. To become familiar with percutaneous vascular access.

4. To learn about the latest trials.

Author Disclosure:

F. Fanelli: Consultant; WL Gore \& Associates, Medtronic, Cook. Speaker; WL Gore \& Associates, Medtronic, Cook, Covidien.

A-097 09:21

C. Imaging follow-up and complications

R. Morgan; London/UK (robert.morgan@stgeorges.nhs.uk)

The standard imaging modality after endovascular treatment for thoracic aortic pathology (TEVR) is CT angiography (CTA). CTA enables the radiologist to assess for the patency of the endografts, the presence of any complications after TEVR, and the development of any new pathology related not only to the aorta, but elsewhere above and below the diaphragm. The role of MR is reduced compared with CTA, particularly in the presence of stainless steel endografts. However, good images can be obtained by MRI and MRA if nitino endografts have been inserted. MR is a useful modality for follow-up in young patients who require lifelong follow-up and, therefore, limited exposure to ionising radiation. Ultrasound has no practical role in the follow-up after TEVR, and although plain thoracic radiographs are routinely performed by some centres post-TEVR, in practice almost equivalent information can be obtained by $\mathrm{CT}$. The main complications after TEVR are endoleaks, type A dissection, paraplegia and stroke. The classification of endoleaks is similar to the classification for EVAR. Type 1 and type 3 endoleaks require treatment by additional endograft coverage with or without supraaortic or visceral artery bypass. Other treatment options include the use of branched, fenestrated endografts or the use of chimneys and snorkels. Type 2 endoleaks are less common than post-EVAR. Similar to the EVAR population, intervention is mandated for an enlarging aortic diameter and involves embolisation in most cases.

Learning Objectives:

1. To learn about available imaging methods for follow-up.

2. To become familiar with complications and failure of EVAR.

3. To learn how to treat endoleaks.

Panel discussion:

What are the best imaging methods for follow-up? 09:44

08:30 - 10:00

Conf. Room M3

Professional Challenges Session

PC 4

Interventional radiology in oncology

A-098 08:30

Chairman's introduction

A. Adam; London/UK (andy.adam@kcl.ac.uk)

Interventional oncology is a discipline that uses imaging-guided procedures in the treatment of cancer patients. When the malignant disease in not curable, interventional techniques such as biliary and oesophageal stenting can provide useful palliation and improve the patient's quality of life. In patients with potentially curable tumours, thermal ablation, cryotherapy and radioembolisation provide useful alternatives to radiotherapy and surgery in carefully selected patients. Interventional radiologists who treat patients with cancer should practise as clinicians, participating in multidisciplinary meetings 
where treatment choices can be discussed with specialists from other oncological disciplines. They should involve themselves in all stages of clinical practice, including pre-treatment consultations, ward rounds and patient follow-up. Radiation oncology and interventional oncology have much in common, including the potential for curing cancer using minimally invasive techniques. Interventional oncologists can usefully adopt many of the principles and methods of practice in radiation oncology, including quality assurance and the collection of robust outcome measures. An alliance between these disciplines would be beneficial to clinical practice and research in both disciplines.

Session Objectives:

1. To understand the contribution of interventional radiology to oncology.

2. To appreciate the areas of overlap between radiation oncology and interventional oncology

3. To understand the need for a clinical pattern of practice in interventional radiology in oncology.

\section{A-099 08:35}

The current scope and future challenges

J.I. Bilbao; Pamplona/ES (jibilbao@unav.es)

Together with chemotherapy, surgery and radiotherapy, interventional radiology (IR) is the fourth pillar in oncology. Transcutaneous and endovascular procedures, guided by imaging, constitute a fundamental knowledge essential for the multidisciplinary management of oncologic patients. The range is wide and covers most of the needs in patient's care. In short: obtaining samples for making an accurate diagnosis and also to know more about its specific biology (biomarkers); IR covers any possibility needed for vascular access; percutaneous needle ablation is the best alternative in selected patients; endovascular embolisation with "vehiculisation" of therapies is an outstanding method for selective treatment, and sometimes precise ablation, of different tumors; IR is a unique way to offer palliation in a wide range of tumoral complications, such as embolisation for bleeding, stenting for vein obstructions or drainage of fluid collections. There is an obvious need of continuous technical refinement. Image-guided therapy is a continuous process that implies many disciplines and that includes every step, from staging through guidance of the procedure to the evaluation of the outcomes, both immediately, during and in the follow-up. Future developments in IR may be focused towards the personalisation of therapies to each patient and to a better understanding of the biological mechanisms of tumour response or progression and their image expression. Finally, radiologists must be actively involved in the whole process of clinical trials, from the creation of new lines to the performance of therapy until the evaluation and diffusion of the data. Learning Objectives:

1. To learn about the range of interventional radiological procedures in the field of oncology.

2. To appreciate the importance of technology and imaging in interventional oncology.

3. To learn about likely future developments in interventional oncology.

Author Disclosure:

J.I. Bilbao: Speaker; Sirtex Medical Europe.

\section{A-100 08:58}

How an oncologist works and why technology alone is not enough

L.M. Kenny; Brisbane/AU (lizkenny@bigpond.net.au)

Radiation treatment has a fundamental role in the multidisciplinary management of people with cancer. The evidence base for radiation therapy is robust and approximately $50 \%$ of people diagnosed with cancer will benefit from radiation treatment. Most of this benefit comes from an increase in cure. The technical quality of delivery of radiation treatment is of great importance in avoiding a geographic miss and also in reducing the amount of normal tissue being irradiated unnecessarily. As well as the excellent technical delivery of any treatment offered, of paramount importance is the intelligent integration of care with attention to evidence base choices for both local and systemic treatment. Multidisciplinary teams are in the best position to integrate surgery, radiation treatment, interventional oncology and systemic therapy in a planned and deliberate fashion. The members of the team need to understand what each has to offer and what choices are available. The full delivery of multidisciplinary care requires input from nursing and allied health. Great technical care, although critical, is insufficient. Caring for people with cancer requires caring for them as a person and being a competent cancer clinician accepting full clinical responsibility before during and after care. Without this, any member of the team is but a technician and the full potential of what they have to offer is likely to be unrealised.

Learning Objectives:

1. To learn about the role of radiation oncology in the local treatment of tumours.

2. To understand the main elements of oncological practice.

3. To understand the importance of primary clinical responsibility in the care of patients with cancer.

\section{A-101 09:21}

Best practice: how to organise an interventional oncology unit A. Gangi, J. Garnon, G. Tsoumakidou; Strasbourg/FR (gangi@unistra.fr)

Interventional oncology is a fast growing field. Interventional oncology is a clinical and technical speciality. Solid clinical knowledge associated with the technical skills is mandatory for a successful practice. The technical skills should be associated to the clinical part. The knowledge of all treatment modalities and the participation of a multidisciplinary team is vital for the best practice. Every single patient should be seen by the interventional oncologist and followed-up. The interventional oncologist is part of a multidisciplinary team, taking care of the patient. The team includes oncologists, radiotherapists, pain specialists, psychologists, surgeons, and anesthesiologists. The interventional oncology unit should include specialised nurses, technician, anesthesiologist, anesthesiologist nurses, and the interventional radiologist. The team should be able to take care of the patient's follow-up and eventual complications. To summarise, high-quality interventional oncology services are essential for safe and effective patient care. The technical skills of the interventional oncologist should be associated with an excellent clinical knowledge of the disease. A multidisciplinary team work is mandatory for a successful interventional team. Harmonisation of training and appropriate credentialing is required for clinical services of the best quality. Interventional radiologists continuously improving their skills and knowledge, patient access to safe and effective minimally invasive treatment options is increasing.

Learning Objectives:

1. To learn how to organise an interventional oncology unit.

2. To understand the clinical role of an interventional oncologist.

3. To learn how interventional oncologists collaborate with other specialists caring for patients with cancer.

Author Disclosure:

A. Gangi: Other; Proctoring Galil Medical.

Panel discussion:

Interventional radiology as a new link between oncology

and imaging

09:44

08:30 - 10:00

Board Room A

Special Focus Session

SF 4

\section{Right heart morphology and function}

A-102 08:30

Chairmen's introduction

M. Remy-Jardin ${ }^{1}$, M. Gutberlet ${ }^{2} ;{ }^{1}$ Lille/FR, ${ }^{2}$ Leipzig/DE

Whereas cardiac $\mathrm{CT}$ and $\mathrm{MRI}$ are routine examinations for specialised radiologists, it has been necessary to wait for the introduction of fast scanning techniques to modify the behavior of nonspecialised radiologists regarding the interpretation of cardiac cavities. The objective of this session is twofold. First, cardiac radiologists will underline the role of these imaging modalities in the assessment of congenital heart disease but also in ischaemic and nonischaemic cardiomyopathies. In the second part of this session, chest radiologists will focus on the diagnostic information accessible at the level of the right ventricle, known to be the target of numerous respiratory disorders. Two practical situations will illustrate this approach: pulmonary thromboembolic and chronic respiratory diseases.

Session Objectives:

1. To make the attendees familiar with the impact of RV dysfunction in various cardiac and respiratory disorders.

2. To describe basic anatomical and functional information that can be deduced from routine cross-sectional imaging.

3. To underline the pros and cons of dedicated cardiac examinations and those carried out using cardiothoracic imaging.

A-103 08:35

Role of cardiac CT and MRI in assessment of RV in congenital heart disease

A.M. Taylor; London/UK (a.taylor76@ucl.ac.uk)

Accurate assessment of the right ventricle (RV) is crucial for the management of congenital heart disease. Echocardiography can provide some information, but as the RV sits directly behind the sternum and as many patients have undergone multiple operations, visualisation can be difficult. Furthermore, for long-term follow-up and treatment planning, accurate measurement of ventricular size and mass, and valvular flow (tricuspid and pulmonary), and assessment of anatomy in 3D and 4D are vital. This presentation will outline 
the role of cross-sectional MRI and CT for assessing the RV, and describing the indications, imaging protocols, technical pitfalls and relevant information for treatment planning.

Learning Objectives:

1. To become familiar with the most frequent congenital heart diseases (CHD) in which the RV is involved.

2. To learn about the most frequent surgical procedures in CHD with right heart involvement.

3. To understand which modality to choose and for which CHD.

Author Disclosure:

A.M. Taylor: Employee; UCL Institute of Cardiovascular Science. Grant Recipient; Siemens, UK NIHR, BHF, Heart Research UK,. Research/Grant

Support; Siemens - Research agreement.

\section{A-104 08:53}

Right heart failure in ischaemic and non-ischaemic cardiomyopathies

M. Grothoff; Leipzig/DE (Matthias.Grothoff@herzzentrum-leipzig.de)

The role of the right ventricle (RV) has been underestimated for many years. Today, we know that the RV is of importance for keeping up a sufficient circulation and that right heart failure in both ischemic and non-ischemic cardiomyopathies can result in hemodynamic compromise. RV ischemia has a higher in-hospital morbidity and mortality but post-infarction chronic right heart failure is a rare finding. As the RV has a different physiology from the left ventricle (LV), also the pathophysiology of ischemia is different here. Using our standard CMR tools T2 weighted imaging for detection of edema and late gadolinium enhancement imaging for detection of necrosis/scar we can find that the rate of myocardial salvage is much higher in the RV than in the LV and that RV failure in the acute phase is basically caused by reversible ischemic effects. In non-ischemic cardiomyopathies like HCM and DCM changes in the RV myocardium mostly resemble changes of the LV. In arrhythmogenic right ventricular cardiomyopathy/dysplasia (ARVC/D) however, there are specific findings in the RV and the role of imaging in diagnosing ARVC has been strengthened by the revised task force criteria published in 2010. Cine imaging should be performed carefully for detection of RV akinesia, dyskinesia or small aneurysms as well as the planimetry of the RV. Findings like the detection of fibrosis in LGE imaging and the detection of fat in T1 imaging can provide additional information, but are not diagnostic for ARVC.

Learning Objectives:

1. To learn about the different $\mathrm{CT}$ and MRI techniques for evaluating right heart morphology and function.

2. To understand the prognostic impact of right heart involvement in ischaemic heart disease.

3. To learn about the basic morphological and functional features of different non-ischaemic cardiomyopathies

\section{A-105 09:11}

Right heart and chronic respiratory diseases: can CT be used as a onestop-shop?

M. Remy-Jardin; Lille/FR (martine.remy@chru-lille.fr)

Over the last decade, CT technology has evolved towards fast scanning capabilities and high temporal resolution, enabling improved evaluation of the heart and surrounding structures in the course of routine thoracic CT imaging This has progressively modified the radiologist's implication in the interpretation of chest CT examinations. Firstly, it is more and more common to integrate the physiological interactions between heart and lung when analysing the CT features depicted on a given examination. Secondly, it is now possible to integrate cardiac functional information into a diagnostic CT examination of the chest, providing prognostic information in the management of patients with a wide variety of chronic respiratory disorders. The purpose of this presentation is to provide non-specialised radiologists with some practical guidelines in the choice of the scanning protocols and range of functional information that can be deducted from chest CT examinations. Right ventricular dysfunction and levels of pressure within the pulmonary circulation are the key points of such approaches.

Learning Objectives:

1. To appreciate the clinical impact of a cardiothoracic evaluation from the same CT examination.

2. To learn about the scanning protocols enabling such a combined analysis

3. To understand its compatibility with other non-invasive modalities.

\section{A-106 09:29}

\section{RV and prognosis in pulmonary thromboembolic disease}

N.J. Screaton; Cambridge/UK (Nicholas.Screaton@papworth.nhs.uk)

Right ventricular failure is the most common cause of early death following acute pulmonary embolism and in patients with chronic thromboembolic pulmonary hypertension. Echocardiography is sensitive in diagnosing and quantifying RV dysfunction. CT is the reference standard for the diagnosis of acute $\mathrm{PE}$ diagnosis and has the potential to provide comprehensive evaluation of the pulmonary arteries, heart and lung parenchyma. CT signs of right ventricular dysfunction which have shown predictive value for adverse outcome include: flattening of the inter-ventricular septum or bowing towards the left ventricle, reflux of contrast medium into the IVC, relative RV/LV diameter ratios on transverse and 4-chamber reconstructions, and ventricular volumetry as assessed both on routine non-gated CTPA and ECG-gated CT. In patients with $\mathrm{CTEPH}, \mathrm{MRI}$ permits imaging of the pulmonary vasculature and functional evaluation of the right ventricle and pulmonary circulation, enabling both morphological assessment and prognostic evaluation.

Learning Objectives:

1. To learn about clinical and imaging factors associated with prognosis of thromboembolic disease.

2. To understand the benefits and weaknesses of different imaging modalities in predicting prognosis in thromboembolism.

3. To discuss the merits of comprehensive imaging evaluation in the routine diagnostic workup of suspected thromboembolic disease.

Panel discussion:

What is the impact of RV function assessment

on patient management? $\quad 09: 47$

08:30 - 10:00

Room P

Joint Session of the ESR and EFSUMB

\section{Advances in diagnostic ultrasound: better results through combinations}

Moderators:

L.E. Derchi; Genoa/IT

C.F. Dietrich; Bad Mergentheim/DE

A-107 08:30

US/MRI fusion to guide prostate biopsies

F.M. Drudi; Rome/IT (FrancescoM.Drudi@uniroma1.it)

Patients should undergo 1.5-3 Tesla MRI using a 6-16 channel surface coil placed over the pelvis or with an endorectal coil. To rperform MRI/TRUS image fusion-guided prostate biopsy, MRI sequences should include tri-planar T2weighted imaging, axial diffusion-weighted imaging with apparent diffusion coefficient mapping, 3D point resolved spatially localised spectroscopy and dynamic contrast-enhanced axial MRI. MRI/TRUS biopsy is performed with electromagnetic tracking of suspicious lesions identified on MRI. An electromagnetic field generator is placed above the pelvis to allow real-time tracking using a probe with a passive electromagnetic tracking sensor. 2D TRUS transverse images of the prostate are recorded to render a 3D US image which can be fused with MRI. Suspicious areas identified on MRI are imported and manually or semi-automatically fused with the US data set. This allows lesions marked on MRI scans to be superimposed on the real-time TRUS image. Electromagnetic tracking allows localisation and mapping of needle trajectories in both random and subsequent targeted biopsies Indications for MRI/TRUS image fusion-guided biopsy include screening of patients at increased risk due to age, elevated PSA blood level and clinical suspicion of prostate cancer. Moreover, this diagnostic method is particularly useful in patients with negative TRUS outcome, as MRI/TRUS fusion permits targeted biopsy of suspicious areas which might have been missed at TRUS. Learning Objectives:

1. To learn about the technologies needed to fuse US/MRI of the prostate.

2. To learn how to perform prostate biopsies with US/MRI fusion image guidance.

3. To understand indications for the use of these technologies.

\section{A-108 08:50}

CT/US and US/MRI images: are they useful for diagnostic applications? C. Ewertsen; Copenhagen/DK (caroline.ewertsen@dadlnet.dk)

Multimodality imaging including ultrasound has within recent years been introduced in several anatomical regions. PET, CT and MRI datasets can after an initial co-registration be fused and shown either side by side or using overlay on screen while doing real-time ultrasound; thus, the PET, CT or MRI dataset is reformatted in a projection to fit the real-time ultrasound images. Several methods of aligning the images are available and have been shown to have a high accuracy. Image fusion involving ultrasound has been tested in phantoms, animals and in patient studies, mainly in abdominal imaging. By combining several imaging modalities, it is possible to benefit from the strengths of each modality. It is useful for characterisation of and intervention on liver lesions, abscesses containing air and lesions in areas with poor overview by ultrasound. Also, the method is useful for characterisation and biopsy of PET-positive lesions, especially in patients with a history of cancer. The background of the method and clinical examples, mainly from abdominal 
applications, will be covered. Also, perspectives and future research topics will be highlighted.

Learning Objectives:

1. To learn about the technologies needed to fuse CT/US and US/MRI images.

2. To understand how to use them in clinical practice.

3. To understand the indications for these technologies in difficult cases.

Author Disclosure:

C. Ewertsen: Speaker; Speaker honorarium from GE.

\section{A-109 09:10}

Imaging tumour perfusion: CEUS compared with CT, MRI and PET

N. Lassau; Villejuif/FR (nathalie.lassau@gustaveroussy.fr)

Contrast-enhanced ultrasound (CEUS) has proved to improve the detection and characterisation of pathologies compared to conventional ultrasound, CT and MRI in a number of indications. Ultrasound contrast agents (UCA), which are purely intravascular, do not show any interstitial diffusion or glomerular filtration like iodinated complexes or gadolinium chelates. Dynamic contrastenhanced ultrasound (DCE-US) allows to display the enhancement of a lesion with a high frame rate after bolus or infusion administration of UCA, and to compare enhancement profiles between normal and abnormal tissue. Quantification of DCE-US is useful to quantify tumour enhancement and to limit intra- and interobserver variability. Mathematical models with several perfusion parameters can be used. DCE-US has shown to be of interest after antiangiogenic therapies as it allows an earlier evaluation of tumour response than usually done with $\mathrm{CT}$ and $\mathrm{MRI}$, which remain mainly based on the RECIST criteria. In clinical applications, recent mono-centric and multi-centric DCE-US studies evaluating anti-angiogenic drugs have demonstrated the relevance of this technique compared to $\mathrm{CT}$ and $\mathrm{MRI}$ with a significant correlation with PFS and OS in different types of tumours (HCC, metastatic RCC, colon cancer, breast cancer, GIST and melanoma). A consensus pape has been recently published by EFSUMB (European Federation of the Societies for Ultrasound in Medicine and Biology), providing recommendations in DCE-US. The development of targeted contrast agents opens the area of molecular ultrasound.

Learning Objectives:

1. To learn how to image tumour perfusion with CEUS

2. To compare the results of CEUS with those of CT, MRI and PET.

3. To understand differences among the techniques, and how to choose from among them

\section{A-110 09:30}

3D/4D ultrasonography: tool or toy?

M. Claudon ${ }^{1}$, S.T. Elliott ${ }^{2} ;{ }^{1}$ Vandoeuvre-les-Nancy/FR,

${ }^{2}$ Newcastle upon Tyne/UK (m.claudon@chu-nancy.fr)

The common perception of 3D ultrasound (US) is of surface-rendered images, especially of the foetus. The same approach, enhanced by new transducer technology and software, can be used to take ultrasonography to another leve in general body imaging, providing different image presentations and working practices similar to those of CT and MR. The technology involved in a US volume acquisition was represented initially by mechanical $3 \mathrm{D}$ transducers, and more recently by purely electronic, matrix probes. This latest technology allows bi-plane real-time acquisitions, fast switch to volume acquisition up to 100 degrees, and 4D acquisitions with acceptable frame rates. Protocols have been described for major abdominal and retroperitoneal organs, with fast optimisation of acquisition settings. The technology supports contrastenhanced ultrasound (CEUS). After volume acquisition, interpretation for volume US datasets requires a post-treatment phase, including multi-planar reconstruction, multislice imaging, and volumetric analysis. Virtual cystoscopy and volume CEUS quantification are new options. The implementation of this new, efficient US modality in a radiology department supposes deep changes in the daily practice with delayed post-treatment reporting times. It should represent a more effective way for comparison between different examinations and a better communication tool with clinicians. The proven and potentia benefits, in terms of clinical accuracy, training, workflow and overall efficiency, will be discussed.

Learning Objectives:

1. To learn about the technologies available to perform 3D/4D ultrasonography

2. To understand their possible role in clinical practice.

3. To understand the advantages in workflow offered by the use of 3D/4D

ultrasonography.

Author Disclosure:

M. Claudon: Consultant; Philips Ultrasound.

Panel discussion

Technical advances in ultrasonography: it is our responsibility to make them work 09:50

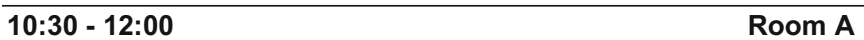

Interactive Teaching Session

$E^{3} 520 a$

Pitfalls in abdominal imaging

\author{
A-111 10:30 \\ A. Liver \\ V. Vilgrain, M. Ronot, A. Kerbaol, O. Bruno; Clichy/FR \\ (valerie.vilgrain@bjn.aphp.fr)
}

The purpose of this lecture is to emphasise some pitfalls in liver imaging Morphologic changes in the liver are usually attributed to chronic liver disease where liver cirrhosis represents the most important cause. However, noncirrhotic diseases may also induce atrophy-hypertrophic changes of the liver The most common mechanisms are related to venous obstruction (either portal or hepatic venous) and biliary obstruction. Multidetector CT and MR imaging are essential to highlight these abnormalities. When dealing with liver tumours, the most important question that has to be solved is tumour characterisation. Yet, it is often difficult to assess whether a large tumour is intra- or extrahepatic. Imaging findings that might be helpful will be shown. Last, some liver lesions can mimic liver tumors. Vascular disorders and focal fatty changes or focal fatty sparing are the most common causes. Some other conditions can be also mimickers and such cases will be shown.

Learning Objectives:

1. To learn about morphological changes in the liver observed in non-cirrhotic diseases.

2. To understand imaging features enabling a distinction between intrahepatic and extrahepatic tumours.

3. To become familiar with liver lesions mimicking liver tumours.

A-112 11:15

B. Pancreas and bile ducts

R. Manfredi; Verona/IT (riccardo.manfredi@univr.it)

The technique and the anatomy of the bile duct and pancreatic duct will be described. The anatomical variants of the biliary duct and the pancreatic duct system will be analysed and their possible role in generating diagnostic imaging pitfalls described. Strategies to avoid pitfalls in diagnostic imaging of the bile duct and the pancreatic duct system will be illustrated, considering the possible source of pitfalls. Diagnostic imaging findings of different diseases involving the biliary ducts and pancreatic duct system will be illustrated, as well as the diagnostic imaging criteria useful for the differential diagnosis. Learning Objectives:

1. To understand the MRI technique for evaluating the pancreatic parenchyma, the pancreatic duct system, the biliary tree, and the functional assessment following secretin stimulation.

2. To become familiar with the signs in MR imaging of the pancreas and bile ducts.

3. To understand the diagnostic imaging criteria useful for differential diagnosis.

$10: 30-12: 00$ Room B

\section{ESR meets Russia}

\section{EM 1}

\section{Crossroads of diagnostic imaging in the} big country

Welcome by the ESR President

G. Frija; Paris/FR

Presiding:

N. Rozhkova; Moscow/RU

V.E. Sinitsyn; Moscow/RU

I.E. Tyurin: Moscow/RU

A-113 10:30

Introduction: Russian radiology today

N. Rozhkova, I.E. Tyurin; Moscow/RU

Diagnostic radiology in the Russian Federation is a fast-growing part of clinical medicine. The implementation of national projects in health care has led to substantial improvement in the facilities of hospitals' modern diagnostic equipment. We have 14,189 radiologists and 29,445 technologists working in 9,000 hospitals. We have about 150 million x-ray-only investigations per year. Over the last two years, the number of CT and MRI units has increased by 
$30 \%$, and modern PET centers and leading centers for radiotherapy have been built. We can identify an approximately $20 \%$ increase in the number of radiological research projects--mainly ultrasound, CT, and MRI investigations. During the last ten years the total number of vascular interventions and MR studies has increased by ten times, four times for $\mathrm{CT}$, and the annual growth of US and $x$-ray investigations is about $10 \%$. Unfortunately, these technical innovations are not always accompanied with PACS and RIS installations; this is a reason why all advantages of digital equipment are not achieved. We see rapid development of private medicine; new private hospitals and ambulances have grown by more than $10 \%$ and investment companies are looking for new projects in this field. However, the professional education of radiologists is not enough to work with new diagnostic equipment. This is a reason why in 2014 we will start a pilot project to create a new system of continuing medical education in clinical medicine, including diagnostic radiology. We hope, this pilot project will increase the efficiency of clinical practise.

Session Objectives:

1. To learn about the organisational structure of radiology in Russia.

2. To become aware of Russian radiologists' involvement in ESR activities.

3. To express gratitude to the ESR for inviting Russia as a guest country to the ECR

\section{A-114 10:35}

\section{CT perfusion in the differential diagnosis of CNS pathology}

I.N. Pronin; Moscow/RU (pronin@nsi.ru)

Differential diagnosis (DD) including tumour and non-tumour brain pathologies is an integral part of neuroradiological diagnostics and in some cases is a challenge to experienced neuroradiologists. More than 5000 patients with different CNS pathologies have been investigated in the Department of Neuroradiology, Burdenko Neurosurgical Institute since 2002. In the vast majority of the cases, there were tumours. We used deconvolution method for CBV,CBF, MTT and PS maps reconstruction on commercially available software (Perfusion 1.2.0, 4.0 protocols, ADW, GE). CT perfusion has demonstrated high level of information in determining the degree of malignancy in glial brain tumours. A strong correlation between CBV and PS and tumour degree has been demonstrated. The use of CT-PWI makes it possible to successfully carry out DD between radiation necrosis and tumour progression. Some histological types of brain lesions are characterised by unique haemodynamic properties which enable using this method as non-invasive "biopsy". CT-PWI has been especially useful in DD of the skull base region, bone and extracranial pathologies. The absence of artefacts created by the skull base bones makes this method especially valuable as compared to the MRI T2*- and ASL- perfusions. CT- PWI makes it possible to successfully differentiate tumours and a number of non-tumour CNS lesions including demyelinating diseases, infectious and non-infectious granulomas, etc. CTPWI is a powerful method for differential diagnosis of brain pathology. CT-PWI can provide unique functional information regarding tumour pathophysiology and haemodynamics, which are not available with routine CT and MRI.

Learning Objectives:

1. To become familiar with the technique of brain perfusion $\mathrm{CT}$.

2. To understand the added diagnostic value of perfusion CT.

3. To learn about input of CT perfusion for assessment of treatment.

\section{A-115 10:55}

\section{Interlude: Development and use of web-based teleradiology in Russia}

O.S. Pianykh; Newton Highlands, MA/US (opiany@gmail.com)

The largest territory in the world, Russia, presents a perfect teleradiology use case of extremely unevenly distributed health care, concentrated in Moscow and spread thin outside over an enormous area and sparse population. This unevenness deeply affects the quality and availability of radiological services, and cries out for efficient and practical teleradiology solutions. However, the same unevenness in medical expertise, financing and IT infrastructure frequently acts against the advancement of teleradiology projects, blocking their natural progress. As a result, technical, financial, educational and clinical matters, interleaved into the nascent Russian teleradiology ventures, require serious and predictable solutions, based on analysis and adaptation of already developed, internationally proven strategies. Currently, Russian teleradiology simply takes advantage of whatever is available to make its progress better, but needs to develop itself up to the tasks it faces.

\section{A-116 11:00}

Tuberculosis in Russia: a challenge for a national radiological service I.E. Tyurin; Moscow/RU (igortyurin@gmail.com)

Approximately, 1.7 billion people worldwide are infected with Mycobacterium tuberculosis, of which 20 million are active cases. The reasons for resurgence of TB infection include the HIV epidemic, a rise in reactivation disease in the elderly, a growing migrant population and spread of drug-resistant strains. The incidence of atypical TB infection is also on the rise, of which Mycobacterium avium intracellulare is the most important human pathogen. The pathologic form of the pulmonary infection depends on the sensitivity of the infected host and is classified as primary or postprimary. Primary TB pattern represents infection resulting from recent contact with the pathogen. Postprimary TB pattern results from reactivation of a dormant focus within the lungs. Thoracic tuberculosis produces a broad spectrum of radiographic abnormalities. The radiological patterns had been described as parenchymal, airway, vascular, mediastinal, pleural, and chest wall lesions. Common causes of a missed diagnosis of thoracic tuberculosis are failure to recognise hilar and mediastinal lymphadenopathy as a manifestation of primary disease in adults, overlooking of minimal productive lesions or reporting them as inactive, and failure to recognise that an upper lobe or superior segment of lower lobe mass might be tuberculosis. In AIDS patients, the imaging features depend on the degree of immune suppression. A pattern of postprimary TB is also usually seen among patients with decreased immunity due to alcoholism, renal failure, diabetes mellitus, ageing, malignancy, and renal and cardiac transplantation.

\section{Learning Objectives:}

1. To understand the scope of the problem with tuberculosis screening in the Russian Federation.

2. To learn about the changing imaging patterns of thoracic tuberculosis

3. To appreciate the current role of imaging in the differential diagnosis and follow-up of this pathology.

A-117 11:20

Interlude: Mystery of Denisov's cave and paleoradiology

M. Mednikova; Moscow/RU (medma_pa@mail.ru)

This study focuses on the anatomical identification of fossil bones from Altai highland in Southern Siberia, their radiological description, and parallels among other hominin fossils. Methods describe the small and isolated tubular bones. We used microCT VersaXRM-500 by Xradia Inc. and digital microfocus $\mathrm{x}$-ray. Denisova Cave is the best studied Paleolithic cave in North Asia Archaeological data suggest that about 300 thousand years before the present, a group of humans migrated to Southern Siberia from the west. Their descendants had lived in the Altai Mountains in relative isolation for 250 thousand years. The taxonomic status of the early inhabitants of Southern Siberia was clarified thanks to palaeogenetic studies. It was concluded that the Altai hominin was genetically twice further from modern humans than were Neanderthals. Phylogenetic analysis suggests that this clade diverged from that ancestral to humans and Neanderthals very early - about 1 billion years ago. Descendants of this clade apparently survived in the Altai refugium until the period 50 - 40 thousand years ago. Due to structural patterns of preserved pedal and manual phalanxes, humans from Denisova Cave could be expected to be the bearer of "archaic" traits. The extraordinarily thick walls of tubular bones in adults reflect a high level of occupational stress. Radiological volumetric microscopy of the Denisovan girl phalanx gave evidence for the histological estimation of the developmental age. Radiological methods seem to be the perspective approach in the study of rare and fragmentary fossil human remains, differentiating between archaic and modern humans.

\section{A-118 11:25 \\ Advances in imaging of pancreatic masses \\ G.G. Karmazanovsky; Moscow/RU (karmazanovsky@ixv.ru)}

Modern diagnostic radiology allows not only to detect pancreatic tumours, but also to carry out their differential diagnosis with high accuracy. This is especially important because many benign tumours of the pancreas do not require immediate surgery. At the same time, many benign tumours may become malignant. The differential diagnosis of the pancreatic cystic lesions using endoscopic ultrasound and magnetic resonance pancreaticocholangiography has now become particularly accurate. Contrast enhancement allows detecting signs of tumor invasion into the main blood vessels and adjacent organs. Modern developments of radiology help to use extended operations for malignant tumours and sparing surgery with minimal removal of intact functioning pancreatic tissue in benign tumours.

Learning Objectives:

1. To become familiar with major types of pancreatic masses and their radiological manifestations.

2. To understand approaches to the selection of diagnostic pathways in pancreatic tumours.

3. To become acquainted with new developments in diagnostic imaging of pancreatic tumours.

A-119 11:45

Interlude: An artistic view of CT

A.L. Yudin; Moscow/RU (prof yudin@mail.ru)

Radiology is a specialty based largely on figurative perception of diagnostic imaging. The radiological picture of many symptoms reminds one of objects and environmental phenomena (for example, vanishing lung, draped aorta, geographic liver, porcelain gallbladder, dirty fat and others). Training of creative mentation promotes lasting assimilation of educational material at the 
associative level. Metaphorical thinking skills can also be improved by searching for amusing fragments in radiological images similar to the objects in everyday life; animals, cartoon characters, and others. The essential help in the study of the physical foundations of radiology and functional capabilities of workstations provide the execution and study of X-ray films, CT or MRI of fruits and vegetables, flowers, various artifacts and other objects in the environment. Eventually, it is just beautiful.

Panel discussion:

Future developments in Russian radiology: which path to take? 11:50

$10: 30-12: 00$ Room E2

Foundation Course: Breast Imaging

\section{$E^{3} 520 b$}

\section{Breast US}

Moderator:

R. Schulz-Wendtland; Erlangen/DE

A-120 10:30

A. Clinical US technique (2D and automated)

G. Ivanac; Zagreb/HR (gordana.augustan@zg.t-com.hr)

Ultrasound of the breast is an established imaging technique with severa advantages. It has been used to differentiate solid from cystic breast lesions for a long time. It has also been increasingly used to visualise small lesions in mammographically dense breasts and is the initial imaging method of the breast in young women. The features of the lesions that need to be analysed are: shape, relation to anteroposterior to laterolateral diameter, margins, internal structure and absorption of sound (distal acoustic phenomena). Color Doppler enables the thorough assessment of flow within the breast lesions. Elastography is established as a useful method to differentiate breast lesions on the basis of their stiffness, which can be quantified. The accurate staging of axillary disease is an important aspect in the management of patients with breast cancer. It is recognised, however, that underlying malignancy can be found in lymph nodes that appear morphologically normal. A variety of morphological features that may be seen in pathological nodes have been described (cortical thickness of $>3.5 \mathrm{~mm}$ and absence of a fatty hilum). The automated breast ultrasound technique has been known for a long time, but with recent improvements now may play an important role. The automated breast volume scanner (AVBS) employs frequencies of 5-14 $\mathrm{MHz}$. AVBS has various major advantages, such as being independent of the experience of the operator as well as supplying consistent and reproducible results. Additionally, for the first time it has become possible to obtain coronal images of the complete breast, which greatly assists in the planning of surgical interventions. Learning Objectives:

1. To understand the role of US in lesion characterisation.

2. To become familiar with the principles of axillary lymph node status assessment.

3. To learn about automated 3D US.

\section{A-121 11:00}

\section{B. US of the breast and axilla}

C.F. Weismann; Salzburg/AT (christian.weismann@inode.at)

To study the technical basics in breast ultrasound (US), a breast scanning phantom helps to understand different US settings of greyscale imaging. Typically, breast US work has a linear array transducer with a minimum field of view of $3.8 \mathrm{~cm}$ and a high frequency of minimum $7 \mathrm{MHz}$. A broadband transducer (e.g. 6-16 MHz) with different bands is preferred. In the central axillary area, a lower frequency band offers a deeper penetration depth. High frequencies improve the tissue contrast and optimise the axial and lateral resolution. The transmitter focus is adapted to the region of interest. Highly sensitive color Doppler detects slow flow rates $(1 \mathrm{~cm} / \mathrm{s}$ and less). For frequencies and power settings used in diagnostic greyscale and color Doppler studies, no safety limitations have to be considered. Breast US is mainly used as a second-line imaging tool to mammography (MG). A correlation between both imaging modalities is crucial. Skin markers and additional MGs may help to solve the correlation problem. In BI-RADS 4 or 5 US lesions with unclear correlation in MG, a marker placement after US-guided large-core needle biopsy helps to clear the problem. The pathomorphological growth pattern of a lesion is the basis to understand different imaging aspects with different modalities. Second-look US after MRI is an important correlation of lesions, which were first visible with MRI. Understanding the complementary imaging criteria will help to reduce the number of false-positive results.
Learning Objectives:

1. To understand basic technical and safety considerations.

2. To learn about radio-pathological correlations.

3. To become familiar with possible ways to decrease the number of biopsies.

A-122 11:30

C. Sonoelastography and ancillary modalities

C.S. Balleyguier, S. Canale, L. Ciolovan, C. Dromain; Villejuif/FR

(balleyguier@igr.fr)

Ultrasonography (US) B-mode is an established and powerful imaging tool in the diagnosis of breast tissue abnormalities. US provides a high degree of sensitivity in differentiating malignancies, nevertheless, false positive results represent a drawback for US. Elastography imaging has been shown to improve specificity of the US evaluation of breast masses, in evaluating tissue stiffness. Most common elasticity imaging techniques are represented by freehand elastography, with requires manual compression on a lesion with the ultrasound probe. This technique is easily feasible with a learning curve, but remains dependant of the operator. Shear-wave elasticity imaging is a new technology which provides qualitative and quantitative analysis on a lesion, less dependant on the operator. Performances of shear-wave elasticity may improve breast lesion characterisation and help to better categorise undetermined lesions such as Bi-Rads $4 \mathrm{a}$ and 3 nodules. Elasticity imaging characteristics will be added in the new version of Bi-Rads ultrasound lexicon. Elasticity imaging principles with an overview of the different elasticity modes which may be used will be presented during this session.

Learning Objectives:

1. To get acquainted with ancillary modalities.

2. To learn about radio-pathological correlations.

3. To appreciate the future use of ancillary modalities.

$10: 30-12: 00$

Room F1

Joint Course of ESR and RSNA (Radiological Society

of North America): Emergency Radiology

MC 522

\section{CNS emergencies}

Moderators:

S.E. Mirvis; Baltimore, MD/US

A. Palko; Szeged/HU

A-123 10:30

A. CNS trauma and neurovascular injury

H.A. Rowley; Madison, WI/US (hrowley@uwhealth.org)

A practical imaging approach to brain and neurovascular trauma will be reviewed, with an emphasis on understanding the correlation between pathophysiology and imaging signs. Guidelines on when to order acute neurovascular studies will be presented. Special technical considerations to help optimise CT and MR imaging protocols for suspected brain injury, CNS haemorrhage, and arterial dissection will be discussed.

Learning Objectives:

1. To become familiar with traumatic brain injury demographics and classification schemes.

2. To learn how to apply appropriateness criteria for head trauma imaging to children and adults.

3. To identify key imaging patterns and pitfalls in the evaluation of brain and neurovascular trauma.

Author Disclosure:

H.A. Rowley: Consultant; GE, Bracco, Guerbet, Gore, Genentech, and Lundbeck.

A-124 11:00

B. CNS non-traumatic emergencies

M. Smits; Rotterdam/NL (marion.smits@erasmusmc.nl)

Neurological emergencies are often associated with high morbidity and mortality, and thus require prompt diagnostic and therapeutic action. Nontraumatic emergencies may however have a subacute onset, and radiological signs may be subtle, which can lead to delay in diagnosis and treatment. Since clinical features are often nonspecific, the radiologist may be the first to point the clinician in the direction of the correct diagnosis. It is therefore of great importance that the radiologist is aware of and familiar with the various imaging findings, on both computed tomography (CT) and magnetic resonance imaging (MRI), of non-traumatic neurological emergencies. These include vascular, infectious and inflammatory diseases. Commonly encountered emergencies are ischaemic and haemorrhage stroke, venous thrombosis, arterial dissection, abscess, acute disseminated encephalomyelitis (ADEM), and encephalitis. 
Radiological findings in rarer diseases may mimic those in the more commonly occurring diseases, but need to be correctly interpreted as therapeutic strategies and prognosis may be entirely different. Such entities include for instance posterior reversible encephalopathy syndrome (PRES), reversible cerebral vasoconstriction syndrome, Susac's syndrome, and status epilepticus Furthermore, initial findings of (impending) complications of brain disease, such as hydrocephalus and herniation of brain structures, may be subtle, while early recognition allows for prompt and adequate intervention. Finally, diagnostic and therapeutic interventions performed in an emergency setting may interfere with the diagnosis and interpretation of clinical and imaging findings. Associated limitations and pitfalls therefore need to be recognised to avoid false-negative or false-positive diagnosis, respectively.

Learning Objectives:

1. To learn about the modalities (CT/MRI) and protocols for non-traumatic neurological emergencies.

2. To learn how to diagnose the main non-traumatic neurological vascular and non-vascular emergencies.

3. To become aware of the pitfalls and limitations of clinical presentation and imaging findings in non-traumatic neurological emergencies.

\section{A-125 11:30}

C. Interactive case discussion

H.A. Rowley ${ }^{1}$, M. Smits ${ }^{2} ;{ }^{1}$ Madison, WI/US, ${ }^{2}$ Rotterdam/NL

Brain and neurovascular trauma cases will be reviewed in an interactive format, with an emphasis on understanding the correlation between pathophysiology and imaging signs. Imaging 'pearls and pitfalls' will be presented using a variety of CT and MR examples. Guidelines on when to order and how to perform acute neurovascular studies will be illustrated. Special technical considerations to help optimise CT and MR imaging protocols for suspected brain injury, CNS haemorrhage, and arterial dissection will be highlighted through use of memorable case examples.

Learning Objectives:

1. To learn about traumatic brain injury (TBI) and non-traumatic neurological emergencies.

2. To become familiar with imaging manifestations of TBI and non-traumatic neurological emergencies.

3. To understand the clinical implications of radiological imaging findings in TBI and non-traumatic neurological emergencies.

4. To learn about the state-of-the-art radiological imaging options for the assessment of acute TBI and non-traumatic neurological emergencies. Author Disclosure:

H.A. Rowley: Consultant; GE, Bracco, Guerbet, Gore, Genentech, and Lundbeck.

\section{$10: 30-12: 00$} Room P

\section{ESR Radiation Protection Session}

\section{Launch of ESR EuroSafe Imaging Campaign: dealing with challenges of radiation protection}

Moderators:

C. Owens; London/UK

P. Vock; Spiegel/CH

\section{A-126 10:30}

\section{ESR EuroSafe Imaging Campaign}

M.M. Rehani; Vienna/AT (madan.rehani@gmail.com)

The current century has brought with it new challenges in radiation protection, many of which follow the development of MDCT which greatly enhanced diagnostic capabilities and substantially reduced scanning time to just a single breath-hold for chest CT, making CT scanning both patient-friendly and the physician's preferred tool. CT has also been added to nuclear imaging systems to create hybrid scanners (PET/CT; SPECT/CT). Skin injuries to patients from CT were for the first time reported in 2005-2009. Surveys in many countries in Europe and elsewhere show wide variations in the radiation dose given to patients for a particular radiological examination, and there are an increasing number of reports of inappropriate examinations. ESR has recently published its position statement on Insights into Imaging on GPS implying: Globalisation (detailing all the steps and involving all stakeholders), Personalised medicine and Safety. The EuroSafe Imaging will involve a number of actions, including: 1. Survey of paediatric CT practices in European countries. 2. EuroSafe Imaging Posters for ECR 2014. 3. Development of individual 10-point lists of criteria for safe radiological examinations for CT, mammography, radiography, etc. Additionally, the criteria for each examination will be applied to specific body parts. The intention is to make the criteria available in all European languages. 4. Orienting chairs and senior radiologists on radiation protection. 5. Session at ECR 2014 to announce EuroSafe Imaging campaign. 6. A column on radiation protection in each issue of the ESR's Newsletter. 7. Actions for residents and fellows using social media. 8. Certification: Is your imaging EuroSafe?

Learning Objectives:

1. To learn about the ESR's new EuroSafe Imaging campaign.

2. To understand the issues that require a European campaign.

A-127 10:40

Image Gently Campaign

M.J. Goske; Cincinnati, OH/US (marilyn.goske@cchmc.org)

Image Gently 5 years later: Lessons learned in radiation protection for children The Alliance for Radiation Safety in Pediatric Imaging was founded in 2007 to promote radiation protection for children worldwide through awareness, education and advocacy. Comprising over 75 medical professional societies and agencies, the Alliance seeks to change practice locally for improved paediatric patient safety in medical imaging. Since its inception, the Alliance has had 6 campaigns in CT, diagnostic and interventional radiology, nuclear medicine, digital radiography and an outreach campaign to parents and 3 educational summits. Yet, there is still work to be done. There is a need for parents and patients to receive information about their imaging test prior to the performance of imaging procedures, particularly those involving ionising radiation. The paediatric medical imaging community should work tirelessly with the manufacturers of imaging equipment to tailor equipment for children to optimise the study and ensure that the users of the equipment are knowledgeable in its safe use in children. The routine display of size-specific dose estimate on CT scanners will better estimate patient dose for quality improvement within facilities and in dose registries. Finally, there is a need for diagnostic reference levels in paediatric radiology. This presentation will update the audience in progress to date regarding these challenges. Learning Objectives:

1. To learn about the Image Gently Campaign for radiation protection of children.

2. To understand the lessons learnt while communicating messages.

A-128 10:50

Image Wisely Campaign

J.A. Brink; Boston, MA/US (JABRINK@partners.org)

The Image Wisely programme continues to move forward, guided largely by its steering committee, composed of two representatives each from ACR and RSNA and one representative each from AAPM and ASRT. The Image Wisely website (www.imagewisely.org) has educational content for imaging professionals (radiologists, medical physicists, and technologists) and referring physicians. A patient tab features links to radiologyinfo.org and the Image Gently website. A tab for "My Equipment" links visitors to radiation dose-related 'microsites' developed by GE, Hitachi, Philips, Siemens and Toshiba. As of August 29, 2013, 18,646 individuals, facilities and associations have taken the Image Wisely pledge. To attract traffic to its website, Image Wisely has recently instituted 'News' and 'Top Reads' sections on the splash page highlighting recent articles and announcement, highlighting breaking news coverage and journal publications on relevant topics. In 2012, content on nuclear medicine procedures was finalised, completing two of the three targeted foci of the initial action plan CT and nuclear medicine. In October 2013, a launch meeting will initiate the process of developing content for the third target area, fluoroscopy. In 2012 and 2013, Image Wisely has concentrated on efforts to engage referring physician communities, with particular interest in paediatricians and emergency medicine physicians. Progress has been slow, but has recently gained traction with emergency medicine in the form of radiologist participation in a 2015 symposium, Diagnostic Imaging in the Emergency Department: A Research Agenda to Optimise Utilisation, organised by the Society of Academic Emergency Medicine.

Learning Objectives:

1. To learn about the Image Wisely Campaign for radiation protection of adults. 2. To understand the lessons learnt while communicating messages.

\section{A-129 11:00}

Lessons from a national approach to patient safety in radiation protection P. Cavanagh ; Taunton/UK (petecavanagh@gmail.com)

In considering a European campaign on radiation awareness, it is useful to gain information from similar campaigns in health care. There have been a number of national patient safety campaigns based on the Institute for Healthcare Improvement (IHI) 100,000 lives campaign initiated in the USA in 2006. Following the success of this, a similar campaign was launched in England. Part of this process included an analysis of the success of the programme and the key interventions and drivers that were considered essential at both the national and local level. These included strong leadership, 
measurable outcomes, continual effective communication and support as well as a clear package of evidence-based interventions.

Learning Objectives:

1. To learn about the national patient safety campaign in the UK.

2. To understand the lessons learnt during the national campaign

\section{A-129/1 11:10}

Current challenges for radiation protection research in Europe

J. Repussard; Paris/FR

Panel discussion 11:20

Moderator: L. Bonomo; Rome/IT

Panellists:

M.M. Rehani; Vienna/AT, M.J. Goske; Cincinnati OH/US, J.A. Brink; Boston, MA/US,

P. Cavanagh; Taunton/UK, J. Repussard; Paris/FR

European Commission, G. Simeonov; Luxembourg/LU

IAEA, R. Chhem; Vienna/AT

WHO, M. del Rosario Perez; Geneva/CH

HERCA and Federal Office for Radiation Protection (Bfs), J. Griebel; Munich/DE

Art. 31 Group of Experts/ICRP, E. Vano; Madrid/ES

COCIR, N. Denjoy; Brussels/BE

EFRS, G. Paulo; Coimbra/PT

EFOMP, J. Damilakis; Heraklion/GR

CIRSE, W. Jaschke; Innsbruck/AT

$12: 30-13: 30$

Room E2

The Beauty of Basic Knowledge: Skeletal Radiology

\section{26B}

\section{Tumoural and pseudotumoural MSK lesions \\ Moderator:

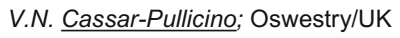

\section{A-130 12:30}

Tumoural and pseudotumoural MSK lesions

K. Verstraete; Ghent/BE (koenraad.verstraete@ugent.be)

1. Bone (pseudo)-tumours: The majority of bone tumours can be detected on plain radiography. The age of the patient, location of the tumour in a bone and history of pre-existing bone abnormality should be included in determining the likely diagnosis. Careful analysis of pattern of bone destruction, periosteal reaction and matrix mineralisation allow for characterisation of most bone tumours and differentiation from pseudotumours. CT may be useful for osteoid osteoma and MRI is the best imaging technique for further diagnosis and staging by displaying tumour composition and extent of bone marrow involvement, including skip lesions, presence and extent of extraosseous soft tissue mass, and involvement of neurovascular bundle, muscle compartments and adjacent joint. This allows to find the best biopsy and surgical approach To evaluate local control of disease and for detection of local recurrence, MR is usually the best imaging technique. 2. Soft tissue (pseudo)-tumours: The majority of soft tissue tumours can be detected on ultrasound. Except for cysts and subcutaneous lipoma, MR will be the next imaging technique for diagnosis local staging, and eventually to find the best biopsy and surgical approach. The age of the patient, location of the tumour and careful analysis of the signal characteristics on MRI allow for characterisation of many soft tissue tumours and differentiation from pseudotumours. Plain radiography and CT may be useful for detection of calcifications and myositis ossificans. To evaluate local control of disease and for detection of local recurrence, ultrasound and MRI are the best imaging techniques.

Learning Objectives:

1. To become familiar with the common pathological features of bone and soft tissue tumoural lesions.

2. To appreciate the imaging hallmarks of pseudotumoural bone and soft tissue lesions.

3. To learn how best to use imaging modalities in differential diagnosis.
$12: 30-13: 30$

Room F1

The Beauty of Basic Knowledge: Interventional Radiology

MC 25B

Percutaneous drainages and nephrostomies

Moderator:

M. Bezzi; Rome/IT

A-131 12:30

Percutaneous drainages and nephrostomies

R. Portugaller; Graz/AT (rupert.portugaller@medunigraz.at)

Image-guided catheter drainage is regarded to be effective if fluid collections are infected, produce symptoms or communicate with fistulas. Coagulopathy and the lack of a safe puncture access route represent contraindications. In the first scenario, coagulation factors are mostly helpful and in the second hydrodissection may be applied to oust organs from the access route. The clinical success of catheter drainage is achieved in about $85 \%$. Complications like septic shock, haemorrhage and bowel or pleural transgression were reported in up to $10 \%$. Criteria for catheter removal comprise improvement of clinical condition, laboratory results and resolution of the cavity in the absence of a fistula. The amount of drained fluid should decrease beyond $10 \mathrm{ml}$ to $20 \mathrm{ml} / 24$ hours. Percutaneous nephrostomy is mostly performed to relieve the urinary tract from benign or malignant obstructions, urinary leakage or fistulas, or to obtain access to the collecting system for manipulations like stone disintegration and removal. Technical success rates of approximately $98 \%$ were reported for obstructed collecting systems, whereas the success declined to $85 \%$ in non-dilated tracts. Severe complications like septic shock and transfusion-demanding haemorrhage occur in $1.3 \%$ to $1.8 \%$ and $2 \%$ to $4 \%$, respectively. In case of pyonephrosis, the rate of septic shock can be as high as $7 \%$. To prevent encrustation of the nephrostomy catheters, outpatient catheter exchanges are usually scheduled at 8-week intervals. If the urinary bladder function is preserved, antegrade double-j ureter-stent insertion via the nephrostomy access may be reasonable. Then, every 3 months transvesical stent exchange is recommended.

Learning Objectives:

1. To understand the indications for percutaneous fluid drainage and nephrostomy.

2. To learn the various imaging modalities for guidance and basic techniques 3. To appreciate the efficacy of the procedures and the appropriate follow-up.

12:45 - 13:15

Room A

Plenary Session

HL 1

Josef Lissner Honorary Lecture

Presiding:

V.E. Sinitsyn; Moscow/RU

A-132 12:45

Research in cardiac imaging: how I do it

A. de Roos; Leiden/NL (A.de_Roos@lumc.nl)

In this honorary lecture, I will review a number of personal impressions that may contribute to the successful performance of research projects in cardiovascular radiology. Also, issues and obstacles in performing successfu research in the field of radiology will be considered. Key factors for success include guidance by inspiring mentor, genuine interest and motivation, some creativity and serendipity. Radiology has developed tremendously with many innovative modalities over the last 25 years, providing ample opportunity to participate in clinical research. Potential issues that should be addressed are subspecialisation, turf battles, competition from other clinicians/ imaging specialists and adequate training in radiology research. The field of imaging research includes traditionally more clinically oriented research by focussing on developing and testing of new modalities and applications, now becoming more basic by including molecular imaging, population imaging, outcome studies and alike.

Learning Objectives:

1. To learn about personal impressions how to succeed in research

2. To learn what makes research exciting. 
14:00 - 15:30 Room E2

\section{Foundation Course: Breast Imaging}

\section{$E^{3} 620$}

\section{MRI of the breast}

Moderator:

F. Pediconi; Rome/IT

A-133 14:00

A. Technique and diagnosis

K. Kinkel; Chêne-Bougeries/CH (karen.kinkel-trugli@wanadoo.fr)

MRI of the breast is ideally performed during the second week of the cycle (day 7-14) when hormonal glandular background enhancement is minimal. The protocol includes T2-weighted imaging for anatomical purposes and T1weighted images for the detection of breast cancer after intravenous injection of contrast media. Fat suppression techniques are important to visualise the enhancing lesion in the presence of $\mathrm{T} 1$ high signal intensity fat. Subtraction techniques (postcontrast image minus precontrast image) require no motion artefact which is obtained through dedicated breast coils with gentle breast compression. Spectral fat suppression is another fat suppression technique often used in 3D sequences allowing for high spatial resolution. Lesion characterisation relies on both morphology and kinetic enhancement characteristics over time. Spiculated borders, rim enhancement and segmental or irregular linear enhancement are typical morphological features of breast cancer, whereas washout kinetic demonstrates high positive predictive value of cancer. Lesion interpretation takes into account the patient's age, hormonal status, personal risk for breast cancer and history of current medical, surgical or radiation therapy. Comparison with prior imaging studies is crucial to identify benign stable disease of the breast and MRI-only lesions changing patient management. Suspicious MRI-only lesions not identified at second-look ultrasound will undergo MRI-guided biopsy with clip positioning

Learning Objectives:

1. To learn how to do it

2. To learn how to read the images.

3. To understand which patients can benefit from it.

Author Disclosure:

K. Kinkel: Research/Grant Support; Clinique des Grangettes.

\section{A-134 14:30}

B. MRI of the postoperative breast

A. Tardivon; Paris/FR (anne.tardivon@curie.net)

Silicone-filled implants (single or double lumen) require a dedicated sequence where silicone exhibits a high signal intensity, whereas signals from fatty and fibro-glandular tissues are cancelled. Diagnosis of intracapsular rupture relies on the detection of a linguine sign (direct sign) or multiple "key-hole" and/or " teardrop" images (indirect signs). Extracapsular rupture is defined as the presence of silicone outside the capsule (breast, soft tissues, lymph nodes). Breast reconstruction after mastectomy may involve autologous tissue flaps (skin, fatty tissue, muscle and fascia). The transferred muscle is inserted in front of the major pectoralis muscle and enhances after contrast medium injection. The most common benign findings seen in such reconstructed breasts and also after a breast-conserving treatment are oedema, seroma haematoma, fat necrosis and fibrosis. Diffuse thickening of the skin and trabeculae is usually observed the first year after completion of radiation therapy. Small focal areas (less than $5 \mathrm{~mm}$ ) of non-mass-like enhancement and thin linear or rim enhancement at the lumpectomy site can all be expected findings. These benign findings decrease progressively, but residual enhancement may be observed in $15 \%$ of women at 5 or more years. In patients who were switched from tamoxifen to aromatase inhibitors, a stronger or re-appearance of a background enhancement may be observed. Before classifying such findings as benign or probably benign, clinical examination and standard imaging data must be taken into account. Highly suspicious lesions in such patients are similar to BI-RADS 5 lesions in the nontreated breast.

\section{Learning Objectives:}

1. To understand the MRI technique used.

2. To become familiar with different implant types.

3. To learn when to raise the flag.

\section{A-135 15:00}

C. MRI in the high-risk population

C.K. Kuhl; Aachen/DE (ckuhl@ukaachen.de)

Prevention strategies for women at high lifetime risk of breast cancer are usually adapted to the individual woman's risk of breast cancer. Women at high risk (BRCA mutation carriers or women with a $30 \%$ likelihood of BRCA heterzygosity) are offered primary prevention, which includes chemoprevention by Tamoxifen or surgery (risk-reducing mastectomy and/or salpingooopherectomy, where the latter also helps reduce the risk of subsequent breast cancer by $30 \%$ ). If women opt for secondary prevention (active surveillance/screening), this has to start at 30 at the latest. MRI is considered a compulsory component of all programs worldwide. Due to the very limited added cancer yield of mammography in this subset of women, and because of the still unsettled issue of possibly increased radiosensitivity of BRCA-mutation carriers, mammographic screening is not recommended until the age of 40 . There is broad agreement that (at least) an annual screening is important; especially for BRCA1 mutation carriers, screening every 6 months may be more appropriate. We recommend interleaved screening, with annual MRI and annual ultrasound ( \pm mammography) phase-shifted by 6 months. There are important differences between BRCA1- and BRCA2-associated cancers in terms of age distribution and incidence rates, histologic features, but also specific imaging features which must be known by radiologists. MRI screening protocols must be adjusted to the specific needs of this (usually very young) screening population. This presentation will review the current evidence with respect to screening of women at high familial risk of breast cancer and will provide information on all aspects mentioned above.

Learning Objectives:

1. To understand breast cancer genetics (genes, DNA, mRNA).

2. To understand the importance of family history, genetic disorders and syndromes with increased incidence.

3. To appreciate the role of MRI in screening and downstream management.

14:00 - 15:30

Joint Course of ESR and RSNA (Radiological Society

of North America): Emergency Radiology

\section{622}

\section{Chest emergencies}

Moderators:

S.E. Mirvis; Baltimore, MD/US

A. Palko; Szeged/HU

A-136 14:00

A. Thoracic injuries

S.E. Mirvis; Baltimore, MD/US (smirvis@umm.edu)

Chest trauma is directly responsible for $25 \%$ of all trauma deaths and is a major contributor in another $50 \%$ of all trauma mortality. Blunt trauma, accounting for $90 \%$ of chest injuries, is the third most common site of injury in polytrauma patients. Plain radiographs still have a role in recognition of some acute thoracic pathology that requires immediate further management, either diagnostically and/or therapeutically, such as tension pneumothorax, major transdiaphragmatic herniation, large hemothorax or obvious mediastinal hematoma. MDCT of the chest is now typically included in a whole body scan with IV contrast to facilitate rapid diagnosis on polytrauma cases using less radiation than selected segmental scans. MDCT is the well-proven diagnostic gold standard for chest injury evaluation. The major advantages of MDCT over other modalities include identification of active bleeding, direct signs of trachea or esophageal injury, direct evidence of major arterial vascular injury, such as pseudoanurysms, pneumo and hemopericardium, location and extent of lung contusion and laceration, and assessment for thoracic spine, shoulder girdle and rib fractures. Diaphragm injuries are well depicted by MDCT, especially on the left by identifying both the torn diaphragm edges, herniation and constriction of abdominal contents at the level of the torn diaphragm (collar sign), and direct contact of herniated structures with the posterior chest wall (dependent viscera). Tracheal injuries are suggested by diffuse and progressive pneumomediastinum, dilated tracheostomy cuff, ectopic endotracheal tube, and direct connection of mediastinal air with the trachea lumen. CT-angiography eliminates the majority of indications for diagnostic catheter angiography.

Learning Objectives:

1. To learn how to differentiate traumatic aortic injuries from congenital variants that mimic injury, to distinguish minor from major aortic injuries, and to understand how injury classification can influence management. 2. To become familiar with the various CT appearances suggesting and verifying major airway injury.

3. To understand the various CT appearances of blood/bleeding in the chest and how the location, quantity of blood/bleeding and patient clinical status determine initial treatment.

4. To appreciate the spectrum of cardiac injuries that can be diagnosed on admission contrast-enhanced CT and those that require urgent intervention. 


\section{Postgraduate Educational Programme}

A-137 14:30

B. Non-traumatic thoracic emergencies

C.M. Schaefer-Prokop; Amersfoort/NL (cornelia.schaeferprokop@gmail.com)

Pulmonary symptoms such as chest pain, shortness of breath or wheezing are common non-traumatic symptoms prompting ER visits. Because clinical symptoms are very non-specific, imaging plays a major role in differentiating life-threatening from less severe diseases and forming a diagnosis. The chest radiograph remains the first imaging despite its limited sensitivity for certain diseases and being prone to inter-observer variability. Comprehensive cardiothoracic CT examinations using most modern CT equipment are well evaluated in their diagnostic accuracy to determine the presence of vascular life-threatening events such as aortic dissection, acute coronary disease or pulmonary embolism. Protocols and literature evidence will be discussed. The main focus of the course, however, will lie on the analysis of pulmonary CT findings and its contribution to the differential diagnosis of pulmonary emergencies (e.g. pneumonia, oedema, pneumothorax, exacerbation of fibrotic or obstructive lung diseases) and how to further integrate imaging findings, laboratory findings, patient history and clinical information to tackle the differential diagnosis. Imaging findings will be discussed by clinical case studies, key findings and also overlapping morphological features of other differential diagnosis will be discussed side by side, illustrating the options and also limitations of imaging findings.

Learning Objectives:

1. To become familiar with protocols and diagnostic performance of comprehensive cardiothoracic CT examinations for determining the presence of life-threatening vascular events such as aortic dissection, acute coronary disease and pulmonary embolism.

2. To understand typical but, also less common, CXR and CT findings in patients with pulmonary or mediastinal diseases causing acute dyspnoea and/or requiring immediate treatment, and to learn about key imaging findings in these patients allowing for a fast differential diagnosis.

3. To learn how to adapt CT protocols to CXR findings and to integrate imaging findings into lab findings, patient history and clinical information in order to make a diagnosis.

\section{A-138 15:00}

C. Interactive case discussion

S.E. Mirvis ${ }^{1}$, C.M. Schaefer-Prokop ${ }^{2} ;{ }^{1}$ Baltimore, MD/US, ${ }^{2}$ Amersfoort/NL

14:00 - 15:30 Room N/O

\section{ESOR Session}

\section{Mentoring in radiology}

\section{Moderators:}

G. Frija; Paris/FR

N. Gourtsoyiannis; Athens/GR

\section{A-139 14:00}

\section{Introduction}

G. Frija; Paris/FR (guy.frija@egp.aphp.fr)

Mentor was first introduced in Greek mythology, and in its modern usage first recorded by a French writer, François Fénelon, in 1699. Since then, this concept has been widely used in the development of mentoring, mentorship and more recently of mentees, with an overarching principle based on accompanying, sowing, catalysing, showing and harvesting. This concept remains today as a fundamental one in all teaching activities and implies generally a close contact between the mentor and the mentee. However, the development of Internet with concomitant e-learning capabilities, which are hugely increasing, raises the very challenging necessity to re-think this concept and to adapt it to this new era. The ESR is very concerned by this endeavour in the context of the striking development of ESOR, on one hand, and with the launching of its e-library, on the other.

\section{A-140 14:05}

\section{ESOR in action 2014}

N. Gourtsoyiannis; Athens/GR (gournick@gmail.com)

The presentation "ESOR in action" gives an overview on all ESOR activities in 2013 and 2014. A detailed report on the ESOR courses and teaching programmes in 2013 is presented, containing statistics about the number of course participants and of scholarship and fellowship grants. Additionally, all educational activities in 2014, including dates, venues, topics and local organisers, are announced.

\author{
A-141 14:20 \\ Evidence-based radiology: the basics \\ F. Sardanelli; Milan/IT (f.sardanelli@grupposandonato.it)
}

Evidence-based medicine (EBM) was derived from clinical epidemiology during the last century. It "is the conscientious, explicit, and judicious use of current best evidence in making decision about the care of individual patients" (Dave L. Sackett, 1996). This means integrating (1) individual clinical expertise with (2) the best available external evidence from systematic research and (3) patient's values and preferences. When considering evidence-based radiology (EBR), the needed expertise is not only clinical, but also technical, while (4) radioprotection issues assume a relevant value according to the "as low as reasonably achievable" (ALARA) principle. EBM (and EBR) can be practised according to the "top-down" model, when using guidelines issued by governmental, professional, or academic bodies, or the "bottom-up" model, when local physicians (1) formulate an answerable question, (2) search for the best evidence; (3) appraise critically; (4) apply findings to practice; and (5) evaluate the performance. A hierarchy of radiological studies has been established according to the investigated matter: 1. technical performance; 2. diagnostic performance; 3 . diagnostic impact; 4 . therapeutic impact; 5 . patient outcome; 6 societal impact. Different degrees of recommendations are based on different levels of evidence, with experts' opinion as the lowest level and meta-analyses of high-quality homogeneous studies and multicenter studies being the best level of evidence. To practise EBR, radiologists should be familiar at least with basic statistics and epidemiology as well as of methods for study design, thus recognising the principal types of bias which can limit the value of published studies.

Learning Objectives:

1. To understand the historical development and general principles of evidence-based medicine (EBM) with its top-down and bottom-up approaches 2. To learn how to apply EBM principles to radiology (evidence-based radiology, EBR) and to illustrate the main reasons for the relative delay in developing EBR, including the challenges of fast technological innovation in medical imaging.

3. To learn about the relationship between levels of evidence and the strength of recommendations for diagnostic imaging and interventional radiology.

4. To learn how radiologists could play a stronger role in building the evidence in favour of diagnostic imaging and interventional radiology, taking the patient's interest as the primary aim of clinical practice and science.

\section{A-142 14:35}

Education in research: action plan

J. Hodler; Zurich/CH

A survey regarding education in research has been commissioned by the ESR and published in Insights Imaging (2012) 3: 535-542. The results indicate that education in research, associated with career models, needs promotion. The Research Committee of the ESR is working on possible future steps to promote research through education. Several problems will have to be addressed, most notably inhomogeneity within Europe, motivation issues, illdefined career paths as well as funding issues. Several stakeholders will play an important role, including scientific societies, although this is the core business of academic radiology chairmen.

Learning Objectives:

1. To become familiar with the current status of research education in radiology.

2. To understand the importance of research education for the long term development of radiology.

3. To learn about possible actions for promotion of education in research. Author Disclosure:

J. Hodler: Equipment Support Recipient; Siemens, General Electric. Grant Recipient; Guerbet, Bayer.

\section{A-143 14:50}

Mentoring in radiology

H.-U. Kauczor; Heidelberg/DE (Hans-Ulrich.Kauczor@med.uni-heidelberg.de)

Most young professionals starting a career in academic institutions are eager to expand their professional and personal aptitudes. An effective way to support young professionals in this regard is by mentoring. Mentorship is a relationship where the mentor supports a junior faculty member (mentee) in personal and professional development. The mentee benefits from early determination and initiation of career-relevant steps, greater productivity in research and publishing activity and developing techniques in networking and collaboration. Moreover, the mentee gains perspectives on how his/her discipline operates academically, socially and politically, improves self-efficacy and deals more confidently with challenging intellectual work. The mentor, a knowledgeable, experienced and regarded person, benefits from fresh ideas, energy and curiosity of mentees, as well as through acceptance as a mentor an enhanced status in the department. It is a trusted relationship of dialogue, 
learning and challenges, based on mutual interests. However, the mentor is not automatically the best friend or the sole exclusive advisor of the mentee. More and more academic medical institutions offer facilitated mentoring programmes, which help the mentoring pairs to create an effective mentoring relationship to accomplish the desired skills. Evaluation of faculty mentoring programmes in academic medical institutions demonstrates the importance of mentoring in research and academic development as well as in patient care. Especially in radiology where over many years there have been challenges in recruiting and retaining talented young professionals, mentoring is considered as an essential way for preparing the next generation of scientific and intellectual leaders.

Learning Objectives:

1. To learn the definition, types and benefits of mentoring

2. To become familiar with the roles and tasks of mentor and mentee.

3. To understand the importance of mentoring for career development.

4. To appreciate potential reservations and gender issues in mentoring. Author Disclosure:

H.-U. Kauczor: Equipment Support Recipient; Siemens, Philips. Speaker; Bracco, Siemens, Boehringer, Bayer, Novartis.

\section{Awards 15:05}

$16: 00-17: 30$ Room A

\section{Interactive Teaching Session}

\section{$E^{3} 720 a$}

\section{Pitfalls in head and neck imaging}

\section{A-144 16:00}

A. Pitfalls in neck imaging

F.A. Pameijer; Utrecht/NL (f.a.pameijer@umcutrecht.nl)

Pitfall: "a hidden or unexpected danger or difficulty". Imaging methods can provide an extraordinary amount of useful data to specialists treating head and neck (cancer) patients. It is crucial that these data are used to full advantage of individual patients. The most important factor in this process is mutual cooperation between the physicians in charge of patient care and the diagnostic imaging specialist. Pitfalls in the head and neck may present in various ways: normal variants may look like disease, incidental findings are frequently encountered and suboptimal technique may obscure important findings. Moreover, many pitfalls are directly related to technical errors. The presentation aims to familiarise general radiologists, who have an interest in head and neck imaging, with common pitfalls encountered on CT and MR studies focussing on the neck. In an interactive setting, examples from daily practice will be discussed.

Learning Objectives:

1. To understand the variations of normal anatomy in the neck that should not be interpreted as abnormal.

2. To become familiar with the incidental findings which are frequently encountered when searching for neck disease.

3. To learn about suboptimal neck studies, or technique related problems, and understand how these may influence interpretation.

\section{A-145 16:45}

\section{B. Pitfalls in maxillofacial and skull-base imaging}

R. Hermans; Leuven/BE (Robert.Hermans@uzleuven.be)

Imaging of the skull base and maxillofacial skeleton requires a meticulous imaging technique and a good knowledge of normal anatomy and possible anatomical variants. Asymmetry in the pneumatisation of the paranasal sinuses, skull base or temporal bone is a common reason for misinterpretation. For example, hypoplasia of the maxillary sinus may be misinterpreted on conventional radiography as maxillary sinusitis or orbital blowout fracture, depending on the context; asymmetric pneumatisation of the petrous apex or mastoid bone may mimic, respectively, a cholesterol granuloma and fluid effusion in the non-pneumatised side on MRI. Vascular variants may also cause interpretation problems. For example, turbulent flow in a large jugular bulb may mimic a jugular foramen tumour on MRI. Variants in the vascular plexus surrounding the trigeminal or facial nerve may occur, and cause asymmetric findings on MRI, possibly mimicking neuritis or perineural tumour spread. Incomplete maturation or arrested development of skull base structures may also cause confusion. Examples are the cochlear cleft, not to be confused with otosclerosis, or arrested pneumatisation of the sphenoid sinus, possibly mimicking a tumoural lesion in the central skull base. To avoid problems, one should keep in mind the existence of such variants, while correlating the imaging findings with the clinical problem; in some cases, an additional imaging study may be needed to exclude a pathological process more confidently.

Learning Objectives:

1. To understand the basic requirements for an optimal imaging study of the skull base and maxillofacial region.

2. To become familiar with anatomical variants potentially mimicking disease.

3. To learn to appreciate incidental findings, thus avoiding unnecessary concern while recognising relevant pathology.

16:00 - 17:30

Room B

\section{State of the Art Symposium}

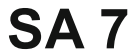

\section{Cardiac computed tomography: from diagnosis to prognosis}

\author{
A-146 16:00 \\ Chairman's introduction \\ M. Dewey; Berlin/DE (dewey@charite.de)
}

This short 5-min introduction by the session chairman will give an overview of the current state of cardiac computed tomography (CT) for diagnosis and prognosis. In addition, the introduction will provide the basis for the subsequent dedicated lectures covering low radiation dose, diagnostic accuracy, and incidental findings in cardiac CT.

Session Objectives:

1. To become familiar with the potential of cardiac CT to diagnose coronary artery disease.

2. To understand current research projects about on non-invasive diagnosis of coronary artery disease.

3. To learn about the facilitation of clinical management decisions in patients with suspected or known disease.

Author Disclosure:

M. Dewey: Author; editor of Coronary CT Angiography and Cardiac CT, both published by Springer. Consultant; Guerbet. Equipment Support Recipient; Institutional master research agreements exist with Siemens Medical Solutions, Philips Medical Systems, and Toshiba Medical Systems. The terms of these arrangements are managed by the legal department. Grant Recipient; Heisenberg Program of the DFG. European Regional Development Fund. German Heart Foundation. German Federal Ministry of Education and Research. GE Healthcare, Bracco, Guerbet, and Toshiba. Speaker; Toshiba Medical Systems, Guerbet, Cardiac MR Academy Berlin, and Bayer (ScheringBerlex).

\section{A-147 16:05}

What are the protocols with the lowest radiation dose in clinical practice? J.-F. Paul; Le Plessis Robinson/FR (pauljf@ccml.fr)

The radiation dose delivered for coronary CT angiography using retrospective gating is high, because only a small part of the total radiation delivered is used for the reconstruction of the image in the retrospective mode. On average, $20 \%$ of the radiation burden is used to reconstruct one phase of the cardiac cycle. ECG-triggered tube current modulation allows reducing the nominal dose by up to $96 \%$ in the systolic phase. Another approach for reducing radiation exposure is to use prospective, sequential acquisition, with this approach being associated with $75 \%$ dose reduction compared to the spiral retrospective acquisition. Exposure time may be also shortened by an increase of pitch, in particular on the dual-source CT. However, applicability to each dose-sparing technique depends on the heart rate and its regularity. Dose-sparing strategies should be modified in case of high or irregular heart rate and radiation dose reduction may be less effective. For coronary CT angiography, individually weight-adapted protocols have been successfully applied, by adjusting $\mathrm{mAs}$ to patient's weight. More recently, settings of $100 \mathrm{kV}$ and even $80 \mathrm{kV}$ have been successfully used for cardiac studies, especially in slim patients or children. Reduction of exposure time and individual adaptation have an additive effect on dose reduction: it has thus been possible to scan down under $1 \mathrm{mSv}$ in selected, slim patients, using this combined approach. In many cases, using the latest technology, radiation dose may be lower than the mean radiation dose associated with conventional angiography.

Learning Objectives:

1. To understand various protocols to lower radiation dose in cardiac CT with regard to the clinical situation and the risk of image quality impairment. 2. To learn how to tailor the radiation dose level to each patient in terms of morphology.

3. To become familiar with modulating exposure windows in regards to a patient's ECG. 


\section{A-148 16:28}

How accurate and prognostically valid is coronary $\mathrm{CT}$ angiography?

F. Cademartiri, E. Maffei; Monastier di Treviso/IT

(filippocademartiri@gmail.com)

The role of cardiac computed tomography in cardiovascular diseases is becoming increasingly important. CCT is a robust and reliable investigation for the detection and exclusion of significant coronary artery disease. In addition, CCT provides reliable noninvasive information concerning coronary plaque burden (severity, distribution, type), morphology and function of left and right ventricle, valves, aorta, and so forth. In the past 5 years, the prognostic role of CCT has been established. The prognostic information that can be extrapolated from CCT is very diverse and most of it is still under investigation. There are converging evidences that CCT can stratify cardiovascular risk better than conventional clinical methods, especially in symptomatic patients. For asymptomatic individuals, the role is still controversial since coronary calcium score alone has a very important role already. Several aspects of CCT information seem to have importance for prognostic stratification: plaque presence, obstruction, plaque type and distribution. More recent studies have started showing the relationship between CCT and pharmacological treatment. This is a very new and appealing topic, since the evidence is towards the fact that the most effective pharmacological treatment (e.g. statin) should be restricted to patients with at least some nonobstructive CAD as detected by CCT. This might become a revolutionary concept in cardiovascular medicine with several implications.

Learning Objectives:

1. To learn about and integrate the prognostic information from coronary CT angiography into conventional methods.

2. To learn about coronary CT angiography and conventional methods.

3. To appreciate the prognostic impact of coronary CT angiography and conventional methods.

\section{A-149 16:51}

Incidental findings in cardiac $\mathrm{CT}$ : how to report and proceed

S. Leschka; St. Gallen/CH (sebastian.leschka@kssg.ch)

Rapid advances in computed tomographic (CT) technology have facilitated the widespread use of CT for cardiac imaging worldwide, and can be considered nowadays an essential part of the clinical workup of patients with suspected coronary artery disease. Albeit that the performed CT study is focussed on the cardiac structures and the coronary arteries, adjacent noncardiac structures are in the field of view whenever a patient undergoes cardiac CT to quantify coronary calcium or to perform a noninvasive coronary angiogram. In addition, the location of the heart near other anatomic regions, including the lungs, mediastinum, upper abdomen, and bones, necessitate that these structures are included when acquiring coronary CT angiographic images. Several publications have demonstrated a high frequency of incidental findings on coronary CT angiography. The availability of this information is part of an ongoing clinical debate. While some radiologists believe that every image should be interpreted completely, others believe that examining noncardiac structures reveal too many incidental findings of uncertain clinical significance, which may harm because additional testing increases risk, cost, and patient anxiety. The aim of this lecture is to separate solid facts from opinions and beliefs.

Learning Objectives:

1. To understand the pros and cons of reporting extracardiac findings at cardiac CT

2. To understand the clinical consequences of incidental findings.

3. To learn when additional testing is reasonable and when it's not. Author Disclosure:

S. Leschka: Speaker; Siemens Healthcare, Guerbet, GE Healthcare.

Panel discussion:

Cardiac CT: ready to go clinical?
16:00 - 17:30 Room C

Controversies in Chest Imaging

MC 724

\section{Diagnosis of pulmonary embolism when CTA is indeterminate or unfeasible}

Moderator:

A. Perrier; Geneva/CH

Teaser:

M. Rémy-Jardin; Lille/FR

A-150 16:00

A. Radiological approach: ultrasound and MR imaging

M.-P. Revel; Paris/FR (marie-pierre.revel@htd.aphp.fr)

MR imaging and ultrasound may be used as alternative techniques for pulmonary embolism (PE) diagnosis, when $\mathrm{CT}$ angiography is indeterminate or not feasible, which occurs in up to $22 \%$ of the cases. Deep venous thrombosis (DVT) and pulmonary embolism are two clinical presentations of the same disease. Finding a proximal DVT in patients suspected of PE is sufficient to warrant anticoagulant treatment without further testing. The only validated diagnostic criterion for DVT is incomplete compressibility of the vein on compression ultrasound (CUS). A positive CUS of the proximal lower limb veins is highly predictive of PE on computed tomography in suspected patients. A large prospective outcome study reported $39 \%$ sensitivity of CUS for the presence of PE on MSCT and 99\% specificity. Including the distal veins increases CUS sensitivity, but decreases specificity to only $84 \%$. The probability of a positive proximal CUS in suspected PE is higher in patients with leg symptoms. MRI diagnosis of PE is mainly based on MR angiography (MRA) sequences. Unenhanced sequences using steady-state-free precession are now available, in addition to traditional gadolinium-enhanced MRA. There are conflicting results regarding the diagnostic value of perfusion sequences, with reported sensitivity reaching $100 \%$ in some series, whereas others report only $75-79 \%$ sensitivity. MR shows very high specificity for the diagnosis of PE, reaching $99 \%$, but still limited sensitivity, except for proximal PE diagnosis. Its main limitation is the high proportion of technically inadequate or inconclusive images, ranging from 25 to $30 \%$, but ongoing developments should overcome this current limitation.

Learning Objectives:

1. To understand the role of ultrasound in the diagnostic strategy for pulmonary embolism.

2. To learn about the technical aspects of MR imaging in the diagnosis of pulmonary embolism.

3. To appreciate the performance and limitations of MRI in the diagnosis of pulmonary embolism.

\section{A-151 16:25}

B. Nuclear medicine approach: combined ventilation-perfusion SPECT and low-dose CT

H. Gutte; Copenhagen/DK (henrikgb@gmail.com)

The diagnosis of pulmonary embolism (PE) is usually established by a combination of clinical assessment, D-dimer testing, and imaging with either pulmonary ventilation-perfusion $(\mathrm{V} / \mathrm{Q})$ scintigraphy or pulmonary multidetector CT angiography (CTPA). V/Q scintigraphy is a functional imaging method using isotopes for the visualisation of ventilation and perfusion distribution in the lungs. In recent years, the imaging techniques for diagnosing PE have improved. Many nuclear medicine centres have adopted the single photon emission computed tomography (SPECT) technique as opposed to the planar technique when diagnosing PE. The introduction of 3-dimensional V/Q SPECT technology instead of 2-dimensional planar V/Q scintigraphy has resulted in fewer indeterminate results and a higher diagnostic value. The latest improvement is the addition and combination of a low-dose CT without contrast to the VIP SPECT (SPECT/CT) technique. The SPECT/CT in combination has been compared to CTPA, planar scintigraphy and SPECT alone, and one study has shown that SPECT/CT in combination had the highest diagnostic accuracy for PE diagnosis. SPECT in combination with low-dose CT without contrast enhancement is superior, especially for small subsegmental emboli; however, consensus is lacking regarding the clinical impact and treatment. SPECT and SPECT/CT may provide alternative diagnosis if PE is refuted and are feasible in almost all patients, because there are no definitive contraindications. In the present lecture, SPECT and SPECT in combination with low-dose CT are discussed in the context of diagnosing PE. Learning Objectives:

1. To understand the principle of hybrid gamma-camera/MDCT system. 2. To appreciate the diagnostic performance and limitations of SPECT-CT for the diagnosis of pulmonary embolism. 
A-152 16:50

Discussion

A. Perrier ${ }^{1}$, M. Rémy-Jardin ${ }^{2} ;{ }^{1} \mathrm{Geneva} / \mathrm{CH},{ }^{2}$ Lille/FR

The discussion will address the following issues:

The choice of imaging modality in cases of indeterminate or unfeasible CTA depends on: patient age, pregnancy, presence or absence of clinical symptoms suggesting limb venous thrombosis, acute or chronic clinical situation, imaging technique availability, level of clinical probability of thromboembolic disease

$16: 00-17: 30$ Room D

\section{Emergency Radiology}

RC 717

\section{ER: basic principles - polytrauma: feel your own pulse first! \\ Moderator: \\ D.R. Kool; Nijmegen/NL}

A-153 16:00

A. Logistics and management

M. Körner; Landshut/DE (markus@dr-koerner.net)

Over the last few years, emergency radiology (ER) has been a subspeciality with growing interest. Because of rapid developments in technology, CT has become the most useful tool in evaluating trauma and other emergency patients. In this lecture, the basics of planning and organising an ER department are presented and discussed. Besides optimising technical equipment and protocols for imaging, different logistic concepts have to be considered in planning and organising ER departments. First of all, logistic concepts have to be considered for obtaining an optimal workflow: the radiology department has to be in close proximity to the emergency department and the admitting area, in particular. The whole workflow must be optimised for speed and accuracy. This also mandates having dedicated and standardised examination and viewing protocols for CT. In contrast to the USA where dedicated emergency radiology departments are well established, nonspecialised radiologists still frequently do the reading of emergency radiology cases in European countries. The radiologic staff involved has to be trained for interpretation of trauma and other emergent cases. This does not only account for residents, but also for consultants and attending radiologists. Since a large number of cases will arrive during after hours and on weekends, staffing has to be adjusted to this fact, which includes attending radiologists to be available during these hours on call or, preferably, on-site. This lecture will give an overview of logistic concepts and organisation of an emergency department and will also discuss critical issues in polytrauma imaging.

Learning Objectives:

1. To understand how an emergency radiology department should be organised.

2. To become familiar with the logistics, staffing and technical equipment of an ER department operating 24/7.

A-154 16:30

B. Polytrauma: choosing the right protocol

S. Wirth, M. Muggenthaler, L. Geyer, K. Weber, F. Mück, M.F. Reiser;

Munich/DE (swirth@med.uni-muenchen.de)

At least during the radiological training in hospitals, every radiologist will personally be confronted with the treatment of polytraumatised patients. It is a fact that standardised whole body MDCT (WB-CT) as an independent predictor is able to save around $20 \%$ of patient lives. Thus, WB-CT is the major diagnostic tool and should be performed as fast as possible. Some institutions bypass the emergency room and directly start patient stabilisation in the CT cabinet. However, radiographs and focused ultrasound remain important in general settings as long as they are performed in a way which does not delay CT diagnostics. After scanning, specially trained radiologists support the whole interdisciplinary team with correct, appropriate and prioritised diagnoses. Besides optimisation of parameters like logistics, patient positioning preferably 'feet first', reading and communication, 'choosing the right protocol' is a crucial factor for ideal radiological patient care. Four types of protocols should be differentiated: 1. Polytrauma 1 - WB-CT with scanning the upper abdomen twice, one time in arterial and one time in portal venous phase; 2 . Polytrauma 2 - WB-CT without scanning the upper abdomen twice, but instead utilising a double bolus protocol for mixed phase contrast enhancement; 3 . WB-CT under resuscitation; 4 . WB-CT as triage tool in the case of mass casuality incidents ( $\mathrm{MCl}$, up to 20 patients per hour).

Learning Objectives:

1. To understand the relationship between emergent imaging and emergency radiology.

2. To learn more about the rational use of $C R$, US and CT according to patient priorities in the emergency setting.

3. To become familiar with established whole-body MDCT protocols and their relation to injuries.

A-155 17:00

C. Whole-body CT: dose reduction and data handling

M. Rieger; Hall i. Tirol/AT (michael.rieger@tilak.at)

There is a wide agreement that for patients with polytrauma, integrating whole body computed tomography (CT) scan into early trauma care significantly increases the probability of survival. Therefore, CT is today considered the most important imaging technique in the diagnostic workup of polytrauma patients. The downside of whole body CT represents the relatively high radiation exposure. To overcome this handicap, the following measures can be taken: 1. positioning of the arms during whole body CT above the head results in reduction of radiation dose; 2 . adaption of scan parameters, especially reduction of tube voltage to $100 \mathrm{kV}$ reduces radiation dose; 3 . installation of recent technical improvements like iterative reconstruction algorithm reduces noise in the image, allowing a downward adjustment in radiation dose to obtain standard diagnostic quality images; 4 . installation of dose control software enables optimisation and improved dose management over time. Another disadvantage of high-resolution whole body $\mathrm{CT}$ is the amount of acquired data. To optimise the workflow in the evaluation of a couple of thousand submillimetre images it is necessary to connect the MDCT scanner with a dedicated workstation for volume image reading, which offers the possibility of quick multiplanar online evaluation.

Learning Objectives:

1. To understand new concepts of CT dose reduction.

2. To become familiar with the correlation of large data sets and the need for rapid data acquisition in the emergency radiology setting.

16:00 - 17:30 Board Room B

\section{New Horizons Session}

\section{NH 7}

\section{The human connectome: a comprehensive map of brain connections}

\section{A-156 16:00}

Chairman's introduction: definitions and basic imaging technique P. Hagmann; Lausanne/CH (patric.hagmann@epfl.ch)

In this introduction I will define the connectome (a comprehensive map of neural connections in the brain) and related concepts such as scaling, structural and functional connectivity as well as the integration-segregation paradigm. The development of MRI-based techniques such as white matter tractography and segmentation of white and gray matter has played a crucia role in the emergence of connectomics by providing tools to map in vivo the entire human structural connectivity at a macroscopic scale. I will explain these methods and how from MR imaging a human connectome can be mapped and represented as a network (set of nodes and edges). Finally, I will also review the validation work related to those techniques and mention the foreseen technical advancements in the field.

Session Objectives:

1. To learn what the human connectome is and how it is affected by brain disease.

2. To understand how the human connectome can be imaged and characterised with MRI.

3. To become familiar with the network formalism and its interpretation. 4. To understand the relationship between brain function and underlying structural connectivity.

5. To understand the conceptual ideas behind connectomics. 6 . To learn about the general workflow, from diffusion imaging to mapping a structural brain network.

7. To become familiar with the robustness of the technique by reviewing validation studies and getting a glimpse of the technical challenges. 
A-157 16:15

The economics of brain networks

E. Bullmore; Cambridge/UK (etb23@cam.ac.uk)

We review the methods and recent results of network analysis of human neuroimaging data. Human functional MRI and structural MRI data can be analysed using mathematical tools drawn from graph theory to quantify the complex (non-random) topological properties of brain networks. These results can be substantiated by meta-analysis of prior neuroimaging results and analysis of non-human nervous systems. Brain networks consistently express complex topological features, such as small-worldness, hubs, modules and rich clubs. Some of these features entail disproportionate biological cost, but may be "worth it" by supporting integrative information processing and adaptive behaviours. Highly connected hub nodes are high cost/high value network components that likely also represent special points of vulnerability for diverse brain disorders. These economical principles of brain network organisation are expressed at the microscopic scale, e.g., in the nervous system of the nematode worm $C$. elegans, and may therefore provide a new axis for translation between macro and micro systems neuroscience. Neuroimaging can be used to elucidate the economical principles of human brain network organisation in health and disease.

Learning Objectives:

1. To understand network metrics through examples of different network types and their characteristics.

2. To appreciate the organisation of the human connectome in health and disease.

3. To become familiar with the relation between network organisation and cognition

Author Disclosure:

E. Bullmore: Employee; GlaxoSmithKline. Shareholder; GlaxoSmithKline.

A-158 16:35

Connectomics in brain pathology

M.P. van den Heuvel; Utrecht/NL (m.p.vandenheuvel@umcutrecht.nl)

Healthy brain function depends on efficient functional communication within a complex network of structural neural connections, a network known as the connectome. Conversely, damage to the brain's network, disrupting local neuronal processes and/or global communication between remote functional systems may lead to brain dysfunction. In the last few years, emerging evidence from a wide variety of studies suggests that connectome abnormalities may indeed play an important role in the aetiology of several brain disorders. In my talk, I will discuss the results of recent studies suggesting an important role for affected connectome organisation in a number of neurological and psychiatric disorders. In particular, I will highlight the findings of affected functional and structural brain network in neurodegenerative disorders such as Alzheimer's and ALS, as well as discuss how the application of network science and connectomics may aid our understanding of the biological basis of psychiatric disorders such as autism and schizophrenia.

Learning Objectives:

1. To understand the role of connectome architecture in (cognitive) brain function and dysfunction using diffusion MRI/functional MRI.

2. To become familiar with connectomics as a tool for examining disease pathology in a wide range of neurological and psychiatric brain disorders.

\section{A-159 16:55}

Linking structure and function: the role of modeling in understanding the pathophysiology

G. Deco; Barcelona/ES (gustavo.deco@upf.edu)

The interplay of the brain's intrinsic activity and the external world has seen revival in the last decade, especially in neuroimaging. A long-held assumption in many of these studies has been that ongoing brain activity is sufficiently random that it averages out in statistical analysis. Hence, imaging studies are termed 'activation' paradigms, where experimental manipulation results in the activation of cerebral circuits that are necessary for performing the task. Nevertheless, a large amount of recent literature reports a body of observations that there are consistently distributed patterns of activity during rest. This fact has led to the suggestion that it might be possible to characterise network dynamics without needing an explicit task to drive brain activity. Indeed, numerous neuroimaging experiments have evidenced the solid existence of spontaneous long-range correlations, i.e. functional connectivity (FC), by fMRI, MEG, and EEG techniques. The functional connectivity is defined as the statistical dependence between remote neurophysiological dynamics. The emergence of resting functional connectivity is intrinsically linked with the underlying anatomical connections between those areas, i.e. the structural connectivity. Whole brain modeling can indeed establish a direct link between structure and function. Furthermore, the modeling can shed light on the origin of pathological functional disfunction by making the link with the underlying anatomy explicit. We will show how, structural DTI tractography, functional imaging and modelling studies can be combined for reaching this goal.

Learning Objectives:

1. To understand large-scale models of the whole brain.

2. To learn about the mechanisms underlying resting and task activity.

3. To become familiar with the fusion of structural (diffusion MRI) and functional (functional MRI/MEG) data.

Panel discussion:

What will connectomics bring to clinicans in the future?

$17: 15$

$16: 00-17: 30$

Room E1

\section{Musculoskeletal}

RC 710

Trauma to the paediatric skeleton

Moderator:

M. Padrón; Madrid/ES

A-160 16:00

A. Pelvis/hips

N. Boutry, B. Herbaux; Lille/FR (nboutry@gmail.com)

Traumas to the paediatric pelvis and hip include traumatic dislocations of the hip, fractures of the femoral neck, fractures of the pelvic ring, acetabular fractures and apophyseal avulsion fractures. Traumatic dislocations of the hip, fractures of the femoral neck, fractures of the pelvic ring and acetabular fractures are rare in children (less than $5 \%$ of paediatric fractures), as compared to adults. These fractures are commonly the result of high-energy trauma. Imaging is based on plain radiographs, but CT and MRI are very useful to precisely assess bone (CT) and cartilage and soft tissue (MRI) lesions. Imaging enables accurate diagnosis, appropriate treatment and detection of potential complications (femoral head osteonecrosis, premature physeal closure ...). In contrast with the previous injuries, apophyseal avulsion fractures of the hip and pelvis are common in children and adolescents, usually associated with athletic activities. In most cases, these fractures are of good prognosis and can be treated conservatively when minimally displaced. Plain radiographs confirm avulsion injuries to ossified apophyses, but MRI and ultrasound are the modalities of choice to demonstrate injuries to nonossified apophyses and to assess apophyseal displacement.

Learning Objectives:

1. To become familiar with the types of injuries seen in the paediatric pelvis/hips.

2. To understand the strengths and weaknesses of different imaging modalities.
A-161 16:30
B. Elbow
K.J. Johnson; Birmingham/UK (karl.johnson@bch.nhs.uk)

The elbow is a very common site for fractures in a child. The challenge for the radiologist is to differentiate normal variants of growth from possible injury and this is usually achieved by having good-quality radiographs and an understanding of normal growth. It is important to recognise those fractures which require surgical intra-operative treatment. In a small number of cases when assessing for vascular integrity, intra-articular extent and injury to cartilaginous structures, ultrasound and MR imaging have a valuable role. This lecture will give an overview of the radiographic appearance of fractures, highlight the features which require orthopaedic intervention and illustrate the use of additional imaging modalities.

Learning Objectives:

1. To become familiar with the types of injuries seen in the paediatric elbow.

2. To understand the strengths and weaknesses of different imaging modalities.

\section{A-162 17:00}

C. Spine

L.B.O. Jans; Ghent/BE (lennartjans@hotmail.com)

The diagnosis of C-spine injury is more complex in children than in adults. Early diagnosis is crucial since delayed diagnosis results in high morbidity and mortality. Leading mechanisms of pediatric C-spine trauma are motor vehicle accidents, sports and pedestrian injuries. Due to their anatomy, children are prone to different types and locations of injuries. Children $<10$ yeas of age are more likely to injure the spinal cord itself and are prone to dislocations and high (C1-C4) bony injuries. Children $>10$ years of age more often sustain C-spine fractures. In children under the age of 2 years, radiographs are rarely helpful. 
AP and lateral radiographs are helpful in children aged between 2 and 8 years. In children aged $>8$ years, additional lateral and odontoid views are obtained. Anatomical variants such as pseudosubluxation of $\mathrm{C} 2-\mathrm{C} 3$, widening of the atlantodental interval and ossification centres may appear to be of concern on imaging, but are normal. Abnormal radiographic findings require additional imaging to differentiate them further with CT on the area of concern. MRI is mandatory if signs of atlantorotary subluxation and spinal cord injury without radiologic abnormality (SCIWORA) are present. MRI identifies injuries to the spinal cord that are not apparent with other modalities, and should be used when a child presents with a neurologic deficit but normal radiographs on CT scan

Learning Objectives:

1. To become familiar with the types of injuries seen in the paediatric spine

2. To understand the strengths and weaknesses of different imaging modalities.

$16: 00-17: 30$ Room E2

Foundation Course: Breast Imaging

\section{$E^{3} 720 b$}

\section{Image-guided breast biopsy \\ Moderator:}

G. Esen; Istanbul/TR

\section{A-163 16:00}

A. US-guided biopsy of breast and axilla

L.J. Pina Insausti; Pamplona/ES (ljpina@unav.es)

Nowadays, ultrasound (US) has become the first choice for performing most breast biopsies. The main advantages of this technique are non-ionising radiation, full control of the needle position in real time and widespread use of US equipments at all centres. US allows access to difficult places (such as the axilla or near the nipple), multiple lesions can be safely biopsied in one single session, the breast is not compressed, there is excellent comfort for patients and radiologists, local anaesthesia and haematoma do not hide the lesion and it is a cost-effective technique. However, the main limitation is that the lesion must be visible on US. US-guided fine-needle cytology was widely used in the past, but today its use has decreased. US-guided core-needle biopsy has proven to be a reliable technique to perform a biopsy for breast lesions, showing a sensitivity value of about $98 \%$. Furthermore, it can be safely used for performing biopsies of axillary lymph nodes. Vacuum-assisted devices can be used not only for diagnostic purposes, but also for therapeutic ones, because small palpable benign lesions (such as fibroadenomas), papillomas and radial scars can be completely removed.

Learning Objectives:

1. To learn how to do it.

2. To become familiar with the possible complications.

3. To consolidate knowledge on the value of axillary FNA vs. core biopsy.

\section{A-164 16:30}

\section{B. Stereotactic vacuum-assisted biopsy}

R.M. Mann; Nijmegen/NL (r.mann@rad.umcn.nl)

Stereotactic breast biopsy is the performance of tissue sampling under mammographic guidance. As with any imaging technique, some lesions are only seen with mammography. When these lesions are suspicious (e.g. BIRADS 3 or higher), there are no imaging techniques that can downgrade this suspiciousness directly, although for BIRADS 3 lesions follow-up might be a viable alternative. In mammography, most lesions that are only visible on the mammogram consist of clusters of microcalcifications. These harbour a likelihood of around $25 \%$ being malignant and should thus be classified as BIRADS 4. This implies that tissue sampling is mandatory. Different from ultrasound-guided biopsy, it is not possible to perform a real-time biopsy under mammographic guidance. Rather, stereotactic biopsy uses triangulation to assess the depth of a lesion within the breast, while the lesion location in the $\mathrm{x}$ and $y$-plane is assessed on a scout view. The needle is positioned using the coordinates thus obtained. Since there is no real-time feedback of the accuracy of needle positioning, it is necessary to obtain substantially more tissue than under ultrasound guidance. Therefore, vacuum-assisted systems are essential. In case of calcified lesions, the biopsy result is controlled by x-ray. Calcifications should be present in the sample. Biopsy complications that occur under stereotactic guidance are usually limited. Most common is the formation of large haematomas. Scarring may also occur.
Learning Objectives:

1. To learn how to do it.

2. To become familiar with the possible complications.

3. To appreciate the advantages of large core needles.

Author Disclosure:

R.M. Mann: Speaker; Bayer Healthcare.

A-165 17:00

C. MRI-guided biopsy

C. Smith; Dublin/IE (csmith@mater.ie)

Breast magnetic resonance (MR) imaging is the most sensitive modality available to evaluate the breast for cancer. It can detect lesions that are occult at mammography and ultrasound. It has a limited sensitivity and the positive predictive value ranges between 15 and $40 \%$. Histopathological assessment of MR-detected lesions is therefore mandatory. Some of these lesions will be visible at second-look ultrasound and can be biopsied sonographically. A significant number however will only be discernable on MR, and MR-guided biopsy is therefore the sampling technique of choice. MR biopsy has become an essential component of any breast imaging practice. Several MR-biopsy techniques are available including the grid-localising, pillar and post and the freehand techniques. The technique for preparing and performing a MR-guided vacuum-assisted biopsy using the grid-localising technique is reviewed. Potential complications, limitations of MR breast biopsy and actions to prevent failure are discussed. Imaging-histologic correlation is essential to ensure accurate sampling. Clip placement and follow-up imaging should be performed to ensure sampling, including ultrasound correlates. Finally, new developments in MR-guided therapeutic interventions are discussed.

Learning Objectives:

1. To learn how to do it.

2. To become familiar with the possible complications.

3. To learn about the potential of MRI-guided treatment.

16:00 - 17:30 Room F1

Joint Course of ESR and RSNA (Radiological Society of North America): Emergency Radiology

MC 722

\section{Abdominal emergencies}

Moderators:

S.E. Mirvis; Baltimore, MD/US

A. $\underline{\text { Palko; }}$ Szeged/HU

A-166 16:00

A. Abdominal injuries

A. Palko; Szeged/HU (palkoand@gmail.com)

Abdominal injuries require a timely and reliable diagnosis to prevent potentially lethal outcomes. The armoury of clinical tools (physical examination, lab tests) does not fulfil these criteria, since they are either not fast or not reliable. Imaging diagnostic modalities help the clinician to acquire the necessary amount of information to initiate focused and effective treatment. However, the selection of the appropriate imaging algorithm, modality and technique, as well as the precise detection and interpretation of essential imaging findings are frequently challenging, especially because the circumstances, under which these examinations are performed (open wounds, bandages, non-removable life-supporting equipment, lack of patient cooperation, etc)., are frequently less than optimal. Knowledge of critical imaging signs, symptoms and the role they play in the evaluation of the patient's condition, as well as fast decision-making and ability to closely cooperate with the clinicians are skills of key importance for radiologist members of the trauma team.

Learning Objectives:

1. To understand the significance of injury mechanism and its role in the formation of abdominal lesions and their complications.

2. To learn about the role of proper imaging techniques and diagnostic algorithms in the prompt diagnosis of abdominal injuries.

3. To learn more about typical and unusual findings of various abdominal traumatic conditions.

A-167 16:30

B. The enemy within: non-traumatic abdominal emergencies

R.J. Zagoria; San Francisco, CA/US (Ron.zagoria@ucsf.edu)

This presentation will describe the technique and important findings for CT stone studies as well as the accuracy of this study. An explanation of when IV contrast should be given to augment a CT stone study and findings of GU and non-GU diseases that can mimic symptoms of stone disease will be provided. Examples and descriptions of acute mesenteric vascular abnormalities, bowel 
obstruction and infectious conditions will be included. Ways to differentiate and categorise emphysematous infections for proper treatment will be described. Lower tract emergencies will also be illustrated and described. A systematic approach to evaluating abdominal CTs will be described to avoid mistakes. Learning Objectives:

1. To learn how to better analyse CT scans for non-traumatic causes of abdominal pain.

2. To learn about the CT signs and causes of bowel ischaemia.

3. To learn about the CT findings of common causes of an 'acute' abdomen.

4. To learn about the imaging findings of acute, non-traumatic urinary tract and GI tract emergencies.

\section{A-168 17:00}

C. Interactive case discussion

A. $\underline{\text { Palko }}{ }^{1}$, R.J. Zagoria ${ }^{2} ;{ }^{1}$ Szeged/HU, ${ }^{2}$ San Francisco, CA/US

Using cases and an audience response system, this segment of the course will go over the optimal imaging approach for patients presenting with acute abdominal pain and abdominal injuries. CT findings will be emphasised. Key imaging findings of traumatic and nontraumatic causes of acute abdominal pain including gastrointestinal tract and urinary tract pathology will be explained. A systematic approach for the imaging evaluation of patients with abdominal emergencies will be illustrated and explained including proper scan protocols and analysis of imaging findings. Imaging diagnosis of blunt and penetrating abdominal injuries, urinary tract obstruction, infection, bowel obstruction, and ischemia will be emphasised.

Learning Objectives:

1. To learn how to better analyse CT scans for traumatic and non-traumatic causes of abdominal pain.

2. To learn about the CT signs and causes of bowel ischaemia and injuries.

3. To learn about the CT findings of common causes of traumatic and nontraumatic 'acute' abdomen.

4. To learn about the imaging findings of acute, traumatic and non-traumatic urinary tract and $\mathrm{Gl}$ tract emergencies.

16:00 - 17:30

Room F2

Special Focus Session

\section{SF 7a}

\section{Renal artery denervation in the management of resistant hypertension}

A-169 16:00

Chairman's introduction

F. Fanelli; Rome/lT (fabrizio.fanelli@uniroma1.it)

Transcatheter renal denervation represents a novel therapy for treating patients with treatment-resistant hypertension, leading to higher risk of major cardiovascular events. An overview of sympathetic nervous system anatomy, physiology and physiopathology will be followed by a description of the technical aspects of renal denervation. First, results in patients with therapyresistant hypertension look very promising. However, these data need to be confirmed. Importantly, long-term efficacy and safety need to be assessed. As a consequence, the treatment cannot be considered, at the moment, standard therapy. Future studies need to address other disease conditions characterised by sympathetic hyperactivity, including heart failure, chronic kidney failure and others. It is a general opinion that in the near future the treatment will also be applied to lesser severe hypertensive patients. Future studies should also include formal cost-effectiveness analyses.

Session Objectives:

1. To understand resistant hypertension and sympathetic nervous system pathophysiology.

2. To understand the principles of patient selection.

3. To become familiar with the principles and the selection of devices.

Author Disclosure:

F. Fanelli: Consultant; WL Gore \& Associates, Medtronic, Cook. Speaker; WL Gore \& Associates, Medtronic, Cook, Covidien.

\section{A-170 16:05}

Resistant hypertension and sympathetic nervous system

M. Sapoval; Paris/FR (marc.sapoval2@egp.aphp.fr)

Hypertension is the most frequent cardio vascular disease and is responsible for most strokes, cardiac failures, and renal failures. Control of blood pressure using medical treatment is usually achieved by employing different classes of drugs. Resistant hyperension is defined by a patient taking 3 drugs and still having blood pressure higher than $140 / 90 \mathrm{~mm} \mathrm{Hg}$. The data obtained from a general population living in the USA between 2003 and 2008 indicate that resistant HT is observed in approximately $9 \%$ of treated HT patients. Patients with resistant HT can suffer from target organ damage and from the early onset of cardiovascular complications, leading to an increase in morbidity and mortality. It has been shown that the $\mathrm{CV}$ prognosis is directly related to the BP level, which justifies the treatment of resistant $\mathrm{HT}$ in order to obtain BP control In resistant hypertension. It is recognised that there is sympathetic over activity and previous experience of surgical denervation has confirmed this physiopathological evidence. Recent experience with percutaenous renal denervation has shown improvement of blood pressure level in patients presenting with resistant hypertension. Many clinical trials are ongoing to try to better understant the role of renal denervation in the management of resistant hypertension.

Learning Objectives:

1. To understand the pathophysiology of hypertension

2. To become familiar with relationship between blood pressure and autonomous nervous system.

3. To learn about the complications associated with resistant hypertension. Author Disclosure:

M. Sapoval: Consultant; Medtronic, Cordis, ReCor, Boston. Investigator; Medtronic, Cordis, ReCor, Boston.

\section{A-171 16:28}

Renal artery denervation: current technologies, indications and contraindications

G. Goh; London/UK (gerard.goh@stgeorges.nhs.uk)

Renal denervation can be achieved by a number of different techniques that include radiofrequency ablation, intraluminal and high-intensity focussed ultrasound ablation, chemical ablation and radiation ablation. Radiofrequency and ultrasound ablation are currently the most commonly used techniques. Most of the evidence to date is derived from studies and trials based on devices that use radiofrequency ablation technology. There are a number of currently CE-marked devices available and each device differs in the catheter design, generator design and energy delivery. These device types include single-point ablation, multi-point ablation, balloon-mounted systems and irrigated technology. The indications for renal artery denervation have been formulated by various international and European societies based on the various clinical trials and studies. These indications include patients with true resistant hypertension, age 18-85 years, eGFR $>45 \mathrm{ml} / \mathrm{min} / 1.73 \mathrm{~m}^{2}$ (MDRD formula), single renal arteries with diameter $>3-4 \mathrm{~mm}$ with a landing zone of 20-21 mm depending on the device, no significant renal artery stenosis $>30 \%$, no renal artery aneurysm, no previous renal artery angioplasty or stenting and the absence of significant valvular heart disease where lowering blood pressure will be dangerous.

Learning Objectives:

1. To understand the principles of renal denervation.

2. To understand the differences between the different systems available.

3. To learn about indications and contraindications.

Author Disclosure:

G. Goh: Consultant; Boston Scientific, Covidien. Research/Grant Support; Covidien. Speaker; St Jude Medical.

A-172 16:51

Procedural technique and clinical results

J.A. Reekers; Amsterdam/NL (j.a.reekers@amc.uva.nl)

Renal denervation of the sympatic nerves around the renal arteries is a new catheter technique to treat patients with resistant hypertension. Resistant hypertension is seen in about $10 \%-20 \%$ of all patients with hypertension. The mechanism of sympatic nerve driven resistant hypertension will be explained. The available techniques for RDN will be discussed, focusing on the pros and cons of each technique. Currently, RF ablation, HIFU and cryo-ablation are the techniques under investigation. The technique and physics of renal denervation with radiofrequency ablation will be explained in more detail, focusing on available devices in the market. Patient selection and nonresponders will be discussed. The current evidence from the available trials will be reviewed. The future of RDN and the role of radiology and the radiologist will be illuminated.

Learning Objectives:

1. To become familiar with the procedural technique.

2. To learn about parameters influencing clinical outcome.

3. To learn how to build a dedicated service.

Author Disclosure:

J.A. Reekers: Advisory Board; J\&J for RDN.

Panel discussion: How do you start a service? 
16:00 - 17:30

Room G/H

\section{Genitourinary}

RC 707

\section{Renal and adrenal tumours \\ Moderator:}

M. Moschetta; Bari/lT

A-173 16:00

A. Adrenal masses: a practical approach

G. Heinz-Peer; St. Pölten/AT (Gertraud.Heinz@stpoelten.Iknoe.at)

The adrenal glands are composite endocrine organs consisting of the steroid hormone-producing cortex and the catecholamine-synthesising medulla. The increased use of imaging modalities has demonstrated the presence of varying sized mass lesions in up to $5 \%$ of individuals subjected to CT studies for reasons unrelated to adrenal dysfunction. Most of these incidentally discovered lesions are non-functioning benign lesions of cortical origin. However, incidentalomas may also represent functioning lesions and malignant masses. Clinical diagnostic and biochemical evaluation is used to further subdivide functional and non-functional adrenal lesions. F-DOPA has been found to be of high sensitivity and specificity in PET imaging of pheochromocytoma. CT and MR imaging are first choice in characterisation of adrenal lesions. Techniques of dual energy CT and histogram analysis may offer additional information. PET-CT has been shown to contribute to the diagnostic power, especially in oncologic patients. Knowledge of the physiologic appearance of adrenal glands in 18-FDG PET is necessary to correctly identify pathologic processes. FDGPET also has the ability to detect metastatic lesions in non-enlarged adrenal glands. In addition, FDG-PET has the advantage of simultaneously detecting metastases at other sites. The role of MR-DWI and MR-spectroscopy in characterising adrenal masses has to be defined by further studies. Differentiating benign from malignant adrenal masses using non-invasive imaging methods can reduce the need for percutaneous adrenal biopsy in patients with underlying malignant disease and the follow-up imaging of incidentally detected adrenal adenomas.

Learning Objectives:

1. To become familiar with the different imaging appearances of adrenal masses including pathological relation.

2. To learn about the different imaging techniques in order to improve evaluation of benign versus malignant adrenal masses.

3. To understand the impact of imaging given the fact that a patient either has or doesn't have a known malignancy.

\section{A-174 16:30}

B. Staging renal cancer

M. Notohamiprodjo

Munich/DE (mike.notohamiprodjo@med.uni-muenchen.de)

Renal cell carcinoma (RCC) is the most common malignant tumour of the kidney. As response rates to radiation and nonantiangiogenic chemotherapy are low, surgical excision, i.e. radical nephrectomy has been the treatment of choice. However novel treatment options have emerged, so that imaging of RCC is of increasing interest. Nephron sparing partial nephrectomy has become an accepted operative option and antiangiogenic agents such as inhibitors of tyrosine kinase (TK) and mammalian target of rapamycin (mTOR) have been approved for treatment of advanced RCC. Thus, it has become important to perform correct local staging and identify patients suitable for partial nephrectomy using standardised scoring systems such as RENAL or PADUA. Furthermore, optimal imaging strategies for monitoring of advanced and metastatic RCC are discussed, as current antiangiogenic therapy evaluation in clinical routine is based only on morphological imaging information, but changes in tumour size may lag behind functional changes. Finally, common side effects, i.e. pneumonitis, of vascular disruptive agents are addressed.

Learning Objectives:

1. To be able to recognise the CT/MRI/US findings for staging.

2. To learn about the optimal imaging protocol for the diagnosis and staging of renal cancer.

3. To understand treatment options and implications.

Author Disclosure:

M. Notohamiprodjo: Investigator; Novartis, GSK.

\section{A-175 17:00}

C. How to deal with small indeterminate renal masses

J.-M. Correas, O. Hélénon; Paris/FR (jean-michel.correas@nck.aphp.fr)

Characterisation of renal masses can be performed using three categories with respect to the lesion size and gross architecture: the indeterminate very small masses, the cystic, and solid renal masses. Lesions with diameters below $10 \mathrm{~mm}$ are usually difficult to classify due to partial volume effect that prevents accurate CT attenuation measurement. In the general population, these lesions are likely to be microcysts and do not require further workup. Better characterisation remains needed in selected patient populations such as patients with hereditary renal tumour disease and previous history of renal carcinoma. In this case, MRI combining T2w, Diffusion-Weighted imaging and Dynamic contrast enhanced sequences or contrast-enhanced US may help differentiate very small cysts from solid neoplasms. Cystic renal mass characterisation still relies on Bosniak's classification with 5 categories: benign (I) and minimally complicated (II) cysts, indeterminate cystic lesions (IIF and III) and malignant cystic masses (IV). Some cystic masses remain unclassified at CT because of atypical attenuation characteristics or enhancement properties. US, contrast-enhanced US, and MRI are now playing a key role by providing additional diagnostic information that help distinguish between atypical fluid filled masses and atypical solid neoplasms, especially poorly enhancing solid papillary RCC. The characterisation of small solid renal tumours starts at CT with the identification of macroscopic fat, a typical feature of angiomyolipoma. In the case of non-fatty indeterminate renal neoploasms, percutaneous-guided biopsy can be performed when accurate characterisation is needed before surgery or when renal metastases or lymphoma are suspected.

Learning Objectives:

1. To become familiar with the various appearances of small indeterminate renal masses.

2. To learn about the respective roles of US, CT and MR imaging in investigating small renal masses.

3. To learn the main pitfalls in assessing small renal masses.

$16: 00-17: 30$ Room I/K

\section{Special Focus Session}

\section{SF $7 b$}

\section{Imaging biomarkers in cancer drug development}

A-176 16:00

Chairman's introduction

B.E. Van Beers; Clichy/FR (bernard.van-beers@bjn.aphp.fr)

Drug development being a complex and costly process, there is an increasing need for imaging biomarkers to take go/no go decisions in the early clinica phases. The RECIST criteria based on tumour size measurements at CT are currently used for this purpose. However, additional functional and molecular biomarkers have been developed to assess the early biological effect of drugs on tumours. Development of imaging biomarkers is a structured process in which new biomarkers are discovered, validated and qualified against biological processes and clinical end points. The validation not only concerns the determination of the sensitivity and specificity, but also the measurement of reproducibility. Reproducibility assessments, standardisation of the acquisition and data analysis methods and quality control are crucial when imaging biomarkers are used in multi-centre trials. Functional and molecular parameters obtained at perfusion imaging, diffusion-weighted MR imaging and PET are being developed and validated. The perspectives (earlier assessment of response to treatment) and limitations (limited validation and standardisation) of these imaging biomarkers in cancer drug development will be presented.

Session Objective:

1. To introduce the general context of imaging biomarkers in drug development.

\section{A-177 16:05}

Qualification of imaging biomarkers in drug development

J.C. Waterton; Manchester/UK (John.Waterton@manchester.ac.uk)

Most imaging tests used in cancer drug development are correctly called biomarkers. Important imaging biomarkers include TNM stage, PFS and OR. More recently, pharmacodynamic imaging biomarkers such as FDG-SUV and $\mathrm{K}^{\text {trans }}$ have been introduced. Drug developers are reluctant to use more exploratory unvalidated imaging biomarkers (i.e. cannot distinguish a true negative from a false negative). The extensive literature on biomarker validation mostly refers to biochemical biomarkers extracted as analytes from biospecimens and is unhelpful to radiologists. Unlike biospecimen biomarkers, the quality and validity of imaging measurements as biomarkers depend crucially on the use of a diagnostic imaging device, in the presence of the patient, in a manner for which the device (a) was not designed, (b) has not received regulatory approval and (c) may be unfamiliar to the user in the trial site. Technical validation and biological validation are orthogonal activities. "Technical validity" is confidence that the imaging biomarker can be measured 
reliably anywhere in the world. "Biological validity" is confidence that the biomarker correctly reports some underlying biology that is important to the patient's future clinical outcome. Since the physician always has access to the patient's clinical status and history, the biomarker is only useful if it provides a better forecast than clinical data alone. (If the forecast is near perfect, the biomarker might be a surrogate end point). Technical and biological validations are massive undertakings best achieved by consortia, and in particular publicprivate partnerships, of which the Innovative Medicines Initiative in Europe and the Biomarkers Consortium in the USA, are prominent examples.

Learning Objectives:

1. To understand the steps and hurdles in drug development and the need for imaging biomarkers.

2. To consolidate knowledge on the steps towards the qualification of imaging biomarkers.

3. To learn about the problem of double validation for the use of biomarkers in drug development.

Author Disclosure

J.C. Waterton: Employee; AstraZeneca. Research/Grant Support;

AstraZeneca. Shareholder; AstraZeneca.

\section{A-178 16:28}

Functional imaging in cancer drug development

N.M. deSouza; Sutton/UK (nandita.desouza@icr.ac.uk)

The standard imaging assessment of tumour response relies on size measurements, which, with predominantly cytostatic targeted agents, may not reflect the drug effect. Functional imaging biomarkers have the potential to quantify the biological characteristics of tumours and measure on-target and off-target effects that indicate early likelihood of response to a specific therapy, which can then be used to guide the optimal biological dose and drug schedule. Serial, non-invasive assessments of whole tumour are possible. This is particularly important in the context of inter and intra-patient tumour heterogeneity, as different parts of the tumour and primary vs metastatic lesions may be biologically different and these characteristics may change with treatment. However, functional imaging end points suffer from variability, which can be very significant in a multicentre setting. Strict quality assurance and quality control measures need to be implemented at the start of a trial and the variability across centres documented. Data acquisition protocols need to take account of equipment variations. Data analysis methodology needs standardisation of software, central review and preferably double reading of scans. Automation may not always prove the most robust and reliable option. This presentation will focus on the factors that are crucial in determining the compatibility of data in multicentre trials with functional imaging end points. Learning Objectives:

1. To learn about the potential role of quantitative imaging in processes related to tumour growth such as cell metabolism, cell death, and vascular function in the assessment of tumour response.

2. To become familiar with the issues of accuracy, reproducibility and standardisation for using functional imaging biomarkers in drug development. Author Disclosure:

N.M. deSouza: Research/Grant Support; CRUK and EPSRC Cancer Imaging Centre in association with MRC and Department of Health (England; grant C1060/A103334), NIHR funding to the Clinical Research Facility in Imaging and the Biomedical Resea.

\section{A-179 16:51}

Quantitative nuclear medicine in drug development

W. Weber; New York, NY/US

Nuclear medicine techniques can detect and quantify very low concentrations of radiolabelled pharmaceuticals in the human body. This allows investigators to use nuclear imaging for various purposes during drug development. Nuclear imaging can visualise drug targets that are only present in nanomolar concentrations and can thus identify patients most likely for therapies directed against these targets. A recent example is folate receptor SPECT imaging for the selection of patients for treatment with a folate-targeted drug conjugate. In addition, nuclear imaging and specifically PET can measure the concentration of pharmaceuticals within tumour and normal organs over time. Nuclear imaging can also be used to monitor target inhibition, for example the blocking of oestrogen receptors by anti-oestrogens. Finally, nuclear imaging can assess tumour response to therapy by measuring changes in tumour metabolism or proliferation, e.g. with FDG- and FLT PET/CT. This allows an earlier and more sensitive detection of tumour response than morphologic techniques. Since PET is a whole body imaging technology, PET imaging can also be used to study the heterogeneity of target expression, tissue pharmacokinetics, target inhibition and response. PET imaging is now widely available in many countries and has become internationally standardised. It is therefore a robust clinical technique that will increasingly be used during drug development.
Learning Objectives:

1. To understand the role and limitations of radiolabelled functional and molecular imaging biomarkers in drug development from the preclinical phase to phase 3.

2. To consolidate knowledge on the specific role of radiolabeled versus other imaging biomarkers.

Author Disclosure:

W. Weber: Advisory Board; Endocyte, MSD. Grant Recipient; Bayer, Philips

Panel discussion:

What new imaging biomarkers are on the horizon in

drug development? 17:14

$16: 00-17: 30$

Room L/M

Interventional Radiology

RC 709

\section{The diabetic foot: contribution of interventional radiology}

\author{
A-180 16:00 \\ Chairman's introduction \\ J. Lammer; Vienna/AT (johannes.lammer@akhwien.at)
}

There are about 80.000 patients per year in Europe who lose their leg. About $70 \%$ are due to diabetic ischaemic and ulcerative leg problems. In diabetic patients the prevalence of foot ulcerations is about $20 \%$ and the risk for developing foot ulcerations is increased four times. Diabetic foot problems may be due to neuropathy and malperfusion or a combination of both. The neuropathic ulcer and the neuroischaemic ulcer are usually at the plantar pedis, have a punched-out appearance and are painless. Bone deformities (Charcot foot) may be associated. The arterial ulcer is usually at the toe, forefoot and ankle, with pale, cold skin and it may be painful. Patients need a multimodality approach involving a diabetologist, vascular specialist (angiologist, vascular surgeon, interventional radiologist) and podiatrist. Pain control, antibiotic and antithrombotic treatment and the treatment of cardiovascular risk factors and other co-morbid disease have to be done first. In case of ischaemic pain and ulceration imaging such as MRA and CTA is the next step. Patients should be referred to a team of vascular specialist early in the course of their disease to plan for revascularisation options. Revascularisation is the optimal treatment for patients with an ischaemic and neuroischaemic diabetic foot.

\section{A-181 16:05}

\section{A. Pathophysiology of the diabetic foot}

V. Bérczi; Budapest/HU (Berczi@hotmail.com)

The incidence of diabetes mellitus, associated with both predisposing genetic and environmental factors, is increasing globally. Several major clinical trials have proved that complications may occur many years following proper glycaemic control. Besides peripheral arterial disease, sensory and motor neuropathy along with an altered response to infection is of crucial importance. Recent studies have showed that microvascular occlusive arterial disease is not a major factor affecting the diabetic foot; infrapopliteal macrovascular disease and microvascular dysfunction (e.g. arteriovenous shunting, precapillary sphincter malfunction, capillary leakage, venous pooling) are major components of impaired perfusion of diabetic foot. There are no randomised controlled trials analysing the major outcome following endovacular or open bypass surgery. The major outcomes, however, were similar in the case series: 1 -year limb salvage showed a median of $85 \%$ (interquartile range $80-90 \%$ ) and $78 \%$ (interquartile range $70.5-85.5 \%$ ) following open surgery and endovascular treatment, respectively. Limb salvage rate, however, was considerably higher with either type of revascularisation compared to medical therapy. Negative pressure wound therapy, hyperbaric oxygen therapy, effective off-loading or nonweight-bearing therapies (total contact casts rather than removable devices) have also shown promising results in recent publications. The International Working Group on the Diabetic Foot (IWDGF) has produced a guideline in 2011 on the diagnosis and treatment of peripheral arterial disease in patients with diabetes and foot ulcers (Diabetes Metab Res Rev 2012;28 (Suppl 1):179-217; Diabetes Metab Res Rev. 2012;28 (Suppl 1):218-24). Diabetic foot ulcers should be managed by a multidisciplinary team (medical, surgical, endovascular, podiatric, nursing and orthotic experts). Learning Objectives:

1. To undertstand the pathophysiology of the diabetic foot

2. To learn about foot care.

3. To understand the outcomes and complications. 
A-182 16:28

B. Imaging and angiosom concept

A. Basile; Catania/IT (antodoc@yahoo.com)

Endovascular arterial revascularisation is today a solid option in the management of CLI with low complication rates and limb salvage rates comparable with surgery. The restoration of adequate blood flow to the foot is crucial to facilitate wound healing, provide pain relief, and avoid whatever amputations. The angiosome concept was first introduced in 1987 by Taylor and further developed by Attinger for planning treatment of ischaemic lesions of the foot. They divided the foot into six distinct angiosomes, arising from tibial and peroneal arteries. Planning the procedure on the basis of this concept will yield the best local results of wound healing, compared with the indirect intervention. A proper pre-procedure assessment through colour Doppler US and DSA of lower limbs is mandatory for guiding the procedure through the vessels of the foot. Several studies have evaluated the efficacy of PTA in the BTK and the reliability of the angiosome model, approaching $100 \%$ with a limb salvage rate of up to $87 \%$ at 12 months. On the basis of these data, we can conclude that PTA in diabetic patients with BTK disease is a safe and effective technique. The first endovascular treatment option is related to the angiosome model, but when not feasible the indirect technique is also a valid and similarly effective procedure.

Learning Objectives:

1. To understand the angiosome concept.

2. To understand variations in blood supply to the foot.

3. To apprciate the impact of the angiosome concept on treatment strategy.

\section{A-183 16:51}

C. Special techniques for crural endovascular revascularisation S.D. Qanadli; Lausanne/CH (Salah.Qanadli@chuv.ch)

To face technical failures, up to $20 \%$ in crural chronic total occlusion a decade ago, different approaches and dedicated devices and technologies have been developed in the last few years. Furthermore, percutaneous revascularisations are gaining more interest, particularly in patients with critical limb ischaemia not only as first-line treatment, but also as the only possible treatment for complex lesions and high-risk patients. Special techniques for crural endovascular revascularisations are by design techniques rarely used in routine practice, but could offer wide possibilities for the interventional radiologist to solve challenging situations and manage complex lesions. These techniques include options for arterial access (trans-popliteal, trans-tibial, trans-pedal), approaches for crossing chronic total occlusion (assisted endoluminal, lambda technique, re-entry technologies) and uncommon routes for angioplasty/recanalisation (trans-metatarsal loop technique, trans-collateral techniques). Combined antegrade and retrograde approaches have also been developed as well as related methods for successful re-entry. Deciding when the patient/lesion is a potential candidate for special techniques is also a crucial issue. The interventional radiologist should be able to convert at any time if necessary the strategy of treatment to another one. He should also be able to select the most appropriate technique for the patient in planning revascularisation. Continuous medical education and training is however mandatory and for some techniques the learning curve is relatively long.

Learning Objectives:

1. To review different tools for crural endovascular revascularisation.

2. To learn about different access points.

3. To discuss how to select the appropriate technique.

Author Disclosure:

S.D. Qanadli: Consultant; BARD, ABBOTT VASCULAR, CORDIS. Patent Holder; Calibrated Vascular Introducer, Multimodality Vascular Phantom. Research/Grant Support; BIOTRONIK, BARD, ABBOTT VASCULAR.

Panel discussion:

Is there a role for pretherapeutic imaging besides catheterisation angiography?
16:00 - 17:30

Room N/O

Head and Neck

RC 708

The orbit:

you can't see what you haven't learnt

Moderator:

U.Y. Ayaz; Mersin/TR

A-184 16:00

A. Anatomy and commonly encountered postoperative findings

N. Hosten; Greifswald/DE (hosten@uni-greifswald.de)

Pathology of the eye and orbit is rare in the radiologist's practice. However, differential diagnosis is not too difficult if the compartment model is applied. In the orbit, different anatomical structures like the optic nerve as part of the CNS, muscles for moving the globe, vascular structures and glandular tissue are present in a very small space. Each anatomical structure is found in a special compartment; each compartment may give rise to a different group of pathologies, and only to these pathologies: glioma in the optic nerve, rhabdomyosarcoma in the eye muscles, varix in the venous vessels (intraconal compartment) and pleomorphic adenoma in the lachrymal gland. Understanding the compartments in the orbit is therefore the key to differentiating different pathological entities. This presentation explains the orbital anatomy, how the compartments are differentiated, and what changes in anatomy treatment may induce.

Learning Objectives:

1. To become familiar with the examination technique according to the clinical presentation.

2. To learn about the anatomic compartments of the orbit.

3. To understand post-treatment imaging findings.

Author Disclosure:

N. Hosten: Author; MRI of the Eye and Orbit Thieme Publisher. Grant Recipient; INTERREG Program European Comunity. Shareholder; Siemens AG.

A-185 16:30

B. Congenital and inflammatory disease

T.A. Ferreira; Leiden/NL (T.A.Ferreira@lumc.nl)

Orbital congenital lesions are uncommon. They can be diagnosed prenatally, at birth or later during childhood. Several orbital components can be involved. We will focus on congenital globe lesions (such as staphyloma, coloboma, persistent hyperplastic primary vitreous, Coats disease), the developmental cysts such as epidermoids and dermoids, and vascular malformations such as Iymphangioma and vascular tumours, e.g. capillary haemangioma. Orbital Inflammatory and Infectious lesions are on the other hand common. Orbital pseudotumour can involve any area of orbit, being one of the great mimickers in the orbit. If located in the orbital apex and/or cavernous sinus, it will be called Tolosa-Hunt syndrome. Other inflammatory processes in the orbit are sarcoidosis and Wegener and Sjogren syndrome. Among infections the most common is the orbital cellulitis, mostly secondary to a sinusitis and frequent in the urgency setting. The role of the radiologist is to assess whether it is preseptal or already postseptal or complicated by a subperiosteal phlegmon or abscess. Also of importance is to know and evaluate the possible intracranial complications. Finally, we will focus on inflammatory and infectious lesions of some specific regions such as the globe and lachrymal gland and give some clues for their differentiation.

Learning Objectives:

1. To appreciate imaging findings in congenital globe and orbit disease.

2. To learn about inflammation and infection

3. To become familiar with the complications and pathways of orbital infections.

A-186 17:00

C. Benign and malignant neoplastic tumours

W. Müller-Forell; Mainz/DE (wibke.mueller-forell@unimedizin-mainz.de)

As more than 100 pathologies can be seen in the orbit, a systematic approach is very important to come to the right diagnosis. The main and most helpful criteria of differential diagnosis of any orbital pathology is the definition of the affected orbital compartment, as some tumours may only or preferentially involve specific orbital structures. The criteria of the most frequent masses of the globe, malignant melanoma and retinoblastoma are presented as well as those of cavernoma and Iymphoma, the main representatives of intraconal tumours. There are numerous extraconal neoplasms, only few arising from the nasal sinuses, and only a little number of tumours of the optic nerve. The 
presentation will include the most frequent as well as rare, but important tumours.

Learning Objectives:

1. To become aquatinted with the differential diagnoses of orbital masses.

2. To learn an approach to differentiating orbital tumours.

3. To appreciate the typical imaging findings of benign and malignant orbital neoplasm.

16:00 - 17:30

Conf. Room M3

Oncologic Imaging: Follow-up of Systemic and Local Therapies

CC 718

\section{Imaging after systemic therapies: the standards \\ Moderator:}

M. Nadrljanski; Belgrade/RS

A-187 16:00

A. RECIST criteria

Y. Menu; Paris/FR (yves.menu@sat.aphp.fr)

The routine practice of oncologic imaging requires standardisation, which means that we need to harmonise technical protocols and agree on the meaning of selected words for the radiological report. The words "Response, "Progression" and "Stable disease" are precisely defined according to internationally accepted thresholds and criteria. Although the rules are quite simple and rather easy to apply, they are very efficient in the classification of the response to treatment, and therefore for the medical decisions. However, the role of the radiologist is not limited to measurements and calculation. The detection of new lesions may be challenging and requires experience. The differential between cancer progression and complications of the treatment might be very difficult and requires an adequate communication with the referring clinician. Overall, most of the decisions taken by the clinician will be related to imaging results, stressing the importance of adequate protocols and reports.

Learning Objectives:

1. To consolidate knowledge on the evaluation of solid tumour response.

2. To learn some tips and tricks to help bring RECIST to everyday practice.

3. To understand the advantages and limitations of RECIST.

A-188 16:30

B. PERCIST: evolving considerations for PET response criteria in solid tumours

T.F. Hany; Zurich/CH (thomas.hany@gmail.com)

In solid as well as non-solid tumours, PET/CT imaging using 18-fluorodeoxyglucose (FDG) has demonstrated the ability to a) correctly stage disease, b) demonstrate therapy response and c) predict therapy outcome. FDG uptake can be measured objectively; however several factors in the standardisation processes of tracer application, image acquisition and post-processing are needed for reproducibility. The term standard uptake value (SUV) measurement is used for compensating the influence of injected dose, decay time and body mass and represents FDG uptake in any selected pixel of the image. For therapy assessment, drop in FDG uptake represents tumour cell kill, notably a negative PET scan does not exclude viable tumour cells but overall has a better outcome. PET response criteria in solid tumours (PERCIST 1.0) have been introduced to refine previously established PET response criteria by EORTC. Major changes concern the use of lean body mass-based SUV (SUL), SULpeak measurement in a fixed ROI, use of only a single target lesion and normalization to liver uptake. Metric measurements in CT component of the PET/CT as an intrinsic asset like in RECIST 1.1 have not yet been introduced, but might be crucial in the future. The proposed PERCIST 1.0 criteria are not yet standard, since several limitations hamper its general use but may improve metabolic tumour response assessment.

Learning Objectives:

1. To learn about the evaluation of solid tumours through metabolic imaging.

2. To understand the benefits of metabolic imaging.

A-189 17:00

\section{Evaluation of brain tumours}

C. Majós; Barcelona/ES (cmajos@bellvitgehospital.cat)

Malignant gliomas (WHO grade III and IV) are the most common primary tumours of the brain. According to consensus guidelines, the standard of care of these tumours includes maximal safe surgical resection followed by combined treatment with chemoradiotherapy. Radiological assessment is critical in the follow-up and should be performed at four different times: 1.
Within 72 hours after surgery, an early post-operative MRI must be done to evaluate residual tumour and to be used as baseline for follow-up. 2. Two to six weeks after completing radiotherapy, a new MRI examination is recommended to evaluate the response to treatment. Four possibilities are envisaged according to the RANO criteria: complete response, partial response, stable disease or progressive disease. The evaluation of this first MRI examination after RT is challenging and the pseudoprogression phenomenon can appear. Advanced MR techniques may be of help in this respect. 3. Additional follow-up should then be performed by MRI every 2 to 4 months to rule out clinically silent progression. 4. In any case, an MRI examination must be completed when there is a clinical suspicion of progressive disease. Several options for treatment can be offered when progressive disease is detected, including antiangiogenic drugs. Evaluation of response to these drugs is challenging too, and the pseudoresponse phenomenon can then appear. Due its complexity, it is recommended that the management of malignant gliomas be performed in the context of multidisciplinary teams and that the radiologists are strongly involved in these teams.

Learning Objectives:

1. To learn about the evaluation criteria for brain tumours

2. To become familiar with the evaluation of brain tumours after treatment with various therapies.

16:00 - 17:30 Board Room A

Special Focus Session

\section{SF 7c}

\section{Educational challenges for radiographers}

A-190 16:00

Chairmen's introduction

G. $\underline{\text { Paulo }}^{1}$, L. Bonomo ${ }^{2} ;{ }^{1}$ Coimbra/PT, ${ }^{2}$ Rome/IT

We are facing complex times with no parallel in human history. The worldwide economic crisis, in combination with a non-regulated process of globalisation, is pressuring countries to change their social and political model. These changes are affecting academic institutions and the health-care sector. Higher education was always more internationally open than most sectors because of its immersion in knowledge, which was never worried about country juridical boundaries. It is well recognised that it was the knowledge created by universities research that contributed to the development of societies. We, as European citizens, must be able to transmit the message that having more knowledge and being capable of understanding better the world we live in will make us stronger, more successful and more competitive. European academic institutions must be prepared for this shift of paradigm and understand that they are no longer educating only for their country but for the globalised world. It is also important to bear in mind that developed health-care systems are suffering several pressures and a call for a new inter-professional collaboration concept is needed, based on each one's roles and responsibilities, towards a better and more efficient health-care delivery for the patients. In this session, the invited speakers will give us an overview about the challenges that academic Institutions will face in the near future and the way they must adapt to become more attractive and at the same time guarantee that radiographers and radiologists are educated and trained according to the highest professional standards.

Session Objectives:

1. To appreciate the challenges that academic institutions must face in order to educate graduates with the ability to work internationally and to actively contribute to the healthcare sector of the future.

2. To explore innovative approaches in education and assessment.

3. To highlight the importance of inter-professional collaboration in order to ensure that graduates are ready to meet the demands of the modern healthcare sector.

A-191 16:05

Challenges in undergraduate education

L.J.O.C. Lanca; Lisbon/PT (luis.lanca@estesl.ipl.pt)

Radiographers operate a wide range of sophisticated equipment and perform a range of techniques in several radiological procedures. They are responsible for the radiation delivered to the patient while assuring safety and image quality at an acceptable level for an accurate diagnosis. Education and training in radiography, in line with the constant technological developments, is a requirement to be fulfilled by higher education institutions (HEI). This is of major importance in undergraduate education where radiographers learn to play an effective role as healthcare providers in planning, preparing and performing accurate and safe radiological procedures within the diagnostic or therapeutic field. The European Qualifications Framework (EQF) for 
radiography, in terms of the development of knowledge, skills and competences, provides a European standard for the development of radiography learning outcomes at EQF level 6 . This constitutes an opportunity to develop, tune, advance and promote standards of radiographic practice, education and research throughout Europe. The EQF provides guidance to increase the educational and professional recognition of radiography in Europe. At a European level, the cooperation between HEI in undergraduate radiography education would be an opportunity to provide discussions of mutual concern, explore new perspectives and views of different cultures, and also share learning and teaching methods. International cooperation is an opportunity to improve and provide the harmonisation of education regarding a professional group that plays an effective role in healthcare in their field of competence and expertise.

Learning Objectives:

1. To understand radiography undergraduate education requirements and how they meet current challenges in healthcare.

2. To appreciate the European Qualifications Framework for radiography in terms of the development of knowledge, skills and competences.

3. To highlight the importance of international cooperation in undergraduate radiography education.

\section{A-192 16:28}

Innovative approaches to educational enhancement: teaching and assessment

J. Mc Nulty; Dublin/IE (jonathan.mcnulty@ucd.ie)

There is a vast amount of evidence available from the published literature that higher education institutions continue to explore innovation in both teaching and assessment practices. This is especially true of health professional programmes as evidenced by the abundance of literature relating to medicine, nursing, radiography and other allied health professions. Such higher education, health professional programmes would appear to be more innovative than many other disciplines often as a direct attempt to mirror the dynamic clinical environment in which most graduates will work. There are however, challenges associated with any such educational innovations and novel approaches to delivery and assessment. These specific challenges must be carefully considered in parallel with the larger challenges facing higher education institutions, both nationally and internationally, along with the professions which they contribute to. Through a case study approach supported by published peer-reviewed literature, institutional reports and research, this presentation will consider international best practice in education along with the value of collaborative, inter-professional approaches to teaching and assessment.

Learning Objectives:

1. To understand the factors influencing the change in approaches to teaching and assessment as evidenced by the published literature.

2. To become familiar with international best practice in teaching and assessment.

3. To appreciate the value of inter-professional collaborative approaches to both teaching and assessment.

4. To understand and appreciate approaches to teaching and assessment from the students perspective.

\section{A-193 16:51}

\section{Perception of changes: the radiologist's point of view}

B. Ertl-Wagner; Munich/DE (Birgit.Ertl-Wagner@med.uni-muenchen.de)

The European Training Curriculum for Radiology is a living document that was last revised in March 2013. It outlines the post-graduate training for physicians who want to specialise in radiology. The ESR recommends a 5 -year postgraduate training period to become a radiologist divided into $3+2$ years (level I and II training). In order to subspecialise in an area of interest, further training is necessary (level III training). With a changing medical environment and increasing economic pressures, both radiologists and radiographers face challenges. In order to succeed, both groups will need to stand together and focus on synergies, while minimising areas of conflict.

Learning Objectives:

1. To become familiar with the European Training Curriculum for Radiology.

2. To evaluate the differences in training for radiologists and radiographers.

3. To understand the best way towards collaboration between radiologists and radiographers.

Panel discussion:

Joint approaches by radiologists and radiographers:

the key to success $17: 14$

\section{$16: 00-17: 30$ \\ Room P \\ Vascular \\ RC 715 \\ Dialysis fistula: imaging and intervention}

Moderator:

H.I. Manninen; Kuopio/FI

\section{A-194 16:00 \\ A. Assessment of vascular access malfunction \\ R. Uberoi; Oxford/UK (raman.uberoi@orh.nhs.uk)}

Haemodialysis accesses have become the most commonly performed type of vascular surgical procedure. However, only $50 \%$ of all haemodialysis accesses remain patent at 3 years. Autogenous fistulas have a higher rate of primary failure compared to prosthetic grafts (50\% vs. $15 \%)$, but the long-term patency of fistulas is superior to that of grafts. Autogenous fistulas fail after a median of 3 to 7 years, whereas prosthetic arteriovenous grafts fail after a median lifetime of only 12 to 18 months. The pathogenic stenoses causing access failure occur in various locations, but the most common site in prosthetic arteriovenous grafts is at the anastomosis between the graft and outflow vein, as identified in $47 \%(23,246)$ to $60 \%$ of cases, or in the outflow vein itself in autogenous arteriovenous fistulas. Clinical assessment alone will detect a large number of failing fistulas. By assessment of the thrill, pulse character, palpation of the fistula itself, excessive bleeding and difficulty needling a fistula may all indicate a failing fistula. If the venous pressures during haemodialysis exceed $120 \mathrm{~mm}$ $\mathrm{Hg}$, fistula flow falls to $<500 \mathrm{ml} / \mathrm{min}$, graft flow decreases to $<50 \mathrm{ml} / \mathrm{min}$, or access blood flow falls by more than $25 \%$. A fistulogram is recommended if monitoring or surveillance suggests that thrombosis is either imminent or has already occurred. Duplex can be used to assess the fistula, but cannot visualise the central veins. MR and CT venography has also been used in many patients successfully.

Learning Objectives:

1. To learn about the imaging features of the malfunctioning vascular access.

2. To learn about screening protocols and the results of screening.

3. To learn about the most common problems and how to detect them.

\section{A-195 16:30}

\section{B. Treating the stenosed access}

D. Henroteaux; Liège/BE (denis.henroteaux@chrcitadelle.be)

Treatment of symptomatic stenosis of vascular access is essential as it can lead to thrombosis and loss of vascular access. But treatment can lead to restenosis. The treatment of stenosis remains a challenge which requires a good knowledge of different materials (guidewire, catheters, balloons and stents). Though the pathophysiological mechanism of stenosis is different for native fistulas, grafts or restenosis, the treatment technique remains the same. The basic principle is the use of high inflation pressure balloon angioplasty. Whatever the material used, the key is to have no residual stenosis greater than $30 \%$. So far, no study has demonstrated the superiority of the "new" tools (cutting balloon, drug-eluting balloon, cryotherapy) regarding restenosis. Except perhaps for anastomotic grafts stenosis, stents should be reserved to treat complications or failure of balloon angioplasty. The stents indications are residual elastic stenosis, wall obstructive damage, acute rupture during angioplasty, aneurysm or restenosis. Complications of stenosis treatment are rare, but can lead to the loss of vascular access. The main complications are thrombosis and acute rupture. As with any treatment, there have been contraindications: infection, distal ischaemia, high flow, newly created or surgically revised access $(<6$ weeks). When dealing with a vascular stenosis access, never compromise on the future, but think about the surgical alternative. So, the management of stenosis vascular access must also be multidisciplinary

Learning Objectives:

1. To learn about treatment techniques, material and equipment.

2. To become familiar with the difference between treating dialysis fistulas and grafts.

3. To learn about complication management. 
A-196 17:00

C. Treating the thrombosed access

L. Turmel-Rodrigues; Tours/FR (luc.turmel@wanadoo.fr)

Percutaneous treatment of a thrombosed dialysis access can be extremely challenging. The major concern does not stem from the fact that the procedure can be complexly long, but arises rather from the management of its complications, which can be serious particularly when the access is branched onto the brachial artery. Access infection is an absolute contraindication. Temporary contraindications include fluid overload and severe hyperkalaemia.

Percutaneous thrombectomy of grafts is very well standardised and predictable: size of $6 \mathrm{~mm}$, well palpable wall which is easy to needle, small average clot burden, and an underlining stenosis almost always found at the venous anastomosis. Thrombectomy of AVFs is subject to variations in approach and technical difficulties depending on their anatomical particularities.

All techniques employed follow 2 rules: first, the removal of thrombi and second, dilation of the stenosis responsible for the thrombosis. Heparin and antibiotics must be injected. The basic principle is to place in the arterialized vein or graft 2 introducer sheaths in opposite directions to work on both the venous outflow and arterial inflow. Thrombus lysis or removal can be achieved by mechanical, pharmacological and pharmaco-mechanical methods. All techniques may work in grafts given that the modest amount of thrombus (3.2 $\mathrm{ml}$ on average) can be simply pushed into the lungs. Prosthetic grafts are thus easier to declot than native fistulas, but they are much more prone to early rethrombosis. Thrombi located at the arteriovenous anastomosis form a firm and rubbery plug, frequently and notoriously resistant to thrombolysis.

Learning Objectives:

1. To learn about treatment techniques, material and equipment.

2. To become familiar with the difference between treating dialysis fistulas and grafts.

3. To learn about complication management. 


\section{Saturday, March 8}


08:30 - 10:00

Room A

\section{Interactive Teaching Sessions}

\section{$E^{3} 820 a$}

\section{Thoracic changes after treatment}

A-197 08:30

A. Drug-related conditions

T. Franquet; Barcelona/ES (tfranquet@santpau.cat)

Drug-induced lung disease is an increasingly common cause of morbidity and mortality. The diagnosis is based on clinical history and consistent radiologic findings. Lung biopsy is performed in a small percentage of cases. Highresolution CT may demonstrate different parenchymal patterns including diffuse alveolar damage, acute or chronic alveolar haemorrhage, nonspecific interstitial pneumonia (NSIP), hypersensitivity pneumonitis, organising pneumonia, and eosinophilic pneumonia. The imaging and histologic manifestations are often nonspecific. A systematic approach to the radiological evaluation of drug-related lung diseases is essential and includes not only chest imaging pattern recognition, but also integration of available clinical information.

Learning Objectives:

1. To understand pulmonary changes related to treatment.

2. To understand the mechanisms of injury to the lung in drug related conditions.

Author Disclosure:

T. Franquet: Author; Amirsys/Elservier.

\section{A-198 09:15}

\section{B. Device-related conditions}

G.R. Ferretti; Grenoble/FR (GFerretti@chu-grenoble.fr)

In this interactive session, we will present and discuss several cases illustrating thoracic changes after instrumental procedures in the thorax such as surgery, radiofrequency ablation, interventional endoscopy, and intensive care. Radiologists have an important role in assessing the results of these procedures as well as in depicting the complications. However, knowledge of the normal appearance after those procedures has to be presented.

Learning Objectives:

1. To understand changes related to lines and wires in ICU patients.

2. To learn about findings related to surgery.

08:30 - 10:00 Room B

Emergency Radiology

\section{RC 817}

\section{Polytrauma: redefining imaging issues for management priorities}

\section{A-199 08:30}

Chairman's introduction: advanced imaging, logistics and management priorities in patients after polytrauma

H. Alkadhi; Zurich/CH (hatem.alkadhi@usz.ch)

The initial imaging workup of polytrauma patients remains challenging. Besides hardware requirements, workflow issues continue to evolve, with the current focus on the introduction of whole body CT into the early resuscitation phase of severely injured patients as a standard and basic diagnostic imaging method. This session is aimed at a thorough discussion on the requirements for advanced imaging in the early clinical situation in emergency radiology. Focus is directed on the issues of radiation dose as well as on CT and contrast media protocols.

\section{A-200 08:40}

\section{A. Chest and abdomen}

M. Scaglione; Castel Volturno/IT (mscaglione@tiscali.it)

Thoraco-abdominal injuries are a significant cause of death in the polytraumatised patients. Early recognition and communication of lifethreatening thoraco-abdominal injuries is the major task of the radiologists involved in the emergency room. Although most of these patients reach the hospital prior to dying, lethality continues to remain high. Heart, thoracic great vessels, trachea, bronchus, pleura, lung, diaphragm, abdominal/retroperitoneal, vascular and solid organ injuries are potential cause of death. Any appropriate surgical/interventional management approach must be carried out "around the clock", before thoraco-abdominal injuries reach the level of clinical evidence. On the other hand, non-operative management has actually become the standard of care for the most serious thoraco-abdominal injuries. These goals become feasible if a correct contrast-enhanced MDCT diagnosis, in a dedicated facility in which the trauma team works effectively 24 $\mathrm{h}$ a day, 7 days a week, is performed. Thus, in this lecture, the most serious thoraco-abdominal injuries will be illustrated, with special emphasis on vascular/injuries as well as the value of post-processing techniques, protocols, pitfalls, tips and tricks. Furthermore, the importance of a rational and integrated imaging approach will be pointed out and, finally, the role of the radiologist in the emergency room will be emphasised.

Learning Objectives:

1. To become familiar with the most important imaging findings and their impact on patient management.

2. To understand common classification systems, trauma scoring systems and their impact on patient management

3. To learn about typical and atypical imaging findings

A-201 09:00

B. Spine and pelvis

G. Schueller; Bülach/CH (gerd.schueller@spitalbuelach.ch)

Spinal and musculoskeletal trauma account for significant morbidity in severely traumatised patients. Traumatic injuries to the spine encompass a variety of frequently occurring disorders and primarily result in stable injury. Although rare, unstable disorders comprise injuries to the bone, the discs, and the ligaments. In the majority of cases, spinal cord injuries result in devastating medical and social consequences. Severe musculoskeletal disorders usually are the result of high-impact accidents, such as motor vehicle accidents and falls from a height. For instance, the injured pelvic ring causes one of the most life-threatening conditions that the trauma team must handle. Pelvic injuries are often associated with severe arterial, venous, and/or osseous bleeding. Radiography is no longer recommended as the primary screening modality in spinal and pelvic trauma for adults. Despite the radiation dose burden of CT, patients with a high risk of spine injury receive MDCT imaging, as it is the method of choice. MR imaging is indicated primarily when patients present with myelopathy, and to search for spinal cord pathology. Interventional radiology plays a major role in the therapy of complex traumatic pelvic disorders. Learning Objectives:

1. To become familiar with the pathomechanisms and classification of spinal and pelvic trauma treatment options.

2. To become familiar with the image interpretation of the spine and the pelvis. 3. To learn about typical imaging findings based on CT and MR imaging

\section{A-202 09:25 \\ C. Extremities \\ U. Linsenmaier, L. Geyer; Munich/DE \\ (Ulrich.Linsenmaier@kliniken-pasing-perlach.de)}

Extremity injuries in patients after polytrauma can be complex and are initially often difficult to be fully diagnosed. Emergency radiology diagnosis is today mostly based on a standardised whole body CT (WBCT), which can be extended with CTA and adapted to cover extremity injuries. Extremity injuries comprise: fractures of (1) long bones, (2) articular joints, (3) complex fractures of hands and feet, (4) vascular, (5) soft tissue, (6) nerve and plexus injuries and (7) amputations. Imaging protocol: *MDCT is indicated in all major and complex bony fractures and is carried out early or integrated with WBCT. CTA using MIPs and MPRs enables a thorough workup. * The role of US and CR is limited. * The role of MR and MRA (in stable patients only) is to evaluate unstable articular injuries, injuries of tendons or major ligaments and nerve and plexus injuries). * The role of DSA is mostly for intervention. Clinical findings and findings from WBCT determine how to proceed, "first things are done first" in a priority-oriented clinical algorithm. Treatment of extremity injuries must therefore be priority oriented and carefully planned in the context of possible concurrent injuries and a possible risk of multi-organ failure (MOF).

Learning Objectives:

1. To become familiar with imaging strategies, the role of $\mathrm{CR}, \mathrm{CT}$ and MR. 2. To understand common classification systems and their impact on patient management.

3. To learn about typical and atypical imaging findings.

Panel discussion: How to speed up your diagnoses 09:45 
08:30 - 10:00

Room C

\section{Interactive Teaching Session}

$E^{3} 820 b$

\section{Central nervous system changes after treatment: what you need to know}

\section{A-203 08:30}

A. Drug-related conditions

F. Barkhof; Amsterdam/NL (f.barkhof@vumc.nl)

Systemic drugs can be used for the treatment of CNS and non-CNS diseases Both classes can affect the brain inadvertently. Common general drugs that affect the brain in a bystander manner are steroids $(\sim 1 \%$ brain volume reduction), recreational drugs (alcohol, cocaine, heroin, XTC), metronidazole and anti-epileptic drugs (corpus callosum demyelination) and immunosuppressants like cyclosporine/tacrolimus (PRES/RPLS) and methotrexate. Mechanisms of action include neurovascular compromise, fluid/metabolite shifts and toxic effects to myelin of other tissue components. Among CNS-targeted drugs, especially immunomodulating agents may cause specific side effects. Cytokine-release syndromes may occur with broad-acting agents such as general $T$ cell antibodies. Specific side effects may occur in multiple sclerosis, where treatment with natalizmumab may cause reactivation of $\mathrm{JC}$ virus leading to progressive multifocal leukencephalopathy (PML). Upon withdrawal of therapy, this may then evolve into an Immune reconstitution inflammatory syndrome (IRIS). In Alzheimer disease, novel antibodies or vaccinations against amyloid may cause amyloid related imaging abnormalities (ARIA), which may present with microhaemorrhage on $2^{*}$ images, or with edema and effusion on FLAIR.

Learning Objectives:

1. To understand changes related to systemic treatment.

2. To learn about CNS treatment agents.

\section{A-204 09:15}

B. Imaging of treated brain tumours

J. Alvarez-Linera; Madrid/ES (jalinera@ruberinternacional.es)

The objective of this session will be to review the diagnostic value of conventional sequences as well as the use of contrast in the monitoring of brain tumours, with special attention to gliomas. Also, we will address the advantages and limitations of advanced techniques: perfusion, diffusion, and spectroscopy. Immediately after surgery, the main objective of neuroimaging is the detection of the remaining tumour. It may also be necessary to rule out complications such as haemorrhage, ischaemia or infection. The fundamental technique is MRI with contrast in the first 48 hours and also diffusion sequences. In late follow-up, the goal is to differentiate the changes secondary to treatment of those related to tumour progression or recurrence. In these cases, conventional sequences present important constraints and are usefu studies of diffusion, perfusion and spectroscopy. During follow-up after chemoradiotherapy, the objective is to assess tumour response. The response according to the new RANO criteria will be revised. The combination of chemotherapy and radiotherapy, as well as the use of anti-angiogenic drugs causes changes that complicate the assessment of the response to treatment, with cases of pseudoprogression and pseudoresponse. In these cases perfusion techniques, diffusion and spectroscopy can provide relevant information, although it is necessary to standardise the quantification to make its wide use possible.

Learning Objectives:

1. To appreciate CNS manifestations after surgery.

2. To understand CNS changes after radiotherapy and other non-surgical treatments.

\section{8:30 - 10:00}

Room D

\section{Genitourinary}

RC 807

\section{Contrast media safety: update}

\section{A-205 08:30}

Chairman's introduction

P. Reimer; Karlsruhe/DE

The speakers in this course will update the audience on contrast media safety such as steps to be taken before contrast administration and present the newest safety guidelines. The first speaker will cover new concepts of non- renal reactions to contrast media explaining which hypersensitivity reactions are allergic and non-allergic. The audience may learn assessing symptoms according to the Ring and Messmer classification and understand the importance of tryptase sampling and skin testing in the follow-up. The second speaker will address NSF. The presentation will review the pathophysiology, risk factors, recent recommendations and prevention of NSF. Patients with GFR less than $30 \mathrm{ml} / \mathrm{min} / 1.73 \mathrm{~m}^{2}$ have increased risk of developing NSF. Lowstability gadolinium contrast media show the strongest association with NSF. Following existing guidelines on the use of gadolinium contrast agents minimises the risk of NSF. Potential long-term harm from gadolinium accumulation in the body and legal issues are discussed. The last speakers will cover contrast medium-induced nephropathy with more recently published guidelines related to that issue. The presentation will include the definition of $\mathrm{CIN}$ and the choice of contrast medium and prophylactic measures. Recent changes in ESUR guidelines will be explained. The risk of CIN is considered significantly lower following iv. CM administration and patients referred for enhanced CT are genuinely at risk if they have an eGFR $<45 \mathrm{ml} / \mathrm{min} / 1.73 \mathrm{~m}^{2}$. Volume expansion with isotonic saline or sodium bicarbonate may be used for preventing $\mathrm{CIN}$ in at-risk patients. Author Disclosure:

P. Reimer: Investigator; Bayer Health. Speaker; Bayer Health, Bracco.

\section{A-206 08:35}

A. Acute non-renal reactions to contrast media: new concepts O. Clément; Paris/FR (olivier.clement@inserm.fr)

Acute immediate hypersensitivity reactions occur within the hour following the administration of contrast media. They can be seen with lodinated and gadolinium-based contrast agents. Over the last ten years, new concepts have emerged in the way of understanding, managing and exploring hypersensitivity reactions. The clinical appearance is best classified by the Ring and Messmer scoring, from Grade 1 (cutaneous and subcutaneous signs) to Grade 4 (cardiovascular arrest). The mechanisms involve either true IgE-mediated hypersensitivity or non-allergic hypersensitivity. The differential diagnosis in favour of allergy is made on a triad: clinical signs (the more severe, the more are the chances to be allergic), elevated tryptase levels in the plasma (indicating mastocyte triggering) and positive skin tests performed one month after the reaction. These new concepts induce important consequences in managing hypersensitivity reactions: be prepared to treat the patient adequately, be prepared to draw blood after the reaction to dose tryptase levels, send the patient to a dedicated allergologist, and forget about the preventive role of premedication against severe reactions.

Learning Objectives:

1. To learn which hypersensitivity reactions are allergic and non-allergic.

2. To learn the clinical symptoms according to the Ring and Messmer classification.

3. To understand the importance of tryptase sampling and skin testing in the follow-up.

Author Disclosure:

O. Clément: Board Member; French society of Radiology.

\section{A-207 08:58}

B. Nephrogenic systemic fibrosis: from pathophysiology to recommendations

H.S. Thomsen; Herlev/DK (henrik.thomsen@regionh.dk)

Gadolinium ( $\mathrm{Gd}$ ) belongs to the lanthanides in the periodic table, all of which affect collagen. The ability to hold the $\mathrm{Gd}$ ion varies from chelate to chelate, with non-ionic linear chelates being the weakest binders. Transmetalation (exchange of Gd with another ion, e.g. zinc) leads to NSF, a horrifying very late adverse reaction to some Gd-CM. Since 2007, the various Gd-CM have been classified into 3 groups: high risk (of NSF), intermediate risk and low risk. The authorities have introduced several restrictions on the use of high-risk agents, which will be followed by every physician in the EU. The agents are contraindicated in 1) patients with severely reduced renal function including dialysis, 2) acute renal insufficiency, 3) neonates and 4) pregnant women. They may only be used with caution in patients with moderately reduced renal function and children less than 1 year old. There must be at least 7 days between 2 injections in those patients. Renal function must always be determined by laboratory methods before use of high-risk agents. Women should stop lactation for 24 hours. These agents should never be given at doses higher than $0.1 \mathrm{mmol} / \mathrm{kg}$ per examination in any patient. For the intermediate and low-risk agents, the restrictions are significantly less; they should only be used with caution in patients with severely reduced renal function including dialysis. If a physician does not follow these rules, he or she will have legal problems as they have been introduced into the SPC by the authorities. 


\section{Postgraduate Educational Programme}

Learning Objectives:

1. To understand the underlying pathophysiology.

2. To learn about the clinical implications of the recent

recommendations/guidelines.

3. To become familiar with legal issues.

\section{A-208 09:21}

C. Contrast medium-induced nephropathy and new guidelines

F. Stacul; Trieste/IT (fulvio.stacul@aots.sanita.fvg.it)

The Contrast Media Safety Committee (CMSC) of the ESUR has updated its guidelines on contrast medium-induced nephropathy (CIN). The ACR recently updated its guidelines as well. New guidelines were produced by nephrological societies. The topics reviewed include the definition of $\mathrm{CIN}$, the choice of contrast medium, and the prophylactic measures used to reduce the incidence of CIN. The CMSC considered it appropriate to keep the definition of CIN that was agreed in 1999. However, nephrologists have recently agreed on a new definition. In the previous guideline, a number of risk factors were listed (raised S-creatinine levels, particularly secondary to diabetic nephropathy, dehydration, congestive heart failure, age over 70 years, concurrent administration of nephrotoxic drugs). The significance of these risk factors has been confirmed and new risk factors were added. The CMSC agreed that the risk of $\mathrm{CIN}$ was significantly lower following intravenous $\mathrm{CM}$ administration and concluded that patients referred for enhanced CT were genuinely at risk if they had an eGFR $<45 \mathrm{ml} / \mathrm{min} / 1.73 \mathrm{~m}^{2}$. The previous CMSC guideline suggested the use of low or iso-osmolar CM in patients with risk factors for $\mathrm{CIN}$ and the CMSC considered that this previous guideline should not be changed. The CMSC considered that there was enough evidence to recommend that eithe volume expansion with isotonic saline or sodium bicarbonate may be used for preventing $\mathrm{CIN}$ in at-risk patients, while the efficacy of NAC and other drugs in reducing the incidence of $\mathrm{CIN}$ remained unproven. Guidelines produced by other societies provide very similar suggestions, thus further validating these recommendations.

Learning Objectives:

1. To become familiar with newest guidelines on contrast-induced nephropathy (CIN)

2. To understand the future clinical implications of the new guidelines.

Author Disclosure:

F. Stacul: Advisory Board; GE Healthcare. Speaker; Bracco, GE Healthcare.

Panel discussion:

What is the most appropriate radiological approach to patients with

falling eGFR and when should contrast media be administered and when should it not? 09:44

08:30 - 10:00 Board Room B

\section{Paediatric}

\section{RC 812}

\section{Autoimmune disorders in children}

Moderator:

M.P. García-Peña; Barcelona/ES

\section{A-209 08:30}

A. The joints in juvenile idiopathic arthritis

L.-S. Ording Müller; Oslo/NO (lilsofie.ording@googlemail.com)

JIA is the most common rheumatic entity in childhood and includes a subset of childhood arthritis, all of which are characterised by chronic synovitis with a potential risk of progressive joint destruction. Radiological investigations in JIA should ideally be able to determine the presence and degree of active inflammation, precursors of bony destructions and established erosions. However, there are many pitfalls in the interpretation of joint pathology in children. Ultrasonography is often the initial tool in the assessment of arthritis and can depict joint fluid and synovitis. Erosions and cartilage destruction of small joints may also be seen. The major problems are standardising the imaging technique and the lack of normal standards of anatomy in US in children. Radiographs can show bone erosions and may depict cartilage loss indirectly through joint space narrowing, but are insensitive to inflammation and early joint destruction. MRI is the only imaging modality that can assess all relevant anatomical structures in joint inflammation and is sensitive to early inflammation and destruction. However, large variations in the amount of joint fluid, bone marrow oedema-like lesions and changes resembling erosions are seen in children and also in healthy individuals. The differentiation between true pathology and normal findings on MRI in children remains a challenge, particularly in early disease. In this lecture, the role of radiographs, ultrasound and MRI and the typical radiological findings in joint pathology in JIA will be presented. Current knowledge on validity and reliability of the different imaging techniques in JIA will be discussed.

Learning Objectives:

1. To learn about the different joint lesions in JIA.

2. To understand when to use US or MRI.

3. To learn how to recognise the typical imaging patterns.

\section{A-210 09:00 \\ B. The digestive tract \\ E. Alexopoulou; Athens/GR}

Inflammatory bowel disease (IBD) is a range of diseases, which includes Crohn disease, ulcerative colitis, and indeterminate colitis. The combined effects of the four basic components seem to result in the disease: environmental changes, genetic factors, intestinal microbiota alterations and immune system deregulation. In children with unknown IBD, US is considered to be the first choice imaging technique which is performed before endoscopy. Bowel wall thickening, location and length of the disease, echogenicity of the bowel wall, the presence or not of normal stratification, ulcers, bowel stiffness, stricture or distention can often be detected with US. Additional information about the surrounding fat and the presence of mesenteric nodes can be obtained. The absence of bowel wall thickening has a strong negative predictive value. MR enterography is the technique of choice in children with known IBD, as it is a reproducible and well-tolerated examination, lacking radiation and providing excellent information about bowel disease. MR enterography clearly illustrates: a) bowel loop appearance (fixed, dilated, strictured, pseudo sacculation appearance); b) bowel wall (thickness, focal lesions such as ulceration, pseudopolyps and mural abscess, type of signal on T2 -W sequences and pattern of enhancement after iv contrast injection); c) extramural signs (fibrofatty proliferation, "comb sign", fistula, abscess, mesenteric nodes). CT should be performed in cases where MRI is contraindicated or in acute-emergency situations, when US is inadequate. It remains also an examination of choice for abscess drainage, to depict its exact extension and select the most appropriate access route.

Learning Objectives:

1. To learn the basics of mechanisms related to autoimmune enteritis.

2. To appreciate the role of US, CT and MRI.

3. To become familiar with imaging findings useful in the diagnosis.

A-211 09:30

C. Multiple sclerosis in children

C. Adamsbaum, B. Husson, K. Deiva, M. Tardieu; Le Kremlin Bicêtre/FR (c.adamsbaum@bct.aphp.fr)

The recent criteria for paediatric MS (Mult Scler 2013, 19, 1261-7) and the revised McDonald criteria for adults and adolescent MS allow the diagnosis of MS at the time of the first demyelinating clinical event. Brain MRI plays an important role in those criteria, as it can demonstrate the classical dissemination in time and space and helps earlier diagnosis, which is of major importance since the present recommendation in children is to start immunomodulating treatments as soon as diagnosis is established. Among paediatric MS, $14 \%$ begin before the age of 6 years and $30 \%$ before the age of 10 years, frequently with an ADEM presentation in young children as initia manifestation of MS. However, only $20 \%$ of the ADEM indicate MS onset and the most predictive factors should be known, i.e. periventricular, deep white matter, corpus callosum high T2 signal lesions and black holes on T1 sequences. MRI evaluation is also instrumental in differential diagnoses such as nonrelapsing ADEM, vasculitis, immunogenetic diseases and occasionally leukodystrophies. Finally, brain MRI is useful to evaluate the risk of more severe MS.

Learning Objectives:

1. To understand the difference between children and adults.

2. To become familiar with imaging findings.

3. To learn about the principle differential diagnosis.

08:30 - 10:00 Room E1

State of the Art Symposium

SA 8

Radiology and obesity

A-212 08:30

Chairman's introduction

S.A. Jackson; Plymouth/UK (simon.jackson1@nhs.net)

The obesity epidemic represents one of the most significant European and public health challenges in the $21^{\text {st }}$ century with prevalence of the disease having tripled in many countries during the past 30 years. This is resulting in an 
ever increasing cost to healthcare systems including hospital and in particular radiology services which face unique challenges when imaging this group of patients. Obesity significantly increases the patient's risk of various comorbid diseases including the incidence of cancer, diabetes, and cardiovascular and liver pathology with a multidisciplinary team approach mandatory for optimal patient care. This multidisciplinary symposium will review the implications of the epidemic with cutting edge, in-depth lectures presented by European experts addressing the epidemiology, role of imaging in the bariatric surgical patient as well as the importance of abnormal fat deposition in the liver.

Session Objectives:

1. To learn about the impact of the obesity epidemic on European healthcare.

2. To appreciate the value of imaging techniques in the management of the post-operative bariatric patient.

3. To understand the role of radiology in fatty liver disease and the importance of imaging during subsequent patient surveillance.

\section{A-213 08:35}

Obesity: causes and consequences to the patient

R. Batterham; London/UK (r.batterham@ucl.ac.uk)

Obesity is one of the greatest $21^{\text {st }}$ century public health challenges. Its prevalence has tripled in many European Union (EU) countries since the 1980 s. Currently, $52 \%$ of EU adult population is overweight and $17 \%$ obese, and the numbers of those affected continue to rise. Overweight and obesity are risk factors for numerous health problems, including hypertension, diabetes, cardiovascular diseases, respiratory problems, musculoskeletal diseases and some forms of cancer. Mortality also increases sharply once the overweight threshold is crossed. Because obesity is associated with higher risks of chronic illnesses, it is linked to significant additional health care costs and is already responsible for $2-8 \%$ of health costs. Changes in our environment are the main driver for this increase in overweight/obese. However, a person's genetic make-up can either increase or decrease their chances of becoming overweight. The gastrointestinal tract is the body's largest endocrine organ producing hormones that regulate bodyweight. Dietary modifications, such as caloric restriction, are the first-line obesity treatments. However, dieting produces only moderate weight-loss with poor weight-loss maintenance. Compensatory gut hormone changes induced by dieting are thought to contribute to the failure of dieting. In contrast, bariatric surgery is an efficacious treatment modality for obesity, producing durable weight-loss, amelioration of obesity-associated co-morbidities and reduced mortality. Consequently, the number of bariatric procedures undertaken within Europe has doubled in the last 5 years with 112,000 procedures undertaken in 2011. There is increasing evidence that surgically-induced alterations in circulating gut hormones mediate the weight-loss and metabolic beneficial effects of bariatric surgery.

Learning Objectives:

1. To understand the epidemiology of obesity and its impact on European healthcare provision.

2. To appreciate the aetiology of obesity and the scientific rational for surgical treatment.

3. To learn about the effects of obesity on health

\section{A-214 08:58}

Imaging of modern surgical procedures and their complications

M. Rengo; Latina/IT (marco.rengo@gmail.com)

We will illustrate the common bariatric procedures, in particular their norma appearance on different diagnostic technique as well as their early and late complications. We will illustrate the correlation between conventional barium studies and advanced imaging with MDCT and MR. We will explain how to optimise MDCT and MR acquisition protocol according to the clinical indication. We will explain what to evaluate before redo surgery, in particular quantitative and functional analysis. We will illustrate the role of interventional radiology in the management of early postoperative complications, in particular in the management of patients subjected to gastric banding.

Learning Objectives:

1. To understand the common bariatric surgical procedures and post operative imaging appearances.

2. To appreciate the role of imaging in the assessment of suspected immediate and long-term postoperative complications.

3. To become familiar with the role of interventional radiology in the

postoperative period, including gastric band follow-up.

\section{A-215 09:21}

\section{Fat: where it shouldn't be}

V. Vilgrain ${ }^{1}$, M. Ronot ${ }^{1}$, B. Leporcq ${ }^{2}$, S. Lambert ${ }^{2}$, B.E. Van Beers ${ }^{1} ;{ }^{1}$ Clichy/FR, ${ }^{2}$ Paris/FR (valerie.vilgrain@bjn.aphp.fr)

Non-alcoholic fatty liver disease comprises a variety of pathological disorders ranging from simple steatosis to steatohepatitis. This condition is common in the Western population and is typically associated with obesity and the metabolic syndrome. Its incidence increases dramatically. Diagnosis of fatty liver and distinction between simple steatosis and steatohepatitis are keys because the latter can lead to extensive fibrosis and cirrhosis with an increased risk of hepatocellular carcinoma (HCC). Imaging plays a crucial role in diagnosing fatty liver. The two most important imaging modalities are ultrasound and MR imaging. Ultrasound can exclude major steatosis but lacks accuracy to precisely quantify fat, while MR is the most accurate for quantification. The technique of reference is MR spectroscopy, but sophisticated sequences based on chemical shift principle have been shown as accurate as MR spectroscopy. Unfortunately, imaging has still limitations to assess the presence of fibrosis and inflammation which are associated with steatohepatitis and functional tools could be of interest. Today, imaging is combined with clinical and biological biomarkers to evaluate the risk of steatohepatitis. Due to the increased risk of HCC and cirrhosis-related complications in patients with steatohepatitis, patients at risk should be enrolled in the surveillance programme.

Learning Objectives:

1. To understand the pathophysiology of fatty liver disease and its link to cirrhosis.

2. To become familiar with the role of imaging in the detection and quantification of fat in the liver.

3. To learn about the importance of imaging in the surveillance of patients with fatty liver disease.

Panel discussion:

How to optimise an imaging service for the obese patient?

$09: 44$

08:30 - 10:00

Room E2

Foundation Course: Breast Imaging

$E^{3} 820 c$

\section{Multidisciplinary team meeting} from Milan, IT

\section{A-216}

Multidisciplinary team meeting from Milan, IT

P. Panizza, R. Agresti, L. Lozza, G. Bianchi, B. Paolini; Milan/IT

(pietro.panizza@istitutotumori.mi.it)

The concept of breast cancer units originates from the need for making available to all women in Europe high-quality breast services where breast disease could be looked after by specialists working as teams. Such teams have to provide all the services related to breast cancer, including genetics and prevention, treatment of the primary tumour, care of advanced disease, palliation and follow-up of previously treated women. The breast unit is made up of a group of dedicated breast cancer specialists including a radiologist, radiographer, surgeon, reconstructive surgeon, pathologist, medical oncologist, radiation oncologist and breast care nurse. The specialists involved in breast cancer units have access to all the facilities required for high-quality care and spend most of their working time dealing with breast cancer. In our hospital, we routinely work as a team and to make it possible we organise weekly meetings involving specialists from different disciplines to evaluate and plan patient care at any step of the diagnostic and therapeutic process. Each year, we take care of about 1.500 newly diagnosed patients with primary breast cancer and of 10.000 patients in follow-up. About 500 are patients who undergo RT. After a short introduction from our fellows from pathology, surgery, oncology and radiation therapy, who will discuss their role in the breast unit, we will try to give a practical demonstration of the everyday work of breast units with particular emphasis on the role of the radiologist.

Session Objectives:

1. To learn about breast cancer management.

2. To understand when imaging is necessary for planning a therapeutic strategy.

3. To appreciate multidisciplinary team work

4. To become familiar with the various breast unit disciplines other than radiology.

08:30

A. Radio-pathologic correlation/cooperation

\section{B. Paolini; Milan/IT}

Learning Objectives:

1. To understand how radiology can help in precancerous conditions.

2. To consolidate knowledge on benign appearing masses which prove to be malignant.

3. To appreciate the pathologist's input at a multidisciplinary meeting. 


\section{Postgraduate Educational Programme}

08:40

B. The surgeon's point of view

R. Agresti; Milan/IT

Learning Objectives:

1. To understand what the surgeon needs to know before operation.

2. To learn how to ensure that postoperative images are understood.

3. To appreciate the surgeon's input at a multidisciplinary meeting.

08:50

C. Oncologist/radiotherapist's point of view

G. Bianchi, L. Lozza; Milan/lT

\section{Learning Objectives:}

1. To understand what the oncologist needs to know before starting treatment.

2. To learn how to ensure that post-treatment images are understood.

3. To appreciate the oncologist's input at a multidisciplinary meeting.

09:00

D. MDT discussion

P. Panizza; Milan/IT

08:30 - 10:00 Room F2

Special Focus Session

\section{SF 8}

\section{Image-guided prostate biopsies: a paradigm shift}

A-217 08:30

Chairman's introduction

J.C. Vilanova; Girona/ES (kvilanova@comg.cat)

MR imaging has been playing an increasing role in the clinical management of prostate cancer. New MR techniques, multi-parametric (mp-MRI) acquisition, are showing improved assessment for prostate cancer detection and characterisation. The implementation of the new methodology on MRI needs to standardise the use and establish the recommendations for the clinical indications, as it has recently been presented. Prostate cancer is usually diagnosed with systematic blinded transrectal ultrasound biopsies. However, this method has low accuracy and for this purpose different approaches have been recently developed to change and improve the approach to diagnose prostate cancer. The challenge is to achieve an optimal and accurate method for an imaging-guided prostate biopsy.

Session Objectives:

1. To learn about the current role of imaging in managing prostate biopsy.

2. To become familiar with the current recommendations and guidelines for multi-parametric technique of prostate MRI and the standardisation of reporting.

3. To learn about different approaches to improving the performance of prostate biopsy.

\section{A-218 08:35}

MRI definition of the target: the ESUR scoring system

P. Zamecnik; Nijmegen/NL (Patrik.Zamecnik@radboudumc.nl)

The European Society of Urogenital Radiology (ESUR) published, in 2012, the new clinical guidelines for evaluation of the multi-parametric MRI of the prostate. This structured reporting/scoring system (PI-RADS) is based on literary evidence and consensus of experts' opinions. The PI-RADS scoring system is similar to that already employed by breast imaging (BI-RADS) and reflects the probability of a prostatic lesion to be significant. Each lesion can be scored in all sequences used in the multi-parametric prostate MRI protocols (T2- and diffusion weighted imaging, dynamic contrast enhanced imaging and MR spectroscopy) based on defined MRI criteria, which are specific for each sequence. Based on the scoring in the particular sequences a final PI-RADS score for each lesion can be assessed. For each lesion a five-grade scoring systems was created where, for example, the score 1 means that clinically significant disease is highly unlikely to be present and score 5 clinically significant disease is highly likely to be present. This lecture elucidates the principles of this scoring system and its impact on the target definition for the invasive diagnostics and therapy.

Learning Objectives:

1. To show the value of standardisation of reading multi-parametric MRI using (PI-RADS).

2. To learn how the PI-RADS reading should be done.

3. To understand how localisation of PI-RADS 4 and 5 lesions can be used to aim biopsy.
A-219 08:53

A targeted-only biopsy strategy: impact on overdiagnosis and overtreatment

N.B. Delongchamps; Paris/FR (nicolasbdl@hotmail.com)

Prostate cancer screening with PSA and extended systematic biopsy protocols have led to the over-detection and over-treatment of small and well differentiated cancers, considered clinically insignificant. These cancers cover up to $70 \%$ of the overall prevalence and may not be of any threat. Multiparametric (mp)-MRI has shown recently its value in the detection, localisation and characterisation of prostatic tumour foci larger than $0.2 \mathrm{~cm}$ and may be of value to address the issue of over-detection and over-treatment. An MRI-targeted biopsy strategy alone, without any additional systematic biopsies, has been suggested to decrease the detection rate of insignificant tumours while increasing that of potentially aggressive tumours. In patients with no evidence of lesion on mp-MRI, the biopsy may probably be deferred. In patients with localised prostate cancer, mp-MRI may also become a cornerstone in the selection, guidance and surveillance of patients managed with focal therapy. The rational for this new modality of treatment is to decrease over-treatment by destroying exclusively the index lesion detected on mp-MRI. The precision of such treatments may be increased with MRItransrectal ultrasound image fusion, allowing for real time navigation during the procedure.

Learning Objectives:

1. To consolidate knowledge of the clinical consequences of PSA screening and extended biopsy strategies.

2. To understand and develop the scientific rational of targeted-only biopsy.

3. To learn about the results of targeted-only biopsy.

4. To learn about the implications for patient management and the selection of focal therapy.

Author Disclosure:

N.B. Delongchamps: Speaker; LUT forum (Astellas), Janssen.

\section{A-220 09:11}

MRI-guided biopsies: pros and cons

B. Hamm; Berlin/DE (bernd.hamm@charite.de)

Allowing accurate detection of high-grade prostate cancer, multiparametric magnetic resonance imaging (mp-MRI) techniques have expanded the role of targeted biopsies of the prostate. MRI-guided prostate biopsy (MRGB) differs from systematic prostate sampling using transrectal ultrasound (TRUS) in that tissue can be specifically sampled from suspicious areas within the prostate. MR image guidance and targeting accuracy are the distinguishing features of MRGB compared with systematic (random) TRUS biopsies. Nevertheless, TRUS-guided biopsy is still the most widely used approach in patients with suspected prostate cancer. With TRUS-guided biopsy, there is an association between the number of tissue cores obtained and the cancer detection rate. However, this association has its limits since some areas within the prostate cannot be sampled at all or are difficult to reach with this biopsy technique. MpMRI, on the other hand, helps in the workup of suspicious prostate lesions by integration of several techniques. These techniques range from cognitive fusion to direct MR-guided biopsy, which means that only targeted tissue samples are obtained. The recent introduction of software-based fusion of MRI datasets with real-time ultrasound images for prostate biopsy combines the diagnostic accuracy of MRI with the wide availability of ultrasound-guided biopsy.

Learning Objectives:

1. To become familiar with the indications for MRI-guided biopsies.

2. To learn about the technical approach.

3. To learn about contraindications and complications.

4. To understand future developments in biopsies guided by MRI/US fusion. Author Disclosure:

B. Hamm: Board Member; Deutsche Röntgengesellschaft, European Congress of Radiology, European Society of Radiology, ESMRMB, European School of Radiology, Deutsche Forschungsgemeinschaft. Consultant; Bayer Schering Pharma, Toshiba. Grant Recipient; Kendle/ MorphoSys AG.

\section{A-221 09:29}

TRUS-guided biopsies with TRUS-MRI image fusion: pros and cons F. Cornud; Paris/FR (frcornud@imagerie-tourville.com)

The accuracy of multiparametric MRI has greatly improved the ability of localising tumour foci of prostate cancer. This property can be used to perform a TRUS-MR image registration, a new technological advance, which allows for an overlay of an MRI onto a TRUS image to target a prostate biopsy towards a suspicious area. Three types of registrations have been developed: cognitivebased, sensor-based and organ-based registration. Cognitive registration consists of aiming at a suspicious area during biopsy with the knowledge of the lesion location identified on multiparametric MRI. Sensor-based registration consists of tracking in real time the TRUS probe with a magnetic device, 
achieving a global positioning system which overlays in real time prostate image on both modalities. Its main limitation is that it does not take into account prostate and patient motion during biopsy Two systems (Artemis and Uronav) have been developed to partially circumvent this drawback. Organbased registration (Koelis) does not aim at tracking the TRUS probe, but the prostate itself to compute in a 3D acquisition the TRUS prostate shape, allowing for a registration with the corresponding 3D MRI shape. This system is not limited by prostate/patient motion and allows for a deformation of the organ during registration. The pros and cons of each technique and the rationale for a targeted-biopsy only policy are discussed.

Learning Objectives:

1. To learn the physical principles of TRUS-MRI image registration.

2. To become familiar with the different types of registration (cognitive, sensorbased, organ-based).

3. To learn about the accuracy of TRUS-MRI image registration-based prostate biopsies.

4. To understand the potential applications of TRUS-MRI image registration to guide focal therapy

Panel discussion:

Are we ready for the standardisation of MRI to manage prostate guided biopsy in routine clinical practice? 09:47

$08: 30-10: 00$ Room G/H

\section{EFOMP Workshop}

Between diagnosis and therapy:

new technology in imaging of the prostate

\section{EF 1}

\section{Imaging of the prostate for radiotherapy}

Moderators

P. Sharp; Aberdeen/UK

J.N. Vassileva; Sofia/BG

A-222 08:30

Welcome address

V.E. Sinitsyn ${ }^{1}$, P. Sharp ${ }^{2} ;{ }^{1}$ Moscow/RU,${ }^{2}$ Aberdeen/UK

\section{A-223 08:40}

Radiologist point of view: clinical and technical requirements for imaging of the prostate

H.-P. Schlemmer; Heidelberg/DE (h.schlemmer@dkfz.de)

Prostate cancer is associated with high morbidity and accordingly high socioeconomic impact. Due to overdiagnosis and overtreatment, avoidable morbidity and costs are feared in a selective but unpredictable patient group. The conventional way of establishing the diagnosis and making individual treatment decisions relies on the individual PSA serum level and pathologic Gleason score from systematic TRUS biopsy samples, which has well-known limitations. Patient stratification for choosing the best individual treatment becomes increasingly challenging, as various less invasive treatment alternatives and active surveillance have been established. Multiparametric MR imaging has been proven to be remarkably advantageous in this context for detecting cancer, characterising its heterogeneity and aggressiveness, targeting the most aggressive part (the dominant intraprostatic lesion, DIL) guiding the biopsy needle to that area and evaluation of local tumour spreading. The gained information supports individualised decision-making concerning treatment selection, planning, guidance, monitoring and follow-up. In case of active surveillance, functional MR parameters additionally yield objective and reproducible biomarkers for monitoring temporal changes of individual tumour aggressiveness during follow-up. PET imaging of prostate cancer with the use of various radiotracers is an ongoing research field applied in clinical studies. The recently developed novel PET/MR technology furthermore opens the window to improved molecular diagnostics concerning biologic cancer characterisation and detection of early cancer spread into lymph nodes and bone marrow. Multiparametric MR and PET/MR imaging will be an important role for comprehensive assessment and staging of prostate cancer and thus for individualised patient management.

\section{Learning Objectives:}

1. To become familiar with conventional diagnostic pathways and different treatment options of prostate cancer.

2. To appreciate the role of multiparametric MR and MR-targeted biopsy for clinical management of prostate cancer patients.

3. To learn about novel developments of PET and PET/MR imaging for improved diagnosis and (re-)staging of prostate cancer.

Author Disclosure:

H.-P. Schlemmer: Advisory Board; Siemens. Research/Grant Support; Siemens. Speaker; Siemens.

\section{A-224 09:10}

Ultrasound imaging in the treatment of prostate cancer W.J. van der Putten; Galway/IE (wil.vanderputten@hse.ie)

Radiotherapy of the prostate typically takes the form of either external beam radiotherapy or alternatively, brachytherapy using radioactive seed implants. In both cases, the use of ultrasound has been proven to be highly useful and this presentation will give an current state of the art overview of ultrasound in external beam and radioactive seed radiotherapy. In the case of external beam radiotherapy, accurate localisation of the prostate is essential to ensure adequate target coverage with minimal damage to normal tissue. Dedicated ultrasound scanners are able to provide sub millimeter localisation of the prostate superior to the imaging traditionally obtained using CT scanners. This positional information can be obtained daily. In addition, ultrasound allows the imaging of patients with metal hip implants which are normally difficult to image using CT. Apart from the benefits of the technique, some pitfalls will also be highlighted. In the case of prostate brachytherapy, the use of ultrasound in the $3-D$ volume imaging of the prostate both prior and during treatment will be discussed. In both cases, some time will be spend discussing the Quality Assurance requirements for ultrasound imaging systems.

Learning Objectives:

1. To learn about the use of ultrasound in image guided radiotherapy.

2. To understand the clinical capabilities of ultrasound guided radiotherapy.

3. To understand and appreciate the limitations of the technology.

\section{A-225 09:35}

Cone beam computed tomography for treatment of prostate cancer A. Torresin, B. Bortolato, M. Brambilla, C. Carbonini, P. Colombo, M. Minella, A. Monti, M. Parisotto, L. Trombetta; Milan/IT (alberto.torresin@unimi.it)

Image-guided radiation therapy (IGRT) with onboard kilovoltage cone-beam CT (CBCT) allows image guidance during radiotherapy treatments for patient setup and dose replanning. All these items will be discussed in connection with other topics: organ dose and image quality. It is compulsory to convert CBCTimage's pixels from arbitrary grey scale to Hounsfield unit (HU). This conversion was obtained using a Catphan 600 phantom. The same phantom is used for image quality evaluation; standard CTDI head and body phantoms and a Farmer chamber were used to measure the CBDI to estimate organ and effective doses by Monte Carlo software of different protocols of acquisition. CTDosimetry software (ver.1.0.4-ImPACT) and PCXMC2.0 Rotation software were used. To verify the dose replanning techniques by $\mathrm{CBCT}$, horizontal and vertical dose profiles were compared with the same obtained from CT. Patient replanning was verified using CBCT vs CT in terms of conformity Index. Image quality parameters of CBCT (in comparison with CT images) are fine for spatial resolution, but are less useful for low contrast. CBDI of $5.7 \mathrm{mGy} / 100 \mathrm{mAs}$ was measured; $4 \mathrm{mSv}$ effective dose, $26 \mathrm{mGy}$ prostate dose and $32 \mathrm{mGy}$ bladder dose were evaluated and discussed during the presentation. Replanning on cylindrical phantom shows a mean percentage difference for each profile $\leq 2 \%$ and a variation on $\mathrm{D}$ mean in all inserts $<3 \%$. The mean percentage difference between parameters characterising CT and CBCT-based plan values is less than $5 \%$. The replanning showed a substantial agreement with doses evaluated on the reference CT-image; patient dose must be evaluated for all radiation sources.

Learning Objectives:

1. To learn about application of digital imaging in cancer treatment.

2. To understand image quality and patient dose in CBCT.

3. To appreciate the interdisciplinary approach of using CBCT in radiation therapy.

4. To become familiar with concept of image quality and patient dose in CBCT.

5. To consolidate knowledge of rule of Medical Physics Expert for planning and verification in cancer treatment. 
08:30 - 10:00

Room I/K

\section{Head and Neck Imaging}

CC 819

\section{How to perform and read a head and neck study (part 1) \\ Moderator:}

J. Olliff; Birmingham/UK

\section{A-226 08:30}

\section{A. Cranial nerve examination made easy}

J. Casselman; Bruges/BE (jan.casselman@azbrugge.be)

Cranial nerves I-VI have an anteroposterior course and are best examined in the coronal plane. Cranial nerves VII-XII run in an anterolateral direction and are therefore best examined in the axial plane. These two planes also allow left-right comparison which makes lesion detection easier Lesions involving the cranial nerve nuclei can be detected on axial proton-density/T2/multi-echoGE images and diffusion-weighted images can exclude acute infarction. The cisternal segment of the cranial nerves is best detected on heavily T2-weighted images (DRIVE/FIESTA/3D-TSE/CISS). At 3-tesla, 3D-sequences (e.g. b-FFE) can be used and it is often possible to cover the cisternal segments of all 12 cranial nerves. In the cavernous sinus, the cranial nerves can only be evaluated on coronal gadolinium-enhanced high-resolution T1-weighted images and similar axial images are needed to evaluate the nerves in the jugular foramen and hypoglossal canal. Axial and coronal Gd-enhanced highresolution T1-weighted with or without fat suppression are used to image the extracranial course of the cranial nerves. Today, the TSE-DIXON sequences provide non-FatSat and FatSat images simultaneously, making cranial nerve imaging faster and easier. Time-of-flight images can be used to study neurovascular conflicts, although these can also be detected on submillimetric Gd-enhanced T1 images. Of course, it is important to know the major anatomy of the cranial nerves and the most frequently occurring lesions. The abovementioned imaging techniques, the most important anatomy and the most frequently occurring lesions will be demonstrated and discussed in this lecture. Learning Objectives:

1. To learn how to perform an examination and evaluation of cranial nerves.

2. To become familiar with some technical issues in obtaining good quality images.

3. To learn some basic information regarding cranial nerves anatomy Author Disclosure:

J. Casselman: Equipment Support Recipient; World reference site for MR equipment Philips. Speaker; Speaker at lunch sessions and meetings organised by Philips.

\section{A-227 09:00}

\section{B. MRI of the lower neck - feasible?}

S. Gerevini; Milan/IT (gerevini.simonetta@hsr.it)

MRI study of the lower neck space includes the study of anatomy and pathologies of the thoracic outlet, or cervicothoracobrachial junction, extending from the cervical spine and the mediastinum to the lower border of the pectoralis minor muscle, the brachial plexus and the supraaortic vessels (carotid, subclavian arteries and veins). For this, use of a dedicated head and neck coil is fundamental to avoid frequent artefacts arising from air and the passage between different surfaces. Only in case of thoracic outlet evaluation, a dynamic angio-MRI study should be performed and this means the use of a surface coil. MRI protocol of the lower neck should include T1- and T2weighted sequences. Both fat-saturated proton density and T2 with STIR sequences can be used to overcome the inhomogeneity of the magnetic field, especially with large FOV. Sand bags can be placed on either side of the neck and suprascapular region of the patient to improve image quality. Also, flow saturation bands can be utilised to limit blood flow artefacts. Also, volumetric sequences are in common use to obtain a quick examination. Breath or cardiac gating can be helpful. Abnormal findings of brachial plexus consist of nerve signal abnormalities with mild or marked hyperintensity on T2-weighted, being aware of magic angle effects and swelling. In case of thoracic outlet syndrome, MRI protocol should define the compression of brachial plexus components arteries and or veins, both in indifferent and dynamic MRI acquisition.

Learning Objectives:

1. To learn how to overcome difficulties in performing a lower neck study.

2. To understand how to avoid the most common pitfalls.

3. To become familiar with differential diagnosis.

\section{A-228 09:30 \\ C. CT and MRI of temporal bone: user's guide \\ F. Veillon; Strasbourg/FR}

CT and MR imaging of the temporal bone must be performed with a precise technique. In CT it is important to locate the box of the study above and not in the orbit to avoid the lenses. Irradiation is divided by 10 compared with a study through the orbit. The axial sections must be parallel to the lateral semicircular canal the coronal, sagittal sections are completed by oblique coronal views through the long process of the incus and the head of the malleus to get the ossicular $\mathrm{V}$. Double oblique sections through the 2 componants of the $\mathrm{V}$ permit a view of the stapes and also the malleus and incus in $3 \mathrm{D}$ (MIP : $3 \mathrm{~mm}$ ). MRI must be performed parallel to the roof of the orbit permiting very good axial sections in the plane of the lateral semicircular canal (T1 and T2). The internal auditory meatus and the inner ear must be analysed with $1 \mathrm{~mm}$ axial sections (SE) after intravenous gadolinium injection completed by a high resolution T2 (gradient echo or SE) 0.3-0.4 MM, depending on the machine (3T, 1,5 T). The middle ear in chronic otitis media must be studied with $\mathrm{T} 1$, diffusion and high resolution T2. There is no need of contrast medium injection apart from the complication: fistula of the lateral semicircular canal, thrombosis of the sigmoid sinus. The different pathologies are then discussed: external auditory meatus and middle ear pathologies: CT first. MRI is useful for postoperative cholesteatomas. Inner ear malformations, otosclerosis, trauma: CT. Labyrinthitis, inner ear hemorragia, schwannomas, internal auditory canal content: MRI.

Learning Objectives:

1. To learn about acquisition protocols for temporal bone examination

2. To learn how to evaluate images and learn which post-processing tools to use.

3. To learn how to report on temporal bone findings.

08:30 - 10:00 Room L/M

Vascular

\section{RC 815}

\section{Imaging and intervention in acute ischaemic stroke}

Moderator:

K.A. Hausegger; Klagenfurt/AT

A-229 08:30

A. Acute stroke imaging

J. Tacke; Passau/DE (josef.tacke@klinikum-passau.de)

Revascularisation time windows for patients with acute ischemic stroke are generally restricted up to 4.5 hours in the anterior and up to 8 hours is the posterior perfusion area. Later treatment attempts require more accurate prediction of risk and benefit, as safety and efficacy at these time strata are less well. Thus, rapid and effective imaging is important for decision-making concerning intrarterial catheter based recanalisation and/or thrombolytic therapy. Advanced imaging techniques identify irreversible infarction as well as tissue at risk. Diffusion-weighted MRI detects ischemia within minutes of onset, whereas perfusion-weighted MRI and CT perfusion studies disclose the ischemic penumbra. Combined, they provide information on mismatched tissue, i.e. potentially salvageable brain. In addition, non-catheter angiographic techniques like CT or MR angiography are a useful adjunct to localise arterial occlusion. As an attempt to a reliable emergency examination, the following protocol has been proven to be robust: for the anterior perfusion area, a non contrast CT may exclude cerebral bleeding and is followed by CT angiography (including supraaortic and intracranial vasculature). If technical available, CT perfusion should be performed in addition. In ischaemic strokes of the vertrebrobasilar region, CTA is essential to exclude basilary stenoses or thrombotic occlusion. If the stroke onset remains unclear or might extend the above mentioned time window, MR stroke imaging (i.e. diffusion, FLAIR and perfusion sequences) is suggested as MR offers a higher sensitivity. Anyway, the best method for each emergency stroke imaging center is depending on clinical availability $24 \mathrm{~h} / 365$ days, technical equipment and - finally - individual experience of the emergency team.

Learning Objectives:

1. To learn about parenchymal and vascular stroke imaging.

2. To become familiar with appropriate imaging protocols for all imaging modalities.

3. To learn about the pros and cons of each modality. 


\section{A-230 09:00}

\section{B. Indication for intervention}

D. Vorwerk; Ingolstadt/DE (dierk.vorwerk@klinikum-ingolstadt.de)

Some recent publications have questioned interventional treatment of stroke as an alternative to iv thrombolysis only. These papers - albeit published in highranking sources - usually do not describe the modern concepts of interventional stroke treatment. Interventional radiologists, therefore, are convinced by their practical experience that the modern concept of combining iv thrombolysis with mechanical thrombectomy by stent retrievers offers a benefit to a subgroup of patients with severe stroke. This includes anatomical level of occlusion - basilar artery or single vertebral artery, proximal carotid occlusion with distal tandem occlusion, carotid T obstruction and M1 occlusion M2 occlusions are debatable. Besides location, the clinical status of the patient before stroke and time of onset, absence of early CT signs of stroke or bleeding and clinical contraindications to iv thrombolysis such as recent surgery influence the decision-making. More difficult than the description of technical success is prediction of clinical success. There are a couple of scores such as Thrive available that may be used for outcome forecasting. The amount of collateral flow is frequently used as a decision tool, but is not always easy to quantify. Mismatch scores and penumbra have been questioned recently. In conclusion, anatomical factors are pretty easy to identify to indicate treatment, but the clinical and functional setting still lacks quick and reliable parameters that allow a clear decision-making, particulary in borderline cases. Learning Objectives:

1. To learn how to define the indication for treatment

2. To learn about the treatment decision-making process.

3. To learn about the classification of lesions and indications for treatment.

\section{A-231 09:30}

\section{Mechanical revascularisation}

A. Dörfler, T. Engelhorn; Erlangen/DE (arnd.doerfler@uk-erlangen.de)

The refresher course addresses the current state-of the-art use of different mechanical revascularisation strategies, devices and potential complications. In addition, multimodal imaging applications with a focus on patient selection for endovascular recanalisation therapies, as well as new techniques to guide endovascular therapy within the angio suite are presented. Finally, some organisational aspects important for providing an interdisciplinary interventional stroke service are discussed. The ultimate goal of an acute endovascular stroke intervention is neurological recovery or improvement. Recanalisation of an arterial occlusion is key in achieving this goal. Clinical data suggest that endovascular stroke treatment results in higher recanalisation rates and may provide superior clinical outcomes when compared with intravenous thrombolytic therapy only. However, these higher recanalisation rates are far away in being paralleled by equally higher rates of favourable outcomes in recanalised patients. Thus, patient selection remains crucial. Besides the careful neurological assessment, brain imaging is here of major importance. The case presentations illustrate that imaging may help in patient selection for subsequent thrombolytic/endovascular therapies by differentiation of patients who may profit from intravenous or interventional therapy in an even extended time window from those who do not. There are a number of key areas supported by evidence-based medicine necessary for a high-level interventional stroke service. As a precondition, a neurointerventional stroke service has to be organised within a multidisciplinary acute stroke team. Inside the hospital, it is all about streamlined pathways. Any possible delays should be minimised at every step.

Learning Objectives:

1. To learn the difference between mechanical and pharmacological stroke treatment.

2. To learn about different mechanical revascularisation techniques.

3. To learn how to manage complications.

08:30 - 10:00 Room N/O

Interventional Radiology

\section{RC 809}

\section{Percutaneous treatment of chronic back pain and sciatica}

\section{A-232 08:30}

\section{Chairman's introduction}

A. Gangi; Strasbourg/FR (gangi@unistra.fr)

Back pain has a diverse etiology and causes pain, suffering and disability which have a great social and economic impact. Minimally invasive percutaneous techniques can improve the quality of life of well selected patients.

Author Disclosure:

A. Gangi: Other; Proctoring Galil Medical.

A-233 08:35

A. Sacroiliac joint syndrome

D.J. Wilson; Oxford/UK (david.wilson@ndorms.ox.ac.uk)

Sacroiliac joint pain may arise from a number of conditions including inflammatory arthritis, degeneration, fractures and tumours. Studies suggest a prevalence of $10 \%$ to $60 \%$ pain arising from the joint in patients with positive clinical signs. Temporary effect is provided by a mixture of local anaesthetics with steroids with a response varying between $50 \%$ and $80 \%$ in reported series. Dual blocks using agents of differing duration are considered more precise, but are less often used in practice. Imaging including MR and scintigraphy are of limited predictive value. Injections may be into the synovial joint, around the joint or adjacent to the nerve innervation of the joint. There is evidence that para-articular sources of pain are common and injection outside the joint may be more effective. Sl-joint injections are performed through a dorsal approach guided by ultrasound, fluoroscopy or low-dose CT. Shortacting agents may have lasting benefit, but radiofrequency ablation has been employed in an attempt to obtain long-term response. The evidence for lasting therapeutic response to intra-articular or periarticular injection of steroids and conventional radiofrequency neurotomy is weak. There is fair evidence of longterm response to cooled radiofrequency neurotomy.

Learning Objectives:

1. To learn about relevant anatomy and clinical presentations of the syndrome. 2. To learn more about the available treatments

3. To learn about clinical results and possible further developments.

A-234 08:58

B. Facet joint syndrome

A.D. Kelekis; Athens/GR (akelekis@med.uoa.gr)

Facet joints account for $25-40 \%$ of all low back pain. They are affected by osteoarthritis, joint space narrowing, intra-articular vacuum phenomenon/fluid, osteophytes, synovial cyst and ligament hypertrophy. Conservative therapy is initially proposed. Percutaneous facet joint steroid infiltrations are minimally invasive procedures involving injection of corticosteroid with or without local anaesthetic inside the joint. They also can provide diagnostic verification of a certain facet joint acting as the pain source. The injectate usually contains a long-acting corticosteroid mixed with a local anaesthetic. Sodium hyaluronate solutions or ozone were tested; however more and extensive studies are necessary. Other options are either percutaneous ablation or surgical arthrodesis. Fluoroscopy, computed tomography or magnetic resonance can be used for guidance. Fluoroscopy has the advantage of real-time imaging Cone-beam CT can also be used. Computed tomography provides better anatomy information, but has increased radiation dose for the patient. Magnetic resonance has higher cost and longer duration. A recent study concluded that it is twice the cost of CT-guided infiltrations. MRI can be used in combination with focal ultrasound for ablation, a technique which is still under investigation. Success depends upon patient selection (59-94\% immediate and $27-54 \%$ long-term relief). The level of evidence is moderate for lumbar spine concerning short- and long-term improvement. However, the most recent guidelines released (American Society of Pain Physicians) state that it is the oldest and most commonly used technique. The success rates with its safety profile and least invasiveness seem to make it an attractive therapy.

Learning Objectives:

1. To understand the difference between facet joint and disc disease.

2. To learn about different treatment options for facet disease.

3. To learn how to manage patients.

\section{A-235 09:21}

C. Intervertebral disc syndrome

N. Amoretti; Nice/FR (amorettinicolas@yahoo.fr)

Low-back (LBP) is a frequent complaint and a leading cause of disability in the general population. Epidemiological studies have identified many individual, psychosocial, and occupational risk factors for its onset, but the primary causative mechanisms of LBP remain largely undetermined. Psychological factors have an important role in the transition from acute to chronic pain and related disability. A variety of minimally invasive techniques have been investigated over the years as treatment of back pain related to disc disease. Besides the classical surgical intervention, new therapeutic options have been proposed secondary to the progress in interventional imaging. Randomised clinical trials are important to assess treatments of painful conditions and low back pain. Due to the expected placebo effect and the subjective nature of pain assessment in general, the variable natural history of hernia and low back pain often responds to conservative care. After the commercial withdrawal of chymopapain, the need for new substances or devices to treat intervertebral 
disk hernias is important. The goal of this communication is to give our point of view and experience in the treatment of symptomatic herniated disks by IR in patients in whom open surgery was indicated.

Learning Objectives:

1. To understand possible treatment techniques for disc disease.

2. To learn more about clinical and imaging findings in treatment.

3. To learn about published results on percutaneous disc treatment.

Panel discussion:

How can imaging methods separate candidates for percutaneous therapy and surgery?

09:44

08:30 - 10:00

Conf. Room M3

Oncologic Imaging: Follow-up of Systemic and Local Therapies

\section{818}

\section{Imaging after systemic therapies: advanced techniques}

Moderator:

K. Brindle; Cambridge/UK

\section{A-236 08:30}

A. What can we expect from biomarkers

B.E. Van Beers; Clichy/FR (bernard.van-beers@bjn.aphp.fr)

Biomarkers are quantitative parameters measured with imaging methods that can objectively assess disease status. Anatomical imaging biomarkers based on tumor size measurements (RECIST) are often used but have limitations because tumor size decrease may be delayed or absent. Therefore, mRECIST, EASL and Choi criteria based on size measurements of viable, contrast-enhancing tumor regions or on tumor attenuation are increasingly used for assessing the effect of targeted treatments. Moreover, there is a growing interest for validating functional imaging biomarkers such as perfusion and diffusion parameters obtained with ultrasound, CT and MR imaging Although promising for early assessment of response to treatment, these newer functional biomarkers need extensive validation and standardisation for their wide clinical use. Validation includes the assessment of reproducibility and accuracy, whereas standardisation concerns image acquisition and postprocessing. The added value of the more complex functional biomarkers relative to the viability parameters should also be shown. Viability and functional imaging biomarkers are evolving and emerging parameters for the early assessment of response to treatment.

Learning Objectives:

1. To become familiar with biomarkers.

2. To consolidate knowledge of various biomarkers and their utility.

\section{A-237 09:00}

\section{B. MRI biomarkers: from acquisition to post-processing}

O. Lucidarme, M. Wagner; Paris/FR (olivier.lucidarme@psl.ap-hop-paris.fr)

MRI biomarkers must be able to show how tumours respond to specific treatment. They need to allow assessment of the effectiveness of new treatment more rapidly than classical clinical end points. These biomarkers must be easy to obtain to facilitate a large spread of the technique. They have to be reproducible. The longest diameter of the tumour remains the easiest biomarker that can be obtained from any kind of morphologic acquisition with no need of post-processing. Additional information about the tissular organisation and cellularity can be now easily obtained using modern scanners through diffusion-weighted sequences. The ease with which those sequences are obtained for a while masked the necessity to perform a more complex postprocessing than the one initially done to get reliable biomarkers. There are numerous MRI biomarkers of microcirculation, reflecting $\mathrm{O}_{2}$ consumption, blood volume, blood flow, vessel permeability and extravascular volume. To get them, we need more sophisticated acquisitions and image processing that take into account the $\mathrm{T} 1$ of the tissue, arterial input function, respiratory motion, etc. Most of these new MRI biomarkers are now used in research and in phase I studies, but have not been validated in more advanced clinical trials or in clinical practice. To use them widely and reliably, we need to perfectly understand the consequences of the choices we make during the acquisition and post-processing of these biomarkers.

Learning Objectives:

1. To become familiar with MRI biomarkers

2. To learn some tips and tricks for MRI biomarker evaluation.

Author Disclosure:

O. Lucidarme: Board Member; Roche, Boehringer Ingelheim. Speaker; Bracco.

\begin{abstract}
A-238 09:30
C. Assessing the precision and accuracy of biomarker imaging: is it reproducible?
\end{abstract}

D.-M. Koh; Sutton/UK

New targeted treatments in cancer can be effective without significantly reducing tumour size. There are already a large number of targeted treatments that are licensed to treat a range of cancers. For some of these cancers, there is currently no reliable method to tell whether the drug is effective and new response, predictive and prognostic biomarkers are required. Functional imaging techniques such as diffusion-weighted MRI, dynamic contrastenhanced MRI and FDG-PET imaging are being developed or applied as response biomarkers. However, for these to be useful in a wider multi-centre setting, the measurements need to be precise, repeatable and reproducible. We discuss these properties in the context of emerging imaging response biomarkers.

Learning Objectives:

1. To understand imaging biomarker precision (repeatability and reproducibility) and accuracy and how it is evaluated.

2. To learn how to interpret biomarker precision and accuracy in the context of the biomarker's intended use.

\begin{tabular}{ll}
\hline 08:30 - 10:00 & Board Room A \\
\hline Cardiac
\end{tabular}

\section{Cardiac}

\section{RC 803}

\section{Quantification of myocardial perfusion: which test is the best (PET, MRI, MDCT)?}

Moderator:

A. Jacquier; Marseille/FR

A-239 08:30

A. PET for evaluation of perfusion, absolute myocardial blood flow and coronary flow reserve

J. Bucerius; Maastricht/NL (jan.bucerius@mumc.nl)

Coronary artery disease (CAD) and its related cardiac disorders are still the number one cause of death in the USA and the Western world. Up to date, single photon computed tomography (SPECT) using traditional radiotracers like thallium-201 or Tc-99m sestamibi is the most utilised imaging technique for the assessment of myocardial perfusion. However, over the past decade, there has been a growing interest in cardiac imaging with positron emission tomography (PET) and, indeed, a paradigm shift has been witnessed in the use of myocardial perfusion imaging (MPI) with PET taking advantage of the superior imaging properties of PET over SPECT. Therefore, PET MPI is now being increasingly used for routine clinical evaluation of patients with known or suspected CAD. Furthermore, it is being used not only at large academic institutions, but also at community hospitals and even in private practice. Several factors contribute to this shift in the use of PET MPI, including the growing availability of combined PET and computed tomography (CT) systems, mainly driven by oncological applications, radiotracer, like rubidium-82 or $18 \mathrm{~F}$ flurpiridaz, which can be used in clinical routine, changes in reimbursement, and the increasing clinical evidence supporting the value of PET/CT MPI. The lecture "PET for evaluation of perfusion, absolute myocardial blood flow and coronary flow reserve" will cover several aspects of the growing field of PET MPI.

Learning Objectives:

1. To understand the molecular tracers used to asses myocardial perfusion by PET.

2. To appreciate the strengths and limitations of PET in evaluating of myocardial perfusion.

3. To become familiar with its role in different clinical scenarios. 


\section{A-240 09:00}

B. Stress perfusion CT imaging for the detection and quantification of relevant coronary stenosis

F. Bamberg; Munich/DE (fabian.bamberg@med.uni-muenchen.de)

Besides the visualisation of coronary morphology, computed tomography (CT) has shown feasibility to also assess myocardial perfusion. Currently, there are two different approaches to CT-based myocardial perfusion imaging: singleshot and dynamic, sequential acquisitions over a predefined scan time. The presentation will cover basic concepts of both approaches and highlight protocol details and findings in these stress acquisitions. Also, emerging scientific results with respect to diagnostic accuracy, the detection of hemodynamically relevant coronary stenosis and prognostic implications will be discussed.

Learning Objectives:

1. To learn about protocol setup and interpretation of stress myocardial perfusion $\mathrm{CT}$.

2. To understand the prognostic implications and therapeutic considerations of CT perfusion parameters.

3. To appreciate the value of stress myocardial perfusion CT for the detection of significant coronary stenosis.

Author Disclosure:

F. Bamberg: Research/Grant Support; Bayer Healthcare. Speaker; Bayer Healthcare, Siemens Healthcare.

\section{A-241 09:30}

\section{Analysis of myocardial perfusion using MRI}

R. Manka; Zurich/CH (robert.manka@usz.ch)

Over the past few years, cardiovascular magnetic resonance imaging (CMR) has been increasingly established as an important method in the diagnosis of cardiovascular disease. Many studies have shown the equality or even superiority of CMR compared to other imaging modalities (e.g. nuclear medicine and echocardiography). CMR offers important advantages like the absence of ionising radiation, high spatial resolution, and the combination of perfusion imaging with tissue characterisation. The main clinical applications in the assessment of coronary artery disease (CAD) include ventricular function, myocardial viability and perfusion. In clinical routine, myocardial perfusion is determined by contrast-enhanced first-pass perfusion techniques during pharmacological stress using coronary vasodilators (e.g. adenosine) or Badrenergic agents (e.g. dobutamine). Non-invasive characterisation of myocardial microcirculation is thought to reflect myocardial tissue supply much better than mere luminographic detection and quantification of epicardial coronary stenosis, and has been shown to be useful for planning of revascularisation procedures and cardiac risk stratification. In several studies on the prognostic value of CMR in CAD assessment, normal stress perfusion CMR was highly predictive for a good prognosis, thus able to identify patients in whom invasive angiography can be deferred safely.

Learning Objectives:

1. To learn about protocol setup and interpretation of stress myocardial perfusion CT.

2. To understand the role of CMR perfusion imaging as a tool for diagnosis and prognosis of coronary artery disease.

3. To discuss how CMR perfusion can be utilised in the assessment of microvascular disease.

\section{8:30 - 10:00}

Room P

\section{Joint Session of the ESR and ESMRMB}

\section{Diffusion MRI: everything you always wanted to know but never dared to ask}

\section{Moderators:}

E.-M.B. Larsson; Uppsala/SE

M. Smits; Rotterdam/NL

\section{A-242 08:30}

\section{Diffusion MRI: the bare basics}

C.A.M. Wheeler-Kingshott; London/UK (c.wheeler-kingshott@ucl.ac.uk)

The purpose of this lecture is to demonstrate how it is possible to sensitise the MR signal to water molecules diffusion in tissue and how to use calculated indices to reflect structural integrity. The concept of diffusion-weighted imaging will be introduced with particular emphasis on the pulsed gradient spin echo (PGSE) sequence. Methods to calculate the apparent diffusion coefficient (ADC) will be described and the concept of the diffusion tensor (DT) will be explained. Image processing of diffusion data includes steps like eddy current distortion correction, model fitting and potentially registration of maps to a reference space. Indices that are reproducible and rotationally invariant will be described, such as fractional anisotropy (FA), mean diffusivity (MD), axial diffusivity (AD) and radial diffusivity (RD). Examples of when to use the ADC maps or the DT indices will be given in relation to pathologies such as stroke, multiple sclerosis and neurodegenerative diseases. Strategies for result presentation such as region of interest approach, histogram analysis and tractbased methods will be shown. Limitations and advantages of diffusion imaging methods such as DT do not preclude the use of this technique in research and clinical radiology for investigating structural changes in disease.

Learning Objectives:

1. To understand the basic principles of diffusion MRI (diffusion-weighted imaging, diffusion tensor imaging)

2. To learn how to select an optimal diffusion-weighted imaging protocol for clinical neuro and body imaging.

Author Disclosure:

C.A.M. Wheeler-Kingshott: Advisory Board; Biogen (BG12). Author; Publications in Peered Reviewed Journals. Employee; University College London. Research/Grant Support; Multiple Sclerosis Society of great britain and Northern Ireland, Biogen, Novartis, Medical Research Council,

Engineering and Physical Sciences Research Council, International Spinal Research TrustInternational Society. Speaker; Invited speaker at national and international conferences and iniversities.

\section{A-243 08:50}

Processing and analysing diffusion MRI data

W. Van Hecke; Antwerp/BE (wim.vanhecke@ua.ac.be)

Diffusion imaging methods have a lot of potential to be used in clinical practice. However, although diffusion-weighted imaging has been increasingly applied, clinical use of diffusion tensor imaging is limited to date. This is in part due to the lack of standardisation and the need for more complex analysis tools to evaluate the data. During this lecture, an overview will be provided of some analysis methods for diffusion-weighted and tensor imaging. I will discuss the different parameters that can be obtained as well as some advantages and limitations of the different analysis methods.

Learning Objectives:

1. To learn about post-processing of diffusion-weighted imaging and the terminology for diffusion parameters.

2. To learn about qualitative analysis of diffusion-weighted images including $T 2$ shine-through.

3. To learn about quantitative measurements of diffusion parameters in clinical radiology.

4. To understand different possibilities for analysing diffusion tensor imaging in clinical radiology.

Author Disclosure:

W. Van Hecke: Founder; icoMetrix.

\section{A-244 09:10}

Clinical applications of diffusion MRI (neuro)

E.-M.B. Larsson; Uppsala/SE (elna-marie.larsson@radiol.uu.se)

It is highly recommended to include diffusion-weighted imaging (DWI) in the routine protocols for MRI of the brain. DWI as well as automatically calculated apparent diffusion coefficient (ADC) map is used for evaluation. The ADC values of gray and white matter are identical in the adult brain. Restricted diffusion (high signal on DWI and low signal on ADC) is seen not only in acute stroke, but can also be present in some brain tumours, the necrotic centre of abscesses, some acute MR plaques, some contusions, encephalitis, Creutzfeldt-Jakob disease, metabolic diseases, etc. This finding is thus not specific, but is very helpful combined with clinical information and the signal pattern on other sequences. Acute stroke has restricted diffusion during the first 7-10 days and the diffusion gradually increases to become very high in a chronic infarct. The diffusion changes in ischemic brain and spinal cord tissue are usually irreversible, but may be reversible. Brain tumours with high cellularity, such as lymphoma, glioblastoma multiforme, medulloblastoma and metastases from small cell lung carcinoma, usually have restricted diffusion. The surrounding vasogenic oedema has increased diffusion. In the central necrosis of a pyogenic abscess the diffusion is restricted, while it is increased in the necrotic centre of a malignant brain tumour. Reversible diffusion restriction in the cortex, the hippocampi and thalami can be seen in patients with status epilepticus. Diffusion tensor imaging has so far had limited clinical use, but may be useful, e.g. for the preoperative evaluation of brain tumours. Learning Objectives:

1. To understand the differential diagnostic possibilities of high signal intensity lesions on diffusion weighted images (DWI) of the brain and spinal cord. 2. To become familiar with the appearance of acute, subacute and chronic stroke on DWI.

3. To learn about the appearance of cerebral tumours, infection/inflammation, neurodegenerative diseases and traumatic lesions on DWI.

4. To understand the present use of diffusion tensor imaging (DTI) and diffusion tensor tractography (DTT) in clinical neuroradiology.

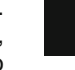


A-245 09:30

Clinical applications of diffusion MRI (body)

H. Ahlström; Uppsala/SE (hakan.ahlstrom@akademiska.se)

The signal intensity in diffusion-weighted imaging (DWI) reflects the cell density in the tissue. DWI, including apparent diffusion coefficient (ADC) maps, can therefore be used to differentiate highly cellular from acellular regions of tumours, distinguish cystic from solid lesions, and monitor change in tumour cellularity over time, reflecting response to therapy. In general, tumours have high signal intensities in DWI, but low corresponding ADC values compared to normal/benign/reactive tissues. DWI has a wide range of clinical applications, which includes cancer imaging, imaging of infections and inflammations, evaluation of trauma and visualisation of peripheral nerves. Of these, the most promising application appears to be in oncological imaging. There is, e.g. now evidence to recommend inclusion of DWI in MRI protocols for lesion detection and characterisation in the liver. The advent of whole body (WB) MRI including WB-DWI (from root of the neck to groin) has introduced tumour imaging with a systemic approach compared to established multi-modal diagnostic algorithms. It has been found valuable for staging, therapy evaluation and surveillance of tumours, especially in children. However, DWI is generally recommended to be incorporated into oncologic MRI protocols of both WB-MRI and of selected organs, because it provides additional valuable information to the conventional MR sequences. DWI of the body is frequently prone to imaging artefacts, which can obscure or mimic lesions. To minimise misinterpretations, analysis of the raw b-value images directly in conjunction with ADC maps and conventional coregistered sequences is recommended.

Learning Objectives:

1. To understand the present indications for whole-body DWI and DWI of selected organs of the body.

2. To learn how to interpret whole-body DWI qualitatively and quantitatively, including tricks and pitfalls.

3. To learn about the oncologic applications of DWI in the body.

4. To learn about the appearance of other diseases of the body on DWI.

Author Disclosure:

H. Ahlström: Research/Grant Support; Astrazeneca.

Panel discussion

09:50

10:30 - 12:00 Room A

\section{Interactive Teaching Session}

\section{$E^{3} 920 a$}

\section{The treated spine and joints}

\section{A-246 10:30}

A. Imaging of the postoperative spine

P.N.M. Tyrrell; Oswestry/UK (prudencia.tyrrell@rjah.nhs.uk)

Spinal surgery is most frequently performed to decompress (disc herniation, stenosis, malignant infiltration), fuse and stabilise (particularly following trauma or infiltrative destructive processes) and correct deformity. Often, there may be a combination of these procedures at one operation. Surgical instrumentation or bone graft is sometimes employed. Patients may present themselves with symptoms early or late following the procedure. This interactive session seeks to address the variety of surgical procedures undertaken and subsequently imaged post-operatively because of symptoms. The session aims to help one to understand and become familiar with the expected post-operative imaging appearances related to the surgical procedure, learn about abnormal pathological features as a cause of symptoms in the acute and more chronic situation and explore the diagnosis and differential diagnosis. This may include post-operative fibrosis versus recurrent disc herniation versus post-operative infection. Failure of fusion due to failure of instrumentation or inadequate take of bone graft can give rise to pseudoarthrosis. Recurrent stenotic symptoms may relate to an inadequate decompression, recurrent disc herniation, postoperative haematoma, extension of a malignant process or ischaemic damage. Learning Objectives:

1. To understand changes related to surgery.

2. To learn about changes related to non-surgical treatments.

\section{A-247 11:15}

\section{B. Imaging of joint replacement}

M. Zanetti; Zurich/CH (marco.zanetti@hirslanden.ch)

Joint replacement surgery for the treatment of arthritis most often offers the patient excellent results. However, there are potential complications that a radiologist should know about. It is essential to understand the importance of pre- and postoperative imaging for evaluating patients. Most commonly, standard radiographs are used to assess the patients after joint replacement.
However, CT, nuclear medicine methods as well as MR imaging play an increasing role in such patients. The radiologist should be aware of the most common type of prosthesis and the most common complications after joint replacement. These complications include postoperative prosthesis loosening, prosthesis fractures, periprosthetic fractures, postoperative infection, rotation failure of the prosthesis, and soft tissue abnormalities such as surrounding tendon tears. This interactive session seeks to address the variety of joint replacements which are undertaken and subsequently imaged postoperatively as a result of symptoms. The session aims to help one to understand and become familiar with the expected postoperative imaging appearances related to joint replacement, learn about abnormal pathological features as a cause of symptoms and explore the diagnosis and differential diagnosis.

Learning Objectives:

1. To learn about changes related to surgery.

2. To understand changes related to non-surgical treatments.

10:30 - 12:00 Room B

\section{ESR meets Serbia}

\section{EM 2}

\section{A guided tour of radiology in Serbia}

Welcome by the ESR President

G. Frija; Paris/FR

Presiding:

M.A. Lucic; Sremska Kamenica/RS

V.E. Sinitsyn; Moscow/RU

\author{
A-248 10:30 \\ Introduction \\ M.A. Lucic; Sremska Kamenica/RS (milos.a.lucic@gmail.com)
}

Serbia is a beautiful country where East and West merged and often collided for centuries, and where nowadays Eastern cultural heritage meets and mixes with the Western heritage in a lovely and unprecedented way, with a lot of charm and with a variety of extraordinarily natural beauty and plenty of both traditional and gastronomical wonders to explore. It extends from the edges of the Pannonian flatland in the north, over the Danube and Sava rivers to the gradually growing wonderful mountains in the south. Already in 1897, the first X-ray unit was installed, and only twenty years later X-ray units are present in most state-owned and private hospitals in Serbia. Nowadays, slightly more than half of the thousand radiologists in Serbia perform a broad variety of diagnostic imaging and interventional techniques in different radiological units, including numerous CT, MRI, and few PET/CT units distributed in five university centers, a number of public clinical centers and hospitals, and also in the private sector. Several centers are fully equipped with PACS and RIS systems, and are capable of performing teleradiology services. There are still significant challenges in Serbian radiology in the field of education and research, planning and implementation of national radiology networks, improvement of standard clinical practice and financing the new equipment. Yet since radiologists' enthusiasm overwhelms most of the difficulties, radiology in Serbia provides nowadays a broad variety of modern and competent diagnostic and interventional procedures, improving the management and treatment of our patients.

Session Objectives:

1. To get a brief insight into the variety of radiological techniques in Serbia today.

2. To learn about interventional radiological techniques in the therapy of varicoceles.

3. To understand the potential of MRI in the diagnosis of non-ischaemic cardiomyopathies.

4. To appreciate the concept and importance of foetal MRI in the early detection of CNS abnormalities.

\section{A-249 10:35}

Interventional treatment of varicoceles

P.M. Bosnjakovic, Z. Radovanovic, D.A. Stojanov, A. Bojanovic; Nis/RS

Varicoceles are considered to be one of the most frequent causes of male infertility. The entity represents the insufficiency of the spermatic vein, which by an unknown mechanism causes inefficient spermatogenesis. A variety of techniques for spermatic vein occlusion have been described since 1966, when the first interventional treatment took place. We aim to describe and review the variety of techniques used by the authors and evaluate our own experiences with more than 2000 treated patients. In 30 years, more than 2000 patients have been treated. The methods have been changing over time taking into consideration developments in interventional tools and experiences of other authors. Successful catheterization was achieved in almost all of the patients. Most frequently, the so-called "sandwich technique" was used 
considering the embolic device at the level of the inguinal ligament, then sclerotic agent followed by another embolic device more proximally. Using this technique it is possible to protect the pampiniform plexus and obliterate all the collateral veins and parallel anastomosis. Gelfoam plugs were used in the vast majority of patients and pushable or detachable coils only when there was a technical demand (large vein, difficult distal positioning of the catheter). Aetoxisclerol was the most frequently used sclerosant. Initial vein occlusion was achieved in all patients. Two puncture site complications occurred. In one patient, the tip of the catheter broke and was flown to the right middle pulmonary artery branch and subsequently percutaneously extracted without clinical consequences. The reccurence rate was within literature limits. The percutaneous treatment of varicoceles has been proven to be safe and efficient.

Learning Objectives:

1. To learn about the different radiological techniques for spermatic vein occlusion during the last 50 years.

2. To understand radiological treatment compared to other treatment options.

3. To appreciate the importance of varicoceles.

\section{A-250 10:55}

Interlude: Radiology in Serbia at a glance

T. Stosic-Opincal; Belgrade/RS (topincal@gmail.com)

Radiology in Serbia, since its beginning, has been inextricably linked to European radiology. There are written documents testifying to the fact that the first X-ray appliances in Serbia had emerged and had been used for medical purposes only two years after Wilhelm Conrad Röntgen discovered x-rays in 1895. The real beginnings of diagnostic radiology in Serbia can be linked to the establishment of the General State Hospital in 1908, where the first x-ray cabinet was installed. Later discoveries in the field of radiological techniques, materials and contrast agents were also immediately accepted by the Serbian radiology community. The first cerebral angiography was performed in 1935 , which may be considered as the beginning of neuroradiology in Serbia. The second half of the twentieth century is characterised by the introduction and wide application of ultrasound, CT and MR diagnostics and outbreak of vascular and nonvascular interventional radiology procedures in the areas of neuroradiology, uroradiology, and cardiovascular and gastrointestinal radiology. For years, Serbian interventional radiology has successfully kept pace with achievements of the most relevant centres worldwide. Nowadays, Serbian radiologists monitor and enforce the most complex modern procedures in different areas of radiology. The rapid development of science and information technology has enabled the daily monitoring of all modern developments, methods and approaches and their increasingly faster introduction into practice.

\section{A-251 11:00}

MRI in non-ischaemic cardiomyopathies

R. Maksimović; Belgrade/RS (ruzica.maksimovic@med.bg.ac.rs)

Non-ischaemic cardiomyopathies (NICM) refer to myocardial diseases caused by mechanical and/or electrical disorder in the absence of significant coronary artery disease, valvular heart disease, hypertension, or congenital heart disease. NICM often present with genetic mutations, but no clinically apparent disease. In some cases, the presence of fibrotic tissue could cause adverse events and, therefore, the use of late enhancement (LE) post-gadolinium technique is necessary in evaluating these patients. LE suggestive of fibrotic tissue could be found in up to $35 \%$ of patients with NICM. In the majority of cases (up to $65 \%$ ), midwall/subepicardial enhancement could be found; patchy enhancement, often at septal junctions, has been registered in $35 \%$ of patients, while the least common pattern of LE was subendocardial $(5 \%)$. In ischaemic cardiomyopathy (ICM), subendocardial or transmural is a typical pattern of hyperenhancement and is related to coronary artery distribution. Furthermore, LE has the potential to provide important information for risk stratification in clinical practice. In NICM, the extent of fibrosis has been shown to be independently associated with increased rates of future adverse events including all-cause mortality, while in ICM myocardium with LE in more than $50 \%$ of wall thickness is unlikely to recover contractile function following coronary revascularisation. As many NICM disorders are associated with the presence of scar tissue, LE in NICM is nonspecific and should be correlated with clinical presentation and history, which is critical in the evaluation of these patients.

Learning Objectives:

1. To learn how to differentiate non-ischaemic and ischaemic cardiomyopathies.

2. To understand and describe patterns of delayed enhancement in non-

ischaemic cardiomyopathies and their prognostic value.

3. To learn how to develop an accurate differential diagnosis of non-ischaemic cardiomyopathies based on MRI features.

\section{A-252 11:20 \\ Interlude: Hop-on, hop-off sightseeing tour of Serbia}

S. Stojanovic; Novi Sad/RS (tupsons@gmail.com)

In the interlude between scientific lectures, you are most welcome to hop on a sightseeing tour around Serbia during which we shall stop at a few stations where the natural and cultural heritage of our country will be shown. Nota bene: some of the sights will be visible to everyone and some only to radiologists. Ten thousand years of human effort and five billion years of European soil refinement will blink in front of your eyes through the window we have made with red churches' walls, azure skies and a silver sparkle of our spirit. All the time, a bit of grayscale shadow will supplement the picture as we in radiology are so used to. Fragments of past and present will make a mosaic, the glance of which will hopefully light up your day. When you hop off our tour, we believe, some pictures will become part of your emotional memory and maybe will make you wish to see them in vivo.

A-253 11:25

The role of prenatal MRI in foetal central nervous system abnormalities: a case-based pictorial review

K. Koprivsek; Sremska Kamenica/RS (katarina.koprivsek@gmail.com)

MRI provides a unique opportunity for studying in vivo central nervous system development. Its higher contrast resolution compared with prenatal sonography allows better visualisation of sonographically occult normal CNS structures as well as structural abnormalities. In clinical praxis, prenatal MRI has become a mandatory tool in evaluating: a) foetuses that have abnormalities suspected on the basis of foetal sonography; b) foetuses with increased risk for brain abnormalities even in the setting of a normal prenatal sonogram (family history of CNS anomalies or a genetic/metabolic disorder); c) foetuses potentially at risk due to maternal illness; d) foetuses with congenital malformation, which are candidates for prenatal or neonatal surgical treatment; e) foetuses that could not be evaluated by sonography due to oligohydroamnion, maternal obesity, difficult position of the foetal head, or US reverberation on foetal calvaria in advanced gestational age. We will present a selection of foetal CNS abnormalities detected in our Institution during the last 8 years, including ventriculomegaly, abnormalities of the posterior fossa, agenesis of the corpus callosum, cerebral cortex developmental abnormalities, spine developmental abnormalities and a variety of encephaloclastic lesions. In all previously listed cases, prenatal MRI could provide either crucial or additional information, which can lead to the accurate diagnosis of different CNS developmental abnormalities (cortical, commissural and posterior fossa malformation), specific disorder or syndrome, further enabling adequate pregnancy management and parents' counselling.

Learning Objectives:

1. To learn about normal anatomical findings and sequences used.

2. To know when, why and how to perform a prenatal CNS MRI study and what to look for.

3. To learn how to confirm the presence of foetal CNS pathology, define its full extent, characterise it and demonstrate associated abnormalities using prenatal MRI.

4. To learn about a selection of relevant cases from our institution, including ventriculomegaly, abnormalities of the posterior fossa, agenesis of the corpus callosum, cerebral cortex developmental abnormalities, spine developmental abnormalities and a variety of encefaloclastic lesions.

5. To assess the potential of prenatal MRI in parent counselling, management of pregnancy and delivery, and postnatal care planning.

Panel discussion:

Radiology in Serbia: frequently asked questions

$11: 45$

$\begin{array}{ll}10: 30-12: 00 & \text { Room E2 }\end{array}$

Foundation Course: Breast Imaging

\section{$E^{3} 920 b$}

\section{How to report}

Moderator:

R. Holland; Nijmegen/NL

\section{A-254 10:30}

A. Mammography

F.J. Gilbert; Cambridge/UK (fjg28@cam.ac.uk)

This lecture will cover the standard mammographic views required, the reporting systems for mammograms together with BIRADS and other European systems, breast density, feature analysis, information the clinician requires in a cancer case and how to approach a B3 lesion. ACR Practice guidelines (2008) recommend obtaining previous mammograms as you can 
dismiss an abnormality that is unchanged for 5 years or that has been previously worked up and are able to detect subtle new lesions more easily, although this has not been confirmed in the screening situation. In a structured report the following information is recommended: indication, breast density, description of lesion, size, location, comparison to previous findings, overall assessment, management recommendations and a score to indicate the level of suspicion. Examples of different common conditions seen on mammography will be shown including soft tissue masses, microcalcification, architectural distortion and asymmetry.

Learning Objectives:

1. To become familiar with BI-RADS mammography descriptors and assessment categories.

2. To understand which information a clinician needs to find in a breast cancer patient's report.

3. To learn about the basic parts of a structured report, specifically in BI-RADS 3 lesions.

Author Disclosure:

F.J. Gilbert: Speaker; Bracco.

\section{A-255 11:00}

\section{B. Breast US}

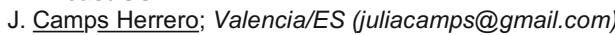

Breast ultrasound is one of the main imaging modalities in breast radiology, it allows us to characterise lesions and also guide interventional procedures. The BI-RADS categorisation of ultrasonographic findings facilitates the diagnostic approach and also helps the radiologist to use a common language, understood not only by the rest of the radiological community, but also by other breast cancer professionals. Ultrasound can be a diagnostic procedure on its own, but is mainly a modality that characterises the findings of other modalities (mammography and $\mathrm{MRI}$ ) and, as such, correlation between all these techniques is the mainstay of everyday clinical practice. This act of correlating and integrating the information of all modalities is what makes a breast radiologist a key actor in the diagnosis, staging and follow-up of breast cancer and other benign or high-risk entities. The final product of this integration will be the radiological report, the means by which we convey all the information we have gathered through all the procedures to our clinical colleagues. This report shall also follow some composition rules to be clear and concise.

Learning Objectives:

1. To become familiar with BI-RADS US descriptors and assessment categories.

2. To learn how to integrate clinical information into radiological findings.

3. To learn how to write a breast US report in a cancer patient.

Author Disclosure:

J. Camps Herrero: Advisory Board; Bayer. Speaker; Bayer.

\section{A-256 11:30}

\section{MRI of the breast}

M.H. Fuchsjäger; Graz/AT (michael.fuchsjaeger@medunigraz.at)

As with any other modalities, breast magnetic resonance imaging (MRI) examinations should be reported in a structured way following the guidelines of the respective national, continental or international societies. Adequate nonclinical information (i.e. patient name, date and type of examination, etc). is indispensable. Any breast imaging report has to follow a stringent structure including indication, clinical history, clinical findings, brief description of technology used, assessment of parenchymal density, detailed description of significant findings, comparison with previous imaging studies and final assessment according to BI-RADS. Indications and contraindications of breast MRI will be discussed. Significant findings at breast MRI include foci (small contrast-enhancing spot, NOS, $<5 \mathrm{~mm}$ ), mass lesions, "non-mass-like enhancement" (no mass lesion, partly diffuse regional contrast enhancement of various size) and associated findings. Furthermore, as breast MRI is a functional study, the different appearance patterns of kinetic contrast enhancement will be presented. The current status and the appropriate use of the BI-RADS MRI lexicon will be discussed. Any breast MRI report should not only follow the guidelines, but also follow a red thread, be consistent, express confidence and be comprehensible to clinicians. The overall final BI-RADS assessment is based on the most worrisome finding, taking into account both breasts and all imaging methods (mammography, ultrasound, MRI) evaluated. Furthermore, adequate communication of the result, as well as do's and don'ts of the report wording will be discussed.

Learning Objectives:

1. To become familiar with BI-RADS MRI descriptors and assessment categories.

2. To learn how to integrate clinical information and conventional imaging findings into a breast MRI report.

3. To learn how to compose a complete report with all morphological and kinetic information obtained.

\section{$10: 30-12: 00$ \\ EFOMP Workshop \\ Between diagnosis and therapy: new technology in imaging of the prostate \\ EF 2 \\ Novel approaches to imaging and treatment \\ Moderators: \\ O. Ciraj-Bjelac; Belgrade/RS \\ R. Padovani; Udine/IT} Room G/H

\section{A-257 10:30}

High intensity focussed ultrasound for the treatment of prostate disease G. ter Haar; Sutton/UK (gail.terhaar@icr.ac.uk)

High-Intensity focused ultrasound provides a method of selectively delivering ablative thermal energy to a predetermined tissue volume whilst leaving surrounding and overlying tissues unharmed. For the prostate, three potential treatment routes are available: trans-perineal, trans-urethral and trans-rectal. The trans-rectal approach, under ultrasound guidance, has been the most commonly used clinically. Whole gland, nerve sparing and partial ablations have all been used. The principles of the HIFU technique, available devices and published clinical results will be discussed.

Learning Objectives:

1. To learn about high intensity focused ultrasound (HIFU) and its use in prostate disease.

2. To appreciate the advantages and disadvantages of HIFU treatments of the prostate.

Author Disclosure:

G. ter Haar: Equipment Support Recipient; Philips.

\section{A-258 11:00}

\section{MR imaging of the prostate}

G. Payne; London/UK (Geoffrey.Payne@icr.ac.uk)

The prostate is a small glandular organ, whose main function is to secrete seminal fluid. In young men it comprises of mainly the peripheral zone $(70 \%)$ and a smaller central gland. However, the central gland enlarges with age to form the larger part. The main diseases of the prostate are inflammation, hyperplasia, and cancer (the most important). Approximately $70 \%$ of cancers arise in the peripheral zone. The main applications of MRI are for cancer detection, staging, and recurrence. MRI is increasingly used for radiotherapy planning owing to its good soft-tissue contrast when compared with CT scanning. Endo-rectal RF receiver coils, usually inflated with air or perfluorocarbon but sometimes of a rigid design, improve signal detection of the prostate. Phased-array surface coils may also be used or (preferably) in combination. $\mathrm{T}_{1}$-weighted imaging helps to distinguish haemorrhage caused by biopsy. On $\mathrm{T}_{2}$-weighted images the peripheral zone appears brighter, owing to a larger proportion of prostatic ducts, while tumours generally appear darker. More sophisticated MR methods are increasingly used to help diagnosis. In diffusion-weighted images (DWI) tumours appear relatively bright, but dark in the corresponding calculated map of Apparent Diffusion Coefficient (ADC) owing to the more cellularly-dense nature of tumours, and hence, reduced diffusion. In dynamic contrast-enhanced MRI (DCE-MRI) tumours appear bright owing to increased vasculature. Magnetic resonance spectroscopic imaging (MRSI) of normal prostate is dominated by high levels of citrate from the prostatic ducts; in cancer this is reduced while choline is increased. Prostatectomy samples allow one to verify image contrast against histology. Learning Objectives:

1. To become familiar with the main methods and applications of MRI in prostate.

2. To understand some of the problems associated with prostate MR.

\section{A-259 11:30}

New developments in molecular imaging of the prostate

M. de Jong; Rotterdam/NL (m.hendriks-dejong@erasmusmc.nl)

Recent studies on prostate cancer have provided a number of relevant targets for imaging and treatment purposes. Today, imaging plays an important role in different aspects of the disease, but its role should grow in answering clinical questions at various phases of the disease. Relevant targets for imaging include metabolites (including glucose, acetate, choline, and amino acids), antigens (including prostate-specific membrane antigen), receptors (GRP receptor, CXCR4, androgen receptor), proliferation, angiogenesis, and hypoxia. In this presentation, a range of radiotracers for potential use in the 
imaging of prostate cancer will be discussed, as well as options to tailor nuclear imaging tracers to the various phases of the heterogeneous disease. Learning Objectives:

1. To learn about biomarkers for molecular imaging of the prostate.

2. To appreciate new ways to improve molecular imaging of the prostate.

$10: 30-12: 00$

Room N/O

\section{Joint Session of ESR/ESMOFIR and ESMI}

Molecular and functional imaging methods for interventions

\section{Moderators:}

P. Brader; Vienna/AT

F.M.A. Kiessling; Aachen/DE

\section{A-260 10:30}

Interventional oncology based on molecular and functional imaging

R. Bale; Innsbruck/AT (reto.bale@i-med.ac.at)

The fusion of morphologic (CT and MRI) and functional (PET, SPECT) imaging modalities (PET, SPECT) has become widely available and part of the standard diagnostic workup in cancer patients. For many years, neurosurgeons have already relied on multimodal imaging data during brain tumour surgery to identify hyper- or hypometabolic tissue. The availability of PET and SPECT data during percutaneous ablation procedures may also be helpful to facilitate treatment planning, probe placement, probe re-positioning, and early detection of residual disease following percutaneous image-guided ablation of cancer. However, up to now conventional US- and CT-guided thermal ablation procedures rely on morphologic information only. Intraoperative implementation of multimodal data requires the application of frame-based or frameless stereotactic techniques, both relying on the establishment of an accurate relationship between the patient's preoperative image space and the intraoperative physical space. In image-guided neurosurgery, $\mathrm{CT/MRT/SPECT/PET} \mathrm{data} \mathrm{are} \mathrm{registered} \mathrm{to} \mathrm{the} \mathrm{patient} \mathrm{using} \mathrm{rigid} \mathrm{body}$ transformation. Due to respiratory motion and different patient positioning during image acquisition and the actual intervention, soft tissue deformations in the liver occur. Thus, the images and the surgical presentation do not match between preoperative imaging and intervention, leading to an extensive degradation of spatial guidance. This presentation illustrates solutions for these fundamental problems that occur during 3D-navigated liver interventions, including patient immobilisation and repositioning, respiratory triggering and intraoperative imaging. In addition, examples of CT/MRI/SPECT/PET-guided stereotactic and robotic liver tumour biopsies and ablations (RFA, MWA, IRE) are demonstrated.

Learning Objectives:

1. To learn about new image-guided therapies using molecular and functional information.

2. To become familiar with MI methods used for guiding interventions.

3. To understand new ablation techniques like electroporation.

Author Disclosure:

R. Bale: Equipment Support Recipient; iSys has supported the Section of Microinvasive Therapy with testing equipment. Patent Holder; RB is a (co-) inventor of the Atlas aiming device and the BodyFix immobilisation device and a (co-)shareholder in its financial returns.

\section{A-261 11:00}

MR-guided focused ultrasound in drug delivery and gene expression

C. Moonen; Utrecht/NL (C.Moonen@umcutrecht.nl)

The goal of ultrasound-triggered, image-guided drug delivery is to increase the therapeutic index and decrease adverse effects of drugs. The bio-effects of focused ultrasound can lead to local tissue heating, cavitation, and radiation force, which can be used for 1) local drug release from nanocarriers circulating in the blood, 2) increased extravasation and cellular uptake of drugs and/or carriers, and 3) enhanced diffusivity of drugs. Thermo-sensitive liposomes have been suggested for local drug release in combination with local hyperthermia more than 25 years ago. Microbubbles may be designed to enhance cavitation effects. Real-time imaging methods, such as magnetic resonance, as well as optical and ultrasound imaging have led to novel insights and methods for ultrasound-triggered drug delivery. Image guidance of ultrasound can be used for: 1) target identification and characterisation; 2) spatio-temporal guidance of actions to release or activate the drugs and/or permeabilise membranes; 3) evaluation of biodistribution, PKPD; 4) physiological read-outs to evaluate the therapeutic efficacy. Liposomes may carry both hydrophilic and hydrophobic drugs in their aqueous interior and lipid bilayer membrane, respectively. The circulation half-life may be increased by incorporating polyethylene glycol (PEG)-lipids in the bilayer. Recent publications have shown that ultrasound-triggered delivery is feasible. Realtime imaging methods, such as magnetic resonance, optical and ultrasound imaging may lead to novel insights and methods for ultrasound-triggered drug delivery.

Learning Objectives:

1. To learn about the basic principle of MR-guided focused ultrasound and drug delivery.

2. To appreciate the potential clinical applications of these methods.

3. To understand how MR-guided focused ultrasound can monitor gene expression.

Author Disclosure:

C. Moonen: Research/Grant Support; Philips Healthcare, Celsion Corp.

\section{A-262 11:30}

Intraoperative imaging: a new dimension of imaging for radiology

V. Ntziachristos; Munich/DE

Up to now, the success of surgical tumour resection has always been limited by the surgeon's vision. The human eye is not an accurate detector of small cancer cell clusters and it cannot accurately differentiate cancerous from healthy tissues due to lack of "visible" contrast. By combining a fluorescent probe targeting the folate receptor and a multi-spectral real-time fluorescence camera to observe the operating field, we found that, using fluorescence molecular imaging, 3-fold more lesions can be identified compared to conventional surgery. These first in-human results point to molecular-based clinical decision-making in surgical and endoscopic procedures as a paradigm shift over decision-making based on human vision. The talk describes current progress with instruments, methods and applications in the field of intraoperative imaging. Pre-clinical and clinical results are presented and the advantages and limitations of the method as well as future directions will be discussed. The emerging field of multispectral optoacoustic tomography (MSOT) is also outlined

Learning Objectives:

1. To learn that intraoperative molecular imaging has the potential to shape the future of surgical procedures.

2. To understand that this techniques can improve the sensitivity, accuracy and contrast of tumour delineation and lymph node interrogation.

3. To learn about further advances in the development and clinical translation of intraoperative imaging modalities.

$12: 15-12: 45$

Room A

Plenary Session

\section{HL 2}

\section{Wilhelm Conrad Röntgen Honorary Lecture}

Presiding:

V.E. Sinitsyn; Moscow/RU

\section{A-263 12:15}

Mysteries of the human brain unveiled: imaging of white matter microstructure and neuroplasticity

P.M. Parizel; Antwerp/BE (paul.parizel@uantwerpen.be)

The human brain remains one of nature's great mysteries, and is considered science's final frontier. This greyish lump of tissue with a weight of $1.5 \mathrm{~kg}$ contains some 100 billion neurons, each of which are connected to thousands of other nerve cells in an intricate network of white matter fibers. In the previous century, the brain was perceived as a fixed three-dimensional landscape, with brain functions confined to certain locations (e.g. motor and sensory cortex, visual cortex, auditory cortex). This concept of neurolocalisationism was in line with the philosophy that there should be "a place for everything, and everything should be in its place". Advanced quantitative MRI techniques for acquisition and data analyis (fMRI, DWI, DTI, VBM), help to unravel white matter connectivity of the human brain, and are able to show that the brain can modify its structure and function in response to changing circumstances (such as learning, memory, hormones). This process, which is known as neuroplasticity, occurs at different levels and different time scales. For example, in response to injury, changes may occur at cellular leve as well as on a larger scale with cortical remapping. Some processes may take months or years (e.g. physical therapy and training) whereas some forms of neuroplasticity happen within hours or days (e.g. changes in brain volume and connectivity during the female menstrual cycle, which have been linked to behavioral changes). In summary, quantitative MRI techniques help to unveil the mysteries of the human brain and have opened exciting new fields of active research such as white matter connectivity and neuroplasticity. Author Disclosure:

P.M. Parizel: Board Member; icoMetrix. Founder; icoMetrix. 
$12: 30-13: 30$

Room E2

The Beauty of Basic Knowledge: Skeletal Radiology

MC 26C

\section{Inflammatory/infectious disorders}

Moderator:

V.N. Cassar-Pullicino; Oswestry/UK

A-264 12:30

Inflammatory/infectious disorders

V.N. Cassar-Pullicino; Oswestry/UK (Victor.Pullicino@rjah.nhs.uk)

There are a host of inflammatory and infective insults that can manifest focally or diffusely within the musculoskeletal system. The appearances of the underlying pathological processes in both the soft tissues and skeleton cover a very wide imaging spectrum. The appearances vary, depending on the timing and degree of inflammatory insult and the host response in the involved tissues. The approach of this lecture will cover the imaging manifestations using all modalities covering radiography, ultrasound, CT, scintigraphy and magnetic resonance imaging. The basic knowledge that is required will be displayed in 4 major musculoskeletal categories covering disorders involving the Soft Tissues, Joints, Bones and Entheses. The imaging manifestations will also be linked with the evolution of the pathological processes covering acute, sub-acute and chronic stages of the inflammatory/infective disorders. By the end of the session the audience should have a clear understanding in making best use of the imaging modalities in the correct diagnosis of a wide variety of inflammatory and infective conditions that can affect the musculoskeletal system.

Learning Objectives:

1. To learn about the pathomechanisms involved in inflammatory and infections disorders.

2. To understand the imaging appearances and their differential diagnosis in the acute, sub-acute and chronic phases of infection.

3. To become familiar with the spectrum of imaging features of inflammatory disorders in the axial and peripheral skeleton.

$12: 30-13: 30$

Room F1

The Beauty of Basic Knowledge: Interventional Radiology

\section{25C}

\section{Basics of arterial PTA/stent}

Moderator:

M. Bezzi; Rome/IT

\section{A-265 12:30}

\section{Basics of arterial PTA/stent}

R. Uberoi; Oxford/UK (raman.uberoi@orh.nhs.uk)

Arterial PTA and stenting is firmly established as primary treatment in the management of athero-occlusive disease, particularly of peripheral limb arterial disease (PAD). Patients are selected on the basis of symptoms such as short distance claudication or critical limb ischaemia for lower limb disease or appropriate ischaemic symptoms in other territories in the renal and mesenteric territories and head and neck vessels. Commonly used grading systems such as the Fontaine or Rutherford scores can be useful in patient selection. Where treatment is required, noninvasive imaging is utilised to look for the site, severity and extent of arterial disease. Most centers use duplex imaging as the primary imaging modality; MRA or CTA are often used with a higher sensitivity and specificity; and invasive angiography is reserved for problem solving or immediately prior to intervention. The TASCII classification has been devised to select patients for either endovascular or surgical intervention. However, most centres will adopt an endovascular approach first. All patients should be on good medical therapy, i.e. aspirin and statins, with good blood pressure and diabetic control where appropriate. When carrying out interventions, an access sheath is used, peri-procedural heparin administered and lesions crossed using selective catheters and commonly hydrophilic guidewires. Subintimal crossing of occlusions can be much simpler than trying to cross lesions luminally. Balloon angioplasty and or stenting may be applied differently depending on the site and extent of the lesions. The evidence for drug-eluting technologies has also been growing with improved patency rates compared to standard balloon angioplasty.
Learning Objectives:

1. To understand the clinical principles of patient selection and preprocedural imaging.

2. To understand how to gain safe access for PTA, and to learn about the range of techniques available for safely crossing arterial stenosis and occlusions.

3. To become familiar with the indications for arterial stenting and correct balloon, or stent, selection.

14:00 - 15:30

Room B

\section{EFRS meets Russia}

EM 5

\section{The role of the radiographer in image} acquisition and processing

Moderators:

S. Geers Van Gemeren; Utrecht/NL

S. Mamedova; Moscow/RU

A-266 14:00

Introduction

S. Geers-van Gemeren 1 , S. Mamedova ${ }^{2} ;{ }^{1}$ Utrecht/NL, ${ }^{2}$ Moscow/RU

During the EFRS meets Russia session, radiographers have the opportunity to get information about the role and education of radiographers in the different European countries. The radiographer is a health-care team member who interacts with other professionals in the primary and secondary health-care environment to provide an optimum diagnostic or therapeutic outcome. Radiographer education therefore requires that the curriculum covers a wide range of scientific, medical, pathological, sociological, ethical and technical subjects together with the development of appropriate clinical skills. The curriculum should also include the development of research and audit skills to ensure the constant improvement of service quality for the benefit of service users. The session will include a panel discussion about the role of the radiographer in image acquisition and processing.

Session Objectives:

1. To understand the background of education and training in Russia.

2. To gain awareness of the importance of education and training in Europe.

3. To understand the common issues among radiographers in Europe.

\section{A-267 14:10}

MRI safety: the radiographers' role

V. Labutin; Chelyabinsk/RU (vlad_lab@mail.ru)

The tasks of the assistant to the radiologist are: filling of the protocol of research, survey of the patient, instructing the patient according to safety measures, positioning the patient, placement of coils of strengthening, beginning of magnetic and resonant research - the research is approved by the doctor, introduction of contrast substance (in the presence of the certificate of the procedural sister) and supervision over the patient during MR research Furthermore, the other tasks are briefing on patient safety, excluding introduction of the patient to metal objects on MR tomography, explaining the procedure of the study, informing about the duration of the study, wearing noise-cancelling headphones, warning about possible vibration and loud sounds, and providing a globular button to call the nurse. Learning Objectives:

1. To understand the safety aspects of MRI practice.

2. To understand possible safety measures in MRI practice.

3. To gain awareness of the radiographers' role in MRI safety.

\section{A-268 14:25}

Contribution of radiographer to success of coronary MDCT

E. Hichina; Moscow/RU (lenahichina@mail.ru)

Today coronary CT-angiography has become a well-established and proven diagnostic modality. Quality of coronary and cardiac CTA datasets has tremendous significance for correct diagnosis. A radiographer has a pivotal role in performance of coronary CTA. Coronary CTA is one of the most challenging examinations for radiographers because even a small mistake at any stage of examination may ruin the whole study. Before the scanning procedure, the radiographer performs a check for possible contraindications to contrast media injection (history of adverse reactions to contrast media and impairment of renal function are the most important ones). The radiographer checks the patient's heart rhythm and takes part in administration of betablockers for control of the heart rate. A detailed knowledge of different scanning modalities of CT-machine is a must. Radiation exposure to the patient from coronary CTA examinations has been one of the major public 
concerns. This is why the radiographer should be aware of different approaches to the performance of low-dose CTA (prospective gating, tube current modulation, high-pitch scanning) according to patient body composition. The radiographer should have knowledge of when and how to use radiation dose control in an appropriate way. Another big issue is injection protocols. Selection of optimal bolus timing and iodine load results in highquality CTA images. The radiographer has to know the basics of CT image reconstruction and interpretation in order to perform different types of reconstructions from the raw data according to the radiologist's requests and be able to recognise image artifacts. Clinical examples of the radiographer's role for performance of coronary CTA will be given.

Learning Objectives:

1. To learn the importance of patient selection and preparation in achieving high quality scans.

2. To understand the practical techniques available for performing of low-dose CTA, injection protocols.

3. To learn how to recognise image artifacts.

\section{A-269 14:40}

Interlude: Russian art and culture

I. Arkhipova, V. Sinitsyn, E. Mershina; Moscow/RU (iarkhipova77@mail.ru)

Every country is famous for its architecture, history, literature, music, museums, etc. However, there are some distinctive features for Russia. "Russia is a riddle wrapped in a mystery inside an enigma." These words by the famous British statesman Winston Churchill aptly throws light on the pulsating art and culture of Russia. The different aspects of Russian art and culture find its best expression in the rich heritage. Just feeling the nature and boundless expanses of the country can understand the mysterious Russian soul, works of art and real masterpieces.

\section{A-270 14:50}

Cardiac MDCT in children with congenital heart diseases

V. Bereznitskiy, K. Serkova; Moscow/RU (vsber@mail.ru)

In CT studies of children with congenital heart diseases, it is mandatory to keep the x-ray dose, amount of contrast agent and study time as low and as short as possible and simultaneously get the best diagnostic image quality. How can we reduce the $x$-ray dose? By minimising the volume coverage, reducing the voltage, minimising the effect of "overranging", use of $x$-ray protective equipment, use of tube current modulation technique, use of iterative reconstruction technique. How can we reduce the amount of contrast agent by volume? By covering of only necessary anatomic volume, minimising scanning time, use of adequate speed injection in $\mathrm{ml} / \mathrm{sec}$, which depends on catheter position and size, blood flow and anatomic structures. How can we reduce the amount of iodine concentration in the contrast agent? By lowering the voltage we may reduce the iodine concentration and get higher absorption CT values $(\mathrm{HU})$. How can we reduce the time of cardiac CT studies? By preparing the study protocol ahead, preparing all necessary equipment in the CT room, warming up of the contrast agent before the study. What is the radiographer's role in cardiac CT studies? To take care along with the responsible radiologist the above-mentioned needs, to take care of adequate room and instrument temperature, to take care that only absolutely necessary people with adequate $x$-ray protection are in the study room, for performing the necessary image post-processing, archiving and distribution of studies.

Learning Objectives:

1. To understand MDCT techniques in cardiac imaging.

2. To learn about the use of MDCT for children with congenital heart disease.

3. To gain awareness of the radiographers' role in cardiac MDCT.

\section{A-271 15:05}

MDCT in polytrauma patients: the radiographers' role

A. Tarasenko, E. lasakova; Krasnodar/RU

The purpose is to evaluate the quality of imaging in forced position of patients with anesthetic support. The role of laboratory technician in CT post processing. We analysed images of unconscious patients on artificial lung ventilation on 24- and 64-slice computer homographs GE and Siemens and monitored them through the infusion. During investigation the patients were in forced positions, as a result they got artifacts from upper extremities on the abdominal cavity. We analysed 157 patients - hands under the butt, 135 patients - hands on the chest (abdomen), 57 patients - arms along the body. According to the images the smallest number of artifacts was identified when the patient was on the table with hands under the butt. Different variations of the patient's hands position and the problem of choice of this position will be offered for researching. The reconstruction the laboratory assistant can execute and also the results of tests will be presented. By order of the doctor the laboratory technician builds $3 D$ and $4 D$ reconstructions of fractured ribs, pelvis, facial skeleton, sagittal and coronary reconstructions of spine in case of its fracture.

Learning Objectives:

1. To understand MDCT techniques.

2. To learn about the use of MDCT for polytrauma patients.

3. To gain awareness of the radiographers' role in image acquisition.

Panel discussion:

The radiographers' role in image acquisition and processing

$15: 20$

\begin{tabular}{ll}
\hline $14: 00-15: 30$ & Room $P$
\end{tabular}

\section{MITIGATE Session}

\section{Molecular imaging and targeted-image guided therapy in gastrointestinal stromal tumours}

Moderators:

S. Aime; Turin/IT

S.O. Schönberg; Mannheim/DE

14:00

Introduction

S. $\underline{\text { Aime }}^{1}$, S.O. Schönberg ${ }^{2} ;{ }^{1}$ Turin/IT, ${ }^{2}$ Mannheim/DE

A-272 14:10

Introduction to the MITIGATE concept

S.O. Schönberg;

Mannheim/DE (stefan.schoenberg@rad.ma.uni-heidelberg.de)

Gastrointestinal stromal tumours (GIST) are a rare disease that metastasises in up to $85 \%$ of patients with subsequent median progression-free survival (PFS) of around 30 months. Tumours are characterised by activating mutations in the KIT or the PDGFRA gene and treatment is mainly based on tyrosine kinase inhibitors designed to block mutated receptors. However, drug resistance is often based on mutations changing the conformity of the receptor, leaving little effective therapeutic options. To date, second line chemotherapy offers a median PFS of 6-9 months and external beam radiotherapy is limited by organs at risk close to the tumour. Alternative approaches like endoradiotherapy or minimally-invasive ablation techniques are effective for local control but are inconsistently used and are not tailored to the individual patient's type of disease. To address these issues, the MITIGATE project proposes a closed-loop personalised treatment concept combining endoscopic-assisted tissue sampling, inline biotechnology and targeted molecular PET imaging probe development combined with minimally-invasive treatment monitored by new MR imaging techniques. A consistent value chain across European research institutes and SMEs will be established for mass spectrometry of tumours, linkage of radiochemical molecular imaging probes, design of new animal models and targeted therapeutic radiopharmaceuticals. This closed-loop platform will minimise fragmentation of treatment approaches by a coherent molecular-based multimodality concept, thus providing new treatment options. The MITIGATE platform can be expanded to further patient cohorts with oligometastatic diseases such as other sarcomas or renal cell carcinoma.

Author Disclosure:

S.O. Schönberg: Research/Grant Support; EU-FP7 Project (2013-2017).

\section{A-273 14:25}

Molecular imaging and targeted endoradiotherapy: key methods to overcome TKI-resistancies?

B. Wängler; Mannheim/DE (Bjoern.Waengler@medma.uni-heidelberg.de)

Gastrointestinal stromal tumours (GIST) are characterised by highly specific genetic mutations. Several specific targets are overexpressed in a majority of the tumours. On the other hand, secondary resistancies limit the usability of highly specific chemotherapeutics such as Imatinib. Instead of applying cascades of alternative TKI inhibitors in order to find an efficient one, quantitative molecular imaging and targeted Endoradiotherapy could be considered. For the detection of GIST by imaging, $18 \mathrm{~F}$ fluorodeoxyglucose (FDG)-PET is widely accepted to visualise the tumour burden. However, as FDG gives no molecular information of potential targets for treatment, new radiopharmaceuticals have to be developed to visualise and quantify other target-structures expressed by GIST that would be able to be addressed. This molecular information can be used both for designing a conventional treatment or an advanced strategy: i.e. targeted endoradiopharmaceuticals. Compared to conventional chemotherapy this method has the great advantage that not only tumour cells expressing the targeting vector are affected but also tumour cells within the range of the radiation of the decaying radiopharmaceutical. This method is used with great success in the case of neuroendocrine tumours using labeled octreotate 
derivatives (a small cyclic peptide binding to the somatostatin-receptor) and non-Hodgkin's lymphoma applying Y-90 labeled Zevalin (a monoclonal antibody binding to the CD20 antigen). The success of these methods relies on the crossfire-effect, meaning that a certain margin of tissue surrounding the cell targeted by the specifically accumulating endoradiopharmaceuticals is destroyed as well, depending on the penetration range of the emitted radiation. Thus, the development of new radiopharmaceuticals for the specific diagnosis and treatment of GIST would strongly improve the outcome of the tumour patient.

\section{A-274 14:40}

Stereotactic radiofrequency ablation of liver tumours: does improved targeting increase tumour response?

R. Bale, G. Widmann, P. Schullian, M. Haidu, W.R. Jaschke; Innsbruck/AT (werner.jaschke@i-med.ac.at)

The purpose is to describe stereotactic radiofrequency ablation (SRFA) and to review its inital results in the treatment of patients with primary and secondary liver tumours. One retrospective review includes 90 consecutive patients with 106 SRFA sessions for the treatment of 72 hepatocellular carcinomas (HCC) and 105 metastases (1). In two other studies the outcome after SRFA of intrahepatic cholangiocellular carcinomas (ICC) (2) and colorectal liver metastases (CRLM) (3) was evaluated. The overall technique effectiveness was $95.5 \%$ with a local recurrence at 1 year of $2.9 \%$. Technique effectiveness was not significantly different for lesions $<3 \mathrm{~cm}(95.9 \%)$ and $3-5 \mathrm{~cm}(100 \%)$ After treatment of 17 inoperable consecutive patients with 52 ICCs a median overall survival of 60 months was achieved. A median OS of 58 months and OS rates of $92 \%, 66 \%$ and $48 \%$ at 1,3 and 5 years in patients with resectable CRLM were achieved. Tumour size did not affect OS and DFS. RFA probes can be precisely planned, positioned and distributed by means of stereotaxy. Especially in large liver tumours the local recurrence rate can be greatly reduced by precise stereotactic placement of multiple radiofrequency probes. These improved local control rates result in better long-term survival rates. Author Disclosure:

R. Bale: Shareholder; Coshareholder Atlas aiming device. Other; Coinventor Atlas aiming device.

\section{A-275 14:55}

Functional and molecular imaging in tumour therapy response assessment

S. Aime; Turin/IT (silvio.aime@unito.it)

In the MITIGATE project further development of currently used MRI and CT techniques will be tackled in order to obtain functional and metabolic information which are mandatory to detect early response to the targeted radiopharmaceutical approach. New Gd-based agents are under scrutiny in order to improve DCE-MRI procedures and attain more precise information on the tumour microvascularity level as well on the vessel permeability and the assessment of extracellular $\mathrm{pH}$ and MMP activity. The X-nuclei MRI $\left({ }^{23} \mathrm{Na}\right.$ sodium) is a non-invasive imaging technique, which enables the measurement of the tissue sodium concentration (TSC) in humans. The direct link of the TSC with the tissue integrity and viability provides a promising approach for monitoring tumour tissue, and could also help to assess cytoxicity and cell death by means of breakdown of the sodium-potassium-pump. However ${ }^{23} \mathrm{Na}$ MRI is challenging because of the low in vivo abundance, the fast transversal relaxation rate and the low gyromagnetic ratio of the ${ }^{23} \mathrm{Na}$ ions in human tissue. New MR measuring sequences for X-nuclei MRI will be developed to establish a standardised TSC measurement method within the EU for better comparability. Furthermore, the X-nuclei MR data will be reconstructed, scaled and fused with further imaging modalities.

Discussion

15:10

$16: 00-17: 30$

Room A

Interactive Teaching Session

$E^{3} 1120$

\section{What to look for after treatment of lung cancer}

\section{A-276 16:00}

A. Imaging of surgically treated lung cancer

C. Heussel; Heidelberg/DE

Chest x-ray, CT, and MRI are shown to demonstrate early, intermediate, and late findings after lung surgery and during follow-up. Complications frequently seen on intensive care unit, "lines and wires", typical change of the chest and caveats are shown, "Problem zones " of imaging after surgery and hints for long-term follow-up are presented.

Learning Objectives:

1. To appreciate the complications after surgery for lung cancer.

2. To understand the anatomic changes which occur after surgery for lung cancer.

Author Disclosure:

C. Heussel: Consultant; Schering-Plough, Pfizer, Basilea, Boehringer Ingelheim, Novartis, Roche, Astellas, Gilead, MSD, Lilly, Intermune, Fresenius. Patent Holder; Methods and Device For Representing the Microstructure of the Lungs. IPC8 Class: AA61B5055 FI, PAN: 20080208038, Inventors: W Schreiber, U. Wolf, A.W. Scholz, C.P. Heussel. Research/Grant Support; Siemens, Pfizer, MeVis. Shareholder; Stada, GSK. Speaker; Gilead, Essex Schering-Plough, AstraZeneca, Lilly, Roche, MSD, Pfizer, Bracco, MEDA Pharma, Intermune, Chiesi, Siemens, Covidien, Pierre Fabre, Boehringer Ingelheim, Grifols, Novartis.

\section{A-277 16:45}

$B$. Imaging of non-surgical treatment of lung cancer

B. Ghaye; Brussels/BE (benoit.ghaye@uclouvain.be)

Lung cancer is the leading cause of death related to cancer. Most patients are inoperable as they present with advanced stage disease or even a localised tumour associated with poor general condition, limited cardiopulmonary function or a too high surgical risk. According to the stage of the disease, chemotherapy, radiotherapy and percutaneous ablation therapies are the current therapeutic options for inoperable patients. It is important that radiologists are familiar with the various response and complication imaging patterns related to each of those treatments. The timeline modifications after radiation therapy and percutaneous ablation and the diagnostic management of drug-induced lung disease will be reviewed. This presentation will summarise the current evidence and how to detect early recurrences after those treatments.

Learning Objectives:

1. To learn about changes after radiotherapy.

2. To learn about sequela after percutaneous ablation of lung tumours

3. To understand changes after chemotherapy of lung cancer

16:00 - 17:30

Room B

ESR meets ESC (European Society of Cardiology)

EM 3

\section{The role of imaging in the cardiac patient}

Welcome by the ESR President

G. Frija; Paris/FR

Presiding:

V.E. Sinitsyn; Moscow/RU

P.E. Vardas; Iraklion/GR

\section{A-278 16:00}

Introduction

P.E. Vardas; Iraklion/GR (cardio@med.uoc.gr)

Cardiovascular medicine owes much of its spectacular development to the parallel evolution of imaging techniques in the last thirty years. The complex contemporary techniques in interventional cardiology, electrophysiology, and cardiac surgery require advanced imaging modalities. In current times, a wide spectrum of imaging techniques - such as fluoroscopy, MRI, non-contact mapping, echo techniques - is performed in organised cardiovascular labs while computed tomography and nuclear techniques are provided by the collaborative departments of radiology and nuclear medicine. It is indeed the collaborative spirit among specialists that bring about the results patients desire.

Session Objective:

1. To better understand the needs of clinicians and what imaging can provide in different clinical settings.

Author Disclosure:

P.E. Vardas: Advisory Board; Menarini, Bayer, Servier. Board Member; ESC Employee; Iraklion University Hospital. Research/Grant Support; Medtronic, Boehringer. Speaker; Menarini, Bayer, Servier, Boehringer, Bristol.

\section{A-279 16:05}

Assessing myocardial viability

M. Lombardi; San Donato Milanese/IT

(massimo.lombardi@grupposandonato.it)

Cardiac magnetic resonance (CMR) is a very versatile imaging technique for the assessment of patients with left ventricular dysfunction and has become a 
central method for characterising the etiology of the dysfunction. Indeed, wall thinning less than $6 \mathrm{~mm}$ and aneurysmal motion are morphological signs of transmural necrosis. Furthermore, a low dosage of dobutamine (10-20 $\mathrm{microg} / \mathrm{min}$ ) is used to detect functional reserve in the case of viable tissue. A further option offered by CMR is late contrast enhancement (LE) imaging, which allows identifying the presence of fibrotic tissue as well as necrotic infarcted myocardium. The sensitivity and specificity of late hyperintensity is, respectively, 0.86 and 0.94 . Similar results can be obtained with $99 \mathrm{mTC}$ tetrofosmina G SPECT. There is a relation between transmural extension of hyperenhancement and the recovery of contractile function after coronary revascularisation. The chance of recovery decreases progressively with the transmural extension of late hyperintensity, since the average value of the latter is $10 \pm 7 \%$ in the segments that recover and $47 \pm 14 \%$ in the segments that do not recover contractile function. CMR was also shown to be able to predict response to cardiac resynchronisation Therapy differentiating between nonischemic and ischaemic cardiomyopathy LE-CMR may allow to precisely delineate the presence and the location of fibrosis, avoiding the placement left ventricular leads in necrotic areas and in particular when the scar is located in the posterolateral area.

Learning Objectives:

1. To learn how MRI can help in assessing patients with CAD.

2. To become familiar with different protocols in CMR, and learn how they can be applied in clinical practice while assessing viability.

\section{A-280 16:23}

\section{Assessing acute chest pain}

R. Rosenhek; Vienna/AT

"No abstract submitted"

\section{Learning Objectives:}

1. To learn what CT can do in the patient with acute chest pain

2. To appreciate the clinical evidence for different applications of CT in this setting.

\section{A-281 16:41}

\section{Assessing valvular heart disease}

S. Muzzarelli ${ }^{1}$, J. Schwitter ${ }^{2} ;{ }^{1}$ Lugano/ $\mathrm{CH},{ }^{2}$ Lausanne/CH

(stefano.muzzarelli@cardiocentro.org)

At the beginning of the presentation, a short overview will be given on the relevance and prevalence of valvular heart diseases with special focus on Europe and the data collected in the EuroHeart Survey. Also, the aetiologies of the various valve diseases will be briefly mentioned. In the second part, special attention will be given to aortic stenosis (AS) and mitral regurgitation (MR), as these two entities are responsible for up to $60 \%$ of all valvular heart diseases. Echocardiography is well accepted as the backbone examination of any valvular heart disease evaluation. Echocardiographic criteria for severe AS and MR are reviewed as well as their importance for the indication of valve repair or valve replacement. In the third part, we will focus on newer "cross-sectional" imaging technologies such as cardiac MR and CT. In particular, the strengths of cardiac MR in the assessment of regurgitant lesions will be demonstrated. In addition, the value of cardiac MR for the pre-interventional evaluation of congenital heart diseases will be discussed. Similarly, the crucial role of cardiac CT in the workup for transaortic valve implantation (TAVI) will be illustrated. Finally, the yield of these novel techniques in the context of combined valvular and ischaemic heart disease will shortly be reviewed.

Learning Objectives:

1. To better understand the role of cardiac MRI in valvular heart disease.

2. To appreciate the added value of MRI vs other imaging modailities.

\section{A-282 16:59}

\section{Assessing interventions}

V. Delgado; Leiden/NL (v.delgado@lumc.nl)

Cardiac resynchronisation therapy (CRT) and transcatheter valve heart (THV) interventions have been important therapeutic breakthroughs for heart failure patients and patients with valvular heart disease and contraindications for surgery. Patient selection is crucial to optimise procedural outcomes and therapeutic efficacy. Multimodality imaging plays a central role in patient selection and procedural strategy planning. Assessment of left ventricular dyssynchrony, cardiac venous anatomy and extent and location of myocardial scar are key to identify heart failure patients who will respond to CRT. Stepwise use of non-invasive imaging modalities, including echocardiography, nuclear imaging, magnetic resonance imaging and multidetector row computed tomography (MDCT), has been common practice to select patients for CRT. Recent technological advances have allowed integration or fusion of imaging modalities to create 3-dimensional models that provide relevant information. Multimodality imaging is also crucial to optimise the results of THV interventions. Detailed anatomical and geometrical evaluation of the mitral valve with current 3 -dimensional imaging techniques has permitted better selection of patient candidates for transcatheter edge-to-edge mitral valve repair and accurate procedural guidance. For transcatheter aortic valve implantation, accurate measurement of the aortic annulus with 3-dimensional echocardiography or MDCT allows us accurate selection of the prosthesis size to minimise the risk of complications, and evaluation of the procedural access (transarterial or transapical) with MDCT permits accurate procedural strategy planning. The learning objectives of this session include: providing an overview of current imaging modalities to select patients for CRT and THV interventions and how to use them in clinical practice.

Learning Objectives:

1. To understand the role of the multimodality approach to cardiac interventions.

2. To understand how imaging helps in selecting, monitoring and following up such patients

Author Disclosure:

V. Delgado: Consultant; medtronic and St.Jude Medical.

Panel discussion $\quad 17: 17$

$16: 00-17: 30$

Room C

Modern Imaging of the GI Tract

CC 1121

Perianal fistula disease:

all you need to know

Moderator:

D. Weishaupt; Zurich/CH

A-283 16:00

A. Perianal anatomy and imaging techniques

K. Horsthuis; Amsterdam/NL (k.horsthuis@amc.uva.nl)

The inner layer of the anal canal consists of squamous and columnar epithelium with the transitional zone at the dentate line. The muscular component of the anal sphincter consists of an inner layer of circular smooth muscle (internal sphincter), extending downwards from the rectum, and an outer striated muscular layer extending downwards from the levator ani muscle, comprising the puborectalis muscle and the external sphincter. Between these layers is the fat-containing intersphincteric space, including the continuation of smooth-muscle fibers of the longitudinal muscle of the rectum. Outside the anal sphincter is the fat-containing ischioanal space. Magnetic resonance imaging (MRI) and endoscopic ultrasonography have become the mainstay for preoperative imaging of perianal fistulas. For complex tracts, MRI seems preferable. MRI can be performed using an endoluminal coil or a phased-array surface coil. A state-of-the-art imaging protocol should include T2 TSE sequences in three orthogonal planes, with the axial and coronal sequences angulated at the anal canal. Addition of a fat-saturated T2 TSE sequence is recommended for optimal conspicuity of inflammatory changes and post-contrast T1-weighted imaging can also be helpful. Intersphincteric infection is the principal feature of perianal fistulas; this is generally not found in other conditions. Veins can be mistaken for fistulas, but in contrast to fistulas, veins usually are thin-walled, tortuous, symmetric structures. A pilonidal sinus may resemble a fistula, but absence of extension to the intersphincteric space helps one to discriminate between them. Haemorrhoids and anal tags may resemble small submucosal fluid collections, but are easily diagnosed at clinical examination.

Learning Objectives:

1. To understand anal canal anatomy.

2. To become familiar with state-of-the-art protocols for imaging the anal canal. 3. To learn about normal variants which mimic anal disease.

A-284 16:20

B. Perianal fistula disease: the basics

S. Halligan; London/UK (s.halligan@ucl.ac.uk)

This lecture will describe the pathogenesis of fistula-in-ano, with a focus on cryptoglandular disease, so that the radiologist can understand how the various classifications of fistulas arise. The Park's classification for fistula-inano will be described with reference to cryptoglandular disease and other aetiologies. The surgical questions that need to be answered by imaging will be presented, followed by a description of what the radiologists need to include in their report for it to be clinically useful. The role of anal ultrasound and MRI for preoperative fistula imaging will be described, with explanation as to why the latter is fundamental to modern management of the disease. 


\section{Postgraduate Educational Programme}

Learning Objectives:

1. To understand the pathophysiology and classification of perianal fistula disease.

2. To become familiar with the role of US and MRI in assessing fistula disease. 3. To learn about the basic reporting of fistula disease.

\section{A-285 16:40}

C. Perianal fistula disease: advanced

F. Maccioni; Rome/IT (francesca.maccioni@uniroma1.it)

Perianal fistulising disease develops in approximately half of the adult and paediatric patients with Crohn's disease (CD), with a relevant impact on their quality of life. In patients with $C D$, particularly those with severe fistulising ileal or colo-rectal disease, complex ramified anal fistulas associated with abscesses (Parks' classification type 3-4 or S.James Hospital classification type 4-5) are more frequently observed than in other patients. Perianal disease thus represents a severe complication of $C D$, which may change disease management, by requiring specific pharmacological and/or surgical treatments A non-responsive disease eventually may lead to total proctectomy with ileostomy. MRI represents the gold standard diagnostic modality, because it provides a comprehensive staging of both enteric and perianal disease, with high accuracy and without invasiveness. Moreover, MRI is the ideal tool for monitoring disease response to therapy, being able to detect the inflammatory activity of perianal fistulas with high accuracy. So far, several clinical indexes have been proposed to measure fistula's activity, including the Perianal Disease Activity Index (PDAI). Similarly, several MRI classifications of fistula's activity, based either on T2-weighted or T1-weighted contrast-enhanced imaging, have been proposed, although a widely accepted imaging severity score is not available yet. Several cases of complex perianal fistulising CD of increasing severity will be shown, with anatomical and clinical correlations, staging and activity description, particularly in patient follow-up and treatment monitoring. Finally, differential diagnoses with other benign conditions which may affect the anal canal, including inflammatory infective diseases, hydradenitis and pilonidal disease will be discussed.

Learning Objectives:

1. To lean about complex perianal fistula disease, particularly in Crohn's disease.

2. To understand the role of imaging in patient follow-up and treatment monitoring

3. To learn about benign inflammatory conditions which may affect the anal canal, including hydradenitis and pilonidal disease and how they are differentiated from perianal fistulae.

\section{A-286 17:00}

\section{Interactive case discussion}

D. Weishaupt; Zurich/CH (dominik.weishaupt@triemli.zuerich.ch)

The interactive case discussion will highlight clinically relevant aspects of perianal fistula disease in selected clinical cases. A patient case of each of the subtopics of the categorical course is presented.

Learning Objectives:

1. To become familiar with typical cases demonstrating the crucial role of imaging perianal fistula disease.

2. To learn about typical imaging findings.

3. To be aware of potential pitfalls in imaging perianal fistula disease

$16: 00-17: 30$

Room D

Chest

\section{RC 1104}

\section{Patterns in chest radiology: are there subtype patterns of ground glass opacity (GGO)?}

\section{A-287 16:00}

\section{Chairman's introduction}

A. Oikonomou; Toronto, ON/CA (anastasia.oikonomou@sunnybrook.ca)

Ground-glass opacity (GGO) is characterised on HRCT by the presence of a hazy increase in lung opacity that does not cause obscuration of underlying bronchial and vascular margins. Although a very common finding, it also constitutes a very nonspecific term since it can be seen in a variety of different intraalveolar and interstitial processes with different histology including inflammatory, infectious and neoplastic diseases that have a common physiologic mechanism: partial displacement of air. Ground-glass opacity may even be seen in normal processes such as poor ventilation in dependant lung areas and in expiration. Moreover, GGO can represent either an ongoing, active and potentially treatable disease or an irreversible process. To interpret correctly this highly nonspecific but very significant finding, it is crucial to attempt to further classify the different large main entities in which this radiological finding appears. Are there specific radiological and HRCT findings that can help us differentiate GGO in autoimmune-inflammatory conditions from infectious and neoplastic processes? Are there associated findings other than GGO, such as nodules, reticulation or focal disease, and distribution of findings that can narrow the differential diagnosis? Systematic evaluation of GGO and associated findings as well as integration with clinical information (acute, subacute or chronic symptoms) is essential in defining GGO subtypes to improve the radiological diagnosis.

Session Objectives:

1. To learn about the prevalence and low specificity of GGO

2. To appreciate the need for a definition of GGO subtypes to improve radiological diagnoses.

\section{A-288 16:05}

A. Ground-glass opacification: why do we see it and what does it mean? S.R. Desai; London/UK (sujal.desai@nhs.net)

Radiologists who regularly review high resolution CT (HRCT) should be aware of the range of patterns and, more importantly, their potential pathological meaning. A pattern of ground-glass opacification is one of the more common HRCT findings but, to the unwary, its interpretation can be problematic. An important underlying principle is that a ground-glass pattern may be caused by any process - physiological or pathological - which partially displaces air Physiological (i.e. non-disease-related) ground-glass opacification is perhaps most commonly seen in subjects who, for whatever reason (e.g. breathlessness, obesity), are unable to maintain or achieve a satisfactory inspiratory effort during scanning. A generally increased lung density (in contrast to adults) is also a feature in infants and young children simply because there are fewer alveoli in the developing lung. Finally, it is worth noting that intravenous contrast administration (presumably because of a relative but temporary increase in capillary blood volume causing partia displacement of air) can unpredictably increase lung density. Disease processes which lead to partial filling of the air spaces, thickening of the interstitium, partial collapsing of alveoli, and/or increased capillary blood volume will also manifest as a pattern of ground-glass opacification. In clinical practice, the recognised causes of ground-glass opacities on HRCT include pulmonary oedema (cardiogenic or otherwise), infections (e.g. pneumocystis jiroveci pneumonia) and some of the idiopathic interstitial pneumonias (e.g. non-specific interstitial pneumonia and respiratory bronchiolitis associated interstitial lung disease). The presentation will review and revise the causes of physiological and disease-related ground-glass opacification on HRCT. Learning Objectives:

1. To appreciate the different physiological conditions which cause GGO pattern.

2. To learn about the associations and reversibility of physiology-related GGO

\section{A-289 16:28}

B. Inflammatory and infectious GGO

K. Marten-Engelke; Göttingen/DE (kmarten@med.uni-goettingen.de)

Ground-glass opacity (GGO) is defined as increased attenuation of the lung parenchyma without obscuration of the pulmonary vascular markings on CT images. GGO may be the result of a variety of interstitial and alveolar infectious and noninfectious inflammatory diseases. As an imaging finding alone, GGO does not usually allow a specific diagnosis. GGO in inflammatory disorders is often present in the company of other interstitial or alveolar findings. However, the number of diseases that cause diffuse isolated GGO or GGO as the predominant finding is relatively small and can be prioritised with clinical information. The most common cause of diffuse isolated GGO in immunocompromised hosts are a variety of diffuse, opportunistic pneumonias, e.g. pneumocystis jiroveci pneumonia (PCP), cytomegalovirus pneumonia (CMV) or herpes simplex pneumonia (HSV), which constitute the first differential. Chronic onset disorders in immunocompetent patients include cellular nonspecific interstitial pneumonia (NSIP), subacute hypersensitivity pneumonitis (HP), organising pneumonia, air-space sarcoid, and drug-induced lung disease. In these disorders, ancillary findings such as an associated reticular pattern with traction bronchiectasis/bronchiolectasis (NSIP), mediastinal lymphadenopathy (sarcoidosis), superimposed nodularity or cysts or may help to refine the diagnosis. In patients with collagen vascular disorders, e.g. scleroderma, GGO secondary to pulmonary involvement needs to be differentiated from drug-induced lung disease. This refresher course will put GGO in the context of outpatients versus inpatients, the acuity of clinical symptoms, e.g. fever, cough and dyspnoea, signs of massive systemic inflammation, and the clinical situation such as inhalational history, pneumotoxic drug administration, immunocompromise, or bone marrow suppression. 


\section{Postgraduate Educational Programme}

Learning Objectives:

1. To learn more about the inflammatory conditions which cause GGO.

2. To appreciate the histopathological correlates of inflammatory and infectious GGO

3. To become familiar with GGO in autoimmune and infectious lung disease.

\section{A-290 16:51}

C. GGO in dysplasia and neoplasia

G.R. Ferretti; Grenoble/FR (GFerretti@chu-grenoble.fr)

In this course, we will review the dysplastic and neoplastic conditions that are associated with persistent GGO in the lung parenchyma. We will separate these conditions into those that are responsible for localised diseases such as single nodular GGO and those responsible for more extended diseases. Nodular GGO can be separated into pure or mixed or part-solid nodules. Pathology-radiology correlations show that nodular GGO are related to atypica adenomatous hyperplasia $(A A H)$, adenocarcinoma (ADC) in situ (AIS), minimally invasive ADC (MIA), and invasive ADC according to the new IASLC/ATS/ERS lung adenocarcinoma classification. Differential diagnosis includes exceptional metastases of angiosarcoma and melanoma as well as infection, inflammation and localised fibrosis. Diffuse GGO related to neoplastic conditions is rare and may be due to lepidic ADC (former advanced ADC with BAC component), diffuse large B-cell non-Hodgkin's lymphoma, intravascular lymphomatosis (IVL) or mucosa-associated lymphoid tissue (MALT) lymphoma. These neoplastic diseases should be differentiated from infectious and inflammatory causes of diffuse GGO. We will review the value of different morphological CT criteria to differentiate benign from malignant localised GGO such as the size, the morphology (round, oval, flat), presence of mixed vs. pure GGO, and the multiplicity of nodular shadows. Discussion will also include the changes that may occur within the nodule as well as the mean doubling time. The role of PET CT and transthoracic biopsy will be discussed. Finally, we will present the current recommendations regarding the management of nodular GGO

\section{Learning Objectives:}

1. To learn more about the dysplastic and neoplastic conditions causing GGO.

2. To appreciate the histopathological correlates of dysplastic and neoplastic

GGO

3. To understand how to estimate malignancy on the basis of GGO pattern.

Panel discussion:

How should we report and manage ground glass opacity?

$17: 14$

$16: 00-17: 30$

Room E1

\section{Musculoskeletal}

RC 1110

\section{The ankle and foot}

Moderator:

S.J. Ostlere; Oxford/UK

\section{A-291 16:00}

A. Ankle sprain: patterns of injury

J.L.M.A. Gielen, P. Van Dyck, J. Veryser; Antwerp/BE (jan.gielen@uza.be)

It is the purpose of this lecture to briefly discuss ankle distortion-related lesions with emphasis on failed radiographic diagnosis, discuss their significance and management implications and define the diagnostic pathways in the acute and subacute stage. Significant lesions are those with no return to sports activities after 12 months; these have our major interest. In the acute setting of ankle distortion, the Ottawa clinical decision rules are accepted to decide whether or not radiographic evaluation is needed; about $35 \%$ of radiographic examinations can be avoided. Additional evaluation is restricted to patients with residual pain during reevaluation one week after trauma. Assessment of fractures with the Lauge Hansen classification has a major advantage, as it defines the stability at the talocrural and distal tibiofibular joint. Only in restricted cases, additional examinations may be needed to rule out instability. Although radiographs easily detect displaced fractures, occult fractures may occur; also, grade II-II ligament lesions and peroneal tendon dislocation may go undetected. Additional imaging evaluation should focus on the detection of significant lesions. Significant lesions are talar fractures and complete calcaneofibular ligament tears. The latter is explained by the instability at the level of the subtalar joint with associated sinus tarsus syndrome. In case of residual local tenderness posterior and inferior to the lateral malleolus, the fibulocalcanear ligament and retinaculum peroneorum lesions are best evaluated with (dynamic) ultrasound. MRI or CT is used to detect occult fractures in case of residual talocrural joint effusion with anterior talar tenderness during endorotation and plantar flexion.
Learning Objectives:

1. To learn more about the imaging appearances of soft tissue and osteoarticular injury.

2. To become familiar with the patterns of bone and soft tissue injury in the ankle and foot.

\author{
A-292 16:30 \\ B. Inflammatory disorders \\ R. Lalam; Oswestry/UK (radhesh.lalam@rjah.nhs.uk)
}

The ankle and foot can be affected by inflammation from a number of diseases. The main focus of this lecture will be inflammation secondary to infection and systemic inflammatory disorders. Due to its function in locomotion and weightbearing, the foot is specifically susceptible to infections secondary to penetrative trauma including foreign bodies or to abnormal repetitive pressure. In addition, both seropositive and seronegative arthropathies can affect the foot. Other unusual causes of inflammation include chronic recurrent multifocal osteomyelitis, osteoarthritis, mechanical disorders and sensory loss. This lecture will deal with the various patterns of inflammation in the context of pathogenesis and discuss the imaging features that aid in diagnosis. The importance of the clinical context in diagnosis will also be discussed. Learning Objectives:

1. To learn more about the imaging appearances of soft tissue and osteoarticular inflammation.

2. To become familiar with imaging findings of specific inflammatory conditions.

A-293 17:00

C. Tumours and tumour-like lesions

I.-M. Noebauer-Huhmann; Vienna/AT (iris.noebauer@meduniwien.ac.at)

Soft tissue tumours of the foot and ankle are rare. This leads to frequent misdiagnosis or diagnostic delay, which is of importance, especially in the few patients with unsuspected malignancy. By the use of ultrasound, some entities which are clearly benign and do not require further imaging can be identified. In the other lesions, MR is the imaging modality of choice; additionally, radiography should be performed. Several common benign soft tissue tumours and those of intermediate type, as well as tumour-like lesions of the foot and ankle region, exhibit typical MR features, e.g. the Morton neuroma, the plantar fibromatosis and aggressive fibromatosis/extra-abdominal desmoids, lipoma, ganglia, and also vascular malformations and extraskeletal chondromas. MR findings of synovial lesions, such as pigmented villonodular synovitis (PVNS) and the giant cell tumour of the tendon sheath (GCTTS), as well as synovial chondromatosis, are also characteristic. It is important not to misdiagnose the most common malignant tumour of that region, the synovial sarcoma, as the growth rate may be slow. The same is true for clear cell sarcoma, which tends to have a high incidence of distant metastases and a high recurrence rate. Lesions that are indeterminate or likely malignant require histologic diagnosis. Biopsy may be performed by ultrasound or MR guidance. The importance of MRI for staging of soft tissue tumours will be discussed, including the recognition of compartmental involvement and relation to adjacent neurovascular structures.

Learning Objectives:

1. To learn more about the spectrum of intra and para-articular soft tissue tumours, and tumour-like soft tissue lesions.

2. To become familiar with US and MRI findings of specific soft tissue lesions.

16:00 - 17:30 Room E2

Multidisciplinary Session: Managing Patients with Cancer

\section{MS 11 \\ Characterisation and treatment of renal tumours: new paradigms}

\author{
A-294 16:00 \\ Chairman's introduction \\ N. Grenier; Bordeaux/FR (nicolas.grenier@chu-bordeaux.fr)
}

This session will be devoted to recent changes of paradigms in the management of renal tumours. Fist, characterisation of solid renal tumours has improved, using an association of morphological and functional information, using US, CT and, more recently, multiparametric MRI, and by extending the indications of percutaneous biopsy in undetermined cases. Second, surgical and radiological treatments have improved with the development of indications and techniques of nephron-sparing procedures (open, laparoscopic or robotised) and of percutaneous thermoablations (RF, cryo, microwaves, electroporation), with a larger consideration of active surveillance for the elderly. Third, antiangiogenic treatments have changed the prognosis of 
metastatic renal tumours, at least for certain tumour types, and may help as neoadjuvant phases before ablation.

Session Objectives:

1. To learn about the actual capabilities of imaging for characterising renal tumours.

2. To become familiar with emerging therapeutic strategies.

3. To understand the imaging requirements for staging metastatic tumours and monitoring new treatments.

\section{A-295 16:05}

Characterising solid renal tumours in 2014: from morphology to function N. Grenier, F. Cornelis; Bordeaux/FR (nicolas.grenier@chu-bordeaux.fr)

Characterising tumours before deciding on the most appropriate treatment is a general rule in oncology. Cystic tumours are now adequately graded based on Bosniak's classification. However, this pre-therapeutic characterisation is not currently systematic in case of solid renal tumours, as it remains difficult in clinical practice due to the high prevalence of renal cell carcinomas (RCC) and the lack of reliable imaging criteria for recognition of benign versus malignan tumours. As a result, approximately $10 \%$ to $20 \%$ of surgically excised renal masses are actually benign. To avoid these unnecessary ablations, pretherapeutic image-guided biopsy has been proposed, but its use is still debated as it is invasive and time consuming. New validated imaging criteria, able to accurately differentiate the most common tumour types but also indolent vs more aggressive malignant lesions, would be useful to reduce the number of unnecessary biopsies or to consider the most appropriate treatment for a tumour or active surveillance. Among these, MR imaging plays a major role based on chemical shift gradient echo (GRE) sequences, signal intensity on T2-weighted images, dynamic contrast-enhanced sequences, diffusionweighted sequences and late contrast-enhanced images. Multiparametric MR imaging is now performed in clinical practice in most primary care centres. Therefore, using different combinations of two or several parameters, it is now possible to distinguish certain renal tumours. A larger validation of all these combinations is still necessary to define those having a clinical significance for routine practice.

Learning Objectives:

1. To learn about the capabilities of multiparametric imaging for characterisation of tumours.

2. To understand the impact of characterisation on biopsy strategies.

3. To become familiar with functional techniques applied to renal tumours.

\section{A-296 16:25}

New therapeutic approaches for localised renal tumours: the urologist's opinion

J.-J. Patard; Le Kremlin-Bicêtre/FR (jean-jacques.patard@bct.aphp.fr)

The widespread use of modern imaging has led to increased detection of small, low-stage, low-grade renal tumours. In this setting, partial nephrectomy (PN) has progressively emerged as a standard of care. PN was first used in absolute indications (solitary kidneys, bilateral tumours, renal chronic disease) and thereafter in elective cases for tumours measuring less than $4 \mathrm{~cm}$. Nowadays, PN is recommended for all T1 tumours, because perhaps it is technically feasible and the negative margins can be safely obtained. In parallel, PN modalities have evolved from open to laparoscopic and now robotic-assisted approach. Robotic PN combines the advantages of open (short ischaemic time) and laparoscopic PN approaches (low morbidity, reduced postoperative pain, short hospital stay). In the meantime, reliable anatomical classifications have helped urologists to predict PN complexity and postoperative morbidity. The use of ablative treatment was first restricted to patients who were not suitable for or who refused surgical treatment. The advantages of these mini-invasive treatments compared to $\mathrm{PN}$ are: possibility of an outcome procedure, percutaneous approach, minimal nephron damage, very low morbidity and the possibility of repeated treatments. The current indications include: small cortical tumours $(\leq 3 \mathrm{~cm})$ in aged people with significant life expectancy, inherited renal or bilateral tumours and solitary kidney. Obtaining histology by percutaneous biopsy before treatment is recommended. Finally, the current indications for active surveillance include: patients $>75$ years with multiple and severe comorbidities, renal mass $\varnothing$ $<4 \mathrm{~cm}$, incidental diagnosis and acceptance for repeated imaging. Learning Objectives:

1. To learn about expansion and the limitations of conservative surgery. 2. To become familiar with actual indications of percutaneous image-guided ablations.

3. To learn about application of an active surveillance strategy to renal tumours.

\author{
A-297 16:45 \\ Impact of imaging in targeted therapy of metastatic renal cancer: the \\ oncologist's opinion \\ A. Ravaud; Bordeaux/FR (alain.ravaud@chu-bordeaux.fr)
}

Targeted therapies such as VEGF mAb bevacizumab, VEGF tyrosine kinase inhibitors (sunitinib, pazopanib, axitinib and sorafenib) and mTOR inhibitors (temsirolimus and everolimus) have been approved and included in European guidelines. Pre-therapeutic imaging prerequisites are mainly based on the natural history of MRCC. This justifies imaging through a thoracoabdominal CT scan. Outside clinical symptoms, there is no need for bone imaging or brain CT scan; the latter nevertheless could be done because of influencing the therapeutic strategy. PET scan should not be done due to low sensitivity. The evolution of metastatic sites by comparison from previous imaging is useful to evaluate the natural history. Outside clinical trials or research imput the strategy is to use the drug in an optimal way to increase PFS. So far, monitoring has to inform about change in RECIST criteria for CR, PR, SD or $P D$, and if $P D$ is a real PD such as RECIST PD or a slowing PD. In conclusion, standard CT scan and RECIST are adapted to real life. Nevertheless, more information on the mode of action of targeted drugs should be interesting for knowledge and research purpose, without any impact on the management of patients. Additional imaging could be done with additional criteria than RECIST on CT scan, by vascular functional imaging and PET scan.

Learning Objectives:

1. To learn about new targeted drugs for metastatic renal cancer.

2. To understand the pre-therapeutic imaging prerequisites.

3. To learn about the role of imaging for monitoring treatments. Author Disclosure:

A. Ravaud: Advisory Board; Member of Global, European and/or French boards in renal cell tumor for Pfizer, Novartis, Bayer, GSK, Aveo, Astellas, BMS. Investigator; international PI for study for Pfizer, Novartis; steering committe for trials for Pfizer, Novartis, BMS. Research/Grant Support; institutional grant support by Pfozer, Novartis, Roche. Other; Travelling and housing suppport for meetings by Pfizer, Novartis.

Case presentation and discussion

$17: 05$

$16: 00-17: 30$

Room F1

Breast

\section{RC 1102 \\ Clinical challenges in breast MRI}

\author{
A-298 16:00 \\ Chairman's introduction \\ F.J. Gilbert; Cambridge/UK (fjg28@cam.ac.uk)
}

Breast MRI is recognised as a useful tool for a number of clinical indications, but remains relatively challenging. Recognising the importance of an evidencebased approach to the development of protocols will influence the further integration of this technique into everyday clinical practice in the management of breast cancer patients. There remain concerns regarding the potential of over-diagnosis from breast MRI. The aim of this integrated RC is to inform the clinical practice with regard to the establishment of breast MRI in specific clinical and imaging scenarios, namely, using MRI for surveillance in high-risk patients, monitoring response to neo-adjuvant chemotherapy and developing protocols for the indeterminate imaging scenario of non-mass-like enhancement. The establishment of protocols to maximise the specificity and sensitivity of the technique of breast MRI in such indeterminate clinical and imaging scenarios will be discussed.

Author Disclosure:

F.J. Gilbert: Speaker; Bracco.

A-299 16:05

A. High-risk patients: establishing clinical protocols

J. Veltman; Almelo/NL (j.veltman@zgt.nl)

Contrast-enhanced MRI has, especially in younger women with dense breasts, a superior sensitivity for detecting breast cancer. Young women usually do not need screening unless they have an increased risk for developing breast cancer. An increased risk can be based on a positive familial history or on a proven gene mutation. The BRCA1 and BRCA2 gene mutations are the most well known related to an increased breast cancer risk. In this group, falsenegative rates up to $62 \%$ have been reported for mammography. Several papers have demonstrated the value of contrast-enhanced MRI in high-risk screening for early detection and even demonstrated a more favourable lymph node status for these women. An upcoming MRI technique for detecting and classifying breast lesions is diffusion-weighted imaging (DWI). The main 
advantages of DWI are the short examination time and that no contrast is needed. In literature, DWI has demonstrated great diagnostic potential. Especially high sensitivities have been reported (80\%-97.8\%). The high sensitivity values reported give DWI the potential to become a fast, noncontrast, and therefore relatively cheap, highly sensitive screening technique The specificity and spatial resolution are however still moderate. At this point, contrast-enhanced MRI is the best available modality for the detection of breast cancer in women with an increased risk. The detection of smaller lesions, patient anxiety, the often benign morphologic characteristics seen in cancers and the often aggressive nature of breast cancer in young women should lower the threshold for MRI-guided-biopsies

Learning Objectives:

1. To become familiar with the literature on the role of breast MRI in screening of high-risk patients.

2. To understand current protocols in clinical practice.

3. To appreciate the challenges of breast MRI imaging in this patient group. Author Disclosure:

J. Veltman: Advisory Board; Hologic.

\section{A-300 16:28}

B. Non-mass like enhancement (NMLE): when to biopsy?

C.K. Kuhl; Aachen/DE (ckuhl@ukaachen.de)

Non-mass like enhancement is a frequent finding in breast MRI. It relates to the fact that enhancement occurs in the normal-appearing fibroglandular tissue that surpasses that of the other parts of the parenchyma. There is no associated space-occupying lesion. In pre-contrast non-fat-suppressed or fatsuppressed T1- and T2-weighted images, there is usually no correlate observable. The differential diagnosis of non-mass-like enhancement is between benign nonspecific fibrocystic disease/adenosis, hormonal stimulation, and subclinical mastitis on one hand, vs DCIS or (less likely) diffusely infiltrating (usually lobular) cancer on the other. Key components of differential diagnosis is configuration of the enhancement (does it follow the orientation of the milk duct or not?) and symmetry (symmetric or asymmetric) Less important criteria are internal enhancement (internal architecture) and enhancement kinetics. It is important to realise that enhancement kinetics can only be used to corroborate the suspicion of DCIS - but they cannot be used to alleviate the indication to biopsy a finding which, based on configuration and asymmetry, is suspicious. Management depends on the different constellation of clinical, mammographic, and MRI findings. It usually includes short-term follow-up (6 months) and, if stable/persistent, MR guided vacuum biopsy.

Learning Objectives:

1. To learn about the clinical significance of NMLE

2. To understand differential diagnosis for NMLE.

3. To become familiar with a work-up algorithm of NMLE cases.

A-301 16:51

\section{Monitoring response to neo-adjuvant chemotherapy}

T.H. Helbich; Vienna/AT (Thomas.Helbich@meduniwien.ac.at)

Assessment of the change in tumour burden is an important feature of the clinical evaluation of cancer therapeutics. Tumour shrinkage and time to the development of disease progression are important end points in cancer clinical trials. However, these end points are useful only if based on widely accepted and readily applied standard criteria. Criteria, known as RECIST, were published and are updated on a regular base. The revised RECIST includes a new imaging appendix and underlines the importance of moving from anatomic unidimensional assessment to either volumetric anatomical assessment or functional assessment with PET or MRI. The goal of the lecture is to discuss the value of the different MR imaging techniques (e.g. MR spectroscopy, diffusion-weighted imaging, angiogenesis mapping, PET-MRI), which evaluate response to treatment in breast cancer. Participants will learn about particular imaging challenges of assessing response to neo-adjuvant chemotherapy. In addition, they will learn to understand clinical aspects of neo-adjuvant chemotherapy based on RECIST.

Learning Objectives:

1. To understand the clinical aspects of neo-adjuvant chemotherapy.

2. To appreciate evidence-based protocols for imaging in this clinical setting.

3. To learn about particular imaging challenges of assessing response to neoadjuvant chemotherapy.

Author Disclosure:

T.H. Helbich: Advisory Board; Philips. Grant Recipient; Siemens, Guerbet.

Panel discussion:

How can the specificity and sensitivity of breast MRI in these indeterminate clinical and imaging scenarios be maximised?

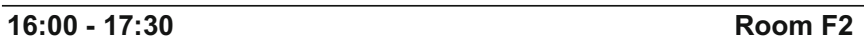

Professional Challenges Session

PC 11

The role of social media in radiology

\author{
A-302 16:00 \\ Chairman's introduction \\ P. Pokieser; Vienna/AT (peter.pokieser@meduniwien.ac.at)
}

Currently, the ESR has been using social media in many successful ways to improve the contacts among members and to boost our fast development as one of the most advanced scientific communities in the world. Our session "The role of social media in radiology" presents the view of 4 experts, acknowledged leaders of international IT projects. It seems to be important to discuss the major implications of social media for radiology, the leading clinica discipline in information technology. Davide Caramella will clarify the relationship of social media to professional societies and especially to the ESR, including their basic features. The "Social media generation" has developed from schoolkids to students and, recently, to teachers as well. Alexander Sachs represents the young professional expert of ELearning in Radiology and will reflect the current user behaviour. Osman Ratib will share his view on how social networks enhance the communication between radiologists, nonradiologists and patients. Critical aspects of the pros and cons of large communication scenarios are followed by some ideas of future developments. Jürgen Brandstätter will indicate the patient's view. Most patients see social network communications with their radiologist as useful for, e.g. appointment management or other non-sensitive communication. Besides communicating personal health data, a wide area of potential useful other possibilities are at hand, like anonymised health data for reviewing purposes, second opinion or education of patients. The fast development of social media may induce hopes and fears; the four speakers of this session will present them in a panel discussion.

Session Objectives:

1. To learn about social networks which are important for the ESR and its members.

2. To understand the intentions and motivations behind social networks.

3. To appreciate the unique services and opportunities offered by different types of networks.

4. To become familiar with the pros and cons of using social networks.

\section{A-303 16:05}

Major social media networks and their relationship to professional societies

D. Caramella; Pisa/IT (davide.caramella@med.unipi.it)

The widespread use of social networks in all professional activities has attracted a great interest in the recent years. In medical applications, the main concerns include the possible consequences in terms of patients' confidentiality protection, perceived role of the physician, patient-physician relationship, and caregivers' distraction during professional activities. There are no doubts, however, that social networks are beneficial in enhancing the information flow between scientific societies and their professional members, without any interference at the point of care. Social networks have proved to be extremely effective in updating members about relevant news concerning their areas of interests and in allowing them to be informed about the educational activities promoted by their scientific societies. This presentation focuses on the social network activities promoted by the ESR via Facebook, YouTube, Twitter and other blogs.

Learning Objectives:

1. To learn about the ESR's relations with to social networks.

2. To understand the basic features of Facebook, YouTube, Twitter and blogs.

3. To learn about the productive use of ESR networking for its members.

\section{A-304 16:23}

Impact of social media on the training of radiologists

A. Sachs; Vienna/AT (alexander.sachs@meduniwien.ac.at)

In modern times, social media have gained more and more important value in exchanging first experiences in radiology from resident to resident. Social networks such as Facebook, Twitter, Google plus and others are used to start discussion groups about cases and share the growing knowledge with different educational concepts in each hospital. Growing e-learning modalities as MOOCs, applications for smartphones or tabs and web-based case series or learning platforms support the training of radiologist in social media. Modern elearning platforms use social media as their first choice of sharing knowledge around the whole world about certain illnesses, cases or different decisions of therapy. Though sharing information seems to be easier and shows several 
advantages than in former times, social media can also highlight new problems. This lecture focuses on user behaviour to improve learning, the pedagogic evidence for large group learning with networks and the importance of using networks to manage time.

Learning Objectives:

1. To learn about the current user behaviour of ESR members in order to improve learning.

2. To learn about paedagogic evidence for large group learning with networks.

3. To appreciate the importance of using networks to manage time.

\section{A-305 16:41}

Social media networks in communication between radiologists, nonradiologists and patients

O. Ratib; Geneva/CH

While social networks have gained unprecedented popularity in our daily environment, they have only limited utilisation in medical applications. The paradigm of communication within a given community for exchange and sharing of imaging data would certainly have its place in a medical environment where there is an increasing need for multidisciplinary collaborative work. The required security and confidentiality in management of sensitive patient data have often precluded the usage of existing public social networks. Professional proprietary systems have emerged in a more restricted way with much less flexibility leading to limited use. The exchange of medical images adds a level of complexity due both to the size of the data and the need for appropriate browsing and visualisation tools. Traditional PACS and teleradiology system offer such features, but in a very limited way, far from the desired convenience of social networking between groups of users. A prototype of such a system aimed essentially at the community of users that need to exchange anonymised medical images and data for academic and research purposes will be presented and discussed. The project nicknamed DICOM SandBox is being tested and evaluated by some users in Europe under the coordination of the ESR subcommittee of eHealth and informatics. Developed with Open Source software components, it allows using existing cloud-based storage services with additional layers of secure DICOM file management and visualisation. It allows the community of users to share DICOM data with all social networking and notification features beyond simple peer-to-peer exchange.

Learning Objectives:

1. To appreciate how social networks enhance communication between radiologists, non-radiologists and patients.

2. To appreciate the pros and cons of large communication scenarios.

3. To learn about the future of social networks.

\section{A-306 16:59}

Interactions between patients and radiologists through social media

J. Brandstätter; Wiener Neudorf/AT (j.brandstaetter@codewerk.at)

Social networks have become integral parts of most citizen's use of the Internet. Especially, the younger generation uses social networks extensively however the way those tools are used should be chosen carefully. It is one thing to use a social network for private interests, but a different one to use it as transport media for sensible data like personal health-care data. The sensitiveness of citizens regarding privacy has been increased recently by different events of misuse or fraud, especially accelerated by the recent privacy affairs caused by governmental agencies. Though most patients see social network communications with their radiologist useful for e.g. appointment management or other non-sensitive communication, personal medical reports or images shall not be shared across this media. Besides communicating personal health data, a wide area of potential useful other possibilities are at hand (e.g. anonymised health data for reviewing purposes, second opinion, education of patients, ...). Future developments shall make use of social media where useful, but always with deep respect to the patient's privacy rights.

Learning Objectives:

1. To learn to appreciate the patient's view.

2. To understand what patients demand from radiologists in social network communications.

3. To become familiar with the patient's view of future developments.

Panel discussion: Social networks: hopes and fears 17:17

16:00-17:30
Neuro
RC 1111
Brain tumours: the central role of
radiology

Room G/H

A-307 16:00

Chairman's introduction

M.A. Lucic; Sremska Kamenica/RS (milos.a.lucic@gmail.com)

Apart from the historical, but ever present clinical expectation from the neuroradiologist is the answer to the most important question if the patient has a brain tumour or not. Nowadays, due to the outstanding technological advances in imaging techniques during the last decades, the stakes are growing much higher and it is the neuroradiologist on whom lies the initial responsibility of answering a large number of questions that are of utmost importance for further brain tumour patient care and treatment. Having the opportunity to provide the usual and generally basic information regarding tumour morphology and exact location and to narrow the differential diagnostic possibilities, neuroradiologic expertise now opens a wide spectrum of techniques, especially those derived from MRI, such as MR spectroscopy, MR perfusion, DWI, DTI, SWI, etc. These provide us the unique possibility of not only obtaining and comparing images, but also of exploring multiparametric brain maps, allowing us to gain insight into the internal architecture of the tumour and the functional and molecular features of the tumorous tissue. This consequently defines the actual stand of neuroradiology, indicating the uprising clinical urge not only to provide the answers regarding the tumour type, grade and possibilities of the most appropriate and patient-individualised therapy modality, whether surgical or nonsurgical, but also to accurately assess the post-treatment tumour behaviour and status, by differentiating the true tumour progression from pseudoprogression, represented by inflammatory responses to the radio- and/or chemotherapeutical treatment, and to monitor other neurooncological response assessment criteria.

\section{A-308 16:05 \\ A. Differential diagnosis and pseudo-tumoural lesions H.R. Jäger; London/UK (r.jager@ucl.ac.uk)}

Conventional contrast-enhanced MR imaging gives an indication about the likely tumour type and histological grade, but advanced MR imaging methods, including perfusion-weighted (PWI) and diffusion-weighted (DWI) imaging, MR spectroscopy (MRS) and PET imaging provide additional information which helps to refine the diagnosis of intrinsic brain tumours. Contrast enhancement can be a feature of low-grade (pilocytic astrocytoma and ganglioneuromas) as well as high-grade gliomas (WHO grade III anaplastic gliomas and WHO grade IV glioblastomas). Infiltrative low-grade (WHO grade II) astrocytomas do not usually enhance, whereas WHO grade II oligodendrogliomas can enhance. Higher-grade glial tumours (WHO grade III and IV) tend to have an elevated relative cerebral blood volume ( $\mathrm{rCBV}$ ) on PWI, a decreased ADC on DWI and increased choline turnover on MRS and PET imaging. A raised rCBV can, however, also be seen in low-grade oligodendrogliomas, particularly in those with a $1 \mathrm{p} / 19 \mathrm{q}$ chromosomal translocation, which is associated with a better response to chemotherapy. PWI and DWI are useful in the differentiation of glial neoplasms from cerebral lymphoma, which has an increasing incidence in the immuno-competent population. DWI and PWI are also very useful in the differentiation of enhancing pseudo-tumoural lesion such as cerebral abscesses, tumefactive demyelination, pseudoprogression and radiation necrosis.

Learning Objectives:

1. To appreciate the differential diagnosis of tumoural lesions in the diagnostic setting.

2. To learn how to differentiate between tumoural and non-tumoural disease using DWI.

3. To consolidate knowledge on how to combine DWI with other advanced MRI tools.

\section{A-309 16:28}

B. Grading and prognosis: role of perfusion imaging

Y. Özsunar; Aydin/TR (yeldaozsunar@gmail.com)

Perfusion imaging plays an important role in the characterisation and management of brain tumours. Low-grade brain tumours may transform into high-grade gliomas at some point, which is a transformation that is highly variable and difficult to predict in an individual patient. Conventional contrastenhanced computed tomography (CT) and magnetic resonance (MR) imaging are found to be insufficient to depict this transformation and angiogenesis and 
therefore to detect malignancy. However, perfusion imaging provides additional information over conventional imaging in terms of tumour physiology and haemodynamics, providing important biomarkers for malignancy and prognosis. Perfusion CT and MR perfusion techniques such as arteriel spin labelling and dynamic susceptibility-weighted perfusion imaging are more practical and widely used compared to nuclear medicine methods. These perfusion techniques should be considered and used in routine clinical workup of brain tumours to asses grading and prognosis.

Learning Objectives:

1. To understand why perfusion imaging is routinely needed for brain tumours.

2. To become familiar with principles, applications, and pitfalls of various perfusion imaging techniques.

3. To consolidate knowledge of perfusion imaging for various brain masses that can be encountered in daily practice.

Author Disclosure:

Y. Özsunar: Research/Grant Support; funding from Adnan Menderes University Research Funding Programme.

\section{A-310 16:51}

\section{Monitoring treatment: real or pseudo progression?}

P.C. Maly Sundgren; Lund/SE (Pia.Sundgren@med.Iu.se)

Maximal safe resection, radiation therapy and temozolomide chemotherapy are the current standard of care for newly diagnosed high-grade gliomas. Still, HGG have a poor survival rate. A contributing factor to the poor survival is the inability of currently available imaging techniques to accurately delineate the tumour, with the result that targeted focal treatment my not be effective. Conventional imaging is not able to give an early assessment of the effectiveness of radiation and/or chemotherapy. In addition, conventional imaging has difficulties in differentiating pseudo-progression, which is a common phenomenon in conventional chemo-radiation therapy, from true progression. Early identification of patients who suffer from tumour recurrence can be of great advantage: it provides the opportunity to adjust individuals more rapidly, sparing patients unnecessary morbidity and delay in the initiation of other, maybe more effective, treatment. In recent years, different functional imaging approaches such as perfusion MRI, diffusion-weighted imaging and spectroscopy, have been complementarily used for imaging evaluation of treatment response. In this lecture, different advanced MR and CT imaging methods used to support differentiation between pseudo-progression and true tumour progression to assess treatment response will be discussed. In addition, a novel recently published promising technique, the parametric response mapping (PRM), a novel voxel-wise analytical method of monitoring physiological and environmental changes in a tumour volume during treatment will be presented and compared to the traditional methods used. Finally, the aim of the lecture is to consolidate the present knowledge and novel ideas in brain tumour imaging for assessment of pseudo-progression versus true tumour progression.

Learning Objectives:

1. To understand the challenges and limitations of routine MRI in monitoring brain tumour treatment.

2. To become familiar with the role of advanced imaging biomarkers for early assessment of treatment response.

3. To learn how to integrate routine and advance MRI into clinical practice after tumour therapy.

Panel discussion:

What is the optimal role of radiology in a tumour

board session? $\quad 17: 14$

$16: 00-17: 30$ Room I/K

Head and Neck Imaging

\section{1119}

\section{How to perform and read a head and neck study (part 2) \\ Moderator}

S. Bisdas; Tübingen/DE

\section{A-311 16:00}

A. CT and MR perfusion study of the head and neck

K. Šurlan Popovič; Ljubljana/SI (katarina.surlan@kclj.si)

The purpose of this lecture is to describe the role of functional radiologica imaging modality-CT and MR perfusion in the evaluation of head and neck tumors. Conventional contrast-enhanced CT and/or MR imaging are the current standard techniques for the diagnosis and treatment evaluation of the head and neck tumors. However, this method is limited in its ability to depict the angiogenesis which is a hall-mark of tumor growth. Perfusion imaging provides a rapid evaluation of tissue perfusion and can be easily implemented in every head and neck CT or MR protocol. The determination of tissue perfusion is based on examining the relationships between the arterial, tissue and potentially the venous enhancement after the introduction of a bolus of contrast material. The quantification of the perfusion values helps to outline the malignant tissue as well as to differentiate recurrent disease from nonspecific post-therapeutic changes and can be used as a therapeutic monitoring tool during and after tumor therapy.

Learning Objectives:

1. To learn how to perform proper CT/MR perfusion.

2. To learn how to interpret the results.

3. To understand which clinical and diagnostic dilemmas can be addressed with this technique.

\section{A-312 16:30 \\ B. MR diffusion study and how to interpret it \\ H.C. Thoeny; Berne/CH (harriet.thoeny@insel.ch)}

In recent years diffusion-weighted MRI (DWI) gained increasing importance and also acceptance in head and neck radiology. Although this functional technique is challenging due to air tissue interfaces, its ability to improve the diagnostic accuracy is widely accepted. DWI can be applied to detect lesions not only in adults, but also in children. It is helpful in lesion characterisation and has a great potential to differentiate recurrence from posttherapeutic changes. Initial promising results to improve lymph node staging however are difficult to reproduce. Image interpretation is performed qualitatively based on visual assessment of the high $b$-value images $\left(b=800-1000 \mathrm{sec} / \mathrm{mm}^{2}\right)$ and the corresponding apparent diffusion coefficient (ADC) map. Quantitative image analysis is based on the measurement of the underlying ADC value. Solid tumors including recurrences are bright on the high b-value images and dark on the corresponding ADC map, whereas posttherapeutic changes are bight on both the high b-value images as well as the ADC map. Cystic and necrotic lesions are typically dark on the high b-value image and bright on the ADC map. Furthermore, malignant lesions tend to have a lower ADC compared to benign and cystic lesions provided that no necrosis is present. Therefore, image interpretation always has to be performed together with morphological images to avoid misinterpretation of functional images.

Learning Objectives:

1. To learn the technique and learn how to perform MR diffusion successfully.

2. To learn how to interpret the results.

3. To appreciate the pitfalls and advantages of this method.

A-313 17:00

C. PET/CT and MR/PET: the true added value of the method

M. Becker; Geneva/CH

PET/CT has established itself as a robust, rapid and reliable technique in head and neck oncology. It is mainly used to stage nodal disease, to detect distant metastases and synchronous tumours, to identify unknown primary tumours in patients with metastatic neck nodes, to assess treatment response and prognosis after chemoradiotherapy and for radiotherapy planning. MRI with diffusion-weighted imaging (DWI) is routinely used for the assessment of submucosal tumour spread, to stage nodal disease, to monitor treatment response and to detect recurrent disease. The information provided by PET/CT and MRI is often complementary and the recent implementation of hybrid MR/PET systems in clinical settings holds promise because it can combine morphologic functional and molecular information. This lecture focuses on clinical applications of PET/CT and MR/PET in the head and neck. Current evidence about the combined use of PET/CT and MRI with DWI is discussed. The principles of MR/PET data fusion are summarised as well as current knowledge regarding the diagnostic performance of MR/PET in the head and neck. Typical radiologic findings of tumour manifestations are reviewed with particular emphasis on the early detection of lesions, their appearance on different imaging modalities and the added value of hybrid imaging techniques. The variable appearance of functional phenomena mimicking disease as well as the potential pitfalls of image interpretation and how to avoid them are equally addressed. Major emphasis will be put on how to report the findings in a comprehensive way.

Learning Objectives:

1. To learn what additional information is acquired by PET.

2. To appreciate the clinical importance of this examination.

3. To learn how to interpret results and avoid mistakes. 
$16: 00-17: 30$

Room L/M

\section{Physics in Radiology}

\section{RC 1113}

\section{Novel developments in CT and their impact on dose \\ Moderators:}

W. Stiller; Heidelberg/DE

A.J. van der Molen; Leiden/NL

\section{A-314 16:00}

A. Impact of $\mathrm{CT}$ on patient dose

R. Bly; Helsinki/FI (ritva.bly@stuk.fi)

Methods to assess patient dose from CT and its importance for collective effective dose to population (population dose) will be presented. Patient dose from CT as an effective dose can be estimated using technical dose parameters, such as dose length product (DLP) and CT dose index (CTDI) and conversion factors. Technical dose parameters can also be used to define diagnostic reference values (DRL) that are used as a tool for assessing if CT procedures are optimised. Population doses from radiodiagnostic procedures in Europe were estimated for the first time in the recent DOSE DATAMED 2 (DDM2) project (www.ddmed.eu) launched by the European Commission. Examples of calculation procedures for CT dose will be presented. Typical effective doses for some procedures will be presented as well as existing DRLs in Europe. The estimated population dose per caput in Europe is $1 \mathrm{mSv}$, which is lower than the corresponding dose in the USA ( $3 \mathrm{mSv}$ ) or Australia (1.7 $\mathrm{mSv}$ ). The highest average contribution is from $\mathrm{CT}, 56 \%$. There are high variations between countries in frequencies and typical effective doses. This presentation will review the available data in European countries using graphs. Learning Objectives:

1. To learn about the European-wide DoseDataMed2 survey.

2. To understand the impact of CT practice on population exposure.

3. To understand the role of diagnostic reference levels in CT.

\section{A-315 16:30}

B. New frontiers in CT: functional and spectral imaging

A. Persson; Linköping/SE (anders.persson@cmiv.liu.se)

Multi-energy and functional imaging CT has reached a robustness allowing the use of this new technology in clinical routine for a variety of different clinical questions. This presentation will discuss the basic principles and the strengths and limitations of the techniques. Implementations of multi-energy methods for material characterisation and of CT methods for functional imaging will be discussed. Contrary to normal single-energy CT systems, multi-energy CT scanners allow simultaneous scanning at two peak X-ray energies. When the attenuation is measured at two energies, their values are not exactly proportional to each other, which open new diagnostic possibilities. Measurements at two spectra can be achieved using multiple kVp and/or filtration or with detectors with energy discrimination. These methods have different pros and cons such as sensitivity to subject motion and dose efficiency. To be able to acquire functional data such as perfusion, images are acquired dynamically following the injection of a contrast agent and physiological models are used to convert the measured contrast agent concentration to perfusion estimates. Methods that acquire multiple images have the potential to increase the radiation dose to the patient, so CT protocols need to be optimised.

Learning Objectives:

1. To learn about new developments in functional and spectral CT imaging

2. To appreciate the impact on patient dose.

\section{A-316 17:00}

\section{New image reconstruction techniques}

\section{J. Sijbers; Antwerp/BE (jan.sijbers@ua.ac.be)}

In computed tomography, 3D images are reconstructed from a number of $x$-ray radiographs that were acquired at different angles. Tomographic reconstruction relates to the generation of a 3D image based on these acquired radiographs. Most tomographic reconstruction methods are based on a technique called filtered back projection (FBP) in which a sharpened version of the radiographs are back projected to generate the $3 \mathrm{D}$ image. While it has been the standard reconstruction method, mostly due to its speed and good quality if many radiographs are available, iterative reconstruction methods are emerging. The reason for that is that FBP suffers from important disadvantages. In this talk, the basic concepts of iterative reconstruction are explained and its virtues are detailed. The flexibility of iterative reconstruction methods is demonstrated for the field of $\mathrm{x}$-ray computed tomography. Finally an outlook is given with respect to future research on tomographic reconstruction.

Learning Objectives:

1. To understand the principles of iterative reconstruction techniques in CT.

2. To learn about advances in iterative reconstruction algorithms.

3. To appreciate the impact on patient dose.

16:00 - 17:30 Room N/O

Interventional Radiology

RC 1109

\section{Gynaecological and obstetrical haemorrhagic emergencies}

\author{
A-317 16:00 \\ Chairman's introduction \\ A.-M. Belli; London/UK (Anna.Belli@stgeorges.nhs.uk)
}

Interventional radiology (IR) has been increasingly applied in the management of obstetric and gynaecological haemorrhage. National reviews of maternal deaths from post-partum haemorrhage have recognised that maternal deaths may be prevented by IR and that all obstetric units should have protocols and arrangements in place to ensure appropriate and timely referral to IR. The important role that IRs play in the management of fibroids has also highlighted how these techniques can be applied to other gynaecological conditions which may result in haemorrhage. This session will start by describing which gynaecological disorders may result in haemorrhage and the technical aspects of treatment followed by the published evidence for embolisation. The session will then focus on obstetric haemorrhage, with an emphasis on prophylaxis and how women deemed at high risk for haemorrhage might be managed safely. The techniques used for prophylaxis of haemorrhage will be described in detail and the published data presented. The final presentation will concentrate on the technical aspects of embolisation when haemorrhage has occurred to ensure the best results by knowing the relevant anatomy and appropriate technique of embolisation. The published evidence of the results of embolisation in PPH will be presented. These presentations will be followed by a panel discussion on how IRs can reduce the radiation dose whilst simultaneously ensuring successful outcomes for their therapies, an important issue in a young and fertile group of women.

Author Disclosure:

A.-M. Belli: Advisory Board; Boston Scientific. Investigator; Covidien, Eurocor, Bayer.

\section{A-318 16:05}

A. Etiology and treatment of benign and malignant gynaecological causes of massive bleeding

O. Pellerin; Paris/FR (olivier.pellerin@egp.aphp.fr)

Gynaecologic massive bleeding related to benign and malignant causes is under the scope of all radiologists because of being diagnostic and also because of its implication in interventional radiology procedures. The causes are uterine fibroids (UF) and post-partum hemorrhage (PPH). However, oncologic disease, arterio-venous abnormalities, or iatrogenic injuries are less commonly known to be eligible for embolisation procedures. Usually, surgical homeostasis attempts or radical hysterectomy is preferred to embolisation procedures. Despite the good clinical outcome for hysterectomy and the hazardous success rate of conservative treatment, both are unsatisfactory to patients. Pelvic artery embolisation (PAE) could dramatically change the patients' clinical issue. The purposes of this lecture are to: revise the pelvic arterial anatomy (possible arterial anastomosis or variation); outline the aetiology of non-obstetric massive menometrorrhagia; expose the typical clinical presenting features; describe the imaging appearances of the etiologies; and discuss patient section, procedure technique, pitfalls and complications.

Learning Objectives:

1. To learn more about causes of massive bleeding due to gynaecological disorders.

2. To learn about diagnostic imaging and therapeutic embolisation.

3. To learn about published results obtained through embolisation.

A-319 16:28

B. Can we prevent post-partum haemorrhage

J.K. Gupta; Birmingham/UK (j.k.gupta@bham.ac.uk)

In my lecture, I shall identify a patient at risk for post-partum haemorrhage and the obstetric complications that can arise as a result. I shall indicate how to become familiar with preventive measures and medication, and discuss more 
about techniques for invasive prevention and the role of uterine artery embolisation as a possible preventive technique

Learning Objectives:

1. To learn how to identify a high-risk patient.

2. To become familiar with preventive measures and medication.

3. To learn more about techniques for invasive prevention.

4. To learn about the results obtained through preventive techniques.

\section{A-320 16:51}

\section{Treatment of post-partum haemorrhage}

A. Krajina, I. Kalousek; Hradec Králové/CZ (antonin.krajina@fnhk.cz)

Obstetric haemorrhage occurring mainly in the postpartum period is a significant cause of maternal morbidity and death. Blood loss of over 500 and $1000 \mathrm{ml}$ after vaginal delivery or caesarean section, respectively, complicates $4 \%$ of deliveries. Major blood loss of more than $1000 \mathrm{ml}$ occurs following less than $1 \%$ of all deliveries. The causes include uterine atony, retained placenta products, placental abnormalities, uterine rupture, lower genital tract laceration, cervical ectopic pregnancy and coagulopathies. Generally, treatment is based on administration of uterotonic drugs, vaginal packing, surgical ligation of uterine arteries and even hysterectomy is sometimes needed. Transcatheter embolisation of uterine, pudendal, vaginal arteries and ovaries is considered to be superior and should be the first-line treatment for intractable obstetric haemorrhage where interventional radiology is available. Angiography reveals extravasation of contrast agent in more than $50 \%$ of these patients. The most frequent extravasation is from vaginal artery, and then from pudendal and uterine arteries. Depending on the extravasation location and haemodynamic stability of a patient, $5 \mathrm{~F}$ catheters or coaxial microcatheter technique is utilised for catheterisation. Gelatin foam, microparticles acrylic glue and microcoils are the most frequent embolic agents. The embolisation procedure usually does not require general anaesthesia and can be repeated if bleeding continues. Primary success rate in bleeding control is reported from 80 to $90 \%$. The most commonly reported long-term side effects after embolisation were transient buttock numbness and urinary frequency. The transcatheter pelvic artery embolisation for obstetric intractable bleeding is a fertility-preserving alternative to hysterectomy.

Learning Objectives:

1. To learn about clinical evaluation of post-partum haemorrhage

2. To become familiar with relevant anatomy.

3. To understand catheterisation and embolisation techniques.

4. To become familiar with results of embolisation.

Panel discussion:

How can radiation doses be reduced during treatment?

$17: 14$

16:00 - 17:30

Conf. Room M3

\section{Head and Neck}

RC 1108

\section{Skull base lesions: imaging studies and differential diagnosis}

Moderator:

S. Kösling; Halle a.d. Saale/DE

A-321 16:00

A. Anterior cranial fossa with special emphasis on olfactory apparatus lesions

T.P.J. Duprez; Brussels/BE (thierry.duprez@uclouvain.be)

Medical imaging has nowadays integrated the diagnostic armamentarium of anosmic patients regarding not only qualitative assessment of the olfactory tract, but also quantitative evaluation of olfactory bulb volumes which are known to closely correlate to the olfactory function. Many clinical studies on various pathological conditions have evidenced the value of such measurements in the workup of olfactory dysfunction for both aetiologic and prognostic purposes. Imaging workup also plays a role in the medico-legal evaluation of post-traumatic anosmia together with electrophysiological and clinical olfactory tests. Technical improvements in fibre tracking (FT) using diffusion-tensor imaging (DTI) and appropriate designs of olfactory stimulation at BOLD-based functional MRI (fMRI) are expected to allow insights into the neurophysiological processes and circuitry of olfaction in the very near future. Imaging workup of the anosmic patients will be the cornerstone of this lecture. The relevance of the different imaging techniques will be detailed. Beyond the workup of anosmia, a comprehensive overview of the most common lesions of the olfactory tract seen in clinical practice will be given. Additional review of lesions observed at the anterior cranial fossa in the near vicinity of the olfactory tract will be done, which comprehensively includes all commonly observed developmental, traumatic, inflammatory and neoplastic conditions not arising from the olfactory tract. Their potential impact on the olfactory function or on other sensory/neurological functions will be underlined. Clues to clinical/radiological differential diagnoses of the most common conditions and radiological features of the anterior cranial fossa will be highlighted.

Learning Objectives:

1. To learn the imaging techniques and anatomy of the anterior skull base with an emphasis on the olfactory apparatus.

2. To become familiar with the imaging findings.

3. To learn how to differentiate between lesions in the anterior skull base.

A-322 16:30

B. Middle cranial fossa pathologies

A. Borges; Lisbon/PT (borgalexandra@gmail.com)

The central skull base, the floor of the middle cranial fossa, has a complex anatomy and is pierced by a variety of foramina and canals providing crossroads for spread of disease between the extra-cranial head and neck and the intracranial compartment. Disease spread into the intracranial compartment indicates a dismal prognosis, as often it becomes inoperable due to involvement of vital structures including cranial nerves, carotid artery and/or brain parenchyma. Imaging plays a pivotal role in the assessment of these structures which are not amenable to clinical inspection and can dramatically change the patient's management. In this complex anatomical area, CT and MR have a complementary role in providing a comprehensive roadmap for treatment planning. Of major importance is the pterygopalatine fossa, the cavernous sinuses and orbital apex. The former, lying between the pterygoid plates and the maxillary sinus, is a major crossroad between the intra- and extracranial compartments and should be carefully inspected. Pathologies affecting the central skull base are varied and may originate from the skul base proper, from the middle cranial fossa or from the extracranial head and neck. By looking at the centre of the lesion, its tissue characteristics and pattern of growth, it is often possible to provide a useful differential diagnosis and, most importantly, to map the lesion's extent. Here, we present a radiologist-friendly approach to central skull base pathology highlighting the most important features in the differential diagnosis and treatment planning. Learning Objectives:

1. To become familiar with imaging strategies and anatomy of the middle cranial fossa.

2. To learn about imaging findings of common lesions.

3. To learn how to differentiate between the lesions in middle cranial fossa.

A-323 17:00

C. Posterior cranial fossa pathologies

H. Tanghe; Rotterdam/NL (tanghe@planet.nl)

1. The jugular foramen: The jugular foramen is an opening in the skull base. The radiologic evaluation requires high-quality imaging with $\mathrm{CT}$ and MR. Angiography is reserved for preoperative embolisation. It is important to recognise the "pseudo lesions". The most common tumour of the jugular foramen is the paraganglioma. The second is the schwannoma of the lower cranial nerve, and the jugular foramen meningioma is the third most common. The differential diagnosis shall be discussed. 2. The cerebellopontine angle (CPA) and the internal auditory canal (IAC): The IAC is a bony conduit for several nerves and a vessel, the neurovascular bundle. The CPA is a cistern of the peripheral cerebral spinal fluid, and several anatomical structures pass through this. It is also the place of some frequent disease processes. Most of the lesions in the CPA are benign tumours with order of frequency: vestibular schwannoma and meningioma. The third most common lesion is a benign cystic lesion: the epidermoid cyst. In the IAC, you can encounter the same benign tumoural lesion as in the CPA, but also inflammatory lesions, viral lesions and malignant lesions. Why is it important in your imaging protocol of the IAC and CPA region to use gadolinium? The differential diagnosis of different lesions shall be discussed.

Learning Objectives:

1. To learn about the imaging techniques and anatomy in the posterior cranial fossa.

2. To become familiar with the imaging findings of common posterior cranial fossa pathologies.

3. To learn how to differentiate between the lesions in the posterior cranial fossa. 
$16: 00-17: 30$

Board Room A

\section{Radiographers}

RC 1114

\section{Evidence-based practice in medical imaging: a tool to improve the quality of care}

Moderators:

C.J. Herold; Vienna/AT

D. Katsifarakis; Athens/GR

\section{A-324 16:00}

A. The radiographer as the interface between patient and technology: clinical judgement and professional accountability

G. Paulo; Coimbra/PT (graciano@estescoimbra.pt)

Health care is characterised by several specific features making it different from traditional markets. Two of the key features are: the impossibility of patients to make choices, due to asymmetry of knowledge between them and their health-care providers; the fact that patients create an agency relationship with health-care providers, delegating to them the decision about the care delivered. Due to these characteristics and to the fact that patients are fragile when they need care, there is a natural tendency for patients to rely on healthcare professionals. It is in this complex socio-technical environment that radiographers must be aware of their roles and responsibilities and ensure professional autonomy and accountability; demonstrate an ethical and knowledgeable understanding of the profession; apply professional practice in securing, maintaining or improving health and well-being; develop knowledge, skills and competences that underpin their education and training, contributing to the wellbeing of the patient; understand that professional advancement arises out of evidence-based practice and is acquired through focused research. This demands for a focus on patient care and safety, based on high professional standards. EBP is the best way to achieve this desideratum, as it combines the best research evidence with clinical knowledge and expertise, addressing the patient in a holistic way. Since the radiographer is the ultimate interface between patient and technology, it is crucial to create a real teamwork concept, using guidelines for the roles and responsibilities of each team member as the pathway to minimise practice error, maximising patient and staff safety

Learning Objectives:

1. To understand the importance of the radiographer in the application of evidence-based practices.

2. To appreciate the need for systematic evaluation of health care technology.

3. To learn about the importance of critical assessment of research data and clinical guidelines.

\section{A-325 16:30}

B. Justification and optimisation in medical imaging

J. Santos; Coimbra/PT (joanasantos@estescoimbra.pt)

The use of ionising radiation in medical exposures is well documented across countries. Recent studies indicate that a substantial fraction (20 to $50 \%$ ) of these procedures is unnecessary when taking into consideration patient clinical indication. Defensive medicine, economic interest, poor patient clinical information, patient pressure and the lack of knowledge about other alternative procedures, taking into account the benefits and risks, are the principal causes of unnecessary radiological examinations. Patients must be referred to a medical imaging department for an examination that should be justified according to clinically appropriate image criteria and informed of the potential benefits and risks. Following justification, the optimisation of practice is essential to ensure the minimal exposure of radiation dose to the patient whilst diagnostic efficacy is maintained. The principal aim of optimisation is getting a narrower dose distribution, with lower mean and $75^{\text {th }}$ percentile values of the diagnostic reference level. Optimisation processes need to consider several factors including the type of examinations, body region, clinical information, available equipment technology and image processing tools. To implement this process, a multidisciplinary teamwork is essential where radiographers, being the pivot between patient and technology, have an essential role. To understand and apply optimisation procedures, radiographers should be aware of the international guidelines, standards and directives. All medical imaging procedures must be audited to analyse if the workflow processes (referral, justification, optimisation and radiation protection principles) are according to the recommendations to create a radiation protection safety culture.
Learning Objectives:

1. To learn about developing and consolidating approaches to justification in medical imaging.

2. To learn about current optimisation in medical imaging.

3. To become familiar with international recommendations, standards and directives.

\section{A-326 17:00}

C. Service development through evidence-based approaches

E. Metsala; Helsinki/FI (eija.metsala@metropolia.fi)

Although we are approaching the year 2014, a lot of clinical radiography is not based on best evidence. Lot of clinical decision-making is based on, for example, traditional practices that we cannot argue as being the best possible ones. In this presentation, barriers to using research and other sources of evidence and facilitators of evidence-based (EB) radiography are described. The role radiographers have in developing EB guidelines and procedures may vary according to educational level and work description. Developing EB guidelines is not the work of every radiographer, but obeying them is. The speaker gives some suggestions on what to do in a situation if there are no EB guidelines and procedures in ones' country or institution and no group that develops them. With the framework of "the new work order" and increasing implementation of new work processes, professionals are faced with new modes of collaboration requiring communicative skills as well as multiprofessional competencies between all the stakeholders across the hospital. Some evidence about the consequences of ineffective communication in the field of radiography is presented. In addition to benefits associated with the procedure of clinical audit, uniform good quality protocols for examinations and uniform national/international criteria help to perform comparisons between radiology units. Clinical audit also works as an educational tool and as a feedback tool for the staff on their performance. It also points out the need for corrective actions.

Learning Objectives:

1. To appreciate the challenges in implementing evidence-based approaches in the radiology department.

2. To appreciate the radiographers' role in developing clinical guidelines and procedures.

3. To understand the importance of enabling effective communication between stakeholders across the hospital.

4. To learn about the importance of audit practices as part of evidence-based service development.

16:00 - 17:30
EDiR Session
European Diploma in Radiology: and the
beat goes on!

A-327 16:00

Chairman's introduction: setting the scene for the European Diploma Y. Menu; Paris/FR (yves.menu@sat.aphp.fr)

Initiatives from professional organisations are probably the most powerfu engines for the construction of Europe, and in many cases open the road for legal and political advances. Many medical specialties felt that there was a need for the harmonisation and standardisation of medical training over Europe. The ESR has shared this opinion and has elaborated a unanimously appreciated training curriculum, which is constantly updated. Establishing the EBR (European Board of Radiology), with the mission to organise a European diploma was the logical next step. Today, 10 examinations have already been organised and more that 350 candidates from European countries and abroad have applied. The trainee who passes the examination proves his professional skills and has reached the objectives assigned in the curriculum. In this session, we will explain how ESR organises the diploma. We will review, capitalising on our current experience, the main lessons learnt from the past editions, the keys for success and reasons for failure, as seen by the examiners. We will also learn about the "Diploma adventure", from the point of view of a successful candidate.

Session Objectives:

1. To learn more about the organisation of the European Diploma (EDiR).

2. To appreciate the benefits of taking the examination.

3. To be informed about the experience acquired by the examiners and the candidates.

4. To understand the development of the EDiR in Europe and abroad. 


\section{A-328 16:05}

Who really needs a European Diploma in Radiology?

W. Schima; Vienna/AT (wolfgang.schima@khgh.at)

National radiology training programmes in Europe differ to a large extent, regarding length of training, content, and the presence or absence of a national board examination for certification. Thus, for cross-border radiologic activities, the level of training and expertise applying to radiologists is difficult to assess under these circumstances. There is an increasing number of medical subspecialties, which organise European diplomas. More than 20 subspecialties (e.g. anaesthesiology, ophthalmology, urology, etc). organise European examinations, which are well received among the respective communities. Growing numbers of residents in training successfully stand this test of knowledge and practical skills. Recognition of European diplomas by national authorities is an issue, which will increase the level of acceptance. The present status of the recognition process in European countries will be summarised.

Learning Objectives:

1. To learn why the European Diploma in Radiology is useful in the era of cross-border radiology and teleradiology.

2. To understand how EDiR contributes to the harmonisation of the training programmes across Europe and, at the same time, sets a standard of quality. 3. To appreciate the experience from other specialties, demonstrating that European diplomas are a general trend in medical specialties.

\section{A-329 16:25}

Learn from past mistakes: lessons to improve your preparation and performance

S. Barter ${ }^{1}$, L. McKnight ${ }^{2} ;{ }^{1}$ Cambridge/UK, ${ }^{2}$ Langland/UK (suebarter@btinternet.com)

Case material for the diploma will test knowledge in general radiology at a standard to be expected at the end of training as outlined in the ESR curriculum. All ten ESR-recognised subspecialty areas of the curriculum will be tested. The written exam comprises two computer-based sections, a multiple response section, and a short case section. There will be 75 multiple response questions. These questions comprise both multiple options format, a single stem with 5 answer options, and short imaging studies where candidates are asked to either annotate the image from a drop-down menu, or select the radiological features displayed or differential diagnosis from the options given. A special scoring system has been developed to prevent guessing. The short case section comprises 4 or 5 case-based questions. You may practice using the self-assessment tests on the ESR website which follow the same format as the written examination. For the oral cases, you must have a systematic method and perform under pressure, which takes practice. You may rehearse with experienced radiologists who feedback on your performance. You can break a radiology report into 3 areas, observations, interpretation and conclusion. Conclusions should take into account the clinical information. Candidates often forget this under pressure, so ask for it to be repeated. Your differential diagnosis should be logical and comprehensive. The 3 commonest causes of an abnormality is a good topic to revise. Suggest if further imaging tests are indicated and give an indication of urgency. Each case is marked separately.

Learning Objectives:

1. To learn how questions are formulated and selected for the written and oral examinations.

2. To become aware of some examples of the most common errors made by

the candidates

3. To learn the best approach to the oral examination and what examiners really expect from the candidates.

4. To learn some tricks that may help candidates prepare for EDiR.

Author Disclosure:

S. Barter: Board Member; Scientific Board EBR.

\section{A-330 16:55}

We made it! The Turkish Board of Radiology values EDiR

S.S. Özbek; Izmir/TR (sureyya.ozbek@ege.edu.tr)

As a well-known statement in the academic world of education, "assessment drives learning". With the help of carefully prepared and performed examinations, it is possible to emphasise and instill the training curriculum. Due to different reasons, training of radiology in European countries is highly varied. Like in our experience in Turkey, a central examination system, accompanied by a neatly prepared training charter and curriculum, is a good starting point to solve the problem. We believe that a common European assessment system like EDiR will contribute a great deal to the harmonisation of radiological training and establishment of quality standard in every country. The need for harmonising the training is not unique to radiology. This is why in more than 30 medical disciplines, European diploma examinations are now being organised. Furthermore, in some disciplines like urology, annual and online "In-Service Assessment" of residents and practising urologists allows the participants to evaluate their current knowledge base against the current European standards, and also provides the trainers and directors knowledge about the teaching and learning process in education centres. Although there are huge diversities among different European centres, the urgent need of harmonising radiology training and setting a standard of quality are felt by all radiologists in Europe. Establishing and institutionalising a common assessment system in European radiology appears to be the shortest way to reach this goal. This is why we should support "European Diploma in Radiology" by any means and work hard to promote its recognition throughout the continent.

Learning Objectives:

1. To learn the reasons why the Turkish Board of Radiology has decided to recognise the EDiR as equivalent to the first part of their national examination. 2. To understand how EDiR contributes to the harmonisation of training programmes across Europe and, at the same time, sets a standard of quality. 3. To appreciate the experience from other specialties, demonstrating that European diplomas are a general trend in medical specialties.

\section{A-331 17:15}

I did it! Lessons from a successful candidate

J. Muscat, A. Mizzi; Birkirkara/MT (jessica2860@gmail.com)

The purpose of this lecture is to outline a candidate's incentive for taking the exam, share my EDiR experience and discuss the EDiR's 'returns' from a candidate's point of view. Hailing from the small island state of Malta, local postgraduate training is a concept almost as new as the EDiR itself. The October 2012 sitting of the EDiR happened to fill in the gap just prior to the UK Final FRCR Part 2B I was preparing to take in the spring. Preparation for the EDiR went, quite simply, in tandem with that for the FRCR consisting of revision cases - online and in textbooks - of imaging-by-system and the vitally important "hot" seat sessions - mimicking the oral exam - with consultant radiologists. Another factor that influenced my taking the EDiR was undeniably the venue - the Journées Françaises de Radiologie in Paris. Though postgraduate training in radiology may vary, as aptly put by Prof Maximillian $\mathrm{F}$. Reiser, the aim of the EDiR is "the enhancement and harmonisation of radiological training in Europe": the EDiR can now be considered a potential European certification in radiology and as such an opportunity not to be missed!

Learning Objectives:

1. To understand the general atmosphere of an EDiR exam from the perspective of a candidate.

2. To understand the candidate's motivation for applying for the examination. 3 . To appreciate what the real and potential benefits of taking the examination are. 


\section{Sunday, March 9}


08:30 - 10:00

Room A

\section{Interactive Teaching Session}

$E^{3} 1220$

\section{Pitfalls in brain imaging}

\author{
A-332 08:30 \\ A. CT \\ L. van den Hauwe, J.W. Van Goethem, C. Venstermans, F. De Belder, \\ T. van der Zijden, M. Voormolen, P.M. Parizel; Antwerp/BE \\ (lucvdhauwe@mac.com)
}

The majority of emergency CT studies reported by junior radiologists or general radiologists out-of-hours comprise brain scans. Brain CT studies are often performed to detect acute life-threatening abnormalities, such as stroke, intraparenchymal or subarachnoid haemorrhage, cerebral edema, etc. Misrecognition of these often subtle, but life-threatening abnormalities can lead to inappropriate patient management and worsen patient outcome. Errors in interpretation can generally be categorised as either perceptual or cognitive in nature. Perceptual errors are those in which the radiologist does not see the abnormality, resulting in a false-negative interpretation (e.g. basilar artery thrombosis, deep cerebral venous thrombosis, PRES,...). Cognitive errors, on the other hand, are those in which an abnormality is identified but the meaning or significance of the abnormality is not recognised. Cognitive errors can result in false-positive interpretation if, for example, a normal anatomic variant is mistaken for a pathologic condition. While the more common normal variants and artefacts often do not present a problem to experienced clinicians and neuroradiologists, less experienced individuals should beware of these diagnostic hazards. To reduce false-negative and false-positive reporting, a checklist of the review areas (blind spots) to be verified on any brain CT scan will be offered and the most frequent normal variants will be discussed.

Learning Objectives:

1. To learn about common pitfalls in CT evaluation of the brain.

2. To become familiar with anatomical variants potentially mimicking disease

A-333 09:15

B. MRI

D. Prayer; Vienna/AT (daniela.prayer@meduniwien.ac.at)

Pitfalls in brain imaging comprise perceptual or cognitive errors of interpretation of imaging findings. Errors of perception refer to a missing of a pathology that is present on an image, while cognitive errors consist of a wrong interpretation of a seen structure or pathology. Interpretation may be false negative or false positive. In addition, a wrong conclusion may be drawn resulting from missing, incomplete or false clinical information, or as a consequence of a lack of knowledge of differential diagnoses. Perception errors are frequently the consequence of incomplete examination protocols where lesions are missed (for instance, acute ischaemic changes without the use of diffusion-weighted Imaging) or signals, for instance, flow-related phenomena are misinterpreted. Asymmetric positioning of the patient's head may also lead to misinterpretation of side differences, especially in the temporal lobe. Another source of perception errors might be reduced attention (especially at night shifts) and have been proved to occur in regions that are "potential blind spots", as the sulci, cavernous sinus, Meckel cave, dural sinuses, brainstem, and skull base foramina. Perceptual errors may be avoided by following a checklist when reading images. All sequences should be used for comparison of signal intensities to distinguish artefacts from pathology. Another very important fact to avoid misinterpretation lies in a profound knowledge of morphologic and metabolic age-related changes of the brain and of anatomical normal variants (such as, for instance, accentuated Virchow Robin spaces in certain locations)

Learning Objectives:

1. To learn about common pitfalls in MRI evaluation of the brain.

2. To become familiar with anatomical variants potentially mimicking disease.
08:30 - 10:00

Room B

\section{Abdominal Viscera}

RC 1201

The many faces of benign liver lesions

Moderator:

A. Ba-Ssalamah; Vienna/AT

A-334 08:30

A. Vascular

M. Karcaaltincaba; Ankara/TR (musturayk@yahoo.com)

Benign hypervascular focal liver lesions include haemangioma, focal nodular hyperplasia, hepatic adenoma, angiomyolipoma, nodular regenerative hyperplasia and haemangioendothelioma. Tips for differentiation of benign and malignant hypervascular liver lesions will be presented. US, CT and MR findings will be summarised with emphasis on typical and atypical findings. Liver imaging is important for noninvasive diagnosis of hypervascular benign liver lesions and confidence in diagnosis can obviate biopsy in routine clinical practice.

Learning Objectives:

1. To become familiar with typical and infrequent manifestations of benign hypervascular focal liver lesions.

2. To learn how to differentiate between benign and malignant lesions.

3. To appreciate the limitations and complementary roles of CT and MR.

\section{A-335 09:00}

B. Cystic

G. Brancatelli; Palermo/IT (gbranca@yahoo.com)

Cystic liver lesions can be classified based on their nature as benign and malignant. In the benign category are, among others, developmental and infectious/inflammatory cysts, while neoplastic cyst can be subdivided into primary and secondary. Developmental cysts originate from abnormal ductal plate malformation and consist of hepatic (bile duct) cyst, bile duct hamartomas, Caroli's disease and polycystic liver disease. Infectious/inflammatory cysts include, among others, abscesses (pyogenic and amoebic) and hydatid cysts. Primitive neoplastic cystic lesions are cystoadenoma and cystoadenocarcinoma. Secondary lesions can originate mostly from mucinous tumours such as colon and ovary. The role of crosssectional imaging in the detection and characterisation of these entities will be discussed, with an emphasis on the differential diagnosis with CT and MR Imaging.

Learning Objectives:

1. To understand the features of congenital and infectious cystic liver lesions.

2. To learn how to differentiate between benign and malignant cystic lesions.

Author Disclosure:

G. Brancatelli: Speaker; Bayer.

A-336 09:30

C. Hepatocellular

F. Caseiro-Alves; Coimbra/PT (caseiroalves@gmail.com)

Benign hepatocellular neoplasms are being recognised with increased frequency using cross-sectional imaging. One of the main goals is to be able to make a clear-cut differential diagnosis between focal nodular hyperplasia $(\mathrm{FNH})$ and hepatocellular adenoma $(\mathrm{HCA})$ since patient management is substantially different. Despite the sound knowledge acquired in the last few years about the morphological features of FNH using various cross-sectional imaging techniques, new players in the field have arisen such as diffusionweighted MR imaging (DWI) and use of hepatobiliary contrast agents. These new biomarkers offer a different view over $\mathrm{FNH}$ and allow a more accurate characterisation even in more atypical cases. Concerning HCA, current knowledge implies that the sub-types of this neoplasm should be known since, again, patient management differs and these patients are no longer seen as compulsory surgical candidates. These sub-types will be further discussed and the role of imaging for risk stratification (haemorrhage and malignant transformation) will be addressed. Further, illustrative cases of combined $\mathrm{FNH} / \mathrm{HCA}$ cases will be demonstrated along with the main imaging clues for a successful differential diagnosis. 


\section{Postgraduate Educational Programme}

Learning Objectives:

1. To understand the typical aspect of hepatocellular benign lesions on US, CT and MRI

2. To learn when a liver specific contrast medium can help us in the proper characterisation of hepatocellular benign liver lesions.

3. To understand the classification of liver adenomas, prognosis and imaging characteristics.

Author Disclosure:

F. Caseiro-Alves: Advisory Board; Euromedic. Consultant; Bayer Schering.

08:30 - 10:00 Room C

Modern Imaging of the GI Tract

CC 1221

\section{Imaging the postoperative patient}

Moderator:

M. Hellström; Gothenburg/SE

A-337 08:30

A. What the surgeon does: surgical procedures and normal postoperative anatomy

M.M. Maher; Cork/IE (m.maher@ucc.ie)

In most upper and lower gastrointestinal surgical units, imaging is frequently required in patients suspected of post-operative complications or in whom recovery would appear delayed. Interpretation of these studies can be challenging and requires an appreciation of expected imaging appearances of the post-operative abdomen as well as the anatomy of common surgica procedures. Imaging findings in the post-operative abdomen can vary significantly, depending on the surgical procedure performed and this can result in pitfalls for the radiologist.

Learning Objectives:

1. To understand common surgical procedures performed in the esophagus, stomach, small bowel and colon.

2. To appreciate the normal appearance of an enteric anastamosis on

fluoroscopic and cross-sectional imaging

3. To understand the normal appearances of haemostatic packing and other surgical devices on radiological investigations.

\section{A-338 08:50}

B. Do I need to re-operate? Postoperative imaging and immediate complications

D.J.M. Tolan; Leeds/UK (djmtolan@doctors.org.uk)

Imaging in the early postoperative period is complex. It is essential for radiologists to know the new anatomic arrangement after surgery and to understand the range of normal postoperative appearances. This latter aspect can be particularly difficult, because the radiological findings are affected by the type of operation performed (open vs. laparoscopic) and the complexity of the surgery (such as the presence of adhesions or haemorrhage) as well as the underlying comorbidity of the patient. Patients may be considered in 4 broad categories: haemodynamically unstable patients who may be bleeding patients with distension or unopened bowels who may have obstruction or ileus; patients with sepsis in whom to search for an intraabdominal collection or leak; and patients who do not follow an expected recovery for which the cause is unclear. CT is the primary imaging technique, with ultrasound reserved predominantly for liver and renal transplants or assessment of perihepatic collections, and fluoroscopy for routine postoperative assessment of upper and lower GI anastomoses. CT protocols should be optimised to detect particular complications with positive luminal contrast for assessment of anastomotic leaks $(8 \%)$, triple phase imaging for bleeding (without positive oral contrast) and delayed phase imaging to detect ureteric injury. Since each operation has a specific range of expected complications, these should be considered when deciding on the protocol and in light of the patient's clinical status. Excellent communication with the surgical team is required to make an accurate diagnosis and in particular where haemostatic compound or mesh for hernia repair was used.

Learning Objectives:

1. To understand the best imaging options for investigating suspected postoperative complications.

2. To appreciate the normal appearances of the abdomen and pelvis after surgery, in particular with regard to free fluid, haematoma and free gas, and when to suspect complications.

3. To become familiar with the radiological appearances of common and more unusual post-operative complications and their interventional radiological management.

Author Disclosure:

D.J.M. Tolan: Equipment Support Recipient; Vital Imaging. Speaker; Bracco.

\author{
A-339 09:10 \\ C. It still hurts: follow-up imaging and long-term complications \\ L. Curvo-Semedo; Coimbra/PT (curvosemedo@gmail.com)
}

Radiologists should be aware of the newer surgical techniques and expected post-operative alterations, to yield a correct interpretation of a post-surgery imaging examination, thus being able to differentiate a normal post-operative finding from a potential complication. In this setting, it is crucial to perform a technically adequate imaging examination, so that post-operative anatomical and functional findings may be evaluated. Therefore, the aim of this lecture is to give an overview of the contribution of various imaging modalities in the diagnosis of late post-operative complications following GI tract surgery. It will include a mention of the technical issues that need to be considered to achieve better diagnostic accuracy, as well as a description and illustration of the main imaging findings of late post-operative complications. Those include diseaserelated (recurrence for malignancy or inflammatory disease) and procedurerelated (anastomotic strictures, internal herniation, adhesions and intussusception, among others) complications. Focus will be placed on crosssectional imaging techniques, which at present constitute the workhorse for detecting and characterising late post-operative complications after $\mathrm{Gl}$ tract surgery. In this way, imaging has an obvious and pivotal role in planning therapy, since some late complications are usually treated in a conservative way while others require a surgical approach.

Learning Objectives:

1. To become familiar with the long-term complications of surgery to the $\mathrm{GI}$ tract.

2. To appreciate the appropriate use of imaging in the assessment of long-term complications.

3. To learn about the radiological investigation of suspected postoperative adhesions.

A-340 09:30

D. Interactive case discussion

M. Hellström; Gothenburg/SE

08:30 - 10:00

Room D

Chest

RC 1204

\section{Lung cancer: staging and personalised imaging in 2014 \\ Moderator:}

E.J.R. van Beek; Edinburgh/UK

A-341 08:30

A. Lung biopsy: who, how and why

F. Laurent; Pessac/FR (francois.laurent@chu-bordeaux.fr)

Lung biopsy of a suspected lung cancer remains a well-established technique for histopathologic examination in patients with suspected lung cancer. Complications can nevertheless occur and therefore indication should be given after carefully balancing the benefits and drawbacks for a particular patient. The method should be clearly considered complementary to alternate methods of tissue sampling, fiberoptic endoscopic, mediastinoscopy or US-guided endoscopy, surgical biopsies and resection during a multidisciplinary discussion. Several steps are needed in the preparation of the biopsy procedure, including informed consent, management of anticoagulation and anti-platelet medications, imaging assessment of the lesion and its environmental lung, and planing of the needle trajectory procedure. The choice of the guiding method and the most appropriate biopsy needle is still largely dependent on local skills and habits. CT-guided percutaneous biopsies using coaxial automated core biopsy needles offer many advantages. Needle manipulation can help to reach difficult targets. Participation of the patient is needed in terms of respiratory manoeuvres and breathhold. Complications may occur such as pneumothoraces haemorrhage and air embolism. Knowledge of the respective risk factors and the potential method of prevention or treatment by operators are essential.

Learning Objectives:

1. To learn when biopsy is necessary in patients with lung cancer.

2. To understand the importance of molecular diagnostic testing in lung cancer.

3. To appreciate the different techniques available for obtaining a histological

diagnosis in patients with lung cancer 


\section{A-342 09:00}

\section{B. Pre and post-treatment tumour assessment}

N. Tacelli; Brussels/BE (ntacelli@gmail.com)

The therapeutic response of lung cancer can be objectively evaluated on the basis of changes in the tumoural size or depiction of metastases, such proposed in the RECIST criteria (Response Evaluation Criteria In Solid Tumours). Recent advances in cancer biology have triggered development of novel targeted therapies designed to disrupt specific biologic pathways. Among them, antiangiogenic drugs represent a promising strategy for non-small cell lung carcinomas (NSCLC). These agents are more cytostatic rather than cytotoxic, explaining the limitations of tumour response assessment based on morphological criteria. Dynamic contrast-enhanced imaging techniques have the potential to quantify tumoural angiogenesis. In particular, dynamic contrastenhanced multidetector-row CT (DCE-CT) represents a promising tool to provide non-invasive and repeatable assessment of the angiogenic process within non-small cell lung cancers (NSCLC), offering the possibility to generate morphological and functional information from the same examination. DCE-CT allows the calculation of regional tumour blood flow, blood volume, flowextraction product, and permeability-surface area product, over the entire tumoural volume, using mathematical models and dedicated softwares. DCECT can depict early changes in lung cancer vascularity, before tumour shrinkage, that could help predict response to antiangiogenic drugs. Becoming familiar with this technique enables the radiologist to integrate it into clinical practice and to assist the thoracic oncologist in the pre-therapeutic and follow-up evaluation of NSCLC patients treated by antiangiogenic drugs. This new approach should improve patient's selection for targeted therapies.

Learning Objectives:

1. To learn about the practical approach for assessing disease response in patients with lung cancer.

2. To become familiar with new therapies in lung cancer.

3. To appreciate new techniques such as perfusion CT in assessing disease prognosis and response in lung cancer.

\section{A-343 09:30}

\section{The role of PET-CT and whole body MRI}

F. Gleeson; Oxford/UK (fgleeson@mac.com)

It is now accepted that PET-CT is of value in the staging of lung cancer and reduces the number of unnecessary thoracotomies. Its role has been further clarified by recent publications on its value in assessing mediastinal nodal involvement, tumour aggressiveness using either standardised uptake value or total glycolytic volume, and response to neoadjuvant chemotherapy. More recently, MRI has been investigated as a tool to detect distant disease using diffusion-weighted imaging, and there are a number of trials that have compared both PET-CT and DWI MRI retrospectively and prospectively. This presentation will summarise the current available data and the likely benefits of either imaging modality

Learning Objectives:

1. To learn about the role of PET-CT in staging and determining prognosis in lung cancer.

2. To appreciate the role of PET-CT in determining disease response in lung cancer.

3. To become familiar with the potential use of whole body MRI and DWI in lung cancer.

\section{Paediatric}

\section{RC 1212}

\section{Paediatric emergencies}

Moderator:

J.-P. Van Nieuwenhuyse; Luxembourg/LU

\section{A-344 08:30}

A. The acute non-traumatic neurological patient: CT or MRI?

E. Vázquez; Barcelona/ES (evazquez@vhebron.net)

Neurological deficits in children are an urgent condition that depends significantly on imaging for a prompt and accurate diagnosis because of the significant overlap present in clinical history, presentation and neurological examination. Different imaging modalities, such as ultrasound, computed tomography (CT) or magnetic resonance imaging (MRI), are utilised dependent on the age of the patient and neurological symptoms. The purpose of the present lecture is to discuss the causes and imaging appearance of acute neurological conditions in childhood, broadly classified into stroke, infection, inflammatory demyelination, metabolic disorder, cerebral neoplasm or neurotoxicity. Imaging also plays an outstanding role in the management of paediatric oncology patients presenting with acute neurological symptoms related to disorders of blood cell counts, immunosuppression, neurotoxicity of therapy, or progression of the primary malignancy. A review of the main indications to perform a neuroimaging procedure in these children will be undertaken as well as a differential diagnosis based on representative cases selected from the daily routine in a paediatric tertiary hospital. Practical algorithms with the preferential use of either CT or MRI will be developed for each section. CT continues to be the first imaging modality in these patients in many centres, despite the accompanying radiation, because of the easy availability and no requirement of sedation. However, MR imaging is nowadays better for imaging these children owing to no radiation, the more completely provided information and the useful advanced techniques that can be used such as diffusion imaging (DI), spectroscopy, arterial spin labelling (ASL), and susceptibility weighted imaging (SWI).

Learning Objectives:

1. To learn about the currently limited role of $C T$ in the non-traumatic acute setting.

2. To become familiar with radioprotection strategies and protocols adapted to children.

3. To consolidate knowledge on the role of MRI as the modality of choice for acute non-traumatic neurologically ill children, with an emphasis on newer techniques.

4. To become familiar with imaging findings and the main differential diagnosis of acute neurological conditions in children.

\section{A-345 09:00 \\ B. Imaging of acute chest pain and distress in children \\ C.E. de Lange; Oslo/NO (clange@ous-hf.no)}

Acute chest pain in children is a common complaint in the emergency department, but patients rarely present with significant distress or lifethreatening symptoms requiring immediate care or resuscitation. The most frequently reported cause is benign musculoskeletal pain followed by respiratory and gastrointestinal causes, while cardiac causes are less frequent. A thorough clinical history and careful physical examination will determine, in most cases, the patients in need of further investigation to establish a diagnosis. In this regard, radiology plays an important role, especially in the emergency setting in patients with more serious associated symptoms like acute breathing difficulties, swallowing problems, fever or sepsis. When choosing the appropriate technique for investigation, the consequences of radiation exposure in children must be considered. Plain radiography and fluoroscopy still remain the most important and frequently used tools to gain information on various acute chest/pulmonary problems. Ultrasonography is the first choice for diagnosis/treatment of pleural effusions. Multidetector computed tomography and magnetic resonance imaging are mainly used for investigating pulmonary/mediastinal masses and congenital abnormalities of the great vessels and the lungs. This lecture will discuss the choice of imaging technique and the urgency of radiological management depending on the symptoms and age of the patient. The imaging characteristics of the different causes of acute chest pain and/or distress in children will be reviewed, represented by the more common conditions involving the chest wall, respiratory tract, oesophagus and the heart, as well as less frequent causes such as tumours, manifestations of congenital malformations and nonaccidental trauma

Learning Objectives:

1. To understand the role of radiographs, US, CT and MRI.

2. To become familiar with the most common conditions that cause acute chest pain and distress in children.

3. To appreciate the different differential diagnoses, depending on the age of the patient.

4. To understand the consequences of delaying their diagnosis and treatment.

A-346 09:30

C. The role of the interventional radiologist in paediatric trauma

J.B. Karani; London/UK (john.karani@nhs.uk)

Outcome data from trauma series have demonstrated improved patient survival by the prompt diagnosis of a range of injuries and the recognition of life-threatening sequels, principally that of active arterial haemorrhage. Widely used trauma scoring systems are applicable in the paediatric population, although the mechanisms and combination of injuries may differ from those encountered in adults. A CT diagnosis of active haemorrhage demands early operative or non-operative intervention with embolisation or use of alternative endovascular techniques including deployment of vascular stents. This principle is applicable to blunt or penetrating liver, splenic, renal or pelvic trauma. It is also applicable in some cases to severe mediastinal and limb vascular injury. Important caveats are firstly the use of CT in an overall trauma service and avoidance of any inappropriate irradiation. Secondly, in paediatric interventional practice, due regard needs to be given to the delivery of implantable devices that may have adverse sequels in later life. Nevertheless, the demand for immediacy in intervention may outweigh any such 
consideration. This presentation will summarise the importance of imaging and intervention in both the early and delayed complications of trauma with an emphasis on abdominal and thoracic trauma.

Learning Objectives:

1. To understand the role of CT, CTA and conventional angiography in the diagnosis of traumatic vascular complications.

2. To understand the importance of vascular and non-vascular intervention following trauma in children.

08:30 - 10:00

Room E1

\section{Musculoskeletal}

RC 1210

\section{Peripheral nerve imaging: MRI and US}

Moderator:

M. Maas; Amsterdam/NL

\section{A-347 08:30}

A. Anatomy and pathology of the brachial plexus

S. Gerevini; Milan/IT (gerevini.simonetta@hsr.it)

Brachial plexus originates from the ventral branches of the cervical nerve roots from C5 to T1. It is composed of roots, trunks, divisions, cords, and branches, topographically divided into supraclavicular (roots and trunks), retroclavicular (divisions), and infraclavicular sections (cords and branches). MRI has the advantages of multiplanar imaging, high tissue contrast, and relative paucity of artefacts. The T1-weighted sequences display topographic anatomy including muscles, blood vessels, and nerves stressed by tissue fat planes. The nerves appear as elongated fibres that are isointense to the scalene muscle, posteriorly and superiorly to the curvilinear flow void of the subclavian artery. The fat-suppressed PD and T2-weighted and STIR sequences detect signal alterations of the BP. General abnormal findings include: loss of fat planes around part or all BP components, nerve signal abnormalities with mild or marked hyperintensity on T2-weighted images that can be associated with swelling, focal or extensive enhancement of the nerves after Gad injection on T1 fat sat images. Differences in signal intensity should be judged by visual inspection, which is susceptible to a subjective point of view; the comparison of both sides of BP has a relevant role in judging the presence of signal alterations, especially when they are smooth and/or focal. The pathology of brachial plexus can be divided into nontraumatic and traumatic brachial plexopathies. Among nontraumatic brachial plexopathies, we find radiation fibrosis, metastatic breast cancer and primary or metastatic lung cancer. Other pathologies are tumours and inflammatory disease or thoracic outlet disease. Learning Objectives:

1. To understand the anatomy of the brachial plexus as seen with MRI

2. To appreciate the range of pathology seen at the brachial plexus.

3. To become familiar with the MRI findings of brachial plexus pathology.

\section{A-348 09:00}

B. Upper limb nerve entrapment

D. Weishaupt; Zurich/CH (dominik.weishaupt@triemli.zuerich.ch)

The peripheral nerves of the upper limb are affected by a number of entrapment and compression neuropathies. These syndromes involve the brachial plexus as well the musculocutaneous, axillary, subscapular, ulnar radial and median nerves. Clinical examination and electrophysiological studies are traditionally the mainstay of diagnostic workup. However, ultrasonography and magnetic resonance imaging (MRI) may provide key information about the exact anatomic location of the lesion or may help to narrow the differential diagnosis. In certain patients with the diagnosis of a peripheral neuropathy, imaging using either ultrasonography or MRI may help establish the cause of the condition and provide information crucial for conservative management or surgical planning. In addition, imaging is particularly valuable in complex cases with discrepant nerve function test results.

Learning Objectives:

1. To become familiar with the strengths and weaknesses of US and MRI for assessing upper limb nerves.

2. To appreciate the imaging findings of upper limb nerve entrapment.

\section{A-349 09:30}

C. Lower limb nerve entrapment

C. Martinoli; Genoa/IT (carlo.martinoli@libero.it)

A variety of peripheral neuropathies can be encountered in the lower limb. Most are entrapment syndromes affecting many nerves, such as the sciatic, gluteal, femoral, lateral femoral cutaneous, obturator and pudendal around the hip, the peroneal and its branches and the saphenous at the knee, the superficial peroneal at the lateral leg, the tibial with its plantar and calcaneal branches at the ankle, the deep peroneal and the interdigital nerves in the foot Although clinical examination and nerve conduction studies are the mainstay of the diagnostic workup of peripheral neuropathies, ultrasound (US) and magnetic resonance (MR) imaging may provide key information about the exact anatomic location of a lesion and the nature of the constricting finding or may help narrow the differential diagnosis. In patients with peripheral neuropathies of the lower extremity, US and MR imaging may provide critical information for planning an adequate treatment strategy. Although US and MR imaging have followed parallel paths for nerve imaging with little comparison of the two modalities, US seems to have some advantages over MR imaging, including higher spatial resolution, time effectiveness, the ability to explore long nerve segments in a single study and to examine tissues in both static and dynamic states.

Learning Objectives:

1. To become familiar with the strengths and weaknesses of US and MRI for assessing lower limb nerves.

2. To appreciate the imaging findings of lower limb nerve entrapment.

$\begin{array}{ll}08: 30-10: 00 & \text { Room E2 }\end{array}$

\section{Neuro}

\section{RC 1211}

\section{Stroke: diagnosis, mimics and treatment}

\section{A-350 08:30}

Chairman's introduction

B.F. Schuknecht; Zurich/CH (image-solution@ggaweb.ch)

Advances in diagnosis of acute stroke have been achieved by brain imaging. CT or MRI distinguish ischaemia from haemorrhage and may identify acute clot (dense artery sign) or vessel wall pathology by "black blood MRI". DWI provides evidence of early infarction. CT and MRA enable identification of large vessel pathology as potential source of embolic or haemodynamic ischaemia. Supplementary techniques are CT or MR perfusion. On MRI perfusion, a mismatch between the area of restricted diffusion and perfusion (PWI) is a signature of the penumbra. The tissue of PWI restriction outside of the DW abnormality represents "tissue at risk". Similarly on CT perfusion, the region of reduced cerebral blood volume presumably represents irreversible infarction and the area of reduced CBF and extended MTT visible beyond the cerebra blood volume abnormality represents potentially reversible ischaemia Morphologic imaging and advanced standardised perfusion/penumbral stroke protocols aim at identifying lesions that are amenable to acute stroke therapy and exclude pathologies that mimic stroke but do not represent vascular disease. Since iv tPA has become an approved therapy from 1996, the number of acute stroke patients entitled for treatment has remained limited $(\sim 5)$ despite extension of the time frame from 3 to $4.5 \mathrm{~h}$. DWI-FLAIR mismatch and an individualised assessment of the ischaemic penumbra may serve in the identification of patients within the appropriate time frame. By selection of patients with advanced brain imaging and initiation of appropriate therapy, a further increase in good outcomes and reduction of the incidence of symptomatic haemorrhage may be achieved.

\section{A-351 08:35}

A. Imaging acute stroke: value of CTA and perfusion

P.M. Parizel, C. Venstermans, F. De Belder, T. Van der Zijden,

J.W. Van Goethem, L. van den Hauwe, M. Voormolen; Antwerp/BE (paul.parizel@uantwerpen.be)

In recent years, the role of imaging in the patient admitted with an acute neurologic deficit has changed significantly, due to the arrival of new treatments for acute stroke, aimed at re-establishing blood flow, reducing infarct size and protecting the brain at risk. The first goal is to differentiate haemorrhagic from ischaemic stroke and to rule out other stroke mimics. This can be achieved by performing nonenhanced CT scan of the brain. The next strategic imaging objectives include: demonstration of major blood vesse occlusion (e.g. by CT angiography) and identification of potentially salvageable brain tissue, the so-called "penumbra" (e.g. by CT perfusion). CT angiography is performed during rapid intravenous bolus injection of a high-concentration iodinated contrast agent. The FOV should cover not only the intracranial arterial circulation, but also the arteries of the neck, to exclude dissection. CT perfusion is also achieved by bolus injection of contrast, followed by a series of fast images or volume acquisition. CT densities change over time and reflect the iodine concentration. Perfusion images are processed to generate parametric maps, which reflect: regional cerebral blood flow ( $\mathrm{rCBF})$, blood volume (rCBV), mean transit time (MTT), and time to peak (TTP). The combination of $\mathrm{CT}$ angiography and $\mathrm{CT}$ perfusion provides a unique insight into the pathophysiology of the cerebral circulation. These techniques are now 
essential tools in the management of acute stroke and in selecting those patients who are potential candidates for advanced therapies such as thrombolysis or thrombectomy.

Learning Objectives:

1. To become familiar with a comprehensive imaging protocol in patients with suspected stroke.

2. To understand the advantages and limitations of CTA and perfusion in the initial work-up of stroke patients.

3. To recognise the different imaging patterns in stroke and their prognostic value.

\section{A-352 08:58}

\section{B. Stroke mimickers and pitfalls}

P. Vilela; Lisbon/PT (ferrovilela@sapo.pt)

"Stroke mimic" is classically used to describe nonvascular diseases that present with acute focal neurologic deficit corresponding to a consistent vascular distribution, which may resemble or may even be indistinguishable from stoke. It is estimated that up to $15 \%$ of stroke-like presentations are due to mimickers. There are several neurological and psychiatric disorders that can have such a clinical presentation, such as seizures and/or postictal status, the most frequent one. The other classical clinical stroke mimickers include: multiple sclerosis, migraine (specially hemiplegic migraine), metabolic disturbances (more commonly hypoglycaemia/hyperglycaemia), intracranial tumours or infections and the conversion reactions. Brain imaging plays a key role in the diagnosis and management of acute stroke by differentiating ischaemic from haemorrhagic lesions, identifying the vessel occluded and estimating the viable brain tissue that is at risk for stroke. Imaging is also important to exclude some of the aforementioned stroke mimics. The more comprehensive stroke imaging protocols, with CT perfusion/angiography and/or with $\mathrm{MRI}$, have reduced significantly the misdiagnosis of stroke. MRIDWI is the most sensitive and specific imaging modality for depicting ischaemic stroke. However, it is also essential to be aware that there are some pathological processes that can mimic stroke in imaging studies. These include some disorders that may present themselves with lesions associated with restricted diffusion, like transitory postictal brain abnormalities, migraine and encephalitis. The author reviews the most common clinical and imaging stroke mimics and highlights the importance of brain imaging in depicting these mimickers and avoiding the potential adverse effects of stroke therapy in these patients.

Learning Objectives:

1. To learn the differential diagnosis of stroke.

2. To understand the role of CT and MRI in the work-up of stroke and related disorders.

3. To recognise imaging patterns that may mimic stroke clinically and radiologically.

\section{A-353 09:21}

C. Treatment: only intravenous?

L. Pierot, S. Soize, S. Emeriau; Reims/FR (Ipierot@chu-reims.fr)

Imaging plays a central role in the management of stroke, which is an important health issue according to the number of patients involved and the severity of the disease. It helps to select patients who will benefit from a revascularisation therapy (IV thrombolysis or endovascular treatment) at the acute phase of stroke. If the value of perfusion/diffusion MRI mismatch in the evaluation of penumbra is still a matter of debate, other imaging factors are associated with the clinical outcome including length of clot, Flair positivity, and collateral circulation. If IV thrombolysis using rtPA is still the reference treatment, endovascular treatment is emerging since several years as a feasible and efficacious alternative singularly since the appearance of stent retrievers. Recent neutral randomised trials comparing IV thrombolysis and endovascular treatment have shown the critical role of appropriate imaging in the selection of patients who can benefit from acute revascularisation as well as the importance of using the most efficacious endovascular devices. Prevention of stroke is partially based on the treatment of cervical and intracranial stenosis. Several randomised trials comparing carotid angioplasty and carotid endarterectomy were initially negative, but a recent meta-analysis showed that below the age of 70 years both techniques were equivalent. The place and value of endovascular treatment of intracranial arterial stenosis is still controversial after the publication of the SAMMPRIS results.

Learning Objectives:

1. To understand how imaging can help select patients for intravenous treatment of acute ischemic stroke.

2. To understand what the current indications are for the endovascular treatment of acute ischemic stroke.

3. To understand the current role of the endovascular treatment in the prevention of stroke.

Author Disclosure:

L. Pierot: Consultant; Codman, Covidien/EV3, Microvention, Penumbra, Sequent.
Panel discussion:

What is the future of stroke prevention and treatment?

$09: 44$

08:30 - 10:00

Room F1

Special Focus Session

SF 12

\section{Imaging of thyroid nodules}

A-354 08:30

Chairman's introduction

T. Beale ${ }^{1}$, S. Colley ${ }^{2} ;{ }^{1}$ London/UK, ${ }^{2}$ Birmingham/UK

(Steve.Colley@uhb.nhs.uk)

The management of thyroid nodules has been continuously evolving. I hope that during this session you will understand the important differences in the upto-date national and international guidelines on thyroid nodule management and understand the role of the radiologist in the multidisciplinary thyroid meetings. The features that are suggestive of benign, indeterminate and malignant nodules will be demonstrated. Advances in both radiological and cytological techniques such as US elastography and BRAF mutation analysis that may help triage patients with thyroid nodules will be covered. The differing biopsy techniques (fine needle aspiration, non-aspiration (FNA and FNNAC) and trucut biopsy) and their roles will be highlighted. There will be time for an open question and answer discussion with all the speakers before the end of the session.

Session Objectives:

1. To become familiar with the management of thyroid nodules.

2. To learn about the imaging features suggestive of both benign and malignant thyroid nodules.

3. To learn how to optimise imaging of thyroid nodules and the role of newer techniques such as US elastography.

4. To understand which nodules require FNA/biopsy.

A-355 08:35

Management

T. Beale ${ }^{1}$, S. Colley ${ }^{2} ;{ }^{1}$ London/UK, ${ }^{2}$ Birmingham/UK

(Steve.Colley@uhb.nhs.uk)

The management of thyroid nodules and the guidelines of the various interested parties, surgeons, endocrinologists, nuclear medicine physicians, radiologists and ultrasound specialists, vary across the world, indeed between different countries within the EU, and are constantly evolving. I will discuss how the thyroid multidisciplinary meeting (MDT) works at our institution and the role of the radiologist in it. The current differing international guidelines on the management of thyroid nodules will be mentioned, highlighting their salient points and differences. The role of the differing imaging modalities used in the management of thyroid nodules will be discussed based on differing clinical scenarios. Finally, the management of the common clinical scenario of the incidental thyroid nodule will also be discussed.

Learning Objectives:

1. To understand the role of the radiologist as part of a multidisciplinary team.

2. To learn about current guidelines on the management of the thyroid nodule.

3. To understand the role of various imaging modalities in the patient with thyroid nodule.

A-356 08:58

US and US elastography

A.S. McQueen; Newcastle upon Tyne/UK (andrewmcqueen7@hotmail.com)

Thyroid disease is ubiquitous and usually benign; therefore accurate imaging of the thyroid is a major challenge for radiology. Significant advances in conventional ultrasonography allow superb visualisation and anatomic characterisation of thyroid pathology and the impact of broader bandwidths, higher frequencies, spatial compound imaging and novel signal processing techniques will be discussed in this session. The key B mode features of thyroid nodules are discussed with reference to relevant clinicoradiological guidelines, specific pitfalls and limitations. A significant number of nodules remain indeterminate after conventional assessment, and imaging advances that enable more accurate, non-invasive thyroid characterisation are highly attractive, potentially reducing unnecessary biopsy and surgery for a large number of patients. Ultrasound elastography offers a potentially useful adjunct to conventional sonography in thyroid disease with a growing evidence base indicating that thyroid malignancies are typically stiffer than benign lesions and that a 'soft' cutoff value with a high negative predictive value is feasible. However, elastography studies have included a variety of different techniques, 
patient/nodule selection and assessment methods; therefore the current role of elastography will be discussed.

Learning Objectives:

1. To become familiar with the US features of thyroid pathology.

2. To understand the role of US elastography in thyroid nodules.

3. To consolidate knowledge on pitfalls in the US assessment of thyroid nodules.

\section{A-357 09:21}

Fine needle biopsy of the thyroid nodule

F. Campoy-Balbontín, M.C. Jurado-Gomez; Sevilla/ES

(fcampoy2000@gmail.com)

Thyroid nodules are very common. The clinical importance of thyroid nodules rests with the need to exclude thyroid cancer, which occurs in 5-15\%. Ultrasound (US) has been widely attempted to differentiate benign from malignant nodules and to guide fine needle biopsy (FNB). However, considerable overlap between benign and malignant characteristics has been found. Nowadays, there are a number of different guidelines for the management of thyroid nodules. At our hospital, we have adopted the guideline proposed by the Society of Radiologist in Ultrasound (2005). The different elements necessary to perform the FNB procedure are demonstrated; the transducer probe, the needles, the slides, the syringe. The way of managing these elements is shown by figures and videos, with different skills and performances to optimise the procedure and get the best sample. UScontrol FNB can be in parallel or perpendicular, both methods are demonstrated. Thyroid FNB is usually non-aspirated, but sometimes it has to be undertaken with aspiration; when and how to change from one method to another is explained. With good technique, the insufficient cytological rate can be significantly reduced, with which the need for core biopsy diminishes. Although the complication rate for core biopsy is low, FNB is safer and if performed correctly has a high diagnostic yield. Core biopsy should be limited to those nodules with a second insufficient FNB cytological result, with US findings suspicious for malignancy.

Learning Objectives:

1. To understand when it is necessary to perform a fine needle biopsy of the thyroid nodule.

2. To understand the different techniques used to perform thyroid $\mathrm{FN}$ and how to optimise them

3. To learn when a biopsy may be required

Panel discussion:

What is the role of the radiologist in the management of thyroid nodules? 09:44

08:30 - 10:00 Room F2

Breast

RC 1202

\section{Multimodality breast imaging}

A-358 08:30

Chairman's introduction

M. Lesaru; Bucharest/RO (mlesaru@hotmail.com)

Multimodality breast imaging is emerging as radiologists have access to new technologies coinciding with the refinement of operative techniques. One of the challenges for radiologist remains the correct preoperative staging, especially the metastatic involvement of axillary lymph nodes. Multimodality imaging has new accurate solutions. The imaging follow-up of patients with sophisticated oncoplastic techniques is another challenge where multimodality breast imaging is the solution. Coincident with this increasing contribution to the diagnostic process is the emerging role of the breast imager as a therapeutic clinician, making use of innovative image-guided procedures. In this integrated $\mathrm{RC}$, experts will present a clinical update on these newer techniques and there will be an opportunity to discuss how the evolution of such techniques is changing the nature of the modern multidisciplinary team meeting.

\section{A-359 08:35}

A. Conventional, functional and interventional lymph node assessment R.M. Pijnappel; Utrecht/NL (r.m.pijnappel@umcutrecht.nl)

Metastatic involvement of axillary lymph nodes has been known to be one of the most important prognostic factors for women with breast cancer. The traditional approach to staging the axilla is either lymph node sampling procedures or sentinel node biopsy. The main diagnostic features of potentially malignant involvement of the axilla are abnormal lymph node morphology (shape and cortical thickness). There has been a concerted effort in recent years to find imaging techniques that might facilitate accurate axillary staging. So far, no imaging technique alone (CT, MRI, scintigraphy and ultrasound) has been capable of differentiating between positive and negative lymph nodes of the axilla in breast cancer. Recent techniques like gadofosveset-enhanced magnetic resonance imaging and high spatial resolution 7-T MRI imaging appear promising; however ultrasound-guided sampling of suspicious lymph nodes by core or FNA remains the standard of care in assessing the axilla prior to treatment planning. Ultrasound-guided axillary lymph node sampling is an effective method of assessing the axilla. A recent meta-analysis shows that ultrasound can be expected to achieve sensitivity approaching $50 \%$ for detecting abnormal nodes in those that eventually prove to be nodal metastatic disease. The false-negative rate where biopsy is performed is around $25 \%$. It is impossible to detect the sentinel node by ultrasound alone. Therefore a new promising technique was introduced, injecting an ultrasound-contrast agent around the nipple. Using this method, it is possible to detect the sentinel node in $90 \%$ and therefore lower the false-negative rate of US-guided biopsy with another $15 \%$.

Learning Objectives:

1. To learn about normal lymph node morphology

2. To understand criteria suggestive of morphological abnormality.

3. To become familiar with factors affecting overall sensitivity of pre-operative lymph node assessment.

Author Disclosure:

R.M. Pijnappel: Advisory Board; Hologic, without financial reimbursement.

\section{A-360 08:58}

B. Multimodality assessment of the breast following oncoplastic surgery M. Torres-Tabanera, S. Pérez-Rodrigo; Madrid/ES

(mtorrestabanera@gmail.com)

Oncoplastic breast procedures were introduced to fill the gap between comprehensive oncologic surgical treatment of breast cancer and the achievement of cosmetic results that fulfill patient expectations in both, body imaging and psychological well-being. Due to its complexity and relevance for the patient, the decision of performing these procedures must be made as part of the multidisciplinary approach of breast cancer treatment. As a consequence, the role of breast radiologists has expanded beyond the anatomic region of the breast and the usual imaging techniques. Basic knowledge of the different oncoplastic techniques is mandatory to understand the spectrum of findings from a multi-modality approach. Implants and/or autologous reconstruction techniques (pedicle, free or perforator flaps, as well as lipofilling techniques) are widely applied. The role of the radiologist in the multidisciplinary team is twofold: assessment during the planning stage, and imaging evaluation at follow-up. The assessment during the planning stage includes the determination of the local extent of the disease that makes the choice of the appropriate surgical technique possible, and the imaging study of the donor site in those cases where autologous reconstruction is elected. Imaging evaluation at follow-up comprises the recognition of changes and potential pitfalls after reconstruction, the identification of short $/ \mathrm{mid} / \mathrm{long}$-term reconstruction complications, and the detection of recurrent/second carcinomas. Controversial aspects will be reviewed, such as the probability of recurrence after oncoplastic surgery, the need to establish multimodality follow-up protocols and the interrelations between the autologous tissues and the mastectomy bed or remaining breast.

Learning Objectives:

1. To learn about the range of oncoplastic breast procedures in current clinical practice.

2. To become familiar with imaging features resulting from oncoplastic surgery. 3. To appreciate the potential pitfalls encountered while imaging such cases.

\section{A-361 09:21}

C. Image-guided therapy in breast lesions: indications and techniques R.M. Mann; Nijmegen/NL (r.mann@rad.umcn.nl)

The use of image guidance for surgical planning has only recently been accepted. The use of intraoperative ultrasound in women undergoing lumpectomy is associated with a higher rate of negative margins. The combination of image guidance and radiological intervention techniques also harbours the possibility of tumour treatment without surgery. Most techniques make use of the placement of needles within the tumour. Treatment is performed by heating, freezing or extracting the tumour tissue. Currently, radiofrequency ablation under ultrasound guidance is being furthest evaluated. However, in RFA even after optical complete tumour ablation, residual vital tumour tissue is still found in many cases. This is mainly dependent on tumour size with poorer results $(50-60 \%$ success rates) in tumour over $2 \mathrm{~cm}$, and better results ( $90 \%$ success rate) in smaller tumours. Adequate tumour size estimation is thus of vital importance. Currently, since remaining vital cells cannot be excluded, the technique is limited to use in patients who cannot undergo surgery due to poor health state. Larger series in which RFA is combined with radiotherapy have not been reported. It is therefore not clear whether it is important to actually excise residual vital tumour. Similar 
restrictions currently hold for cryotherapy, laser ablation and hifu. Large vacuum-assisted needles and the breast lesion excision system can be used to remove the tumour through a minimal incision in the skin. However, margin evaluation is difficult. Nevertheless, these techniques are already optional for the removal of high-risk lesions and can potentially be combined with (focused) radiotherapy.

Learning Objectives:

1. To understand indications for therapeutic interventions in malignant and benign lesions.

2. To learn about current image-guided therapeutic techniques in malignant and benign lesions.

3. To appreciate potential future developments of therapeutic interventions. Author Disclosure:

R.M. Mann: Speaker; Bayer Healthcare.

Panel discussion:

How is the evolution of multimodality breast imaging changing the nature of the multidisciplinary meeting (MDM)? 09:44

08:30 - 10:00

Room G/H

\section{Genitourinary}

RC 1207

\section{Female imaging: how I do it?}

Moderator:

V. Panebianco; Rome/IT

\section{A-362 08:30}

\section{A. Imaging female congenital anomalies}

G. Restaino; Campobasso/IT (gennares@hotmail.com)

The Müllerian ducts are paired embryologic structures that undergo fusion and resorption between the $6^{\text {th }}-11^{\text {th }}$ weeks of gestation to give rise to the uterus, fallopian tubes, cervix, and upper two-thirds of the vagina. Non-development, defective vertical or lateral fusion, or resorption failure of the Müllerian ducts can result in formation of Müllerian duct anomalies (MDAs), whose reported prevalence ranges between $0.4 \%$ and $8 \%-10 \%$. The classification revised by the American Society for Reproductive Medicine in 1988 is the most widely accepted. Obstructive MDA can occur in infants as palpable pelvic mass, at menarche with cyclic pelvic pain, or pelvic mass, and primary amenorrhea. Complications include hematocolpos, hematometra, hematosalpinx, endometriosis, pelvic adhesions, and obstruction of urinary tract. MDAs are commonly associated with renal and other anomalies; thus, identification of both kidneys is important. Accurate diagnosis of an MDA is essential because of the high associated risk of infertility, endometriosis and miscarriage, and since the management approach varies depending on the type of malformation. Hysterosalpingography (HSG) is routinely used in evaluation of infertility. Because a key component of MDAs characterisation is the external uterine fundal contour, HSG is limited for this purpose. Ultrasonography (US) is initially performed to evaluate uterine anatomy and to assess for associated renal abnormalities. MRI is reserved for complex or indeterminate cases to characterise the uterine anomaly and evaluate the possibility of a vaginal abnormality, or after a seemingly normal US when clinical opinion ensures its use, or to make decisions on management strategies and preoperative planning.

Learning Objectives:

1. To learn about the embryology of normal and abnormal female genital tract.

2. To understand the role of different available imaging techniques for female congenital anomalies: ISG, TV-US and MRI.

3. To learn about the examination technique and understand the imaging

findings of MRI in the different classes of female congenital anomalies.

\section{A-363 09:00}

B. PET-CT in the female pelvis: how I do it?

A.G. Rockall; London/UK (a.rockall@imperial.ac.uk)

This refresher course will focus on the radiologist's approach to using PET-CT within the context of the gynaecologic oncology multidisciplinary meeting. The current indications for using FDG-PET/CT will be discussed for cervix endometrial and ovarian cancer as well as the potential use of FDG-PET/CT in rare gynaecologic cancers. The value of adding FDG-PET/CT to the patient management pathway will be presented alongside the potential pitfalls in interpretation as well as controversies. Future developments, including potential new tracers, will be mentioned.
Learning Objectives:

1. To understand the role of PET-CT in staging of various pelvic tumours and detection of tumour recurrence.

2. To learn about the advantages and limitations of PET-CT in imaging the female pelvis.

3. To appreciate the role of PET-CT with respect to other imaging techniques in the diagnostic algorithms of pelvic tumours.

Author Disclosure:

A.G. Rockall: Research/Grant Support; Merck and Co. Speaker; Novartis, Guerbet.

\section{A-364 09:30}

C. How to image cystic tumours of the ovary

R. Forstner, M. Meissnitzer; Salzburg/AT (r.forstner@salk.at)

Cystic lesions account for the vast majority of ovarian masses and are detected incidentally in $5-18 \%$. Their spectrum ranges from non-neoplastic cysts to benign tumours, e.g. cystadenomas and dermoids to the rare ovarian cancer. Diagnostic criteria in US, CT and MRI are based on the clinica background (age, menopausal status, medical history, and tumour markers) and the morphology of the ovarian mass. Typical of a benign cystic lesion is its pure cystic structure or thin septations, fatty or haemorrhagic contents, and mild enhancement of solid components in a complex solid and cystic ovarian mass. Thus, patients can be categorised into 3 different risk groups Sonography has been established as the first-line imaging modality to assess the ovaries. Recently, management guidelines have been adopted for cystic ovarian lesions. In these guidelines, also cystic adnexal incidentalomas are included. MRI is most useful as a complementary technique in sonographically indeterminate masses. An algorithmic approach will render a specific diagnosis in the vast majority of cases. In complex cystic lesions, integration of DWI and dynamic contrast-MRI allows differentiation of rare benign complex tumours, e.g. cystadenofibroma from ovarian cancer. Pitfalls of cystic ovarian tumours include cystic fibroids, peritoneal cysts, and extraperitoneal cystic tumours. Compared to US, both CT and MRI are superior in assessing large cystic pelvic masses. Criteria to differentiate between intra- and extraperioneal origin include displacement patterns and identification of the vascular pedicle. Careful analysis of imaging and clinical findings usually allows differentiation of inflammatory tumours from ovarian cancer.

Learning Objectives:

1. To learn about specific imaging algorithms of ovarian cystic tumours.

2. To understand certain imaging features that can differentiate ovarian from non-ovarian cystic tumours in the pelvis.

3 . To become familiar with the pitfalls in imaging of ovarian cystic tumours and the lessons to be learned from them.

08:30 - 10:00 Room I/K

Head and Neck Imaging

\section{1219}

\section{What do I see? Clinical radiological interaction (part 1) \\ Moderator:}

S. Robinson; Vienna/AT

A-365 08:30

A. Temporal bone: conductive hearing loss and cholesteatoma

B. De Foer, F. Offeciers; Wilrijk-Antwerp/BE

The diagnostic methods available in patients with conductive hearing loss are: personal and family history, otoscopy, audiology, imaging, surgical inspection and counseling. The most important reasons for imaging are: atypical history, suspicion of congenital hearing loss, suspected otoscopic image, asymmetric bone conduction thresholds, profound mixed hearing loss and suspected tympanometry. CT can be used to detect otosclerosis, tympanosclerosis, posttraumatic ossicular lesions, superior semicircular canal dehiscence and cholesteatoma. MRI can be used to exclude schwannoma, congenital cholesteatoma and residual and recurrent cholesteatoma after prior surgery. This lecture will illustrate the value of $\mathrm{CT}$ and MRI in the evaluation of cholesteatoma patients as well as prior to first stage surgery and prior to second look surgery. Different subtypes of surgery will be highlighted and illustrated. The growing importance of MR imaging and diffusion weighted (DW) imaging in particular will be illustrated in the evaluation of cholesteatoma patients. The role of echo-planar and non-echo-planar DW imaging will be discussed as well as the eventual use of delayed gadolinium-enhanced T1weighted imaging. 


\section{Postgraduate Educational Programme}

Learning Objectives:

1. To become familiar with pathologies causing $\mathrm{CHL}$.

2. To understand what the clinician needs in order to perform surgery.

3. To learn about possible pitfalls and complications.

\section{A-366 09:00}

B. Temporal bone: sensorineural hearing loss and deafness J. Casselman ${ }^{1}$, F. Offeciers ${ }^{2}$, B. De Foer ${ }^{2} ;{ }^{1}$ Bruges/BE, ${ }^{2}$ Antwerp/BE

MR is the modality of choice in patients with congenital or acquired sensorineural hearing loss ( $\mathrm{SNHL}$ ) and the complete auditory pathway from the cochlea to the auditory cortex must be studied in these patients. The labyrinth, internal auditory canal (IAC) and cerebellopontine angle (CPA) are best studied using submillimetric heavily $\mathrm{T} 2 \mathrm{~W}$ images, showing the intralabyrinthine fluid and nerves. But submillimetric Gd-enhanced T1W images or 3D-Flair images remain more sensitive. The auditory pathway in the brainstem is best studied with a multi-echo sequence (m-FFE/medic/merge) or T2W TSE sequence and the auditory cortex is best studied on T2W TSE images. The clinical information provided by the otologist will direct the radiologist to a certain part of the auditory pathway or will let him use certain imaging techniques. For instance, congenital malformations in the labyrinth and IAC must be excluded in case of congenital $\mathrm{SNH}$, and in case of mixed hearing one will start with CT to exclude otosclerosis. The most frequent pathology in the labyrinth (labyrinthitis, intralabyrinthine schwannoma, congenital malformation, post-traumatic lesions), the IAC and CPA (cochleovestibula schwannomas, meningiomas, epidermoid tumours and facial nerve neuritis) and central auditory pathway (MS, infarction, tumor and trauma) will be illustrated using clinical examples and the important "otologist-radiologist" team work will be stressed.

Learning Objectives:

1. To become familiar with causes of $\mathrm{SNHL}$ and diagnostic possibilities.

2. To learn which pathologies can be successfully treated and what information is needed from the radiologist.

3. To learn about interesting cases highlighting the role of diagnostic imaging in treatment planning.

\section{A-367 09:30}

\section{Laryngeal and hypopharyngeal cancer}

R. Maroldi, P. Nicolai; Brescia/lT

For tumours that nearly always arise from the investing mucosa, like in the larynx and hypopharynx, it is endoscopy that directly maps the superficial extent and grades the functional impairment (arytenoid and vocal cord movement, pyriform sinus distensibility). In addition to white light, the recent use of narrow band imaging endoscopic technology, enhancing fine capillaries in the mucosal surface, enables the detection of neoangiogenesis caused by early squamous cell carcinoma. Conversely, submucosal spread is the domain of cross-sectional imaging techniques. Therefore, it is the integration of the two classes of data, along with the information about patient conditions, which permits the choice of the most proper treatment strategy. In early and advanced glottic cancer, treatment planning is modulated by information about deep neoplastic invasion into the paraglottic space, the cartilage or the suprasubglottis subsites. This information can be derived by discriminating the tumour tissue from intra-laryngeal muscles, fat spaces and the cortical rim of ossified cartilages. Though MR shows a greater contrast resolution than $\mathrm{CT}$, it is hampered by a significantly longer acquisition time. This means more artefacts caused by breathing and movement. Nevertheless, MR has the potential to unravel the signals from submucosal tissues, separating tumour from oedema, muscles, fat and ossified cartilages. Advanced laryngeal and hypopharyngeal cancers need to preliminary assess local (nodes) and distan (metastases) spread. CT-PET is the more accurate tool. The multidisciplinary approach to the processing of the whole frame of data is essential to offer the patient the best care.

Learning Objectives:

1. To learn about different types of laryngeal cancer and treatment options.

2. To understand the role of diagnostic imaging in treatment planning.

3. To review clinical cases in order to highlight the role of clinical-radiological cooperation.
08:30 - 10:00

Room L/M

\section{Physics in Radiology}

RC 1213

Cone-beam CT

Moderators:

D. Bor; Ankara/TR

S.J. Golding; Oxford/UK

\section{A-368 08:30}

A. Fundamentals of cone-beam CT

M. Kachelrieß; Heidelberg/DE (marc.kachelriess@dkfz.de)

Traditionally, x-ray CT scanners were equipped with a single or a few detector rows only, image reconstruction was assuming parallel slices, which was a good approximation for these fan-beam systems. About a decade ago the number of slices increased to 16 or more and the parallel slice approximation was no longer valid. The cone-beam nature of these multi-slice CT (MSCT) scanners had to be taken into account by specific cone-beam reconstruction algorithms. While these MSCT systems - today they are simultaneously acquiring up to 320 slices - are cone-beam CT systems in a general sense, the notion of cone-beam CT (CBCT) is also associated with a specific kind of conebeam CT, namely those equipped with flat detectors, i.e. the non-diagnostic or non-clinical CT systems. MSCT, in contrast, is a cone-beam CT system for diagnostic use, i.e. a clinical CT system. This distinction between MSCT and CBCT is useful, as there are decisive differences in application, performance, and in image reconstruction. These differences will be reviewed in the presentation, with a particular focus on why image quality in MSCT is so much better than in CBCT.

Learning Objectives:

1. To understand the principles of volumetric image formation with flat detectors.

2. To understand the difference between CBCT and MSCT.

3. To learn about reconstruction techniques and image processing.

4. To become acquainted with the important image quality parameters.

A-369 09:00

B. Medical applications of cone-beam CT

M. Grass; Hamburg/DE (michael.grass@philips.com)

Cone-beam (CB) imaging with flat detectors is performed on a variety of different imaging systems. The most important area is CB imaging using interventional $\mathrm{C}$-arm systems. The minimally invasive procedures carried out on these systems benefit from the availability of high-resolution 3D images for intervention planning, guidance and outcome control. Interventional CB imaging was first applied in neuroradiology using rotational angiography acquisitions. Today, it is used for a variety of procedures in interventiona radiology, cardiology and oncology based on angiographic and soft tissue protocols. One of the inherent advantages of this approach is the direct registration of the volume images into the interventional procedure. Other application areas of CB imaging using different scanning systems are onboard imaging in radiation therapy or the integration of flat detector tomography and SPECT. This part of the course teaches the generation of $3 \mathrm{D}$ volume information from flat detector systems and its utilisation in different clinical applications. Image acquisition protocols, scan modes, and system design parameters are explained. Application-specific calibration and processing steps are introduced to show how CB imaging is tailored for specific clinical applications. Examples are presented for angiographic and soft tissue volumetric imaging. Medical applications utilising the tomographic images for diagnosis, intervention planning, guidance, or outcome control are discussed. Learning Objectives:

1. To become acquainted with the applications of CB imaging.

2. To learn about systems design and parameters.

3. To understand image quality characteristics.

4. To learn how to use cone-beam images in image-guided interventions. Author Disclosure:

M. Grass: Employee; Philips Research.

A-370 09:30

C. 3D dentomaxillofacial imaging

K. Horner; Manchester/UK (keith.horner@manchester.ac.uk)

In the last decade, three-dimensional dentomaxillofacial imaging through conebeam CT (CBCT) technology has become widely available. Dental CBCT (digital volumetric tomography; DVT) equipment is compact and often affordable to dentists. The driver for dental CBCT was implant dentistry, but its use has spread to other areas, including paediatric applications. Radiation doses are variable. Some systems offer a fixed, large, field of view and others 
fixed exposure factors, obstructing attempts at optimisation. Typically, doses are at least an order of magnitude greater than for "conventional" imaging. Image quality is also variable, which means that certain equipment may be unsuitable for some clinical applications. There is often scope for lower exposure factors to be used than those recommended by manufacturers. A key aspect of using CBCT relates to justification. Imaging in three dimensions may be perceived by dentists as inevitably superior, a view which is often implicitly encouraged by those selling equipment. The research on diagnostic efficacy is, however, limited. In 2012, the European Commission published "Radiation Protection 172: Evidence-based Guidelines on Cone Beam CT for Dental and Maxillofacial Radiology". This provides a comprehensive set of recommendations, including referral criteria, a quality assurance programme and optimisation strategies. Priorities for future research relate to exposures to the necessary image quality requirements for specific clinical applications, along with research on the impact on clinical outcomes of CBCT-based treatments. It is clear that much work remains before the place of CBCT in dentistry can be established.

Learning Objectives:

1. To become acquainted with CBCT systems for dentomaxillofacial imaging.

2. To learn about image quality characteristics and patient dose compared with other techniques.

3. To learn how to access CB images and influence the clinical outcome.

08:30 - 10:00 Room N/O

Interventional Radiology

RC 1209

\section{Thoracic nonvascular interventions}

\section{A-371 08:30}

Chairman's introduction

O. Akhan; Ankara/TR (akhano@tr.net)

The role of nonvascular interventional radiology of thorax for both diagnosis and treatment has significantly increased in the last 20 years. Although these procedures are technically easy with highly successful results, thoracic nonvascular interventional procedures are not unfortunately routinely performed in all of the interventional units. The most commonly performed procedures generally under CT guidance are transthoracic biopsies of the lung, mediastinal lesions and pleura. The others include percutaneous drainage of thoracic collections such as pleural effusions, empyema and mediastinal collections. Image-guided tumour ablation performed by either radiofrequency ablation (RFA) or microwave (MW) as a nonvascular thoracic intervention has become a serious treatment alternative in patients with lung tumours (either non-small cell lung cancer or lung metastasis) since the year 2000. Interventional radiologists are expected to understand the clinical indications of these procedures at the first step. In addition, the techniques of the procedures and possible risk of complications in their management methods should be learnt by interventional radiologists as well. This refresher course will help us to discuss the main indications, techniques and complication management of these procedures.

\section{A-372 08:35}

\section{A. Lung biopsy}

T. Sabharwal; London/UK (Tarun.Sabharwal@gstt.sthames.nhs.uk)

Lung biopsy is most commonly now performed under CT guidance. It has a high technical success rate. Common complications include pneumothorax, bleeding, sepsis, pain and failure. Air embolism is a rare complication. PET scan is a useful tool to guide for appropriate target. Ultrasound is useful for biopsying pleural-based lesions.

Learning Objectives:

1. To understand the role of lung biopsy.

2. To learn how to properly plan a lung biopsy.

3. To understand common complications and their management.

\section{A-373 08:58}

B. Percutaneous drainage of thoracic fluid collections

I. Vollmer; Barcelona/ES (ivollmer@hospitaldelmar.cat)

Drainage of thoracic fluid collections is a relative emergency. We will review the different thoracic fluid collections that can be drained by radiologists: pleural (parapneumonic, empyema, and malignant effusions), mediastinal, chest wall, pulmonary (lung abscesses and infected tumours) and pericardial effusions. We will answer the following questions about the main thoracic fluid collections: how to diagnose, when and how to drain, which guidance (ultrasound or CT-fluoroscopy), and procedures to perform before and after the drainage. The choice of imaging to detect thoracic fluid collections and guide drainage depends on the availability of the modalities, the imaging characteristics, location of the collection, and the comfort and expertise of the operator with the specific modalities. We will also review the main complications of percutaneous drainage of thoracic fluid collections (pneumothorax, pain, drain dislodgement and drain blockage) and compare these complications with those related to large-bore chest catheters inserted by thoracic surgeons.

Learning Objectives:

1. To learn the imaging features of thoracic fluid collections.

2. To learn when and how to drain thoracic fluid collection.

3. To understand patient management.

\section{A-374 09:21}

C. RFA of lung tumours

P.L. Pereira; Heilbronn/DE (philippe.pereira@slk-kliniken.de)

The incidence of lung cancers continues to increase and primary lung cancer remains the leading cause of cancer-related deaths in both women and men. Therapy of lung tumours includes resection, radiation therapy, chemotherapy, thermal ablation or a combination of these treatment modalities. Image-guided percutaneous thermal ablation therapies are minimally invasive techniques established in the local treatment of hepatic, renal, or osseous tumours. Among these techniques, radiofrequency ablation (RFA) has now attained consideration for therapy of small pulmonal tumours. Other ablation techniques that have been used for treatment of lung tumours include cryoablation, laser (LITT) and more recently microwave ablation (MWA) and irreversible electroporation (IRE). Ablative techniques may produce a complete and irreversible tumour tissue destruction through application of either hot or cold thermal energy, or through permeabilisation of the cell membrane while using IRE. CT is currently the imaging modality that is most widely used for planning, monitoring the ablation's course and for assessment of treatment response. Only patients with stage I and stage II lung cancer and those with small metastases are potential candidates for ablation. The medical history and physical examination of the patient as well as recent imaging with CT should be evaluated to determine the indication for thermal ablation. Percutaneous lung RFA is considered as a safe procedure with an overall morbidity rate from $15.2 \%$ to $55.6 \%$ and mortality rate from $0 \%$ to $5.6 \%$; it requires less costs and offers faster recovery, and reduced morbidity and mortality.

Learning Objectives:

1. To learn about the best indications for RFA in comparison to other treatment options.

2. To appreciate the imaging problems during and after lung ablation.

3. To learn about outcome after RFA.

Author Disclosure:

P.L. Pereira: Advisory Board; Terumo. Consultant; Terumo. Research/Grant Support; Celon Olympus, Siemens Medical Solutions. Speaker; Angiodynamics, Terumo.

Panel discussion:

Is there a role for other imaging modalities besides CT?

$09: 44$

08:30 - 10:00

Conf. Room M3

Oncologic Imaging: Follow-up of Systemic and Local Therapies

\section{1218}

\section{Assessing HCC response to ...}

Moderator:

C. Bartolozzi; Pisa/IT

\section{A-375 08:30}

\section{A. Systemic therapies}

V. Vilgrain, M. Ronot, M. Zappa, S. Faivre, E. Raymond; Clichy/FR (valerie.vilgrain@bjn.aphp.fr)

Sorafenib, a tyrosine kinase inhibitor, has shown clinical efficacy in patients with hepatocellular carcinoma ( $\mathrm{HCC})$ and is the standard of care for patients with advanced-stage HCC. Nowadays, many targeted therapies are evaluated in HCC either as sole treatment or in combination with other treatments such as tumour ablation, chemo-embolisation, and surgical resection. Therefore, there is a need to assess the efficacy of targeted therapy in HCC. RECIST is the reference method to evaluate treatment efficacy in solid tumours, but does not seem appropriate in evaluating targeted therapy as objective responses are seen in very few cases in patients treated with sorafenib or sunitinib. New criteria have been proposed to evaluate the treatment efficacy of nonsurgical treatments in patients with HCC. The most common ones are the Choi criteria, the EASL criteria, and the modified RECIST criteria. All these criteria mainly focus on internal tumour changes such as appearance of necrosis or disappearance ot tumour hypervascularity. Many examples will be shown 
during the lecture. Another approach is based on functional imaging and especially perfusion-related imaging. Contrast-enhanced ultrasound, CT perfusion and dynamic contrast-enhanced MR imaging have the capability to assess perfusion changes in patients under treatment. The advantages and disadvantages of these modalities will be discussed. Lastly, other functional tools that are not routinely used will be presented.

Learning Objectives:

1. To learn about difficulties in HCC assessment with local therapy.

2. To become familiar with various evaluation criteria of $\mathrm{HCC}$.

\section{A-376 09:00}

B. Endovascular therapies

R. Lencioni; Pisa/IT (riccardo.lencioni@med.unipi.it)

Tumour response in oncology is usually measured according to Response Evaluation Criteria in Solid Tumors (RECIST). The RECIST model - developed in 2000 and revised in 2009 with the publication of the version 1.1 - was designed for evaluation of cytotoxic agents. It offers a simple approach to determining anatomic size and lesion changes during treatment as an indicator of response. However, it does not address measures of antitumour activity other than tumour shrinkage and was shown to be unable to capture the anticancer activity of tumour-directed therapies used in the treatment of HCC such as transarterial chemoembolisation (TACE) and radioembolisation. Given the poor correlation observed between response evaluation by conventional metrics and overall survival, a group of experts in $\mathrm{HCC}$ clinical trials convened by the American Association for the Study of Liver introduced the concept of viable tumour in HCC response assessment, and proposed specific amendments to standard RECIST. The resulting criteria were named modified RECIST (mRECIST) for HCC. The mRECIST criteria have been endorsed by several scientific societies and organisations and have been increasingly used in HCC clinical research. A growing body of scientific evidence suggests that mRECIST- designed for response assessment in clinical trials - may translate into a tool for clinical practice.

Learning Objectives:

1. To appreciate the value of different imaging techniques for assessment of intra-arterial HCC therapies.

2. To become familiar with post-treatment imaging after intra-arterial therapies according to type of therapy.

\section{A-377 09:30}

\section{Ablative therapies}

C. Ayuso; Barcelona/ES (cayuso@clinic.ub.es)

The evaluation of treatment efficacy is a key issue with prognostic and patient survival implications. It is crucial to have objective and reproducible criteria for specific groups of patients. The goal of ablative therapies of HCC is to induce tumoural tissue destruction. Complete response (CR) after initial chemical and thermal percutaneous ablation, defined as the absence of contrast enhancement of the treated tumour at CEUS, dynamic CT or dynamic MR, has been reported to correlate to long-term survival. Nevertheless, the clinical effectiveness of imaging techniques to assess initial treatment success differs according to tumour size. The success rate of RF has been demonstrated to be superior to $\mathrm{PEI}$ in $\mathrm{HCCs}>2 \mathrm{~cm}$, and depends on the ability to ablate all viable tumour tissue including an adequate tumour-free margin all around the lesion of 0.5 to $1 \mathrm{~cm}$. Thus, the effectiveness of RF directly depends on the tumour location and size. RF is considered an effective treatment in lesions $\leq$ $3 \mathrm{~cm}$ and its effectiveness is progressively reduced along with tumour size and it is not effective in lesions $>5 \mathrm{~cm}$. CEUS beyond 1 month may confirm or detect residual tumour, deserving a final ablation procedure. CT and MR are more effective in the follow-up to confirm CR and detect local recurrence, or additional HCC lesions in the liver parenchyma. The presence of transient hyperaemic inflammatory changes in the periphery of the treated area is a common finding that should be considered to avoid overestimation of the recurrence rate.

Learning Objectives:

1. To learn about the value of different imaging techniques for the assessment of ablative HCC therapies.

2. To become familiar with post-treatment imaging after ablative therapies according to type of therapy.
$08: 30-10: 00$

Board Room A

\section{Radiographers}

RC 1214

How important are state-of-the-art displays to radiology?

\author{
A-378 08:30 \\ Chairman's introduction \\ C. Vandulek; Kaposvár/HU (cvandulek@gmail.com)
}

One of the major characteristics of medical imaging in the twenty-first century is the dramatic influx of novel technology. This impact of new technology and techniques is experienced in all imaging modalities. The continuous development and implementation of highly sophisticated medical products and devices is key to the evolution of medical imaging leading to the improvement of patient care in terms of quality and positive outcomes. This session will analyse state-of-the-art displays (monitors, smartphones, tablets) and their applications in modern imaging. The session will provide a brief overview of displays in the current imaging chain. It will then evaluate the various specifications and parameters associated with displays. The session will evaluate current $\mathrm{QA}$ standards of modern displays as well as the challenges surrounding such novel technologies.

Session Objectives:

1. To appreciate the technical parameters associated with display devices and why we need to be familiar with them.

2. To consider the evolution of display devices since the introduction of digital imaging techniques.

\section{A-379 08:35 \\ A. Primary class displays \\ W.A. Hummel; Leeuwarden/NL (willy.hummel@kcl.znb.nl)}

Image quality can be described in terms of resolution and contrast. An image displayed on a monitor consists of differences in brightness. The display monitor is at the end of the image chain in radiology, which starts at the X-ray tube. There are several factors in this chain that have influence on the contrast displayed on the monitor. Diagnostic image quality can be described as observed contrast, which is not only dependent on the displayed image, but is also influenced by ambient conditions. For the primary class displays, typical characteristics of display monitors; such as resolution, homogeneity, luminance, contrast, monochrome or color, must be appropriate for the radiologist to execute a reliable diagnosis. Calibration of display monitors is also important. Which parameters are significant and what is the effect of variation of these parameters. There are standards like DICOM GSDF and technical standard from ACR-AAPM-SIIM.

Learning Objectives:

1. To understand the range of display devices currently on the market for medical imaging purposes.

2. To explore current legislation and guidelines related to the use of display devices in radiology.

3. To become familiar with current legislation and guidelines on quality control and quality assurance procedures.

\section{A-380 08:58}

\section{B. Is a smartphone all that is required?}

R. Toomey; Dublin/IE (rachel.toomey@ucd.ie)

Both within and outside the clinical environment, the use of smartphones and tablet computers is increasing. Reported applications range from teaching and education to navigation in surgical procedures. Interest in the use of smartphones and tablets for viewing radiological images has been growing. However, these portable displays can differ significantly from conventional radiological displays. In order to determine where and how smartphones and tablets can be used appropriately, it is important to understand these displays and the challenges associated with them. This presentation will outline the current and potential uses of smartphones and tablet computers in radiology and compare their performance with other radiological displays and standards. The possible limitations/benefits of smartphones and tablets as display devices for radiological images will be discussed. Recent research in the field will be reviewed, with emphasis on studies of diagnostic efficacy.

Learning Objectives:

1. To become familiar with the growing evidence base that both supports and opposes the use of hand-held display devices in medical imaging. 
2. To learn about ongoing developments in radiology display devices and whether or not these can currently be justified.

3. To learn about examples of appropriate and inappropriate use of state-ofthe-art-display devices in radiology.

\section{A-381 09:21}

C. Clinical requirements for image display

D. Pekarovic, U. Zdešar; Ljubljana/SI (dean.pekarovic@kclj.si)

In modern clinical environment, diagnostic monitors have replaced glowboxes and films and have become an important part of the imaging chain. The required specifications and the quality of monitors largely depend on their purpose, beginning from quite simple monitors used for the preview of radiographic images placed on modality or in control room to monitors used by high-end diagnostic workstations. Decision about the kind of monitor for certain workplace is also very important from the economic standpoint as there are large differences in their prices. Besides monitor specifications, also ambient conditions and ergonometry in the reading rooms are also very important issues which need to be considered to ensure optimal environment for clinical image reading. Some of good and bad examples will be given in the presentation. As with other radiological equipment, also diagnostic monitors need to be controlled to ensure their optimum performance. Quality control begins with monitor acceptance testing and setting up measurement parameters which are to be followed. Practically all monitor vendors include some QC software within their workstations which can, together with some independent measurements, form an efficient quality control programme. In the last steps, how to deliver all information regarding image quality and possible presets on the diagnostic monitor and how not to confuse the user are important.

Learning Objectives:

1. To become familiar with clinical requirements in terms of the primary class displays used.

2. To appreciate the practical steps involved in the implementation of a quality assurance programme for radiology displays.

3. To understand the key considerations in evaluating the appropriateness of display devices.

Panel discussion:

The pros and cons of cutting-edge display devices 09:44

\begin{tabular}{l}
\hline $08: 30$ - 10:00 \\
EDiR Workshop \\
European Diploma in Radiology: what a \\
hearty meal!
\end{tabular}

\section{A-382}

Appetiser: what is behind the curtain

Y. Menu; Paris/FR

Sitting an examination is always a source of anxiety. The EDiR exam is no exception. This workshop is dedicated to those who want to take the EDiR exam, or become examiners, and would like to learn more about its format and discover some tips and tricks. The EDiR organisers and examiners have decided to build an interactive, friendly and enjoyable session in order to teach the essentials. Some will play the role of the examiners, some the role of the candidate. In order to simulate the candidate's experience, this session will have no safety net. Some senior members will expose themselves to real difficult questions in general radiology. Fortunately, we hope that the attendees will be able to help the "candidates" to succeed. Hopefully, you will enjoy this session and get some very useful information for your EDiR exam.

\section{A-383}

Pièce de résistance: live EDiR performance

L. McKnight ${ }^{1}$, J. Vilar ${ }^{2}$, Y. Menu ${ }^{3} ;{ }^{1}$ Langland/UK, ${ }^{2}$ Valencia/ES, ${ }^{3}$ Paris/FR

\section{A-384}

Catch of the day: fun and nasty tricks to succeed or to fail

S. Barter ${ }^{1}$, W. Schima ${ }^{2}$, L.E. Derchi ${ }^{3}$, M.M. Thurnher ${ }^{2} ;{ }^{1}$ Cambridge/UK,

${ }^{2}$ Vienna/AT, ${ }^{3}$ Genoa/lT

\section{A-385}

Nightcap: the EDiR tomorrow

Y. Menu; Paris/FR

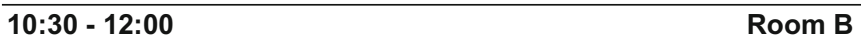

ESR meets Mexico

EM 4

Oncology imaging in Mexico

Welcome by the ESR President

G. Frija; Paris/FR

Presiding:

C. Rodriguez Treviño; Mexico/MX

V.E. Sinitsyn; Moscow/RU

J. Tanus Haij; Mexico/MX

\section{A-386 10:30}

Introduction

C. Rodríguez Treviño, J. Tanus-Haji; Mexico/MX

The Mexican Society of Radiology and Imaging, SMRI, was the first radiology society in the country. It was constituted in 1926, first as Mexican Society of Electroradiology, and in 1942 transformed to Mexican Society of Radiology; adding Imaging in the first years of 1970. The Mexican Federation of Radiology and Imaging, FMRI, was established in 1972 and is constituted by all the 32 national radiology societies. Simultaneously, the Mexican Board of Radiology began to work in relation to radiologist's certification. The presidents of these institutions have a two-year term. As presidents of SMRI and FMRI, we mention our appreciation for the invitation to the European Congress of Radiology-European Society of Radiology so that our institutions can participate in this important programme: ECR Meets Mexico. Since our Society and our Federation were established many decades ago, among our main objectives have been promoting continual medical education in radiology and contributing to the development of standards of excellence and reliable and personalised radiology in a framework of professionalism. For some years, we have had the support of radiologist members of ESR-ECR in Mexican courses, academic meetings and congresses. The last International Congress of Radiology was set in Cancun, Mexico, in 2002, and was organised by FMRI in conjunction with the International Society of Radiology, ISR, and the European Society of Radiology. For this and other academic support, we also want to express our special thanks.

Session Objectives:

1. To express our appreciation to the ECR for inviting Mexico to participate.

2. To learn about Mexican radiology organisations.

3. To learn about Mexico's participation in international radiology meetings.

A-387 10:35

Abdominal imaging in oncology

L.A. Sosa-Lozano; Mexico City/MX (antonsosarad@gmail.com)

Abdominal malignancies are an important health problem in Mexico. Like in many other middle-income countries, there has been an epidemiological transition with an overall increase in oncologic diseases. Colorectal, gastric and liver carcinomas are some of the frequent abdominal malignancies. Colorectal cancer is a disease that is curable if detected early and even preventable if precursor polypoid lesions are removed. Imaging plays a critical role in staging at diagnosis. Additionally, virtual colonoscopy is an accepted modality in cancer screening. High-resolution magnetic resonance imaging (MRI) has become a pivotal modality in the pretreatment assessment of recta carcinoma. Computed tomography (CT) is an excellent modality in the preoperative staging of gastric cancer and follow-up after treatment. Positron emission tomography (PET) combined with CT (PET/CT) is particularly helpful for gastric and colonic carcinoma staging. Hepatocellular carcinoma $(\mathrm{HCC})$ is also common. Cirrhosis related to alcohol and hepatitis $\mathrm{C}$ infection are by far the commonest aetiologies. Ultrasound (US) in conjunction with alpha-protein is the most widely used modality for screening purposes. CT and MR are confirmatory modalities. The advent of hepatospecific gadolinium contrast agents has been very helpful in the characterisation of focal lesions in cirrhotic patients. The use of multidetector CT (MDCT) in pancreatic carcinoma for the detection of vascular and adjacent organ invasion is crucial in treatment planning. Over the last few decades, there has been an important improvement in imaging techniques resulting in better quality images. Imaging is critical not only for the diagnosis, but also for treatment planning and follow-up.

Learning Objectives:

1. To learn about the imaging of common oncologic gastrointestinal diseases.

2. To understand the importance of imaging in liver and colon cancer.

3. To learn how imaging supports oncologic institutions. 


\section{Postgraduate Educational Programme}

\section{A-388 10:55}

\section{Interlude: Meet Mexico}

J. Ramírez-Arias; Mexico/MX (jlramirez.arias@saludangeles.com)

The presentation has the objective of giving the audience a synthesised panorama of our country situated in North America, with more than 110 million inhabitants and a large prehispanic history based on the Aztec, Toltec and Maya cultures, and also of interesting facts related to the three centuries of colonial existence under the Spain influence and dominium until the 1810 war of Independence. After 1880, Mexico has never had an international war; the last was against the northern border country, the United States. Also, we will give important data on how in 1910 the Mexicans rebelled against a 30-year dictator, General Porfirio Diaz. The Mexican Revolution was a long and cruel war, but later opened the doors to democracy and a complex developmental era began that is still in process. Some demographic and contributions to health, science and culture issues will be mentioned and also the works of Mexican scientists, writers and philosophers who have been presented awards including the Nobel Prize. In relation to well-known Mexican artists such as Diego Rivera and Frida Khalo, some facts of their works will be presented. Learning Objectives:

1. To learn some Mexican history.

2. To learn about the demographics of Mexico.

3. To learn about Mexican culture in the $21^{\text {st }}$ century.

\section{A-389 11:00}

Interventional radiology in oncologic patients

G. Elizondo-Riojas; Monterrey/MX (elizondoguillermo@hotmail.com)

Interventional radiology (IR) is becoming an increasingly prominent subspecialty in the care of oncologic patients. Its role extends from initial diagnosis to minimally invasive treatment of the malignancy and its complications. Image-guided biopsies are increasingly performed using minimally invasive techniques. Also, an integral part of care of these patients is vascular access as a means of medication, chemotherapy or parenteral nutrition, and interventional radiologists can place required the devices with well-established safety and efficacy. IR also plays a substantial role in the therapy of oncologic patients, through local tumor treatments such as transarterial chemo-embolisation and locoregional control with radiofrequency/cryo ablation, as well as management of complications of malignancy such as pain, obstruction (biliary, ureteral, etc)., venous thrombosis and drainage of thoracic and abdominal collections. In Mexico, IR is a growing subspecialty, and more medical students nowadays want to be radiologists and eventually become interventional radiologists. This is a paradigm shift. More and more radiologist wants to be involved with patient management and to be more than just "observers" in the process of patient care. We have to be prepared to offer this opportunity to our residents; otherwise other specialties will "have to fill the empty space" that we have left. Interventional oncology has all the advantages to fulfil this opportunity. It is our chance to contribute to the advancement of medical care.

Learning Objectives:

1. To appreciate the role of interventional radiology in the management of oncologic patients.

2. To learn how interventional radiology changes the quality of life for patients with cancer.

3. To understand the impact of interventional radiology procedures in the outcome of some neoplastic diseases.

\section{A-390 11:20}

\section{Interlude: Origins and development of radiology in Mexico}

M.E. Stoopen-Rometti; Mexico/MX (mstoopen@clinicalomas.com.mx)

Mexican radiology started in 1896, just a few months after the discovery of $x$ rays. During the last few decades, as well as in other countries, there has been a great development both in public and private sectors, which will be described in this interlude.

Learning Objectives:

1. To learn about the history of radiology in Mexico.

2. To learn about the development of radiology in Mexico.

3. To learn about the present and future of radiology in Mexico.

\section{A-391 11:25}

\section{Modern issues in oncologic ultrasound}

J. Tanus-Hajj; Mexico/MX (jtanus59@hotmail.com)

Advances in ultrasound (US) technology allow confident characterisation of masses. These include harmonic imaging, compound imaging, power Doppler faster frame rates, higher resolution transducers, three-dimensional (3D) US US contrast agents and, more recently, elastography and fusion imaging. Highfrequency transducers provide superb spatial and soft-tissue resolution permitting substantially improved differentiation of subtle lesion, margin resolution, and lesion conspicuity in the background of normal tissue. Elastography features such as size ratios, shape, homogeneity, and maximum lesion stiffness complement conventional US in the analysis of lesions. Ultrasound contrast agents have overcome some of the limitations of Doppler ultrasound techniques with demonstration of irregular branching central or penetrating vascularity within a solid mass raising suspicion of malignant neovascularity (neoangiogenesis). Ultrasound contrast agents can provide important information in the assessment of lesions to be treated by locoregional therapies, which include ablation (feeding vessels), trans-arterial chemo/radio-embolisation, detecting viable tumour persistence following this treatment; facilitation of needle positioning in cases of poor lesion delineation, and assessment of local tumour progression. It facilitates needle positioning in cases of incomplete or poor lesion delineation on unenhanced ultrasound. US contrast agents are an important key during the evaluation of the immediate treatment effect of ablation and guidance for immediate re-treatment of residual tumour.

Learning Objectives:

1. To understand the role of ultrasound in the management of oncology patients.

2. To learn how ultrasound is used in large oncology centers.

3. To learn about the modern concepts of ultrasound in oncology patients.

Panel discussion:

Mexican requirements for international collaboration for research programmes, including requirements for visiting professor programmes 11:45

$10: 30-12: 00$ Room C

Modern Imaging of the GI Tract

CC 1321

\section{Inflammatory bowel disease}

Moderator:

S.A. Taylor; London/UK

A-392 10:30

A. Cross-sectional imaging protocols

M.A. Patak; Zurich/CH (Michael.Patak@hirslanden.ch)

The aim of this lecture is to give an overview of the techniques for imaging inflammatory bowel disease (IBD) of the small bowel and the colon with either ultrasound (US), multidetector row computed tomography (MDCT) or with magnetic resonance imaging (MRI) and compare the different modalities for its strength and weakness. Optimal imaging of the bowel begins with the preparation phase. The small bowel has to be distended for a concise examination. This is mainly done orally, which is named enterography. A solution of $2.5 \%$ mannitol seems to be the one preparation technique mostly used for small bowel distension. Another technique is the enteroclysis, application of contrast after intubation of the small bowel. The comparative advatages and disatvantages of the two preparation methods will be discussed. This intraluminal contrast gives a neutral contrast in CT and a biphasic signal in MR. The colon can be prepared in a fashion similar to colonoscopy meaning total cleansing. Another possibility is the so called fecal tagging whereas the stool will be contrasted with an additive to standardised food. Therefore no cleansing is needed for preparation. Imaging parameters will be discussed for MR and CT. The aim of imaging for the bowel should be to establish the following: 1) presence, severity, and extent of disease; 2) activity of the disease and 3) extra-intestinal complications. US, MR and MDCT have proven to be a good tool to evaluate the extent, the activity of the disease and the presence of extraluminal complications. Pros and cons will be discussed when to use which technique.

Learning Objectives:

1. To understand state-of-the-art MRI, CT and US protocols for imaging IBD.

2. To appreciate the comparative advantages and disadvantages of enterography and enteroclysis protocols.

3. To learn about protocol modifications when evaluating the colon.

\section{A-393 10:50}

\section{B. Small bowel disease}

J. Stoker; Amsterdam/NL (j.stoker@amc.uva.nl)

Classification of small bowel Crohn's disease is helpful for assessing disease activity and treatment monitoring. Similar to clinically based classification, also imaging-based classification systems have been developed, of which some have been externally validated. Important imaging features for determining disease activity include bowel wall thickness and vascularity/enhancement; at MRI, also wall oedema plays a role. Stenoses, fistulas and abscesses are important sequels. For assessment and monitoring of small bowel disease 
activity, ultrasound is readily available and gives detailed local information but is limited by the restricted field of view, communication of results to clinicians and comparison of examinations on time. Computed tomography (CT) is fast, readily available and gives a detailed, reproducible overview, but radiation exposure and contrast resolution are limitations. Magnetic resonance imaging combines a good, reproducible overview with high contrast resolution, dynamic information and no radiation exposure and is therefore preferable in many situations, but the longer examination times than for CT, availability and costs are limiting factors. For the diagnosis of stenoses, fistulas and abscesses, either technique can be used, although the unrestricted view of CT and MR favour these techniques in many situations. In acute situations, US and CT are more accessible than MRI. In that setting US can be considered, but when the examination is inconclusive or the patient has clear inflammatory signs, CT is preferable.

Learning Objectives:

1. To learn about the classifications of small bowel Crohn's disease.

2. To become familiar with cross-sectional imaging signs of disease activity and complications.

3. To understand a rational deployment of cross-sectional imaging techniques according to clinical indication, highlighting the main advantages and disadvantages of CT, MRI and US.

Author Disclosure:

J. Stoker: Consultant; Robarts Clinical Trials.

\section{A-394 11:10}

\section{Colitis}

J. Rimola; Barcelona/ES (jrimola@clinic.ub.es)

Endoscopy is currently considered the reference standard for the evaluation of colonic pathologies, including colitis. It allows direct visualisation of the mucosa and in obtaining tissue sample. However, it has major limitations including the invasiveness of the technique, incomplete endoscopy and risk of perforation. Endoscopy cannot, therefore, help to estimate the depth of involvement of transmural inflammation and extraluminal complications. By contrast, an evolving role for cross-sectional imaging in the evaluation of patients with colitis has been increasingly recognised, especially in the setting of Crohn's disease (CD), since cross-sectional imaging has been demonstrated to have a high diagnostic accuracy not only for assessing the presence and extension of luminal disease, but also for evaluating CD-related acute or chronic complications. Establishing the ultimate cause of colitis may sometimes be challenging and histology cannot be conclusive. However, cross-sectional imaging may provide additional information that is useful in the workup of colitis.

Learning Objectives:

1. To learn about the cross-sectional imaging features of colitis.

2. To become familiar with differentiating infectious, inflammatory, ischaemia and autoimmune conditions based on cross-sectional imaging criteria.

3. To appreciate an integrated approach to the use of cross-sectional imaging in colonic inflammatory bowel disease.

\section{A-395 11:30}

\section{Interactive case discussion}

S.A. Taylor; London/UK (csytaylor@yahoo.co.uk)

The use of cross-sectional imaging has been increasing in the evaluation of inflammatory bowel disease. Following their presentations on imaging protocols and features of small bowel disease and colitis, each speaker will present a case to illustrate the learning points covered in their lectures. The session will be moderated by the chairman and the audience will have full opportunity to ask questions of all the panel.

Author Disclosure:

S.A. Taylor: Research/Grant Support; NIHR HTA. Other; study reader for Robarts.

\begin{tabular}{ll}
\hline 10:30 - 12:00 & Board Room B \\
\hline RTF - Radiology Trainees Forum &
\end{tabular}

\section{TF 1}

\author{
Highlighted Lectures \\ Moderators: \\ V.H. Koen; Harleem/NL \\ N. Pyatigorskaya; Paris/FR
}

\author{
A-396 10:30 \\ Imaging hepatocellular carcinoma at the era of multimodality and hybrid \\ imaging \\ A. Luciani, F. Legou, J. Chalaye, M. Chiaradia, F. Pigneur, M. Djabbari, \\ C. Costentin, E. Itti, A. Rahmouni; Creteil/FR
}

Multimodality imaging is essential in a wide variety of oncology situations Anatomic imaging, whether using US, CT or MRI, is mandatory for tumour localisation. Moreover, the evaluation of treatment response mostly relies on size assessment, whether uni- or bi-dimensional. With the advent of new targeted bio-therapies, functional imaging has progressively been integrated in the imaging strategies whether for better tumour characterisation or for optimal evaluation of treatment response. Hence, assessment of molecular targets by PET is supplemented by the recent developments of diffusion MRI, reflecting tissue architecture and cellularity, tissue perfusion, reflecting angiogenesis, and magnetisation properties of tissues. HCC is a primary liver tumour where the use of multimodal anatomic, functional and metabolic imaging appears of particular interest. According to EASL and AASLD recommendations, noninvasive diagnosis of HCC can be performed using dynamic contrast enhanced cross sectional imaging. Liver MRI provides optimal sensitivity and specificity for the detection of as small as $1 \mathrm{~cm}$ large HCC. Recent reports have suggested that the prognosis of $\mathrm{HCC}$ lesions could be anticipated based on the combined analysis of metabolic 18 F -FDG PET CT, as well as with diffusion weighted imaging. The objective of this lecture will be to highlight the potential of combining both metabolic and multimodal cross sectional imaging in order to improve the management of patients with HCC.

Learning Objectives:

1. To appreciate the role of combined multimodal imaging for the management of patients with HCC.

2. To better understand the role of metabolic imaging for the management of patients with $\mathrm{HCC}$

3. To understand the role of multimodal functional imaging for prognosis assessment in HCC patients.

\section{A-397 11:00}

The (extra)ordinary night shift at the ER

K. Petrovic; Novi Sad/RS (smakap@sbb.rs)

Besides the accuracy, working at the ER requires maximal shortening of the diagnostics time, which has a significant influence on the patient's outcome. Thus, it is the radiologist's duty to be familiar with the pathophysiological mechanism of the disorders, specific protocols, possibilities and limits of certain examinations, and also to be aware of all possible pearls and pitfalls, to make the correct diagnosis. Pathology encountered at the ER has an extremely wide range and requires expertise in all imaging modalities from head to toe. The more the radiologist is aware of all possible situations, the more is the diagnosis accurate, and information given to the clinician is more valuable. The aim of this presentation is to point out the possibility of encountering different pathological conditions in different body regions. Moreover, it is important to be reminded that in radiology there are different modalities, each of which has its own indications and limits. This lecture reviews the selected series of cases from the ER Department of Clinical Center of Vojvodina, pointing out the most common possible differential diagnosis and possible diagnostic mistakes, which often occur due to the lack of experience.

Learning Objectives:

1. To point out the in-depth knowledge and experience in all imaging modalities and all body regions required for a radiologist to perform a routine work at the ER.

2. To present a series of cases that are most and less often encountered at the ER, discuss the possible differential diagnosis and mistakes which could potentially be made.

A-398 11:30

Diagnosis and management of pancreatic cystic lesions

R.J. Méndez; Madrid/ES (ramiro.mendez@salud.madrid.org)

Pancreatic cystic lesions are frequently diagnosed in patients with clinically suspected pancreatic disease, but they are also increasingly detected on imaging studies performed due to other abdominal processes. The prevalence 
of pancreatic cysts increases with patient's age. Most symptomatic cysts will be surgically resected, whereas asymptomatic lesions should be managed depending on the likelihood of causing harm to the patient. Cystic lesions can be inflammatory or neoplastic. The incidence of pseudocysts is low without a history of pancreatitis. Some pancreatic cystic neoplasms are malignant, but even benign lesions can become symptomatic depending on its size and location in the pancreas. The radiologist detects most of the pancreatic cystic lesions and should also play an important role in the clinical decision-making process. Cyst size, morphology, calcifications, contrast enhancement, location in the pancreas, relation with pancreatic ducts, patient's age and gender are important data to classify the lesion. A specific radiological diagnosis is not possible in every patient, but in many cases this information is enough to decide on a conservative approach with imaging follow-up. If a more aggressive lesion is a concern, then endoscopic ultrasound and fluid analysis can help to better characterise some pancreatic cystic lesions. Cyst puncture is only recommended if fluid analysis results will modify the patient's management. Patient's age, status and preferences should also be taken into account. When follow-up is recommended, the preferred imaging technique and interval should be indicated.

Learning Objectives:

1. To learn about the prevalence of pancreatic cystic lesions in the general population and how frequently they are detected on imaging studies.

2. To understand the different types of pancreatic cystic lesions, with emphasis on the more frequent aetiologies.

3. To become familiar with the radiological signs helping to classify the pancreatic cysts.

4. To learn about the consensus guidelines for the management of pancreatic cystic lesions.

$10: 30-12: 00$

Room N/O

Professional Challenges Session

PC 13

\section{Doing good not harm - understanding audit and its role in reducing patient dose}

\section{A-399 10:30}

Chairman's introduction

P. Cavanagh; ; Taunton/UK (peter.cavanagh@gmail.com)

The Chair will set the scene for the need for effective radiation protection in clinical radiology and highlight the role of clinical audit as a tool in achieving this.

Session Objectives:

1. To appreciate the case for improved radiation protection in diagnostic radiology.

2. To understand the principles of clinical audit.

3. To learn how clinical audit can be an effective tool in radiation dose reduction.

4. To recognise the relative merits of both internal and external Audit.

\section{A-400 10:35}

Internal audit in the UK

S. Barter; Cambridge/UK (suebarter@btinternet.com)

Clinical audit is defined as a professionally led method of improving patient care through the systematic examination of systems, processes and outcomes against chosen and agreed standards. It can readily be carried out by individuals, groups or whole departments. If properly conducted, clinical audit can be an effective way of examining what we do to provide reassurance on performance, and to improve patient care. The Clinical Radiology Audit Committee (CRAC) of the Royal College of Radiologists (RCR) co-ordinates national radiology audit activity. It promotes and facilitates audit through nominated audit leads in each hospital, who act as a link between the RCR and their department. At least one national audit is carried out annually, with data collected via electronic submission and the anonymised results presented at an annual Audit Forum. Individual departmental results are analysed using statistical process control (SPC) methodology. This enables identification of departments underperforming against the national mean, and recommending corrective action, by redesigning the process being audited, or by identifying and eliminating specific root causes locally. The Committee has developed a web-based tool for facilitating local audit, "AuditLive", a fully searchable collection of templates which can be downloaded and adapted. Fellows are also able to submit their own templates for publication hence sharing best practice. Trainees are encouraged to participate in audit through Audit Poster competitions at National Radiology Scientific Meetings. Our experience leads us to believe that audit succeeds when relevant, locally owned and properly structured, and multi-professional, and the RCR model encourages this. Learning Objectives:

1. To comprehend the role of clinical audit in radiology practice.

2. To understand how to facilitate clinical audit locally and nationally

3. To become familiar with some of the systems developed in the UK by the

Royal College of Radiologists.

Author Disclosure

S. Barter: Board Member; Chair Case Collection Committee European Board of Radiology, Member of Scientific Board European Board of Radiology. Employee; Cambridge University Hospitals Foundation NHS Trust.

\section{A-401 10:58 \\ Models of external audit in the Netherlands \\ S. Geers-van Gemeren; Utrecht/NL}

In the Netherlands clinical audit is legally obligatory for healthcare professions in order to be able to practise since 2010. Clinical audit is a tool designed to improve the quality of patient care, experience and outcome through formal review of systems, pathways and outcome of care against defined standards, and the implementation of change based on the results. The quality of the provision of care by professionals is assessed by peers. In the fields of Radiology, Nuclear Medicine and Radiotherapy, different models of clinical audit are used. For radiotherapy a multidisciplinary audit has been used since 2003. For Nuclear medicine a multidisciplinary audit has been implemented since 2013. For radiology the clinical audit for radiologists and for radiographers are separate. Implementation of the multidisciplinary audits needs requirements and adjustments of the audit system. This process is complex and needs approval of the members of all involved societies. To support the clinical audit a web based tool ADAS (General Digital Audit System) is used. The development of professional standards is a prerequisite to start clinical audit. The use of ADAS in multidisciplinary audits is a requirement to be able to audit different professions and focus on the content and the quality of their contribution to patient care. Clinical audit is a good tool to improve the quality of patient care. Important are the professional standards, the culture of learning and willing to improve by the professionals. "Every defect is a treasure."

Learning Objectives:

1. To learn about the different Dutch models of external clinical audit.

2. To comprehend the importance of professional standards, the culture of learning and willingness to improve.

3. To become familiar with the use of a digital audit system to support multidisciplinary clinical audit.

\section{A-402 11:21}

External regulatory audit in Finland

R. Seuri; Helsinki/FI (raija.seuri@hus.fi)

Directive 97/43/Euratom states that the clinical audit of medical imaging should be carried out in accordance with national procedures. The Finnish solution has been regulatory external audits every five years by radiological professionals with special training for audits. All radiological units have so far been audited at least twice, and the third time is performed on site in the unit. Although the purpose is to audit the process of radiological practises compared to "good practise", special focuses are recommended by the Finnish advisory committee for clinical audit, set by the National Institute for Health and Welfare. At the beginning focus was on the organisation and documentation of the structure and processes of imaging practise like justification, optimisation and quality control, but also encouragement and guidance to self-assessment. Later special focus has been on paediatric imaging and CT, and the third cycle will target deeper to CT practises. The auditing group includes at least a radiologist and a radiographer to audit a small unit, and a medical physicist if the unit has high-dose modalities like CT. During the audit the auditors work for an independent organisation. Multi-professionality gives both insight and the possibility to contact all professionals in imaging practise. The focus of clinical audit is on quality improvement; it is a way to both give and get feed-back and education. It is not control or inspection, but encouragement and guidance to self evaluation and implementation of good practises.

Learning Objectives:

1. To comprehend the supportive role of clinical audit.

2. To understand the different roles of clinical audit and technical quality control.

3. To become familiar with the Finnish clinical audit system. Author Disclosure:

R. Seuri: Consultant; STUK - Radiation and Nuclear Safety Authority, Finland. Other; Auditor: Labquality, Finland.

Panel discussion:

How can we use audit more effectively in reducing patient dose? 
12:15 - 12:45

Room A

Plenary Session

HL 3

\section{Samuil A. Reinberg Honorary Lecture \\ Presiding: \\ V.E. Sinitsyn; Moscow/RU}

A-403 12:15

The tempestuous genesis of MRI: credit and discredit

M.A. Meyers; East Setauket, NY/US (jimenez1234@optonline.net)

We often think of the scientific researcher as detached, objective, and dispassionate, nobly labouring without any expectation of reward. Nothing could be further from the truth. Scientists are as subject to ambition, competitiveness, envy and even guile as any person. Lifting the veil on scientific discovery reveals to us the human dramas that underlie not only the coveting of recognition, but also great conflicts over priority and credit. Nowhere are the stakes higher than for that pinnacle of scientific honour: the Nobel Prize. MRI has had a notably tempestuous genesis - a cogent episode that has much to teach us. It involved an explosive clash of personalities; deceit and rampant self-interest; challenges to the basic concept of scientific behavior; legal suits and validation by the United States Supreme Court; and declamations of denunciation in the international press. It persisted as a 30 year battle - a prize fight, a blood feud - between two protagonists: one whose seminal contribution established the biologic basis of MRI, and the other whose flash of insight served as a cornerstone of diagnostic imaging. One was awarded the Nobel Prize in medicine in 2010. The other was excluded. Why? Learning Objectives:

1. To understand the human elements in the process of discovery.

2. To understand the factors that determine the attribution of priority and credit.

3. To understand the courage to think on entirely new lines with innovative combinations of insights from different fields.

4. To understand the challenge of being in a race with another researcher.

5. To understand the power of entrenched dogma.

6. To understand the temptation of scientific misconduct.

$\overline{12: 30 ~-~ 13: 30 ~ R o o m ~ E 2 ~}$

The Beauty of Basic Knowledge: Skeletal Radiology

MC 26D

\section{Metabolic, endocrine and marrow disease \\ Moderator: \\ V.N. Cassar-Pullicino; Oswestry/UK}

\section{A-404 12:30}

Metabolic, endocrine and marrow disease

B. Vande Berg, A. Larbi, V. Perlepe, J. Malghem, F.E. Lecouvet; Brussels/BE (bruno.vandeberg@uclouvain.be)

1. Metabolic disorders of the skeleton involve the mineralised components of the skeleton. They affect all bone components histologically, but involvement patterns may vary depending on the age of the patient (growing versus adult skeleton) as well as the type of bone (cortical versus trabecular bone). They can be depicted on radiographs and CT images, but remain occult on MR images because the bone marrow is spared in the vast majority of these disorders. MR imaging can help in the assessment of local complication such as fractures. 2. Metabolic disorders of the bone marrow can affect either red (anemia...) or yellow marrow as well as the balance between these 2 marrow types in the body. Medical imaging plays a limited role in the assessment of these disorders, but marrow changes associated with these metabolic conditions must be recognised to avoid confusion with neoplastic conditions. Learning Objectives:

1. To appreciate the musculoskeletal manifestations of systemic disorders and their underlying pathomechanisms.

2. To understand the pathological processes involved in these imaging abnormalities.

3. To appreciate the strengths and weaknesses of imaging modalities in assessing these disorders.

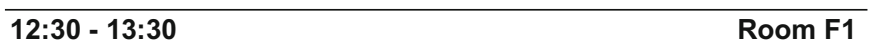

The Beauty of Basic Knowledge: Interventional Radiology

MC 25D

Tumour ablation: a primer

Moderator:

M. Bezzi; Rome/IT

A-405 12:30

Tumour ablation: a primer

T.K. Helmberger; Munich/DE (Thomas.Helmberger@klinikum-muenchen.de)

In primary and secondary malignant tumours of solid organs, in most of the cases surgical resection is considered the curative treatment. However, this is possible only in about $20 \%$ of the cases with tumour-dependent recurrence rates of $60 \%$ and even more. The main components for adjuvant, neo-adjuvant and finally palliative therapy are the permanently growing number of chemoand antibody therapies. Nevertheless, in many cases these therapies provide no definitive or long-lasting success, necessitating multimodality treatment concepts. In the meantime, local ablative techniques represent the main components of these concepts. Three main minimally invasive tumour-ablative techniques can be differentiated: chemo-ablation (percutaneous alcohol injection, transarterial chemo-embolisation, chemotherapy, chemo-perfusion), thermo-ablation (radiofrequency-, microwave-, laser-ablation, high-intensified focused ultrasound, cryo-ablation) and radio-ablation (radio-embolisation, interstitial brachytherapy, percutaneous stereotactic radiation). These ablation techniques differ significantly among each other with respect to their mode of action and indications; nevertheless, their benefit is high tolerance and at the same time high therapeutic efficacy, which can easily be combined with other treatment modalities. The different minimally invasive techniques will be discussed together with specific short- and long-term results and complications.

Learning Objectives:

1. To understand the indications for tumour ablation.

2. To learn about the technique and devices for image-guided ablation.

3. To learn about results, complications and follow-up strategies.

14:00 - 15:30 Room A

Interactive Teaching Session

$E^{3} 1420$

Common and uncommon errors in plain film and CT imaging of the chest: how to improve your performance

\footnotetext{
A-406 14:00

Common and uncommon errors in plain film and $\mathrm{CT}$ imaging of the chest: how to improve your performance

D. Tack ${ }^{1}$, N. Howarth' ${ }^{2},{ }^{1}$ Baudour/BE, ${ }^{2}$ Chêne-Bougeries/CH

Missed lung lesions are one of the most frequent causes of malpractice issues. Chest radiography plays an important role in the detection and management of patients with lung cancer, chronic airways disease, pneumonia and interstitial lung disease. Among all diagnostic tests, chest radiography is essential for confirming or excluding the diagnosis of most chest diseases. However, numerous lesions of a wide variety of disease processes affecting the thorax may be missed on a chest radiograph. The chest radiograph will also help narrow a differential diagnosis, help to direct additional diagnostic measures, and serve during follow-up. The diagnostic usefulness of the radiograph will be maximised by the integration of the radiological findings with the clinical features of the individual patient. CT has a tremendous spatial resolution that helps detect lesions in the chest and has proven to be more sensitive and specific than chest radiographs. However, missing lesions or misinterpreting lesions in CT of the chest is not uncommon. In this session, we will provide interactive cases of chest examinations (radiographs and CT) in which lesions have been missed and or misinterpreted, with a special focus on how correlation with MDCT of missed lung lesions can help improve interpretation of plain chest radiographs.
} 


\section{Postgraduate Educational Programme}

Learning Objectives:

1. To learn about the common reasons for errors in the interpretation of plain film and CT imaging.

2. To understand how a side-by-side comparison of the chest $\mathrm{x}$-ray and MDCT with missed lesions can help reduce the busy radiologist's error rate.

3. To learn about the skills required for accurate interpretation of plain film and $\mathrm{CT}$ imaging of the chest.

$14: 00-15: 30$ Room B

\section{New Horizons Session}

\section{NH 14}

\section{Imaging in precision medicine}

\section{A-407 14:00}

Chairman's introduction

H. Hricak; New York, NY/US (hricakh@mskcc.org)

This session will explore the value of imaging for facilitating precision medicine in which molecular data (including genomics, proteomics and metabolomics) is used to classify patients into subpopulations and tailor treatments to the specific molecular characteristics of their diseases. Imaging already plays a limited role in precision medicine, primarily through the use of molecular imaging techniques (e.g. PET/CT, MRI/PET and hyperpolarised MRI) and theranostics (the combination of an imaging agent with a therapeutic entity). In the near future, the role of imaging in precision medicine will increase at a gradually accelerating pace through the development of new molecular imaging probes and theranostic agents and through progress in radiogenomics (the correlation of radiologic findings with genomic features). This session will discuss recent findings and methodologies in radiogenomics as well as the use of nanotechnology for designing novel molecular imaging probes and theranostics. In addition, as theranostics is often considered the essence of precision medicine, the session will provide an in-depth look at current and emerging theranostic strategies and their special benefits for treatment selection, assessment of dose distribution of targeted therapies, and treatment follow-up

Session Objectives:

1. To understand the importance of imaging in precision medicine.

2. To learn about the emerging field of radiogenomics.

3. To appreciate the value of nanotechnology for molecular imaging and theranostics.

\section{A-408 14:05}

\section{Radiogenomics and personalised (precision) medicine}

G.P. Krestin; Rotterdam/NL (g.p.krestin@erasmusmc.nl)

"Precision medicine" as well as related notions, such as personalised medicine, or stratification medicine, all revolve around the idea that the consideration of individual characteristics - molecular and otherwise - can improve medical research and practice. Precision medicine is a multi-faceted approach to medicine that integrates molecular and clinical research with patient data and outcomes. Individual assessment of the location and extent of an alteration is and always has been the basis of medical imaging, whether the 'alteration' is a disease, a malformation, or an injury. As such, medical imaging intrinsically enables "precision medicine". The addition of genomic data in the last twenty years allows new correlations to be made between cellular genomics and tissue-scale imaging. Structural and functional imaging and the automated analysis of large amounts of image data have only recently reached a stage where they can be used on a large scale and in a population setting. Identifying common genetic variants that contribute to explain variance in imaging phenotypes by a systematic analysis of the genome is based on genome-wide association studies (GWAS). The power of GWA analyses has been recently demonstrated with the identification of susceptibility genes involved in a range of imaging phenotypes like coronary artery calcifications, intracranial volume, or size of the hippocampus. These developments highlight new etiological pathways and are expected to improve the understanding of the molecular basis of some diseases. Correlation between genotype and imaging phenotypes may be relevant for further characterising the development of disease states.

Learning Objectives:

1. To understand new concepts of precision medicine.

2. To learn about the potential correlation between genomics and imaging

phenotypes.

3. To appreciate the future role of imaging in precision medicine.

Author Disclosure:

G.P. Krestin: Advisory Board; Technion University, LMU,. Consultant; GEHC

Europe. Grant Recipient; Siemens, GEHC, Bayer HC, Philips HC, Bracco SA.

\section{A-409 14:28 \\ Use of nanotechnology, imaging and therapy}

T. Lammers; Aachen/DE (tlammers@ukaachen.de)

Advances in nanotechnology and chemical engineering have led to the development of a significant number of novel materials for diagnosis and therapy. Many different diagnostic and therapeutic nano- and micro-materials have been designed and evaluated over the years, including, e.g. gadoliniumcontaining dendrimers, USPIO nanoparticles and microbubbles for functional and molecular imaging, and drug-loaded liposomes, polymers and micelles for temporally and spatially controlled drug delivery to tumors and to sites of inflammation. In the literature, such advanced nano- and micromaterials are generally claimed to be highly useful and broadly applicable. A critical reflection on their specific capabilities, however, as well as on their pharmacokinetic properties, biodegradability and toxicity is often lacking. In the present lecture, I will briefly introduce the rationale for using diagnostic and therapeutic nanomaterials. I will highlight several clinically relevant examples in which nano- and micromaterials hold potential for improving disease diagnosis and detail how combining diagnostic and therapeutic properties within a single 'nanomedicine' formulation can be used to individualise and improve (chemo-) therapeutic treatments.

Learning Objectives:

1. To learn about the design and construction of nanoparticles for use in imaging and therapy.

2. To appreciate both the advantages and limitations of using nanoparticles as agents for both imaging and therapy.

3. To learn how nanotechnology enables multimodality imaging and therapeutic agents.

\section{A-410 14:51}

Theranostics

W. Weber; New York, NY/US

Precision medicine requires diagnostic tests that predict the effectiveness of specific therapeutic interventions for individual patients. This close interaction between therapeutics and diagnostics is expressed in the term "theranostics". A frequently used example for a theranostic is the staining of tumours for HER2 expression prior to therapy with HER2 antibodies, such as trastuzumab or pertuzumab. Theranostic has also been practised since many years in nuclear medicine by using radioiodine scans to select patients for treatment with iodine-131. A more recent example is the selection of patient for peptide receptor-targeted radiotherapy (PRRT) by somatostatin receptor imaging. Imaging is highly attractive for theranostics, because it allows investigators to study the spatial and temporal heterogeneity of target expression, whereas in vitro assays generally analyse a small part of the tumour at one point in time. Several molecular imaging probes, such ${ }^{89} \mathrm{Zr}$-trastuzumab or folate receptor targeting ligands, are in clinical development as in vivo companion diagnostics. Imaging companion diagnostics can also be used to assess tissue pharmacokinetics and thereby help to determine the optimal dose and dose schedule of therapeutic agents. They can also identify unexpected interactions between two therapeutic agents. For example, studies with ${ }^{11} \mathrm{C}$-docetaxel have shown that bevacizumab can markedly decrease the intratumoral delivery of docetaxel. Finally, imaging-based companion diagnostics can assess target inhibition, as demonstrated by the inhibition of uptake of the androgen receptor ligand ${ }^{18} \mathrm{~F}-\mathrm{FDHT}$ in metastatic prostate cancer treated with anti-androgens. Imaging-based companion diagnostics are therefore expected to play an important role in establishing precision medicine.

Learning Objectives:

1. To become familiar with current and emerging theranostic strategies and technologies.

2. To understand the value of theranostics for assessing dose distribution and the efficacy of targeted therapy.

3. To understand the key benefits of theranostics for treatment selection and follow-up.

Author Disclosure:

W. Weber: Advisory Board; Endocyte, MSD. Grant Recipient; Bayer, Philips.

Panel discussion:

Imaging in precision medicine: extracting simplicity

from complexity $\quad 15: 14$ 
14:00 - 15:30

Room C

\section{Modern Imaging of the GI Tract}

CC 1421

\section{Rectal cancer}

Moderator:

L.C.O. Blomqvist; Stockholm/SE

A-411 14:00

A. Imaging protocols

S. Gourtsoyianni; London/UK (sgty76@gmail.com)

ERUS technique as well as the state-of-the-art MR imaging protocols tailored according to the presenting rectal tumor site, allowing trained radiologists to obtain all necessary information for appropriate treatment decision-making, will be described. Normal cross-sectional appearances as well as morphological and signal changes encountered in pelvic structures/tissues that may be involved in primary rectal cancer will be illustrated. The influence of imaging findings on initial therapeutic approach and potential limitations of different imaging techniques will be discussed.

Learning Objectives:

1. To become familiar with state-of-the-art MRI and US protocols for rectal cancer staging.

2. To understand the normal appearances of the rectum and perirectal tissues on cross-sectional imaging.

3. To understand the advantages and disadvantages of imaging techniques in evaluating the rectum.

\section{A-412 14:20}

B. How to stage lymph nodes in rectal cancer

G. Brown; Sutton/UK (gina.brown@rmh.nhs.uk)

The staging and categorisation of malignant lymph nodes in patients with rectal cancer is a topical issue and has resulted in some degree of confusion. Much enthusiasm has been expended in determining whether mesorectal nodes are, or are not, involved in the primary disease process, as there is a perception that nodal disease is an important determinant of local recurrence. This incorrectly perceived association of nodal status and a high risk of pelvic recurrence have propagated the concept that this should be the primary indication for neoadjuvant therapy regardless of whether TME surgery is to be performed. Furthermore, misinterpretation of involved local nodes results in over-treatment of patients, if firstly involved nodes alone are considered as a poor prognostic factor, and secondly the optimal treatment of involved nodes is pelvic radiotherapy plus a non-systemically acting but radiosensitising dose of chemotherapy within chemoradiotherapy (CRT) schedules. In this lecture, the evidence for risk factors associated with nodal disease and validated risk factors for local recurrence will be reviewed. The evidence for both the optimal techniques and objective criteria for assessing lymph nodes with magnetic resonance imaging (MRI) will be presented.

Learning Objectives:

1. To learn about the importance of lymph node staging in rectal cancer.

2. To become familiar with an approach to assessing lymph nodes and be aware of pitfalls.

3. To appreciate the influence of nodal status on treatment outcomes.

\section{A-413 14:40}

C. Assessment after treatment

R.G.H. Beets-Tan; Maastricht/NL (r.beets.tan@mumc.nl)

The standard treatment for advanced rectal cancer is preoperative chemoradiotherapy (CRT) followed by standard resection of the rectum and mesorectum. Neoadjuvant CRT allows downsizing and downstaging of the tumour, leading to improved resectability and local control. While the role of $M R I$ in rectal cancer treatment is recognised and MRI is recommended as part of the standard staging workup, its role for restaging after preoperative treatment is more controversial. This lecture will provide an understanding of whether and how MRI can assess treatment response in rectal cancer and how it may impact treatment decision. The attendees will learn about the difficulties in image interpretation and learn about potential new imaging techniques to improve its performance.

Learning Objectives:

1. To understand the role of imaging in treatment response assessment of rectal cancer.

2. To learn about imaging features which identify viable disease after chemoradiation.

3. To appreciate the role of imaging in the modern management of patients with rectal cancer.
A-414 15:00

D. Interactive case discussion

L.C.O. Blomqvist; Stockholm/SE

\begin{tabular}{l}
\hline 14:00 - 15:30 \\
\hline Chest \\
RC 1404 \\
Pulmonary infections: the old and the new \\
Moderator: \\
N. Sverzellati; Parma//T
\end{tabular}

A-415 14:00

A. Mycobacterial infections

C. Beigelman; Lausanne/CH

Primary TB typically presents with consolidation in the middle and lower lobes, and necrotic lymphadenopathy. Fibrosis, tuberculomas and calcified nodes may result as sequelae. Cavitation and tree-in-bud appearance involving the apical and posterior segments of the upper lobes and the superior segments of the lower lobes are the hallmarks of reactivation TB is typically seen in immunocompetent patients. In immunocompromised patients, primary TB findings are more commonly observed, as miliary or disseminated disease or atypical manifestations in case of severe immunosuppression. Pleural effusion and tracheobronchial involvement may be observed. The "classical" appearance of non-TB mycobacteria $\left(\mathrm{N}^{\mathrm{TM}}\right)$, less common and more indolent than TB, typically affects males more than 50 years old with pre-existing pulmonary disease or underlying immunologic disorder. Despite a great overlap in the radiologic appearances of both infections, the presence of cavities on sites other than the upper lobes should suggest an $\mathrm{N}^{\mathrm{TM}}$ infection. The "nonclassical" form related to MAC infection that predominantly affects elderly women with no pre-existing pulmonary disease mainly consists in mild bronchiectasis and centrilobular nodules predominantly located in the lingula and middle lobe. A high rate of lymphadenopathy and disseminated disease are seen in immunocompromised patients. The radiologist has to ensure the diagnosis of active TB and mention scarring before starting specific treatments; to suggest $\mathrm{N}^{\mathrm{TM}}$ infections, immune reconstitution inflammatory syndrome with paradoxical worsening in HAART treated HIV-infected patients as multi-drug resistant TB; to appreciate the extent of disease and follow-up; to diagnose the complications; to propose MRI or PET scans in some situations.

Learning Objectives:

1. To appreciate the imaging features of primary and post-primary infections in TB.

2. To learn about the similarities and differences between TB and non-TB mycobacterial infections.

3. To understand the radiologist's role in diagnosis.

\section{A-416 14:30}

B. Infectious disease in immuno-compromised patients

C. Heussel; Heidelberg/DE

The appropriate investigational technique, frequently targeted differential diagnosis, and the special needs of immunocompromised patients need to be understood by the referring physician as well as by the radiologist. Thus, an intensive interdisciplinary co-operation on a patient basis, as well as on a department basis is essential. Early detection of a focus is the major goal in febrile neutropaenic patients. As pneumonia is the most common focus, chest imaging is a special radiological task. The sensitivity of chest $x$-ray, especially in the supine position, is known to be low. Therefore, the very sensitive thinsection multislice-CT became the gold standard in neutropenic hosts and might be cost-effective in comparison to antibiotic treatment. The infiltrate needs to be localised, so that this information can be used as guidance for invasive procedures for further microbiological workup. Furthermore, the radiological characterisation of infiltrates gives a first and rapid hint to differentiate between different sorts of infectious (e.g. typical bacterial, atypical bacterial, fungal) and non-infectious aetiologies. Follow-up investigations need careful interpretation according to disease, recovery, and concomitant treatment. Due to a high incidence of fungal infiltrates, interpretation of the follow-up of an infiltrate must use further parameters besides the lesion size. Besides the lungs, also other organs systems such as brain, liver and paranasal sinuses need attention and are to be imaged with the appropriate technique.

Learning Objectives:

1. To appreciate the various types of immuno-deficiencies and their related infections.

2. To become familiar with the role of the radiologist in immuno-compromised patients.

3. To learn how to follow pulmonary infections in immuno-compromised patients. 


\section{Author Disclosure:}

C. Heussel: Consultant; Schering-Plough, Pfizer, Basilea, Boehringer Ingelheim, Novartis, Roche, Astellas, Gilead, MSD, Lilly, Intermune, Fresenius. Patent Holder; Methods and Device For Representing the Microstructure of the Lungs. IPC8 Class: AA61B5055 FI, PAN: 20080208038, Inventors: W. Schreiber, U. Wolf, A.W. Scholz, C.P. Heussel. Research/Grant Support; Siemens, Pfizer, MeVis. Shareholder; Stada, GSK. Speaker; Gilead, Essex, Schering-Plough, AstraZeneca, Lilly, Roche, MSD, Pfizer, Bracco, MEDA Pharma, Intermune, Chiesi, Siemens, Covidien, Pierre Fabre, Boehringer Ingelheim, Grifols, Novartis.

\section{A-417 15:00}

\section{Emerging infections}

T. Franquet; Barcelona/ES (tfranquet@santpau.cat)

Despite advances in diagnosis and treatment, new pulmonary infections have been diagnosed. Streptococcus pneumoniae remains the main aetiological agent in outpatients with community-acquired pneumonia (CAP). Elderly patients or those with toxic habits and various comorbidities favour the development of severe CAP. In addition, the development of nucleic acid amplification techniques has emphasised the role of concomitant bacterial and viral pneumonia in the outcome of CAP in elderly patients. Healthcareassociated pneumonia has been recently defined as a different infectious condition by the American Thoracic Society/Infectious Diseases Society of America (ATS/IDSA). The main concern of this new disease is the risk of having an infection due to multidrug-resistant pathogens. With the advent of HAART and increased long-term survival of HIV-positive patients, the range of pulmonary manifestations has also evolved. In patients with haematological malignancies or after HSC transplant, Aspergillus is a common infection. Actually, Aspergillus spp. isolation from LRT samples in COPD may indicate an increased diagnosis possibility of IPA. New emerging viruses such as Human metapneumovirus (hMPV), SARS-associated coronavirus, and Avian influenza caused by the H5N1 virus have been diagnosed. In 2009, an outbreak of a novel swine-origin influenza A $(\mathrm{H} 1 \mathrm{~N} 1)$ virus was reported. The clinical diagnosis of new pulmonary infections as well as the presence of concomitant bacterial and viral infections has been significantly enhanced by improved laboratory methods. A systematic approach to the radiological evaluation of lung infections is essential and includes not only chest imaging pattern recognition, but also integration of available demographic, clinical and laboratory information.

Learning Objectives:

1. To learn more about emerging infections.

2. To become familiar with radiological patterns and conditions which suggest viral infections.

3. To learn how to improve diagnostic accuracy.

$14: 00-15: 30$ Board Room B

\section{Paediatric}

\section{RC 1412}

\section{Normal variants in paediatric imaging: not to be confused with disease}

\section{Moderator:}

M. Raissaki; Iraklion/GR

A-418 14:00

\section{A. Brain}

A. Rossi; Genoa/IT (andrearossi@ospedale-gaslini.ge.it)

The paediatric central nervous system is a complex structure undergoing rapid development. As such, there is a rapid, continuous modification of what is "normal" in relation to age and the stage of development. Knowledge of the normal patterns of brain development in the clinically relevant ages from 0 to 18 years is necessary to interpret neuroimaging findings correctly. Knowledge of embryology and normal variants is also greatly helpful. MR imaging equipment and parameters need to be adjusted and optimised for paediatric studies. Pitfalls often occur from the misunderstanding of normal conditions that are perceived as abnormal based on a comparison with the appearance of the normal brain in adults. This includes, for instance, the evaluation of the brain in the first 2-3 years of life during the course of the process of myelination. A summary of the most frequent conditions that may lead to misinterpretation of findings will be provided.

Learning Objectives:

1. To learn about normal variants in the neonatal and child's brain.

2. To understand the typical imaging characteristics of normal variants that should suffice for correct interpretation.

3. To become familiar with the differentiation between normal variants and disease.

\author{
A-419 14:30 \\ B. Chest and abdomen \\ S.G.F. Robben; Maastricht/NL (s.robben@mumc.nl)
}

A wide spectrum of anomalies of thoracic and abdominal organs may be encountered on radiologic evaluation. These anatomic variants and developmental anomalies can all pose a diagnostic challenge to the radiologist. Familiarity with these anomalies, the imaging techniques available for their study, and their variable imaging manifestations is necessary for differentiating them from pathology. A basic understanding of the embryologic development and normal anatomy of thoracic and abdominal organs and vessels is also essential for identifying these anomalies. This review explores the wide variability in appearance of the normal thoracic and abdominal organs during imaging, stressing a thorough understanding of normal anatomy to recognise normal variants.

Learning Objectives:

1. To learn about normal variants in the neonatal and paediatric chest and abdomen.

2. To become familiar with the imaging appearances of common normal variants.

3. To understand how to differentiate between normal variants and disease.

\section{A-420 15:00}

\section{Musculoskeletal}

F. Saez; Barakaldo/ES (fersaez@yahoo.com)

The skeleton of a child is a developing system with a variety of changing normal appearances. Imaging studies, especially plain films, are requested for many clinical reasons, and the radiologist is in the position to determine if an image is a normal finding or we are dealing with a lesion. The way the physis and epiphysis grow, ossify, and fuse constitutes a great source of physiologically bizarre appearances, which the radiologist must be familiar with. This talk will concentrate on the plain film diagnosis of some of the most common musculoskeletal variants. Other imaging modalities will also be shown when appropriate for the case. Irregularities, asymmetries, partial fusions, hypo- or hyper-dense bone areas, accessory bones, prominent normal structures, external artifacts, and potential fracture lines are the most often encountered pseudolesions. A defective radiological technique may also be potentially misleading. Patient age, location of the supposed "abnormality" and lack of significant local symptoms are key factors. Usually plain films, correlated with regional clinical findings, are the only imaging method that is required. However, in certain doubtful situations, ultrasound, CT, MRI, bone scan, or even biopsy may be needed to reach the right diagnosis. Unnecessary overuse of these imaging modalities, and the subsequent family anxiety that ensues from this overuse, should be avoided with careful analysis of the X-ray and clinical findings.

Learning Objectives:

1. To learn about normal variants in the neonatal and paediatric musculoskeletal system.

2. To become familiar with the imaging appearances of common normal variants.

3. To understand how to differentiate between normal variants and disease. 4. To learn how to integrate age, location and clinical history with the radiological features before establishing a diagnosis.

$14: 00-15: 30$

Room E1

\section{Musculoskeletal}

\section{RC 1410 \\ How I do and report}

Moderator:

F. Kainberger; Vienna/AT

\section{A-421 14:00 \\ A. MRI of the hip \\ J. Teh; Oxford/UK (jamesteh1@googlemail.com)}

The purpose of this lecture is to cover the clinical presentation, underlying pathological processes and essential MRI features of relatively common conditions affecting the hip. As the hip is afflicted by different conditions according to age, this is how the various pathological entities will be presented. A simple imaging algorithm is presented showing the role of MRI. The role of MR arthrography in the assessment of the dysplastic hip and femoroacetabular impingement is covered. A structured approach to MRI reporting is outlined. 


\section{Postgraduate Educational Programme}

Learning Objectives:

1. To learn about standardised imaging.

2. To understand the MRI specific findings that aid diagnosis.

3. To learn a structured approach to reporting.

\section{A-422 14:30}

B. MRI of the spine and sacroiliac joints

A. Feydy; Paris/FR (antoine.feydy@cch.aphp.fr)

$M R I$ is the preferred imaging modality for spinal and SIJ imaging in patients with suspected or established spondyloarthropathies (SpA). There is a need to perform standardised and efficient MR protocols in this clinical setting. The main goals are to detect inflammatory active lesions and also structural lesions. In addition, the MRI should be able to diagnose other diseases such as disk degenerative disease, DISH, or metastases in patients with nonconfirmed SpA. A systematic approach is recommended for MRI reading The radiologist report has to focus on the more specific MRI findings of SpA, if present, and to give a conclusion regarding the activity and the structural damages.

Learning Objectives:

1. To learn standardised imaging.

2. To understand the MRI specific findings that aid diagnosis

3. To learn a structured approach to reporting.

A-423 15:00

C. MRI of the hand

C. Schueller-Weidekamm; Vienna/AT

(claudia.schueller-weidekamm@meduniwien.ac.at)

MRI of the hand requires high spatial resolution, perfect immobility, and homogeneous fat suppression. The hand can either be placed in the Superman position, which often is uncomfortable for the patients, or both hands can be placed above the abdomen next to each other while the patient is in the supine position. Standard sequences are axial PD, followed by coronal T1 TSE, and T2 TSE FS in the coronal and sagittal planes. Additional 3D gradient echo sequences with fat saturation are recommended to assess ligaments and cartilage, as well as the triangular fibrocartilage complex (TFCC). In certain cases, MR arthrography with thin-slice T1 FS is helpful for further evaluation of the wrist ligaments. In inflammatory diseases, such as rheumatoid arthritis, static or dynamic T1 FS sequences are useful to assess tenosynovitis and synovitis. The most common injuries and inflammation of the hand and wrist are discussed with special emphasis on key findings for an accurate diagnosis. The awareness of advantages and drawbacks of other imaging modalities, such as conventional radiographs and CT, should be strengthened. The clinical impact of the radiological report is discussed to strengthen the importance of proper terminology for the description of pathological findings. The structure of the report should be clear and concise, and should allow an interaction and broad communication with clinicians.

Learning Objectives:

1. To learn about standardised imaging.

2. To understand the MRI specific findings that aid diagnosis.

3. To learn a structured approach to reporting.

$14: 00-15: 30$

Room E2

\section{Oncologic Imaging}

RC 1416

\section{Gastro-entero-pancreatic neuro-endocrine tumours (GEP-NET): a multidisciplinary update}

\section{A-424 14:00}

Chairman's introduction

C. Matos; Brussels/BE (cmatos@ulb.ac.be)

Gastro-enteropancreatic neuroendocrine tumours (GEP-NET) are a heterogeneous group of cancers that differ in their biology and clinical presentation. Diagnosis of these tumours has been improved by advances in pathology and classification and by the combined use of structural imaging and functional imaging modalities. Multimodal imaging is increasingly recognised both in detecting and staging disease and also in characterising biological patterns of lesions that may be relevant to the selection and delivery of therapy. In this course, the complex nature of GEP-NET and the intrinsic uses and limitations of each diagnostic imaging modality will be underlined. Insights to hybrid structural and molecular imaging techniques will be provided and discussed.

\author{
A-425 14:05 \\ A. Tumour biology, pathogenesis and classification \\ B. Wiedenmann; Berlin/DE (bertram.wiedenmann@charite.de)
}

Neuroendocrine tumour cells are characterised by the coexpression of neuronal and epithelial proteins and cellular organelles such as synaptic vesicles containing synaptophysin of neurons and intermediate filaments/cytokeratins of the epithelial cells. Based on the presence of secretory vesicles and the continous, uncontrolled vesicular release of biogenic amines, neuropeptides and hormones, patients suffer in half of the cases of so called functional symptoms and syndromes. Examples are the carcinoid syndrome (excessive release of serotonine) or the Zollinger-Ellison syndrome (excessive release of gastrin). Activation or inhibition of certain G-protein coupled receptors (e.g. somatostatin receptors) or channel proteins (R-type calcium channels) can lead to the control of the hypersecretion or so called functionality of the affected patients. Medical interference with signal transduction pathways involving tyrosine kinase receptors as such mTor and channel proteins can lead to an inhibition of cellular and tumour growth. These observations have led to the establishment of new therapies, especially for pancreatic NETs using especially $\mathrm{mT}$ Tor and tyrosine kinase inhibitors. Whereas the tumorigenesis is unknown in the case of sporadic NETs, hereditary NETs appear to develop via a menin mutation through the intermediate stage of hyplasia before they develop the full metastatic potential. Based on the above given tumour biological and histopathological findings, a rather robust classification for NETs has been developed by the European Neuroendocrine Tumor Society (ENETS) using a TNM-classification together with a gradingsystem. Furthermore, the formerly used terms such as APUDOMa, Neurocrestoma, Carcinoid, etc. have been largely replaced by the meanwhile generally accepted term neuroendocrine tumour.

Learning Objectives:

1. To learn about the basic aspects of GEP-NET biology, pathogenesis and classification.

2. To understand the epidemiology and current treatment options.

3. To become familiar with rational clinical management.

Author Disclosure:

B. Wiedenmann: Advisory Board; Novartis, IPSEN Biotech, Pfizer. Speaker; Novartis, IPSEN Biotech.

A-426 14:28

B. The current role of nuclear medicine

C. Deroose; Leuven/BE (christophe.deroose@uzleuven.be)

This presentation will focus on the pivotal role of nuclear medicine in the diagnosis and treatment of neuroendocrine tumours (NETs). The metabolic and molecular imaging capabilities of both positron emission tomography (PET) and single photon emission tomography (SPECT) have made a great impact on the clinical management of patients with these tumours. A poster child of molecular imaging in oncology is scintigraphy of the somatostatin receptor (SSR). ${ }^{111}$ In-DTPA-octreotide has a substantial value in detection, diagnosis and staging of NET and more particularly in gastroenteropancreatic NETs. Newer SSR-binding radiopharmaceuticals have been developed for PET, mainly with gallium-68 as radiolabel, with higher affinity and more advantageous pharmacokinetics. When used with high-resolution and rapidthroughput multimodal whole body imaging of modern PET/CT cameras, these tracers provide the current state-of-the art SSR imaging. We will also discuss the clinical value of metabolic tracers, such as $\left.{ }^{18} \mathrm{~F}\right]-F D G$ for glucose metabolism imaging, $\left[{ }^{18} \mathrm{~F}\right]$-DOPA and $\left[{ }^{11} \mathrm{C}\right]$-HTP $\left(\left[{ }^{11} \mathrm{C}\right] 5\right.$-hydroxytryptophan $)$ for amino acid metabolism imaging. The clinical merits and indications of these tracers will be explained. The continuously evolving quest to develop tracer for other receptor systems expressed on NETs will be illustrated, e.g. bombesin, VIP, CCK and glucagon-like peptide receptor ligands. Finally, the role of imaging as selection for metabolic and peptide receptor radionuclide therapy will be discussed.

Learning Objectives:

1. To learn about the cellular properties and GEP-NET used in molecular imaging.

2. To become familiar with the different modalities and new tracers being used.

3. To learn about the performance of the different methods available.

Author Disclosure:

C. Deroose: Advisory Board; Merck; Endocyte.

A-427 14:51

C. Anatomical imaging: transabdominal US, endoscopic US, MDCT and MRI: which is the most appropriate imaging approach?

V. Vilgrain, M.-P. Vullierme, P. Ruszniewski, A. Sauvanet; Clichy/FR (valerie.vilgrain@bjn.aphp.fr)

Gastroenteropancreatic neuroendocrine tumours (GEP-NETs) are a heterogeneous group of neoplasms that arise from cells of the diffuse neuroendocrine system and may present with a wide spectrum of clinical 
presentations. Their prognosis is mainly related to their biology, proliferation and differentiation. The main goals of imaging are the diagnosis and the staging of these tumours. The diagnostic challenge is very different in functional tumours where clinical presentation and laboratory parameters are of utmost importance and in nonfunctional tumours where imaging may show characteristic features such as hypervascularisation and calcifications. Staging is also essential as locoregional involvement and distant metastases (such as liver metastases) may change the therapeutic approach and are major prognostic factors. Multimodal workup included morphological imaging modalities with CT and MR imaging, and endoscopic ultrasound was the most useful and functional imaging. The latter includes somatostatin receptor scintigraphy, FDG PET and more recent functional tools such as PET using $68 \mathrm{Ga}$ and $18 \mathrm{~F}-\mathrm{DOPA}$. Imaging may also play a role in assessing prognosis in combination with tumour differentiation and tumour proliferation, obtained from pathologic examination. Last, imaging is useful in evaluating tumour response after treatment. Although surgery remains the only potentially curative therapy for patients with primary GEP-NETs, other available treatments include chemotherapy, interferon, somatostatin analogues, and targeted therapies. Imaging criteria rely not only on changes in tumour size, but also on internal tumour changes.

Learning Objectives:

1. To learn how to recognise the specific imaging features of GEP-NET.

2. To learn about the strengths and weaknesses of the different imaging modalities.

3. To understand the optimal use of the different imaging modalities in relation to tumour location and staging.

Panel discussion:

The future of hybrid imaging

$15: 14$

$14: 00-15: 30$ Room F1

Special Focus Session

\section{SF 14a}

\section{Assessment of brain perfusion with arterial spin labelling (ASL)}

\section{A-428 14:00}

\section{Chairman's introduction}

X. Golay; London/UK (x.golay@ucl.ac.uk)

The purpose of this session is to bring the audience up to date with a novel MRI method capable of measuring brain perfusion. This method is called arterial spin labelling (ASL) and provides a complete non-invasive means to quantitatively assess cerebral blood flow (CBF). As with any MRI technique, ASL relies on proper setting of many technical parameters to provide an adequate image quality, with minimal influence from potential artefacts. A recent position paper, co-signed by members of the Perfusion Study Group from the International Society for Magnetic Resonance in Medicine and the European COST-funded Action on 'ASL in Dementia', has established a series of simple guidelines to help promote this technology in clinical practice. These guidelines will be provided within this session. The created CBF maps will then be analysed, and a simple description of the main features and clinical characteristics of these physiological images will be given to the audience. In particular, a proper difference in the meaning of CBF in several diseases will be highlighted, as it can be either causative, such as e.g. in stroke and cerebrovascular diseases, where a reduced CBF leads to a breakdown in tissue homeostasis, or consequential, as in e.g. dementia, in which a reduction of $\mathrm{CBF}$ is the mere reflection of impaired metabolism, combined or not with an underlying brain atrophy. Following this session, the radiologist or clinician should be able to better appreciate ASL-based SBF maps in several neurological conditions.

Session Objectives:

1. To understand which elements are key to ensuring the optimal estimation of cerebral blood flow by ASL.

2. To become familiar with the imaging features of ASL.

3. To understand the clinical role of ASL in neurological diseases.

Author Disclosure:

X. Golay: Research/Grant Support; Philips Healthcare.

\section{A-429 14:05}

An introduction to ASL, its jargon and methods

M. Guenther; Bremen/DE (Matthias.Guenther@mevis.fraunhofer.de)

Perfusion is an important parameter to assess the status and liability of organs and tissue. Typically, exogenous contrast agents are administered to measure this quantity. Arterial spin labelling (ASL) is capable of estimating perfusion without the use of exogenous contrast media by labelling inflowing blood magnetically. This labelling process can happen either in a short time over an extended region or over a longer time period, but in a localised area. These techniques are called pulsed ASL (PASL) or continuous ASL (CASL), respectively. For quantification, a critical parameter is the time the labelled blood takes to flow from the region of labelling to the imaging region. This time is typically called bolus arrival time (BAT) or arterial transit time (ATT). It is important to either reduce the influence of this transit time on measured signal intensity or directly estimate it. Applying proper bio-physical models, the perfusion-weighted data can then be converted in absolute measures of perfusion. More sophisticated methods allow measuring of the main feeding artery of each imaging voxel (vascular territory mapping) or assessing other parameters beyond perfusion, allowing more detailed assessment of tissue status and function."

Learning Objectives:

1. To learn the basics of ASL, its methods and proposed implementation in clinical platforms.

2. To understand the influence of parameter choices on perfusion images.

3. To become familiar with basic quantification techniques in ASL.

Author Disclosure:

M. Guenther: CEO; mediri Gmbh, Heidelberg, Germany. Research/Grant Support; Siemens Healthcare, Erlangen, Germany.

A-430 14:28

The use of ASL in cerebrovascular disease

J. Hendrikse; Utrecht/NL (j.hendrikse@umcutrecht.nl)

Obvious applications of arterial spin labelling in clinical MR protocols are cerebral blood flow measurements in patients with acute or chronic cerebrovascular disease. In patients with acute stroke, the cerebral blood flow measurements may indicate the infarct core, with severely decreased perfusion and the infarct penumbra, with decreased perfusion but still viable brain tissue. In chronic cerebrovascular disease, arterial spin labelling CBF measurements show the regionally impaired haemodynamics distal to a carotid obstruction. With adequate collateral blood flow, these areas may be relatively small and with a failure of compensatory mechanism the cerebral blood flow may fall below a critical level. In acute stroke patients, ASL MRI may show compensatory hyperperfusion in stroke regions after the recanalisation of an occluded artery. Other applications of arterial spin labelling are clinical MR protocols in children. In addition to CBF-weighted images, ASL MRI has also the ability to measure timing parameters: typically, the time it takes for the magnetically labelled arterial blood to flow from the arteries in the neck to the brain tissue, which may be delayed in patients with cerebrovascular disease. Furthermore, ASL MRI has the ability to visualise the (collateral) perfusion territories of the brain feeding arteries in many clinical applications. In patients with cerebrovascular disease, a pitfall may be the absence of label in brain regions due to delayed (collateral) flow, which also may result in high ASL signals in (collateral) arteries.

Learning Objectives:

1. To appreciate the different roles of ASL in cerebrovascular diseases.

2. To become familiar with the different types of calculated images obtained

from multi-time points and selective pulses ASL.

3. To become familiar with the limitations and pitfalls of ASL.

\section{A-431 14:51}

The use of ASL in non-vascular brain disease

M. Smits; Rotterdam/NL (marion.smits@erasmusmc.nl)

Arterial spin labelling (ASL) is a non-invasive MRI technique with which cerebral blood flow (CBF) can be measured quantitatively. Although the first publications of ASL MRI date over 10 years back, it is only now that ASL is commercially available on MRI systems from all major vendors and is making its way into clinical practice. The main areas of interest for current and future nonvascular clinical application of ASL MRI of the brain are dementia and neuro-oncology. ASL is proposed as a diagnostic alternative to fluorodeoxy-Dglucose (FDG)-PET in the workup of dementia patients. ASL has several advantages over FDG-PET. Crucially, it can easily be added to the routinely performed structural MRI examination. Feasibility studies show that ASL provides reliable CBF maps in dementia. In patients with established Alzheimer's disease and frontotemporal dementia, hypoperfusion patterns are seen that are similar to hypometabolism patterns with FDG-PET. Current and future studies in this field will need to demonstrate the validity of ASL in the diagnostic workup of the individual patient, early in the disease process. Studies on ASL in brain tumour imaging indicate a high correlation between areas of increased CBF as measured with ASL, and increased cerebral blood volume as measured with dynamic susceptibility contrast-enhanced perfusion imaging. The major advantages of ASL for brain tumour imaging are the fact that CBF measurements are not influenced by breakdown of the blood brain barrier, as well as its quantitative nature, facilitating multicentre and longitudinal studies. 
Learning Objectives:

1. To understand the role of ASL in non-vascular brain disease.

2. To become familiar with current and future indications for the use of ASL in non-vascular brain disease.

3. To appreciate the limitations and pitfalls of ASL.

Panel discussion:

How relevant is ASL in clinical practice today?

$15: 14$

$14: 00-15: 30$

Room F2

Breast

RC 1402

\section{Update on BI-RADS}

Moderator:

M.G. Wallis; Cambridge/UK

A-432 14:00

A. Mammography

U. Bick; Berlin/DE (Ulrich.Bick@charite.de)

The Breast Imaging Reporting and Data System (BI-RADS®) for mammography of the American College of Radiology (ACRß) consists of several components, a standardised lexicon of terms to be used during reporting, a 4-step coding system for the mammographic density as a surrogate parameter for the mammographic sensitivity, and a group of assessment categories ranging from 0 to 6 for structured communication regarding the recommended further management. The goal of BI-RADS $₫$ is to improve the quality of breast imaging reporting and communication. In addition, by providing structured reports it facilitates regular quality assurance measures. The BI-RADS $®$ atlas for mammography is currently in its fourth edition and was released in 2003. The upcoming $5^{\text {th }}$ edition is expected soon and will be incorporated into the course as it becomes available.

Learning Objectives:

1. To learn about the BI-RADS lexicon.

2. To understand the usefulness of BI-RADS system.

3. To become familiar with the mammography descriptors.

A-433 14:30

B. Ultrasound

F. Thibault; Paris/FR (fabienne.thibault@curie.fr)

The Breast Imaging Reporting and Data System (BI-RADS) was first developed by the American College of Radiology for standardising the reporting of mammography. Since its first publication in 1993, new editions have also addressed breast ultrasound (US) and MR imaging examination. The new (fifth) edition will be published soon. On the form, this new version was designed to include a web-based format. Substance includes updates in lexicon descriptors, e.g. masses, calcifications, associated features (now comprising surrounding tissue with stiffness assessment), and special cases. US descriptors will be reviewed in the lecture. Of note, guidance on how to link BI-RADS descriptors with management recommendations has been added in the report section. A new approach to outcome assessment (audit section) is being proposed for screening US. The appropriate use of descriptors is expected to increase the accuracy of imaging interpretation. For relevant patient management, US analysis is to be integrated with other available imaging, as well as with clinical data.

Learning Objectives:

1. To learn about the BI-RADS lexicon.

2. To understand the usefulness of BI-RADS system

3. To become familiar with the ultrasound descriptors.

\section{A-434 15:00}

\section{MRI}

K. Pinker-Domenig, P.A.T. Baltzer; Vienna/AT

(katja.pinker@meduniwien.ac.at)

Dynamic contrast-enhanced magnetic resonance imaging (DCE-MRI) of the breast is a well-established non-invasive imaging technique. It has clinical application in the screening of high-risk patients, diagnosis and staging of breast cancer, monitoring neoadjuvant chemotherapy and post-treatment follow-up. To standardise the reporting of DCE-MRI of the breast and minimise false-positive results without compromising sensitivity, the American College of Radiology (ACR) introduced the Breast Imaging-Reporting and Data System (BI-RADS $尺$ ) MRI lexicon in 2003. BI-RADS relies on the combined analysis of morphological appearance and lesion enhancement kinetics. It is widely used for reporting DCE-MRI of the breast and is applicable at any given field strength. It aims to provide an up-date report on the BI-RADS $₫$ lexicon and instill confidence in using BI-RADS $₫$ descriptors. The BI-RADS $®$ system may be placed in a broader clinical context to highlight its value for standardised reporting of DCE-MRI of the breast.

Learning Objectives:

1. To learn about the MRI BI-RADS $®$ lexicon.

2. To learn how to recognise the descriptors defined in the MRI BI-RADS $®$ lexicon.

3. To understand the clinical relevance of the BI-RADS $®$ system and apply the BI-RADS $®$ lexicon when reporting MRI of the breast.

\begin{tabular}{l} 
14:00 - 15:30 \\
\hline Special Focus Session \\
SF 14b \\
Treatment with MR-guided focused US \\
(FUS)
\end{tabular}

A-435 14:00

Chairman's introduction

W.M.W. Gedroyc; London/UK (w.gedroyc@imperial.ac.uk)

MR-guided focused ultrasound is a new therapeutic modality which can allow selective destruction and or heating of tissues in deep body areas under close image guidance control. These talks will introduce the field and allow you to understand the areas of current work and areas of future development in this rapidly expanding field.

Session Objectives:

1. To become familiar with MR-guided focused ultrasound

2. To understand the advantages of focused ultrasound.

3. To learn in which areas focused ultrasound is evolving successfully.

A-436 14:05

Description of technique

C. Moonen; Utrecht/NL (C.Moonen@umcutrecht.nl)

High-intensity focused ultrasound (HIFU) is the only clinically viable technology that can be used to achieve a local temperature increase deep inside the human body in a non-invasive way. MRI guidance of the procedure allows in situ target definition and identification of nearby healthy tissue to be spared. In addition, MRI can be used to provide continuous temperature mapping during HIFU for spatial and temporal control of the heating procedure and prediction of the final lesion based on the received thermal dose. The primary purpose of the development of MR-guided HIFU was to achieve safe non-invasive tissue ablation. The technique has been tested extensively and is now accepted in the clinic for ablation of uterine fibroids. MR-guided HIFU for ablation shows conceptual similarities with radiation therapy. However, thermal damage generally shows threshold-like behaviour with necrosis above the critical thermal dose and full recovery below. MR-guided HIFU is being clinically evaluated in the cancer field. This presentation will cover the basic technologies for treatment of stationary tissues, and some advances towards treatment of mobile abdominal organs.

Learning Objectives:

1. To learn how MR-guided focused ultrasound is generated.

2. To understand the current physical limitations of this technique.

3. To learn how the technology is being improved to overcome the limitations of administering focused ultrasound to patients.

Author Disclosure:

C. Moonen: Research/Grant Support; Philips Healthcare.

A-437 14:28

Uterine fibroids and adenomyosis

Y. Inbar; Tel Hashomer/IL (Yael.Inbar@sheba.health.gov.il)

Magnetic resonance imaging-guided focused ultrasound (MRgFUS) for the noninvasive treatment of uterine fibroids has been used in a clinical setting since 2003. During the past decade, many improvements have been made in the treating systems, making treatments easier, faster, safer and fitting a wider range of patients. In this presentation, I will talk about the advances made over the years in the MRgFUS systems, helping to overcome many previously encountered obstacles. We will discuss patient selection and suitability and review the current success rates of the procedure. In addition, we will address the issue of adenomyosis, another uterine pathology frequently encountered that can produce symptoms similar to uterine fibroids. For these patients treatment options are extremely limited, making MRgFUS an attractive treatment option. 
Learning Objectives:

1. To learn which patients are suitable for MR-guided focused ultrasound of uterine fibroids.

2. To understand the current success rates of this procedure.

3. To appreciate the outcome of FUS is in the treatment of adenomyosis of the uterus.

Author Disclosure:

Y. Inbar: Research/Grant Support; Insightec.

\section{A-438 14:51}

Transcranial FUS

E. Martin; Zurich/CH (Ernst.Martin@kispi.uzh.ch)

Transcranial focused ultrasound (tcMRgFUS) is a novel technique for noninvasive neurosurgical interventions, that is magnetic resonance imaging guided for target definition and treatment planning with continuous temperature measurements in real time. TcMRgFUS has no trajectory restrictions and does not involve ionising radiation. The ability to focus ultrasound energy through the intact skull precisely to desired targets, while avoiding collateral brain tissue damage, thus, minimising adverse effects by mistargeting is certainly attractive. Since no device is implanted, there is no restriction to future diagnostic workup with high-field MR imaging. TcMRgFUS allows treating a variety of chronic, therapy-resistant neurological diseases by focusing highintensity ultrasound energy (HIFU) to the thalamus, subthalamus or basa ganglia. Ongoing clinical studies on over 130 patients with neuropathic pain essential tremor, Parkinson's disease and obsessive compulsive disorder are very encouraging. They promise tcMRgFUS to become a noninvasive alternative treatment option for patients with therapy-resistant neurological and neuropsychiatric disease. Moreover, ablation of pathological tissue as in brain tumours and brain metastases is urgently needed to supplement traditional neurosurgical procedures. Future trends: preclinical studies using low-intensity focused ultrasound (LIFU) have shown to silence dysfunctional neuronal circuits and to transiently modulate neural functions. This eventually may lead to better targeting during functional neurosurgical interventions such as FUS and DBS. Moreover, in combination with microbubbles, LIFU pulses enable opening of the blood-brain-barrier, facilitating drug delivery to specific targets in the brain. Conclusion: TcMRgFUS promises new therapeutic approaches for a variety of brain diseases that may change patient management.

Learning Objectives:

1. To understand how focused ultrasound can be utilised in the brain

2. To learn which conditions can be treated successfully.

3. To understand how this technique will evolve and what role it will play in the management of chronic and acute neural conditions.

Panel discussion:

How can this technology be spread more widely?

$15: 14$

$14: 00-15: 30$ Room I/K

\section{Head and Neck Imaging}

CC 1419

\section{What do I see? Clinical radiological interaction (part 2)}

Moderator:

B. Ozgen Mocan; Ankara/TR

\section{A-439 14:00}

A. Presurgical and postsurgical imaging: free flap reconstructions A. Trojanowska, P. Trojanowski; Lublin/PL

The use of microvascular free flaps for reconstructions allowed for the development of extensive resections of head and neck tumours. Radiological evaluation of patients subjected to reconstructive operations using free flaps requires the in-depth knowledge of flap structure and anatomy. Imaging allows delineation of the tumour and planning the extent of resection. Availability of reconstructive surgery extends the scope of surgical intervention, but requires meticulous consideration of the choice of flaps. It is necessary to investigate with imaging the donor site to ensure adequate flap size, shape and vasculature. At the same time it is of paramount importance to assure that flap harvesting does not endanger functionality and blood supply to the donor site. Imaging studies of the donor site can reveal vascular abnormalities and therefore prevent acute and chronic donor site ischemia. In the follow-up of patients with transplanted tissues, specific difficulties in detecting recurrence exist because there is an altered anatomy. This lecture is based on the interpretation of CT and MR cases illustrating follow-up of microvascular free flaps used for the reconstruction of defects resulting from head and neck cancer surgery, with particular attention to the role of radiologic-clinical cooperation for the appropriate interpretation of imaging findings. Learning Objectives:

1. To become familiar with major flap types used for head and neck reconstructions and the importance of reconstructive surgery.

2. To understand how to evaluate images for surgery planning.

3. To learn how to perform follow-up studies in patients after free flap reconstructions in order to avoid possible pitfalls.

A-440 14:30

B. Sinonasal imaging for functional endoscopic sinus surgery (FESS) V. Lund, G. Madani; London/UK

Computerised tomography offers the gold standard in terms of imaging the extent of disease and the fine detailed anatomy, both pre-requisites to the safe practice of endoscopic sinus surgery (ESS). A variety of protocols minimising radiation dose to the lens whilst providing high-quality images have been described, depending on the scanner and whether image guidance is being used. In general for ESS in chronic rhinosinusitis with or without nasal polyps, coronal and axial views are sufficient though sagittal cuts are recommended when surgery on the frontal sinus is contemplated. The anatomy varies considerably on an individual basis and includes a range of anatomic variants, which although do not predispose to the disease, require careful evaluation pre-operatively. MRI plays a limited role in benign inflammatory disease, though it may be undertaken secondarily when the diagnosis is unclear. Learning Objectives:

1. To learn about common sinonasal pathologies.

2. To understand why we use FESS and what information is needed to plan a surgery.

3. To learn about several interesting cases in order to understand the importance of cooperation between the radiologist and clinician.

A-441 15:00

C. Sinonasal tumours: key clinical questions

V. Lund ${ }^{1}$, N.J.M. Freling ${ }^{2} ;{ }^{1}$ London/UK, ${ }^{2}$ Amsterdam/NL

The analysis of a sinonasal tumour requires MRI for differentiation between tumour and secretions or polyps, to assess the local extension and spread into adjacent compartments (skull base, intracranial, orbit, deep facial compartments) and to depict perineural spread. T2, T1 and high-resolution postcontrast series preferably in three, but at least in two different planes, are needed. CT provides additional information of bony structures and serves as a road map for surgical navigation. A fascinating benign sinonasal tumour is inverted papilloma, which has a high recurrence rate if not removed in toto and may harbour carcinoma in $<5 \%$ of patients. CT may show the attachment of the tumour as an area of increased sclerosis, which directs the surgeon to plan the operation. In patients with recurrent inverted papilloma, providing information to the radiologist about its presumed localisation is important, the more so in patients suffering from polyposis. Malignant tumours are uncommon and of a large histologic variety. About $80 \%$ arise in the maxillary sinus; $70 \%$ are squamous cell carcinomas. They are often large at diagnosis, because clinical symptoms of nasal obstruction or headache are non-specific. Diagnosis is by biopsy. In view of the rapid development of advanced endoscopic techniques, preoperative imaging is extremely important to assess accurately tumour extension. The skull base and dura as well as the lamina papyracea should be scrutinised for tumour locations to foresee an additional cranioendoscopic approach to a standard endonasal endoscopic approach. Finally, imaging may play a role in differentiating non-nasal disease, e.g. primary bone tumours, from mucosal disease.

Learning Objectives:

1. To learn about the most important sinonasal tumours and techniques for their evaluation.

2. To become familiar with key surgical questions and contraindications for surgery.

3. To learn about several cases in order to understand the role of clinicalradiological cooperation. 
14:00 - 15:30

Room L/M

\section{Physics in Radiology}

\section{RC 1413}

\section{Good radiation and bad radiation? How to assess and communicate radiation risk to patients and referring physicians}

Moderators:

M.M. Rehani; Vienna/AT

P. Vock; Spiegel/CH

\section{A-442 14:00}

A. Radiation risk: a patient's perspective

N. Bedlington; Vienna/AT (nicola.bedlington@eu-patient.eu)

To discuss risk/benefit considerations in medical imaging from the perspective of patients and highlight some of the pre-requisites to ensure trust and confidence. This presentation will draw on the work of the European Patients' Forum on patient safety, quality of care, health literacy, and patient empowerment, to explore what are the key factors to consider in radiation risk analysis, from a patient's perspective, recognising that 'one size does not fit all'. It will examine what constitutes quality information in this environment effective dialogue between the patient and the radiologist, and the interdisciplinary team, informed consent and transparency of data. It will also highlight some of the particular challenges regarding vulnerable patients, and those requiring on-going treatment due to chronic disease (s).

Learning Objectives:

1. To understand the fears of patients.

2. To learn what's expected from physicians and techs.

3. To learn about ideal communication strategies.

\section{A-443 14:15}

B. Radiation risks for patients and staff

P. Gilligan; Dublin/IE (PGilligan@materprivate.ie)

The justification for medical procedures is based on the assumption that the benefit outweighs the risk. This presentation looks at the radiation risk factors from recent high-profile papers (Pearce et al., 2012) and publications from international bodies such as BEIR and ICRP. At lower radiation doses $(<100$ $\mathrm{mSv}$ ), quantifying, understanding and communicating radiation risk to staff, patients and referring clinicians presents a range of challenges. Advances in health information systems will facilitate more precise dose risk relationships. There are a number of approaches to stochastic radiation risk assessment such as organ-/age-based assessment and effective dose equivalent assessment. At-risk groups, such as children and pregnant women, need special focus. EU directives demand special attention for high-dose interventional procedures and CT scans. These techniques, particularly if repeated, require further risk assessment regarding potential deterministic effects such as erythema, hair loss, and radiation-induced cataractogenesis. This paper looks at strategies in consenting, monitoring, and follow-up of such high-dose effects. The practical implications of the new occupational ICRP eye dose limits for interventional practice are also examined.

Learning Objectives:

1. To get the latest information on stochastic risks in radiology.

2. To understand the risks for children as compared with adults.

3 . To get the latest information on tissue reaction to medical procedures.

4. To learn about radiation cataract and its dose dependence.

\section{A-444 14:40}

C. Risk in MRI

R. Peeters; Leuven/BE (ronald.peeters@uzleuven.be)

With the advent of higher field scanners in clinical practice and the construction of 'MRI compatible' implanted devices, the list of the do's and don'ts while performing an MRI examination changes constantly. In this presentation basic safety guidelines and rules will be explained regarding static magnetic field effects, time varying magnetic field effects, radiofrequency field effects and acoustic noise effects both with regard to the patient as well as the personnel using the equipment. Due to the advances in medical technology the list of possible 'safe' and 'unsafe' items changes almost daily. Therefore it is very important to have all the information about the patient's condition and implants prior to the MRI procedure in order to asses possible contraindications in advance. While until a couple of years ago cardiac pacemakers and neurostimulators were contraindicated in the MRI environment, the advent of 'MRI compatible' pacemakers and other implanted devices introduces challenges in patient safety. In fact these devices are only safe in certain configurations and also in a lot of cases specific MRI scan sequences and RF antennas are only allowed. Following the European EMF directive, where the MRI part is derogated, the protection of staff working with EM fields also became a topic of debate. What are the possible risks for staff working with MRI magnets and how can one implement practical rules for the safe use of the MRI equipment.

Learning Objectives:

1. To learn about the risks for patients from MRI procedures

2. To learn about the contraindications for MRI scans.

3. To learn about risks for staff in an MRI department.

\section{A-445 15:05}

D. Communicating risks to patients and the public

N. Leitgeb; Graz/AT

Risk communication is much more than just talking about an issue. It requires specific background knowledge and skills. It needs to be based on adequate facts of risk assessment such as provided by SCENIHR, the strength of evidence and remaining uncertainties. While evidence for health-relevant effects is convincing for X-ray imaging, and heating effects due to absorption of radio-frequency electromagnetic fields (RF-EMF) are well understood in MRI, the existence of adverse stochastic side-effects from exposure to MRI RF-EMF is less clear since they have been classified as possibly carcinogenic (class 2B) by WHO's IARC. Little is known about potential health risks from ultra-high static magnetic fields used in MRI. The still frequent use of gynecologic ultrasonic imaging for non-medical purposes such as for generating videos for parents (baby cinema) in spite of the ban in established guidelines demonstrates that risk awareness varies among physicians and parents.

This can be explained by the fact that risk perception mechanisms are complex and risk perception almost uncoupled from quantitative risk levels. Consequently, in addition to sound factual knowledge, risk communication requires specific skills to help avoiding the numerous communication pitfalls such as using inadequate language, in particular gobbledygook, incorrect simplifications, inadequate parameters, misleading wording, tricky comparisons and ambiguous messages. Emotions and confusing mind models such as about the vulnerability of newborn and children or about dose-response relationships need also to be taken into account. Another challenge is targeting risk communication to stakeholders, in particular to lay people such as in communicating risks from inadequate behavior to patients with electronic implants.

Learning Objectives:

1. To become familiar with communicating risk according to the imaging modality.

2. To become familiar with important rules in communication.

3. To understand the relationship between threat/hazard and parents' perception regarding imaging for their child.

4. To learn how to select a risk communications strategy suited to parents and children.

Author Disclosure:

N. Leitgeb: Other; European Commission's Scientific Committee of Emerging and Newly Identified Health Risks (SCENIHR).

\section{4:00 - 15:30 Conf. Room M3 \\ Oncologic Imaging: Follow-up of Systemic and Local Therapies \\ CC 1418 \\ Follow-up of thermal ablation (part I) \\ Moderator: \\ P. Wiggermann; Regensburg/DE \\ A-446 14:00 \\ A. The zone of cell death and collateral phenomena in cross-sectional imaging: from histopathology to the standardisation of terms \\ A. Denys; Lausanne/CH (Alban.Denys@chuv.ch)}

The field of image-guided ablation has expended recently with new ablation techniques like micro-wave, irreversible electroporation, cryoablation. Nevertheless, after each treatment, whatever the technique used, we will leave in place in the treated organ, a scar instead of the tumour. Follow-up of these treated areas are done by radiologists using CT of MR or contrast enhanced ultrasound. Standardisation of terms has been done in 2003 by an international committee presided by Dr. Goldberg. This allows reporting criteria identical from one center and from one country to another. Furthermore, it allows a more homogeneous literature and evaluation of success. Nevertheless, techniques are not all the same and the cellular and tissue damage in the tumour and around it in the healthy parenchyma are not identical and does not have the same evolution over time. 
Learning Objectives:

1. To understand histopathological changes after various ablation techniques.

2. To become familiar with post-ablation imaging based on histopathological

correlation of ablation zones in various organs.

3. To learn about the correct terms for reporting and publishing on post-

ablation imaging

A-447 14:30

B. PET/CT for the evaluation of ablative therapy: Who? Where? When? Does it help?

D. Deandreis; Villejuif/FR (Desiree.DEANDREIS@igr.fr)

Thermal ablation is frequently used to treat liver, lung and bone tumoural lesions because of its safety, efficacy and tolerability. One issue is the lack of reliable imaging modality to assess treatment efficacy and to detect early relapse. Several papers have demonstrated that 18 FDG PET/CT is a useful tool to follow-up ablated liver tumour, detecting residual disease easily and earlier than conventional imaging. Few reports evaluating the usefulness of 18 FDG-PET/CT in the follow-up of ablated lung lesions are available, but prospective studies show promising results, with a high negative predictive value of this technique. Very few data are available on bone lesions and further studies are needed to assess the role of 18 FDG-PET/CT in the follow-up of these lesions. In general, the in vivo typical aspect of completely ablated lesion on 18 FDG-PET/CT is a ring shape, diffuse, peripheral, homogeneous 18 FDG uptake at the treated lesion; on the other hand, the presence of heterogeneous and focal uptake is more frequently related to disease relapse. SUVmax (Standardised Uptake Value) is not a reliable indicator. It is higher in case of disease relapse than in completely ablated lesions, but also in case of important inflammatory reaction. Finally, the best time point to evaluate ablation efficacy still remains to be defined, but 18 FDG-PET/CT should be performed at least 3 months after treatment to avoid dubious or inconclusive findings due to inflammatory reaction.

Learning Objectives:

1. To understand who are the best candidates for a PET/CT follow-up of ablation therapies.

2. To learn what is the optimal timing of PET/CT in the follow-up of ablative therapies.

\section{A-448 15:00}

C. Everyday practice: $\mathrm{MRI}$ and $\mathrm{CT}$ for evaluating response to thermal ablation

C. Dromain, C. Caramella, L. Vilcot, S. Ammari, F. Deschamps; Villejuif/FR (dromain@igr.fr)

The aim of thermal ablation treatment is to generate an area of thermocoagulation whose diameter is larger or at least equivalent to that of the tumour. This necrotic scar usually shrinks with time, but most often very slowly. Therefore, the criteria of response based on size measurement cannot be applied. The pattern of thermal ablation is similar whatever the thermo-ablation technique used. On CT imaging, thermal ablation areas are well circumscribed and oval shaped. The criterion commonly used to assess the efficacy is the absence of enhancement in the thermal ablation necrosis, which corresponds to tissue devoid of viable tumour. On MR imaging, the thermo-ablation areas are typically hyperintense on unenhanced $\mathrm{T} 1$ due to the presence of proteinaceous material, and hypointense on T2, explained by the dehydrating effect of thermal damage. The residual tumour is typically round in shape and located at the periphery of the necrotic area or in contact with large vessels. MR imaging allows earlier detection of residual liver tumour than CT imaging. Learning Objectives:

1. To become familiar with the imaging aspects of successful ablation.

2. To learn about the main pitfalls of post-ablation imaging.

3. To consolidate knowledge of the imaging aspects of most common complications.

Author Disclosure:

C. Dromain: Advisory Board; Roche, Novartis. Consultant; GE Healthcare. Research/Grant Support; GE Healthcare. Speaker; GE healthcare, Roche, Guerbet, Pfizer. C. Caramella: Advisory Board; Roche. F. Deschamps: Speaker; GE Healthcare.

\begin{tabular}{l}
\hline 14:00 - 15:30 \\
\hline Radiographers \\
RC 1414 \\
Cutting-edge imaging: translating research \\
into clinical reality
\end{tabular}

A-449 14:00

Chairman's introduction

C. Malamateniou; London/UK (christina.malamateniou@kcl.ac.uk)

Neurodegenerative diseases, cancer and heart disease perhaps summarise the major challenges to medicine in this century, a challenge in accurate diagnosis and also in treatment. Recent technological advances in medica imaging technology mean that these challenges can be dealt with more efficiently. Anatomy can be studied faster, safer and with higher spatia resolution and precision. In addition to anatomy, the study of function has become feasible; more sophisticated hardware and software are being used to get the required data to map out the functionality and understand how this relates to anatomy to offer a more comprehensive view of normal development and explain pathological conditions. At the same time, researchers and clinicians are urged to create a platform for reciprocal communication to facilitate translation of the research findings to a graspable clinical benefit for the patients. This translational platform will allow flagging the major clinical questions, inform current and future research experiments and at the same time help utilise recent research findings in the clinical setting. This session will review state-of-the-art MRI techniques and document different approaches to translational imaging. It will also allow understanding the obstacles and remedies in implementing translational medicine.

Session Objectives:

1. To appreciate the impact of continued technological developments in medical imaging on patient care.

2. To understand the challenges of translating cutting-edge imaging technologies from research into clinical reality.

\section{A-450 14:05}

A. Advanced imaging of neurodegenerative disease

E. Hughes; London/UK (emer.hughes@kcl.ac.uk)

Many neurological diseases are associated with progressively reduced cognitive function. Recent evidence suggests that proficient cognitive function depends on an appropriate interaction between large-scale cognitive control networks in the brain. It is hypothesised that damage to white matter microstructure, as found in many neurological diseases, disrupts the integrated operation of these networks and results in impaired cognitive function. Diffusion tensor imaging (DTI) has been used to investigate alteration to white matter micro-structure and resting state fMRI (rSFMRI) has proven to be a useful technique to assess brain function in multiple cortical systems. However, the combined use of these techniques in the clinical setting has not yet taken hold. In this presentation, I will demonstrate the flexibility of these MRI techniques in assessing brain structure and function and address their feasibility of use in a clinical setting.

Learning Objectives:

1. To become familiar with advances and developments in neuro-MRI techniques.

2. To learn about state-of-the-art neuro-MRI research and the steps required to make these techniques a clinical reality.

3. To appreciate the role of the radiographer in advanced neuro-MRI.

\section{A-451 14:28}

B. Advanced oncological imaging

L. Martí-Bonmatí; Valencia/ES (marti_lui@gva.es)

Imaging in oncology is a growing field within radiology. Different tomographic techniques are used either isolated or as multimodality-multivariable imaging as there is an increasing need to combine morphological and functional information. Different cutting-edge modalities, such as PET-CT, DW-MR, CEMR and MR-spectroscopy, are standard in most clinical scenarios. New images have advantages providing excellent soft-tissue contrast and multidimensional functional, structural and morphological information. The development of new diagnostic imaging research areas, mainly in the field of oncology, cardiology and neuropsychiatry, will impact the way medicine is performed today. Both clinical and experimental multimodality studies, in humans and animals, will have to demonstrate an efficient use of the imaging information provided by the modalities to affect the future of medical imaging. We will comment on the impact of continued technological developments in medical imaging on patients with cancer (diagnosis, staging and follow-up) and 
the challenges of imaging technologies from research into clinical reality. The recent advances and developments in measurements and biomarkers which have led to a greater understanding of cancer will be commented, together with the morphologic, metabolic and functional oncological imaging research and clinical practice. Finally, the role of the radiographer in advanced oncological imaging techniques will be evaluated.

Learning Objectives:

1. To become familiar with advances and developments which have led to a greater understanding of disease processes, improved monitoring and better clinical outcomes.

2. To learn about state-of-the-art oncological imaging research and the steps required to make these techniques a clinical reality.

3. To appreciate the role of the radiographer in advanced oncological imaging techniques.

\section{A-452 14:51}

\section{Advanced cardiac imaging}

M.L. Dijkshoorn; Rotterdam/NL

In the last 15 years cardiac $\mathrm{CT}$ imaging has developed from a high radiation dose research technique in a selected population into a low-dose clinica routine examination. The CT scanners have made great advances in applicability, image quality and radiation dose reduction. But does this mean that now we should provide every patient with potential coronary stenosis a CT-Angiography scan? Research on the topic of coronary CTA also has made a development over time. At first the potential of the technique in limited populations on single vendor scanners was investiged. Later larger multicenter studies together with protocol optimization techniques were performed. Results have led to guidelines on patient selection and technique suitability. All the technical developments in this field have led to a wide diversity of scan protocols for different patients with the same clinical question. The role of the radiographer in this field is challenging because standard protocols are more difficult to apply and more individual patient based protocol/parameter choices should be made. Until now cardiac CT imaging has mainly focused on anatomic evaluation of the coronaries for calcium scoring and rule-out of coronary stenosis. Just being able to provide nice images of the coronaries is not enough for every patient. A lot of patients get additional functional exams like SPECT or an exercise test. However, new functional CT imaging techniques like CT-Perfusion and CT-FFR (Fractional Flow Reserve) are on the horizon. Further research is in progress to clarify the potential and clinical position of these new techniques.

Learning Objectives:

1. To become familiar with advances and developments in cardiac CT that have led to a greater understanding of disease processes, earlier diagnosis, improved monitoring and better clinical outcomes.

2. To explore current state of the art cardiac CT imaging research and the steps required to make these techniques a clinical reality.

3. To appreciate the role of the radiographer in advanced cardiac CT imaging techniques.

Author Disclosure:

M.L. Dijkshoorn: Consultant; Clinical training consultant Siemens Healthcare, The Netherlands

Panel discussion:

Bridging the gap between research and clinical reality:

a team approach $15: 14$

$14: 00-15: 30$

Room P

\section{Special Focus Session}

\section{SF 14c}

\section{Dental imaging}

\section{A-453 14:00}

\section{Chairman's introduction}

J. Fruehwald-Pallamar; Vienna/AT

(julia.fruehwald-pallamar@meduniwien.ac.at)

When imaging the head and neck region with CT or MRI, teeth are always present. We frequently encounter radiolucent or radiopaque lesions of the jaw on scans performed for other indications. Familiarity with typical dental conditions is necessary to subject the patients to the right therapy. To correctly evaluate and describe those lesions, this special focus session on dental imaging gives the radiologist a tool to report dental lesions. The anatomy of panoramic and CT images will be reviewed in the first talk. Technical details as well as pitfalls will be presented. In the second talk, the focus is on pre- and post-surgical imaging with CT where typical lesions of the jaw will be presented. An insight into imaging the teeth with MRI will be given by the third speaker. The discussion on the impact of radiologists on dental images will complement this special focus session's theme.

Session Objectives:

1. To learn about the potential of cone-beam CT and MRI in dental imaging.

2. To become familiar with maxillofacial anatomy.

3. To understand postsurgical changes.

A-454 14:05

Review of dentomaxillofacial anatomy with panoramic views and conebeam CT

A. Suomalainen; Helsinki/FI (anni.suomalainen@hus.fi)

Panoramic radiography produces a single image of the maxilla, mandible, teeth, temporomandibular joints and maxillary sinuses. During the exposure, the $x$-ray source and the detector rotate synchronously around the patient producing a curved surface tomography. This horseshoe-shaped zone of sharp image is called the focal trough/image layer. Advantages of this technique are a good overview of lower facial hard tissues, the convenience of an extraoral examination, a low patient radiation dose, and low costs. Disadvantages are limited width of the sharply imaged layer, variable magnification, and oblique projection, especially in the upper premolar region. Patient positioning is most important for obtaining a detailed panoramic radiograph. In addition to patient position errors, patient movement during exposure and metal objects can cause artefacts. Also, ghost images caused by structures located between the $\mathrm{x}$-ray source and the rotation centre can hamper diagnostics. CBCT is a radiographic imaging method that allows accurate $3 \mathrm{D}$ imaging of hard tissues. During a CBCT scan, the scanner rotates around the patient's head $\left(180^{\circ}\right.$ $360^{\circ}$ ) obtaining multiple sequential planar projection images by a $2 \mathrm{D}$ detector The scanning software collects the data which is processed to create a volumetric data set with isotopic voxels with varying FOV. Disadvantages of CBCT imaging are poor soft tissue contrast and artefacts. In addition to normal panoramic and CBCT anatomy, after this presentation the audience will be familiar with the panoramic and CBCT techniques as well as common errors and pitfalls of these techniques.

Learning Objectives:

1. To learn about normal panoramic and cone-beam CT anatomy.

2. To understand techniques of image acquisition.

3. To become familiar with common errors and pitfalls.

A-455 14:28

Pre-surgical and post-surgical imaging with MDCT and cone-beam CT

A. Gahleitner; Vienna/AT (andre.gahleitner@meduniwien.ac.at)

Multislice-CT and more recently cone-beam-CT have become an established method for anatomic imaging of the jaws prior to dental implant placement. Commonly referred to as "dental-CT", these high-resolution imaging techniques have gained importance in diagnosing dental-associated diseases of the mandible and maxilla. Especially in patients with inflammations, cysts, oro-antral fistulas, odontogenous sinusitis, impacted teeth and dental fractures, new indications have emerged. Unfortunately, most radiologists have had little experience in this area and many of the dental-CT findings remain undescribed. Hence, we will determine the CT appearance of frequent dentalrelated diseases of the jaws and demonstrate typical pre- and postoperative findings. This presentation reflects our experiences from 18 years of performing dental-CT with 36.000 investigations, closely working together with dentists and oral surgeons.

Learning Objectives:

1. To learn about the typical findings from pathological conditions of the jaw, which confront dentists and oral surgeons.

2. To understand these findings in cross-sectional imaging like multislice and cone-beam CT.

3. To learn about the usual treatments for these conditions in order to know how they are used in dental radiology.

\section{A-456 14:51}

Dental MRI

S. Rohde; Dortmund/DE (stefan.rohde@klinikumdo.de)

This lecture will discuss the potential of dental MRI in the field of inflammatory and neoplastic disease of the periodontal space. Experimental and preliminary clinical data from high-field dental MRI will be presented with a special focus on the visibility of critical anatomical structures on high-resolution sequences. The results will be compared to high-resolution cone-beam CT. Learning Objectives:

1. To become familiar with the technical requirements

2. To learn about the advantages and disadvantages of dental MRI in comparison to cone-beam-CT.

3. To appreciate its potential and future prospective.

Panel discussion:

What is the impact of radiologists in dental imaging? 15:14 
$16: 00-17: 30$

Room A

\section{Interactive Teaching Session}

\section{$E^{3} 1520$}

\section{Thoracic emergencies}

A-457 16:00

A. Vascular

E. Castañer; Sabadell/ES (ecastaner@tauli.cat)

Based on representative cases, we will review the main causes of thoracic non-traumatic vascular emergencies (acute aortic syndrome, pulmonary thromboembolism and haemoptysis); MDCT angiography has become the firstline imaging test for the diagnosis of these entities. As acute aortic disease is the most common fatal condition in patients with chest pain and prompt recognition and treatment are of paramount importance, we will review the spectrum of acute aortic pathology focusing on the distinctive findings of each entity (classic dissection, intramural haematoma and penetrating aortic ulcer) and upgrading the clues for their diagnosis. Acute pulmonary embolism (PE) remains a common clinical challenge. MDCT pulmonary angiography has become the first-line imaging study in the diagnosis of PE because of its speed, accuracy, low interobserver variability and ability to provide alternative diagnoses. We will review the role of MDCT in the evaluation of acute thrombotic PE: findings of acute PE (including how to evaluate the severity of an episode of PE at CT pulmonary angiography) and some potential pitfalls Massive haemoptysis is a life-threatening condition that is associated with a high mortality rate. Haemoptysis usually involves bleeding from the bronchial arteries or, less frequently, from nonbronchial systemic arteries. Haemoptysis of pulmonary arterial origin is rare, estimated at less than $10 \%$ of haemoptysis cases. MDCT angiography permits noninvasive, rapid, and accurate assessment of the cause and consequences of haemorrhage into the airways and helps guide subsequent management.

Learning Objectives:

1. To become familiar with the main causes of thoracic vascular emergencies.

2. To understand the role of MDCT angiography in thoracic emergencies.

3. To learn about the radiological signs in vascular thoracic emergencies and its impact on the management of these patients.

\section{A-458 16:45}

B. Pulmonary

C.M. Schaefer-Prokop; Amersfoort/NL (cornelia.schaeferprokop@gmail.com)

Acute respiratory failure can have multiple underlying causes including infection, fluid overload, immunological diseases or exacerbation of preexisting lung disease. Since the clinical symptoms are nonspecific, imaging plays an important role. The first imaging method is mostly the chest radiograph, easy to access and to obtain, but non-diagnostic in many cases. (HR)CT offers more possibilities to define the differential diagnosis. The option of this interactive workshop will be to get familiar with the spectrum of diseases that can cause acute respiratory failure and learn about key findings in radiography as well as $\mathrm{CT}$ to reduce the differential diagnosis. The interaction between preexisting lung disease, clinical information (e.g. chemotherapy, rheumatoid arthritis, COPD) and imaging findings will be discussed using clinical case studies. Options and also limitations of imaging findings will be illustrated. The following scenarios will be taken into account: acute cardiac failure and various appearances of oedema; acute immunological-toxic disorders including druginduced lung disease and inhalational injuries; exacerbations of preexisting lung disease including fibrotic and obstructive lung disorders; severe infections causing respiratory failure and their complications.

Learning Objectives:

1. To learn about the role of imaging in severe attacks of obstructive lung disease.

2. To become familiar with direct and indirect signs of pleural disease causing respiratory failure with special emphasis on the intensive care patient.

3. To learn about imaging features which are helpful for the differential diagnosis of pulmonary consolidations causing respiratory failure. 4. To understand the interaction between comorbidity, age and extent of pulmonary disease resulting in severe respiratory failure and the role of imaging in it.

\begin{tabular}{l}
\hline 16:00 - 17:30 Room B \\
\hline Abdominal Viscera \\
RC 1501 \\
Technical advances in liver and pancreatic \\
imaging \\
Moderator: \\
D.J. Lomas; Cambridge/UK
\end{tabular}

A-459 16:00

\section{A. CEUS and elastography}

G.H. Mostbeck; Vienna/AT (gerhard.mostbeck@wienkav.at)

Contrast-enhanced ultrasoud (CEUS) and elastography are evolving US techniques that have already found their ways into clinical practice (CEUS) or will do so in the next few years (elastography). CEUS is performed with $2^{\text {nd }}$. generation US contrast media, but these contrast media are not available worldwide. In many European countries, SonoVue ${ }^{\circledR}$ (Bracco, IT) is the only available contrast, consisting of micro-bubbles less than the size of red blood cells. In contrast to CT and MR contrast media, these bubbles are strictly intravascular. For US imaging, low mechanical index US techniques are used to see the resonance of sound by these bubbles. CEUS is excellent for the differential diagnosis of focal liver lesions based on vascularisation and specific contrast uptake (imaging the wash-in and wash-out of US contrast over time with excellent temporal resolution) and increases US sensitivity to liver metastases in colorectal cancer patients. However, CEUS is only useful when there are excellent US conditions. In pancreatic US, CEUS allows to differentiate adenocarcinoma from neuroendocrine tumours based on vascularisation (US contrast uptake). Whereas elastography (strain elastography or shear wave elastography) is established for the diagnosis of liver fibrosis, there are no general recommendations for using these various techniques to measure the "hardness of a focal lesion" in focal liver and pancreas lesions.

Learning Objectives:

1. To become familiar with novel technical applications that are useful in liver and pancreatic diseases.

2. To understand the underlying pathophysiologic processes.

3. To learn about the strengths and limitations of CEUS compared with CT and $\mathrm{MRI}$ in the study of focal liver lesions and pancreatic diseases.

A-460 16:30

B. Diffusion and perfusion

M.A. Bali; Brussels/BE (mbali@ulb.ac.be)

Diffusion-weighted MR imaging (DW-MRI) provides information on tissue cellularity, extracellular space tortuosity and integrity of cell membranes by measuring the motion of water molecules in tissues modified by flows (blood vessels, glandular ducts,.). and interaction with cellular components. Integrated in conventional-MR acquisition protocol, DW-MRI increases sensitivity for lesion detection. The apparent diffusion coefficient (ADC) can be calculated using a mono-exponential relationship between signal attenuation and $b$-value. This fitting is influenced by microcapillary perfusion. A more sophisticated approach (intravoxel incoherent motion, IVIM) would enable estimation of parameters that separately reflect tissue diffusivity and microcapillary perfusion. Using IVIM-analysis, the derived quantitative parameters describe tissue diffusivity, perfusion and perfusion fraction. By providing qualitative and quantitative information, DW-MRI is applied in oncology to characterise malignancy, including lesion aggressiveness, and to monitor treatment response. Perfusion imaging is a useful tool to achieve information concerning tissue vasculature, microvascular permeability and interstitial space characteristics. Dynamic contrast-enhanced techniques (DCE-CT, DCE-MR) are based on the analysis of the contrast agent (tracer) biodistribution in tissues. Quantitative parameters are obtained using pharmacokinetic models, as transfer constant Ktrans, extracellular-extravascular space fraction ve, vascular tissue fraction vp and the rate constant Kep. Quantitative DW-MR and DCE-MR parameters are increasingly used as imaging biomarkers to predict tumour response and/or to monitor the effects of therapy, especially when anticancer agents with novel models of action are used (anti-angiogenic, tyrosine kinase inhibitors and several others). Therefore, these new imaging biomarkers may have a pivotal role in correctly evaluating tumour response and stratify and managing cancer patients.

Learning Objectives:

1. To learn about the technical requirements necessary for diffusion-weighted MRI and how to avoid artefacts.

2. To learn about the technical requirements for $C T$ and MRI perfusion.

3. To become familiar with imaging biomarkers useful in tumour evaluation. 


\section{Postgraduate Educational Programme}

\section{A-461 17:00}

C. Liver specific contrast agents

B.J. Op de Beeck; Antwerp/BE (bart.op.de.beeck@uza.be)

For state-of-the-art MR liver and pancreatic imaging, a field strength of at least $1.5 \mathrm{~T}$ is required. All non-blood pool gadolinium chelate-based contrast agents are suitable for dynamic liver and pancreatic MRI. All gadolinium chelates should be routinely administered at a rate of $1-2 \mathrm{ml} / \mathrm{s}$ followed by a $20 \mathrm{ml}$ saline flush at 1-2 ml/s using a power injector. To obtain hepatobiliary phase imaging in addition to dynamic phase imaging, the use of liver-specific contrast agents is required. Gd-EOB provides the highest hepatocyte enhancement, but an overlap between delayed phase and hepatocyte phase has to be considered during dynamic evaluation. The hepatocyte phase can be considered adequate when contrast is detected in the intrahepatic bile ducts. Hepatobiliary phase imaging benefits from a gradient echo high flip angle, depending on magnet field strength. In the absence of liver function impairment and biliary obstruction, contrast-enhanced MR cholangiography can be obtained with $\mathrm{Gd}$ EOB at 20-40 minutes, and with Gd-BOPTA at 60-120 minutes. When the differential diagnosis is primarily between solid benign lesion vs. metastasis, the use of a liver-specific CA is recommended, due to the ability to diagnose FNH confidently. The combined interpretation of dynamic and hepatobiliary phase improves diagnostic accuracy of MR imaging for the detection of HCC. Hemangiomas and intrahepatic CCC result in relative hypointensity in the late vascular phase after Gd-EOB administration. When combined with T2weighted MRCP, contrast-enhanced MRC allows morphologic and functional assessment of the biliary system.

Learning Objectives:

1. To learn about the principals and recent technical advances in the use of

liver specific contrast agents for liver imaging.

2. To appreciate the similarities and differences between the classes of contrast agent

3. To become familiar with indications of liver specific contrast in biliary diseases.

4. To learn about future directions of MRI contrast agents.

16:00 - 17:30 Room C

Modern Imaging of the GI Tract

CC 1521

\section{Gastrointestinal stromal tumours (GIST)}

Moderator:

M. Zins; Paris/FR

\section{A-462 16:00}

A. Pathology and treatment options

B. Seddon; London/UK (beatrice.seddon@uclh.nhs.uk)

Gastrointestinal stromal tumours (GIST) are rare malignant tumours arising within the wall of the gut from the interstitial cells of Cajal, which act as pacemaker cells controlling peristalsis. The crude annual incidence of clinically detected GISTs is approximately 10 cases per million in Europe. The median age at diagnosis is approximately 63 years, and $80 \%$ of patients are older than 50 years. However, a small number of cases do occur in younger people and children, and these are usually syndromic GISTs. GISTs can occur at any site of the gut from the oesophagus to rectum, although they can also arise in the extra-gastrointestinal abdominal and pelvic locations, so-called e-GISTs. The commonest location is the stomach $(55 \%)$, followed by the small intestine $(35 \%)$ and rectum (5\%). Diagnosis is by biopsy, with spindle cell or epithelioid morphology, and immunohistochemical staining for CD117 (the protein product of the KIT gene) and/or DOG- 1 receptors. Approximately, $80 \%$ of GISTs have mutations in the KIT gene, $10 \%$ have mutations of the PDGFRA gene, and the remaining $10 \%$ have no mutation (wild-type) or rare gene mutations. Early stage disease is managed by surgery, followed by 3 years of adjuvant imatinib (a tyrosine kinase inhibitor with activity against KIT and PDGFRA receptors) for cases at high risk of relapse. Advanced metastatic disease is treated with imatinib, with a median duration of response of approximately 2 years. At disease progression, second-line treatment is with sunitinib, with a median duration of response of approximately 6 months.

Learning Objectives:

1. To learn about the prevalence and typical location of GIST tumours.

2. To understand the histological and genetic characteristics of GIST tumours and the differential diagnosis.

3. To become familiar with the treatment options for GIST tumours.

Author Disclosure:

B. Seddon: Author; Author on paper on sunitinib in GIST.

\author{
A-463 16:20 \\ B. Disease staging and treatment planning \\ C. Hoeffel; Reims/FR (choeffel-fornes@chu-reims.fr)
}

Imaging plays a major role in the detection, characterisation and staging of gastrointestinal tumours (GISTs). Imaging of GISTs depends on the mode of presentation and the local availability. Endoscopic ultrasound and computed tomography (CT) are the most widely used imaging methods. GISTs have a spectrum of radiological appearances depending on tumour size and site of origin, but often show certain key radiological features. Multiphase multidetector row CT is necessary to detect and stage GISTs and multiplanar imaging is important so as to define the likely organ of origin of the mass, which may be a challenging task. The clinical diagnosis of a GIST is based mainly on imaging, as biopsy runs the risk of tumour rupture or seeding of the biopsy tract. In patients with an unresectable primary tumour or metastatic disease at presentation, biopsy confirmation is important before starting medical treatment. CT or/and $\mathrm{MRI}$ are used to assess primary tumour extension, local invasion and the presence of metastases, with main sites of metastasis being the liver and the omentum.

Learning Objectives:

1. To appreciate the imaging modalities available for staging GIST tumours, and to understand the advantages and disadvantage of each.

2. To learn about optimised acquisition protocols.

3. To become familiar with the imaging differential diagnosis of GIST tumours and the role of biopsy.

4. To appreciate the radiological staging of GIST tumours, including typical sites of metastatic disease.

A-464 16:40

C. Treatment response assessment and disease follow-up

A. Graser; Munich/DE (Anno.Graser@med.uni-muenchen.de)

Gastrointestinal stromal tumours (GISTs) are treated with targeted therapy regimes. These treatment strategies are based on the suppression of tumour vasculature using the tyrosine kinase inhibition pathway. Drugs like imatinib and sunitinib exhibit specific changes in tumours and metastases that can be detected on cross-sectional imaging. These changes differ significantly from standard treatment effects of cytotoxic chemotherapy. While the latter leads to destruction of tumour cells and thereby to a reduction of tumour size, the former will cause a decrease in vascularity and thereby in attenuation and enhancement of lesions while the size may remain unchanged. Radiologists have to be aware of these specific patterns of response to treatment. This presentation will include a review of the Choi criteria which have been developed for the assessment of GIST lesions under treatment. Also, current imaging strategies focusing on time-resolved CT imaging (CT perfusion imaging) will be presented. The aim of this lecture is to provide a practical approach to response imaging in GIST patients integrating both existing and novel strategies

Learning Objectives:

1. To understand the role of imaging in disease treatment response assessment and the pros and cons of available modalities.

2. To appreciate the imaging criteria used in the assessment of disease response.

3. To learn how imaging can help guide appropriate therapy.

Author Disclosure:

A. Graser: Grant Recipient; Bayer Healthcare, Siemens Healthcare. Research/Grant Support; Bayer Healthcare, Siemens Healthcare. Speaker; Siemens Healthcare, Bracco Diagnostics, Pfizer Pharma, GlaxoSmithKline.

A-465 17:00

D. Interactive case discussion

M. Zins; Paris/FR

$16: 00-17: 30$

Room D

Cardiac

RC 1503

\section{Cardiac CT and MRI in difficult patients: what to do?}

A-466 16:00

Chairman's introduction

M. Hamilton; Bristol/UK (Mark.Hamilton@UHBristol.nhs.uk)

Performing high-quality examinations is easy in the ideal patient. However many subjects present challenges that if not overcome may lead to a failed examination. For example, the obese, the breathless, the arrhythmic, the 
elderly and the young can all present special problems that require a greater depth of understanding to obtain a diagnostic study. This session will help you in your quest for reliable high-quality investigations for all.

\section{A-467 16:05}

A. Coronary CTA in patients with severe arrhythmias and high heart rate C. Loewe; Vienna/AT (christian.loewe@meduniwien.ac.at)

Besides all advances in scanner technology, heart rate remains a critical issue for coronary CTA, and motion artefacts due to cardiac function represents still the most frequent reason for limited diagnostic image quality in cardiac CTA Thus, the target heart rate is defined depending on the scanner system used and ranged between below $60 \mathrm{bpm}$ and below $70 \mathrm{bpm}$. However, in a number of patients heart rate control is not possible or not successful (i.e. children, emergencies, heart transplant recipients), whereas a relevant proportion of CTAs are performed in patients with heart rates higher than the target frequency. During this presentation, the possibilities for heart rate control by beta blocker injection and beyond will be discussed and possible workflows will be presented. Furthermore, examination strategies for patients with high heart rates will be presented including reverse dose modulation, high-pitch scanning, systolic scanning and more. Finally, tools for reconstruction and assessment of patients scanned at higher heart rate will be introduced. Different to high heart rates and even more critical with regard to image quality is arrhythmia. Due to the complexity of cardiac synchronisation, a CT suite is not the place for cardiac resynchronisation, whereas the indication to CTA has to be reevaluated depending on referring diagnosis and severity of arrhythmia. Since there are patients undergoing CTA because of arrhythmia including patients prior to ablation treatment, strategies for examination and image reconstruction in case of arrhythmic patients have to be established and will be presented. Learning Objectives:

1. To understand the criteria for optimal patient selection and preparation to achieve best results.

2. To learn about acquisition techniques in patients with arrhythmias and tachycardia.

3. To become familiar with the post-processing techniques available for optimising image quality following the scan.

\section{A-468 16:28}

B. Severe coronary calcinosis and stents: tips and tricks in image acquisition and interpretation

F. Pugliese; London/UK (francesca.pugliese@libero.it)

Cardiac computed tomography (CT) has become a widely available diagnostic tool used in a range of heart conditions. The commonest application of this technique is in the evaluation for coronary artery patency (coronary CT angiography) in patients with chest pain. When coronary arteries are heavily calcified, or post-coronary angioplasty with stent implantation, diagnostic problems can occur. In these circumstances, the evaluation of the coronary arteries on CT is hampered by the occurrence of high-density artefacts caused by calcifications and stent struts. These artefacts may preclude the appropriate assessment of the coronary lumen. The presence of motion artefacts in the dataset or image noise in very large patients may exacerbate the problem. In this scenario, accurate patient selection and preparation remain key to ensure that the diagnostic yield of the cardiac CT study is good. Optimisation of scan parameters $(\mathrm{kV})$, contrast injection protocol and use of appropriate postprocessing techniques (e.g. dedicated convolution filters) play an important role in daily clinical practice. Recent technical developments include dualenergy scan techniques and gemstone spectral detector systems that acquire simultaneously high and low kilovoltage datasets. This is done to achieve tissue differentiation. In principle, by using monochromatic image reconstruction, the effect of high-density artefacts may be decreased. Using a similar principle, high-density structures can be subtracted from the image. The introduction of iterative reconstruction algorithms may play a role in that these algorithms are theoretically more accurate in the modeling of physical noise and tissue geometries.

Learning Objectives:

1. To understand the challenge of calcification and stents when performing coronary CTA.

2. To become familiar with technical features that maximise image quality in this patient group.

3. To be aware of the accuracy of coronary CTA in stented or calcified vessels with the optimum imaging techniques.

\section{A-469 16:51}

C. Cardiac MRI: potential problems and how to avoid them

E. Mershina; Moscow/RU (elena_mershina@mail.ru)

Lack of movement artefacts is one of the major prerequisites for good image quality in cardiac MR. It is crucial to have the minimum possible image acquisition time. It is important to make both TE and TR as short as possible. Data acquisition should be synchronised with patient's ECG or pulse. Special attention should be paid to good quality of ECG recordings (good contact of electrodes with the skin, electrodes positioning, using of dielectric pad). In patients with arrhythmia, prospective ECG synchronisation should be used instead or a retrospective one, or special protocols may be applied for arrhythmia correction. Breathing artefacts are usually not a problem for cardiac $\mathrm{MRI}$, because most sequences are acquired during a single breath-hold. Using SSFP and parallel imaging allows obtaining a complete set of cine MR images through the whole heart in 1-8 short breath-hold periods. The technique of realtime cardiac MR is a good way to perform successful examinations even in difficult patients, especially the ones with heart failure. Other systemic MRartefacts (aliasing, chemical shift, magnetic susceptibility, off-resonance) should be recognised and diminished or eliminated by the operator. These artefacts are more prominent in case of $3 \mathrm{~T}$ systems. Late-enhancement studies with Gd are very dependent on the correct selection of the TI time. Phase-contrast MRI requires VENC calibration in cases of valve stenoses. To meet the challenges and the benefits of cardiac MRI, one must balance the constraints of signal- and contrast-to-noise ratios, spatial and temporal resolution, scan time and image quality.

Learning Objectives:

1. To learn about the importance of patient selection and preparation in achieving high-quality scans.

2. To understand the practical techniques available for performing a successful scan in difficult patients such as those with arrhythmia or poor breath-holding ability.

3. To learn how imaging parameters can be changed for optimal results.

Panel discussion:

When to scan and when not to?

How can we make the best of what we have?

$17: 14$

16:00-17:30
Paediatric
RC 1512
Paediatric MSK radiology: what is
important?
Moderator:
R.A.J. Nievelstein: Utrecht NL

A-470 16:00

A. Scoliosis: what the radiologist needs to know

A. Geoffray, J.-L. Clément; Nice/FR (a.geoffray@lenval.com)

Scoliosis, a common spinal deformity in teenagers, especially girls, progresses during their growth until the end of puberty. Therapeutic choices, which are either medical - with corset - or surgical, depend on this progression. Follow-up is mainly clinical. However, radiographs are often indicated to give precise information: AP and lateral views of the spine in standing position remain the basis of this follow-up. Obtaining a good image quality at all levels of the spine has been a technical challenge for a long time, and the question of radiation dose is also a main concern, as these radiographs require a high radiation dose. Fortunately, radiographic techniques have greatly improved over the past few years: From the conventional 90 or $120 \mathrm{~cm}$ film to digital screens and now flat screens, we now obtain better quality images with less radiation. Today, EOS system using Xenon chamber is the best to give good AP and lateral views. It allows $3 \mathrm{D}$ reconstructions with minimal radiation dose. On these radiographs, measures can be performed, essentially Cobb's angle. It helps in evaluating progression of the curves and making surgical decisions. Other imaging techniques are requested only in particular situations as in the presence of neurological symptoms or general disease such as neurofibromatosis, or in young children with malformative scoliosis and sometimes preoperatively. CT is best to study the vertebrae; it objectivates vertebral malformations (agenesis, hypoplasia, blocks). MR will be requested to study the cord, nervous roots, craniospinal junction and the soft tissues. Collaboration between the radiologist and spinal surgeon is essential. Learning Objectives:

1. To understand how to balance the need for information on the progression of scoliosis against the exposure to ionising radiation.

2. To become familiar with the essential measurements in the scoliotic spine, their reliability and validity.

3. To understand the role of the radiologist in scoliosis imaging.

A-471 16:30

B. Benign bone tumours and pseudotumours in children: the pitfalls D. Dumitriu; Brussels/BE (dana.dumitriu@uclouvain.be)

Benign bone lesions in children are commonly an incidental radiographic finding. They may also cause pain, a palpable mass or pathological fracture. It 
is the radiologist's role to follow a diagnostic algorithm helping him decide on the following points: Is the lesion a tumour or not? Is a normal variant excluded? If it is a tumour, does it show signs of malignancy? Is the radiological aspect typical for a "don't touch lesion" or are other investigations necessary? The key elements in the diagnostic process, as for most bone lesions, are: the age of the patient, the type of bone and bone segment involved, the location within the bone, the presence of signs of aggressiveness (type of osteolysis, limits, presence and type of periosteal reaction). Conventional x-rays should never be absent from the initial workup of a focal bone lesion in a child, as they provide essential features for differential diagnosis and, in most cases, eliminate the need for other more costly techniques. The need and priority for other imaging techniques, such as CT, MRI and bone scintigraphy, are decided on a case-by-case basis and sometimes a combination of these techniques, which complement each other, is needed. It is the radiologist's responsibility to recommend histological analysis of a lesion via biopsy whenever the imaging aspect is not characteristic or indicates signs of possible malignancy.

Learning Objectives:

1. To recognise the most common benign bone tumours and pseudo-tumours.

2. To understand the differences between benign bone tumours, pseudo-

tumours and malignancies in children.

3. To understand imaging modalities that could help in the differential diagnosis of benign bone tumours and pseudo-tumours in children.

\section{A-472 17:00}

\section{Congenital bone dysplasias}

A.C. Offiah; Sheffield/UK (amaka.offiah@nhs.net)

The 2010 Nosology and Classification of Genetic Skeletal Disorders subdivides 456 conditions into 40 groups defined by molecular, biochemical and/or radiographic criteria. 316 conditions are associated with mutations in at leas one of 226 different genes. Some of these conditions are rare or even extremely rare (occurring in fewer than 1 in 100,000 of the population). Clearly, the general paediatric radiologist cannot be expected to correctly recognise and diagnose all of these conditions. The approach is to perform a set of radiographic images dependent on the patient's age/size and to develop a standard system for reviewing these images. The standard set of images for a patient over 2 to 3 years of age consists of AP and lateral skull, AP chest, AP pelvis, lateral thoracolumbar spine, AP one upper limb, AP one lower limb and DP left hand. Variations on this routine dysplasia skeletal survey will be discussed and a standard system for interpreting the images will be presented using radiographs of the commoner skeletal dysplasias as examples. Learning Objectives:

1. To understand the role of the radiologist in diagnosing congenital bone dysplasias.

2. To understand how to classify bone dysplasias

3. To learn how to report radiological studies related to bone dysplasias. Author Disclosure:

A.C. Offiah: Advisory Board; BioMarin - Management of Spinal Cord Compression in Patients with MPS IVA. Author; Fetal and Neonatal Skeletal Dysplasias (Published by Radcliffe Ltd). Speaker; Over the past 10 years I have given multiple lectures in relation to the imaging of skeletal dysplasias. Other; I am Chairperson of the Skeletal Dysplasia Group for Teaching and Research. Room E1

\section{Special Focus Session}

\section{SF $15 a$}

\section{Body composition: a new string to the radiologist's bow}

\section{A-473 16:00}

\section{Chairman's introduction}

G. Guglielmi; Foggia/lT (giuseppe.guglielmi@unifg.it)

In the last few years, interest in body composition (BC) analysis, which is the quantification and characterisation of relative amounts of muscle, fat, bone, and other vital parts composing the human body, has grown rapidly for clinical, research and epidemiological purposes. Studying body composition has gained great importance for the comprehension and decoding of a multitude of patho-physiological processes (e.g. obesity, diabetes and endocrine diseases and also gastrointestinal, renal, nervous, infectious diseases, etc). and physiological and para-physiological conditions as in athletes or growth and ageing processes. Although the main imaging techniques, which include dualenergy $x$-ray absorptiometry (DEXA), computed tomography (CT), and magnetic resonance imaging (MRI), offer a differentiated and attractive analysis of BC, they still need to find a definite position in clinical practice. Session Objectives:

1. To become familiar with abnormalities involving fat mass, lean mass and bone.

2. To understand the importance of body composition for clinical purposes, research purposes and epidemiological purposes.

3. To learn about the predictive value of body composition variables.

A-474 16:05

Body composition: why and how?

J.E. Adams; Manchester/UK (judith.adams@manchester.ac.uk)

Through 'programming' low birth weight is associated with increased rates of coronary heart disease, stroke, hypertension \& non-insulin dependent diabetes. Prediction of morbidity and mortality from body composition, particularly fat, stimulates the importance and application of accurate and precise methods for quantitative assessment of body composition; the gold standard for which is cadaver analysis, but other methods have to be implemented in vivo. Body composition alters in many chronic diseases, starvation cases, metabolic syndromes, and senescence stages, and is a key component of health. Several techniques are available which vary in simplicity and complexity of use. Each make assumptions and may not measure body composition directly, but make predictions from other measurements made. Skinfold thickness measurements assess regional fat and are quick and simple to perform at all ages. Reproducibility is good, but less so in obesity. There is some limitation of reference data to express results as standard deviation scores (SDS). Body mass index (BMI; weight/height ${ }^{2}$ ) is widely used as an index of relative weight expressed as SDS for gender, age and ethnicity. Waist circumference (WC) is a measure of central fat, and can also be expressed as a ratio to hip circumference. Bioelectric impedance analysis (BIA) measures body impedance to a small electric current, which estimates total body water (TBW) from which can be derived fat free mass (FFM). TBW can also be measured by neutron activation. Air displacement plethysmography (Bod Pod) is a new method for body composition assessment.

Learning Objectives:

1. To understand normal body composition in children and adults.

2. To learn how body composition can be altered by disease.

3. To appreciate the clinical relevance of assessing body composition.

4. To understand the non-imaging methods available for measuring body composition, with their advantages and limitations.

\section{A-475 16:25}

Computed tomography: what does it measure and how?

J. Damilakis; Iraklion/GR (damilaki@med.uoc.gr)

CT allows measurement of total body fat and enables differentiation of subcutaneous from visceral adipose tissue. Body fat can be estimated using the conventional technique of manual planimetry. Using planimetry, the user delineates manually the boundaries of subcutaneous and visceral fat in each CT image. However, this method is labour intensive, time-consuming, and therefore, not easily applicable in routine clinical practice. Another CT method used for the estimation of abdominal fat is the semiautomatic measurement of adipose tissue area. Abdominal fat is assessed in a CT slice by using a fixed range of Hounsfield units to define the area of adipose tissue. A limitation of this method is that the attenuation range of fat may vary among individuals. The stereological volume estimation method is based on the Cavalieri's principle. According to this principle, the volume of an object can be measured by cutting it into equally spaced slices and measuring the area of the object on each slice. To measure body fat using stereology, a square grid of test points is randomly superimposed on each CT section. All points lying inside the fat tissue region are selected by the user and the software automatically calculates the total number of points hitting the fat. Stereology provides the possibility for efficient fat volume assessment. However, research studies are needed to optimise the stereological estimation of fat and compare stereological measurements with those from other adipose tissue measurement $\mathrm{CT}$ techniques.

Learning Objectives:

1. To appreciate the role of $\mathrm{CT}$ imaging in body composition analysis. 2. To learn how to measure visceral and subcutaneous adipose tissue using CT.

3. To understand the advantages and limitations of CT techniques (planimetry, thresholding, stereology) in evaluating adipose tissue.

A-476 16:40

MRI: current and future applications

D.C. Karampinos; Munich/DE (dimitrios.karampinos@tum.de)

MRI has been emerging as an ionising radiation-free imaging modality to measure fat distribution in the human body and fat content in different organs. The present talk will first introduce MRI methods to measure fat distribution, 
including the well-established T1-weighted imaging and the emerging Dixon imaging approaches. The challenges related to the data acquisition and image post-processing of the two imaging approaches in the study of fat distribution will be reviewed. Examples will be shown from the current use of MRI in investigating adipose tissue distribution alterations or differences in patients with metabolic disorders, including obesity and diabetes. Quantitative MRI methods enabling the measurement of fat content with high spatial resolution will be then presented, with a focus on water-fat separation methods. The technical challenges associated with the establishment of MRI-based fat content, as an accurate and reproducible imaging biomarker will be discussed. The selection of pulse sequence parameters and image reconstruction algorithm in a state-of-the-art water-fat separation experiment will be explained. Applications will be shown from the growing literature of MRI-based fat quantification in abdominal organs (e.g. liver) and in skeletal muscles, aiming to show the great premise of quantitative water-fat MRI in quantifying fat content with high spatial resolution in different body parts. At the end of the talk, the potential of quantitative water-fat MRI will be discussed in the emerging application of measuring bone marrow fat content and its association with bone health and metabolic disorders.

Learning Objectives:

1. To become familiar with MRI techniques for measuring fat content and fat composition.

2. To learn about the current use of MRI in measuring adipose tissue

distribution in metabolic disorders.

3. To learn about the potential of MRI for measuring bone marrow fat content in osteoporosis.

Author Disclosure:

D.C. Karampinos: Research/Grant Support; Philips Healthcare.

\section{A-477 16:55}

DXA: technical aspects and application

A. Bazzocchi; Bologna/IT (abazzo@inwind.it)

The key role of dual-energy x-ray absorptiometry (DXA) in the management of metabolic bone diseases is well known. The role of DXA in the study of body composition and in the clinical evaluation of disorders which directly or indirectly involve the whole metabolism as they may induce changes in body mass and fat percentage is less known or less understood. DXA has a range of clinical applications in this field, from assessing associations between adipose or lean mass and the risk of disease to understanding and measuring the effects of pathophysiological processes or therapeutic interventions, in both adult and paediatric human populations as well as in pre-clinical settings. DXA analyses body composition at the molecular level that is basically translated into a clinical model made up of fat mass, non-bone lean mass, and bone mineral content. DXA allows total and regional assessment of the three abovementioned compartments, usually by a whole body scan. Since body composition is a hot topic today, manufacturers have steered the development of DXA technology and methodology towards this. New DXA machines have been designed to accommodate heavier and larger patients and to scan wider areas. New strategies, such as half-body assessment, permit accurate body scan and analysis of individuals exceeding scan field limits. Although DXA is a projective imaging technique, new solutions have recently allowed the differential estimate of subcutaneous and intra-abdominal visceral fat. The transition to narrow fan-beam densitometers has led to faster scan times and better resolution; however, inter- or intra-device variation exists depending on several factors

Learning Objectives:

1. To appreciate the role of DXA in the study of body composition.

2. To understand potential limitations and pitfalls of DXA in the analysis of body composition.

3. To learn about technical elements and methods, and to become familiar with biomarkers in DXA.

Panel discussion:

How to rationalise the use of body composition analysis in radiological practice $\quad 17: 15$

\begin{tabular}{l}
\hline 16:00 - 17:30 \\
\hline State of the Art Symposium \\
SA 15 \\
Tumour response assessment in clinical \\
practice
\end{tabular}

A-478 16:00

Chairman's introduction

E. De Kerviler; Paris/FR (eric.de-kerviler@s/s.aphp.fr)

In cancer patients, disease-free survival is a good indicator for tumour response, but for many common cancers, treatment of disseminated disease is often noncurative. The increased duration of survival is related to changes in tumour size after treatment. However, the anatomical determination of tumour response has some limitations, especially when non-cytotoxic targeted therapies are used. With these new treatments options, the lack of progression may be associated with a good improvement in outcome, even in the absence of major shrinkage of tumours. New imaging biomarkers are therefore needed to assess therapeutic response. Molecular imaging is now playing a prominent role in the monitoring of cytostatic targeted therapies. PET-CT, dynamic contrast enhancement studies or diffusion-weighted imaging are the most promising ones. The aim of this session is to present the state of the art in tumour response assessment with regard to new therapeutic regimens. Session Objectives:

1. To understand the role of the radiologist in cancer patient management.

2. To appreciate the key issues of tumour assessment in oncology.

3. To become familiar with the most common response criteria used in practice and in clinical trials.

4. To learn about future imaging biomarkers in response assessment.

\section{A-479 16:05}

Endpoints for therapy response assessment: the oncologist's perspective

J. $\underline{\text { Carles; }}$ Barcelona/ES (jcarles@vhio.net)

In early oncologic times, treatments of different malignancies were reported in different ways. C. Gordon Zubrod and others first articulated the model of multicentre clinical trials and argued for standards to be agreed for included and excluded subjects, the method of assigning treatment and in measuring response. Nowadays, we have better knowledge of tumour biology; however, we continue using the classical response criteria (WHO and RECIST) and overall survival as a primary goal for effective treatment in most of the malignancies. In the last few years, different criteria for responses have been reported to evaluate the disease and a lot of discussions between authors have been reported. Now, we can use the RECIST criteria v1.1, implement the PERCIST criteria and also forget the IMMUNOTHERAPY criteria. Furthermore in the last few years, different treatments such as vaccines, antiangiogenics and targeted therapies have increased our arsenal in the treatment of different malignancies such as kidney cancer, prostate cancer, breast cancer and so on. Another problem for our patients is that those diseases have a long evolution after the first-line treatment. We have to be able to define in each disease the treatment line and type of treatment to determine which evaluation criteria are necessary and which is the objective of treatment, PFS or OS. We have to be aware that if we are not able to define such topics, we may be losing active treatments.

Learning Objectives:

1. To learn about prognostic implications in survival.

2. To understand new endpoints in targeted therapies.

3. To appreciate differences between chemotherapies and targeted therapies.

A-480 16:23

Revised RECIST guidelines: principles, practical applications and pitfalls L.S. Fournier; Paris/FR (laure.fournier@egp.aphp.fr)

RECIST 1.1 are the criteria most often used in studies to evaluate response to treatment in solid tumors. On each follow-up examination, response is defined by a combination of unidimensional measurement of 'target lesions', qualitative evaluation of 'non-target' lesions, and presence/absence of 'new lesions'. CT and MRI are the preferred modalities for RECIST evaluation, and evaluations must be performed using the same modality, in the same plane. We will review anatomic locations which should be avoided, and how to deal with intercurrent events resulting in the impossibility of measurement. Cases when RECIST seems inadequate will be discussed, including evaluation of bone metastases, and focal therapy. Finally, though these criteria were developed for drug trials they may provide a frame for reading examinations and writing reports in routine practice. Reproducible, objective and quantitative criteria help to define 
a common language between radiologists and clinicians, so that the latter can make an informed treatment decision based on sound conclusions.

Learning Objectives:

1. To learn about the RECIST 1.1 criteria.

2. To become familiar with the conditions necessary to implement them

3. To understand the limits of their application.

4. To learn useful lessons from these criteria for routine clinical practice.

Author Disclosure:

L.S. Fournier: Author; Pfizer, Merck. Speaker; Pfizer.

A-481 16:41

Criteria beyond RECIST: Choi, mRECIST, EASL-AASLD, PERCIST

S. Gwyther; Redhill/UK (GwytherSJ@aol.com)

Radiological response evaluation using anatomical imaging was developed in the 1980s in early Phase II clinical trials. Potential new anti-cancer agents were assessed by measuring tumour size before and during therapy and a predetermined reduction in size constituted response. Radiological response represents an objective surrogate for patient benefit and was the primary end point in early Phase II studies. If a pre-defined percentage of patients achieved the required response, then the agent proceeded to large Phase II and III studies where clinical time to progression or progression-free survival comprised the primary end point. Between 1980 and 2000, there were extraordinary advances in cross-sectional imaging techniques which became widely available. Different imaging techniques were introduced piecemeal with different co-operative groups adding different requirements, so meaningful comparisons from one trial to another could not be made. In 2000, the RECIST criteria were introduced requiring specific imaging stipulations and a minimum baseline tumour size to unify the different criteria and enable meaningful comparisons from one study to another. Further advances continue to be made regarding anatomical and functional imaging. Not all anti-cancer agents are cytocidal and many studies now use time to progression or progression-free survival defined by radiological imaging as the primary end point. Certain tumour types respond in a unique manner requiring the employment of specific response criteria in these tumour types to adequately demonstrate response. Some of these tumour types and their specific response criteria will be discussed.

Learning Objectives:

1. To learn about the role of radiological response evaluation in clinical trials.

2. To understand how radiological response evaluation has evolved and continues to evolve.

3. To understand which response criteria are utilised in specific tumour types.

\section{A-482 16:59}

Emerging biomarkers for response assessment: pros and cons

M.C. Roethke; Heidelberg/DE (m.roethke@dkfz.de)

Non-invasive response assessment of new specific anti-cancer therapies is an emerging field of oncologic imaging. In the past few years, large efforts were undertaken to develop new functional biomarkers to enable an earlie diagnosis, improved risk stratification and treatment monitoring of oncological diseases. Imaging biomarkers reflect changes in tumour biology, which can be differentiated into certain categories (e.g. cell density, tumour heterogeneity, iron concentration, elastic properties, or cellular receptors). In a first step, alternative qualitative and quantitative imaging biomarkers for the different modalities (MRI, CT, PET) are elucidated. For magnetic resonance imaging new techniques with potential for treatment response monitoring such as diffusion-weighted imaging, susceptibility-weighted imaging, and elastography will be assessed. Then, quantification of iodine uptake of contrast-enhanced CT as an imaging biomarker will be addressed. Novel PET imaging strategies for therapy monitoring will be mentioned with focus on receptor targeting tracers (e.g. PSMA, DOTATOC). Furthermore, the role of PET-CT/MRI is mentioned to facilitate functional techniques in oncological imaging with imaging biomarkers. The potential clinical use of prior introduced biomarkers is demonstrated for several cancer entities (e.g. HCC, prostate cancer, malign melanoma, multiple myeloma, glioblastoma). Advantages and disadvantages of the illustrated imaging biomarkers are discussed during this part of the lecture.

\section{Learning Objectives:}

1. To become familiar with alternative biomarkers for therapy monitoring.

2. To understand different categories of imaging biomarkers.

3. To learn about the use of emerging biomarkers for tumour assessment in selected cancer entities.

4. To appreciate the advantages and disadvantages of emerging imaging biomarkers for tumour response assessment.

Panel discussion:

Personalised response criteria for personalised therapies?

\section{6:00 - 17:30 \\ Special Focus Session \\ SF $15 b$ \\ Evidence-based radiology, comparative
effectiveness research, and health
technology assessment}

Room F1

A-483 16:00

Chairman's introduction

M.G.M. Hunink; Rotterdam/NL (m.hunink@erasmusmc.nl)

In today's health-care climate, there is an ever-increasing need to justify what we do. Evidence-based radiology (EBR), comparative effectiveness research (CER), and health technology assessment (HTA) provide tools that help us justify imaging decisions. The tools include a hierarchy of study designs to evaluate diagnostic tests, checklists to appraise diagnostic studies, observational studies, clinical trials, decision modelling, simulation studies, and economic evaluation. Understanding these concepts is essential to use the published results in justifying what we do. The goal is to image the right patient, at the right time, using the right test and with the right interpretation so that patients will be advised on the right therapy. Ultimately, the aim is to maximise patient outcomes at an affordable cost. This session will cover the state of the art in EBR, CER, and HTA and demonstrate how the results are used in imaging decisions.

Session Objectives:

1. To understand what is meant by evidence-based radiology (EBR),

comparative effectiveness research (CER), and health technology assessment (HTA).

2. To understand the concept of imaging the right patient at the right time,

using the right test.

3. To learn how to use evidence for justified imaging decisions in day-to-day clinical practice.

Author Disclosure:

M.G.M. Hunink: Advisory Board; NIPED Prevention Compass, Netherlands. Author; Decision Making in Health and Medicine. Employee; Adjunct appointment, Harvard University. Research/Grant Support; ZonMW and STW, Netherlands.

\section{A-484 16:05 \\ Evidence-based imaging \\ F. Sardanelli; Milan/IT (f.sardanelli@grupposandonato.it)}

The application of evidence-based medicine to medical imaging was relatively delayed in comparison to other specialties. Radiologists should be aware of the necessity to justify radiological examinations and imaging-based interventional procedures on the basis of the best available evidence. Diagnostic tests can be evaluated with studies exploring their value in terms of: 1 . technical performance; 2 . diagnostic performance; 3 . diagnostic impact; 4 . therapeutic impact; 5. patient outcome; 6. societal impact. Notably, this is a one-way logic chain. While improvement at the upper levels implies improvement at some of the lower levels, the vice versa is not always true (e.g., a better diagnostic performance does not always imply a therapeutic impact or a better patient outcome). Moreover, different degrees of recommendations are based on different levels of evidence, with experts' opinion as the lowest level of evidence and meta-analyses of high-quality homogeneous studies and multicentre studies being the best level of evidence. The quality of studies needs to be evaluated in terms of internal and external validity, the former regarding study implementation (protocol violations, reference standard, measurements, and readers' independence), the latter regarding study planning (study design, subjects selection, methods, and statistical analysis). Biases influencing the internal validity limit the intrinsic value of study results, while those influencing external validity limit the generalisability of study results to clinical practice. Finally, high-quality research must be planned to build the evidence in favour of radiological procedures, especially for new technologies which tend to enter the market without any preliminary demonstration of efficacy.

Learning Objectives:

1. To understand the different levels of evidence in the medical literature.

2. To become familiar with the hierarchy of studies for diagnostic tests.

3. To understand the concepts of internal and external validity.

4. To learn how different forms of bias can influence reported results in the literature. 


\section{Postgraduate Educational Programme}

\section{A-485 16:28}

State-of-the-art methods in EBR, CER, and HTA relevant to imaging U. Siebert; Hall i. Tirol/AT

More and more, clinical guideline developers and reimbursement decision makers need to base their work and decisions on solid evidence. This presentation will introduce the key concepts and principles of evidence-based medicine (EBM), comparative effectiveness research (CER), and health technology assessment (HTA) and their application to the field of radiology. In particular, it will be discussed which role observational studies, clinical trials, and decision-analytic modeling play in EBR, CER, and HTA and when each of these study types is needed to assess imaging technologies. Specific topics include the assessment of bias, optimising cutoffs and multiple test strategies, making causal inferences, explicitly weighing benefits, risks and costs, and considering ethical, legal and social implications (ELSI).

Learning Objectives:

1. To become familiar with state-of-the-art methods used in EBR, CER and HTA.

2. To understand how observational studies, clinical trials, and decisionanalytic modeling all play a role in EBR, CER and HTA.

3. To understand how these methods can be applied to the systematic and evidence-based evaluation of imaging tests.

Author Disclosure:

U. Siebert: Other; Department of Public Health and Health Technology Assessment, UMIT - University for Health Sciences, Medical Informatics and Technology and ONCOTYROL - Center for Personalized Cancer Medicine, Hall i., Department of Health Policy and Management, Harvard School of Public Health and Department of Radiology, Massachusetts General Hospital, Harvard Medical School, Boston, MA, USA

\section{A-486 16:51}

Translating EBR, CER, and HTA results into imaging decisions in daily practice

F.J. Gilbert; Cambridge/UK (fjg28@cam.ac.uk)

The excitement generated by the discovery of $x$-rays led to the early adoption of this technology in many hospitals around Europe. Over the last three decades, the explosion of new medical imaging technologies together with the recognition by clinicians of the value of these for their patients has meant that imaging budgets have increased exponentially. Evidence based radiology (EBR) is a relatively new approach designed to inform clinicians of the most appropriate technique to use in a given clinical scenario. The comparative effectiveness research (CER) is used by healthcare systems to develop a strategic approach to rationalise the availability of imaging investigations. The Health Technology Assessment (HTA) funding approach is a mechanism to assess new and emerging imaging technologies in a systematic timely manner. The UK has a nationally funded healthcare system which is designed to deliver equitable care for the population free at the point of delivery. The challenge for the UK has been to ensure the highest quality service by delivering the most appropriate technology and care for patients in a timely manner. The National Institute for Clinical Excellence (NICE) is an independent body which gives guidance on new drugs and medical devices. This body requires robust scientific evidence on which to base their decisions. To create the evidence base, the imaging studies need to be of a certain standard to be included (CONSORT, STARD, etc). An example from oncologic imaging will be used to show how EBR influences daily practice.

Learning Objectives:

1. To learn how EBR, CER, and HTA contribute to imaging decisions.

2. To understand how EBR, CER, and HTA influence daily practice through an example from oncologic imaging.

Author Disclosure:

F.J. Gilbert: Speaker; Bracco.

Panel discussion:

Can EBR, CER, and HTA results provide justification for imaging decisions and how do we implement the results? $\quad 17: 14$ \begin{tabular}{ll}
\hline $16: 00 ~-~ 17: 30$ & Room F2
\end{tabular}

Special Focus Session

SF $15 \mathrm{c}$

Childhood abdominal emergency 2014: the dos and don'ts

\section{A-487 16:00 \\ Chairman's introduction \\ E. Alexopoulou; Athens/GR}

Acute abdomen and abdominal trauma are both emergencies with potential fatal sequela when under/ misdiagnosed. Imaging plays a crucial role in the diagnosis and management. US is the imaging technique of choice in most cases, as it is rapid, portable, lacks radiation and there is no need for sedation in young infants. US accuracy can be enhanced by iv contrast agents administration. However, limitations may occur in obese children, in deep structures, or because of gas filled bowel loops. On the other hand, CT provides excellent visualisation without limitations by obesity, gas, or deep lesions. However, there is always the radiation exposure risk of the radiosensitive paediatric patient, while sedation is required in non co-operative young children. The aim of this session is to understand "which" is the modality of choice and "why", for the most common paediatric abdominal emergencies. Session Objectives:

1. To learn about the most common paediatric abdominal emergencies. 2. To become familiar with the advantages and disadvantages of CT and ultrasound in abdominal emergencies.

3. To understand which is the modality of choice for abdominal emergencies.

A-488 16:05

The acute abdomen: US is the answer

S.G.F. Robben; Maastricht/NL (s.robben@mumc.nl)

Several paediatric diseases are real abdominal emergencies. Any delay in diagnosis and treatment may result in death or severe morbidity. These diseases are highly age dependent. Newborn infants may have congenital diseases as Hirschsprung's disease or meconium ileus or may develop necrotising enterocolitis, incarcerated inguinal herniation and midgut volvulus. Infants and preschool children have intussusceptions, urinary tract infections and (rare) haemolytic uraemic syndrome. Children and adolescents have appendicitis, genito-urinary infections, ovarian torsion and Henoch Schonlein purpura. Considering radiation dose in children and the excess value of ultrasonography (US) in small individuals, US plays an important role as initial diagnostic modality in paediatric abdominal emergencies. Sensitivity and specificity for US in diagnosing intussusception, midgut volvulus, urinary tract abnormalities and appendicitis is over $90 \%$. Conventional abdominal radiographs or fluoroscopy are valuable in Hirschsprung's disease, meconium ileus, malrotation and necrotising enterocolitis. I consider CT as an additional technique when the initial techniques (US and conventional radiography) are inconclusive. MRI is seldom indicated in paediatric patients with abdominal emergencies because of motion artefacts in anxious children and sometimes limited MR capacity. Delay in diagnosis of the above-mentioned diseases may be devastating, ranging from life-long disability to death. Therefore, it is important to consider these diagnoses and to initiate prompt adequate diagnostic workup.

Learning Objectives:

1. To learn about the most common and devastating paediatric abdominal emergencies.

2. To understand the value of ultrasonography in acute abdomen.

3. To understand the consequences of any delay in diagnosis.

A-489 16:23

The acute abdomen: $C T$ is the answer

A. Paterson; Belfast/UK (anniezunz@gmail.com)

Abdominal pain is a common symptom in children, and whilst the majority will have a self-limiting condition, in some the pain may indicate an acute medical or surgical condition that requires prompt investigation and treatment. In the paediatric setting, the primary imaging modality for those with an acute abdomen is ultrasound. However, there are certain patient groups - often older children or adolescents - in whom CT plays an important role. It is well acknowledged that $\mathrm{CT}$ is non-operator dependent: an important factor for children presenting to emergency departments outside children's hospitals. The speed of a CT exam is valuable in the acute setting, as is the capability to obtain images without having to touch the tender abdomen of a distressed child. CT offers a global perspective of the abdomen, and image quality is not hindered by the presence of excessive bowel gas, an abnormal body habitus 
or in the obese patient. CT is especially helpful in those with suspected retroperitoneal pathology, in the investigation of immunocompromised patients and those with complex post-operative problems. CT guidance may be required to facilitate percutaneous abscess drainage, particularly for deep pelvic collections. In addition, CT plays a vital role as a 'problem-solver' when ultrasound has failed to fully answer the clinical question posed. This short presentation will utilise clinical studies to illustrate the benefits of CT in the investigation of the acute abdomen, both as the initial imaging modality and as a second line tool following ultrasound.

Learning Objectives:

1. To understand the advantages of CT as a primary imaging modality for children with acute abdomen.

2. To learn about clinical scenarios in which CT is relevant

3. To appreciate the use of CT as an adjunct to ultrasound.

\section{A-490 16:41}

Abdominal trauma: US is better

V. Miele; Rome/IT (vmiele@sirm.org)

Ultrasonography (US) is a reliable technique whose advantages are rapidity, portability and accuracy in depicting intraperitoneal fluid without interrupting resuscitation and without radiation exposure. In the haemodynamically unstable paediatric patients, it represents the first line together with the x-ray evaluation. US for trauma has become more standardised and is worldwide known with the acronym of FAST (focused abdominal sonography for trauma) or E-FAST (extended-FAST) used in depicting also pleural and pericardial effusions and pneumothorax. Nevertheless, many parenchymal injuries are not correctly visualised at baseline US and some traumatic solid organ lesions can occur without hemoperitoneum. In case of haemodynamically stable patients, who have suffered a low-energy trauma, the greater time available allows the use of specific US contrast agents, enabling a better identification of traumatic organ injuries. Contrast-enhanced ultrasonography (CEUS) has a greater sensibility and specificity in the identification of parenchymal traumatic lesions, both in the first evaluation and follow-up, and could avoid unnecessary radiation and iodinated contrast medium exposure. In case of haemodynamically stable paediatric patients, who have suffered a high-energy trauma, US is not recommended as first-line investigation, because CE-MDCT should be performed first. Only in the follow-up, CEUS can be considered an alternative to CT. In conclusion, E-FAST and CEUS should be considered as a useful tool in the assessment and monitoring of paediatric trauma. This examination can be performed at the patient's bedside, representing a useful alternative to CT in the paediatric traumatised patients and in the follow-up of a known abdominal injury.

Learning Objectives:

1. To understand the differences in the diagnostic paths of paediatric patients.

2. To learn about the diagnostic efficacy and limitations of ultrasonography.

3. To become familiar with the use of the contrast-enhanced ultrasonography (CEUS).

\section{A-491 16:59}

\section{Abdominal trauma: CT is better}

M. Raissaki; Iraklion/GR (mraissaki@yahoo.gr)

CT has been considered a sensitive, specific, and accurate test in the identification and grading of injuries, especially in the severely injured child. It should be overemphasised that traumatised children differ from adults: haemodynamically stable children may actually be actively bleeding and rapidly deteriorate. Conversely, children have smaller calibre vessels, stronger vasoconstriction, and stronger solid organ capsules; bleeding may stop spontaneously, organ rupture is more difficult and delayed rupture is rare. This is why few children will undergo laparotomy or trans-arterial embolisation. The goal of imaging is to clear the abdomen in multi-traumatised children, identify those that may rapidly deteriorate because of clinically silent active bleeding increase the surgeon's confidence level and define short- and long-term medical management. Due to children's increased radiosensitivity, all CT scans should be indicated based on appropriate early clinical evaluation, assessment of risk factors for abdominal injuries and evaluation of the closely monitored child. ALARA includes the availability of outside scans upon admission, avoiding non-contrast scans and multiple phases and applying age- and weight-dependent exposure parameters. CT should not be performed as follow-up unless there is clinical deterioration. CT has the advantage of rapidly identifying and delineating with high resolution solid organ, vascular mesenteric, bowel injuries and pending oligaemic collapse (hypoperfusion complex or shock bowel). Important fractures and thoracic injuries may be simultaneously revealed with CT. The objective documentation of intraabdominal injuries is extremely important in suspected child abuse.

Learning Objectives:

1. To understand that children are not small adults.

2. To learn about trauma CT protocols according to the ALARA principle.

3. To become familiar with types of injuries, which can be appropriately evaluated with $\mathrm{CT}$.
Panel discussion:

US or CT in paediatric abdominal emergencies?

$17: 17$

\begin{tabular}{l} 
16:00 - 17:30 \\
\hline Neuro \\
RC 1511 \\
Spine, cord and intervention \\
Moderator: \\
C. Hoffmann: Tel Hashomer/L
\end{tabular}

A-492 16:00

A. Intramedullary pathology

M.M. Thurnher; Vienna/AT (majda.thurnher@meduniwien.ac.at)

Evaluation of MR imaging in a patient with an intramedullary lesion should focus on key features: a) the location of the lesion on the cross-sectional area of the cord, best evaluated on axial images, b) the length of the lesion evaluated on sagittal images, c) the presence of cavitation and cysts, d) signal intensity on $\mathrm{T} 2-\mathrm{WI}, \mathrm{e})$ the presence of enhancement and enhancement type, and $f$ ) associated leptomeningeal enhancement. The knowledge of the presence or absence of the brain lesions is important information for narrowing the differential diagnosis. The clinical picture and the onset of symptoms will help the differentiation between the neoplastic and inflammatory and vascular lesions. In this lecture, the imaging characteristics and typical patterns of intramedullary lesions will be presented. A diagnostic algorithm, which includes imaging, clinics and CSF analysis, will be discussed.

Learning Objectives:

1. To become familiar with the differential diagnosis of intramedullary lesions.

2. To learn how to differentiate between inflammatory, vascular and neoplastic cord disease.

3. To recognise the most common intramedullary lesions.

A-493 16:30

B. Spondylodiscitis, degenerative or tumoural?

G. Lycklama à Nijeholt; The Hague/NL (g.lycklama@mchaaglanden.nl)

A common clinico-radiological situation is the differentiation between spinal infection, degenerative changes and osteopaenia, and spinal tumour. In most cases, conventional CT and/or MRI will allow a certain differentiation, and additional biopsies are mainly used to obtain tissue for bacteriological or histopathological classification. Advanced MR techniques such as DWI may sometimes help in increasing diagnostic certainty, but are seldomly used in day-to-day practice. Spinal infections typically present around the intervertebral disc, but may also present as isoloated spondylitis without disc involvement, and sometimes the infection quickly spreads to the epidural space. Degenerative changes my sometimes mimic infection due to strong gadolinium enhancement. The main differential diagnosis of spinal tumorous processes is osteopaenic changes (oedema), and CT may be helpful in such cases, for example to demonstrate degenerative gas in the vertebral body. Rare causes of spinal abnormalities such as atypical pathogens and rare tumours may cause diagnostic problems; often the fastest road to a diagnosis is biopsy in those cases.

Learning Objectives:

1. To understand the pathogenesis of degenerative and infectious spinal disease.

3. To become familiar with the diagnostic algorithm of patient selection.

4. To understand the natural evolution and treatment effects of spinal disease.

\section{A-494 17:00}

C. Percutaneous treatment of spinal diseases

M. Muto; Naples/IT (mutomar2@gmail.com)

The correct clinical diagnostic approach to spine disease is mandatory in the selection of patients to be treated. The anatomy and the relationship between different structures must be known in detail to understand the source of the pain and so to treat it percutaneously. MR, CT, dynamic x-ray and NM bone scan in selected cases can be used to understand the reason for the pain. MR with T2 STIR sequence or T2 Fat SUPP technique is mandatory to show bone marrow oedema and to decide on which metamer to perform the treatment. CT is often necessary after MR in primary and secondary spine tumours. Disk disease can be seen either on CT or MR. Two major treatments must be considered: discogenic pain and vertebrogenic pain, and for this reason disk treatment and vertebral treatment. Disk treatment includes many mini-invasive systems to use; however, no treatment has been shown to be superior to the others, with good clinical results in $75-80 \%$ of the cases, even at long-term follow-up. VP and assisted techniques are available for the treatment of not 
only porotic fragility fractures, but also of primary and secondary spine tumours such as traumatic fractures.

Learning Objectives:

1. To understand the clinical indications for percutaneous treatment of spinal diseases.

2. To learn which diagnostic modality to suggest.

3. To become familiar with the different percutaneous techniques available.

$16: 00-17: 30$ Room I/K

\section{Head and Neck Imaging}

CC 1519

\section{Tailoring head and neck examinations to the clinical symptoms (part 1)}

Moderator:

M.I. Furmanek; Warsaw/PL

A-495 16:00

A. Impaired eye movements

M.G. Mack; Munich/DE (m.mack@radiologie-muenchen.de)

A wide variety of lesions in and around the orbita can impair eye movement. CT and MR imaging is frequently used to confirm or exclude lesions in and around the orbit in patients with impaired eye movement. First of all, it is very important to know the exact clinical history of the patients. CT is excellent for confirming a mass; however MRI is more sensitive and arrives often at a single most likely diagnosis. Characteristic imaging features may help distinguish among lesions that have overlapping clinical presentations. This review focuses on some of the common orbital masses. In this lecture common benign and malignant lesions will be discussed. Vascular lesions include capillary (infantile) haemangioma, cavernous haemangioma, and lymphangioma. Benign tumours include optic nerve sheath meningioma, schwannoma, and neurofibroma. Malignancies that are reviewed include: lymphoma, metastasis, rhabdomyosarcoma, and optic glioma. In addition, benign and malignant lesions affecting the eye movement from outside of the orbit (e.g. from the paranasal sinuses, nasopharynx and skull base) will be discussed.

Learning Objectives:

1. To learn about impaired eye movement and understand its causes.

2. To learn about the imaging algorithm.

3. To become familiar with differential diagnosis.

\section{A-496 16:30}

\section{B. Trigeminal neuralgia}

A. Borges; Lisbon/PT (borgalexandra@gmail.com)

Trigeminal neuralgia is a unique form of facial pain, defined by the Internationa Association for the Study of Pain as a sudden, unilateral, brief, stabbing, recurrent pain in the distribution of one or more trigeminal nerve branches triggered by a specific event. Additional diagnostic criteria of the International Headache Society include paroxysmal, stereotypic pain attacks and absence of associated neurological deficits. It is due to trigeminal nerve compression at the root entry zone, a transitional zone between central and peripheral myelination, where the myelin sheath is thinner and more prone to compression and electrical stimulation. The most common cause is vascular compression, by aberrant loops of the SCA, AICA, PICA or vertebro-basilar dolichoectasia, leading to a neurovascular conflict. Small size of the prepontine and CPA cisterns has been identified as a predisposing factor. Occasionally, expansile lesions of the skull base or CNS compress the root entry zone and lead to atypical trigeminal neuralgia. MR imaging is the modality of choice to evaluate these patients and requires specific tailored protocols to depict its causes, including $3 \mathrm{D}$ heavily $\mathrm{T} 2 \mathrm{~W}$ images reconstructed in the three planes and an angiographic sequence for the depiction of neurovascular conflicts. As neurovascular contacts are quite common in the general population, strict imaging criteria for the diagnosis of NVC have emerged. The offending vessel needs to cross the nerve perpendicularly and deviate or indent its course at the REZ. A pictorial review of the most common causes of trigeminal neuralgia and trigeminal neuropathy will be presented.

Learning Objectives:

1. To understand the causes of trigeminal neuralgia and its clinical symptoms.

2. To learn about the relevant anatomy and appropriate imaging methods.

3. To become familiar with the relevant radiological images, differential

diagnosis and limitations of the method.

\author{
A-497 17:00 \\ C. Facing problems of the face: facial pain, tics and palsy \\ B. Verbist; Leiden/NL (b.m.verbist@/umc.nl)
}

Facial impairments may be due to neuropathies of the trigeminal and facial nerve. This lecture will focus on the seventh cranial nerve. This nerve is a complex, mixed nerve with motor fibres, parasympathetic fibres, sensory fibres to the external auditory canal and special sensory (taste) fibres to the tongue. The most common presentation of facial nerve dysfunction is facial palsy. The paresis or paralysis of facial muscles may be caused by several conditions such as developmental abnormalities, infectious or inflammatory disease or tumoural lesions. Other presentations of facial nerve dysfunction are facial tics or hemifacial spasm and pain. The indications for imaging, choice of imaging modality and possible imaging findings in case of facial palsy, facial tics and facial nerve-related pain will be discussed

Learning Objectives:

1. To understand the possible causes of facial impairments.

2. To become familiar with the cranial nerves involved.

3. To learn how to choose appropriate imaging modality and which critical structures to evaluate.

16:00 - 17:30
Physics in Radiology
RC 1513
IT tools for dose tracking and workflow
Optimisation

Moderators:

P. Mildenberger; Mainz/DE

A. Trianni: Udine/IT

\section{A-498 16:00 \\ A. DICOM and IHE standards \\ D. Peck; Detroit, MI/US (donaldp@rad.hfh.edu)}

The development of the DICOM Standard is done through Working Groups of the DICOM Standards Committee. The Working Groups perform the majority of work on the extension and corrections to the Standard. The extensions are made through development of Supplements and corrections are done through Change Proposal (CP) mechanism. In this presentation, the procedures used to create the Standard will be reviewed and some of the most recent changes discussed. As a collaboration of professional societies and companies, Integrating the Healthcare Enterprise (IHE, www.ihe.net) seeks to establish methods wherein health computer systems can communicate to achieve specific functional objectives. These involve development of methodologies to implement standards such as DICOM, HL7, etc. In this presentation, the procedures used to create the IHE profiles will be reviewed.

Learning Objectives:

1. To understand the current DICOM standard.

2. To learn about new DICOM efforts that will significantly impact imaging systems operations.

3. To understand how IHE coordinates the integration and management of DICOM objects.

\section{A-499 16:30}

B. Patient dose tracking: a must have?

W. Pavlicek; Scottsdale, AZ/US (pavlicek.william@mayo.edu)

Patient Dose Tracking is rapidly growing in the USA as a large number of commercial products are available in response to user demand. These products leverage the work that has gone into the development of the DICOM Dose Structured report and the proliferation of digital imaging systems. Medical physicists are crucial team members as they are most capable of performing patient dose measurements and calculations. Noteworthy is that the 'toolbox' of the medical physicist is expanding. Informatics with Patient Dose Tracking tools invariably includes familiarity at some detail with DICOM structure and tags, Dose Structured Reports (SR), SR Readers, RIS content and orders, modality work list broker content, modality (imaging) specific content as well as PACs, Archive rules for storage/retrieval and EMR. Patient-specific information and the highly detailed specifics of the individual dose prescription are necessary for advanced estimates of information. A Dose Tracking system (a QA tool outside of the normal imaging device that uses content provided by Dose SR and other information systems) usually strives to elevate the quality of the dose estimate, using informatics tools such as patient and organ models, dosimetry engines, and predictive statistics. General use cases for Dose Tracking will be presented including advanced dose estimations for individual patient exams and QA review for both CT and fluoroscopy. Examples of 
tracking of prescriptions by patient, protocol/procedure and operator for determination of patient dose history, 'outliers', continuous improvement (using DMAIC tools) or for meeting regulatory or accrediting bodies will be included. Learning Objectives:

1. To identify informatics and tools for tracking patient radiation dose.

2. To learn about some possible use cases in clinical practice.

3. To learn about some examples of patient radiation dose tracking.

\section{A-500 17:00}

C. To identify pitfalls and support technique optimisation

E. Vaño; Madrid/ES (eliseov@med.ucm.es)

With the introduction of digital radiology, it is possible to have automatic systems to collect and archive patient dose data individually, in addition to demographic, geometric, and other procedural parameters, as part of the DICOM header or through other DICOM services. These automatic systems mean significant benefits for patient dosimetry and quality control. Different approaches were used, depending on the availability and level of implementation of the DICOM standard, including extracting the technical information from the headers, using the radiation dose structured reports (which contain accumulated dose over several irradiation events), analysing the MPPS messages sent by the modalities to the radiology information system, and implementing optical character recognition techniques on saved screen images. Before issuing a formal patient dose report, the medical physicists should verify and correct all patient dose data. All these approaches allow managing more information and provide better capacity to audit the full imaging procedure and to help with the optimisation. The current level of technology allows doing so at a reasonable cost and with a great benefit for the clinical practice. Automatic detection of abnormal patient doses or mistakes in the technical parameters used and their prompt correction is possible. Diagnostic reference levels will be effortlessly reviewed with such systems. Some examples of pitfalls and possible optimisation actions will be presented. Learning Objectives:

1. To learn about sources of information that could help in identifying possible pitfalls.

2. To understand how to interpret the data stored.

3. To learn about some examples of pitfalls and possible optimisation in clinical practice.

16:00 - 17:30 Conf. Room M3

Oncologic Imaging: Follow-up of Systemic and Local Therapies

\section{1518}

\section{Follow-up of thermal ablation (part II)}

Moderator:

R. Bale; Innsbruck/AT

\section{A-501 16:00}

A. Follow-up imaging of thermal ablative therapies for lung tumours

T. De Baere, F. Deschamps, L. Tselikas; Villejuif/FR (debaere@igr.fr)

Within a few minutes following ablation, CT of the ablation zone will demonstrate an extensive area of ground glass opacity (GGO). A GGO of at least $4.5 \mathrm{~mm}$ peripheral to the tumour is predictive of complete ablation. As early as 24 hours post-treatment, the entire ablated region usually appeared as a well-demarcated homogeneous dense opacity on CT that corresponded to necrotic tissue and its surrounding rim of granulation tissue. This zone of ablation is the "baseline post-ablation imaging" for follow-up. Then, a relatively slow involution of the ablation zone will occur with various patterns, including nodular, fibrosis, disappearance, cavitation and atelectasis. Morphologic features of local tumor progression are an increase in the overall size or a change in the shape of the ablation zone (even without enhancement). It is generally considered that an ablation volume that does not increase in size on subsequent imaging after the baseline post-ablation imaging is a complete ablation. The relatively slow decrease in size of the ablation zone renders CT morphologic evaluation responsible for late discovery of local tumour progression. PET/CT is able to discover incomplete ablation earlier than CT at a stage the disease remains small. Patients who have very early evaluation with $\mathrm{PET} / \mathrm{CT}$ are at risk of either false-positive result due to early inflammation process, or false-negative result due to early inflammation masking active tumor foci. 3 or 6 months after ablation can be a reasonable time for PET/CT. Learning Objectives:

1. To consolidate knowledge on the imaging aspects of successful ablation

2. To become familiar with the main pitfalls of post-ablation imaging.

3. To learn about the imaging aspects of most common complications.
A-503 17:00

C. Follow-up imaging of thermal ablative therapies for kidney tumours D.J. Breen; Southampton/UK (David.Breen@uhs.nhs.uk)

Image-guided ablation of kidney tumours has been increasingly set to become the standard of care treatment for smaller $(<5 \mathrm{~cm})$ renal tumours, yet ablation remains a non-extirpative technique and is therefore paramount, in this curative setting, that imaging follow-up should reliably confirm complete tumour eradication. Early on in radiofrequency ablation (RFA), control remained problematic and inadequate treatment was evidenced by residual marginal crescents of viable, enhancing disease. In the current era of more definitive ablation with multipolar techniques, cryo- and microwave ablation (MWA), this pattern of treatment failure should rarely, if ever, be encountered. Typically following adequate ablation imaging should confirm a completely nonenhancing tumour and a related cortical ablation zone. Whilst nonenhancement is an accepted surrogate of tumour non-viability, most practitioners look for additional collateral features such as the 'halo' sign, appearing as a soft tissue ring in the adjacent peri-renal fat, usually a sound marker of complete tumour eradication. Follow-up data have shown that cryoablation (CRA) can yield robust outcomes, but often incurs notable haemorrhagic change and a 'rind-like' feature around the ablation zone. CRA also appears to induce faster involution of the treated tumour, whereas RFA and MWA can induce a persistent granulomatous mass which only very slowly involutes over a number of years. Late local recurrence is increasingly rare at around $2-3 \%$, but can occur as nodules of enhancing disease as late as 3-6 years after the initial treatment. Subtraction MR can be useful, but to date PET and perfusion techniques still lack resolution and specificity.

Learning Objectives:

1. To learn about the post-ablation aspects, according to the ablation technique used.

2. To become familiar with the imaging aspects of successful ablation.

3. To appreciate the imaging aspect of most common complications.

A-502 16:30

B. Follow-up imaging of percutaneous local treatment of bone tumours A. Gangi, J. Garnon, G. Tsoumakidou; Strasbourg/FR (gangi@unistra.fr)

Multiple percutaneous image-guided therapies are currently available for thermal ablation of bone tumours. Thermal sources for these treatment modalities include high-intensity ultrasound, laser, microwave, radiofrequency, and cryotherapy. The predictability of thermal ablation is adequate to limit collateral damage and complications, however, is limited by biologic and anatomic variability of tissue. Clinical evaluation is essential in symptomatic tumours. Close imaging follow-up with CT, MRI, bone scan, and PET plays a vital role in the management of the post-thermal ablation patient and detection of complications. Recurrences or regrowth can be considered for repeated thermal ablation if the lesion is discovered early, before the tumour geometry, location, or distribution become unfavourable. The imaging features could vary with the different ablation method. The radiologist reporting the follow-up imaging should be familiar with different ablation methods. MRI with dynamic contrast enhanced imaging and subtraction allows to detect recurrences in hypervascular tumour. Contrast enhancement on T1-weighted MRI imaging seems to be predictive of clinically unsuccessful ablation. On CT scan, bone reconstruction can be visualised but is a slow process, particularly in adults. $\mathrm{PET}$ scan is an excellent morphologic and metabolic image to follow-up the tumours. However, the inflammation produce after ablation can be misleading, particularly during the first three months. Imaging follow-up plays an essential role in the management of the post-thermal ablation patient and detection of complications.

Learning Objectives:

1. To learn about the post-ablation aspects, according to the ablation technique used.

2. To consolidate knowledge on the imaging aspects of successful ablation from various types of follow-up imaging, including CT and MRI.

Author Disclosure:

A. Gangi: Other; Proctoring Galil Medical. 
16:00 - 17:30

Board Room A

\section{Radiographers}

RC 1514

\section{The interventional suite of 2014: responsibilities and challenges}

Moderators:

K. Haller; Wiener Neustadt/AT

B. Marincek; Cleveland, $\mathrm{OH} / \mathrm{US}$

\section{A-504 16:00}

A. Today's technology: requirements for a cutting-edge interventional suite

D. Catania; Milan/IT (cataniadiego@hotmail.com)

Interventional radiology (IR) is an evolution of radiology that treats many diseases, originally treated by traditional surgery. The clinical importance of IR has been demonstrated over the years by performing procedures that offer the benefit of therapeutic treatments, competitive from the point of view of a shorter hospitalisation time. Indeed, thanks to IR, patients are treated in a less invasive manner, hospitalisation is limited and thus promotes the containment of social costs. To maintain standards clinical oversight needs to focus upon correct organisational, functional and technological appropriateness of practice. To achieve correct IR performance, it is necessary to guarantee that all the instrumental guides (fluoroscopy, ultrasound, computed tomography equipment) function optimally, the procedural equipment including guides/catheters/stents, etc. are chosen according to their technical features and their characteristics are appropriate for use, incorporating cost considerations. As the complexity and variety of interventional procedures grow, there is an increasing need to ensure the appropriate training of specialised IR staff. Consideration of dedicated education and continued professional development options for radiographers working in IR is of priority. The Italian Association of Interventional Radiographers (AITRI) is very important in sharing guidelines in a multidisciplinary environment to standardise and harmonise the knowledge and skills of the team in hybrid theatre and interventional suites. Interventional radiology technology is rapidly expanding and, to maintain safe and efficient practice, careful consideration of current and potential future organisational needs and the training of staff within an IR suite require detailed consideration.

Learning Objectives:

1. To learn about interventional procedures and their impact on economic factors and outcome.

2. To become familiar with the range of technologies in interventional radiology suites and hybrid theatres.

3. To appreciate the staffing requirements associated with interventional radiology suites and hybrid theatres.

4. To appreciate the education and training needs of radiographers working in interventional radiology.

\section{A-505 16:30}

B. Radiation risk and radiation protection considerations

A. Widmark; Østerås/NO (anders.widmark@nrpa.no)

The lecture will discuss where new and current knowledge about radiation risk and effects are coming from. A brief overview of the different sources of knowledge, including Hiroshima and Nagasaki Life Span Study, will be given. The most important stakeholders involved in research and formulating guidelines for radiation protection, with special emphasis on the new main publication from the International Committee on Radiological Protection (ICRP), and the impact on radiographic practice will be discussed. New tissue weighting factors for breast glandule gives a significant higher risk for females today, especially in the pubertal age. On the other hand, they have the risk for hereditary effects significantly decreased. Estimation of risk, both in general and in the individual, will be discussed. Regarding staff protection, there is a new recommendation for annual dose limit for the eye lens. The previous annual dose limit was $150 \mathrm{mSv}$, and this is now recommended to be decreased to $20 \mathrm{mSv}$ averaged over five years with no single year exceeding $50 \mathrm{mSv}$. The new recommendation can be a limiting factor in interventional radiology and cardiology, if sufficient protection is not used.

Learning Objectives:

1. To understand the importance of radiation protection in interventional radiology for patients and staff.

2. To appreciate the latest recommendations for staff radiation protection in interventional radiology.

3. To appreciate the important role of the interventional radiographer in radiation protection.

\author{
A-506 17:00 \\ C. Changing professional roles \\ B. Hallinan; Dublin/IE (barryhallinan@beaumont.ie)
}

The improvement of patient care and aims for the highest possible levels of service are to the forefront of the modern "interventional suite". Role development experiences will be explored, considering need, effect and management of development. Service demands, the need to extend those provided, the limited number of radiologists, financial constraints and the requirement for continuous professional development at state level provide much stimulus for professional radiographic role development. Increased job satisfaction, reduced waiting lists and improved patient experience all provide largely positive results it appears. Resistance from radiologists, multidisciplinary team members and radiographers themselves however, can provide unnecessary barriers to change. Once abridged the blurring of professional lines and insecurity within multidisciplinary team members may also occur. Various experiences would suggest that only by allowing ownership of change by all the clinical stakeholders involved, thus empowering them, leads to successful development. On examination clear guidelines and distinct protocols must be established, taking account of current workload, to create the ideal scenario for good multidisciplinary team ethos, where members have overlapping yet clearly defined roles. Professional accountability, responsibility and an understanding of those role's are all key, but without correct training, including support and resources, no regime for role development can be achieved successfully. Clinical audit not only ensures the delivery of high quality and effective care in line with best practice, whilst ensuring cost effectiveness, but also provides the confidence for all involved, especially the interventional patient.

Learning Objectives:

1. To understand the factors influencing change in professional roles within interventional radiology.

2. To learn about the potential impact of enhanced professional roles and a multidisciplinary team approach on service delivery and patient outcomes.

3. To understand the need for clear protocols and guidelines along with appropriate training and audit of practice when implementing such changes.

$16: 00-17: 30$

Room P

\section{ENCITE Session}

\section{ENCITE based insights for molecular imaging in guidance of therapy \\ Moderator:}

M. Neeman; Rehovot/IL

16:00

Introduction

M. Neeman; Rehovot/IL (michal.neeman@weizmann.ac.il)

A-507 16:05

A dual-modality gene reporter for in vivo imaging

K. Brindle; Cambridge/UK (kmb1001@cam.ac.uk)

There have been many attempts to develop gene reporters for MRI, however these give relatively modest image contrast that can be difficult to detect. I will describe in this talk a reporter that gives intense and positive contrast in MR images (up to $\sim 8 \mathrm{x}$ increase in signal), which can also be used with radionuclide imaging, thus combining the sensitivity of radionuclide imaging with the spatial resolution of $\mathrm{MRI}$. The contrast obtained is directly related to the degree of gene expression and is readily reversible, thus allowing longitudinal studies of changes in expression.

Author Disclosure:

K. Brindle: Research/Grant Support; GE Healthcare.

\section{A-508 16:20}

MR imaging for pancreatic cells transplantation

M. Hájek, D. Jirák, V. Herynek; Prague/CZ (Milan.Hajek@medicon.cz)

The transplantation of pancreatic islet into the liver is an excellent example of successful cell therapy. Transplanted islet visualisation in vivo using a noninvasive imaging method, for example MRI, is necessary to prove technical success. Monitoring of transplanted islets in vivo and long-term tracking their fate using MRI requires their labelling by a suitable contrast agent in vitro prior to transplantation. Under ENCITE project we successfully performed animal experiments, which proved therapy potential of labelled pancreatic islets. Labelled islets implanted into the rat liver were viable and induced long-term normoglycemia in diabetic rats. MRI proved their viability and even distribution in the host tissue. These results allowed performing a clinical study on a group of 12 patients, which were transplanted by iron labelled pancreatic islets from cadaver donors. The successful pilot experiment enabled introducing of 
transplantation of native islets into routine clinical practice. Search for better alternative sites, improved immunosuppression and alternative insulinproducing cells require multimodal and multifunctional molecular probes. Currently we test polymer meshes as artificial transplant sites, effect of mesenchymal stem cells as a supportive net for beta cells, and novel cellular probes combing $1 \mathrm{H}, 19 \mathrm{~F}$ labels and fluorescent ones.

\section{A-509 16:35}

Optical imaging in the clinic

J. Dijkstra; Leiden/NL (j.dijkstra@lumc.nl)

Recently a lot of developments have been done in the field of optical imaging New sensitive devices made it possible to use both visible and near infrared light as diagnostic tool and guidance tool. The advantage of near infrared is that the penetration depth in tissue is much better than for visible light, allowing to see deeper. By using techniques like augmented reality, the data from infrared, both anatomical and functional, can be presented to the operator in real-time. Optical imaging is used e.g. as an evaluation tools for mammography where the optical spectral properties of the tumor cells is used to monitor the effectiveness of chemo treatment. The spectral properties of the breast are measured using a laser at different wavelengths and a camera to create tomographically a 3D volume. Dedicated near infrared probes which bind to certain tissue types make it possible to look for e.g. remaining tumor tissue in resection margins during surgery. This techniques also allow to visualise structures which should be avoided during procedures like nerves. By adding multispectral imaging, the endogenous contrast can be used. Different tissues have their own absorption spectrum which can be shown as additional information, for instance the ration $\mathrm{Hb} / \mathrm{HbO} 2$. Optical imaging also allows for acquiring images at near microscopic resolution real time in-vivo by using Optical coherence tomography. This modality is being tested to provide information about the presence of certain cell types very fast where otherwise histology is needed.

\section{A-510 16:50}

Mesenchymal stem cells constructs for image-guided cell therapy in myocardial ischemia and digestive fistulas

O. Clément, E. Blondiaux, G. Rahmi, L. Pidial, A. Silva, F. Gazeau, C. Wilhelm, G. Autret; Paris/FR (olivier.clement@inserm.fr)

Regenerative medicine has recently emerged as a potential therapeutic tool. A number of preclinical and clinical trials have been conducted in many diseases ranging from diabetes to myocardial infarction and neuro-regenerative diseases that assess the feasibility and benefits of injecting stem cells However, outcomes relating to graft survival remain generally unsatisfactory, whether the process of injection is direct, intravenous or catheter-guided. To overcome such issues, tissue engineering has potential to improve cell engraftment and therapeutic response including functional parameters. This work aimed to evaluate mesenchymal stem cell constructs for image-guided cell therapy in myocardial ischemia and digestive fistulas. We tried to options: 1. Fibrin patches based on fibrinogen monomers polymerised with thrombin and seeded with cells. 2. Constructs based on 3D multilayers of confluent cells sheets. Tissue constructs were labelled with iron oxide particles and evaluated in a model a myocardial infarction for the fibrin patches or digestive fistula for the 3D constructs. MRI at 4.7 was performed at various time points after treatments using a high resolution coil. Fibrin patches could induce a therapeutic effect by increasing the left ventricular ejection fraction compared to sham. 3D constructs induced an increased number of fistula healing and enhanced micro-vasculature density compared to controls. MRI of labelled stem cell constructs allowed a good evaluation of the models and showed increased therapeutic efficacy. The proposed paracrine mechanisms will be discussed.

\section{A-511 17:05}

Is cell imaging relevant for the clinic? Lessons to be learned from preclinical research

U. Himmelreich; Leuven/BE (Uwe.Himmelreich@med.kuleuven.be)

Non-invasive imaging of therapeutic cells has become a popular field of research over the last decade. This interest was mainly based on the hope that the location, migration but also function of immune, stem and other cells can be visualised over time in individuals. The development of novel contrast agents and mechanisms for MRI but also other imaging methods has resulted in exciting basic research findings. In particular, the application of relatively biotolerant iron oxide based nanoparticles has fostered the hope for direct translation into clinical research and general practice. However, pre-clinical research has also highlighted several limitations of nanoparticle based cell imaging by using $\mathrm{MRI}$ including the generation of unspecific contrast, difficulties to quantitatively image engrafted cells, unambiguous contrast adverse effects on cell biology, limitations for longitudinal follow-up or the lack of functional information. Such shortcomings are traditionally overcome in preclinical research by combining MRI with other imaging modalities like bioluminescence imaging or positron emission tomography. Our research focuses on the optimisation of cell labeling strategies for robust, sensitive and potentially quantitative visualisation of stem and progenitor cells in therapy models in vivo to assess cell behavior after engraftment. The sensitivity, stability, toxicity and adverse effects on the cell biology by the labeling procedure were studied for iron oxide based particles. The potential of Gdchelates and ${ }^{19} \mathrm{~F}$ labeled compounds for cell labeling has been assessed in vitro and in vivo. Based on our preclinical research finding the potential of future applications in patients will be explored.

Discussion

$17: 20$ 


\section{Monday, March 10}


$08: 30-10: 00$ Room A

\section{Interactive Teaching Session}

\section{$E^{3} 1620$}

\section{Pitfalls in heart imaging}

\section{A-512 08:30}

A. CT

G. Bastarrika; Toronto, ON/CA (bastarrika@unav.es)

Cardiac CT is becoming the imaging modality of choice for an increasing number of clinical indications, not only to rule out coronary artery disease but also to evaluate cardiac morphology and function, and to determine patient outcome after coronary artery revascularisation. However, as with any other imaging tools, appropriate interpretation of cardiac CT examinations is required to assess the clinical value of this newly established diagnostic imaging modality. This process requires performance of thorough cardiac CT acquisition protocols, detailed knowledge of standard cardiac anatomic and physiologic terminology, as well as appropriate postprocessing, reading and reporting. In particular, radiologists need to recognise and be aware of the imaging findings that may confound and lead to interpretation errors. This lecture will summarise the practical aspects of postprocessing, reading and reporting of non-invasive cardiac CT examinations. The value and limitations of every available CT postprocessing technique including two-dimensional multiplanar reformations, curved multiplanar reformats, maximum intensity projection (MIP) and volume rendered images will be explained. Moreover, hints for improving reading results by recognising technical causes for various artefacts in cardiac CT will be elucidated and reading approaches to diminish false positives, false negatives and inaccuracies when assessing coronary artery stenosis will be suggested.

Learning Objectives:

1. To learn about practical aspects of post-processing, reading and reporting non-invasive cardiac CT examinations.

2. To learn how to improve reading of results by recognising technical causes for various artefacts in cardiac CT.

3. To become familiar with approaches to reducing inaccuracies and misinterpretations when assessing coronary artery stenosis.

\section{A-513 09:15}

\section{B. MRI}

M. Francone; Rome/IT (marco.francone@uniroma1.it)

Cardiac magnetic resonance (CMR) is a complex imaging technique due to the intrinsic anatomical and technical peculiarities of the exam. These include the non-orthogonal cardiac orientation within the chest cavity requiring dedicated acquisition planes and the complex respiratory and cardiac motion to which the heart is subject and requiring a combination of ECG-gated and breath-hold sequences. Potential additional anatomical pitfalls also include norma structures and variants like the moderator band, papillary muscles, and the presence of prominent crista terminalis or myocardial trabeculations, whose recognition is mandatory and may mimic in some cases a pathological condition. Technical issues of CMR concern the continuous intracavitary inflow of protons and the associated "slow-flow" artifacts (limiting visualisation of endomyocardial border in some cases) pitfalls related to ECG gating, like inadequate synchronisation or the T-wave swell phenomenon; and finally a series of specific artifacts intrinsically related to the use of different pulse sequences that may interfere with image quality. An additional, more complex issue to consider is also the widespread diffusion of high-field magnets which have further enhanced those aspects. Knowledge of the spectrum of those CMR peculiarities is mandatory to approaching and providing a correct diagnosis according to the main clinical request. The present lecture will review the most important anatomical and technical pitfalls of CMR examination and offer, when possible, practical solutions to overcome those limitations. Learning Objectives:

1. To learn about common pitfalls in MRI evaluation of the heart.

2. To become familiar with cardiac anatomical variants potentially mimicking disease.

Author Disclosure:

M. Francone: Speaker; Bracco Medical Imaging.
08:30 - 10:00 Room B

Special Focus Session

SF $16 a$

Shoulder imaging and intervention

\author{
A-514 08:30 \\ Chairman's introduction \\ I. Beggs; Edinburgh/UK (ian.beggs@/uht.scot.nhs.uk)
}

Shoulder imaging and intervention are becoming increasingly important in clinical practice. This session considers the indications, techniques, imaging findings and relative merits of diagnostic ultrasound and MRI of the shoulder. The indications, techniques and results of US-guided interventional procedures are also considered. A panel discussion will deal with controversies in shoulder imaging such as the accuracy of US and MRI in assessing rotator cuff tears, tendinosis, impingement and muscle atrophy, and the efficacy of US-guided interventions. Audience participation in the discussion will be welcome. Session Objectives:

1. To learn how to perform US and MRI of the rotator cuff.

2. To appreciate the advantages and limitations of both techniques.

3. To become familiar with US-guided interventions of the shoulder.

A-515 08:35

US of the rotator cuff

A. Plagou; Athens/GR (athena.plagou@gmail.com)

The shoulder is an anatomic area that is very commonly evaluated with musculoskeletal ultrasound. Ultrasonography is widely recognised as a reliable means of assessing rotator cuff disease with accuracies reaching $100 \%$ for fullthickness tears and $90-95 \%$ for partial-thickness tears. Diagnostic accuracy depends mostly on the skills and experience of the examiner. A comprehensive ultrasound (US) examination requires, first of all, sound knowledge of the anatomy. A specific scanning protocol must be adopted in every US examination in patients with shoulder disease, because focal symptoms do not correlate with the location of the disease. The greatest importance of ultrasonography in rotator cuff assessment lies in its dynamic character. Several dynamic manoeuvres can reveal pathologies such as subacromial impingement. Last, but not least there are several scanning pitfalls such as anisotropy. Anatomy key structures as well as the US technique and scanning protocol will be presented in this lecture. Dynamic manoeuvres will be described and demonstrated with videos. Scanning pitfalls will be emphasised. Advantages and disadvantages of the US examination compared to other imaging modalities will be discussed.

Learning Objectives:

1. To understand the indications for ultrasound in rotator cuff disease.

2. To appreciate the dynamic aspect of ultrasound and its diagnostic importance.

3. To become familiar with the ultrasound examination protocol. 


\section{Postgraduate Educational Programme}

\section{A-516 08:58}

\section{MRI of the rotator cuff}

S. Waldt; Munich/DE (Waldt@roe.med.tum.de)

Guidelines concerning the MR examination technique of the rotator cuff will be presented in this session. On intermediate-weighted MR images, tendinopathy and partial and complete tears of the rotator can be differentiated with high diagnostic accuracy. Indications for MR arthrography, especially for the detection of small articular sided partial tears in athletes, will be presented as well. Established classification systems for the description of rotator cuff tears will be discussed. Besides characterisation of rotator cuff lesions, especially in view of therapeutic decision-making, recognition of the underlying pathomechanism is necessary. Therefore, the role of imaging is to detect different structural findings that are suggestive of a possibly underlying impingement syndrome. In primary impingement syndromes imaging abnormalities of the rotator cuff, the overlying bursa and the coracohumeral arch represent the centre of imaging findings. Primary extrinsic impingement is caused by structural abnormalities of the coracoacromial arch, whereas secondary extrinsic impingement is related to glenohumeral instability. Types of internal impingement (posterosuperior and anterosuperior impingement) are secondary to rotator cuff and/or capsular dysfunction. Posterosuperior impingement can be diagnosed on MR arthrograms by identification of the socalled "kissing lesion" pattern, with corresponding lesions of the undersurface of the rotator cuff, posterosuperior labrum, greater tuberosity and superior glenoid. Imaging abnormalities of this condition will be discussed.

Learning Objectives:

1. To become familiar with MR imaging features of rotator cuff tears.

2. To learn about an optimised MR imaging protocol for the diagnosis of rotator cuff tears.

3. To understand basic pathomechanisms and MR classification systems for categorising rotator cuff tears.

\section{A-517 09:21}

\section{US-guided interventions of the shoulder}

L.M. Sconfienza; San Donato Milanese/IT (io@/ucasconfienza.it)

The shoulder is one of the joints in the human body that is most subject to a number of pathologic conditions, both in young and in elderly subjects, such as subacromial-subdeltoid bursitis, calcific tendinopathy, and degenerative conditions. Being inexpensive, readily available, and radiation-free, ultrasound is the imaging modality of choice to guide interventional procedures around the shoulder. Thanks to its high resolution and multiplanar capabilities, ultrasound can be used to guide needles precisely in the tendons of the rotator cuff or within the joint space, both gleno-humeral and acromio-clavicular. This approach can be used to perform a number of different procedures. When dealing with bursitis, a needle can be guided within the subacromial bursa to aspirate fluid and to inject anti-inflammatory drugs. In case of calcific tendinopathy, one or two needles can be used to dissolve calcium deposit and drain it, providing patients prompt relief. In selected patients with overuse tendinopathy, ultrasound can be used to guide intratendinous injection of platelet-rich plasma that has been reported to be helpful in stimulating tendon healing. Although minimally invasive, these procedures should be performed in an ultrasound ward with a high degree of sterility, as risk of infection can be concrete.

\section{Learning Objectives:}

1. To become familiar with US-guided interventional techniques for the shoulder.

2. To understand the indications for interventions.

3. To appreciate the types and doses of therapeutic agents.

Panel discussion:

Controversies and confusion in shoulder imaging $\quad 09: 44$

08:30 - 10:00 Room C

Modern Imaging of the GI Tract

CC 1621

\section{Oesophageal and gastric cancer}

\section{Moderator:}

W. Schima; Vienna/AT

\section{A-518 08:30}

A. Modern imaging: an update

A. Ba-Ssalamah; Vienna/AT (ahmed.ba-ssalamah@meduniwien.ac.at)

Multi-detector computed tomography (CT) offers new opportunities in the imaging of the gastrointestinal tract. Its ability to cover a large volume in a very short scan time, and in a single breath-hold with thin collimation and isotropic voxels, allows the imaging of the entire oesophagus, stomach, and the whole chest and abdomen with high-quality multiplanar reformation and threedimensional reconstruction. Preparation of the patients by fasting from solid food approximately 6 hours prior the examination is important. Proper distention of the oesophagus and stomach by oral administration of effervescent granules and water, and optimally timed administration of intravenous contrast material are required to detect and characterise the disease. Preoperative staging of oesophageal and gastric carcinoma appears to be the main indication for MDCT and may replace endoluminal ultrasound (EUS) in the staging of advanced cancers. The use of various reconstruction techniques, including virtual gastroscopy (VG) using a volume-rendering (VR) technique, is promising for the detection of early gastric cancer. The application of the texture analysis technique to distinguish between the different types of gastric and esophageal tumors is still evolving. Finally, the introduction of FDG PET, in combination with MDCT, has resulted in further optimisation of the diagnostic workup of oesophageal cancer, as well as specific types of cases of gastric cancer. By providing morphologic and functional information in the same setting, this technique has come to be the modality of choice, when available.

Learning Objectives:

1. To learn about the appropriate use of modern imaging techniques in the staging of oesophageal and gastric cancer.

2. To become familiar with optimised imaging acquisition protocols, including patient preparation.

3. To appreciate the use of emerging visualisation techniques, including virtual gastroscopy.

\section{A-519 08:50}

\section{B. How to provide the perfect staging report}

R.M. Mendelson; Perth/AU (richard.mendelson@health.wa.gov.au)

The diagnosis of oesophageal and gastric cancer is usually based on endoscopic findings accompanied by biopsy. However, staging is a matter for diagnostic imaging and is the major determinant of disease management. This should be discussed within a multidisciplinary forum (multidisciplinary team [MDT] meeting) in which the radiologist plays a crucial part. Accurate staging usually based on the TNM staging criteria - is essential and the radiologist's report should reflect this pivotal role. The TNM staging of oesophageal cancer and gastric cancer will be discussed in detail. The phases in staging is essentially a filtering process which seeks to initially exclude distant metastasis and/or advanced local disease, initially by optimally protocolled CT scanning, If CT shows advanced disease, treatment is palliative, but even under these circumstances imaging will help determine the method of palliation. Conversely, if CT demonstrates localised disease, 18 F FDG-PET scanning for oesophageal cancer and for selected cases of gastric cancer is indicated. If this, too, shows no nodal or distant metastasis, accurate $T$ staging with EUS will help determine whether the patient proceeds directly to surgery or undergoes neo-adjuvant chemo/radiotherapy prior to surgery, or in the case of oesophageal cancer may be suitable for EMR. The role of staging laparoscopy in gastric cancer will also be discussed. In summary, the radiologist and nuclear medicine physician are crucial in determining treatment. Their reports are lynch pins in the MDT discussion of patient management. It is therefore essential that the imaging report should optimally inform this discussion. Learning Objectives:

1. To learn about the latest TMN staging in oesophageal and gastric cancer. 2. To appreciate the imaging criteria for local, nodal and metastatic disease, and understand the accuracy of imaging staging.

3. To become familiar with the structure of a perfect imaging report.

A-520 09:10

C. Assessment after treatment

A.M. Riddell; London/UK (Angela.Riddell@rmh.nhs.uk)

It is now established that for the majority of patients with oesophageal and advanced gastric cancer, there is survival benefit from the use of neoadjuvant therapy. Therefore, there is a requirement for imaging to accurately restage the tumour and to assess the response to neoadjuvant therapy, to provide prognostic information and to direct future management. Restaging following therapy is challenging, as differentiating treatment-related fibrosis/oedema from viable tumour is problematic with both $\mathrm{CT}$ and endoscopic ultrasound. The $\mathrm{T}$ and $\mathrm{N}$ staging accuracy for both modalities falls following neoadjuvant therapy. Inconsistencies in measurements due to alterations in the degree of gastric/oesophageal distension can also limit the accuracy of RECIST criteria to determine the response. Functional imaging techniques such as PET-CT offer an improved method for assessing response. Alterations in the Standardised Uptake Value (SUV) occur much earlier than changes in size; therefore a metabolic response can be detected sooner, allowing for more rapid alterations in treatment strategies. Acute complications following oesophagogastrectomy generally occur within the thorax and are either related to a leak at the anastomosis/mediastinitis or respiratory complications such as pneumonia or a pleural effusion. Intra-abdominal collections may develop 
following oesophagogastrectomy and gastrectomy. Late complications following both procedures are often due to tumour recurrence: locoregional such as lymph node recurrence or at the anastomosis; metastatic spread such as haematogenous spread or via the peritoneum or pleura. Currently, there is no consensus on the most appropriate timing or frequency of postoperative imaging.

Learning Objectives:

1. To understand the role of imaging in treatment response assessment.

2. To appreciate the imaging signs of viable or recurrent tumour after therapy.

3. To learn about the common surgical procedures and potential pitfalls in interpreting postoperative imaging.

A-521 09:30

D. Interactive case discussion

W. Schima; Vienna/AT

08:30 - 10:00 Room D

Chest

\section{RC 1604}

\section{Pulmonary arterial hypertension}

Moderator:

A.P. Parkar; Bergen/NO

\section{A-522 08:30}

A. An overview of pulmonary artery hypertension

N.J. Screaton; Cambridge/UK (Nicholas.Screaton@papworth.nhs.uk)

Pulmonary hypertension is defined by increased mean pulmonary arterial pressure $>25 \mathrm{mmHg}$ at rest or $>30 \mathrm{mmHg}$ during exercise. $\mathrm{PH}$ causes significant mortality and morbidity, but commonly presents with non-specific clinical signs and symptoms resulting in significant delay in accurate diagnosis and specific treatment. Untreated $\mathrm{PH}$ is progressive with increased pulmonary vascular resistance leading to right ventricular failure and ultimately death. The current Dana Point classification of pulmonary hypertension is clinically based. It groups diseases with similar pathophysiological mechanisms and therapeutic approaches. Groupings include conditions characterised by diffuse small vessel narrowing (Group 1 and Group 1'), PH secondary to left sided cardiac disease (Group 2), chronic hypoxic pleuro-parenchymal disease (Group 3), chronic thrombo-embolic pulmonary hypertension CTEPH (Group 4), and a miscellaneous group of diseases with either unclear or multi-factorial aetiologies (Group 5). In the Dana Point classification, small vessel diseases are subdivided into Group 1 which primarily affect the pulmonary arterioles and Group 1' affecting the capillary/venous pulmonary circulation (pulmonary capillary haemangiomatosis and pulmonary veno-occlusive disease). The differentiation of Group 1 from Group 1' diseases is important since in group 1' arteriolar dilatation treatments can cause life-threatening pulmonary oedema Group 4 is synonymous with CTEPH with other causes of large vessel obstruction (vasculitis and pulmonary artery tumour) being considered as Group 5 disorders. Recent advances include an increased understanding of molecular mechanisms underpinning $\mathrm{PAH}$, facilitating targeted therapy development, a rapidly expanding role of surgical pulmonary endarterectomy in proximal $\mathrm{CTEPH}$, and recognition of imaging as a potential therapeutic end point.

Learning Objectives:

1. To learn about the epidemiology of pulmonary artery hypertension.

2. To become familiar with the clinical symptoms, signs and causes of pulmonary artery hypertension.

3. To appreciate the importance of and difficulties in treating pulmonary artery hypertension.

\section{A-523 09:00}

B. CT in pulmonary artery hypertension

M.-P. Revel; Paris/FR (marie-pierre.revel@htd.aphp.fr)

CT allows depicting pulmonary hypertension $(\mathrm{PH})$ and helps identifying its cause, therefore playing a crucial role in the diagnostic workup. CT features of pulmonary arterial hypertension include dilatation of the pulmonary artery trunk, with a diameter greater than or equal to $29 \mathrm{~mm}$, a ratio to the aortic diameter greater than $1: 1$ and a segmental artery-to-bronchus ratio greater than 1:1 in at least three pulmonary lobes. On ECG-gated CT, right pulmonary artery distensibility shows the best diagnostic value with $86 \%$ sensitivity and $96 \%$ specificity for a cutoff value of $16.5 \%$. Among the various causes of secondary $\mathrm{PH}, \mathrm{CT}$ is especially useful for detecting signs of chronic thromboembolic pulmonary hypertension, including wall-adherent thrombi, bands, webs or chronic arterial occlusion, mosaic lung attenuation and systemic collateral supply. CT shows signs of pulmonary edema, such as thickening of the interlobular septa, centrilobular ground glass opacities, mediastinal lymph node enlargement and pleural effusion in $\mathrm{PH}$ caused by pulmonary veno-occlusive disease, left heart diseases or mediastinal fibrosis. Signs of lung parenchyma diseases may be indentified on $\mathrm{CT} ; \mathrm{PH}$ is a late complication in patients with pulmonary fibrosis, sarcoidosis or chronic obstructive lung disease, but may affect systemic sclerosis patients with limited lung parenchyma involvement. Congenital cardiac abnormalities with untreated right-to-left shunting resulting in Eisenmenger syndrome, such as ventricular or atrial septal defect and patent ductus arteriosus are easily recognised on CT. Conversely, signs of peripheral pulmonary arteriovenous shunting in portopulmonary hypertension and $\mathrm{PH}$ caused by hepatopulmonary syndrome are more difficult to assess.

Learning Objectives:

1. To learn about the CT diagnosis of pulmonary artery hypertension.

2. To become familiar with the causes of pulmonary artery hypertension on CT.

A-524 09:30

C. MRI in pulmonary artery hypertension

J. Biederer; Heidelberg/DE (Juergen.Biederer@med.uni-heidelberg.de)

For the assessment of pulmonary arterial hypertension (PAH), the dedicated $30 \mathrm{~min} \mathrm{MRI}$ protocol would comprise a free breathing and noncontrastenhanced examination, short T2-w sequences, dynamic contrast-enhanced perfusion imaging, a high-resolution angiogram, a 3D breath-hold acquisition, dynamic steady-state free precession or gradient echo sequences of the heart and a study of myocardial late enhancement. The morphologic sequences show typical features of $\mathrm{PAH}$ : right atrial/ventricular dilatation, enlargement of the pulmonary trunk/main pulmonary arteries and peripherally attenuated pulmonary vessels. Incidental infiltrates, nodules or masses of the lung, mediastinum and chest wall would be covered. The first pass contrastenhanced perfusion imaging demonstrates an increased mean transit time/decreased pulmonary blood flow, but a relatively homogeneous lung perfusion (important to differentiate from CTEPH, where multiple segmental perfusion defects would be expected). The cardiac part shows right ventricular mass, wall thickness and functional changes correlating with elevation of pulmonary arterial pressure: distortion of the interventricular septum, area change of the pulmonary trunk, right ventricular volume/stroke volume as well as pathologic right/left ventricular end-diastolic volume indexes. Late enhancement of the right ventricular wall would correlate with myocardia fibrosis. Furthermore, optional experimental velocity-encoded sequences (ideally for multidirectional flow visualisation, "4D flow") show a decreased pulmonary artery blood flow velocity, increased retrograde flow and inhomogeneous velocity profiles. In conclusion for the near future, given the availability of scanner time and appropriate experience of the team, thoracic $\mathrm{MRI}$ is probably the most comprehensive and effective single examination for the diagnosis and follow-up of $\mathrm{PAH}$.

Learning Objectives:

1. To learn about the MRI diagnosis of pulmonary artery hypertension.

2. To appreciate the role of MRI in the assessment of pulmonary artery hypertension.

3. To become familiar with MRI techniques in the assessment of pulmonary artery hypertension.

Author Disclosure:

J. Biederer: Equipment Support Recipient; Siemens. Speaker; Siemens.

08:30 - 10:00

Board Room B

\section{Special Focus Session}

\section{SF $16 b$}

\section{Multisystemic malignancies in organ- based radiology}

A-525 08:30

Chairmen's introduction

I.E. Tyurin ${ }^{1}, \mathrm{H}$. von Tengg-Kobligk $\underline{2}^{2} ;{ }^{1} \mathrm{Moscow} / \mathrm{RU},{ }^{2} \mathrm{Berne} / \mathrm{CH}$

In the era of organ-based radiology, the group of diseases known as multisystemic malignancies represents an obvious challenge to both radiologists and oncologists. The necessity to match the growing possibilities of different imaging modalities with widespread multisystemic pathology and clinical sufficiency resulted in upgrading well-known diagnostic algorithms. The precise knowledge of clinical staging systems and classification, and the pathologic and physiologic mechanisms of the disease pathways are important for planning imaging modalities and specific protocols. Perfect imaging of multisystemic malignancies now includes not only traditional anatomic-based modalities, but also much more often different types of whole body scanning such as CT, MRI, PET and their combination in SPECT/CT, PET/CT and now even PET/MRI. New imaging modalities and growing possibilities of traditional imaging techniques obviously influence the current clinical guidelines for this 
pathology. Finally, the perfect assessment of clinical course of the disease and possible outcomes, the understanding of tumor response criteria and therapyinduced changes are significant for image interpretation in patients with multisystemic malignancies.

Session Objectives:

1. To appreciate the complexity of the clinical management of patients with multisystemic malignant diseases.

2. To learn about the potential of whole-body CT and MRI, including pitfalls and limitations.

3. To become familiar with the clinical role of PET/CT and MR/PET, including radiotracers

\section{A-526 08:35}

Multiple myeloma

M.-A. Weber; Heidelberg/DE (MarcAndre.Weber@med.uni-heidelberg.de)

Multiple myeloma is a haematologic disorder characterised by the infiltration and proliferation of monoclonal plasma cells mainly in the bone marrow. The main symptoms are hypercalcaemia, renal impairment, cytopaenia/anaemia and bone disease - summarised as CRAB-criteria. Symptomatic multiple myeloma is consistently preceded by asymptomatic premalignant stages called monoclonal gammopathy of undetermined significance and smoldering multiple myeloma. These stages also present with detectable monoclonal protein and/or monoclonal plasma cells in bone marrow, but do not show any end organ impairment. Staging of multiple myeloma is based on the measurement of the monoclonal protein in serum and urine as well as the assessment of impairment of haematopoiesis, renal function and mineralised bone. In the last decade, the development of novel therapeutic agents has led to an increase in response rates and survival time of patients with multiple myeloma, which further stresses the value of response assessment by imaging. Cross-sectional imaging like MRI, CT and PET-CT is currently replacing conventional radiological surveys in the initial workup and follow-up of patients with monoclonal plasma cell diseases. Recent studies demonstrate the added value of MRI in improving initial staging by unravelling a diffuse infiltration of bone marrow by plasma cells, a focal pattern or a combination of both. Also, PET-CT can detect response to therapy earlier than conventional response criteria Furthermore, recent studies revealed that a complete remission of myeloma confirmed by MRI or PET-CT goes along with a better prognosis compared to a complete response based only on serological parameters.

Learning Objectives:

1. To learn about the role of clinical staging systems and imaging in work-up and classification of plasma cell disorders with a focus on multiple myeloma and to learn about parameters of disease activity.

2. To understand the pathophysiologic mechanisms of multiple myeloma and to learn about the effects of abnormal plasma cells on bone remodeling cells.

3. To become familiar with response criteria and therapy-induced changes and to compare the significance of imaging and serological markers for response evaluation in multiple myeloma.

4. To learn about recent studies on imaging based response assessment and prognostic significance.

\section{A-527 08:58}

Lymphoma

A. Mikhaylov; Moscow/RU (azatmihailov@gmail.com)

Over the last 40 years, there have been numerous efforts to classify lymphoid malignancies, culminating in the WHO classification introduced in 2008 . So it is clear that malignant lymphomas require a sophisticated diagnostic approach based on clinical features, morphology, immunophenotyping and genetic analysis. It is essential that such an approach underpins the clinical management of these diseases, many of which are amenable to cure. The same situation is present in radiology staging procedures - it has a multidisciplinary approach. A wide range of staging procedures can include al radiology methods from $\mathrm{CXR}$ and chest and abdominopelvic computed tomograph to whole body MRI, scintigraphy and PET-CT scans.

Learning Objectives:

1. To learn about the current classification and staging of lymphomas.

2. To become familiar with classic imaging patterns of lymphomas.

3. To understand the role of imaging in the follow-up of lymphoma patients.

\section{A-528 09:21}

\section{Melanoma}

M.A. Fischer; Zurich/CH

Malignant melanoma (MM) originates from the melanocytes of the basal layer of the epidermis showing a rising incidence and high mortality in the white, Western population. Risk factors of melanomagenesis like sun exposure are well known and early detection with surgical resection of MM is essential for patient prognosis. Staging of MM is based on tumour thickness, ulceration, lymph node and distant metastases. Lymph flow and chemotaxis are though to be responsible for the homing of MM cells, resulting in satellite or in-transit lesions of the regional lymph node system. Distant spread of MM is thought to transpire intra- and extravascularly (along the external vessel lattices) supported by specific mechanisms of extravasation and protection of MM cells against the local immune system. To date, many imaging methods, such as ultrasound, computed tomography (CT), magnetic resonance imaging, and positron emission tomography/CT, are used in the diagnosis of $\mathrm{MM}$ and its metastases, whereas lymphoscintigraphy has become an important tool for workup of the lymph node drainage patterns. Radiological challenges include improved staging and re-staging of multi-systemic MM. Accordingly, current imaging guidelines and the role and applications of the different imaging modalities in advanced MM (stage III / IV) are reviewed with regard to their usefulness in patient management. However, standardised imaging protocols and integration of functional information to morphological imaging are needed in the future to allow for improved detection and guidance of treatment in patients with MM.

Learning Objectives:

1. To learn about the prognosis factors of melanoma.

2. To understand the metastatic pathways and time course of melanoma.

3. To become familiar with imaging guidelines in cutaneous melanoma.

Panel discussion:

What is the role of imaging in the staging of malignancies?

$09: 44$

08:30 - 10:00

Room E1

\section{Abdominal Viscera}

\section{RC 1601}

\section{Pancreatic inflammation}

\section{Moderator:}

R.J. Méndez; Madrid/ES

\section{A-529 08:30 \\ A. Acute pancreatitis \\ M. Zins; Paris/FR (mzins@hpsj.fr)}

The original Atlanta Classification of acute pancreatitis established in 1992 is outdated. In 2012, an international working group has modified the Atlanta Classification for acute pancreatitis to update the terminology and provide new morphologic classifications. The revised classification of acute pancreatitis identified two phases of the disease: early (first week) and late (after the first week). Acute pancreatitis is subdivided into two types: interstitial oedematous pancreatitis and necrotising pancreatitis. If the diagnosis of acute pancreatitis is established by abdominal pain and by increases in the serum pancreatic enzyme activities, a contrast-enhanced CT is not usually required for diagnosis in the early phase. This revised classification introduces new terminology for pancreatic fluid collections. Depending on the presence or absence of necrosis, acute collections in the first 4 weeks are called acute necrotic collections or acute peripancreatic fluid collections. Once an enhancing capsule develops, persistent acute peripancreatic fluid collections are referred to as pseudocysts and acute necrotic collections, as walled-off necroses. All can be sterile or infected. This classification of acute pancreatitis allows a consistent, worldwide classification and should avoid confusion in the terminology of pancreatic fluid collections.

Learning Objectives:

1. To learn how imaging should be scheduled for patients with acute pancreatitis.

2. To become familiar with the imaging features and the terminology proposed in the Atlanta classification.

\section{A-530 09:00}

\section{B. Chronic pancreatitis and IPMN}

C. Matos; Brussels/BE (cmatos@ulb.ac.be)

The role of diagnostic imaging in chronic and inflammatory pancreatitis and IPMNs is to detect structural changes of the ducts and of pancreatic parenchyma, assess the functional integrity of the gland, detect associated complications, and assist in management. These goals are generally fully achieved using MDCT and MR imaging. In this lecture, the advantages and limitations of each technique will be discussed and illustrated. Key features allowing differential diagnosis of both entities will be underlined.

Learning Objectives:

1. To learn the classical aspect of IPMN on imaging

2. To learn how it merges with chronic pancreatic disease and how can we differentiate both

3. To understand the role of different imaging techniques in assessing both diseases. 
A-531 09:30

\section{Paraduodenal and autoimmune pancreatitis}

S.A. Jackson; Plymouth/UK (simon.jackson1@nhs.net)

Autoimmune pancreatitis (AIP) was first described in 1961 and represents a rare form of immune mediated chronic pancreatitis which is characterised by a marked infiltration of lymphocytes and plasma cells into pancreatic tissue. Whilst the majority of cases present with diffuse gland involvement, approximately $30 \%$ of patients demonstrate either segmental or focal involvement of the pancreas. Clinical presentation is very variable with patients describing a range of symptoms; severe pain however is uncommon. Imaging plays a central role in the diagnosis and management of AIP and knowledge of the radiological appearances, which can vary significantly due to the various degrees of fibrosis and inflammatory infiltrate, is critical. Cardinal features include focal or diffuse pancreatic enlargement with the loss of normal lobular architecture. In addition, pancreatic duct involvement as demonstrated by single or multiple focal strictures with limited more proximal dilatation is common. Whilst these appearances may suggest a diagnosis of AIP correlation with clinical history, serology and histopathology is mandatory to accurately diagnose atypical cases. In contrast, paraduodenal pancreatitis is a specific and rare form of segmental chronic inflammation characterised by fibrous tissue formation in the "groove" area between the duodenum, head of pancreas and lower common bile duct. The pathology was first described in 1973 and has since been subdivided into pure, segmental and non-segmental forms. Whilst the radiological diagnosis of both AIP and paraduodenal pancreatitis remains challenging, the presentation will emphasise relevant imaging strategies as well as provide tips and tricks for accurate diagnosis.

Learning Objectives:

1. To become familiar with the clinical presentation and the radiological signs that may be observed in autoimmune pancreatitis.

2. To become familiar with the diagnostic criteria for the disease.

3. To learn about the strategies for managing doubtful clinical situations.

08:30 - 10:00

Room E2

\section{Oncologic Imaging}

RC 1616

\section{Management of bone metastases}

A-532 08:30

Chairman's introduction

H. Ahlström; Uppsala/SE (hakan.ah/strom@radiol.uu.se)

Bone scintigraphy is often used in combination with CT and clinical information for staging and therapy response evaluation of bone metastases. Magnetic resonance (MR) imaging is a technique that is known to be valuable both for detection of bone metastases and for evaluation of their response to treatment. Integrated positron emission tomography with computed tomography (PET/CT) combines the functional data from PET with anatomic information of $C T$ in single examination. $18 \mathrm{~F}$-fluoride is a non-specific PET tracer that has recently shown very high sensitivity for bone metastases. For accurate response evaluation, it is important to recognise not only changes in bone metastases, but also the effects of treatment on unaffected, normal parts of the bone marrow. These latter therapy-related benign changes could otherwise be misinterpreted as disseminated disease or vice versa. Despite the fact that the methods mentioned above, alone or in combination, have improved the assessment of bone metastases, they often remain non-specific. A specific diagnosis can in many cases only be achieved with a bone biopsy. This refresher course will give you an overview of the present imaging methods for detection and characterisation of bone lesions. Indications for, and how to perform, ablation and cementoplasty of bone metastases will be presented. Optimal algorithms for treatment follow-up of bone metastases and advantages and disadvantages with different imaging methods will be reviewed. Also, future development of diagnostic and therapeutic methods will be included in the presentations, e.g. potential role of emerging PET imaging probes for assessing bone malignancies.

Author Disclosure:

H. Ahlström: Research/Grant Support; Astrazeneca.

\section{A-533 08:35}

A. Diagnostic approach: the role of hybrid imaging

K. Herrmann; Würzburg/DE (Herrmann_K1@klinik.uni-wuerzburg.de)

This presentation will include a short review of the epidemiology of primary and metastatic bone disease, its classification and staging definitions. Then we discuss the role of radionuclide imaging in initial and subsequent treatment strategy considerations. Another aim is to provide guidelines for the appropriate use and applications of diagnostic radionuclide approaches for the assessment of bone diseases. The focus of this presentation will be on the role of radionuclide imaging in metastatic prostate, breast and lung cancer in the context of other available diagnostic imaging approaches. The ability of hybrid imaging technologies to achieve improved diagnostic accuracy will be emphasised and when appropriate the potential role of emerging PET imaging probes for assessing bone malignancies discussed. If feasible, the availability and effectiveness of radionuclide-based therapeutic approaches for malignant bone diseases will be reviewed.

Learning Objectives:

1. To learn about the standard MRI pulse sequence protocol for the assessment of bone metastases.

2. To understand the physiological and metabolic tumour properties which can be assessed with different PET tracers.

3. To become familiar with potential pitfalls and abnormalities that mimic metastases.

\section{A-534 08:58}

$B$. Therapeutic radiological approaches: from ablation to cementoplasty T. De Baere, L. Tselikas, F. Deschamps; Villejuif/FR (debaere@igr.fr)

Bone metastases occur in about $20 \%$ of cancer patients and can induce pain and/or a pathological fracture. Cementoplasty and percutaneous therma ablation techniques (radiofrequency, cryotherapy) can help in pain relief and consolidation. Cementoplasty is mostly performed in vertebral and acetabular metastases (even if other bony structures can be treated) with a clearly demonstrated analgesic effect. The role of cementoplasty versus radiotherapy and/or opioid is still debated. The proof of benefit in consolidation of bone by cementoplasty is not well demonstrated, and cementoplasty is still inadequate in structures subject to high mechanical stresses such as long bones. For such purpose, percutaneous deployment of orthopaedic hardware such as percutaneous screw fixation has been developed. Last, cementoplasty is not an antitumour treatment and cannot therefore be offered as a cure for bone metastasis. Thermal ablation is currently performed for pain palliation of bone metastases, in selected patients, namely in locations difficult to treat with cementoplasty. In a subset of bone metastases, thermal ablation can also be curative. Indeed, out of 88 curative intent thermal ablation of 122 bone metastases, the 1-year complete treatment rate was $67 \%$ [95\% Cl: $58 \%-75 \%$ ]. In multivariate analysis, the good prognostic factors for complete local treatment were: metachronous bone metastasis $(p=0.004)$, no cortical erosion $(p=0.01)$, small metastasis size $(p=0.001)$, no neurological structures in the vicinity $(p=0.002)$.

Learning Objectives:

1. To understand the clinical relevance and indications for ablation and cementoplasty of bone metastases.

2. To learn how to perform state-of-the-art ablation and cementoplasty.

3. To become familiar with possible complications of these interventions.

\section{A-535 09:21}

C. Assessment of tumour response: techniques and timing

G. Cook; London/UK (Gary.Cook@kcl.ac.uk)

Skeletal metastases are associated with significant morbidity and have high impact on health costs. Effective palliative treatments are available, but less than $50 \%$ of patients respond in terms of tumour markers, pain or quality of life and all treatments are associated with some toxicity. It is generally accepted that the current imaging techniques are inadequate for measuring treatment response in a clinically relevant time frame such that skeletal metastases are usually considered as non-measurable disease in clinical routine and trials. There is therefore a need for non-invasive, objective methods to evaluate treatment response of skeletal metastases at earlier time points to guide clinical care. In addition to imaging structural changes in bone (e.g. X-ray, CT), it is now possible to image a number of biological characteristics of bone metastases including tumour metabolism, tumour cellularity, and osteoblast and osteoclast activity. Methods currently available, or being actively investigated, include ${ }^{99 \mathrm{~m}} \mathrm{TC}-\mathrm{MDP}$ SPECT/CT, ${ }^{18} \mathrm{~F}$-fluoride PET/CT, ${ }^{18} \mathrm{~F}$-FDG $\mathrm{PET} / \mathrm{CT},{ }^{11} \mathrm{C} /{ }^{18} \mathrm{~F}$-choline PET/CT, RGD-SPECT/PET and DW-MRI. Some of the tracer methodologies will potentially be applicable for and complementary to PET/MRI. The timing of follow-up imaging after commencing treatment is still under investigation. It is likely that tumour-specific methodologies may reflect response/non-response earlier than bone-specific methods due to a prolonged flare in reactive bone in responding metastatic sites.

Learning Objectives:

1. To understand the techniques and quantitative parameters used for treatment response evaluation.

2. To learn the optimal timing for follow-up imaging after treatment.

3 . To become familiar with common pitfalls in the interpretation of posttreatment MRI and PET.

Panel discussion:

How to differentiate between bone metastases and chronic inflammatory or treatment-induced changes 09:44 
08:30 - 10:00

Room F1

\section{Special Focus Session}

\section{SF $16 c$}

\section{Structured reporting}

\section{A-536 08:30}

Chairman's introduction

A. Palko; Szeged/HU (palkoand@gmail.com)

Structured reporting is an opportunity and a challenge at the same time. Expert speakers of this session will present and discuss their views on the subject, contributing to the clear vision of the members of audience on this complicated subject, convincing us to take this technique as an opportunity to improve and standardise the quality of our reporting activities.

Session Objectives:

1. To become familiar with the concept of structured reporting.

2. To learn more about the various techniques and technical requirements of structured reporting.

3. To understand how structured reporting and decision support tools may assist the work of radiologists.

Author Disclosure:

A. Palko: Advisory Board; Euromedic.

\section{A-537 08:35}

Structured reporting: from clinical needs to technical challenges M. Fatehi; Tehran/IR (fatehi@irsr.org)

Even though structured reporting (SR) has been appreciated as one of the effective methods of electronic reporting, there are not that many real applications to facilitate utilisation of this technique in practice. Why it is so? The radiologists want a flexible tool to create clear, concise reports in a reasonably short time. Like all template-based reporting systems, the application must be tailored to preferences of multiple users in a radiology department. The report templates should also be flexible in terms of length to avoid inappropriately detailed reports. The final appearance of the report is critical for acceptance by clinicians. Accordingly, the technical challenges of development of SR systems may be grouped as: "user interface", "database structure", and "output file/document" problems. Flexibility to comply with different user preferences will lead to both user interface design and database structure challenges. One of the major technical challenges is the transformation of structured data to a final text to be acceptable and applicable for the referring physician. Also, exporting of reports to DICOM format using DICOM-SR concepts is still a challenge. Multi-media formats are becoming more and more popular specially in SR reports. The purpose of this presentation (based on examples from published and personally developed SR solutions) is to follow through the clinical needs of a radiologist to the technical challenges of implementing a clinical-, not a research-, structured reporting application.

Learning Objectives:

1. To become familiar with different approaches to structured reporting.

2. To understand the technical difficulties of developing a flexible structured reporting application.

3. To learn about structured reporting tools available for radiology practice.

\section{A-538 08:53}

Structured reporting in the US

C.E. Kahn; Milwaukee, WI/US (kahn@mcw.edu)

Effective communication of radiology results is critical to high-quality health care. To that end, the Radiological Society of North America (RSNA) has developed a library with more than 200 best-practice radiology reporting templates. The templates are freely available online (www.radreport.org) and have been accessed more than 750,000 times. The new IHE "Management of Radiology Reporting Templates" (MRRT) profile builds upon the web's Hypertext Markup Language version 5 (HTML5) to provide an international standard for describing and exchanging reporting templates. This presentation will describe the RSNA report template library, introduce the international MRRT standard for report templates, and describe opportunities for ESR members and affiliated societies to participate. The broad goals of this initiative are to improve the quality of radiology reports, increase the productivity of radiologists, promote the use of practice guidelines, and advance data-driven health care.
Learning Objectives:

1. To explore a library of 'best practices' radiology reporting templates. 2. To become familiar with international standards for radiology reporting templates.

3. To understand how structured reporting will promote the use of practice guidelines and will advance data-driven health-care.

\section{A-539 09:11 \\ Structured reporting in Europe: the ESR initiative O. Ratib; Geneva/CH}

The RSNA has initiated a new initiative called RadReport with the aim of helping promote standard reporting and improve reporting practices by creating a library of clear and consistent report templates (http://reportingwiki.rsna.org/). This project also supported in part by the National Institute of Biomedical Imaging and Bioengineering (NIBIB) has already gained the participation of numerous countries around the world, contributing with reports in different languages. These report templates are "structured" in the sense that they incorporate reusable knowledge, or meaning, to the clinical reporting process. They are free and not subject to licence restrictions on their reuse. Recently, the ESR eHealth and Informatics Subcommittee has decided to engage in promoting this initiative in Europe and supporting its development. Professor Charles Kahn from Medical College of Wisconsin, who is an active member of the RSNA working group on standard reporting, has joined the ESR subcommittee to help promote this initiative in Europe as well. There are already over 250 templates and the number of views is increasing every day (over 150,000 downloads so far). Most of the templates are in English, but there are several also in Arabic, Chinese, Turkish, Spanish, etc. There is also interest in translating the templates to other languages. In an effort to support this worldwide initiative, the ESR eHealth and Informatics Subcommittee decided to encourage National Societies to contribute to the submission and translation of templates and participate in the Subspecialty and Allied Sciences Societies to create reporting templates.

Learning Objectives:

1. To review the adoption of structured reporting in Europe.

2. To understand the challenges and differences in local regulation and practices.

3. To learn about the joint efforts of the ESR with RSNA and how to participate.

A-540 09:29

Implications for radiological practice and decision support tools

J.O. Barentsz; Nijmegen/NL (jelle.barentsz@radboudumc.nl)

Structured interpretation and reporting for detecting significant prostate cancer is crucial, as it allows comparing inter-observer interpretation variability, reduces this variability by stimulating discussion of the individual scores, enhances communication with the clinicians in a uniform way, facilitates quality assurance plus research, and in this way improves patient outcome. In this presentation, the PI-RADS scoring system will be shown and discussed, and fast effective structured reporting using computer software will be presented. Learning Objectives:

1. To understand why structured reporting is needed, and what the advantages are for clinical care.

2. To learn how structured reporting should be carried out

3. To become familiar with the content of a structured report.

4. To learn how computer programmes can help.

Author Disclosure:

J.O. Barentsz: Owner; The Radboudumc department of radiology has CE approval of PROCAD software. Other; The Radboudumc department of radiology owns and produces Sinerem/Combidex.

Panel discussion:

Structured reporting in the daily practice: friend or foe?

$09: 47$

08:30 - 10:00

Room G/H

Neuro

\section{RC 1611}

\section{Neuroimaging in dementia}

\author{
A-541 08:30 \\ Chairman's introduction \\ F. Barkhof; Amsterdam/NL (f.barkhof@vumc.nl)
}

In a rapidly ageing society, dementia prevalences are sharply increasing. Furthermore, knowledge about disease mechanisms is constantly evolving. A more nosologic approach in the workup of dementia is required to improve prognostication, patient management as well as select appropriate treatment 
Radiologists will be confronted with a marked increase in the number of requests for imaging. This requires a thorough knowledge of the relevant brain anatomy, choice of appropriate structural and molecular imaging modalities and interpretation of MRI and PET/SPECT in the most prevalent disorders in a structured fashion.

\section{A-542 08:35}

A. Anatomy of the limbic system

T.A. Yousry; London/UK (t.yousry@ucl.ac.uk)

The term "limbic" has been used in many different connotations. To understand the anatomy, we have to define the term first. The clearest definition stems from Broca: "Le grand lobe limbic". Defined as such, the limbic lobe is composed of 2 main structures: the limbic and the intralimbic gyri. 1. The limbic gyrus is composed of the subcallosoal gyrus anteriorly, followed in an arc by the cingulate gyrus, isthmus and parahippocampal gyrus. The latter is composed of 2 parts: i) a posterior narrow segment, the superior surface of which is called subiculum, and ii) a more voluminous anterior segment, also known as the piriform lobe. The latter consists of the anterior part of the uncus and the entorhinal area. 2. The intralimbic gyrus arches within the limbic gyrus. It is divided into 3 parts: i) anterior (prehippocampal rudiment); ii) superior (indusium griseum); and iii) inferior (hippocampus). The latter consists of 2 lamina rolled inside each other: the cornu ammonis and the dentate gyrus, with the cornu ammonis consisting of 4 neuronal fields (CA1-4). To understand the function, we have to understand the connections that define this function. The interplay with the insula is of particular importance. In summary, we will review the MRI characteristics of the components of the limbic lobe, their connections, and their function, using $1.5 \mathrm{~T}, 3 \mathrm{~T}$ and $9.4 \mathrm{~T}$ high field imaging. At the end of this lecture, you will know definition (s) of the limbic lobe, its major subdivisions, connections and functions.

Learning Objectives:

1. To understand the architecture of the limbic system.

2. To recognise the structure and connections of the hippocampus and medial temporal lobe.

3. To become familiar with the imaging characteristics at various field strengths and pulse-sequences.

Author Disclosure:

T.A. Yousry: Advisory Board; Biogen Idec. Author; T Yousry. Research/Grant Support; UCL/UCLH Biomedical Research Centre, GlaxoSmithKline, MS Society of Great Britain and Northern Ireland, MRC, Wellcome Trust, Stroke Association, British Heart Foundation, Novartis, Biogen Idec. Speaker; ESOR, King Abdullah Medical City.

\section{A-543 08:58}

\section{B. Structural MRI and CT: beyond exclusion}

M.P. Wattjes; Amsterdam/NL (m.wattjes@vumc.nl)

Structural neuroimaging is increasingly used in the diagnosis of neurodegenerative diseases. In a memory clinic setting, computed tomography (CT) and magnetic resonance imaging (MRI) have become the most important paraclinical diagnostic tools. Recent guidelines on the diagnosis and management of disorders associated with dementia state that neuroimaging should be performed at least once during the diagnostic workup. In the past, neuroimaging had been performed to exclude potentially treatable causes of dementia. However, this exclusionary approach of neuroimaging in the diagnosis of dementia has been replaced by an inclusionary approach driven by conclusive evidence that $\mathrm{CT}$ and MRI can substantially support the clinical diagnosis by the demonstration of a distinct atrophy pattern and the assessment of vascular (co)morbidity. For these purposes, the use of visual rating scales allows a fast and reproducible assessment of global/regional cortical atrophy and vascular white matter pathology. More recently, new imaging markers such as cerebral microbleeds and superficial siderosis haven been identified and linked to Alzheimer pathology. In addition, next to structura MRI, advanced and quantitative MRI methods have been introduced in the clinical setting. MRI perfusion techniques such as arterial spin labelling (ASL) demonstrated a diagnostic value in memory clinic patients, particularly in those showing no or inconclusive pathology on structural MRI.

Learning Objectives:

1. To become familiar with pathophysiology and clinical manifestations of common dementing disorders.

2. To learn how to define a comprehensive imaging protocol for dementia work-up.

3. To appreciate the role of modern imaging techniques for defining structural signs of dementia.

Author Disclosure:

M.P. Wattjes: Advisory Board; Biogen Idec. Board Member; European Radiology.

\author{
A-544 09:21 \\ C. Molecular imaging: when is it needed? \\ H. Urbach, P.T. Meyer; Freiburg/DE (horst.urbach@uniklinik-freiburg.de)
}

Cognitive decline is a clinical symptom in many neurodegenerative disorders. $\mathrm{MRI}$ is typically performed in the initial diagnostic workup of these patients. As a structural imaging modality, MRI is often unrevealing while several molecular imaging modalities show disease-associated alterations: ${ }^{18} \mathrm{~F}$-FDG-PET shows reduced glucose metabolism in neocortical association cortices, mainly in the posterior cingulate, precuneus and temporoparietal regions. Since glucose metabolism is normally higher in the posterior cingulate and precuneus, reduced glucose metabolism must be specifically sought for or highlighted by voxel-based analysis. ${ }^{18} \mathrm{~F}$-FDG-PET aids in the diagnosis of frontotemporal lobar degeneration (FTLD), cortico-basal degeneration (CBD) and dementia with Lewy bodies (DLB) by showing fronto-temporal, asymmetric fronto-parietal or temporo-parieto-occipital hypometabolism, respectively. Amyloid imaging with ${ }^{11} \mathrm{C}$-PiB and ${ }^{18} \mathrm{~F}$-labelled compounds is of great research interest. Although the first compound has recently been approved for clinical use in the USA and EU, availability is still limited. Nigrostriatal degeneration highlighted by ${ }^{18} \mathrm{~F}$ fluorodopa-PET or (dopamine transporter) ${ }^{123}$ I-FP-CIT-SPECT is present in Parkinson's disease (PD), CBD, DLB, multiple system atrophy (MSA), and progressive supranuclear paralysis (PSP), but absent in essential tremor or drug-induced parkinsonism. Patients with vascular parkinsonism may show focal defects to normal tracer uptake. In MSA and less so also CBD and PSP.

${ }^{123}$ l-iodobenzamide-SPECT shows a loss of striatal dopamine receptors, while cardiac ${ }^{123}$-metaiodobenzylguanidine uptake (i.e., post-ganglionic sympathetic innervation) is normal in contrast to Parkinson's disease. However, recent studies demonstrate that ${ }^{18} \mathrm{~F}$-FDG-PET is superior for the differential diagnosis of parkinsonism showing striatal and cerebellar hypometabolism in MSA and mainly frontal (mesial and premotor cortex), thalamic and brainstem hypometabolism in PSP.

Learning Objectives:

1. To become familiar with the molecular pathology of common dementing disorders.

2. To appreciate technical aspects and interpretation of SPECT and PET in dementia.

3. To understand the interpretation and significance of molecular imaging in the diagnosis of dementia.

Panel discussion:

How to integrate imaging, clinical and CSF findings into a dementia clinic? 09:44

08:30 - 10:00

Room I/K

\section{1619}

\section{Tailoring head and neck examinations to the clinical symptoms (part 2)}

Moderator:

P.-Y. Marcy; Nice/FR

A-545 08:30

\section{A. Horner's syndrome}

D.L. Reede; Brooklyn, NY/US (Deborah.Reede@downstate.edu)

Horner's syndrome (HS) occurs when there is interruption of the oculosympathetic pathway (OSP). This presentation reviews the anatomy of the OSP and clinical findings associated with lesions along this pathway. The imaging findings of lesions associated with HS at various levels of the OSP, classified as preganglionic HS (first- and second-order neuron HS) or postganglionic HS (third-order neuron HS) are demonstrated. Imaging and clinical findings of common lesions encountered at various levels of the OSP are presented to demonstrate the role of imaging in the evaluation of patients who present with HS. The clinical symptoms of HS may cause little if any functional impairment in most patients. However, since both benign and malignant processes are associated with $\mathrm{HS}$, a thorough clinical evaluation is required. Once a lesion is localised clinically within the OSP by a combination of physical examination and pharmacological testing, the radiologic examination can be appropriately tailored.

Learning Objectives:

1. To understand the importance of this syndrome as well as its common and uncommon causes.

2. To become familiar with crucial anatomical landmarks.

3. To learn about the imaging findings. 


\section{A-546 09:00}

\section{B. Dysphagia}

P. Pokieser; Vienna/AT (peter.pokieser@meduniwien.ac.at)

Dynamic imaging of swallowing from the mouth to the duodenum is the only clinical test providing a fast and accurate diagnostic overview of the upper $\mathrm{GI}$ function. Swallowing disorders are divided into those with dysphagia that means problems during eating or drinking, with suspicion of aspiration as an important subgroup and those with sensations like the globus syndrome or noncardiac retrosternal pain. Oropharyngeal dysphagia in patients with neuromuscular diseases or postoperative conditions can be evaluated precisely and within the same session as oesophageal motility disorders, stenoses or postoperative problems are detected and in a complementary fashion to endoscopy, $\mathrm{pH}$ monitoring and manometry. The exclusive ability of dynamic imaging of swallowing is to diagnose combined disorders of the pharynx and the oesophagus, to detect and localise subtle and multiple benign stenoses of the upper GI tract and to combine visualisation of pathophysiology and bolus transport as well. Radiologic evaluation concentrates on 7 functional units, which represent the visible "moving areas" of deglutition, where evident pathologic conditions can be found: 1) oral cavity and tongue, 2) soft palate, 3) epiglottis, 4) hyoid and larynx, 5) pharyngeal constrictors, 6) pharyngoesophageal segment, 7) oesophagus and stomach. This lecture intends to explain the common causes of dysphagia, the approach to a tailored examination and the use of an advanced imaging technique. Further, the basics of taking the swallowing history and the structure of reporting swallowing disorders along the seven functional units will be provided.

Learning Objectives:

1. To understand the common causes of dysphagia.

2. To learn how to tailor radiological examinations to the symptoms

3. To become familiar with advanced imaging techniques helpful in the evaluation of swallowing difficulties.

\section{A-547 09:30}

\section{Hoarseness}

F.A. Pameijer; Utrecht/NL (f.a.pameijer@umcutrecht.nl)

Hoarseness is defined as a rough or noisy quality of voice. The possible causes of hoarseness are manifold, ranging from benign diseases such as the common cold to malignant tumours. Therefore, hoarseness as a symptom should always be taken seriously. Evaluation of a patient with hoarseness by a head and neck specialist will always starts with a history and a physical examination including laryngoscopy. After these examinations, a diagnosis can be established in most patients without additional imaging. The aetiologies of hoarseness are: 1) inflammation/infection, 2) trauma, 3) neoplasms, 4) others (including medical conditions, e.g. hypothyroidism) and 5) vocal cord paralysis This presentation will focus on imaging of vocal cord paralysis. Vocal cord paralysis should not be considered a diagnosis, but as a symptom of (possible) underlying disease. When vocal cord palsy is discovered, this is frequently a reason to perform cross-sectional imaging. The radiological workup needs to include the full course of the vagus nerve. Moreover, reporting radiologists need to be able to recognise the radiological signs of vocal cord palsy in the absence of hoarseness, since many patients with cord palsy are asymptomatic (up to $30 \%$ ). The anatomy of the vagus nerve and its laryngeal branches that innervate the intrinsic muscles of the larynx will be discussed. Then the (expected) course of the vagus nerve and recurrent laryngeal nerve will be shown. Finally, radiological signs of vocal cord palsy will be discussed using examples from daily practice.

Learning Objectives:

1. To understand the underlying causes of this clinical symptom and their importance.

2. To learn what to look for and which imaging method to use

3. To become familiar with differential diagnosis and the limitations of diagnostic imaging studies. Room L/M

\section{Special Focus Session}

\section{SF 16d}

\section{Imaging follow-up of vascular interventions}

\section{A-548 08:30}

Chairman's introduction

M. Szczerbo-Trojanowska; Lublin/PL (mst@radiology.com.pl)

In contemporary medicine, endovascular techniques often replace conventional methods of treatment, including surgery, in the management of an increasing range of diseases. This requires development of specific methods assessing the effectiveness of treatment and able to detect complications that may be much different from those occurring in conventional therapy. This is first of all seen in the endovascular treatment of patients suffering from vascular lesions and neoplastic diseases. Commonly, there is a high initial technical success of endovascular treatment, but the durability is not always satisfactory and needs to be further examined. Endovascular treatment is bound to the risk of specific complications, often without clinical symptoms. Therefore, there is a need to use imaging follow-up of these patients. Interventional radiologists are aware of the importance of those issues and should play an important role in developing and deciding about the follow-up scheme and choice of the best imaging methods for these patients. Besides indepth knowledge of the treatment, radiologists are also familiar with the possible complications and can offer an optimal, minimally invasive and cost effective diagnosis and treatment.

Session Objectives:

1. To learn about the long-term results of vascular interventions.

2. To become aware of the complications in vascular interventions in general.

3. To become familiar with the endovascular treatment of complications

following vascular interventions.

\section{A-549 08:35}

Imaging follow-up and treatment of complications of angioplasty and stenting

R. Morgan; London/UK (robert.morgan@stgeorges.nhs.uk)

The potential imaging methods for follow-up after angioplasty (PTA) and/or stenting include Doppler ultrasound, CTA and MRA. Although excellent images can be obtained by CTA and MRA, the drawbacks of these studies for routine follow-up are obvious. (access to CT and MR equipment, cost, contrast medium, ionising radiation, etc). In the UK, the most common imaging method is duplex ultrasound. There is no established role for the use of ultrasound contrast agents. Ultrasound is freely available in the majority of centres, is inexpensive and complication free. Follow-up by ultrasound may be undertaken by radiologists, although more usually by technologists in vascular laboratories. In practice, few patients are followed up by any imaging study at all unless they develop symptoms. The main category of patients who undergo some form of imaging follow-up after PTA/stenting are patients with surgical bypass grafts, who are usually routinely followed up whether they have undergone angioplasty/stenting or not. In view of the fact that this is a lecture on follow-up after intervention, procedural complications are excluded from the discussion. The complications of angioplasty and stenting are similar. The main early complications are restenosis or reocclusion. Other early complications are related to the arterial puncture site and include haematoma, dissection, occlusion and pseudoaneurysms. The main late complications are restenosis and reocclusion. Treatment of restenosis and reocclusion usually involves additional angioplasty or stents using conventional or drug-eluting balloons or stents. Cutting balloons, atherectomy and cryotherapy have a controversial role based on limited evidence.

Learning Objectives:

1. To learn about selection of imaging methods used for follow-up.

2. To become aware of the early and delayed complications.

3. To become familiar with endovascular methods used to treat complications.

\section{A-550 08:58}

Imaging methods for postprocedural evaluation and follow-up of EVAR E. Brountzos; Athens/GR (ebrountz@med.uoa.gr)

Imaging after EVAR primarily focuses on the prevention of aneurysm rupture. Other late complications include limb occlusion, graft migration, graft infection, and endoleaks. CT angiography (CTA) at standardised intervals has been the modality of choice. Recently, concerns regarding radiation exposure, contrastinduced nephropathy and increasing costs advocate for less CTA imaging and/or for changing the surveillance protocols. Ultrasonography accurately measures aneurysm size, while CEUS detects endoleaks with high sensitivity. No endoleaks requiring interventions will be missed by ultrasound. Ultrasound only can be used in patients with appropriate body habitus, without EVARrelated problems (as demonstrated on earlier CTAs), or in patients with shrinking or stable aneurysms. Use of duplex only will not lead to an increased occurrence of adverse events such as rupture, graft migration or limb occlusion, and can lead to a significant reduction of costs related to EVAR follow- up by $29 \%$. The presence of endoleak at 30 days is associated with an increase in secondary procedures; absence of early endoleak is associated with a reduced risk for subsequent aneurysm-related morbidity. Patients with aneurysm sacs that are stable or shrinking at 1 year and no evidence of endoleak on a CT scan performed at 1 year do not require regular CT scanning anymore and can be imaged with ultrasonography.

Learning Objectives:

1. To learn about imaging protocols used for follow-up.

2. To become aware of complications of EVAR.

3. To become familiar with methods used to treat complications. 
A-551 09:21

Imaging follow-up of oncologic patients after embolisation procedures J.I. Bilbao; Pamplona/ES (jibilbao@unav.es)

The term "embolisation" groups different procedures in which imaging guidance is fundamental for both performing the procedure and for the early detection of complications. It has been demonstrated by several studies that "tumoural response" is tightly associated with survival, thus it is of major interest to measure accurately how the tumour is modified by the treatment. The "World Health Organisation" - WHO - first published the objective criteria for the evaluation of response. These criteria were slightly modified in 2000 and named as "Response Evaluation Criteria for Solid Tumors"- RECIST. Accordingly, "complete response" (CR) corresponds to the disappearance of the tumour, "partial response" (PR) is defined when there is a decrease in the tumour size equal to or higher than $30 \%$ and "progressive disease" (PD) is applied when there is an increase equal to or higher than $20 \%$. "Stabilisation of the disease" (SD) corresponds to measurements in between $>30 \%(P R)$ and $<20 \%$ (PD). Tumours (until five nodules in the same viscera), no matter if necrotic or viable, were evaluated with one measure (its largest diameter). A new version ("1.1 RECIST) has introduced modifications, among which is the number of nodules ( 2 instead of 5 ) that needs to be evaluated. In some circumstances, when morphologic criteria (RECIST and WHO) fail to predict the outcome, then new functional criteria have been recently established. "mRECIST" (which only measures the tumour that uptakes contrast) is an accurate classification for HCC. New methods "beyond RECIST" have been established for some specific treatments such as targeted therapies.

Learning Objectives:

1. To learn how to assess results

2. To understand how to diagnose and treat early complications.

3. To learn how to manage recurrent oncological bleeding.

Author Disclosure:

J.I. Bilbao: Speaker; Sirtex Medical Europe.

Panel discussion:

Cost-effectiveness of imaging follow-up after endovascula interventions 09:44

08:30 - 10:00 Room N/O

\section{Special Focus Session}

\section{SF $16 e$}

\section{Elastography as a new tool}

\section{A-552 08:30}

Chairman's introduction

A. Wieczorek; Lublin/PL (rtg@dsk.lublin.pl)

In this session, the audience will have a comprehensive overview of the elastography, its principles and current state-of-the-art clinical applications. The session will deliver an overview of currently available methods of both ultrasound elastography (USE) and magnetic resonance elastography (MRE), their physical principles, technical and equipment requirements as well as the influence of various factors for obtained results. Following this session, the audience will have insight into clinical applications of elastography in liver, prostate and brain diagnostics. The strengths and weaknesses of elastographic modalities will be compared and discussed under consideration of more established imaging modalities. The session will present potentia developments of USE and MRE and possible future clinical applications. Panel discussion will aim at defining the place of elastography in current and future diagnostic algorithms

Session Objectives:

1. To learn about the basic idea of elastography.

2. To understand the methods of acquiring elastographic images.

3. To learn about the common indications.

Author Disclosure:

A. Wieczorek: Speaker; Speaker supported by B-K Medical Workshops and Lectures.

\section{A-553 08:35}

Principles of US and MR elastography

I. Sack; Berlin/DE (ingolf.sack@charite.de)

The well-known sensitivity of the palpating hand for detecting lesions is related to the incredibly wide range of values of the shear modulus in the body, spanning over eight orders of magnitude. Elastography was invented to exploit this high variability of constants by introducing the shear modulus into the image contrast of medical ultrasound or magnetic resonance imaging. Today, both ultrasound elastography (USE) and magnetic resonance elastography
(MRE) are in clinical use, predominantly for staging liver fibrosis or discriminating tumours in the breast, liver, and prostate. The talk reviews the physical principles, technical requirements and current methods of both USE and MRE. Furthermore, the strengths and weaknesses of elastographic modalities are compared and discussed under consideration of more established imaging modalities. An outlook of elastography is given, highlighting the real-time feasibility of USE and the sensitivity of MRE to tissue pressure.

Learning Objectives:

1. To learn what elastography is good for and why we need it.

2. To understand the advantages and disadvantages of ultrasound-based elastography (USE) compared with MR elastography (MRE)

3. To become familiar with the different methods in both USE and MRE. 4. To learn about the clinical applications.

\section{A-554 08:53}

US elastography of the prostate

F. Aigner; Innsbruck/AT (friedrich.aigner@uki.at)

Suspicion of prostate cancer (PCa) exists in the case of elevated PSA serum values and/or suspicious findings on digital rectal examination (DRE). Systematic biopsy (SB) will be done to confirm or rule out PCa. Unfortunately, an elevation of PSA serum values often is caused by benign changes, e.g. prostatitis or adenomyomatosis and not every cancer is palpable on DRE. Furthermore, SB may miss clinically significant disease in up to $35 \%$. Therefore, a more targeted approach would be desirable and imaging of $\mathrm{PCa}$ is currently under strong effort. One possibility for visualisation of $\mathrm{PCa}$ is the representation of tissue elasticity. Usually, cancers have higher cell and vesse density than the normal surrounding tissue and therefore are associated with increased stiffness. In contrast to DRE, where only the posterior parts of the prostate can be reached, RTE does not have this problem, since all anatomical regions can be evaluated. Furthermore, this noninvasive technique is time-and cost-effective and targeted biopsy or focal therapy of the prostate can be done under real-time conditions. Another important issue is that RTE can be performed by both urologists and radiologists. Nevertheless, RTE is of limited value in the detection of small cancer lesions and there may be problems in visualising $\mathrm{PCa}$ with predominantly Gleason pattern 3 . The lack of data about inter- and intraobserver variability and of multicentre studies (now in process) are probably the reasons why RTE has not been included in the guidelines of urological societies so far.

Learning Objectives:

1. To understand histopathological patterns of prostate cancer $(\mathrm{PCa})$ in correlation with imaging

2. To appreciate the advantages of elastography compared with systematic biopsy.

3. To become familiar with the limitations and potentials of elastography for $\mathrm{PCa}$ detection.

\section{A-555 09:11}

MR elastography of the brain

J.-T. Wuerfel; Göttingen/DE (jens.wuerfel@charite.de)

Cerebral tissue structure is altered in many neurodegenerative diseases, but also during physiological processes like maturation or ageing. Structural changes directly affect the mechanical tissue properties. Magnetic resonance elastography (MRE) is an imaging technique capable of assessing biomechanical brain parenchymal properties non-invasively. Viscoelasticity can be quantified by analysing the propagation of mechanically elicited shear waves in the investigated tissue. Thus, MRE could be a helpful tool to detect physiological or pathological processes influencing the cerebral tissue integrity. Learning Objectives:

1. To appreciate the advantages and limitations of a virtual palpation of the brain.

2. To become familiar with mechanical wave induction and detection in cerebral measurements.

3. To learn about current and potential clinical applications.

4. To learn about the relationship between microscopic and macroscopic, as well as focal and globally diffuse biomechanical brain parenchymal alterations. Author Disclosure:

J. Wuerfel: Advisory Board; Novartis Advisory Board.

A-556 09:29

US and MR elastography of the liver: assessment of liver stiffness with elastography techniques

S.F. Bensamoun; Compiegne/FR (sabine.bensamoun@utc.fr)

Ultrasound (US) and magnetic resonance elastography (MRE) techniques are used in clinical practice for liver exam. These new techniques enable a noninvasive follow-up of treatment and therapy. The objective of this talk is to show the different steps of US and MR elastography methods performed by the radiologist to diagnose the level of fibrosis. Briefly, elastography techniques 
are based on the generation of shear waves inside the liver tissue with specific MR liver drivers and ultrasound probes. Subsequently, phase images revealing the displacement of the waves will be investigated for healthy and pathological (alcoholic fibrosis) livers. Moreover, the development of phantoms mimicking the stiffness of biological soft tissues (liver, muscle, ...) will be also presented. The viscoelastic (elasticity and viscosity) properties of healthy and fibrotic livers were measured, and the relevance of these parameters, used as a liver diagnostic marker, was analysed. In parallel, an increase of the wavelength as a function of the stiffness was obtained for the developed set of phantoms. The characterisation of the elastic properties is an excellent marker to differentiate minor and major fibrosis. A summary of the advantages and disadvantages of US and MR elastography techniques will be presented.

Learning Objectives:

1. To learn how MR elastography and elastography techniques function.

2. To understand how to evaluate the liver with elastography.

3. To appreciate the diagnostic value of elasticity and viscosity.

4. To become familiar with US and MR elastographic imaging techniques for non-invasive follow-up of treatment and therapy.

Panel discussion:

Elastography - a revolutionary technique or a waste of time?

09:47

08:30 - 10:00

Conf. Room M3

Oncologic Imaging: Follow-up of Systemic and Local Therapies

CC 1618

\section{Follow-up of local treatments of breast} cancer

Moderator:

M.H. Fuchsjäger; Graz/AT

\section{A-557 08:30}

A. MRI-guided HIFU therapies in the breast

M. Sklair-Levy; Tel Aviv/IL (mirisklair@gmail.com)

Magnetic resonance high-intensity focused ultrasound is an innovative, noninvasive tumour ablation technique. MRI and focused ultrasound are combined allowing real-time anatomic guidance and temperature mapping during treatment. MRI-g FUS treatment is performed under local anaesthesia. Imaging: T2-W to define the tumour and T1-W with contrast to verify the efficacy after MRgFUS. MRI-guided FUS ablation of breast cancer studies demonstrates variable results with complete tumour necrosis in $20 \%$ to $100 \%$. MRI-guided FUS procedure is well tolerated, with only a minor complication such as skin burn. Several issues need to be further investigated for successful cancer treatment with MR-guided focused US, including patient selection, definition of treatment margins and optimal transducer technology. In summary, FUS ablation has the potential to become an important modality for noninvasive image-guided treatment of localised breast cancer.

Learning Objectives:

1. To learn about HIFU in breast tumours.

2. To become familiar with the post-HIFU aspects of various types of imaging

\section{A-558 09:00}

B. Pre-imaging and post-imaging appearance of breast lesion excision system (BLES) lesions

S.D. Allen; Sutton/UK (steven.allen@rmh.nhs.uk)

The breast lesion excision system (BLES) has evolved as a breast radiological technology over the last decade and is now in widespread use in Europe and across the world. It was designed as a large biopsy device, but more recently due to its unique capability to obtain a single large breast tissue specimen in only a few seconds by utilising a radiofrequency cutting and cauterising wave, it has increasingly been explored in the therapeutic setting. It is easy to use under ultrasound or mammographic guidance with procedures taking a similar length of time to that of a vacuum biopsy, and with patient preparation and anaesthetic essentially identical. The technical aspects of performing these procedures will be detailed as well as its use specifically to perform excisional biopsies. This is limited by patient and lesional factors, all of which will be discussed in more detail. Following a BLES, the post-procedure appearances need to be considered so that follow-up imaging can be interpreted accurately. The risks and complications of this procedure are outlined as well as a discussion made of the latest papers in this field that may suggest future applications and developments.

Learning Objectives:

1. To understand the mechanism of the BLES technique.

2. To become familiar with the post-BLES aspects of various types of imaging.

\author{
A-559 09:30 \\ C. Common features and pitfalls in imaging the treated breast \\ J. Camps Herrero; Valencia/ES (juliacamps@gmail.com)
}

During the past few years, it has been shown that there is not a single disease entity called "breast cancer". There are different subtypes that entail diverse recurrence risks. This is the first issue to take into account, and patients will be stratified accordingly before any follow-up is planned. Imaging findings in a patient treated for breast cancer will depend on the type of treatment: breast conserving therapy (BCT), mastectomy (and all the reconstructive techniques), radiotherapy and minimally invasive techniques. To differentiate between fat necrosis and other common post-treatment changes from relapse, it is important to know the timeline when all these changes take place and also schedule the follow-up imaging procedures accordingly. Other important issues to take into account are the limitations and indications of the different modalities (mammography, ultrasound and MRI). Although ultrasound and mammography have traditionally been used in the follow-up of these patients, MRI is being used more and more often due to its superior multiplanar capabilities and the functional information not supplied by the other techniques. Learning Objectives:

1. To learn about the postsurgical and post-radiation therapies aspects of the breast and their timing.

2. To learn how to diagnose a recurrence in the treated breast and its differential diagnoses.

3. To become familiar with the imaging findings of post-ablation (RFA) of breast.

Author Disclosure:

J. Camps Herrero: Advisory Board; Bayer. Speaker; Bayer.

08:30 - 10:00

Board Room A

Vascular

\section{RC 1615}

\section{Venous thrombosis: imaging and management}

\section{A-560 08:30 \\ Chairman's introduction \\ O. Pellerin; Paris/FR (olivier@pellerin.as)}

Venous thrombosis is a serious health issue. Two clinical presentations exist and both are under the scope of the radiologist. Despite a high efficacy rate of anticoagulation, treatment failure or complication occurs. The prevention of pulmonary embolism and the management of proximal vein occlusion could be done by the interventional radiologist (vena cava filtration, ilio-femoral venous network recanalisation). The chronic phase of venous thrombosis is responsible for post-thrombotic syndrome. This complication needs to be known by the radiology community, because of its insidious presentation and the dramatic results of recanalisation when indicated. The goal of this session is to point out the clinical importance of venous thrombosis imaging, by showing how to diagnose acute or chronic presentation, and to show the rationale of the technique and results of interventional radiologic procedure for vena cava filtration, and acute and chronic vein occlusion.

Author Disclosure:

O. Pellerin: Advisory Board; Siemens Interventional Oncology Advisory Board. Employee; Assistance Publique - Hôpitaux de Paris.

\section{A-561 08:35 \\ A. Ultrasound, CT and MR venography \\ M. Das; Maastricht/NL (m.das@mumc.nl)}

Blood clot formation in the veins is referred to as venous thrombosis (VT). VT is most common in the deep veins of the legs and pelvis. If the thrombus dislocates to the pulmonary arteries this is referred to as pulmonary embolism $(P E)$. The combination of deep vein thrombosis (DVT) and PE is referred to as venous thromboembolism (VTE). The diagnosis of DVT and VTE is essential, as VTE is potentially fatal. Furthermore, undiagnosed, non-fatal DVT may result in negative long-term pathologies (e.g. post-thrombotic syndrome). Major risk factors for the development of VTE are thrombophilia, history of DVT, age $>60$, surgery, obesity, long travel, cancer, immobility and pregnancy. In addition, underlying congenital abnormalities or compression syndromes (MayThurner syndrome) are also important to diagnose. Diagnosis of VTE is not always easy. Primary diagnosis includes clinical (Wells Score) and lab testing (D-Dimer). First line imaging testing is done by leg ultrasound (US). If PE is suspected CT is indicated immediately. CT of the pulmonary arteries (CTPA) may be combined with indirect CT venography (CTV). The question of when to combine CTPA and CTV still remains. A favourable approach is to add CTV in 
a negative CTPA. Recently MR venography (MRV) is emerging as comprehensive imaging tool especially in patients with chronic DVT to assess the extent and underlying causes. In this respect imaging without radiation dose with high spatial delineation of vascular abnormalities facilitates intervention for thrombolysis or interventional therapy including recanalisation and stenting.

Learning Objectives:

1. To learn about the pros and cons of US compared to CTV and MRV

2. To become familiar with imaging parameters, contrast media protocols, flow dependent techniques and flow independent techniques.

3. To understand pitfalls and artefacts characteristic of the respective imaging modalities.

Author Disclosure:

M. Das: Consultant; Bayer Healthcare, Siemens Healthcare. Research/Grant Support; Institutional Grant from Bayer, Siemens.

\section{A-562 08:58}

B. Epidemiology, prevention and treatment

C. Binkert; Winterthur/CH (christoph.binkert@ksw.ch)

1. Epidemiology: acute deep vein thrombosis (DVT) is a common disease affecting 70-100 patients per 100'000 per year. A feared complication of DVT is pulmonary embolism (PE). 2. Prevention. a) Medication: low molecular heparin $(\mathrm{LMH})$ is widely used for immobilised patients to minimise the risk of DVT. Alternatively, intravenous unfractionated heparin is used in situations when a quick reversal is necessary. b) Non-medication: early mobilisation has been widely adopted to activate the muscle pump. If early mobilisation is not possible, compression stockings and/or pneumatic compression boots are applied to better empty the veins. 3. Treatment: a) Anticoagulation: the main goal of anticoagulation is to prevent progression and recurrence of DVT. Typically, anticoagulation is started with $\mathrm{LMH}$, followed by vitamin $\mathrm{K}$ antagonists (Coumadin). The first DVT is generally treated for 3-6 months; recurrent DVT for 12 months. b) Compression therapy: compression stockings ameliorate pain and leg swelling of acute DVT. In addition, the development of a post-thrombotic syndrome (PTS) should be reduced. c) Thrombolysis: the main goal of thrombus removal is prevention of PTS. In addition, thrombolysis is used for severe ilio-femoral DVT, especially with phlegmasia coerulea dolens. There are different ways of thrombolysis: systemic thrombolysis was abandoned because of high bleeding complications. Catheter-based thrombolysis has shown to reduce the bleeding risk, but it is quite time and resource consuming. The latest development is pharmaco-mechanical catheter thrombus removal which combines thrombolytic drugs with a mechanical maceration. Recent studies (CaVent,TORPEDO) have shown a reduction of PTS compared to anticoagulation.

Learning Objectives:

1. To learn about the epidemiology and clinical presentation of venous disease

2. To become familiar with effective prevention measures.

3. To learn about the different endovascular treatment options.

Author Disclosure:

C. Binkert: Speaker; Cook, Covidien

\section{A-563 09:21}

C. IVC filters

G.J. O'Sullivan; Galway/IE (gerard.osullivan2@hse.ie)

The use of inferior vena cava filters (IVCFs) is a controversial method for the prevention of pulmonary emboli. There are large variations in their usage; often with ten to one hundredfold differences in placement between different countries. Ideally, IVCF usage is based on sensible protocols derived from clinical experience and trials. In practice, this is less frequently the case. Indications, contraindications and questionable indications (mainly during venous thrombolysis) will be discussed. Familiarity with ultrasound guidance will be stressed. The key technical steps in the insertion and removal of IVCFs will be discussed.

Learning Objectives:

1. To learn the rationale for IVC filter placement.

2. To become familiar with the key technical steps and learn how to avoid complications during IVC filter placement.

3. To become familiar with the key technical steps and learn how to avoid complications during IVC filter retrieval.

Author Disclosure:

G.J. O'Sullivan: Consultant; Covidien Healthcare, Cook Medical, Marvao

Medical, Optimed, Veniti Medical, Bard, Medtronic, Boston Scientific.

Panel discussion:

Current indications for interventional venous thrombosis

treatment 09:44

\section{8:30 - 10:00 \\ Room P \\ Computer Applications \\ RC 1605 \\ Improving workflow efficiency and quality}

\author{
A-564 08:30 \\ Chairman's introduction \\ D. Caramella; Pisa/lT (davide.caramella@med.unipi.it)
}

The question that this refresher course is aimed to answer is whether novel IT tools may actually help in improving quality and workflow efficiency in daily radiological practice. In fact, since the early installations of PACS, IT tools have often been considered as mere productivity tools rather than enabling technologies for fostering quality in medical care. The three distinguished lecturers in this course will address the following topics: improving quality and efficiency of computerised order entry through decision support, improving quality and efficiency of reporting through structure and templates, and improving quality and efficiency of dose management through exchange between modalities and registries. They will cover all aspects of the radiological workflow: from the selection of the most appropriate diagnostic procedure, to the efficient reporting of diagnostic and non-diagnostic data (such as radiation dose information and contrast media information). They will demonstrate how newly adapted IT tools may provide assistance throughout the radiological workflow, with potentially enormous gains in terms of patients' safety and total quality management.

Session Objectives:

1. To appreciate the need for IT tools to ensure quality control.

2. To understand how to collect data concerning radiation dose.

3. To learn about the integration of contrast media injectors into PACS.

\section{A-565 08:35}

A. Improving the quality and efficiency of computerised order entry through decision support

P. Mildenberger; Mainz/DE (peter.mildenberger@unimedizin-mainz.de)

Electronic communication of requests for radiological procedures is a logical and relevant extension to integrated information systems in health care. This communication of orders and the entry (OE) into RIS improve the workflows. But, quality improvements require additional efforts to link order-entry-solutions with health knowledge for ordering the appropriate imaging procedure. These Clinical Decision Support (CDS) tools should be based on generally accepted and implemented criteria, e.g. evidence-based medicine. It is known that the acceptance of such systems, if successful and efficient implementations are given, is very good. IT concepts for CDS are well known and standards for classifications are available, but semantic interoperability is still a developing area. Actually, clinical information systems provide different levels of integration of OE and CDS. Further developments could be an implementation of interoperability profiles and the representation of CDS knowledge in webbased services of non-commercial organisations.

Learning Objectives:

1. To understand the relationship between decision support tools and evidence-based medicine.

2. To learn how decision support tools can be implemented for requesting radiological studies.

3. To appreciate the potential effects of decision support tools on workflow efficiency.

\section{A-566 08:58}

B. Improving quality and efficiency of reporting through structure and templates

E. Neri; Pisa/IT (emanueleneri1@gmail.com)

The report represents the final stage of the diagnostic imaging process, for which the radiologist is responsible. This process is driven by an accurate selection of the diagnostic tests, based on the clinical question. Therefore, the report should be an expression of this path, depending on the question asked by the clinician. The structure of a radiology report for the same clinical problem and the same method of investigation varies from country to country and between different radiologists. This diversity can be a problem in the globa world where a patient can have imaging tests from different places with different reporting languages, or when patient data is transmitted and shared between different centres. There is, therefore, the need to standardise the report as much as possible; finalising its structure and contents to a specific clinical problem, and possibly using a standard terminology. An attempt to solve this problem is in progress with the joint initiative on structured report ESR- RSNA (http://www.rsna.org/Reporting Initiative.aspx), aimed at improving reporting practises by creating a library of clear and consistent report 
templates. The main expected benefits of structured reporting templates are report uniformity and improvement of communication between referring physicians. Literature evidences that structured reporting can also quicken report turnaround, and reduce costs and lexical errors; thereby improving the quality and safety of radiological practise.

Learning Objectives:

1. To learn about clinical requirements for structured reports.

2. To become familiar with the IT requirements for report templates.

3. To appreciate the potential for generating data for evidence-based radiology.

\section{A-567 09:21}

C. Improving quality and efficiency of dose management trough exchange between modalities and registries

E. Vaño; Madrid/ES (eliseov@med.ucm.es)

The European directive on Medical Exposures requires the assessment and evaluation of patient doses, especially in procedures involving high doses to the patient. In the current draft of the new directive on Basic Safety Standards, some requirements on patient dosimetry in diagnostic and interventional radiology have been reinforced: X-ray systems should provide dosimetric information with the capability of being transferred to the examination report (for all CT and interventional systems). Diagnostic reference levels (DRLs) shall be reviewed regularly. These requirements will push the industry and the users to develop better strategies to evaluate patient doses, transfer these values to patient reports (contributing to the patient exposure tracking system) and also use available software to process these dosimetric data and perform some automatic analysis. This analysis should: a) include periodic calibration factors for patient dose quantities, b) include automatic detection of high dose values (especially relevant for interventional procedures), c) include statistical analysis to update DRLs and compare with the existing ones and d) suggest corrective actions to fulfil the quality assurance programmes and the clinical audit requirements. DICOM radiation dose structured reports represent a significant advantage, but more efforts will be necessary for the automatic process of the relevant data contained in the report to verify that the radiological risk is acceptable and to suggest, if appropriate, corrective actions to improve clinical practice. Without these last steps, patient dosimetry efforts and European regulations for radiation safety could only have a moderate impact.

Learning Objectives:

1. To learn about current European regulatory requirements and standards in patient dosimetry.

2. To become familiar with the dose-reporting evolution and structured dose reporting.

3. To appreciate the potential for dose analysis and reporting, as well as future registries.

Panel discussion:

Will novel IT tools really improve quality and efficiency in daily

radiological practice?

09:44

$10: 30-12: 00$ Room A

\section{Interactive Teaching Session}

\section{$E^{3} 1720$}

\section{Changes of the gastrointestinal tract after treatment}

\section{A-568 10:30}

A. Gastrointestinal tumours

A. Laghini; Latina/IT (andrea.laghi@uniroma1.it)

Gastrointestinal tumors include a variety of lesions, with the most frequent being adenocarcinoma of the small and large intestine, small bowel lymphoma, neuroendocrine tumours (NETs) and gastrointestinal stromal tumours (GISTs). According to histology, location and initial imaging staging, those lesions may require completely different therapies: surgery alone, adjuvant chemotherapy ( $\mathrm{CHT}$ ), neoadjuvant $\mathrm{CHT}$ followed by surgery or a combination of neoadjuvant chemoradiotherapy (CRT) followed by surgery. After treatment, imaging follow-up is mandatory. The most common post-operative findings after small bowel or colonic resections will be presented, together with clues for early detection of recurrence. In those cases which underwent adjuvant $\mathrm{CHT}$, imaging findings and diagnostic criteria related to the use of either cytotoxic or cytostatic drugs will be shown, to facilitate the interpretation of radiologists in assessing response to therapy. Finally, tissue changes following neoadjuvant
CRT will be discussed, particularly in rectal cancer. The possibilities of different imaging modalities in assessing either complete or partial response to therapy will be presented, with a critical analysis of different imaging findings. Learning Objectives:

1. To become familiar with common findings after medical treatment of gastrointestinal tumours.

2. To become familiar with changes after radiotherapy for gastrointestinal tumours.

3. To understand the changes which occur after surgery for gastrointestinal tumours.

Author Disclosure

A. Laghi: Speaker; Bracco, General Electric Healthcare, Im3d.

A-569 11:15

B. Non-tumoural intestinal diseases

J. Rimola; Barcelona/ES (jrimola@clinic.ub.es)

Unlike infectious enteritis, in patients with Crohn's disease (CD) the changes in clinical activity have poor correlation with changes in inflammatory lesions after medical treatment. Mucosal healing $(\mathrm{MH})$ and improvement in patient's symptomatology are usually considered the main objectives of medical treatment. However, improvement in clinical symptoms is not always associated with mucosal healing and the persistence of severe inflammatory lesions augurs a more aggressive disease course in terms of flare-ups, hospitalisation requirements and the need for surgery. Endoscopy is the gold standard for the assessment of luminal lesions in CD, but given the good correlation shown between endoscopy and cross-sectional imaging in assessing the activity and severity, imaging is increasingly introduced as a too to monitor medical treatment. There is some evidence indicating that crosssectional imaging can be considered a responsive and reliable tool as it detects meaningful changes in patients' status over time after therapeutic interventions. Besides this, cross-sectional imaging is capable of monitoring the colon and the small bowel and penetrating lesions that cannot always be assessed by endoscopy. Becoming familiar with the radiological changes during and after treatment will be key in the decision-making process. Learning Objectives:

1. To become familiar with useful signs after medical treatment of Crohn's disease.

2. To understand the changes which occur after recurrence in Crohn's disease. 3. To understand the alterations which occur after treatment of intestinal infectious processes. Room E2

The Beauty of Basic Knowledge: Skeletal Radiology

MC 26E

Degenerative disease

Moderator:

V.N. Cassar-Pullicino; Oswestry/UK

A-570 12:30

Degenerative disease

K. Bohndorf; Vienna/AT

Degeneration is routinely defined as a morbid change of cells, tissues and even organs. In the MSK system degeneration occurs in bones, joints (synarthrosis and diarthrosis), muscles and tendons. Primary degeneration implies ageing as well as overuse, based on mircrotrauma. The latter may be related to constitutional, professional or sports-related factors. A distinction between ageing and overuse is not possible with the use of histology and imaging. Only the intensity of the changes, which may be inadequate in relation to the age of the patient, may give a hint. On the contrary, the term "secondary degeneration" should be used for clearly defined events or diseases, which finally will result in the degeneration of tissues. The causes are inflammation (RA, infection), metabolic disease (e.g. CPPD) or trauma. Other ways to classify degeneration of the MSK system are related to the anatomy. Accordingly, degeneration of the particular parts of the peripheral or axial skeleton, muscles and tendons has to be discussed. One has to have in mind that bones, cartilage, muscles and tendons form a complex unit. The single elements of these complex units jointly undergo degenerative deterioration, e.g. bone, cartilage, fibrocartilage and capsule are jointly involved in case of OA of the knee joint. The same holds true for the synchondroses of the spine. At the rotator cuff not only the tendons, but also the underlying bone and the muscle tissue are changed. 


\section{Postgraduate Educational Programme}

Learning Objectives:

1. To learn about the underlying pathomechanisms involved in degenerative processes.

2. To understand the imaging features of degenerative disease in the peripheral skeleton.

3. To understand the imaging features of degenerative disease in the axial skeleton.

$12: 30-13: 30$ Room F1

The Beauty of Basic Knowledge: Interventional Radiology

MC 25E

\section{Essentials of transcatheter embolisation}

\section{Moderator:}

O.M. van Delden; Amsterdam/NL

A-571 12:30

Essentials of transcatheter embolisation

O.M. van Delden; Amsterdam/NL (o.m.vandelden@amc.uva.nl)

There are many different types of embolisation, but all share many basic principles. The specific approach, technique, materials and equipment used may differ depending upon many variables such as the type of vascular territory involved (venous, arterial, small vessels, large vessels), clinical setting (elective or emergent procedure), and type of underlying disease to be treated (e.g. tumor embolisation, acute haemorrhage, vascular malformation, arteriovenous fistula, etc). Pre-procedural imaging and planning are essential for most embolisation procedures and intra-procedural navigation can be done with fluoroscopy, DSA, and new 3D-techniques. With proper imaging workup prior to embolisation procedure time, radiation dose and contrast load can be significantly reduced. Embolisation materials can be roughly divided into liquid agents (alcohol, glue, polymers), particulate agents (PVA, calibrate microspheres, drug-eluting beads) and coils (detachable, non-detachable) and plugs (Amplatzer®-plugs). Plugs and coils are deployed at the exact site of destination and usually easy and safe to use. Particles and liquid agents reach their site of destination by flow guidance and can be more difficult and unpredictable in their use. Complications include puncture-site complications (thrombosis, dissection, haematoma), systemic complications (contrastinduced nephropathy, allergic reactions) and specific embolisation-related complications (non-target organ embolisation, end-organ ischaemia, postembolisation syndrome). When used with expertise and proper experience, most types of embolisation have good results with acceptable complication rates.

Learning Objectives:

1. To understand the basic concepts and indications for embolisation

2. To learn about the practical aspect of embolisation, the techniques of

endovascular navigation and the deployment of embolisation products.

3. To learn about results and complications.

$16: 00-17: 30$ Room C

GI Tract

\section{RC 1901a}

\section{From my workstation: difficult cases on review}

\section{A-572 16:00}

Chairman's introduction

A. Maier; Vienna/AT (andrea.maier@meduniwien.ac.at)

Although there has been advancement in imaging techniques, several pitfalls in the assessment of diseases of the pancreas, small bowel and rectum remain. The differentiation and the correct characterisation of tumours and inflammatory disease in some cases may be difficult. Also, atypical presentation of common tumours or uncommon tumours persists and is a challenge. Problematic situations are constantly represented as intestinal bleeding and unexpected findings. In this session, we point out the procedure of the best imaging modality and protocols of challenging cases.

\section{A-573 16:05}

\section{A. Pancreas}

C. Triantopoulou; Athens/GR (ctriantopoulou@gmail.com) that are now in use, a possibility for pitfalls remains. These pitfalls are related either to the inability of the early recognition of a lesion, or to the wrong characterisation of a "mass". Both pitfalls are of great importance, taking into account the lethal pancreatic cancer and the possible complications that may follow an unnecessary pancreatic surgery. There are many variants of the pancreatic parenchyma, the ducts or even vessels, and deep knowledge of the pancreatic anatomy and embryology is needed, to be able to recognise these variations in imaging. A pancreatic variant may not only be misinterpreted as a severe pancreatic disease (e.g. ectopic spleen vs neuroendocrine tumour), but may also be the underlying cause of pancreatic inflammatory lesions (e.g. ectopic pancreas on the duodenal wall causing groove pancreatitis). Other pitfalls are related to the atypical presentation of a common lesion (e.g isoattenuating pancreatic adenocarcinoma), the presence of uncommon tumours, diffuse or multifocal diseases, the co-existence of two different entities and the heterogeneous or overlapping appearance of cystic lesions. In any challenging case, a focused methodology should be applied and the diagnostic procedure based on predefined imaging parameters, taking also into consideration the clinical and laboratory findings. Excluding pancreatic cancer should be the first goal and every attempt should be made to differentiate between surgical and non-surgical cases.

Learning Objectives:

1. To appreciate the challenging variants in pancreatic anatomy.

2. To learn about the most important pitfalls in pancreatic imaging

3. To understand the management of these challenging cases.

\section{A-574 16:28 \\ B. Small bowel \\ E. Biscaldi; Genoa/IT (ennio.biscaldi@gmail.com)}

Endoscopy is the gold standard in small bowel diseases, but it is a challenging technique: the PillCam is a commonly used tool and multidetector CT is a diffuse investigation technique. CT enteroclysis/enterography is a worldwide tool in intestinal exploration. Throughout the investigation of the small bowel, morphologic anomalies or embryonal development defects may be present (Meckel's diverticulum, duplications of intestinal tracts). Artefacts may be determined and there may be misunderstanding. MDCT is the first tool in emergency diagnosis; imaging pitfalls are possible. Unsuspected diseases may be revealed: we will see a case of GIST in a patient affected by intestinal bleeding of an obscure origin. CT enteroclysis may find unknown or unsuspected diseases: correct technical parameters have to be respected, as artefacts may be derived from an improper acquisition technique, such as a limited distension or poor intravenous enhancement In follow-up, MR is preferred to investigate the intestine, mainly in the young population. Unexpected findings may be due to rare pathology: we met a case of endometriosis, with ambiguous presentations; patients affected by autoimmune pathologies (pyoderma gangrenosum or lupus erythematosus), with unknown inflammatory intestinal chronic diseases. In oncology, PET-CT imaging is the main diagnostic tool, but there are abdominal pitfalls that limit its effectiveness (intestinal hot spots, artefacts from peristalsis): the small bowel may frequently obstruct an easy diagnosis. In case of emergency, if the radiological diagnosis is not clear, the surgeon determines the management of the patient. In case of a defective study technique, a correct examination may be repeated. Endoscopy or PillCam may help to detect mucosal lesions invisible at CT. Learning Objectives:

1. To learn about the normal anatomy and normal variants.

2. To learn about the most important pitfalls in imaging assessment of small bowel lesions.

3. To understand the management of these challenging cases.

\section{A-575 16:51}

\section{Rectum}

D.M. Lambregts; Maastricht/NL (d.lambregts@mumc.nl)

MRI plays a key role in the assessment of disease of the rectum and in particular for the staging (and restaging) of rectal cancer. In this session, the relevant MR anatomy of the rectum will be discussed. Furthermore, the strengths and weaknesses of $\mathrm{MRI}$ in the assessment of rectal cancer during primary staging and restaging after chemoradiotherapy will be highlighted using various rectal cancer cases. Finally, we will discuss some rare tumours of the rectum (including GIST and carcinoid).

Learning Objectives:

1. To learn about normal rectal anatomy and normal variants.

2. To learn about the most important pitfalls in imaging assessment of rectal cancer or rare tumours of the rectum.

3. To understand the management of these challenging cases

Panel discussion:

What can we learn from challenging cases? 


\begin{tabular}{ll}
\hline $16: 00-17: 30$ & Room D
\end{tabular}

Chest

\section{RC 1904}

\section{Take a deep breath and hold: why and why not}

\section{A-576 16:00}

Chairman's introduction

P.A. Grenier; Paris/FR (philippe.grenier@psl.aphp.fr)

Optimisation of CT and MR techniques has provided new challenges for imaging in the assessment of airway and lung diseases. Phenotypic abnormalities which can be recognised on visual and quantitative evaluation of CT images in COPD patients may improve the diagnostic accuracy, help optimise treatment and provide a framework for clinical trials. Although expiratory CT helps assess air trapping due to small airway obstruction and excessive dynamic collapse of large airways, potential tricks in technique and interpretation of $\mathrm{CT}$ images must be known. Owing to multiple and successive improvements over years, functional and morphologic evaluation of the lung using MRI has become reality for clinical practice. Already regarded as a favoured cross-sectional imaging in paediatric chest radiology, MRI of the lung is gaining new indications also in lung diseases occurring in adults.

\section{A-577 16:05}

A. New insights into COPD on HRCT

N. Sverzellati; Parma/IT (nicolasve@tiscali.it)

The potential for high-resolution computed tomography (HRCT) to uncover several morphological subtypes that come under the umbrella term chronic obstructive pulmonary disease (COPD) is now more familiar to both radiologists and specialist respiratory physicians. A complete approach to the classification of COPD would ideally assimilate several parameters through a combined visual-quantitative HRCT analysis. The insight that some subjects given the label of COPD have "pure" airways diseases or emphysema can be readily provided by a simple visual evaluation. The subtypes - centrilobular, panlobular, and paraseptal emphysema - can be reliably distinguished on HRCT images. The visual assessment of bronchial abnormalities and accompanying smoking-related interstitial lung disease may also complete the phenotypic classification of COPD. Furthermore, it is now possible to objectively quantify the global extent of emphysema, gas-trapping, and bronchial metrics by two types of softwares which are now increasingly available on latest CT workstations.

Learning Objectives:

1. To learn about the classification of COPD.

2. To appreciate the role of HRCT in the classification of COPD.

3. To become familiar with different methods of analysis of HRCT in COPD

\section{A-578 16:28}

B. Using inspiratory and expiratory CT

A. Devaraj; London/UK

This presentation will examine the role of expiratory CT in the diagnosis of small and large airways diseases. Small airways disease or air trapping may be a feature of asthma, COPD and hypersensitivity pneumonitis, and is typically characterised by a mosaic attenuation pattern on CT. Tracheobronchomalacia and excessive dynamic airway collapse (EDAC) are large airways conditions that may be recognised by tracheal narrowing on inspiratory and/or expiratory CT. The typical clinical and CT manifestations of these diseases will be reviewed. Additionally, a number of important caveats with regard to CT in small and large airways disease will be discussed: 1) the comparative strengths and limitations of $\mathrm{CT}$, bronchoscopy and lung function testing in making the diagnosis; 2) the overlap in appearances that exists between diseased and healthy individuals; 3 ) the variability in definitions of conditions such as tracheobronchomalacia. The optimal CT technique for imaging of airways disease, including the role of dynamic expiratory CT, will also be reviewed.

Learning Objectives:

1. To learn when inspiratory and expiratory CT may be of value in patients with lung disease.

2. To become familiar with different diseases and their appearances on inspiratory and expiratory $\mathrm{CT}$.

\section{A-579 16:51 \\ C. Pulmonary disease assessment with MRI \\ H.-U. Kauczor; Heidelberg/DE (Hans-Ulrich.Kauczor@med.uni-heidelberg.de)}

For routine clinical applications, a standardised MRI protocol has been widely implemented. It addresses the major challenges of MRI of the lung, i.e. low proton density, susceptibility artefacts as well as respiratory and cardiac motion. Beyond visualisation of lung morphology, which is done in an inspiratory breath-hold, functional imaging has become an integral part of the routine protocols: perfusion, blood flow, ventilation, respiratory motion, diffusion. For perfusion imaging, inspiratory or expiratory scans can be used blood flow measurement should be performed during shallow breathing, and ventilation and diffusion are mainly assessed in inspiration. Respiratory motion is best assessed by dynamic image acquisitions. Continuous breathing can also be combined with triggering or navigators to improve image quality or derive functional maps. MRI is recommended as the first-line cross-sectional imaging modality in paediatric chest radiology, including cystic fibrosis, complicated pneumonia, any pulmonary or cardiovascular anomaly, as well as the assessment of mediastinal masses in children and adults. MRI is also challenging PET/CT in the staging of lung cancer (TNM) as well as CT and echocardiography in the assessment of pulmonary arterial hypertension. MRI even without contrast - is an alternative in the diagnosis of acute pulmonary embolism if iodinated contrast medium is contraindicated or in pregnant women. MRI should also be used in complicated pneumonia, e.g. in immunocompromised patients as well as for the functional assessment of chronic obstructive pulmonary disease (COPD) or severe asthma. The role of $\mathrm{MRI}$ in interstitial lung disease stills needs to be determined.

Learning Objectives:

1. To learn about different MRI techniques used to assess pulmonary disease. 2. To appreciate when MRI may be of value in patients with pulmonary disease.

Author Disclosure:

H.-U. Kauczor: Equipment Support Recipient; Siemens, Philips. Investigator; Boehringer. Speaker; Siemens, Bracco, Bayer, Boehringer, Novartis.

Panel discussion:

Correlating imaging with lung function and pulmonary

disease genotypes $17: 14$

16:00 - 17:30 Board Room B

\section{Paediatric}

RC 1912

\section{Imaging the head and skull base \\ Moderator: \\ J.F. Schneider; Basle/CH}

\section{A-580 16:00 \\ A. Faciocraniosynostoses revisited \\ F. Di Rocco; Paris/FR}

Unlike the simple forms of cranisynostosis whose modalities and timing of surgical correction are well established, the management of faciocraniosynostoses requires a multispecialistic and repeated evaluation of the single patients. Their evolution, in fact, depends on the specific genetic anomalies but also on the phenotype, which can vary in time, of their clinical expression which may lead to different degrees in severity of the associated functional disturbances (CSF dynamics impairment, visual deficits, respiratory anomalies, etc). Consequently these conditions may require several surgical steps the timing of which are dictated by the exact clinical diagnosis and the evaluation of the functional status at different ages. Current management of faciocraniostenoses is thus based not only on the radiological studies but also on the functional assessment of the brain function (e.g. MRI imaging studies, metabolic and cerebral blood circulation investigations, respiratory pathways volume evaluation, sleep recording). Today's surgical multidisciplinary management may benefit a large variety of techniques which extend from free bone cranioplasty with intraoperative active fixed expansion to more dynamic and less invasive methods which exploit the physiological brain expansion in infants or the gradual cranial vault expansion mechanically stimulated by springs or distractors. In the present report, we will review the decision making process and the rationale on which, at the Necker-Enfants Malades, we base the use of the currently available techniques for skull expansion and correction of cranial malformations according to the accurate dynamic radiological and functional evaluation at different stages of the disease.

Learning Objectives:

1. To learn about the imaging patterns of faciocraniosynostoses.

2. To learn how and when to image.

3. To become familiar with associated brain anomalies. 
A-581 16:30

B. All about the paediatric pituitary gland

M.I. Argyropoulou; Ioannina/GR (margyrop@cc.uoi.gr)

MR is the imaging modality of choice for the assessment of the pituitary gland (PG) and the hypothalamus. The normal adenohypophysis is bright during the first two months of life and appears isosignal to the brain parenchyma afterwards. The neurohypophysis is bright, provided that the child is well hydrated. The PG height decreases during the first year of life and then increases until puberty. Adenohypophyseal deficiency has been associated with a small pituitary size, an ectopic neurohypophysis, a hypoplastic or absent pituitary stalk, hypothalamic gliomas, craniopharyngiomas and iron overload states. Precocious puberty may be idiopathic, but it has also has been associated with hypothalamic hamartomas, craniopharyngiomas and hydrocephalus. Diabetes insipidus may be secondary to histiocytosis $X$, germinomas and trauma causing pituitary stalk interruption. The hypothalamopituitary axis is evaluated by using sagittal and coronal T1-weighted images without and with contrast administration. Dynamic contrast enhancement may be useful in the evaluation of disorders responsible of diabetes insipidus. Magnetisation transfer imaging may be useful in the evaluation of pituitary deficiencies or precocious puberty.

Learning Objectives:

1. To become familiar with age-related changes of the normal pituitary gland.

2. To learn about congenital and acquired pathology of the hypothalamopituitary axis.

3. To understand the pathophysiologic substrate of different imaging patterns.

\section{A-582 17:00}

C. Imaging of the orbit: the globe and the lachrymal gland

P.C. Maly Sundgren; Lund/SE (Pia.Sundgren@med.Iu.se)

Familiarity with orbital and ocular anatomy is crucial to the understanding of disease processes of the orbit. Lesions of the orbit may be divided into those which are intraocular and those which are extraocular. The underlying aetiology and geneses of many different lesions that can occur in the orbits vary depending on the location of the lesions and sometimes on additional nonorbital conditions. CT and MRI play crucial roles in the evaluation of orbital pathology where often MRI still is a complement to CT examination in the evaluation of orbital lesions Familiarity with the radiologic appearance of common orbital lesions is important, as many of these lesions will not be seen on physical examination. After a very brief anatomic overview, most of this lecture will focus on the more common benign and malignant lesions as well as differential diagnosis involving the orbit and lachrymal gland in both the paediatric and adult population. The lecture will also present suggested imaging protocol and standard of care with respect to imaging.

Learning Objectives:

1. To understand the embryology and imaging findings of the most common malformations.

2. To learn about space-occupying lesions and the differential diagnosis of tumours and inflammatory conditions.

3. To be aware of the role of conventional and advanced MR sequences in the diagnostic approach to lesions in the orbit.

$16: 00-17: 30$ Room E1

\section{Musculoskeletal}

\section{RC 1910}

\section{The hand and wrist}

Moderator:

S. Bianchi; Geneva/CH

\section{A-583 16:00}

\section{A. Patterns of injury}

A. Navas Canete; Leiden/NL (a.navas_canete@lumc.nl)

Injury to the wrist and hand is an important clinical problem. First, such injury is relatively common. Second, the spectrum of abnormalities is complicated. The purpose of this presentation will be to learn more about the imaging appearances of soft tissue and osteoarticular injury of the wrist and hand using multiple imaging techniques. Emphasis will be placed upon pathomechanics and characterisation of greater and lesser arc injury patterns of the wrist. The various posttraumatic instability patterns of the wrist will be also discussed. Learning Objectives:

1. To learn more about the imaging appearances of soft tissue and osteoarticular injury.

2. To become familiar with the patterns of bone and soft tissue injury in the hand and wrist.

\author{
A-584 16:30 \\ B. Inflammatory disorders \\ H. Guerini, J.-L. Drapé; Paris/FR (henri.guerini@gmail.com)
}

Rheumatoid arthritis (RA), psoriatic arthritis (PsA) and other inflammatory disease can be diagnosed and sometimes differentiated in the early stages of the disease on the basis of MRI and/or PDUS features of the hand and wrist. Rheumatoid arthritis (RA) activity is closely correlated with inflammation. The synovial membrane is the principal site of inflammation in which the inflammatory process enhances capillary perfusion and permeability. Doppler ultrasonography (DUS), using the amount of colour pixels in the region of interest, and dynamic magnetic resonance imaging (DE-MRI) are both able to detect this inflammation in the wrist and hand. Although these techniques are both capable of monitoring synovium inflammation modifications after RA treatment, PDUS has become an essential tool for RA joint monitoring in routine practice in view of its sensitivity in the detection of synovitis, feasibility in outpatient clinics, and low cost.

Learning Objectives:

1. To learn more about the imaging appearances of soft tissue and osteoarticular inflammation.

2. To become familiar with imaging findings of specific inflammatory conditions.

A-585 17:00

C. Tumours and tumour-like lesions

E. Llopis ${ }^{1}$, V. Higueras ${ }^{2}$, E. Belloch ${ }^{1} ;{ }^{1}$ Valencia/ES, ${ }^{2}$ Alzira/ES

(ellopis@hospital-ribera.com)

Radiological study of the wrist and hand is challenging due to its complex anatomy with many small structures and the number of normal bone and soft tissue variants that might mimic injuries. Moreover, many findings can be asymptomatic. Their knowledge is important to avoid misdiagnosis. During this lecture we will also review the role of the different imaging modalities, such as plain films for wrist alignment and bone structures as well as the important role of US and MR in differentiating tumour from tumour-like conditions. We will become familiar with some specific radiological findings that allow us to make accurate diagnoses of soft tissue and bone lesions.

Learning Objectives:

1. To learn more about the spectrum of intra and para-articular soft tissue tumours, and soft tissue tumour-like lesions.

2. To become familiar with US and MRI findings of specific soft tissue lesions.

16:00 - 17:30 Room E2

\section{Oncologic Imaging}

\section{RC 1916}

\section{Managing incidental findings in cancer patients}

A-586 16:00

Chairman's introduction

C. Pfannenberg; Tübingen/DE (christina.pfannenberg@med.uni-tuebingen.de)

With recent advances in cross-sectional imaging, the frequency of detecting "incidental findings" has markedly increased. Incidental findings (=incidentaloma) are defined as unexpected, asymptomatic abnormalities detected by imaging performed for an unrelated reason. These incidentalomas have created a management dilemma for both radiologists and clinicians, particularly in the cancer patient in whom any mass warrants further evaluation. Discovery of incidental findings often leads to a cascade of additional tests that is costly, provokes anxiety and exposes patients to the risk of unnecessary radiation and intervention. The workup of incidentalomas varies widely by physicians, and strategies for optimising patient management are only beginning to emerge. In this course, guidelines concerning a rational approach to some of the more common incidental abnormalities in cancer patients are presented. Basic principles and tools for interpretation of incidental findings, common pitfalls and protocol issues regarding the differentiation of benign and malignant lesions as well as treatment-induced abnormalities will be addressed with a focus on the liver, lung and skeletal system. At the end of the session, the speakers will discuss the role of functional imaging techniques for characterisation of incidental lesions.

Session Objectives:

1. To become familiar with basic principles and common pitfalls in interpretation of incidental findings in the lung, abdomen and skeletal system. 2. To learn how functional imaging (DWI, PET/CT, BS) can help in lesion characterisation.

3. To provide attendees with clear and practical messages for the management of the most common incidental findings in cancer patients. 


\section{A-587 16:05}

A. Abdomen: common pitfalls and protocol issues

A. Ba-Ssalamah; Vienna/AT (ahmed.ba-ssalamah@meduniwien.ac.at)

An incidentaloma is quite a frequent diagnosis in medicine, in general, and in radiology, in particular. By definition, an incidentaloma is an abnormality or a tumour found by coincidence without clinical symptoms or suspicion. These incidental findings have long posed challenges to physicians, and particularly to radiologists as well as health-care providers due to the resulting high costs. The scope and scale of these challenges have increased with the introduction of new technologies, in particular cross-sectional imaging, such as MDCT and MRI using sub-millimetre thin slices. Incidentalomas still cause a management dilemma for clinicians, radiologists and even for the patients themselves. This dilemma is particularly pertinent to oncology patients. Therefore, a precise knowledge of the broad spectrum of incidental findings is crucial. Based on this knowledge, we can choose the appropriate radiologic examination that will provide a confident diagnosis. This way, needless uncertainty, for both the patient and the physician in charge, is avoided. Furthermore, invasive procedures, such as biopsies, with the potential for complications, as well as cost-intensive follow-up examinations, can be reduced. Furthermore, MRI is usually used to detect the side effects of chemotherapy of the liver. Simple steatosis is usually reversible, unless the liver receives a 'second hit' of damage from other causes leading to other manifestations, including chemotherapy-associated steatosis, sinusoidal obstructive syndrome, nodular regenerative hyperplasia, veno-occlusive disease, peliosis, pseudo-cirrhosis, and sclerosing cholangitis largely due to microvascular injury. Finally, the effects of chemotherapy on peritoneal and mesenteric structures after performing intraperitoneal chemotherapy will be discussed.

Learning Objectives:

1. To become familar with the most common benign lesions and pseudolesions of the liver, the adrenal glands, and the pancreas.

2. To understand the CT and MRI characteristics by which the abovementioned lesions and malignant diseases can be distinguished.

3 . To learn how the liver is generally affected by chemotherapy and how to make a correct assessment.

4. To become familiar with the effects of chemotherapy on peritoneal and mesenterial structures.

\section{A-588 16:28}

\section{B. Chest: CT follow-up and advanced techniques}

A.A. Bankier; Boston, MA/US (abankier@bidmc.harvard.edu)

This presentation will discuss the complex topic of incidental findings in thoracic imaging of oncological patients. Incidental findings will be presented with respect to their respective organs and anatomical regions. The presentation will emphasise the clinical relevancy of the findings as well as the specific of risk estimation in oncological patients. Finally, the presentation will discuss the usefulness (or lack thereof) of current management guidelines for incidental findings, as designed for the general population. Illustrative clinical scenarios will be discussed.

Learning Objectives:

1. To become familiar with the CT characteristics of the most common benign and inflammatory/infectious lung lesions, as well as treatment-induced abnormalities.

2. To learn how to distinguish signs of congestive heart failure from tumour spread.

3. To learn how to distinguish benign parenchymal consolidations from malignant pulmonary nodules.

4. To become familiar with the criteria of the Fleischner society for the follow-up of indeterminate pulmonary nodules in cancer patients.

5. To understand the indications for, and the limitations of PET for the characterisation of pulmonary nodules.

Author Disclosure:

A.A. Bankier: Author; Elsevier. Consultant; Olympus. Grant Recipient; NIH. Speaker; ATS.

\section{A-589 16:51}

\section{Skeletal system: is MRI sufficient?}

F.E. Lecouvet; Brussels/BE (frederic.lecouvet@uclouvain.be)

Radiographs and CT often detect incidental observations in bones corresponding either to normal variants or benign conditions, which require additional imaging to rule out malignant conditions. MRI most often provides nonambiguous explanation for these observations. Beside this, MRI, targeting either the whole body or only the axial skeleton, often including diffusionweighted (DWI) sequences, is increasingly used for bone tumour detection in many "osteophilic" cancers and haematologic malignancies, without need for contrast material injection and without irradiation, with unparallelled sensitivity to bone lesions. An important advantage and also a challenge for musculoskeletal radiologists are the all organ screening capabilities of DWI, demanding careful study of the huge information provided and knowledge extending far beyond bones in terms of organs and metastatic spread of different cancers. A second major advantage is its ability to detect lesion changes under therapy. However, benign lesions and non-neoplastic conditions, as well as artefacts may also lead to confusing observations on $\mathrm{MRI}$, and particularly on DWI. Anatomical MR sequences as well as other imaging modalities are of utmost help to recognise these pitfalls. Whole body MRI including DWI sequences has to position itself among other diagnostic tools, bone scan, SPECT, and especially PET with its variety of tracers, in the currently evolving strategy of bone screening techniques, which will most likely vary according to the primary cancer.

Learning Objectives:

1. To present most frequent $\mathrm{x}$-ray and CT pseudo lesions and benign conditions and show how MRI often enables straightforward interpretation of these abormalities.

2. To highlight the strengths of MRI with diffusion weighted imaging (DWi) for malignant lesion detection and characterisation within bones, but also beyond bones.

3. To highlight the typical appearance of malignant lesions on projectional and cross-sectional imaging.

4. To learn to differentiate these lesions from normal marrow variations, stress lesions, bony pseudo-lesions and other benign conditions, and to become familiar with treatment induced changes within lesions and their environment. 5. To situate MRI amongst other functional imaging techniques (PET, SPECT, $\cdots)$.

Panel discussion:

The role of functional imaging techniques in lesion

characterisation $\quad 17: 14$

16:00 - 17:30

Room F1

Abdominal Viscera

\section{RC 1901b \\ Colorectal cancer liver metastases: assessing tumour response}

\author{
A-590 16:00 \\ Chairman's introduction \\ P.R. Ros; Cleveland, OH/US (Pablo.Ros@UHhospitals.org)
}

Colorectal cancer is the $3^{\text {rd }}$ most commonly diagnosed cancer in the world with $60 \%$ of cases diagnosed in the developed world. With an estimated 1.3 million new cases clinically diagnosed and over 600,000 people killed worldwide by this disease on an annual basis, colorectal cancer is a true public health concern. Survival of colorectal cancer is directly related to the extent of disease and specifically presence of liver metastases. Imaging plays a key role in the initial staging of colorectal cancer and is the gold standard in evaluating extra colonic disease, primarily liver metastases. Imaging is also widely used for therapy monitoring and staging. CT, MRI, PET/CT and PET/MRI are the key imaging modalities. In addition, tumour response therapy assessed with morphological and functional biomarkers is increasingly used by advanced gastrointestinal oncologic programs. Furthermore, image-guided therapy is widely used for patients with unresectable lesions. Liver colorectal metastases were the first liver metastases to be treated with image-guided ablation techniques. A gamut of ablative techniques exists ranging from transarterial embolisation, radiofrequency ablation, highly intensified focused ultrasound brachitherapy etc. In short, diagnosing, characterising and finally assessing tumour response after neoadjuvant and image-guided therapy are all functions where imaging plays a pivotal role. This course is divided into three logical segments dealing with current treatment options, morphological and finally functional biomarkers and it is followed by a panel discussion which includes audience participation.

Author Disclosure:

P.R. Ros: Advisory Board; Philips Healthcare Radiology Medical Advisory Network, KLAS, Medical Advisory Board. Research/Grant Support; Departmental Research support from Philips Healthcare, Siemens, Toshiba Medical Systems and SECTRA.

\section{A-591 16:05}

\section{A. Current treatment options}

T.K. Helmberger; Munich/DE (Thomas.Helmberger@klinikum-muenchen.de)

Hepatic metastases in colorectal cancer may occur in $20 \%$ to $80 \%$ of the cases. Considering the general oncological (isolated hepatic tumour load; prognostic benefit), and technical (size, number, location of hepatic metastases; expected hepatic functional reserve) framework, surgical resection is still considered to be the method of choice - even if this statement never had 
been verified by RTCs. Nevertheless, in clinical reality only $20-30 \%$ of patients with liver metastases may qualify for resection. In consequence, the majority of patients need other or at least modified therapeutical pathways including adjuvant or neoadjuvant chemotherapy and more and more image-guided local ablative therapies. The latter encompass chemo- (transarterial chemoperfusion/-embolisation), thermo- (radiofrequency-, laser-, microwave ablation, high intensified focused ultrasound), and radio-ablative (radio embolisation, interstitial brachytherapy, etc). techniques. Particularly, thermalablative techniques gained wide acceptance over the last years, since ample evidence could be presented showing that this method can be applied as primary and also complementary therapies in resectable and non-resectable metastatic disease. Furthermore, recent data confirm that in multimodality therapy concepts, progression free survival and overall survival in patients with primarily unresectable and with unfavourable prognosis is comparable to surgery with 5 -year survival rates more than $50 \%$. Minimally invasive, imageguided therapies will not replace surgical resection; however, these therapy modalities are eligible in a large number of cases and should be implemented consequently in multimodality treatment regimens according to the interdisciplinary consensus of oncologists, interventional radiologists, and surgeons.

Learning Objectives:

1. To understand the different scenarios in which liver metastases may present in relation to patient prognosis and therapeutic options.

2. To understand the existing therapeutic approaches to liver metastases in different scenarios

\section{A-592 16:28}

B. Morphological biomarkers

S. Skehan; Dublin/IE (s.skehan@st-vincents.ie)

Colorectal liver metastases are typically identified as low attenuation lesions on portal venous phase CT. Planning of optimal therapy depends on accurate localisation and characterisation of all focal liver lesions and this is best achieved with MRI, which should include diffusion-weighted imaging and use of hepatocyte-specific contrast medium. The typical MRI signature of colorecta metastases includes low signal on T1w, moderately increased signal on T2w, irregular peripheral arterial phase enhancement with low signal on porta venous phase, restricted diffusion and absent hepatocytes. Morphological tumour response can be assessed with either modality. Size-based systems for assessing tumour response are widely applied, with RECIST 1.1 the most widely used at present. RECIST 1.1 includes several modifications that make it more user friendly than RECIST 1.1. More advanced morphological criteria have been described for new targeted and molecular therapies, including overall attenuation, the tumour-liver interface and the appearance of the peripheral rim of enhancement. As hepatic surgeons become more aggressive in their approach to resection in patients with liver metastases, it is important to understand that disappearance or calcification of liver metastases after treatment does not necessarily equate to a complete pathological response. The timing of imaging is therefore critical in assisting the surgeon to remove all previously affected hepatic segments after chemotherapy.

Learning Objectives:

1. To learn about the algorithm for detecting and characterising liver metastases.

2. To understand conventional imaging criteria for assessing tumour response.

3. To learn about the rationale for monitoring patients after radical and palliative treatments.

\section{A-593 16:51}

\section{Functional biomarkers}

D.-M. Koh; Sutton/UK

Conventional size measurement criteria remain the most widely used method to determine the response of colorectal liver metastases to treatment However, tumour size reduction is assessed relatively late (e.g. 12 weeks after treatment) and new targeted treatment may be effective without reducing tumour size. New functional imaging techniques can be applied to quantify different aspects of tumour biology and to develop response, and predictive and prognostic biomarkers. We discuss the use of diffusion-weighted MR imaging, dynamic contrast-enhanced MR imaging and FDG-PET imaging in the evaluation of treatment response in patients with colorectal liver metastases.

Learning Objectives:

1. To understand the different functional imaging techniques that can be used to monitor response to therapy including perfusion, diffusion MRI and PET/CT.

2. To learn about efficient algorithms for assessing therapeutic response of

liver metastases that influence prognosis.

3. To understand how functional biomarkers are incorporated into multicentric trials.

Panel discussion:

The "vanishing" lesions: what should you do

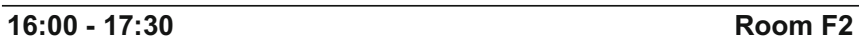

Breast

RC 1902

Emerging breast imaging technologies

Moderator:

F. Pediconi; Rome/IT

A-594 16:00

A. Digital breast tomosynthesis (DBT)

P. Skaane; Oslo/NO (PERSKA@ous-hf.no)

Digital breast tomosynthesis (DBT) is a promising technique for breast imaging based on a full-field digital mammography (FFDM) platform. The x-ray tube moves through a proscribed arc, and several low-dose projection images are acquired. Images are reconstructed into a stack of $1 \mathrm{~mm}$ slices. The radiation dose is about the same or slightly higher than for FFDM. The most important advantage of DBT is the elimination of superimposed tissue, which improves detection of lesions otherwise hidden by dense breast parenchyma and reduces the interpretation problems caused by overlapping tissue. DBT is suggested to improve sensitivity as well as specificity in women with dense breast. DBT is superior to FFDM in tumour size assessment and for mass visibility and cancer conspicuity. DBT is comparable to FFDM for evaluation of microcalcifications. DBT is superior for the detection of spiculated masses and architectural distortion. The potential to improve sensitivity and specificity is of interest for screening. An open question is whether DBT should be applied in one or two views. Two-view DBT would mean a doubling of radiation dose, but a solution is the implementation of synthetic $2 \mathrm{D}$ images reconstructed from the 3D dataset of DBT. The synthesised images are created by summing and filtering the stack of reconstructed DBT slices. Using synthetic 2D allows "combo mode" to be implemented in screening with the same radiation dose as for conventional 2D. Results using DBT in breast cancer screening are very promising. Different study design of published studies may explain the variations of results.

Learning Objectives:

1. To become familiar with the technique of DBT.

2. To understand the results of DBT in screening and diagnostics.

3. To learn about the potential role of DBT in the breast radiological algorithm. Author Disclosure:

P. Skaane: Equipment Support Recipient; Hologic, Inc. sponsored the Oslo tomo trial through the use of equipment. Research/Grant Support; Hologic, Inc. sponsored the Oslo tomo trial through financial support for additional overtime interpretations.

\section{A-595 16:30}

B. Multiparametric high-field MRI and more

P.A.T. Baltzer, K. Pinker-Domenig; Vienna/AT (patbaltzer@gmail.com)

Magnetic resonance imaging of the breast provides a multitude of techniques for detection of breast cancer and differentiation of benign from malignant lesions. In the last few years, significant improvements of scanner hardware and equipment in terms of coils as well as sequence software have been achieved. To date, multiparametric quantitative measurement of perfusion, diffusion and tissue chemistry is possible at field strengths up to 7 Tesla. Although higher field strengths and improved sequence protocols provide higher signal and allow faster imaging, specific technical issues have to be considered to avoid artefacts and pitfalls. This talk aims to demonstrate the possibilities and limitations of multiparametric and (ultra-)/high-field MRI. Furthermore, these new techniques are put into the broader clinical context to determine the potential value for breast lesion detection and differentiation. Learning Objectives:

1. To understand the management of breast lesions based on current MRI applications.

2. To learn how to improve the specificity of breast MRI by adding new developing techniques.

3. To appreciate future developments and limitations.

\section{A-596 17:00}

C. High-resolution radionuclide breast imaging (PEM and molecular imaging)

T. Wagner; London/UK (Thomas.Wagner@nhs.net)

Positron emission mammography is a recently developed imaging device using positron emission technology. After injection of a positron-emitting radiotracer, the radiation is detected by a dedicated high-resolution camera, providing high spatial resolution. Commercially available systems include cameras where the breast is compressed and which display a 2D image similar in its layout to a mammogram and hanging breast devices where a 3D image similar in its 
layout to MRI is produced. These devices have been compared to PET/CT and have shown higher spatial resolution, accuracy and sensitivity in detecting malignant breast lesions. The most widely studied tracer in PET/CT and in PEM is fluorodeoxyglucose (FDG). FDG is a glucose analogue and has had a major impact on oncology imaging with PET/CT. Breast carcinomas demonstrate increased glucose consumption and the intensity of the uptake has been correlated with prognosis, hormonal receptor and HER2 status, Ki67 and nodal involvement. Current potential indications for PEM are detection and characterisation of breast lesions, local staging in patients with known breast cancer and baseline assessment prior to neoadjuvant chemotherapy. Future developments of PEM will involve new tracers and fusion with other breast imaging modalities. Fluorothymidine is a proliferation tracer that seems promising in early assessment of response to chemotherapy. Fluoroestradiol has the potential to image oestrogen receptor status in vivo. The possibility of PEM-guided biopsy is a very exciting development and has the potential to select lesions with the highest intensity of uptake. Fusion with MRI, US and mammography will increase the diagnostic accuracy.

Learning Objectives:

1. To become familiar with the current status: what is available and how does it work?

2. To understand the indications: local staging, distant staging, therapy

monitoring and limitations.

3. To learn about future prospects: PET-MRI, targeted tracers.

\section{Neuro}

\section{RC 1911}

\section{Ruling out disease: a radiologist's headache? \\ Moderator:}

P. Demaerel; Leuven/BE

\section{A-597 16:00}

\section{A. Vasculo-ischaemic lesions: inappropriate for age?}

S. Haller; Geneva/CH (sven.haller@me.com)

The presentation will focus on two frequent findings on MRI with increasing incidence during ageing, notably "unspecific" hyperintense lesions on T2/FLAIR and cerebral mircobleeds (CMBs) on T2* or SWI images. Clinicoradiologic studies suggest that these T2/FLAIR lesions are associated with increased risk of cognitive decline, stroke and death. The clinical relevance of these lesions probably depends on periventricular versus deep white matter location. Radiologic-histopathologic studies correspondingly indicate that the degree of histopathologically confirmed demyelination also depends on the location of these T2/FLAIR lesions. Cerebral mircobleeds (CMBs) are punctate hypointense lesions on T2* or SWI images. While multiple CMBs are associated with diseases such as cerebral amyloid angiopathy (CAA), clinicoradiologic studies demonstrate increased incidence of sporadic CMBs in mild cognitive impairment $(\mathrm{MCl})$ and Alzheimer dementia, indicating a degrading effect of $\mathrm{CMBs}$ on cognition. On the other hand, radiologic-histopathologic studies show that not all black spots on $\mathrm{T}^{*}$ or SWI correspond to CMBs. CMB mimics include micro-calcifications. The presentation will demonstrate typical manifestations of these "unspecific" T2/FLAIR lesions and CMBs during ageing and provide tips for the interpretation of these lesions during daily clinical routine.

Learning Objectives:

1. To understand the aetiology and risk factors for small vessel disease.

2. To appreciate the prognostic impact of extensive vascular white matter lesions.

3. To learn how to interpret vascular white matter lesions according to age and decide what is abnormal.

\section{A-598 16:30}

B. Radiologically isolated syndrome: silent MS?

A. Rovira-Cañellas, C. Auger; Barcelona/ES (alex.rovira@idi-cat.org)

With the increasing use of MRI, it has become more common to discover incidental brain findings. These findings may be non-specific or have some morphological characteristics and geographical distribution highly suggestive of a demyelinating disease. The term "radiologically isolated syndrome" (RIS) was introduced by Okuda in 2009 to describe those asymptomatic patients with radiographic abnormalities highly suggestive of multiple sclerosis (MS). Sometimes, these lesions may even meet the MRI criteria for dissemination in space, which are currently used to predict future development or conversion to MS in patients presenting a clinically isolated syndrome (CIS). However, we must not forget that these radiological criteria should be applied only when the patient had experienced any clinical manifestations suggestive of a demyelinating disease. Therefore, what happens in those patients in whom we found highly suggestive lesions but have never had symptoms? Due to lack of knowledge about the natural history or evolution of this new syndrome, clinical and therapeutic management of these patients is not well established, nor is the risk of conversion to MS. Recent data, however, indicate that the presence of gadolinium-enhancing cervical cord and cortical lesions significantly increases the risk of conversion to CIS or MS. All these data have improved the characterisation of RIS subjects and in our understanding of risk factors for initial symptom development.

Learning Objectives:

1. To understand the 2010 revision of the McDonald criteria for MS and meaning of RIS

2. To become aware of differential diagnosis of MS and red-flags in the diagnostic process.

3. To understand the use of gadolinium and spinal cord imaging in a diagnostic setting.

A-599 17:00

C. Incidental findings: what to report and what not to report?

A. van der Lugt; Rotterdam/NL (a.vanderlugt@erasmusmc.nl)

Incidental findings on brain imaging are defined as previously undetected abnormalities of potential clinical relevance that are unexpectedly discovered and unrelated to the purpose of the imaging. Incidental findings are increasingly detected in clinical practice, with screening, and in the research setting. Data on the prevalence of these abnormalities are scarce, the clinical course of the findings is often unknown, and the management of such lesions is not clear. The prevalence of incidental findings can be expected to vary depending on the purpose of the imaging exams. With improvements in imaging technology (higher field scanners, new pulse sequences), the number of detected incidental findings will increase dramatically. Another important point is the advent of imaging biomarkers. Advanced techniques for postprocessing and analysis, such as automated segmentation of brain structures or voxel based morphometry, will lead to the discovery of imaging biomarkers. Once the predictive value of these markers has been established, most MRI studies of the brain, both in the clinical and research setting, will reveal information that might be relevant for the well-being of patients or participants. Although still incidental, these findings can unfortunately no longer be considered unexpected. We will soon face large medical, ethical, and practical problems as a result of technical improvements. In this lecture, the most important incidental findings and their prevalence will be reported. The management of incidental findings in the clinical and research setting will be discussed. Finally, recommendations for follow-up will be provided. Learning Objectives:

1. To become familiar with the frequency of incidental findings on brain MRI.

2. To understand the clinical relevance of incidental findings - can they cause headache?

3. To learn how to report incidental findings and when to recommend clinical or imaging follow-up.

Author Disclosure:

A. van der Lugt: Board Member; European Society of Magnetic Resonance in Medicine and Biology. Investigator; GE Healthcare. Speaker; GE Healthcare.

16:00 - 17:30

Room N/O

\section{Head and Neck}

\section{RC 1908}

\section{Imaging laryngeal cancer \\ Moderator:}

B. Banko; Belgrade/RS

\section{A-600 16:00}

\section{A. What matters in staging laryngeal cancer}

R. Maroldi; Brescia/IT (maroldi@med.unibs.it)

The primary assessment of laryngeal cancer is provided by endoscopy, which is accurate in delineating both the superficial extent and functional impairment. Imaging techniques are recommended to grade submucosal invasion, local and distant spread. In the last few decades, open and endolaryngeal surgical procedures as well highly sophisticated RT techniques have been developed to treat the tumour while preserving critical laryngeal functions. For example, early glottic tumours (Tis-T2) can be treated by endoscopic laser excision or RT. The integration of information acquired by endoscopy and imaging is essential for proper treatment planning. The radiologist has to know that the site of origin (supraglottis vs glottis or subglottis) and local extent (superficial and submucosal) are the key tumour-related factors in planning the type of treatment. Submucosal invasion has a variable impact depending on the 
location of the primary tumour. The 5 key issues in glottic cancers include the invasion of: the posterior paraglottic space, crico-arytenoid joint, and anterior commissure and contralateral true vocal cord, and vertical spread towards supra and/or subglottis, thyroid or cricoid cartilage. The 5 key issues in supraglottic cancers encompass the invasion of: base of the tongue, preepiglottic space, piriform sinus, vertical spread into glottis and subsequent cartilage invasion (observed exclusively in transglottic cancers). Functionsparing surgical techniques - as supracricoid laryngectomies - can be applied in advanced cancers when sparing of the posterior commissure, the ipsilateral crico-arytenoid joint, the mucosa investing the upper cricoid and the outer perichondrium of the thyroid cartilage is demonstrated by endoscopic and imaging mapping.

Learning Objectives:

1. To become familiar with the typical findings of T1-T4 laryngeal cancer.

2. To learn about the patterns of local nodal involvement.

3. To understand possible imaging pitfalls in staging.

\section{A-601 16:30}

B. How to evaluate the post treatment larynx: clinical and histological alterations after initial treatment

P. Zbären; Berne/CH (peter.zbaeren@insel.ch)

The detection of recurrent laryngeal carcinoma is often challenging. To better appraise the expected findings after initial surgery of laryngeal cancer, the classical partial laryngectomy and laser laryngeal surgery are briefly presented. (Chemo)radiotherapy has become an important treatment modality for laryngeal carcinoma. The recurrence rate in T2-T4 laryngeal carcinoma is reported to be between $25 \%$ and $50 \%$. The difficulty in differentiation between radiation reaction (such as oedema, fibrosis and soft tissue and cartilage necrosis) and recurrent disease is discussed. A histologic study on wholeorgan sections of 29 recurrent glottic carcinomas is also presented: recurrent disease presents with different tumour spread than does a primary carcinoma, resulting in difficulties in estimating the extent of tumour recurrence. Currently, selected recurrences of early glottic carcinoma are, whenever possible, treated by larynx-preserving salvage surgery. In those cases, a precise evaluation of the recurrent tumour extent is mandatory for planning larynx-preserving salvage surgery. This is especially true in critical regions such as the preepiglottic space, infiltration into cartilage, contralateral tumour spread and subglottic tumour extension. The accuracy of crT-classification is relatively low: many patients with signs and symptoms suggesting tumour recurrence present with post-radiotherapy changes; small tumor foci, often localised in the subglottic region, are undetectable by imaging studies.

Learning Objectives:

1. To appreciate the expected findings after surgery of laryngeal cancer.

2. To become familiar with findings after non-surgical treatment.

3. To know about the possible imaging pitfalls due to the histopatholgical patterns of disease recurrence.

\section{A-602 17:00}

C. Can we rule out cancer recurrence?

F.A. Pameijer; Utrecht/NL (f.a.pameijer@umcutrecht.nl)

Tissue changes in the neck treated by surgery and/or radiotherapy (RT) make the detection of residual or recurrent tumour more difficult. Clinical evaluation of the neck is also hampered by these changes. RT-induced fibrosis and postsurgical scar tissue make palpation of the neck very difficult. Endoscopy is hampered by endolaryngeal oedema which can persist for many months postRT. Therefore, any (non-invasive) method helping in the detection of recurrence is welcome. Patients at risk for local failure after RT can be successfully identified by a post-RT CT (or MR) study between 1 to 6 months after RT. The optimal time point to perform such a 'baseline study' seems to be about 3 to 4 months post-RT. Patients with indeterminate findings are candidates for 'imaging surveillance'; i.e. follow-up imaging every 3 to 4 months up to a period of 2 years after RT. However, ongoing studies suggest that metabolic imaging (FDG-PET) may detect local recurrences with a higher accuracy than 'conventional' anatomically based imaging techniques, such as CT and MR. CT and/or MR-findings in the treated neck are frequently inconclusive. At present, new techniques are available to detect recurrent cancer. PET-CT is widely applied in the post-treatment setting. Also, advanced MR-techniques such as diffusion weighted imaging (DWI) and dynamic contrast-enhanced MR (DCE-MR) can be applied. Focusing on the larynx, the advantages of these techniques as well as the limitations will be shown using imaging examples from daily practice.

Learning Objectives:

1. To understand the limitations of clinical examination.

2. To appreciate the value of different imaging techniques in the detection of recurrence.

3. To become familiar with the imaging findings of recurrent laryngeal cancer

\section{$16: 00-17: 30$ \\ Molecular Imaging \\ RC 1906 \\ Molecular imaging in oncology}

Conf. Room M3

Moderator:

E.J. Rummeny; Munich/DE

\section{A-603 16:00 \\ A. New PET-tracers for oncology \\ W. Weber; New York, NY/US}

During the last 10 years, several hundred new radiotracers for PET imaging of cancer have been developed and tested preclinically. These tracers are based on proteins (e.g. antibodies and antibody fragments), peptides (e.g. G proteincoupled receptor ligands) or small molecules (e.g. radiolabelled protein kinase inhibitors or metabolic substrates). Using these radiotracers, the expression and function of a variety of proteins can be studied in patients, including for example glutamine/glutamate metabolism, neutral amino acid transport, PSMA, GRPr, SSTr, CXCR4, folate and alpha-v beta-3 integrin expression. Some of these radiopharmaceuticals are potential companion diagnostics and may allow selection of patients for treatment with radiopharmaceuticals or targeted drug conjugates. Preclinical and initial clinical data are promising for several new radiotracers, but the key challenge is the clinical validation and dissemination of these compounds. Validation and dissemination have been hampered so far by regulatory hurdles as well as by the lack of standardised trial designs for the validation of imaging agents and the lack of accepted and appropriate end points to prove clinical utility. Addressing these issues will be critical for the future clinical use of PET and other molecular imaging technologies.

Learning Objectives:

1. To learn about the new specific tracers that can be used in oncologic patients.

2. To appreciate their potential impact on patient management.

3. To understand their potential and limitations for practice.

Author Disclosure:

W. Weber: Advisory Board; Endocyte, MSD. Grant Recipient; Bayer, Philips.

\section{A-604 16:30}

\section{B. Potential of MRI for molecular imaging in oncology}

F. Gallagher; Cambridge/UK (ferdia.gallagher@doctors.org.uk)

Imaging targets in cancer range from simple size measurements to more specific biomarkers on functional, cellular, metabolic and molecular levels. As our understanding of basic tumour biology has advanced, techniques have been developed to exploit this information to produce increasingly specific molecular imaging tools. The biodistribution of these molecular imaging probes should be more specific in diagnosing and assessing cancer than the morphological information acquired using anatomical imaging alone. This lecture will discuss current and emerging functional and molecular imaging techniques using MRI and their applications in oncology. Functional measures of tumour blood flow and vascular permeability can be made using dynamic contrast-enhanced MRI. Diffusion-weighted imaging is a surrogate for the cellular content of the tumour and emerging methods can be used to probe features of the extracellular space such as tumour $\mathrm{pH}$ and stromal content. On the molecular level, cell surface expression of specific proteins and enzyme activity within the cell can be imaged; labelled probes have been developed which bind to these proteins and a new MR technique is being developed for assessing tumour glucose in a similar way to PET. Hyperpolarisation methods are emerging to overcome the major limitation of MR: low sensitivity. One such approach is dynamic nuclear polarisation, which can probe carbon metabolism non-invasively in patients with cancer. Functional and molecular imaging techniques with MRI will increasingly be used in radiology in conjunction with anatomical imaging methods to improve diagnosis and prognosis, target biopsies, as well as predict and detect response to treatment.

Learning Objectives:

1. To become familiar with the different approaches to molecular imaging with MRI.

2. To understand the role of molecular imaging in oncology.

3. To learn about emerging MRI techniques for molecular imaging Author Disclosure:

F. Gallagher: Research/Grant Support; GE Healthcare. 
A-605 17:00

\section{Emerging molecular imaging techniques}

F.M.A. Kiessling; Aachen/DE (fkiessling@ukaachen.de)

With MR-spectroscopy, diffusion-weighted imaging, PET- and SPECT-CT, as well as PET-MRI, molecular imaging has already become part of clinical routine diagnosis. Also, some contrast agents such as SPIO for MPS-labelling and scavenger-receptor binding gadolinium chelates are clinically applied for liver and lymph node imaging. However, there are still a number of highly promising novel tools that are expected to emerge clinically in the near future. In this context, the first part of the talk will give an overview on how optical imaging basically works and which future clinical applications can be expected. In this context, RAMAN spectroscopy, fluorescence optical reflectance imaging, fluorescence mediated tomography, photoacoustic imaging and Cerenkov luminescence imaging will be addressed. The second part of the talk introduces molecular ultrasound imaging as a safe and preclinically wellevaluated method. Besides the diagnostic use of molecular ultrasound contrast agents, which have been already evaluated in clinical trials, the potential of this method also for theranostic purposes will be highlighted.

Learning Objectives:

1. To become familiar with optical imaging techniques and probes.

2. To learn about the potential of targeted US contrast agents.

3. To learn about emerging hybrid imaging techniques.

16:00 - 17:30

Board Room A

Cardiac

RC 1903

\section{Practical approach to cardiovascular risk stratification with $\mathrm{CT}$ and MRI \\ Moderator: \\ G.I. Kirova-Nedialkova; Sofia/BG}

\section{A-606 16:00}

A. Modern views on value of coronary calcium scoring for risk assessment

A. Stadler; Vienna/AT (alfred.stadler@meduniwien.ac.at)

The assessment of the amount of arterial calcification with computed tomography is a standard method in the risk stratification of coronary heart disease. Coronary calcium detection by CT has been shown to identify atherosclerotic plaque and to quantitatively assess coronary calcium. Many studies have demonstrated the association between the degree of coronary calcium, the burden of atherosclerosis, and the risk for cardiovascular events associated with coronary calcium. The CAC scoring can provide individual risk assessment and reclassify the low and particularly intermediate Framingham risk cohort into lower- and higher-risk strata. The absence of CAC is associated with a very low risk of future cardiovascular events, with modest incremental value of other diagnostic tests in this very low-risk group. The role of CAC in the workup of symptomatic patients is under discussion and there is evidence that the absence of CAC has a high negative predictive value for ruling out acute coronary syndrome. Although CAC measurement is highly standardised, some technical aspects have to be considered and all possibilities of dose reductions utilised. This presentation will show the technical and epidemiological fundamentals of CAC scoring and discuss the clinical applications of the method.

Learning Objectives:

1. To understand what kind of information calcium scoring can deliver and the appropriate indications.

2. To become familiar with its role in an asymptomatic and in a symptomatic population.

3. To learn about the current data and guidelines supporting its use in the assessment of cardiac risk.

\section{A-607 16:30}

\section{B. Prognostic value of coronary CTA}

R. Vliegenthart; Groningen/NL (r.vliegenthart@umcg.nl)

Coronary CTA has gained an important role in the evaluation of patients with chest pain, suspected of coronary artery disease (CAD). In comparison to invasive coronary angiography, coronary CTA with $\geq 64$-multidetector CT has an excellent sensitivity for coronary stenosis. Specificity for stenosis is less excellent, but has improved with newer CT generations. The strongest impact of coronary CTA has been on the exclusion of stenosis in symptomatic patients at low-intermediate pre-test probability. In contrast to invasive angiography, coronary CTA yields more information than just the severity of stenosis. Coronary CTA also allows determination of plaque types (calcified, non- calcified, partly calcified) and quantification of the atherosclerotic plaque burden. Also, new developments point to the possibility to assess the haemodynamic significance of stenosis based on CT density measurements in the coronary arteries, or evaluation of myocardial perfusion. Thus, coronary CTA yields quantitative imaging biomarkers that could be used for risk stratification in the future. Recent studies have shown that whether or not a patient has stenosis and plaque, as well as the type and burden of plaque, affects the risk of myocardial infarction and mortality. Therefore, the impact of coronary CTA imaging biomarkers reaches beyond the mere assessment of stenosis for symptomatic treatment and could potentially alter medical management of patients for improvement of long-term prognosis. Learning Objectives:

1. To understand the accuracy of CTA in the diagnosis of coronary disease.

2. To become familiar with the strengths and limitations of the various quantification techniques.

3. To appreciate the prognostic value of CTA in coronary disease. Author Disclosure:

R. Vliegenthart: Grant Recipient; Supported by a grant from the Netherlands Organisation for Scientific Research.

A-608 17:00

C. Added value of carotid and peripheral artery imaging for risk assessment

L. Natale; Rome/IT (Inatale@rm.unicatt.it)

Technical protocols optimisation of MRA and CTA of carotid and peripheral arteries is crucial for image quality and to complete the analysis of both lumen and vessel wall. Furthermore, MRA protocol needs to be integrated with dedicated pulse sequences in order to obtain vessel wall imaging that is already included in CTA datasets. New CT and MR scanners allow this optimisation in terms of radiation exposure, contrast media dose reduction and spatial resolution. All these parameters will be discussed for both techniques. According to international guidelines, non-invasive imaging indications will be discussed, with particular attention to high risk population (e.g.: diabetic patients). Some possible imaging biomarkers of plaque composition will be discussed.

Learning Objectives:

1. To become familiar with optimal technical considerations for performing CTA and MRA of the carotid and peripheral arteries.

2. To understand clinical indications for carotid and peripheral CTA and MRA.

3. To discuss the role of CTA and MRA parameters including plaque imaging as biomarkers of cardiovascular disease.

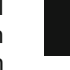

